\title{
X-RAY ABSORPTION SPECTROSCOPIC STUDIES OF THE DINUCLEAR IRON CENTER IN METHANE MONOOXYGENASE AND THE SULFURE AND CHLORINE CENTERS IN PHOTOGRAPHIC MATERIALS*
}

\author{
Jane G. DeWitt \\ Stanford Linear Accelerator Center \\ Stanford Synchrotron Radiation Laboratory \\ Stanford University. Stanford, California 94309
}

December 1992

Prepared for the Department of Energy under contract number DE-AC03-76SFO0515 and the NIH, Biomedial Resource Technology Program, Division of Research Resources

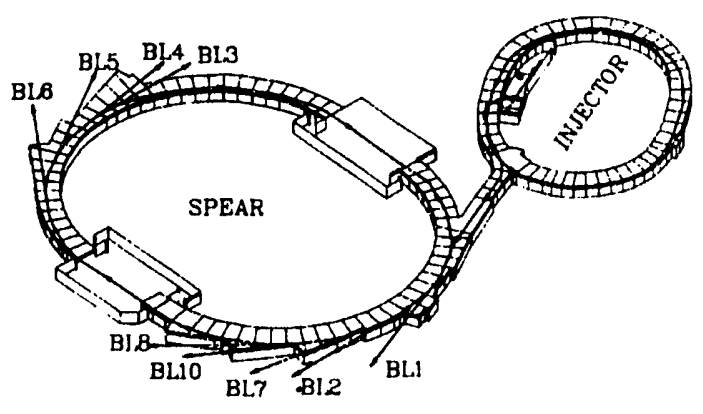

Printed in the United States of America. Avallable from the National Technical Information Service, U.S. Department of Commerce, 5285 Port Royal Road, Springfield, Virginia 22161

*Ph.D. thes'

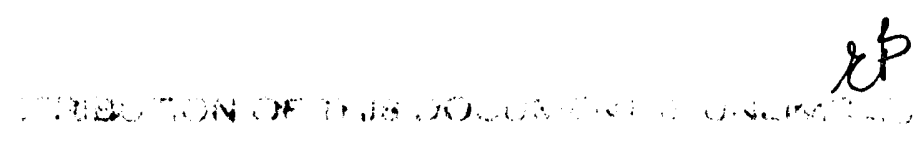




\section{Abstract}

The Diiron Center in Methane Monooxygenase. The dinuclear iron center of the hydroxylase component of soluble methane monooxygenase (MMO) from Methylococcus capsulatus (Bath) and Methylosinus trichosporium (OB3b) has been studied by X-ray absorption spectroscopy. Analysis of the Fe K-edge EXAFS revealed that the first shell coordination of the $\mathrm{Fe}(\mathrm{III}) \mathrm{Fe}$ (III) oxidized state of the hydroxylase from $M$. capsulatus (Bath) consists of approximately $6 \mathrm{~N}$ and $\mathrm{O}$ atoms at an average distance of 2.04 $\AA$. The Fe-Fe distance was determined to be $3.4 \AA$. No evidence for the presence of a short oxo bridge in the iron center of the oxidized hydroxylase was found, suggesting that the active site of MMO is significantly different from the active sites of the dinuclear iron proteins hemerythrin and ribonucleotide reductase. In addition, the results of the first shell fits suggest that there are more oxygen than nitrogen donor ligands.

The active sites of the photoreduced $\mathrm{Fe}$ (III)Fe(II) semimet form of the hydroxylase from both $M$. capsulatus (Bath) and $M$. trichosporium (OB3b) consist of approximately 6 $\mathrm{N}$ and $\mathrm{O}$ atoms at an average distance of $2.06-2.09 \AA$ with an $\mathrm{Fe}$ interaction at $3.41-3.43$ $\AA$. This implies that the diiron center of the hydroxylase from the two species are structurally similar. In addition, the results of the second shell fits to the hydroxylase suggest that there is a shell of low- $Z$ atoms at $\sim 3.0 \AA$ in both the diferric and semimet active sites. Upon reduction to the $\mathrm{Fe}(\mathrm{II}) \mathrm{Fe}$ (II) reduced form, the average first shell distance increased to $2.15 \AA$ and the Fe-Fe interaction was no longer detected.

The Fe K-edge EXAFS showed only minor metrical changes in the coordination environment of the hydroxylase iron center due to the presence of substrate and component $\mathrm{B}$, the regulatory protein of the MMO enzyme system. This finding was true for the complexes of semimet and reduced hydroxylase. The changes seen occured in the first coordination sphere. In particular, the presence of component B seemed to have an effect on the distance distribution of first shell atoms. No evidence of a $\mathrm{Br}$ contribution was seen in the EXAFS of the hydroxylase in the presence of a brominated substrate. This suggests that the site of interaction between the hydroxylase and substrate is more than $4 \AA$ from the iron center. The presence of substrate and component B was found to modify the Fe Kedge spectra of the hydroxylase. The change seen in the spectra of the semimet samples is consistent with an increase in the covalency of the iron center. The appearance of the edges of the reduced forms of the hydroxylase suggest that the presence of substrate or component $B$ inhibits the reduction of the diferric hydroxylase to a diferrous state. These studies suggest that the changes seen in the hydroxylase diiron site in the presence of 
substrate or component B result from subtle perturbations in the coordination environment of the iron atoms accompanied by changes in the electronic structure of the iron center.

Diiron Model Complexes. The analysis of the second shell data of the hydroxylase suggested that the Fe-Fe distance determined may depend on the model compound used in the fits. A detailed investigation of the apparent model bias was therefore performed using nine structurally characterized di- and tri-bridged iron dimers representing a variety of bridging modes and $\mathrm{Fe}-\mathrm{Fe}$ distances. In general, two minima were found in fits to the second shell data: one at the correct Fe-Fe distance and one $\sim 0.4$ $\AA$ away. The best fit to the second shell data, however, corresponded in every case to the Fe-Fe distance most similar to that of the model compound used to extract the Fe amplitude and phase parameters employed in the fit. This bias reflected only the $\mathrm{Fe}-\mathrm{Fe}$ distance of the model compound from which the parameters were obtained, and was independent of the number or nature of bridges in the diiron center except as those factors determine the Fe-Fe distance. A strong correlation between the second shell $\mathrm{Fe}$ and $\mathrm{C}$ parameters was also observed, impacting both the coordination numbers and the distances of the $\mathrm{Fe}$ and $\mathrm{C}$ contributions. These results illustrate that caution must be used in the interpretation of the results of fits to second shell data from dinuclear iron centers. Similar caution should be used in the analysis of other dinuclear metalloprotein systems as well. Finally, other information must be used in conjunction with EXAFS analysis before any final conclusions can be reached regarding the second shell coordination.

The appearance of Fe K-edge XANES spectra for di- and tri-bridged iron dimers was related to the ligation of the iron center and to the nature of the bridging groups. The position of the edge moved to lower energy with increasing average first shell distance. The spectra of some oxo-bridged models had a shoulder on the rising edge that was not present in the spectra of the non-oxo-bridged models. The appearance of this shoulder in the oxo-bridged dimer spectra was correlated to an increase in the $\mathrm{N}$ ligation relative to the $O$ ligation. A reasonable interpretation of this feature is that it is a shake down feature associated with the $1 \mathrm{~s} \rightarrow 4 \mathrm{p}$ transition, and reflects an increase in the covalency of the iron site due to the change in the ligation. The appearance of the pre-edge feature $(1 \mathrm{~s} \rightarrow-3 \mathrm{~d}$ transition) was characteristic of the nature of the bridge in the diferric models, but not of the number of bridges in the diiron site. In oxo-bridged compounds, the pre-edge feature was asymmetric, while in the non-oxo-bridged compounds, the pre-edge feature was clearly resolved into a doublet. The splitting or asymmetry of this feature is consistent with an assignment of the transition to the ${ }^{5} \mathrm{~T}_{2 g}$ and ${ }^{5} \mathrm{E}_{\mathrm{g}}$ molecular states for the excited state $\mathrm{d}^{6}$ configuration. Greater overall intensity of the pre-edge feature was found for the oxobridged models, reflecting the increased distortion of the iron site due to the presence of the 
short $\mathrm{Fe}-\mathrm{O}_{\text {oxo }}$ bridge. The appearance of the edge and pre-edge regions of the edge spectra for the oxidized hy droxylase of MMO is consistent with the absence of a $\mu$-oxo bridge in the diiron site and with 6-coordinate iron atoms.

Photographic Materials. The sulfur and chlorine centers in spectral sensitizing dyes and chemical sensitizing centers of importance to the photographic system were characterized by $\mathrm{S}$ and $\mathrm{Cl} \mathrm{K}$-edge $\mathrm{X}$-ray absorption spectroscopy. This technique was found to be sensitive to the different environments of $S$ in the compounds investigated, as well as to the nature and extent of the interaction between $\mathrm{S}$ and $\mathrm{Au}$ or $\mathrm{Ag}$ metals atoms. In particular, a sharp, intense pre-edge feature seen in the spectra of compounds containing exocyclic S (thione or thiol), is not seen in the spectra of thiazole-containing (cyclic S) compounds. The use of oriented single-crystal studies permitted the assignment of the exocyclic $\mathrm{S}$ pre-edge feature as a transition to a $\mathrm{S} p \pi^{*}$ orbital. The main feature in all $\mathrm{S} \mathrm{K}$ edge spectra was assigned as a transition to a $\mathrm{S} \mathrm{p} \sigma^{*}$ orbital. $\mathrm{Cl}$ is present as a substituent on the ring system of the dye molecules. The $\mathrm{Cl} \mathrm{K}$-edge spectra changed little, reflecting the similar environments the $\mathrm{Cl}$ atom occupies in the compounds studied. Dramatic changes occured in the S K-edge spectra of covalent $\mathrm{Ag}$ and $\mathrm{Au}$ metal complexes, while ionic interactions with metal atoms resulted in little change in the $S$ spectra. Polarized surface measurements at a glancing angle configuration were done on $\mathrm{S}$ species adsorbed on $\mathrm{AgBr}$ sheet crystals. These studies clearly show that a bond is not formed between the metal and $\mathrm{S}$ atoms. The interaction between the dye molecules and the $\mathrm{AgBr}$ substrate is therefore of a physical rather than a chemical nature. In addition, the sensitivity of XAS to the polarization properties of the $\mathrm{S}$ and $\mathrm{Cl} \mathrm{K}$-edge features provided insight into the orientation of the dye molecules on the $\mathrm{AgBr}$ substrate. 


\section{Acknowledgements}

My graduate school experience was marked by good fortune. I joined the Hodgson group because I thought it would be an interesting and unique opportunity to do synchrotron radiation research, and I was absolutely right. There was nothing quite like the sense of accomplishment I felt when I really knew how to run an XAS experiment. The MMO project opened the door to the fields of environemental science and bioremediation, areas of research in which I plan to continue working. Because of our close affiliation with SSRL, our group had the valuable opportunity to learn more about and be more deeply involved with many different aspects of synchrotron radiation research, as well as to interact with other groups doing research at the facility. Some of us were lucky enough to go through the 1989 NSLS experience. The bright side of NSLS was that after mystery meat pizza on X19A, beamtime could only improve in the future.

I am grateful to a host of people who gave me the support, encouragement and friendship I needed to stay and succeed. First and foremost, I would like to thank my adviser, Keith Hodgson, for the trust he exhibited in my ability to achieve this goal and succeed in this work. His confidence in me bolstered my confidence in myself, an invaluable gift. Keith expects his students to work hard and to be independent, and gives us the freedom to develop as individuals, both scientifically and personally. He provided a working environment in which I could work at my own pace, with enough pressure to insure progress but enough guidance to avoid feeling overwhelmed. The work I did was challenging and rewarding, and I am grateful that I had the good fortune to work with Keith. I am also grateful for the many opportunities Keith gave me to present my work at conferences and to interact with other researchers in the field. I wish him continued years of success, and most importantly, of health.

We are very fortunate in the Hodgson group to be able to work closely with two remarkable people, Britt Hedman and Pat Frank. Britt is a wonderfui mentor. She always seems to have the answer, but when asked a question has the unique ability to teach you the answer instead of just telling it to you. Pat always has another way to think about a problem or a result. He reminded me that the more I think I know about something, the more important it is that I question my knowledge and my method of thinking, so that I do not overlook something important. Keith, Britt and Pat represent integrity and dedication in science, and I am so grateful to have learned about the scientific process from them.

I also want to acknowledge and thank the people in the Hodgson group: David Eliezer, Grace Tan, Chrisie Stanfel, Lingling Chen, Kent Nakagawa, Tami Westre, Isaac Liu and Susan Shadle. They are terrific people and I am glad that I was fortunate enough 
to work with them and get to know them. I wish them all successful and speedy experiments in the future. I also want to acknowledge the contributions of the collaborators on the MMO project: Prof. Stephen J. Lippard, Dr. James G. Bentsen, and Amy C. Rosenzweig. I thank them for the reliable delivery of MMO samples. I hope the crystal structure is coming along well. In addition, I want to thank the staff at SSRL, especially Robert Mayer. They are a talented, dedicated group of people and make beamtime a much more successful and enjoyable experience.

Terre Smith was the collaborator on the photo project and continues to be a good friend. She and I always managed to find the worst restaurant, and always managed to pick the worst thing on the menu. I look forward to finding bad restaurants in New Mexico with her. Claire Conway never stopped trying to get me to come up to the city. I've enjoyed getting to know her better and am thankful for her sympathetic ear. I hope that we can find each other again when she gets back from her trip around the world. Brooke Hemming, Tami Westre and Susan Shadle have saved my sanity and given me more support and shored up my self-esteem more than they know. I thank them for the fun, interesting and spirited conversations and for the sense of community and growth that their friendship provides me. I am especially grateful for Susan's friendship and honest perspective on the world. Carl Brown contributed ABBA, Led Zeppelin, disco and lima beans to my stay at Stanford. I have appreciated his friendship and sense of humor. I thank Erin Grant, who has always been and continues to be a source of honesty and friendship, for rescuing me more than once during these past few years. Larry Meixner is very special to me, and I thank him for his unfailing belief in me, for his support and encouragement and his friendship all of these years.

Finally, I want to thank my family for all that they have given me. It has been very important to me to have been within two hours of my family during these years. I thank my sister and brother-in-law, Karen DeWitt-Mattei and Scott Mattei; my step-sister and uncle, Tracy and Tom Barber, and my nephews and niece, Nick Mueller and Donny and Jessica Barber; for all of the support and fun and distractions and for being there for me. For their unconditional support and faith, I thank my mom and step-dad, Marsha and Fred Mueller. I thank my dad, Grigg DeWitt, for his constant curiosity and encouragement. I did this for myself, but I could not have done this without them. They gave me the courage to start on this path, and so this work is dedicated to my family. 


\section{Contents}

Abstract $\quad$ ii

Acknowledgements $\quad \mathrm{V}$

Contents vii

List of Tables $\quad$ xi

List of Figures xiii

List of Abbreviations $\quad$ xvii

Chapter 1 Introduction to the Methane Monooxygenase Enzyme System and the EXAFS Analysis Method 1

1.1. Scope and Organization of this Dissertation 2

1.2. Methanotrophic Bacteria 3

1.3. The Soluble Methane Monooxygenase Enzyme System 5

1.4. Spectroscopic Studies of Dinuclear Non-Heme Iron Centers 7

1.5. X-ray Absorption Spectroscopy 9

1.5.1. Extended X-ray Absorption Fine Structure 11

1.5.2. Data Reduction and Analysis 15

1.6. References and Notes 21

Chapter 2 X-ray Absorption Spectroscopic Studies of the Dinuclear Non-Heme Iron Center in the Hydroxylase Component of Methane Monooxygenase and the B2 Subunit of Ribonucleotide Reductase

2.1. Introduction 26

2.2. Experimental 28

2.2.1. EXAFS Sample Preparation 28

2.2.2. Data Collection, Reduction and Analysis 28

2.3. Results of XAS Experiments 31

2.3.1. Photoreduction of the Oxidized Hydroxylase 35

2.3.2. Results of Fits to the Hydroxylase EXAFS Data 35

2.3.2.1. First Shell Fits 35

2.3.2.2. Second Shell Fits 39

2.3.2.3. Wide Shell Fits 45 
2.3.2.4. Model Dependence of EXAFS Results 45

2.3.2.5. Fits to the Unfiltered Data 50

2.3.2.6. Fits Over a Shorter Data Range 50

2.3.3. Results of Fits to the Ribonucleotide Reductase EXAFS Data 52

2.4. Discussion 52

2.4.1. Nature of the Hydroxylase Active Site 52

2.4.2. Comparisons to Proteins Containing Diiron Cores 56

2.5. Conclusions $\quad 58$

2.6. Acknowledgements $\quad 59$

2.7. References and Notes 60

Chapter 3 Structural Studies of the Diiron Center in Methane Monooxygenase in the Presence of Substrate and the Regulatory Protein of the MMO Enzyme System 64

3.1. Introduction 65

3.2. Experimental 67

3.2.1. EXAFS Sample Preparations 67

3.2.2. EXAFS Data Collection, Reduction and Analysis 68

3.3. Results of XAS Experiments $\quad 70$

3.3.1. Photoreduction of the Oxidized Hydroxylase Samples $\quad 76$

3.3.2. Results of Fits 76

3.3.2.1. First Shell Fits 76

3.3.2.2. Second Shell Fits 83

3.3.2.3. Wide Shell Fits 89

3.3.2.4. The Origin of the Metrical Differences in the EXAFS 93

3.3.2.5. Fits to the Non-Filtered Data 93

3.3.3. X-ray Absorption Near Edge Spectra 102

3.4. Discussion 107

3.4.1. The Empirical Fitting Proceedure 107

3.4.2. The Effect of Substrate and Component B on the Structure of the Diiron Center 109

3.4.2.1. Changes in the Coordination Sphere of the Iron Center 


\subsubsection{Interpretation of the Hydroxylase}

Edge Spectra 115

$\begin{array}{ll}\text { 3.5. Conclusions } & 117\end{array}$

3.6. Acknowledgements 117

3.7. References and Notes 118

Chapter 4 An Investigation of the Model Dependency of EXAFS Data Analysis for Dinuclear Non-Heme Iron Systems 122

4.1. Introduction 123

4.2. Experimental 124

4.3. Results of Fits to the Model Data 130

4.3.1. A Description of the Trends Seen in First Shell Fits 131

4.3.2. The Dependence of Second Shell Fits on Choice of Model Compound 144

4.3.2.1. Investigation with Model Data. 144

4.3.2.2. Correlations with Second Shell Low-Z Atoms 152

4.3.2.3 Investigations with Hydroxylase Data $\quad 157$

4.4. Discussion 158

4.4.1. Interpretation of First Shell Fits 158

4.4.2. Model-Dependent Results of Second Shell Fits 162

4.4.2.1. Model Compounds 162

4.4.2.2. The Hydroxylase Active Site 167

4.4.2.3. Survey of Other Second Shell Analysis Methods 168

$\begin{array}{ll}\text { 4.5. Conclusions } & 169\end{array}$

4.6. Acknowledgements 170

4.7. References and Notes 172

Chapter 5 Fe K-Edge X-ray Absorption Edge Spectra of Iron Models Relevant to Dinuclear Non-Heme Iron Enzyme Systems 175

5.1. Introduction 176

5.1.1. Interpretation of Transition Metal XANES Spectra 176

5.2. Experimental 177 
5.3. Results and Discussion 179

5.3.1. Description of the Edge Spectra. 180

5.3.1.1. Models Containing a $\mu$-oxo Bridge $\quad 180$

5.3.1.2. Non-oxo Bridged Models 185

5.3.1.3. Changes in Spectra with Oxidation State $\quad 187$

5.3.2. Interpretation of Edge Spectra 190

5.3.2.1. Changes in the Edge Spectra with Bridging Geometry 190

5.3.2.2. Changes in the Edge Spectra with Changes in Ligation 194

5.3.2.3. Pre-edge Feature 194

5.3.2.4. Comparisons with Hydroxylase

Edge Spectra 196

5.4. Conclusions 198

5.5. Acknowledgements 199

5.6. References and Notes 200

Chapter 6 Sulfur and Chlorine K-Edge X-ray Absorption

Spectroscopic Studies of Photographic Materials 202

6.1. Introduction 203

6.2. Experimental 205

6.2.1. Single-Crystal Polarized Studies 221

6.2.2. Polarized Surface Measurements 222

6.3. Results 224

6.3.1. S K-Edge Spectra of Dye Molecules 224

6.3.1.1. Cyanine Dye Molecules 224

6.3.1.2. Merocyanine Dye Molecules 227

6.3.2. Cl K-Edge Spectra of Dye Molecules 234

6.3.3. Au and $\mathrm{Ag}$ Complexes with Sulfur-Containing Ligands 237

6.3.4. Surface Measurements 243

6.4. Discussion 250

6.5. Conclusions 255

6.6. Acknowledgements 255

6.7. References and Notes 256 


\section{List of Tables}

\section{Chapter 2}

Table 2.1. Sample and Data Collection Summary 29

Table 2.2. Results of First Shell Fits to the Hydroxylase Data 37

Table 2.3. Results of Second Shell Fits to the Hydroxylase Data 41

Table 2.4. Iron Fits to Second Shell Data for Oxidized and Semimet Hydroxylase Samples 43

Table 2.5. Results of Wide Shell Fits to the Hydroxylase Data 46

Table 2.6. Results of Fits to the Non-Filtered Hydroxylase EXAFS 51

\section{Chapter 3}

Table 3.1. Sample and Data Collection Summary 69

Table 3.2. Results of First Shell Fits to the Hydroxylase Data 77

Table 3.3. Results of Second Shell Fits to the Hydroxylase Data 84

Table 3.4. Results of Wide Shell Fits to the Hydroxylase Data 90

Table 3.5. Results of Fits to the Non-Filtered Hydroxylase EXAFS 97

Table 3.6. Comparison of the Results of Fits to the Hydroxylase Data 111

\section{Chapter 4}

Table 4.1. Summary of Information for Dinuclear and Mononuclear Model

$\begin{array}{ll}\text { Compounds } & 125\end{array}$

Table 4.2. Results of First Shell Fits to the Model Data 138

Table 4.3. A Comparison of the Average First Shell Distances by EXAFS and Crystallography

Table 4.4. Fits to Second Shell Model Data 146

Table 4.5. Fits to Second Shell Model Data with Fe and C 155

Table 4.6. Fits to Second Shell Hydroxylase Data 159

Table 4.7. Average First Shell Distances as a Function of First Shell Ligation 163

\section{Chapter 5}

Table 5.1. Summary of Samples 
Table 5.2. Energies of Features in XANES Spectra of Diiron Models

Table 5.3. Comparison of Average First Shell Distance with Edge Position

\section{Chapter 6}

Table 6.1. Energies of Transitions in S K-Edge Spectra of Cyanine Dyes and Dye Intermediates

Table 6.2. Energies of Transitions in S K-Edge Spectra of Compounds Containing Exocyclic $S$

Table 6.3. Energies of Transitions in Cl K-Edge Spectra of Dyes and Dye Intermediates

Table 6.4 Energies of Transitions in Silver- ard Gold-Sulfur Complexes 


\section{List of Figures}

\section{Chapter 1}

Figure 1.1. An Fe K-Edge X-ray Absorption Spectrum

\section{Chapter 2}

Figure 2.1. EXAFS Data of the Hydroyxlase of MMO 32

Figure 2.2. Fourier Transforms of the Hydroyxlase EXAFS Data 33

Figure 2.3. A Co:nparison of EXAFS Data of Hydroxylase and Model Samples 34

Figure 2.4. The Edge Positions of Diferric, Semimet and Diferrous Hydroxylase Samples 36

Figure 2.5. Fits to Oxidized Hydroxylase Second Shell Data with Iron 44

Figure 2.6. Fits to the Fourier Filtered Oxidized Hydroxylase Data 48

Figure 2.7. Fits to the Fourier Filtered Semimet and Reduced Hydroxylase Data 49

Figure 2.8. A Comparison of Fourier Filtered Second Shell Fe and C EXAFS 54

\section{Chapter 3}

Figure 3.1. EXAFS of Semimet Hydroxylase Samples 71

Figure 3.2. Fourier Transforms of the Semimet Hydroxylase EXAFS 72

Figure 3.3. EXAFS of Reduced Hydroxylase Samples 74

Figure 3.4. Fourier Transforms of the Reduced Hydroxylase EXAFS 75

Figure 3.5. Fourier Transforms of Second Shell Fits to Semimet Hydroxylase Data $\quad 88$

Figure 3.6. Fits to the Non-Filtered EXAFS data for Semimet Hydroxylase Sample $\quad 92$

Figure 3.7. Comparison of First Shell EXAFS Data for Semimet Hydroxylase Samples 94

Figure 3.8. Comparison of Second Shell EXAFS Data for Semimet Hydroxylase Samples $\quad 95$

Figure 3.9. Comparison of Interaction Between First and Second Shell EXAFS Data 96

Figure 3.10. Fits to Non-Filtered Semimet Hydroxylase EXAFS Data 100

Figure 3.11. Fourier Transforms of Fits to Non-Filtered Semimet Hydroxylase 
$\begin{array}{ll}\text { EXAFS Daia } & 101\end{array}$

Figure 3.12. Fits to Non-Fïtered Reduced Hydroxylase EXAFS Data 103

Figure 3.13. Fourier Trans yorms of Fits to Non-Filtered Reduced Hydroxylase $\begin{array}{ll}\text { EXAFS Data } & 104\end{array}$

Figure 3.14. Edge Spectra of Semimet Hydroxylase Samples 105

Figure 3.15. Comparison of Semimet and Reduced Hydroxylase Edge Spectra 106

Figure 3.16. Edge Spectra of Reduced Hydroxylase Samples 108

Figure 3.17. Comparison of Fourier Transforms of Fe Model and Brominated Derivative

\section{Chapter 4}

Figure 4.1. EXAFS of Tribridged Model Compounds 132

Figure 4.2. EXAFS of Dibridged Model Compounds 133

Figure 4.3. EXAFS of Monomeric Model Compounds 134

Figure 4.4. Fourier Transforms of Tribridged Model EXAFS 135

Figure 4.5. Fourier Transforms of Dibridged Model EXAFS 136

Figure 4.6. Fourier Transforms of Monomeric Model EXAFS 137

Figure 4.7. Fits to the First Shell EXAFS Data of FEHBPZOH 142

Figure 4.8. Comparison of Model-Dependent Fits to Second Shell $\begin{array}{ll}\text { EXAFS Data } & 150\end{array}$

Figure 4.9. Comparison of Second Shell EXAFS Data 151

Figure 4.10. Fits to the Second Shell EXAFS Data of FE2CO2 153

Figure 4.11. Fits to the Second Shell EXAFS Data of Oxidized Hydroxylase Sample 160

Figure 4.12. Comparison of Average First Shell Distance with O and N Ligation 164

\section{Chapter 5}

Figure 5.1. Fe K-Edge XANES Spectra of Oxo-Bridged Model Compounds 183

$\begin{array}{lll}\text { Figure 5.2. } & \text { Pre-Edge Features of Iron Model Compounds } & 184\end{array}$

Figure 5.3. Fe K-Edge XANES Spectra of Non-Oxo-Bridged Model $\begin{array}{ll}\text { Compounds } & 186\end{array}$

Figure 5.4. Changes in Edge Spectra with Change in Iron Oxidation State 188 Figure 5.5. Changes in Pre-Edge Feature with Change in Iron Oxidation State 189

Figure 5.6. Comparison of Average First Shell Distance with Position of Edge 192 
Figure 5.7. Comparison of Oxidized Hydroxylase Edge Spectrum with Model Spectra

Figure 5.8. Comparison of Pre-Edge Feature of Hydroxylase Samples with Model Compounds

Chapter

Figure 6.1. Schematic of Surface Measurement Geometry

Figure 6.2. S K-Edge Spectra of Cyanine Dyes and. Dye Intermediates

Figure 6.3. Single-Crystal Polarized S K-Edge Spectra of a Cyanine Dye Molecule

Figure 6.4. S K-Edge Spetra of Merocyanine Dyes and Dye Intermediates

Figure 6.5. Single-Crystal Polarized S K-Edge Spectra of a Merocyanine Dye Molecule

Figure 6.6. Comparison of Edge Spectra of Cyanine and Merocyanine Dye Molecules

Figure 6.7. S K-Edge Spectra of Thioureas, Mercaptotetrazoles and Mercaptotriazoles

Figure 6.8. Comparison of Edge Spectra of Molecules Containing a Thione, Thiol, and Thiolate Exocyclic S

Figure 6.9. Cl K-Edge Spectra of Dyes and Dye Intermediates 235

Figure 6.10. Single-Crystal Polarized Cl K-Edge Spectra 9,10-Dichloroanthracene 236

Figure 6.11. S K-Edge Spectra of Silver-Sulfur Complexes

Figure 6.12. S K-Edge Spectra of Gold-Sulfur Complexes

Figure 6.13. S K-Edge Spectra of Silver and Gold Sulfides

Figure 6.14. Single-Crystal Polarized S K-Edge Spectra of a Silver-Sulfur Compound

Figure 6.15. The Effect of Ionic and Covalent Metal Interactions on S K-Edge Spectra

Figure 6.16. Surface S K-Edge Spectrum of AgBr Sheet Crystal Treated with a Cyanine Dye

Figure 6.17. Surface S K-Edge Spectra of AgBr Sheet Crystal Treated with a Merocyanine Dye

Figure 6.18. Surface Cl K-Edge Spectra of AgBr Sheet Crystal Treated with a Cyanine Dye

Figure 6.19. Surface $\mathrm{Cl}$ K-Edge Spectra of $\mathrm{AgBr}$ Sheet Crystal Treated with a 


\section{Merocyanine Dye}

Figure 6.20. Surface S K-Edge Spectra of Chemically Sensitized AgBr Sheet Crystals

Figure 6.21. Changes in the Appearance of the S K-Edge Spectra with Changing Electronegativity of Substituents 


\section{List of Abbreviations}

acac

BIPhMe

Chel

Dipic

EXAFS

$\mathrm{HB}(\mathrm{pz})_{3}$

Hr

MMO

PAP

RR

RRB2

salmp

TPA

Uf

XANES

XAS acetylacetonate (2,4-pentanedionate)

2,2'-bis(1-methylimidazolyl)phenylmethoxymethane

4-hydroxo-2,6-pyridinedicarboxylate

2,6-pyridinedicarboxylate

extended X-ray absorption fine structure

[tri-1-pyrazolylborate] $]^{1-}$

hemerythrin

methane monooxygenase

purple acid phosphatase

ribonucleotide reductase

ribonucleotide reductase, subunit B2

[2-bis(salicylideneamino)methylphenolate $]^{3-}$

is, 2-pyridylmethyl)amine

uteroferrin

$\mathrm{X}$-ray near edge structure

$\mathrm{X}$-ray absorption spectroscopy 


\section{Chapter 1}

Introduction to the Methane Monooxygenase Enzyme System and the EXAFS Analysis Method 


\subsection{Scope and Organization of this Dissertation}

This dissertation focuses on the results of experiments conducted over the past 5 years to characterize the dinuclear iron site in the hydroxylase of soluble methane monooxygenase (MMO) from Methylococcus capsulatus (Bath). This work has been the result of an extensive and productive collaboration with the group of Prof. Stephen J. Lippard at the Massachusettes Institute of Technology, although very early on, Prof. Howard Dalton's group at the University of Warwick in England provided hydroxylase samples. The MIT group was responsible for isolation and purification of the hydroxylase component from Methylococcus capsulatus (Bath), and the early part of their effort focused on improving the purification procedure developed by the Warwick group. All of the hydroxylase samples discussed in this thesis were prepared by the MIT group, as were some of the model compounds which were indispensible to the analysis of the protein data. In addition, the MIT group characterized the hydroxylase by spectroscopic techniques other than X-ray absorption spectroscopy (XAS). The results of these early accomplishments have been published ${ }^{1}$ and the non-XAS results will not be discussed in this thesis. More recently, the collaboration has focused on characterizing the interaction between the hydroxylase, the regulatory protein of the MMO system, and substrate.

Only the results as obtained by $\mathrm{X}$-ray absorption spectroscopy will be presented. Each of the experimental chapters will have a brief introduction focusing specifically on the aspect of the project to be discussed. A more general introduction to the methane monooxygenase enzyme system is presented in Chapter 1, along with a discussion of the EXAFS technique and the data reduction and analysis method employed. The EXAFS analysis of the MMO hydroxylase in its three oxidation states is presented in Chaper 2. Chapter 3 discusses the EXAFS analysis of the hydroxylase in the presence of substrate and the regulatory protein of the MMO system. A detailed investigation of the analysis method applied to structurally-characterized dinuclear iron model compounds is presented in Chapter 4. In Chapter 5, the relationship between the structure of the model compounds and the appearance of the edge spectra is examined.

In addition to the biological work described above, a materials project was undertaken as a collaboration with Dr. Teresa A. Smith of Eastman Kodak Company. Xray absorption spectroscopy was used to characterize the sulfur and chlorine centers in photographic materials. The interaction between $S$ in these materials and $\mathrm{Ag}$ and Au metals was also characterized using XAS. The project included single-crystal polarized studies as well as surface measurements using a glancing-angle configuration. The last chapter of this dissertation presents the results of these investigations. 


\subsection{Methanotrophic Bacteria}

Methane is used by methanotrophic bacteria as their primary and sometimes sole source of carbon for growth and energy. ${ }^{2}$ Methanotrophic bacteria are found in close association with populations of methanogenic bacteria, which are anaerobic organisms that produce methane as a waste product of their metabolism. The overall oxidation of methane to carbon dioxide is achieved in a series of enzyme-catalyzed reactions, however the first and most difficult step in this pathway is the insertion of an oxygen atom from dioxygen into a $\mathrm{C}-\mathrm{H}$ bond of methane yielding methanol (eq. 1). This initial oxidation uses NADH as the source of electrons, and is catalyzed by the enzyme system methane monooxygenase (MMO).

$$
\mathrm{CH}_{4}+\mathrm{O}_{2}+\mathrm{NADH}+\mathrm{H}^{+} \stackrel{\mathrm{MMO}}{\longrightarrow} \mathrm{CH}_{3} \mathrm{OH}+\mathrm{H}_{2} \mathrm{O}+\mathrm{NAD}^{+}
$$

The next step in the oxidation pathway is the conversion of methanol to formaldehyde via methanol dehydrogenase. Formaldehyde is then either used as a source of carbon for cellular biomass, or as a source of cellular energy by further oxidation to $\mathrm{CO}_{2},{ }^{3}$ which generates the NADH required for methane oxidation.

Methanotrophs are classified according to the pathway used to assimilate carbon from formaldehyde into biomass. ${ }^{3}$ Type I methanotrophs use the ribulose monophosphate pathway (RMP) in which formaldehyde is condensed with ribose 5-phosphate to eventually yield triose phosphate. Type II methanotrophs use the serine pathway, producing serine from the condensation of glycine with the formaldehyde-derived methylenetetrahydrofolate. In addition to the utilization of different carbon assimilation pathways, Type I and Type II methanothrophs exhibit different intracytoplasmic membrane structures. Type I methanotrophs possess uniformly distributed arrays of stacked membranes, whereas Type II methanotrophs possess paired peripheral membranes. The differences in the membrane structure has been assumed to be correlated to differences in the initial metabolism of methane, however the methane monooxygenase enzyme system is responsible for the initiation of methane utilization for both Type I and Type II methanotrophs. ${ }^{3}$

Methane monooxygenase activity is associated with the cellular membranes of methanotrophic bacteria for all methanotrophs, and with the soluble fractions of cell-free extracts for some Type I and Type II methanotrophs. ${ }^{4}$ The availability of copper in the growth media was shown to trigger a switch in the cellular location of monooxygenase activity. 5 As the concentration of copper in the growth media increased from $1 \mu \mathrm{M}$ to 5 
$\mu \mathrm{M}$, a change from soluble to particulate $\mathrm{MMO}$ was detected in cultures of type II methanotroph Methylosinus trichosporium (OB3b) and type I methanotroph Methylococcus capsulatus (Bath). ${ }^{6}$ In addition, oxygen limitation is believed to play a role in the cellular location. It is interesting to note that increasing the concentration of copper (and oxygen) also causes an increase in the amount of intracytoplasmic membrane structure. ${ }^{7}$ It is not entirely clear if the presence of copper induces the particulate form of MMO which in turn causes the increase in membrane content, or if the increase in membrane content induces expression of particulate MMO. The particulate form of MMO does contain a copper protein, and the activity of particulate MMO fractions can be increased by the addition of copper salts. Particulate MMO has not been as well-characterized as the soluble form of MMO, but it is currently the subject of many ongoing investigations. ${ }^{8}$

The soluble and particulate forms of MMO differ in almost every way except for their monooxygenase activity. The particulate MMO system consists of a CO-binding cytochrome $c$, a copper-containing protein and a small protein which has not been well characterized, ${ }^{9}$ while the soluble MMO system consists of an $\mathrm{Fe}_{2} \mathrm{~S}_{2}$-flavoprotein, a dinuclear non-heme iron protein, and a small regulatory protein. ${ }^{10}$ Soluble MMO requires $\mathrm{NADH}$ (or NADPH) as a source of electrons for methane oxidation, ${ }^{11}$ but electrons from ascorbate or $\mathrm{NADH}$, or from the methanol/formaldehyde dehydrogenase pathways can be utilized by the particulate enzyme system. 9 Particulate MMO activity is sensitive to inhibiting agents such as metal chelators, electron transport inhibitors and other molecules, ${ }^{9}$ whereas only 8-hydroxyquinoline and acetylene inhibit the soluble system. ${ }^{12}$

Particulate and soluble MMO from Type I and Type II organisms are non-specific enzyme systems, and catalyze the oxidation of a wide variety of compounds in addition to methane. Some of these compounds are used as altemate growth substrates, and some are cometabolized during oxidation of the primary growth substrate. The soluble MMO system is capable of catalyzing the oxidation of alkanes, alkenes, alcohols, aromatic and alicyclic hydrocarbons, amines and chlorinated hydrocarbons. ${ }^{13}$ This lack of specificity is similar to that found in cytochrome P-45014 and is a trait which enables the methanotrophic bacteria to adapt to changing environmental conditions. The particulate MMO system is not capable of oxidizing as wide a variety of substrates as the soluble system, and is limited to alkenes with 5 carbons or less (the limitation for soluble MMO is $\mathbf{8}$ carbons) and cannot oxidize alicyclic or aromatic compounds. $5 c$ In addition, the products of oxidation are somewhat different, with the n-alkanes being oxidized to primary and secondary alcohols by the soluble system, while only secondary alcohols result from particulate MMO oxidation. 
The mechanism of oxidation for the soluble MMO system has been studied by identifying the products of oxidation for a variety of substrates. Loss of stereochemistry for the oxidation of cis-1,4- and cis-1,3-dimethycyclohexane, allylic rearrangements observed in methylene cyclohexane oxidation, the NIH shift observed for aromatic hydroxylation and the opening of cyclopropyl rings all suggest a non-concerted reaction mechanism of hydrogen abstraction followed by hydroxylation proceeding via carbocation or radical intermediates. ${ }^{15} \mathrm{~A}$ large kinetic isotope effect has been reported for the oxidation of methane, suggesting that breaking the $\mathrm{C}-\mathrm{H}$ bond is rate-limiting. ${ }^{16}$

The soluble form of MMO has been the focus of much of the research of monooxygenase activity because of its broader substrate specificity and the greater ease of isolation and purification of soluble extracts of $\mathrm{MMO}$ as compared to membrane-bound forms. In general, however, the ability of MMO to catalyze the oxidation of methane to methanol has generated interest in this enzyme system, or in models of this enzyme system, as a biocatalyst for methane conversion and alkane oxidation. ${ }^{17} \mathrm{~A}$ more recent interest in the methanotrophic bacteria from which MMO is isolated concerns the ability of MMO to catalyze the oxidation of halogenated hydrocarbons. Trichloroethylene (TCE) is a source of considerable groundwater pollution and a suspected carcinogen. 18 The byproducts of the naturally occurring anaerobic degradation of TCE (via reductive dehalogenation) are even more recalcitrant to anaerobic processes, and vinyl chloride, a known carcinogen, accumulates in anaerobic TCE-contaminated aquifers. ${ }^{19}$ Under aerobic conditions, the degradation of TCE occurs readily, and the susceptibility of the byproducts to aerobic degradation is greater than that of TCE. 20 TCE is not a growth substrate for methanotrophs, however at low concentrations, TCE is cometabolized by methanotrophic populations grown on methane (high concentrations of TCE is toxic to methanotrophs). The rate and extent of TCE oxidation depends on a number of factors, including growth conditions and availability of reducing power for the catabolic process. 21 With the proper approach, however, undesirable solvents in groundwater supplies can be cometabilized by methanotrophic populations. 22

\subsection{The Soluble Methane Monooxygenase Enzyme System}

Soluble methane monooxygenase has been isolated and purified from Type I methanotroph Methylococcus capsulatus (Bath), 10a,11a and Type II methanotrophs Methylosinus trichosporium (OB3b) $10 \mathrm{c}, 12$ and Methylobacterium sp. Strain CRL-26. 10b MMO from $M$. capsulatus (Bath) and $M$. trichosporium (OB3b) was resolved into three components consisting of a hydroxylase, a reductase and a small regulatory protein 
(component B). The soluble MMO system from Methylobacterium sp. Strain CRL-26 was resolved into only two components, the hydroxylase and the reductase. The hydroxylase component ${ }^{10 c, 23}\left(\mathrm{M}_{\mathrm{r}} 250 \mathrm{kDa}\right)$ consists of three polypeptide subunits in an $\alpha_{2} \beta_{2} \gamma_{2}$ arrangement and contains 2-4 non-heme iron atoms per protein unit depending on conditions of growth, harvesting and purification. EPR studies of the hydroxylase component from $M$. capsulatus (Bath) have suggested that the protein is a non-heme dinuclear iron protein. ${ }^{24}$ The reductase component ${ }^{25}\left(\mathrm{M}_{\mathrm{r}} 39 \mathrm{kDa}\right)$ contains one FAD and one $\mathrm{Fe}_{2} \mathrm{~S}_{2}$ cluster, while the regulatory protein $26\left(\mathrm{M}_{\mathrm{r}} 16 \mathrm{kDa}\right)$ contains no metal atoms or prosthetic groups. All three protein components are required for efficient oxidation of substrate for the MMO enzyme systems from both $M$. capsulatus (Bath) and $M$. trichosporium. (OB3b). The reductase from $M$. capsulatus (Bath) and Methylobacterium sp. CRI_-26 are essentially identical, giving rise to the same absorption spectrum (band at $460 \mathrm{~nm}$ with a shoulder at $395 \mathrm{~nm}$ ), EPR spectrum (rhombic with $\mathrm{g}$ values of $1.86,1.96$, 2.04 typical of an $\mathrm{Fe}_{2} \mathrm{~S}_{2}$ center) and redox potentials $\left(-247,-195,-250 \mathrm{mV}\right.$ for the $\mathrm{Fe}_{2} \mathrm{~S}_{2}$, FAD/FADH, FAD/FADH 2 couples from Methylobacterium sp. CRL-26; -220, -150, -260 $\mathrm{mV}$ for the same couples from $M$. capsulatus (Bath)). 25

The reductase component from $M$. capsulatus (Bath) rapidly accepts electruns from the NADH and transfers those electrons to the hydroxylase component. 27 Studies of the oxidation of $\mathrm{NADH}$ by apo- $\mathrm{Fe}_{2} \mathrm{~S}_{2}$ and apo- $\mathrm{Fe}_{2} \mathrm{~S}_{2}$-apo-FAD reductase demonstrated that the FAD center interacts directly with $\mathrm{NADH}$ accepting 2 electrons. These electrons are transferred one at a time to the $\mathrm{Fe}_{2} \mathrm{~S}_{2}$ ceriter, which can carry only one electron, 28 and it is the $\mathrm{Fe}_{2} \mathrm{~S}_{2}$ center which interacts with the hydroxylase component relaying single constantpotential electrons. Intermolecular transfer of electrons from $\mathrm{NADH}$ to the hydroxylase via the reductase occurs in the presence or absence of substrate.

EPR studies of the hydroxylase component in the presence of ethene and cyanomethane, in which the EPR spectra sharpened and increased in intensity, suggested that the hydroxylase interacted directly with the substrate. 29 Studies with a high specificactivity form of the hydroxylase from $M$. trichosporium (OB3b) have shown that in the absence of reductase and component $B$, single turnover is achieved when the hydroxylase is reduced to the diferrous form non-enzymatically and exposed to dioxygen. ${ }^{30}$ This confirms that the hydroxylase component contains the site where substrate binding and oxidation occur, and the $2 \mathrm{e}^{-}$reduced form of the hydroxylase is the catalytically important state. While there is little doubt about the role of the reductase and hydroxylase component, there is some question about the role of component $\mathrm{B}$ in the substrate oxidation process. 
As noted above, electron transfer between the reductase and the hydroxylase occurs in the absence or presence of substrate for the $M$. capsulatus (Bath) enzyme system, resulting in the reduction of $\mathrm{O}_{2}$ to $\mathrm{H}_{2} \mathrm{O}$. Substrate oxidation did not occur unless component $\mathrm{B}$ was present. 27 In the absence of substrate and the presence of component $\mathrm{B}$, electron transfer between the hydroxylase and the reductase decreased dramatically, or no longer occurred. This suggests that component B serves to convert the hydroxylase from an oxidase to an oxygenase by regulating the transfer of electrons from the reductase to the hydroxylase to the presence of oxidizable substrate, perhaps as a conservation mechanism for reducing equivalents. In contrast, oxidation products were rietected for a system consisting of a high specific-activity hydroxylase of $\mathrm{MMO}$ from $M$. trichosporium (OB3b), $\mathrm{O}_{2}$, NADH and reductase, with no component $\mathrm{B}$ present. 30 This difference in the role of component $B$ as a requirement for substrate oxidation could reflect a true difference in the mechanism of oxidation for the MMO complex from the two types of methanotrophs, or the difference in the activity of the hydroxylase as isolated from $M$. trichosporium (OB3b), or the differences may arise from the different relative concentrations of the various proteins used to execute the studies. As noted above, the MMO system from Methylobacterium sp. Strain CRL-26 consists of only the hydroxylase and reductase, hence these three species could reflect specializations in the development of the MMO system in response to specific environmental conditions.

Component B interacts with the $\alpha$ subunit of the hydroxylase, while the reductase interacts with the $\beta$ subunit. ${ }^{31}$ The presence of B perturbs the EPR spectrum of the hydroxylase. In addition, the distribution of the oxidation products of the hydroxylation reaction was found to change when $B$ was present. 32 The interaction of the components of the MMO enzyme system therefore has an effect on the diiron site in the hydroxylase component. Understanding the nature of the change at the iron center is an important step in developing an understanding of the mechanism of oxygen activation.

\subsection{Spectroscopic Studies of Dinuclear Non-Heme Iron Centers}

EPR studies of the hydroxylase component of soluble $\mathrm{MMO}^{24}$ have suggested that this protein belongs to the class of dinuclear non-heme iron proteins which includes hemerythrin (Hr), ribonucleotide reductase subunit B2 (RRB2), purple acid phosphatase from beef spleen (PAP) and uteroferrin from porcine uterine linings (Uf). ${ }^{33}$ These proteins serve very different functions: hemerythrin is an oxygen transport protein, ribonucleotide reductase converts ribonucleotide diphosphate to deoxyribonucleotide diphosphate for DNA synthesis, the purple acid phosphatases hydrolyzes phosphate esters, and methane 
monooxygenase is an oxygen activation protein; however, they have many similar spectroscopic features ${ }^{33}$ suggesting that they have similar active site structures as well. Understanding the structural basis of the spectroscopic similarities and functional differences of these protein systems has been a major focus of research in a number of laboratories.

Structurally characterized inorganic model compounds which mimic the spectroscopic and/or catalytic properties of the dinuclear non-heme iron protein active site are an important tool in understanding structure-function relationships in these protein systems. As a result, a number of multiply bridged non-heme iron models have been synthesized and spectroscopically characterized, and Kurtz has published an extensive review of the models of dinuclear non-heme iron sites. ${ }^{34}$ These models are di- or tribridged with either an oxygen atom (oxo-bridge) or a hydroxide group (hydroxo-bridge) as one of the bridging units, with the balance of the bridges being made up of carboxylate, alkoxo, or hydroxo groups. 35 The dominant structural feature of these model compounds is the presence (or absence) of a $\mu$-oxo bridge between the iron atoms $\left(\mathrm{Fe}-\mathrm{O}_{\mathrm{oxo}}\right.$ distance of $\sim 1.8 \AA ; \mathrm{Fe}-\mathrm{O}_{\text {hydroxo }}$ distance $\sim 2.0 \AA$ ), which imparts characteristic spectroscopic features. The Fe-O-Fe unit gives rise to strong electronic absorption bands between $300-400 \mathrm{~nm}$, which have been assigned as ligand-to-metal (oxo-to-iron) charge transfer transitions, 36 and a pair of weak bands between $440-510 \mathrm{~nm}$, attributed to ligand field transitions which gain intensity by mixing of oxo-to-iron charge transfer transitions. ${ }^{37}$ Hydroxo-bridged models have relatively featureless electronic absorption spectra. The symmetric vibrational stretch of the Fe-O-Fe occurs between 380 and $450 \mathrm{~cm}^{-1}$, and the asymmetric stretch between 725 and $885 \mathrm{~cm}^{-1}$. The hydroxylase of methane monooxygenase exhibits none of these absorption bands. ${ }^{1}$ The $\mathrm{Fe}-\mathrm{OH}-\mathrm{Fe}$ stretch is reported to occur between 3400 and $3600 \mathrm{~cm}^{-1}$.

Diferric oxo- and hydroxo-bridged models exhibit isomer shifts in the range of $0.35-0.60 \mathrm{~mm} / \mathrm{s}$ by Mössbauer spectroscopy, characteristic of 5- or 6-coordinate high-spin iron atoms ( $\mathrm{S}=5 / 2$ ground state). Quadrupole splittings $\left(\Delta \mathrm{E}_{\mathrm{Q}}\right)$ greater than 1 are characteristic of the presence of an oxo-bridge; hydroxo-bridged models have quadrupole splittings $<1$. Magnetic susceptibility measurements indicate that in almost every case, the diferric iron atoms exhibit antiferromagnetic coupling $(J<0)$. Values of the coupling constant, $J$, for oxo-bridged compounds are in the range of 80 to $120 \mathrm{~cm}^{-1}$, while hydroxobridge models have coupling constants in the 7 to $17 \mathrm{~cm}^{-1}$ range. The coupling constant for the semimet hydroxylase has been determined to be $J=-32 \mathrm{~cm}^{-1}{ }^{1}$ The large difference in the magnitude of $J$ is an indication of the ability of the oxo-bridge to mediate strong spinexchange coupling in these systems, and therefore of the involvement of oxo-bridge 
orbitals in a superexchange pathway between the iron atoms. The presence of the other bridges in the center has little effect on the magnitude of $J$, and therefore play a negligible role in the spin-exhange coupling. A correlation has been shown to exist between the magnitude of the coupling constant, $J$, and the shortest superexchange pathway in the molecule, which in every case would involve only the short $\mathrm{Fe}$-oxo bridge (Fe- $\mathrm{O}_{\text {oxo }}$ distance $\sim 1.8 \AA$ ) instead of the longer Fe-carboxylato bridge (Fe-O $\mathrm{O}_{\text {carboxy }}$ distance $\sim 2.0$ $\AA) .38$

The diferric forms of $\mathrm{Hr}, \mathrm{RRB2}$, Uf, and MMO consist of 5- or 6-coordinate highspin iron atoms which are antiferromagnetically coupled. All of the proteins mentioned above have been structurally characterized by X-ray absorption spectroscopy, 39 and $\mathrm{Hr}$ and RR have been characterized by crystallography as well. 40 These studies have shown that the iron active sites of these proteins have in common octahedrally-coordinated high spin iron atoms which are antiferromagnetically coupled. $\mathrm{Hr}$ has a ( $\mu$-oxo)bis( $\mu$-carboxylato) tribridged diiron core, an open coordination site for dioxygen binding and $\mathrm{N}$ ligation from histidines (total of 5 histidine groups) completing the coordination sphere. ${ }^{40 a, b}$ The RR diiron center has a ( $\mu$-oxo $)(\mu$-carboxylato) dibridged iron core with only two histidine atoms coordinating and the rest of the coordination sphere consisting of O-donating carboxylate groups. ${ }^{40 c}$ The iron core in Uf is suggested based on EXAFS analysis to also be dibridged, but no evidence for an oxo-bridge was found. Instead, a ( $\mu$ hydroxo/alkoxo)( $\mu$-carboxylato) core is suggested for the Uf active site.

The differences in the structures of the dinuclear iron centers of the proteins described ahove contribute to the different roles they serve. The characterization of the active site of MMO using $\mathrm{X}$-ray absorption spectroscopy will provide additional information about the variations in the structure of dinuclear iron centers in biology which lead to different functions.

\subsection{X-ray Absorption Spectroscopy}

$\mathrm{X}$-ray absorption spectroscopy (XAS) involves the measurement of the absorption coefficient, $\mu$, of an element of interest as a function of energy (eV). Sharp discontinuities in the absorption coefficient, called edges, arise when a photon is absorbed with energy equal to the ionization energy of an electron in the absorbing species. The X-ray edges are named according to the Buhr atomic level from which the photoionized electron originates. For example, a K-edge refers to the ionization of a $1 \mathrm{~s}$ electron. The spectrum obtained in an XAS experiment is divided into three regions (Figure 1.1). The structure in the edge region, sometimes referred to as the $\mathrm{X}$-ray absorption near-edge structure (XANES), 


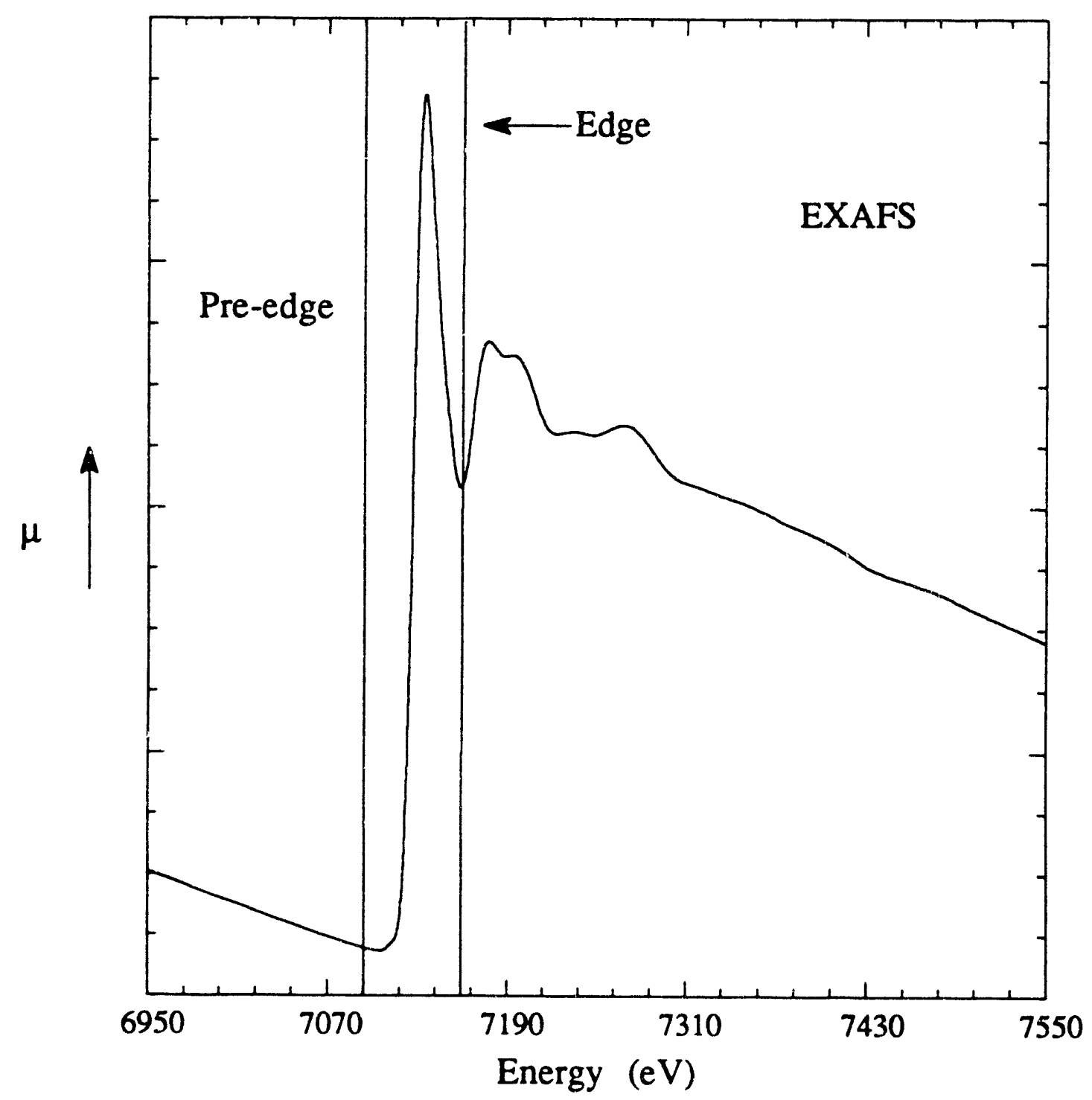

Figure 1.1. An Fe K-edge X-ray absorption spectrum. $\mu$ is the absorption coefficient. The data were measured in transmission mode. The slope in the pre-edge and EXAFS region : - the background caused by the absorption of lower $\mathrm{Z}$ atoms in the sample, as well as by absorption from air tape and windows. 
contains information about the electronic structure of the absorbing atom. Above the edge region, the modulation in the absorption coefficient is referred to as the extended $\mathrm{X}$-ray absorption fine structure (EXAFS) and contains information about the local geometric structure of an absorbing atom. 41 The absorption edges for most biologically relevant transition metal ions are accessible with the current $\mathrm{X}$-ray sources available, but the use of high-intensity synchrotron radiation over a broad range of energies has been essential for the application of XAS to the study of dilute metalloprotein samples.

XAS has proven itself to be a valuable probe of the active site of metalloproteins $\mathrm{s}^{42}$ because it specifically probes the environment around the metal center and can be used on non-crystalline samples of dilute metalloproteins. The $\mathrm{X}$-ray absorption edge region contains transitions to bound atomic and molecular states localized on the absorbing atom, as well as to localized and delocalized continuum resonances. 43 The position the edge is directly related to the oxidation state of the absorbing atom, and variations $i x$ : "position of the edge features among samples in the same oxidation state therefore reflect changes in the covalency of the metal site. In addition, the appearance of the edge is indicative of the coordination number and site symmetry of an absorbing atom. The analysis of the edge structure of dinuclear iron systems will be presented in Chapter 5 along with a more detailed discussion of the interpretation of XANES (X-ray absorption near edge structure) data.

Above the edge region, the photoelectron is ejected into the continuum. Analysis of the modulation pattern of the data in this region produced by backscattering of the X-ray excited photon from neighboring atoms gives information about the type, number and distances of nearest neighbors to the absorbing atom. ${ }^{44}$ The edge thresholds for first row transition metals are separated by several hundred $\mathrm{eV}$, which allows specific information about different metal-containing sites within a single protein to be obtained. The theory and use of EXAFS, and a description of the data reduction and analysis technique are presented below. Details of the design and execution of an EXAFS experiment have been extensively reviewed 44,45 and will not be presented here.

\subsubsection{Extended X-ray Absorption Fine Structure}

EXAFS analysis gives information about the average local structural environment of an absorbing atom. The modulations in the absorption coefficient result from a scattering process involving the neighboring atoms and therefore depend on the coordination environment of the absorbing atom. The analysis below considers only the single scattering interaction between the photoelectron and neighboring atoms. Single 
scattering describes the process in which the photoelectron propagates away from the absorbing atom, scatters off of a neighboring atom, and travels directly back to the absorbing atom The standard EXAFS equation for the $\mathrm{K}$-edge and single scattering is given by: 46

$$
\chi(k)=\sum_{\mathrm{S}} \mathrm{N}_{\mathrm{s}} \frac{\left|\mathrm{f}_{\mathrm{s}}(\pi, k)\right|}{k \mathrm{R}_{\mathrm{as}}^{2}} \exp \left(-2 \sigma_{\mathrm{as}}^{2} k^{2}\right) \exp \left(\frac{-2 \mathrm{R}_{\mathrm{as}}}{\lambda}\right) \sin \left[2 k \mathrm{R}_{\mathrm{as}}+\alpha_{\mathrm{as}}(k)\right]
$$

where $\mathrm{N}_{\mathrm{S}}$ is the number of scattering atoms at an average distance $\boldsymbol{R}_{\mathbf{a s}}$ from the absorbing atom. $\left|\mathrm{f}_{\mathrm{s}}(\pi, k)\right|$ is the backscattering amplitude and $\alpha_{\mathrm{as}}(k)$ is the phase shift of the scattering atom; both are dependent on backscattering atom type. $\lambda$ is the mean free path which accounts for the finite lifetime of the photoelectron, and $k$ is the photoelectron wavevector, given by equation 3 , below. The Debye-Waller factor, $\exp \left(-2 k^{2} \sigma^{2}\right.$ as $)$ accounts for thermal and/or structural disorder in a given coordination sphere, with $\sigma_{\text {as }}$ defined as the rootmean-square variation of atomic distances from the average $R_{a s}$. The EXAFS for any absorbing atom/scattering atom pair can be represented as a damped sine wave with amplitude $\left(\left[\mathrm{N}_{\mathrm{S}} \mid \mathrm{f}_{\mathrm{s}}(\pi, k) \operatorname{lexp}\left(-2 \sigma^{2}{ }_{\text {as }} k^{2}\right) \exp \left(-\mathrm{R}_{\mathrm{as}} \lambda\right)\right] /\left[k\left(\mathrm{R}^{2}{ }_{\mathrm{as}}\right)\right]\right)$, frequency $\left(2 \mathrm{R}_{\mathrm{as}}\right)$ and phase shift $\left(\alpha_{\mathrm{as}}(k)\right)$ characteristic of the atoms involved. The total EXAFS is given by the summation of the individual sine waves which describe each absorbing atom/scattering atom interaction. The modulation of the absorption coefficient is therefore the interference pattern resulting from the superposition of the various sine waves originating from the interaction of the photoelectron with neighboring atoms, as measured at the location of the absorbing atom. The frequency of the EXAFS contains information about the distance to neighboring atoms, the amplitude has information about the numbers of neighboring atoms, and information about the types of scattering atoms can be obtained from both the amplitude and phase of the EXAFS. Analysis of the EXAFS data therefore yields information about the geometric structure of an absorbing atom site. 47

The amplitude of the EXAFS weakens at higher $k$ due to the $1 / k$ dependence of the EXAFS expression, from damping by the exponential Debye-Waller term, and from the lifetime limit represented by the mean free path. This damping effect can be compensated for in the data analysis by $k$ weighting $\chi(k)$. In addition, the backscattering amplitude, $\left|f_{\mathrm{S}}(\pi, k)\right|$, describes an amplitude function that is characteristic of the atom type and reaches a maximum at some value of $k$, representing the region of the EXAFS data to which the atom contributes most significantly. Therefore, $k$ weighting of the data can also increase the relative contribution of atoms which scatter more strongly at higher $k$ values and allow this information to be enhanced in the EXAFS analysis. Among the atoms which 
contribute to higher $k$ data are transition metals, therefore the weighting scheme is particularly important for metalloprotein data. Additionally, the single-scattering approximation which is used for the analysis of the EXAFS data is valid at higher $k$. In all of the analysis presented in this dissertation, the EXAFS analysis is based on $k^{3}$-weighted data. This weighting scheme has proven to provide the best compromise between enhancing the contribution of high $k$ data without overemphasizing the increased noise level of the data in the high $k$ region.

As shown above, EXAFS is expressed as a function of $k$, the photoelectron wave vector, however the absorption data is measured as a function of energy. The relationship between energy and $k$ is given by:

$$
k=\left[2 m_{e}\left(E-E_{0}\right) / \hbar^{2}\right]^{1 / 2}
$$

where $m_{e}$ is the electron mass, $E$ is the photon energy, $\hbar$ is Planck's constant divided by $2 \pi$, and $E_{0}$ is the threshold energy, where $k$ is defined to be zero, with units of $\AA^{-1}$. The contribution of each shell of atoms to the EXAFS data is represented as a damped sine wave with a frequency and amplitude characteristic of the scattering pair. The individual contributions can be conveniently visualized by performing a Fourier transform on the $k^{3}$. weighted EXAFS data (from $\AA^{-1}$ to $\AA$ space) which results in a peak corresponding to the frequency of each sine wave component in the EXAFS. Since the frequency of the sine wave is directly proportional to the distance between the absorbing and scattering atoms (frequency $=2 R_{a s}$ ) the generated Fourier transform represents the radial distribution of atoms around an absorbing atom.

Each peak in the Fourier transform of the EXAFS data corresponds to a shell of coordinating atoms, however the positions of the peaks do not give accurate distance information because of the $k$-dependent phase shift, $\alpha_{a s}(k)$, in equation $2 . \alpha_{a s}$ will contribute to the frequency of the sine wave by an amount $a_{1}$, where $a_{1}$ is the coefficient of the $k$-weighted term in the expansion of $\alpha_{\text {as }}$ (see equation 6 , below). The true frequency of the sine wave is then $\left(2 R_{a s}+a_{1}\right)$ so the position of the peak is shifted from the correct distance $R_{a s}$ by an amount $a_{1} / 2$. The magnitude of this phase shift depends on the types of atoms present in the center. Nevertheless, the Fourier transform allows the principal frequency contributions to the EXAFS data to be isolated and independently analyzed which simplifies the initial curve-fitting procedure described in the following section.

The EXAFS amplitude is proportional to $\left(R_{a s}\right)^{2}$, therefore the same atom at longer distances from the absorbing atom contribute less strongly to the EXAFS than one nearby. EXAFS analysis can therefore yield structural information only within a limited radius of 
the absorbing atom. Structural disorder also contributes to the distance limitation of EXAFS analysis by increasing the Debye-Waller term and therefore decreasing the EXAFS amplitude. For dilute metalloprotein systems, structural information within $4 \AA$ is typically accessible by EXAFS. In addition, if an atom is in more than one environment in a sample, the structural information obtained will be an average over all absorbing atom sites.

The above description of the EXAFS does not include the contributions from multiple scattering pathways. These pathways involve angle-dependent scattering of the photoelectron which contribute to outer shell EXAFS data and involve the interaction of the photoelectron with two or more atoms before returning to the absorbing atom. In general, multiple scattering contributions are weaker than single scattering contributions within the same coordination shell, but may be significant compared to single scattering processes involving a more distance shell of atoms. Specifically, multiple scattering pathways may contribute significantly to the second shell data of dinuclear metal systems and therefore interfere with the analysis of the second shell data using the single scattering form of the EXAFS equation described above. Further discussion of the multiple scattering process is presented in Chapter 4. The first shell data will have no contributions from multiple scattering pathways due to the length of the pathways involved. The low $k$ region of the EXAFS data frequently can have dominant contributions from multiple scattering effects and is not included in data analysis in the absence of a theoretical approach.

There are certain experimental considerations that impact the quality of experimental EXAFS data. First and foremost is the signal-to-noise level of the data which is primarily a function of the concentration of the absorbing atom in the samples. The EXAFS signal is weak compared to the absorption edge (often a few percent of the intensity), therefore in an EXAFS analysis, information is being extracted from a weak signal on a large background. As noted above, the EXAFS signal is damped at high $k$ and must therefore be weighted to obtain an equal contribution over the entire $k$ range. However, the noise contribution is also increased by the weighting scheme, and will limit the amount of data available for further analysis, whihc in turn limits the structural information which can be obtained. In addition, the shorter the $k$ range of the data, the less well-resolved are the frequency contributions to the Fourier transform of the EXAFS data, and therefore the EXAFS analysis will not be able to distinguish between shells of atoms in the same coordination sphere at slightly different distances. For example, for data to $k=14 \AA^{-1}$, the differences in the distances of coordinating shells of atoms greater than $\sim 0.11 \AA$ can be resolved, but for data to $10 \AA^{-1}$, the distance differences have to be greater than $\sim 0.16 \AA$. In general, obtaining data to a high $k$ range will improve the resolution of distance contributions within the same shell. 
The most effective way to improve the signal-to-noise level is to maximize the concentration of the absorbing species in the sample, sometimes difficult for biological samples due to increasing viscosity of concentrated samples and dangers of precipitating the protein. The signal-to-noise level for dilute systems is improved by collecting numerous scans which are averaged together for further analysis. The quality and range of data is then limited by the amount of time available to collect data on the sample. Additionally, the intensity of the incoming beam is an important factor and use of a wiggler beamline greatly improves the quality of the data compared to a bending magnet beamline. 45 Another factor which limits the range of data available for analysis is the damping of the EXAFS resulting from disorder in the structure. There are two contributions to the Debye-Waller factor, a dynamic and a static term. The static term arises from structural disorder in the sample such as a variety of distances to atoms in the first coordination sphere. The dynamic disorder involves vibrational motion of atoms relative to each other. The vibrational contribution to the Debye-Waller factor can be minimized by collecting data at low temperatures, thereby decreasing the damping of the EXAFS amplitude and improving the quality of the data to some degree. In addition, maintaining biological samples at low temperatures may reduce radiation damage and slow photoreduction of the sample caused by the formation of hydrated electrons in aqueous solutions which react with the metal site.

\subsubsection{Data Reduction and Analysis}

The initial steps in data reduction involve energy calibration of each scan, inspection and averaging of individual scans, and removal of monochromator glitches if necessary from the averaged data set. These methods are discussed in the experimental sections of following chapters. The more critical steps in the data reduction procedure are background subtraction and normalization which allow the EXAFS to be extracted from the averaged data file.

EXAFS $(\chi(k))$ is defined as the modulation of the absorption coefficient, $\mu$, and can be expressed as:

$$
\chi(k)=\left(\mu-\mu_{0}\right) / \mu_{0}
$$

where $\mu_{0}$ is the free-atom absorption coefficient which would be observed if the sample contained only the absorbing atom without any neighboring scattering atom. In equation 4 , the EXAFS modulations are isolated from, and normalized to, the free-atom absorption, 
effectively yielding information on a per atom basis. In an XAS experiment, the absorption (or fluorescence) measured consists of the absorption coefficient, $\mu$, plus a background contribution, $\mu_{\text {back}}$, arising from absorption from lower $\mathrm{Z}$ atoms in the sample or scatter from the sample, as well as absorption from windows, tape or air in the beam path. In order to obtain $\mu$ from the experiment, the background must be subtracted from the measured data. The absorption background, $\mu_{\text {back}}$, decreases monotonically with increasing energy and can be approximated by a polynomial which is fit to the data in either the pre-edge or post-edge region of the spectrum, extrapolated through the rest of the spectrum and subtracted. Alternatively, the absorption of a blank sample containing everything except the absorbing species of interest can be measured and then subtracted from the sample absorption. In practice, creation of an accurate blank is a difficult task, so the polynomial fitting method is typically used. This method does not result in complete removal of the background and will leave some residual background, $\mu_{\text {res, }}$, in the data which is removed in the next step.

The free-atom absorption, $\mu_{0}$, cannot be measured and must therefore be approximated in some way. The free-atom absorption would be a smooth curve upon which the EXAFS oscillations are superimposed and can be modeled by a curve obtained by fitting a polynomial spline to the data in the post-edge region. This method will also model and remove $\mu_{\text {res}}$, the residual background left from the absorption correction procedure described above. A different value of $\mu_{0}$ must be used in the denominator of equation 2 to normalize the EXAFS data because the curve will include $\mu_{\text {res. }}$ The value of $\mu_{0}$ used for normalization is usually calculated by using the Victoreen formula. ${ }^{48}$

The determination of $\mu_{0}$ by the spline curve-fitting method is not a trivial task. In general, a three or four region spline is used depending on the range of data available, and the fitting procedure involves changing the lengths of the various regions and the orders of the polynomials used within each region. The criteria which must be matched is that the polynomials must meet at the border of a region with equal value and equal slope. Care must be taken to insure that the curve used to mimic the background does not distort the EXAFS when it is subtracted from the data. This is generally done by monitoring both the appearance of the spline, the EXAFS and the Fourier transform of the EXAFS during the spline-fitting procedure. The curve chosen to represent $\mu_{0}+\mu_{\text {res }}$ should maximize the intensity of the peaks in the Fourier transfom and minimize the low $\mathbf{R}$ noise, and the amplitude of the EXAFS above and below the zero line should show no ultra-lowfrequency structure. Improper background subtraction can result in errors in the structural information obtained by EXAFS analysis. Distortion of the EXAFS can be manifested as changes in both the frequency and the amplitude of the data, thereby leading to incorrect 
distance and coordination number determinations. For that reason, it is best to use few regions for the spline fitting technique and low orders of polynomial within the regions to prevent excessive curve in the spline. Using nodes in the EXAFS data as the end points of the curve-fitting regions and distributing the regions evenly in $k$ space has proven to be a reasonable approach for determining the spline.

The experimental factors which contribute to the background include harmonic contamination of the incident beam, effects from ice in the solution samples, and absorption from low $\mathrm{Z}$ atoms in, or scatter from, the sample as mentioned above. These effects can be reduced by using proper experimental technique. Harmonic contamination can be minimized by detuning the monochromator, which very slightly misaligns one of the two monochromator crystals. This results in a decrease in the contribution to the intensity from the higher harmonic reflection relative to the contribution from the primary reflection due to the narrower shape of the higher-harmonic rocking curve. Ice effects, which range from increased noise to diffraction peaks, can be minimized by adding ethylene glycol or glycerol to the buffer/solvent system for solution samples to facilitate the formation of glasses. Alternatively, if ice effects are a problem in the data, thawing and rapidly freezing the sample may reduce the size of the ice crystals, thereby reducing the diffraction effects. The absorption from low $\mathrm{Z}$ atoms in the sample for data collected in transmission mode can only be eliminated by the background subtraction method discussed above. In general, transmission measurements are used for concentrated samples, because the signal should be large compared to the background. For dilute samples, the transmitted signal is weak compared to the background, so fluorescence detection is used. In fluorescence mode, contributions to the background from scatter can be reduced by using a filter which absorbs the scatter at energies below the fluorescent signal of interest. The easiest way to achieve this is to make a filter of the element one atomic number lower than the element of interest (Z-1 filter) for first row transition metals. Solid state fluorescence detectors allow electronic windowing of the signal of interest, so that only photons from the appropriate $\mathrm{K} \alpha$ line are detected. 49

Normalization of the data scales the spectra to give a value of one for the absorption edge and allows the data to be interpreted on a per atom basis. Data for different samples can then be directly compared to one another. The edge region is sensitive to the chemical environment of the absorbing atom, therefore the point at which the data is scaled must lie above the edge region so that the normalization procedure is independent of the nature of the sample. In practice, the data is normalized at $E_{0}$, defined as the beginning of the continuum region of the XAS spectrum. The scale factor which is used to normalize the 
data corresponds to the difference between the curve fit to the postedge region and the curve used to mimic and remove the absorption background, as measured at $\mathrm{E}_{0}$.

The first step in extracting metrical information from the normalized, backgroundsubtracted EXAFS is to Fourier transform the $k^{3}$-weighted EXAFS data, thereby revealing the phase-shifted radial distribution function. The individual contributions to the EXAFS are isolated from the Fourier transform by applying a window, or filter, to isolate the region of interest in the transform which is then backtransformed into $k$ space for further analysis. Fourier filtering will distort the data somewhat due to artifacts introduced by truncating the data. These effects can be minimized by using a window smoothing function. For that reason, a Gaussian window of width $0.1 \AA$ was used to smooth the window used to isolate the EXAFS data for transformation to $R(\AA)$ space, and the peaks in the Fourier transform for backtransformation to $k\left(\AA^{-1}\right)$ space for curve-fitting analysis.

Analysis of the structural information contained in the EXAFS data involves the simulation of the EXAFS for a hypothetical arrangement of atoms around the absorbing atom. The EXAFS interaction between a given absorbing atom/scattering atom pair is constructed with the use of the appropriate amplitude and phase functions $\left(\left|\mathrm{f}_{\mathbf{s}}(\pi, k)\right|\right.$ and $\alpha_{\text {as }}(k)$ in equation 2$)$, distances ( $\left.R_{a s}\right)$, coordination numbers $\left(N_{s}\right)$, energy shifts $\left(E_{0}\right)$, and Debye-Waller factors ( $\sigma^{2}$ as). In addition, an amplitude reduction factor may be required. A non-linear least squares curve fitting technique is used fit the hypothetical EXAFS to the data by allowing certain parameters to vary, depending on the method of curve fitting used. Theoretical amplitude and phase functions are tabulated for the plane-wave ${ }^{50}$ as curvedwave approximations. 51 These functions often require the use of an amplitude reduction factor and edge shift, $\Delta \mathrm{E}_{0}$, to scale the theoretical amplitude and phase functions. These values are sometimes determined with the aid of structurally characterized models in an approach called FABM, or fine adjustment based on models. 52

A second approach involves the use of appropriate, structurally characterized models to empirically determine amplitude and phase functions for a given scattering pair, 53 and is the method used for the EXAFS analysis presented in this thesis. The amplitude and phase functions required are parameterized according to:

$$
\begin{aligned}
& \left|f_{\mathrm{s}}(\pi, k)\right|=c_{0}\left(\exp \left[c_{1} k+c_{2} k^{2}\right] k^{c_{3}}\right. \\
& \alpha_{\mathrm{as}}(k)=\frac{\mathrm{a}_{-1}}{k}+\mathrm{a}_{0}+\mathrm{a}_{1} k+\mathrm{a}_{2} k^{2}
\end{aligned}
$$

The values of $c_{1}$ and $a_{-1}$ are zero in the work described herein. The method involves fitting the Fourier-filtered EXAFS data for the model compound (to isolate the shell of interest), 
using the correct distance and coordination number and optimizing the initial $\mathrm{c}$ and a parameters in an iterative method. Initially, all 6 parameters are varied in a fit to the data. The optimized parameters are then used as the starting point for the next iteration by successively allowing just the amplitude or the phase parameters to vary in the fits to the data. Once the sets of amplitude and phase parameters no longer changed during the fits (after 4 cycles), all of the parameters were allowed to vary for the final iteration. These empirically-derived amplitude and phase parameters for a scattering pair are then used to fit the EXAFS of interest by letting the distance and coordination number vary in the fit to the unknown .

Not surprisingly, the choice of the model compound from which to extract the amplitude and phase functions in this manner is critical. The assumption implicit in the use of amplitude and phase parameters as obtained by model compounds is that the parameters describe a generic absorbing atom/scattering atom interaction and are therefore transferable to other models; however, this is not always true. For that reason, models from which the amplitude and phase backscattering parameters are extracted should be chosen based on a reasonable similarity to the unknown. The compound chosen to model the absorbing atom/scattering atom interaction should have an equidistant distribution of only the scattering atom in the coordination shell of interest and the single scattering process should dominate. In general, parameters obtained from a first shell analysis of a model compound are not transferable to the second shell or vice versa. The transferability of the parameters must be tested in fits to other structurally characterized models before fits to an unknown are done. Similar Fourier transform ranges, windows and fitting ranges in both $k\left(\AA^{-1}\right)$ and $R(\AA)$ space should be used for extracting empirical parameters from models and fitting the unknown both to minimize truncation artifacts due to the Fourier filtering technique, and because of the $k$-dependence of the parameters. In addition, data on the models and the unknown should be collected at the same temperature due to the dependence of the amplitude function and Debye-Waller term on temperature.

Non-linear least-squares curve fitting techniques are subject to variable correlation, especially between $R_{a s}$ and $E_{0}$ and $N_{s}$ and $\sigma^{2}$ as. For that reason, two of the four variables are generally fixed (one from each pair of correlated variables) and the other two are allowed to float during the fitting procedure. The parameters varied in the FABM technique are generally the distance and coordination number or Debye-Waller factor, using a value of $\mathrm{E}_{0}$ and an amplitude reduction factor from model compounds to calibrate the theoretical phase and amplitude functions, respectively. For the empirical method used here, $\mathrm{E}_{0}$ and the Debye-Waller factor are fixed and the distance and the coordination number are varied. The Debye-Waller factor is represented by $c_{2}$ in the amplitude parameter set and is therefore 
determined using model compounds. The determination of $E_{0}$ is arbitrary, but the value used consistently for $\mathrm{Fe}, 7130 \mathrm{eV}$, has allowed the determination of accurate distances. Using this technique in applications to structurally-characterized model compounds, errors of $\pm 0.03 \AA$ in the distance determination, and of $25 \%$ in the coordination number are estimated. $44,47,49,53$

When using a non-linear least squares fitting technique care must be taken in the interpretation of fit results. It is important to step through distances and coordination numbers in the fitting procedure to insure the identification of a global, rather than a local, minimum corresponding to the best fit. A fitting parameter, $F\left(F=\left\{\left[k^{6}(\text { data }-f i t)^{2}\right] /(\right.\right.$ no. points) $\}^{1 / 2}$ ) is calculated for each fit attempt and the magnitude of $F$ indicates the "goodness" of the fit with smaller values suggesting a better fit, although one must still inspect the data and the fit and not rely only on the value of F calculated. The fits are not constrained, therefore chemical intuition must be used to evaluate the reasonableness of any fit result. An examination of the application and limitation of the empirical curve-fitting technique in fits to the first and second shell of dinuclear iron model compounds is presented in Chapter 4. 


\subsection{References and Notes}

1. DeWitt, J. G.; Bentsen, J. G.; Rosenzweig, A. C.; Hedman, B.; Green, J.; Pilkington, S.; Papaefthymiou, G. C; Dalton, H.; Hodgson, K. O.; Lippard, S. J. J. Am. Chem. Soc. 1991, 113, 9219-9235.

2. Anthony, C. The Biochemistry of the Methylotrophs; Academic Press: London, 1982.

3. Higgins, I. J.; Best, D. J.; Hammond, R. C.; Scott, D. Microbiol. Rev. 1981, 45, 556-590.

4. (a) Dalton, H.; Prior, S. D.; Leak, D. J.; Stanley, S. H. Microb. Growth on C1 Cmpd., Proc. Int. Symp. 1983; Am. Soc. Microbiol.: Washington D. C, 1984; pp. 75-82.

5. (a) Colby, J. D.; Stirling, I.; Dalton, H. Biochem. J. 1977, 165, 395-402. (b) Prior, S. D.; Dalton, H. J. Gen. Microbiol. 1985, 131, 155-163. (c) Burrows, K. J.; Cornish, A.; Scott, D.; Higgins, I. J. J. Gen. Microbiol. 1984, 130, 3327-3333.

6. Stanley, S. H.; Prior, S. D.; Leak, D. J.; Dalton, H. Biotech. Lett. 1983, 5, 487492.

7. Prior, S. D.; Dalton, H. J. Gen. Microbiol. 1985, 131, 155-163.

8. (a) Akent'eva, N. P.; Stukan, R. A.; Prusakov, V. E.; Tsuprun, V. L.; Gvozdev, R. I.; Shusnenecheva, E. V. Biocatalysis 1990, 4, 39-53. (b) Akent'eva, N. P.; Gvozdev, R. I. Biokhimiya 1988, 53, 91-96. (c) Korshunova, L. A.; Akent'eva, N. P.; Gvozdev, R. I.; Shushenacheva, E. V. Biokhimiya 1989, 54, 1652-1657.

9. Tonge, G. M.; Harrison, D. E. F.; Higgins, I. J. Biochem. J., 1977, 161, 333344.

10. (a) Colby, J.; Dalton, H. Biochem. J. 1978, 171, 461-468. (b) Patel, R. N. Microb. Growth on Cl Cmpd., Proc. Int. Symp. 1983; Am. Soc. Microbiol.: Washington D. C, 1984; pp. 83-90. (c) Fox, B. G.; Lipscomb, J. D. Biochem. Biophys. Res. Comm. 1989, 154, 165-170.

11. Colby, J.; Dalton, H. Biochem. J. 1976, 157, 495-497.

12. Stirling, D. I.; Dalton, H. J. Biochem., 1979, 96, 205-212.

13. (a) Colby, J.; Stirling, D. I.; Dalton, H. Biochem. J. 1977, 165, 395-402. (b) Stirling, D. I.; Colby, J.; Dalton, H. Biochem. J. 1979, 177, 361-364. (c) Leak, D. J.; Dalton, H. J. Gen. Microbiol. 1983, 129, 3487-3497. (d) Fox, B. G.; Borneman, J. G.; Wackett, L. P.; Lipscomb, J. D. Biochemistry, 1990, 29, 6419-6427. (e) Hou, C. T.; Patel, R. N.; Laskin, A. I.; Barnabe, N. FEMS Microbiol. Lett. 1980, 9, 267-270.

14. Ortez de Montellano, P. R., Ed. Cytochrome P-450: Structure, Mechanism, and 
Biochemistry; Plenum Press: New York, 1986.

15. (a) Jezequel, S. G.; Higgins, I. J. J. Chem. Tech. Biotechnol. 1983, 33B, 139-144.

(b) Green, J.; Dalton, H. J. Biol. Chem. 1989, 264, 17698-17703. (c) Ruzicka, F.; Huang, D.-S.; Donnelly, M. I.; Frey, P. A. Biochemistry, 1990, 29, 1696-1700.

16. Green, J.; Dalton, H. Biochem. J. 1989, 259, 167-172.

17. (a) Murch, B. P.; Bradley, F. C.; Que, L. Jr. J. Am. Chem. Soc. 1986, 108, 5027-5028. (b) Vincent, J. B.; Huffman, J. C.; Christou, G.; Li, Q.; Nanny, M. A.; Hendrickson, D. N.; Fong, R. H.; Fish, R. H. J. Am. Chem. Soc. 1988, 110, 68986900. (c) Kitajima, N.; Fukui, H.; Moro-oka, Y. J. Chem. Soc., Chem. Commun. 1988, 485.

18. Westrick, L.L.; Mello, J. W.; Thomas, R. F. J. Am Water Works Assoc. 1984, 76, 52-59.

19. (a) Bouwer, E. J.; McCarty, P. L. App. Environ. Microbiol. 1983, 45, 1286-

1294. (b) Parsons, F.; Wood, P. R.; DeMarco, J. J. Am Water Works Assoc. 1984, 76, 56-59. (c) Vogel, T. M.; McCarty, P. L. Appl. Environ. Microbiol. 1985, 49, 10801083. (d) Vogel, T. M.; Criddle, C. S.; McCarty, P. L. Environ. Sci. Technol. 1987, $21,722-736$.

20. Wilson, J. T.; Wilson, B. H. Appl. Environ. Microbiol. 1985, 49, 242-243.

21. (a) Henry, S. M.; Grbic-Galic, D. Microb. Ecol. 1990, 20, 151-169. (b) Henry, S. M.; Grbic-Galic, D. Appl. Envircn. Microbiol. 1991, 57, 236-244.

22. Semprini, L.; Robers, P. V.; Hopkins, G. D.; McCarty, P. L. Groundwater, 1990, 28, 715-727.

23. (a) Colby, J.; Dalton, H. Biochem. J. 1979, 177, 903-908. (b) Woodland, M. P.; Dalton, H. Anal. Biochem. 1984, 139, 459-462. (c) Lund, J.; Dalton, H. Eur. J. Biochem. 1985, 147, 292-296. (d) Patel, R. N.; Savas, J. C. J. Bacteriol. 1987, 169, 2313-2317.

24. (a) Woodland, M. P.; Patil, D. S.; Cammack, R.; Dalton, H. Biochim. Biophys. Acta 1986, 873, 237-242. (b) Fox, B. G.; Surerus, K. K.; Münck, E.; Lipscomb, J. D. J. Biol. Chem. 263, 10553-10556.

25. (a) Colby, J.; Dalion, H. Biochem. J. 1979, 177, 903-908. (b) Lund, J.; Dalton, H. Eur. J. Biochem. 1985, 147, 292-296. (c) Prince, R. C.; Patel, R. N. FEBS 1986, 203, 127-130. (d) Patel, R. N. Arch. Biochem. Biophys. 1987, 252, 229-236.

26. Green, J.; Dalton, H. J. Biol. Chem. 1985, 260, 15795-15801.

27. (a) Lund, J. ; Woodland, M. P.; Dalton, H. Eur. J. Biochem. 1985, 147, 297 305. (b) Green, J. ; Dalton, H. Biochem. J. 1989, 259, 167-172. 
28. Orme-Johnson, W. H.; Beinert, H. J. Biol. Chem. 1969, 244, 6143-6148.

29. (a) Dalton, H. Adv. Appl. Microbiol. 1980, 26, 71-87. (b) Dalton, H. Microbial Growth of C1 Compounds; Heyden Press: London, 1980, pp 1-10.

30. Fox, B. G.; Froland, W. A.; Dege, J. E.; Lipscomb, J. D. J. Biol. Chem. 1989, 264, 10023-10033.

31. Fox, B. G.; Liu, Y.; Dege, J. E.; Lipscomb, J. D. J. Biol. Chem. 1991, 266, 540-550.

32. Froland, W. A.; Andersson, K. K.; Lee, S.-K.; Liu, Y.; Lipscomb, J. D. J. Biol. Chem. 1992, 267, 17588-17597.

33. (a) Lippard, S. J. Angew. Chem. Int. Ed. Engl. 1988, 27, 344-361.

Sanders-Loehr, J. Iron Carriers and Iron Proteins; VCH Publishers Inc.: New York, 1989;

pp. 373-466. (c) Que, L., Jr.; True, A. E. Prog. Inorg. Chem. 1990, 38, 97-200.

34. Kurtz, D. M. Jr. Chem. Rev. 1990, 90, 585-606.

35. The generalizations made for hydroxo-bridged models in the following discussion also hold for alkoxo-bridged models, in which an alkoxide group replaces the hydroxide group. The spectroscopic values reviewed are for diferric model compounds. Models containing porphyrin ligation are not included in this discussion.

36. Reem, R. C.; McCormick, J. M.; Richardson, D. E.; Devlin, F. J.; Stephens, P. J.; Musselman, R. L.; Solomon, E. I. J. Am. Chem. Soc. 1989, 111, 4688-4704.

37. (a) Sanders-Loehr, J.; Wheeler, W. D.; Shiemke, A K: Averill, B. A.; Loehr, T. M. J. Am. Chem. Soc. 1989, 111, 8084-8093. (b) Sanders-Loehr, J.; Loehr, T. M.; Mauk, A. G.; Gray, H. B. J. Am. Chem. Soc. 1980, 102, 6992-6996.

38. Gorun, S. M.; Lippard, S. J. Inorg. Chem. 1991, 30, 1625-1630.

39. (a) Hendrickson, W. A.; Co, M. S.; Smith, J. L.; Hodgson, K. O.; Klippenstein, G. L. Proc. Natl. Acad. Sci. USA 1982, 79, 6255-6259. (b) Elam, W. T.; Stern, E. A.; McCallum, J. D.; Sanders-Loehr, J. J. Am. Chem. Soc. 1982, 104, 6369-6373. (c) Elam, W. T.; Stern, E. A.; McCallum, J. D.; Sanders-Loehr, J. J. Am. Chem. Soc. 1983, 1919-1923. (d) Zhang, K.; Stern, E. A.; Ellis, F.; Sanders-Loehr, J.; Shiemke, A. K. Biochemistry 1988, 27, 7470-7479. (e) Scarrow, R. C.; Maroney, M. J.; Palmer, S. M.; Que, Jr., L.; Roe, A. L.; Salowe, S. P.; Stubbe, J. J. Am. Chem. Soc. 1987, 109, 7857-7864. (f) Scarrow, R. C.; Maroney, M. J.; Palmer, S. M.; Que, L., Jr.; Roe, A. L.; Salowe, S. P.; Stubbe, J. J. Am. Chem. Soc. 1986, 108, 6832-6834. (g) Bunker, G.; Petersson, L.; Sjöberg, B.-M.; Sahlin, M.; Chance, M.; Chance, B.; Ehrenberg, A. Biochemistry 1987, 26, 4708-4716. (h) Kauzlarich, S. M.; Teo, B. K.; Zirino, T.; Burman, S.; Davis, J. C.; Averill, B. A. Inorg. Chem. 1986, 25, 2781-2785. 
(i) True, A. E.; Scarrow, R. C.; Holz, R. C.; Que, L., Jr. Inorg. Biochem. 1991, 43, 545.

40. (a) Stenkamp, ‥ E.; Sieker, L. C.; Jensen, L. H. J. Am. Chem. Soc. 1984, 106, 618-622. (b) Stenkamp, R. E.; Sieker, L. C.; Jensen, L. H.; McCallum, J. D.; SandersLochr, J. Proc. Natl. Acad. Sci. USA 1985, 82, 713-716. (c) Nordlund, P.; Sjöberg, B.-M.; Eklund, H. Nature 1990, 345, 593-598.

41. An excelient, detailed discussion of the theory, practice and applications of X-ray absorption spectroscopy appears in X-ray Absorption: Principles, Applications, Techniques of EXAFS, SEXAFS, and XANES; Koningsberger, D. C., Prins, R., eds.; John Wiley and Sons Inc.: NewYork, 1988.

42. Cramer, S. P. in X-ray Absorption: Principles, Applications, Techniques of EXAFS, SEXAFS, and XANES; Koningsberger, D. C., Prins, R., eds.; John Wiley and Sons Inc.: NewYork, 1988; pp 257-320.

43. Bianconi, A. in X-ray Absorption: Principles, Applications, Techniqu's of EXAFS, SEXAFS, and XANES; Koningsberger, D. C., Hrins, R., eds.; john Wiley and Sons Inc.: NewYork, 1988; pp 573-662.

44. Scott, R. A. Methods Enzymol. 1985, 117, 414-459.

45. Heald, S. M. in X-ray Absorption: Principles, Applications, Techniques of EXAFS, SEXAFS, and XANES; Koningsberger, D. C., Frins, R., eds.; john Wiley and Sons Inc.: NewYork, 1988; pp 87-162.

46. Stern, E. A. in X-ray Absorption: Principles, Applications, Techniques of EXAFS, SEXAFS, and XANES; Koningsberger, D. C., Prins, R., eds., John Wiley and Sorıs Inc.: NewYork, 1988; pp 3-51.

47. Cramer, S. P.; Hodgson, K. O. Prog. Inorg. Chem. 1979, 25, 1-39.

48. MacGillavry, C. H.; Rieck, G. D., Ed.: International Tables for X-ray Crystallography Vol. III, Kynoch Press: Birmingham, England, 1968.

49. Cramer, S. P.; Tench, O.; Yocum, M.; George, G. N. Nucl. Instrum. Methods Phys. Res. 1988, A266, 586-591.

50. Teo, B.-K.; Lee, P. A. J. Am. Chem. Soc., 1979, 101, 2815-2832.

51. McKale, A. G.; Veal, V. W.; Paulikas, A. P.; Chan, S.-K.; Knapp, G. S. J. Am. Chem. Soc. 1988, 110, 3763-3768.

52. Teo, B.-K.; Antonio, M. R.; Averill, B. A. J. Am. Chem. Soc. 1983, 105, 3751 3762 .

53. Cramer, S. P.; Hodgson, K. O.; Stiefel, E. I.; Newton, W. E. J. Am. Chem. Soc. 1978, 100, 2748-2761. 


\section{Chapter 2}

\section{$\mathrm{X}$-ray Absorption Spectroscopic Studies of the Dinuclear Non-Heme Iron Center in the Hydroxylase Component of Methane Monooxygenase and the B2 subunit of Ribonucleotide Reductase}




\subsection{Introduction}

Methanotrophic bacteria oxidize methane to carbon dioxide for growth and energy. 1 The initial and most difficult step in the reaction is the incorporation of oxygen into methane to yield methanol. This step is catalyzed by methane monooxygenase (MMO), a multicomponent enzyme present in both type I and type II methanotrophs. Type I and type II methanotrophs, which differ in their intracytoplasmic membrane structure and their carbon assimilation pathways, ${ }^{2}$ express $\mathrm{MMO}$ in either a particulate or soluble form depending primarily on the concentrations of copper and oxygen present during bacterial growth. ${ }^{3}$ Particulate MMO is a copper-containing membrane-bound protein, ${ }^{4}$ while soluble MMO contains a non-heme iron center and is associated with the soluble fractions of cellular extracts. 5 MMO in both the particulate and soluble form is a non-specific enzyme that catalyzes the oxidation of a wide variety of substrates, including aliphatic, aromatic, cyclic and halogenated compounds, ${ }^{6}$ a property which makes methanotrophs interesting as a possible biocatalyst.

Soluble MMO from Methylococcus capsulatus (Bath), ${ }^{7}$ a type I methanotroph, and Methylosinus trichosporium $\mathrm{OB} 3 \mathrm{~b},{ }^{5 \mathrm{c}}$ a type II methanotroph, has been resolved into three components, which are called the hydroxylase, the reductase, and component B. Soluble MMO from Methylobacterium sp. Strain CRL-26, also type II, has been resolved into only two components, identified as the hydroxylase and the reductase. ${ }^{8}$ The reductase component ${ }^{9}$ ( $\left.\mathrm{Mr} 42,000\right)$, formerly protein $\mathrm{C}$, is an iron-sulfur flavoprotein containing one FAD and one $\mathrm{Fe}_{2} \mathrm{~S}_{2}$ cluster. The reductase mediates the transfer of electrons one at a time from NADH to the hydroxylase component ${ }^{8 \mathrm{a}, 10}(\mathrm{Mr} \mathrm{250,000})$, via its FAD and $\mathrm{Fe}_{2} \mathrm{~S}_{2}$ redox centers. ${ }^{11}$ The third component, component $B^{12}$ (Mr 15,700), contains no prosthetic groups or metals and is believed to serve a regulatory role, linking electron transfer from the reductase to the hydroxylase to substrate oxidation. Recent studies of a very high specific activity hydroxylase from $M$. trichosporium $\mathrm{OB} 3 \mathrm{~b}$ has verified that the hydroxylase is the site of substrate binding and oxygen activation, and that monooxygenase activity is associated with the fully reduced form of the hydroxylase. $10 \mathrm{~b}$

The hydroxylase component (formerly Protein $\mathrm{A}$ ) is a non-heme protein containing 2-4 irons per unit depending on growth and harvesting conditions. It contains no acidlabile sulfur, and is made up of 3 smaller subunits in an $\alpha_{2} \beta_{2} \gamma_{2}$ polypeptide arrangement. EPR studies ${ }^{13}$ of the hydroxylase component of $M M O$ have suggested that the protein belongs to the class of non-heme dinuclear iron proteins which includes hemerythrin $(\mathrm{Hr})$, ribonucleotide reductase (RRB2), purple acid phosphatase (PAP) and uteroferrin (Uf). ${ }^{14}$ Structural studies on the dinuclear iron centers in these proteins are critical to understanding 
the functional differences between the spectroscopically similar active sites (for a review of the spectroscopic properties, see reference 14b).

Structural studies of the non-heme dinuclear iron center in the hydroxylase component of MMO along with relevant model compounds using extended X-ray absorption fine structure (EXAFS) have been initiated. 15 EXAFS has proven to be very sensitive to the presence or absence of $\mu$-oxo bridges in dinuclear iron proteins and model compounds. 16 Most notably, the EXAFS modulations of $\mu$-oxo bridged diferric models are remarkably similar to each other and different from the EXAFS of $\mu$-hydroxo bridged diferric models. Also, the short $\mathrm{Fe}-\mathrm{O}$ distance of the $\left[\mathrm{Fe}_{2} \mathrm{O}\right]^{+4}$ core in model compounds and proteins is distinctly resolved from the longer first shell N/O ligands in the fits to the EXAFS data and a short $\mathrm{Fe}-\mathrm{O}$ contribution (at $\sim 1.8 \AA$ ) is required to obtain an adequate fit to the data. ${ }^{16}$

Previous EXAFS experiments have been done on the oxidized and reduced forms of the hydroxylase of MMO from $M$. capsulatus (Bath) ${ }^{15}$ and on the oxidized form of the hydroxylase from $M$. trichosporium $\mathrm{OB} 3 \mathrm{~b} .{ }^{15 \mathrm{~b}}$ The oxidized protein samples from these experiments were photoreduced by the X-ray beam to the semimet state. Analysis of the EXAFS data on the semimet protein suggested that the protein does not contain a $\mu$-oxo bridge in its dinuclear iron center. An EXAFS study on the oxidized hydroxylase of MMO from Methylobacterium sp. Strain CRL-26 did not conclusively determine the presence or absence of a $\mu$-oxo or $\mu$-hydroxo bridge due to the limited $k$-range of the EXAFS data, although the iron-iron distance determined $(3.05 \AA)$ is in the range of those found for some $\mu$-oxo bridged models and proteins as well as some dibridged models. ${ }^{17}$

Fe K-edge EXAFS data has been collected on samples of the hydroxylase of MMO from Methylococcus capsulatus (Bath) in its oxidized and reduced forms to add to the information obtained in the previous studies on the photoreduced semimet forms of the hydroxylase. EXAFS data on a diferric form of the protein was collected, with no photoreduction of the protein to its semimet state. In addition, EXAFS data on the reduced form of the iron center in the B2 subunit of ribonucleotide reductase has been collected. The results of the experiments on the hydroxylase samples, including details about protein purification and characterization by Mössbauer and EPR spectroscopy have been reported elsewhere. 18 Details of the analysis of the Fe K-edge EXAFS of these samples and the earlier samples, as well as the results of the analysis for the ribonucleotide reductase data will be presented here. 


\subsection{Experimental}

\subsubsection{EXAFS Sample Preparation}

Soluble hydroxylase from Methylococcus capsulatus (Bath) and Methylosinus trichosporium (OB3b) were obtained as discussed before. Purified hydroxylase was diluted in buffer and frozen at $-80^{\circ} \mathrm{C}$ until further use. The hydroxlase was then thawed and concentrated to approximately $11 \mathrm{mg}$ protein $/ \mathrm{ml}$ using an Amicon PM 10 membrane. The hydroxylase complexes were dialyzed into a $50 \%$ ethylene glycol solution of an appropriate buffer ${ }^{18}$ and further concentrated on a Centricon centrifugal microconcentrator. The samples were then degassed and brought into a wet box. Samples EXAFS2, EXAFS4, and EXAFS5 were loaded into lucite EXAFS cells $(23 \mathrm{~mm} \times 2 \mathrm{~mm} \times 3 \mathrm{~mm}$, $\sim 140 \mu \mathrm{l}$ volume) with $25 \mu \mathrm{m}$ Kapton windows. EXAFS3 and EXAFS6 were reduced to the diferrous state by incubating the samples for 25 minutes with a 10 -fold molar excess of dithionite in the presence of $100 \mu \mathrm{M}$ methyl viologen and $10 \mu \mathrm{M}$ proflavin, and then loaded into the lucite EXAFS cells. The samples were immediately frozen in liquid nitrogen upon removal from the wet box and stored in a $\mathrm{LN}_{2}$ refrigerator. EXAFS1 was prepared as previously described. 15 a The sample of ribonucleotide reductase was reduced in the same manner as described above for the hydroxylase samples.

\subsubsection{Data Collection, Reduction and Analysis}

A summary of the samples is presented in Table 2.1. All protein sample data were measured in fluorescence mode at $10 \mathrm{~K}$ maintained by using an Oxford Instruments continuous flow liquid helium CF1208 cryostat. The EXAFS1 sample was run at SSRL on focused beamline 2-2 during dedicated conditions ( $3 \mathrm{GeV}, 50-65 \mathrm{~mA}$ ) by using a $\mathrm{Si}(111)$ double-crystal monochromator tuned $100 \%$ at $7850 \mathrm{eV} .19$ The fluorescence signal was detected with an Ar-filled ionization chamber, 20 equipped with Soller slits and a $\mathrm{Mn}$ filter. EXAFS2, EXAFS3, and EXAFS4 samples were run at SSRL on focused wiggler beamline 4-2 during dedicated conditions by using a Si(111) double-crystal monochromator tuned $100 \%$ at $7968 \mathrm{eV}$, and the same detector setup as for EXAFS1. EXAFS5 sample was run at NSLS on unfocused beamline X-19A (2.5 GeV, 90-200 $\mathrm{mA}$ ) by using a $\mathrm{Si}(220)$ double-crystal monochromator detuned for harmonic rejection to $66 \%$ of the maximum at $7375 \mathrm{eV}$. The fluorescence signal was monitored by using a 13element Ge solid state array detector 21 windowed on the $\mathrm{Fe} \mathrm{K} \alpha$ signal. During the experiment, count rates of approximately $37,000 / \mathrm{s}$ (total per element) were measured at 


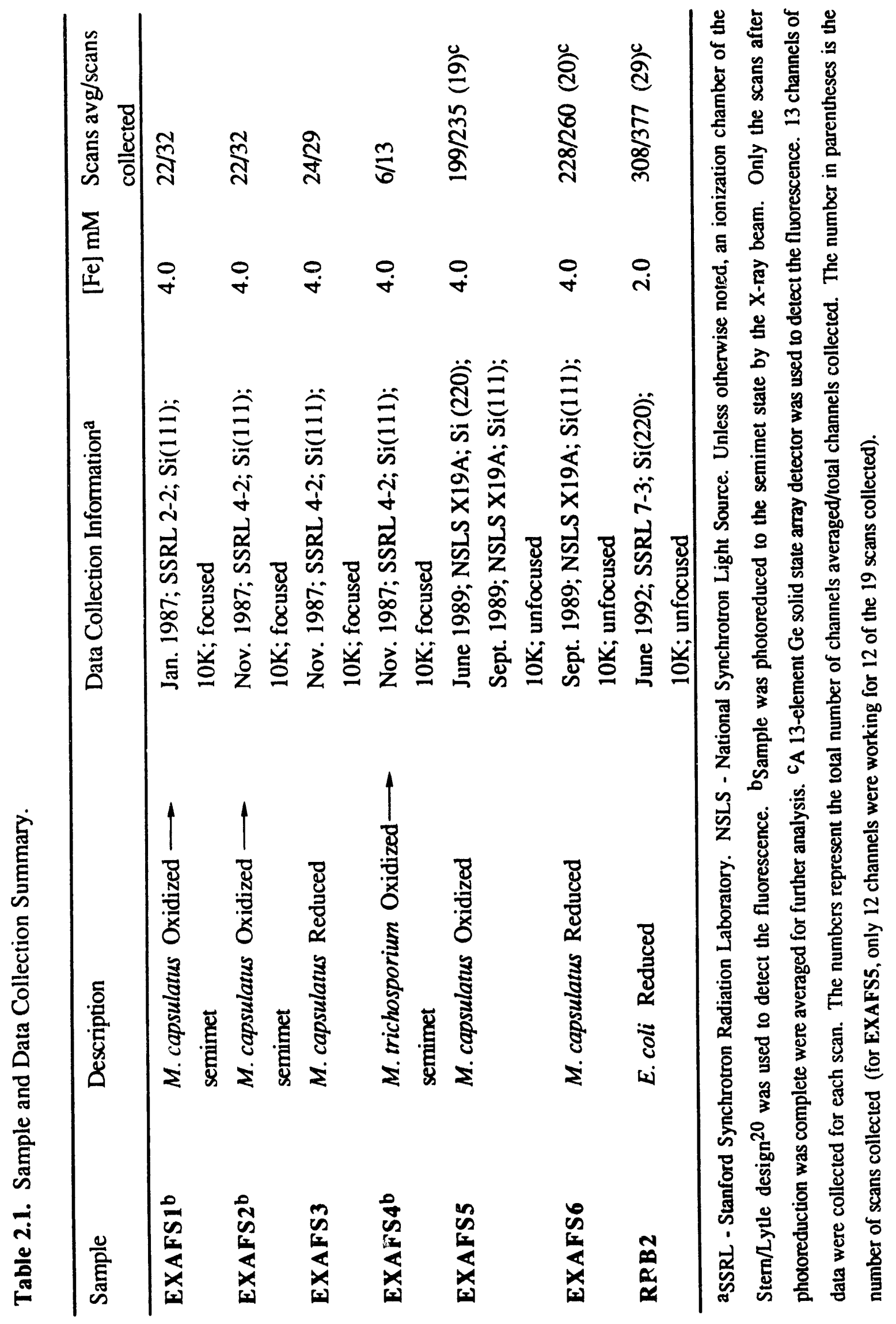


$7375 \mathrm{eV}$. The EXAFS5 sample was again measured at NSLS on beamline X-19A along with EXAFS6 under the same conditions as the first EXAFS5 experiment, except that a $\mathrm{Si}(111)$ double-crystal monochromator detuned to $50 \%$ of the maximum at $7820 \mathrm{eV}$ was employed. Count rates of approximately $28,000 / \mathrm{s}$ (total per element) at $7800 \mathrm{eV}$ were measured for the Ge detector during this experiment. The RRB2 data was measured at SSRL on beamline 7-3 using a $\mathrm{Si}(220)$ double-crystal monochromator detuned $45 \%$ at $7997 \mathrm{eV}$ at $10 \mathrm{~K}$ using the Ge fluorescence detector.

Data reduction was performed according to methods described in detail elsewhere 22 but briefly summarized here. Energies were calibrated by using an internal iron foil standard, ${ }^{23}$ assigning the first inflection point of the Fe absorption edge as $7111.2 \mathrm{eV}$. Calibrated scans were inspected individually and rejected if the signal-to-noise level was too high compared to the other scans as a result of beam instabilities or poor detector statistics, or because a beam loss occurred during the scan. In the case of the photoreduced samples EXAFS1, EXAFS2 and EXAFS4, the edge shifted during the first approximately four hours of irradiation. These scans were excluded from the subsequent weighted average of scans used in the data analysis.

The same oxidized protein sample (EXAFS5) was run twice with different monochromators (Table 2.1), giving rise to different background functions : , litch effects. An average of each data set was done separately (108/144 "scans" for the June 1989 data and 91/91 "scans" for the September 1989 data), the two averages were normalized to each other, and the EXAFS of the two files were averaged before fits were performed on the merged data. Monochromator glitches were edited out of the EXAFS5 individual averages, as well as the EXAFS3 and EXAFS6 average files using a single point replacement method $(2,4$ and 2 points total edited, respectively).

A pre-edge subtraction was performed by fitting the EXAFS region with a smooth polynomial which was extrapolated into the pre-edge region and subtracted. A threesegment spline was fit to the EXAFS region and subtracted and the data normalized to an edge jump of one. The spline was chosen so that it minimized residual low frequency background but did not reduce the EXAFS amplitude as checked by monitoring the Fourier transform of the EXAFS during the background subtraction process. The normalized, background-subtracted data were converted to $k$ space, where $k$ is the photoelectron wavevector defined by $\left[2 m_{e}\left(E-E_{0}\right) / \hbar^{2}\right]^{1 / 2}$. In this expression, $m_{e}$ is the electron mass, $E$ is the photon energy, $\hbar$ is Planck's constant divided by $2 \pi$, and $E_{0}$ is the threshold energy, $7130 \mathrm{eV}$ (where $k$ is defined to be zero).

Analysis was performed with non-linear least squares curve fitting techniques 22,23 using empirical phase and amplitude parameters, as described previously. ${ }^{22}$ The following 
models were used to obtain the empirical $\mathrm{Fe}-\mathrm{X}$ backscattering parameters of interest: $\mathrm{Fe}-\mathrm{O}$ and $\mathrm{Fe}-\mathrm{C}$ from $\left.[\mathrm{Fe} \text { (acetylacetonate) })_{3}\right] ; 24 \mathrm{Fe}-\mathrm{N}$ from $\left[\mathrm{Fe}(1,10 \text {-phenanthroline })_{3}\right]\left(\mathrm{ClO}_{4}\right)_{3} ; 25$ $\mathrm{Fe}-\mathrm{Fe}$ from the tribridged models $\left[\mathrm{Fe}_{2}(\mathrm{OH})(\mathrm{OAc})_{2}\left(\mathrm{HB}(\mathrm{pz})_{3}\right)_{2}\right]\left(\mathrm{ClO}_{4}\right)^{26 a}(\mathrm{FEHBPZOH})$ and $\left[\mathrm{Fe}_{2} \mathrm{O}(\mathrm{OAc})_{2}\left(\mathrm{HB}(\mathrm{pz})_{3}\right)_{2}\right]^{28 \mathrm{~b}}$ (FEHBPZO). Data for these model compounds were collected as described previously. ${ }^{15 a}$

For all the data presented, Fourier transforms (from $k$ to $\mathrm{R}$ space) of $3.5-12.5 \AA^{-1}$ and $3.5-10.8 \AA^{-1}$ were performed and two data ranges, from $4.0-12.0 \AA^{-1}$ and 3.8 $10.0 \AA^{-1}$, respectively, were fit. A gaussian window of $0.1 \AA$ was used for all transforms. The window widths used in the backtransforms (from $\mathbf{R}$ to $k$ space) are listed in the tables. They were kept as similar as possible to each other, as well as to the windows used to extract amplitude and phase parameters from the models, to minimize artifacts introduced by the Fourier filtering technique. All curve fitting was based on $k^{3}$-weighted data and applied to individual filtered shells as well as to wide-range filtered shells and to the raw data. Only the structure-dependent parameters, i.e. the distance and the number of atoms in the shell, were varied except where otherwise noted. For example, in the first shell fits the same Debye-Waller factor as determined for the models was used in fitting the proteins and the coordination number was varied. A "goodness-of-fit" parameter, $F$, was calculated as $\mathrm{F}=\left\{\left[k^{6}(\text { data }- \text { fit })^{2}\right] /(\text { no. of points })\right\}^{1 / 2}$ for each fit.

\subsection{Results of XAS Experiments}

The $k^{3}$-weighted EXAFS of the protein samples EXAFS2-EXAFS6 are presented in Figure 2.1 and the Fourier transforms in Figure 2.2 (for EXAFS1, see reference 15a). The oxidized protein EXAFS are compared to the EXAFS of the model compounds FEHBPZOH and FEHBPZO in Figure 2.3, and shows strong similarity to the FEHBPZOH EXAFS. This suggests that the structure of the active site of the protein resembles the iron center of the hydroxo-bridged model compound. The EXAFS of the semimet protein samples (Figure $2.1 \mathrm{~b}, \mathrm{c}$ ) have maxima (at $\sim 7$ and $11 \AA^{-1}$ ) that are only shoulders in the oxidized protein sample EXAFS. The overall features of the EXAFS of the semimet protein samples from the two bacterial species are similar, despite the higher noise-level for the $\boldsymbol{M}$. trichosporium OB3b sample (EXAFS4), as are the Fourier transforms (Figure 2.2b,c) indicating that the active sites of the hydroxylise from the two species are similar. The reduced protein EXAFS maximum around $k=6 \AA^{-1}$ (Figure $2.1 \mathrm{~d}, \mathrm{e})$ is shifted to lower $k$ relative to the semimet and oxidized protein EXAFS and the first shell peak in the Fourier transform is shifted to higher $R$ (Figure 2.2d,e) suggesting that the first shell coordination around $\mathrm{Fe}$ is at a longer distance in the reduced form of the 


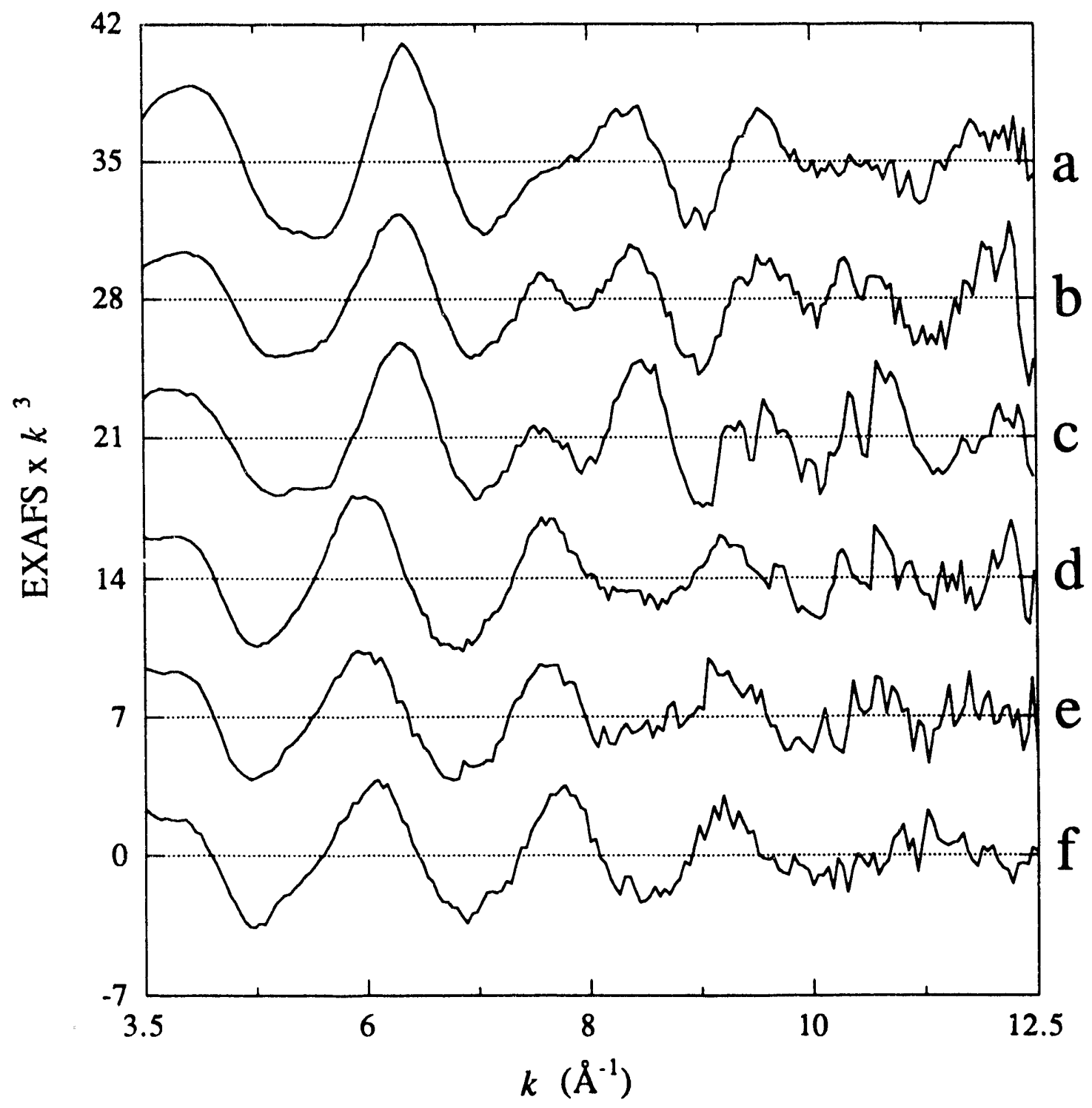

Figure 2.1. EXAFS data of the hydroxylase of MMO: (a) EXAFS5, (b) EXAFS2, (c) EXAFS4, (d) EXAFS3, (e) EXAFS6; and the B2 subunit of RR: (f) RRB2. Note the simpler metrical details of the reduced forms (d-f) over the oxidized and semimet forms. The data shown are the data used for Fourier transforms. 


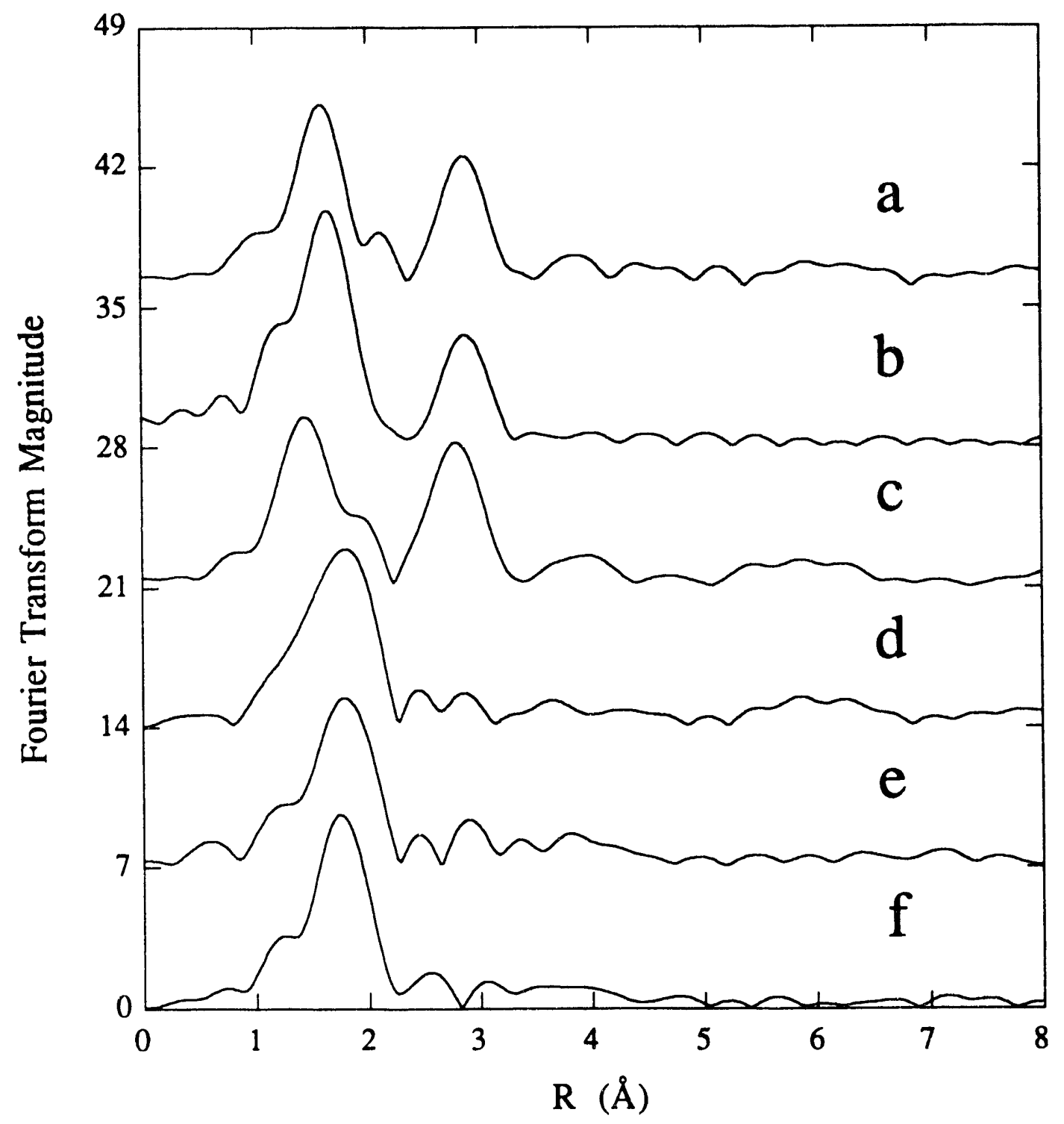

Figure 2.2. Fourier transforms of the EXAFS data presented in Figure 2.1. (a) EXAFS5, (b) EXAFS2, (c) EXAFS4, (d) EXAFS3, (e) EXAFS6, (f) RRB2. Note the absence of the second shell peak in the reduced sample data (d-f) 


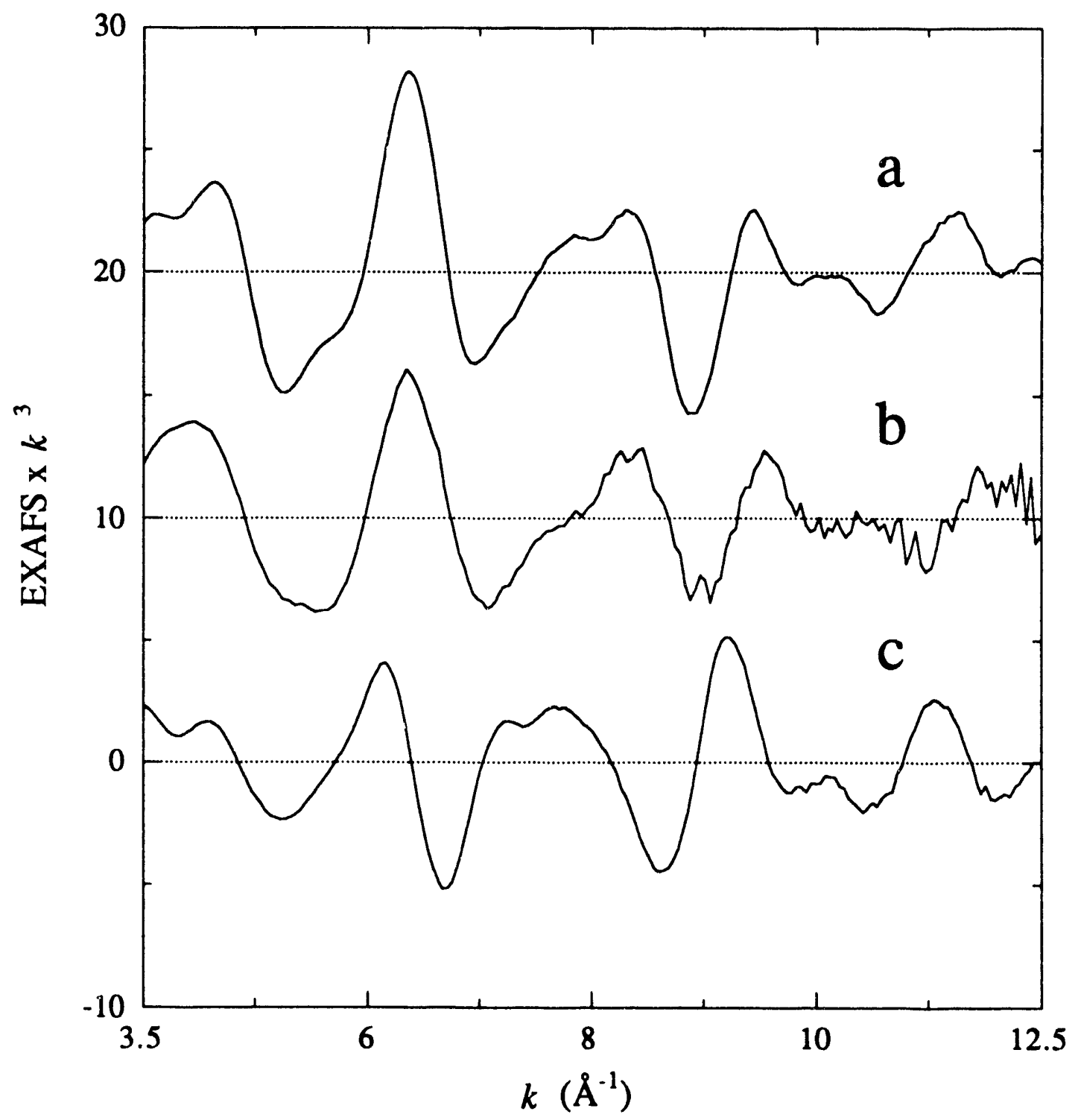

Figure 2.3. A comparison of the EXAFS data for the oxidized hydroxylase data and model compounds: (a) hydroxo-bridged FEHBPZOH, (b) EXAFS5, (c) oxo-bridged FEHBPZO. Note the similarity of the hydroxo-bridged model and hydroxylase EXAFS. 
hydroxylase relative to the semimet and oxidized forms. Only one peak is seen in the Fourier transform of the reduced protein EXAFS; the peak attributed to $\mathrm{Fe}-\mathrm{Fe}$ backscattering is absent, and the overall structure of the reduced protein EXAFS is less complicated than that of the semimet and oxidized protein EXAFS.

\subsubsection{Photoreduction of the Oxidized Hydroxylase}

The edge position of the first three oxidized protein A samples (EXAFS1, EXAFS2, and EXAFS4) shifted approximately $1.5 \mathrm{eV}$ to lower energy during the first four hours of exposure to the $\mathrm{X}$-ray beam. This shift in energy is due to a $1 \mathrm{e}^{-}$ photoreduction of the samples to the semimet state by the beam. For the same sample runs, the Fe foil calibrations insured that these shifts were well outside any experimental error. EPR studies on the photoreduced protein samples have verified that the dinuclear iron center was intact after the XAS experiment, giving rise to a typical $\mathrm{Fe}_{2}$ (II,III) spectrum with $g$ values of 1.92, 1.85, and 1.72.18 In Figure 2.4, the high resolution edge spectra of the non-photoreduced oxidized protein sample (EXAFS5), a reduced protein sample (EXAFS6, see Chapter 3), and a photoreduced protein sample (EXAFS7, see Chapter 3) are presented. Photoreduction of the oxidized protein sample did not occur, due either to the lower incident flux at NSLS compared to SSRL (unfocused bendir." magnet vs. focused wiggler beamlines) or because the new purification procedure ${ }^{18}$ used for this particular sample removed impurities that somehow mediated the photoreduction.

\subsubsection{Results of Fits to the Hydroxylase EXAFS Data}

2.3.2.1. First Shell Fits. The results of fits to the Fourier filtered first shell data are presented in Table 2.2. The widths of the windows used to isolate the first shell data are listed in Table 2.2. A single $\mathrm{N}$ or $\mathrm{O}$ contribution could not adequately fit the data, indicating that the first shell contains backscattering atoms at more than one distance and possibly of more than one type. The data could be fit with two contributions, however more than one minima was found, depending on the initial relative $\mathrm{Fe}-\mathrm{N}\left(\mathrm{R}_{\mathrm{N}}\right)$ and $\mathrm{Fe}-\mathrm{O}$ $\left(R_{O}\right)$ bond lengths. EXAFS can not normally discriminate between backscatterers of similar strength as is the case with $\mathrm{N}$ and $\mathrm{O}$ (which differ only by one in atomic number), giving rise to the multiple minima obtained from the fits. The $\mathrm{N}$ and $\mathrm{O}$ parameters were correlated over the range of data available, so the fit results reported will correspond to coordination-weighted average distances. The accuracy of this approach has been confirmed in fits to a number of dinuclear non-heme iron models (see Chapter 4). 


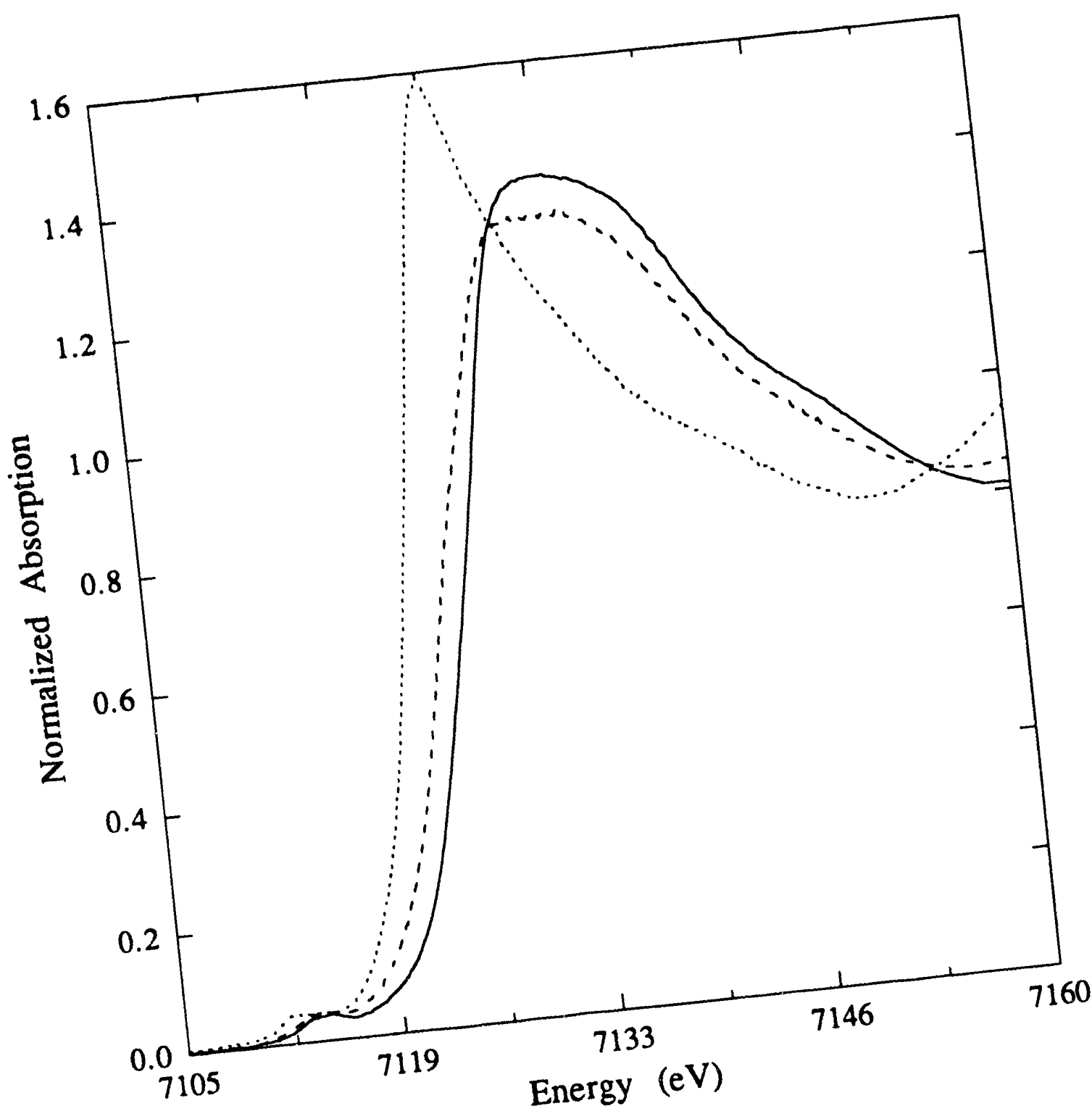
Figure 2.4. The edge positions of the diferric (EXAFS5, solid), photoreduced semimet
(EXAFS7, dash) and diferrous (EXAFS6, dot) forms of the hydroxylase of MMO from M. capsulatus (Bath). 


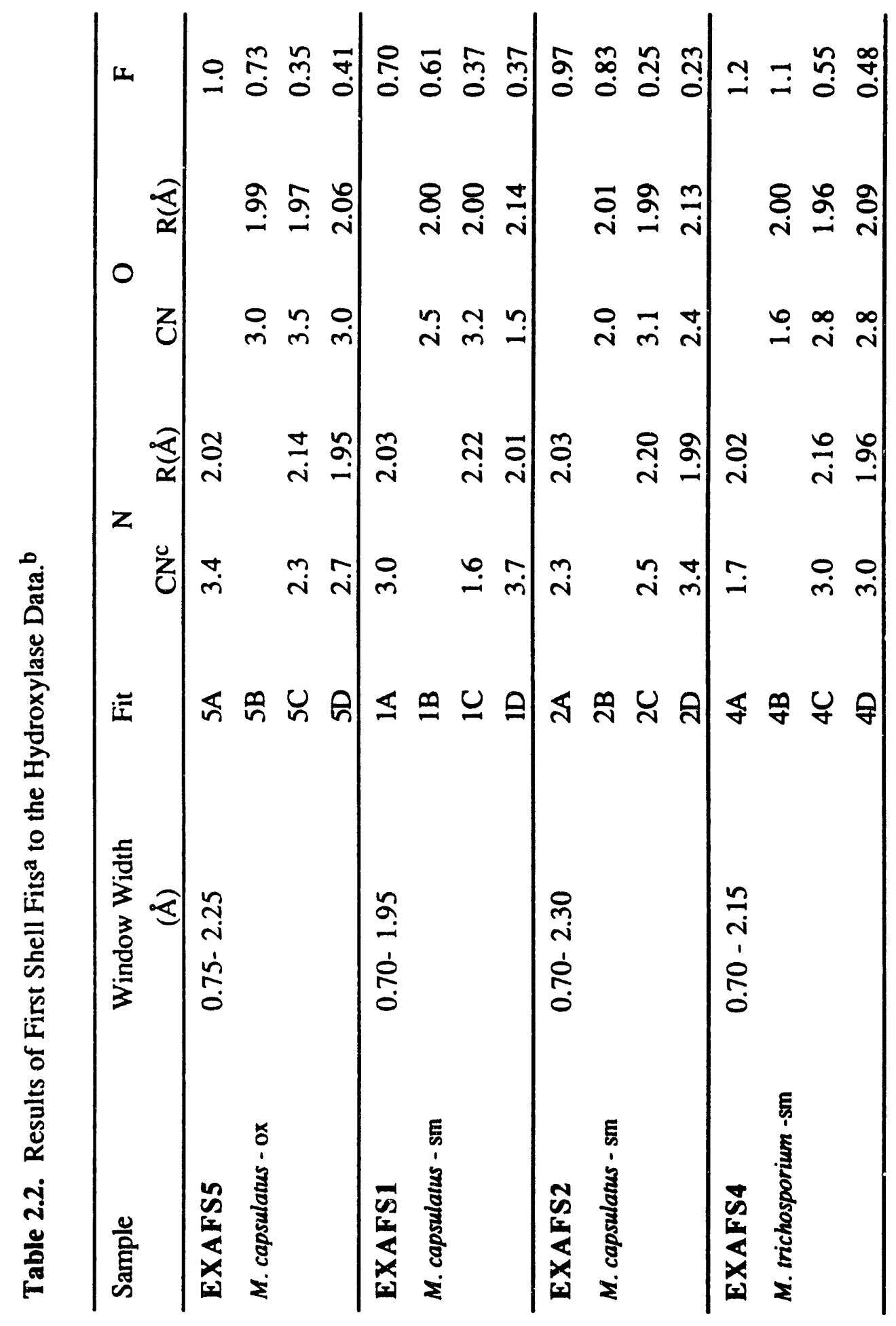




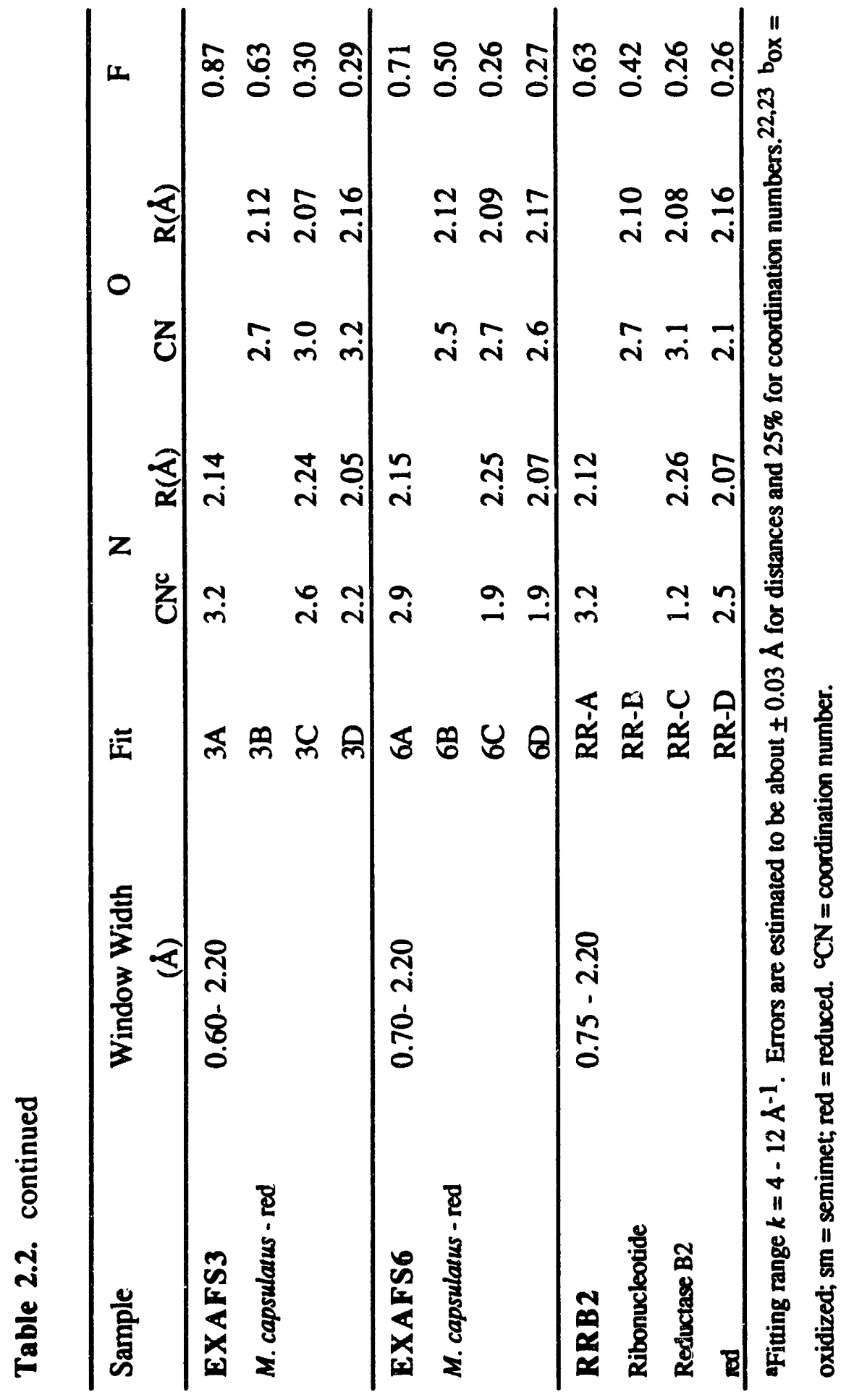


The average of the $\mathbf{R}_{\mathbf{N}}>\mathrm{R}_{\mathbf{O}}$ minima will be reported because Fe-N distances are generally longer than the $\mathrm{Fe}-\mathrm{O}$ distances in the model compounds of the dinuclear nonheme center. The coordination-weighted average first shell coordination for the $\mathbf{R}_{\mathbf{N}}<\mathrm{R}_{\mathrm{O}}$ minima was similar to the average of the other minima, with slightly lower total coordination number and average distance. For the oxidized hydroxylase sample (EXAFS5), an average first shell coordination of 5.8 N/O at $2.04 \AA$ was found (Table 2.2 , fit $5 \mathrm{C}$ ). Upon photoreduction to the semimet state, the first shell coordination distance increased to $4.8 \mathrm{~N} / \mathrm{O}$ at $2.07 \AA$ for EXAFS1 (Table 2.2, fit 1C), to $5.6 \mathrm{~N} / \mathrm{O}$ at $2.08 \AA$ for EXAFS2 (Table 2.2, fit 2C), and to $5.8 \mathrm{~N} / \mathrm{O}$ at $2.06 \AA$ for EXAFS4 (Table 2.2, fit 4C). The quality of the fits to the $M$. trichosporium $\mathrm{OB} 3 \mathrm{~b}$ data set is worse because of the higher noise-level in the data compared to that of $M$. capsulatus (Bath) (only 6 scans were averaged for the $M$. trichosporium $\mathrm{OB} 3 \mathrm{~b}$ ). The average first shell coordination of the reduced protein samples (EXAFS3 and EXAFS6) was found to be $5.1 \mathrm{~N} / \mathrm{O}$ at $2.15 \AA$ (Table 2.2, fits 3C and 6C). Although the average distance determined is the same for both reduced protein samples, the average coordination number for EXAFS3 was more than 5 N/O while that for EXAFS6 was less than 5 N/O, with the difference accounted for primarily in the coordination number of nitrogen.

Fits were also performed on all the protein samples to probe the presence of a short $(\sim 1.80 \AA) \mathrm{Fe}-\mathrm{O}$ distance, indicative of an oxo bridged center. When a short $\mathrm{Fe}-\mathrm{O}$ distance was included, either negative coordination numbers resulted, or the total oxygen contribution was split between two oxygen waves at distances on the order of $2.0 \AA$. Given the previously shown high sensitivity of EXAFS to the presence or absence of the short oxo bridge, 16 this result, together with the similarity of the oxidized hydroxylase EXAFS spectra to that of FEHBPZOH and the dissimilarity to that of FEHBPZO (Figure 2.3), clearly indicates that the hydroxylase of methane monooxygenase does not have an oxo bridge in its dinuclear iron center.

2.3.2.2. Second Shell Fits. The FEHBPZOH model was chosen as a model for the Fe-Fe interaction in MMO for two reasons: first, in this model the distribution of atoms in the second shell is such that the iron is more isolated from second shell carbon and nitrogen than in the analogous oxo-bridged model, FEHBPZO (the nearest $\mathrm{C} / \mathrm{N}$ shell is $0.26 \AA$ in FEHBPZOH and $0.05 \AA$ in FEHBPZO ${ }^{16}$ ); second, since the first shell fits indicate that there is not a $\mu$-oxo bridge in the iron center, a non- $\mu$-oxo bridged model should provide more reliable parameters to use in fits of the protein active site.

Using the empirical amplitude and phase parameters obtained from the FEHBPZOH model to fit the second shell hydroxylase data, two Fe-Fe minima were 
found, one at $\sim 3.0 \AA$ and one at $\sim 3.4 \AA$, depending on the initial Fe-Fe distance used in the fit (Table 2.3). In a subsequent series of Fe-only fits, stepping the initial $\mathrm{Fe}-\mathrm{Fe}$ distance value in intervals of 0.1 or $0.2 \AA$ from 2.6 to $4.2 \AA$, it was found that the calculated iron wave moved into phase with the maximum of the amplitude envelope of the second shell data roughly every $0.4 \AA$, with the best fit (minimum in F and best similarity in shape) and maximum coordination number occurring at the $3.4 \AA \mathrm{Fe}-\mathrm{Fe}$ distance for EXAFS1, EXAFS2 and EXAFS5. The same series of fits was performed by fixing the coordination number at 1 and varying the distance and the Debye-Waller factor, and a minimum in both F and the Debye-Waller factor was found at $3.4 \AA$ (Table 2.4, Figure 2.5) for EXAFS1, EXAFS2 and EXAFS5. For the noisier EXAFS4 data the results were ambiguous, with the fits giving nearly equal preference to the 3.4 and $3.0 \AA$ distances (see comment on shorter data range fits below). As shown in Table 2.3, the two minima obtained for Fe-only fits for EXAFS1, EXAFS2, EXAFS4, and EXAFS5 were essentially identical, $3.41-3.42 \AA$ and $3.03-3.04 \AA$ ('Table 2.3, fits 1E-F, 2E-F, 4E-4F, and $5 \mathrm{E}-\mathrm{F}$ ). The longer $\mathrm{Fe}-\mathrm{Fe}$ distance gave a better fit with a larger coordination number and lower $\mathrm{F}$ value than the shorter $\mathrm{Fe}$-Fe distance, however the metrical details of the data were not fully explained by the iron contribution, suggesting that something in additon to iron needed to be added to the fits.

The addition of a carbon contribution to the second shell fits, while improving the quality of the fits, confused the details of the fits. The iron and carbon shells were strongly correlated, affecting both the Fe coordination number and distance. The results were once more quite similar for EXAFS1, EXAFS2 and EXAFS5, but the Fe-Fe distance was shortened to 3.35 - $3.38 \AA$ and the Fe coordination dropped by a factor of 2 or more when a short ( $3.0 \AA) \mathrm{Fe}-\mathrm{C}$ contribution was included (Table 2.3, fits $1 \mathrm{~J}, 2 \mathrm{~J}$, and $5 \mathrm{~J}$ ). Attempts to fit the data with the $3.4 \AA \mathrm{Fe}$-distance and a longer $\mathrm{Fe}-\mathrm{C}$ distance resulted in a second, less well-defined fit with an $\mathrm{Fe}-\mathrm{C}$ distance of $\sim 3.3 \AA$, and increased $\mathrm{Fe}-\mathrm{Fe}$ distances of 3.45 - $3.47 \AA$ and coordination numbers (Table 2.3, fits 1I, 2I, and 5I). Fits with a short $\mathrm{Fe}-\mathrm{Fe}$ distance and a short $\mathrm{Fe}-\mathrm{C}$ distance resulted in chemically unreasonably short $\mathrm{Fe}-\mathrm{Fe}$ distances and a decrease in the $\mathrm{Fe}$ coordination number (Table 2.3 , fits $1 \mathrm{~K}, 2 \mathrm{~K}$, and $5 \mathrm{~K}$ ). For EXAFS4 the $3.4 \AA$ Fe contribution was essentially overshadowed by the $3.04 \AA \mathrm{Fe}$ $\mathrm{C}$ wave, and showed very high correlation of the coordination numbers for the fit consisting of the long Fe/long $\mathrm{C}$ distances (Table 2.3, fit $4 \mathrm{I}$ ). The best fit occurred at a very short Fe-Fe distance with the $3.04 \AA \mathrm{Fe}-\mathrm{C}$ distance (Table 2.3, fit 4J). C-only fits to the data revealed two Fe-C fit minima at $\sim 3.0$ and $3.4 \AA$ (Table 2.3, fits 1G-H, 2G-H, 4G$\mathrm{H}$, and $5 \mathrm{G}-\mathrm{H}$ ), with a strong preference for the minimum corresponding to the $3.0 \AA$ 


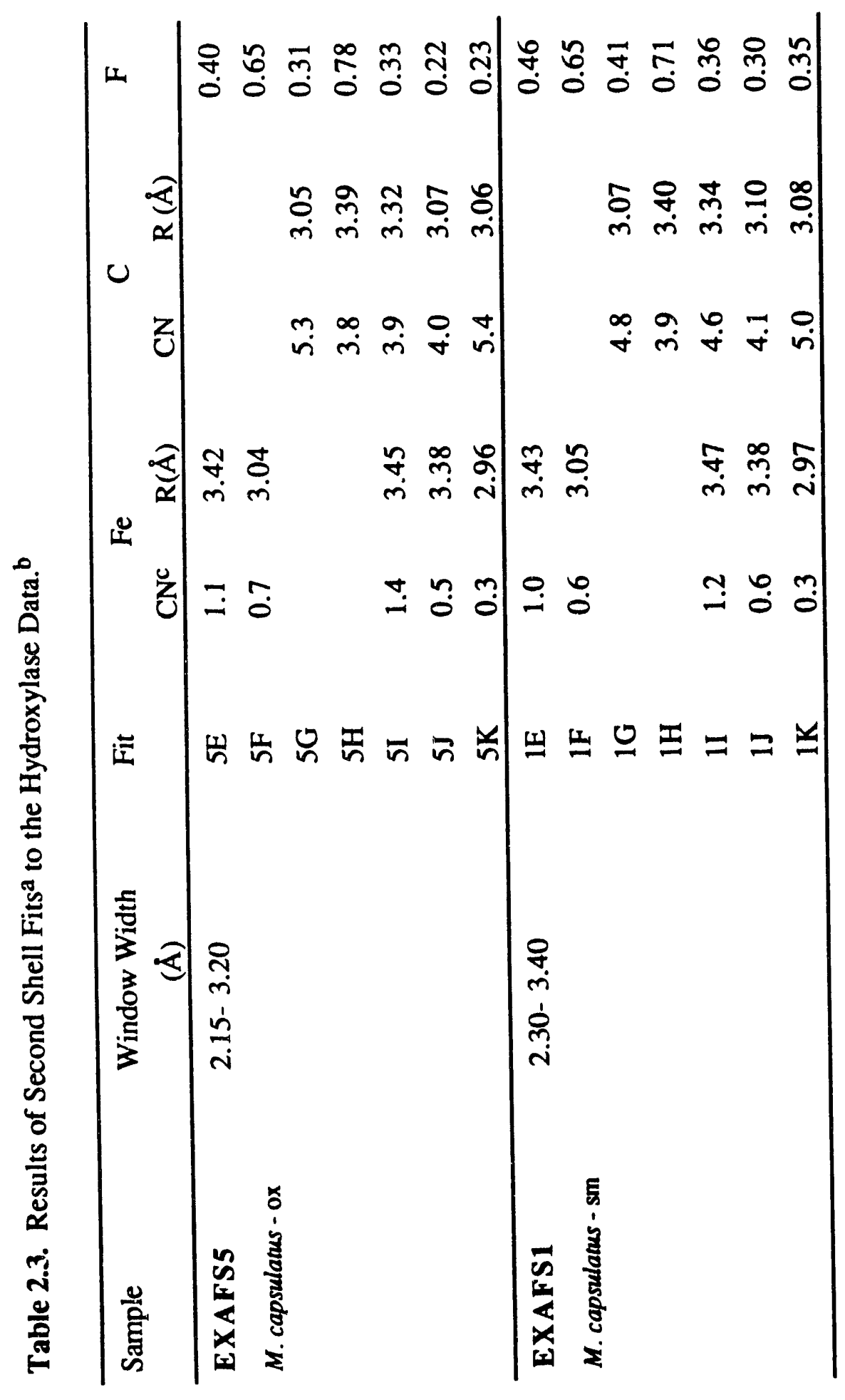




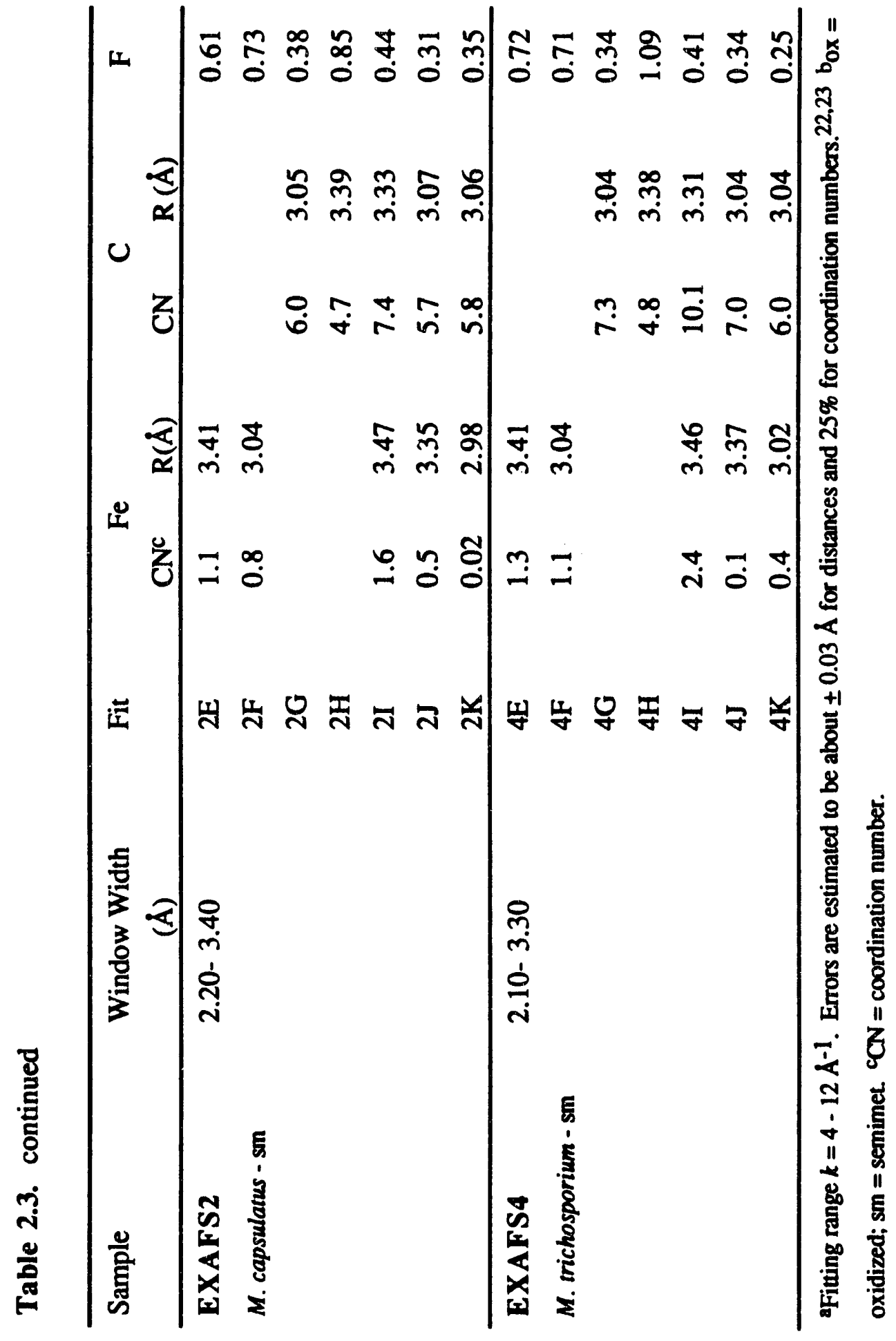


Table 2.4. Iron Fits to Second Shell Data for Oxidized and Semimet Hydroxylase Samples. ${ }^{\mathrm{a}}$

\begin{tabular}{lcclcccccc}
\hline \multicolumn{3}{c}{ EXAFS5 } & \multicolumn{3}{c}{ EXAFS2 } & \multicolumn{3}{c}{ EXAFS4 } \\
\hline $\begin{array}{l}\text { Fe-Fe } \\
(\AA)\end{array}$ & DWb & F & $\begin{array}{l}\text { Fe-Fe } \\
(\AA)\end{array}$ & DW & F & \multicolumn{2}{c}{ Fe-Fe } & DW & F \\
\hline 3.03 & 0.08240 & 0.67 & 3.04 & 0.07891 & 0.75 & 3.04 & 0.07452 & 0.68 \\
3.42 & 0.07578 & 0.40 & 3.41 & 0.07404 & 0.59 & 3.40 & 0.07229 & 0.70 \\
3.84 & 0.09105 & 0.88 & 3.83 & 0.09141 & 1.03 & 3.82 & 0.08795 & 1.20 \\
\hline
\end{tabular}

2For these fits, the coordination number was set to 1 and the Fe-Fe distance and Debye-Waller factor were varied. ${ }^{b} D W=$ Debye-Waller factor. Initial value $=0.07743$. 


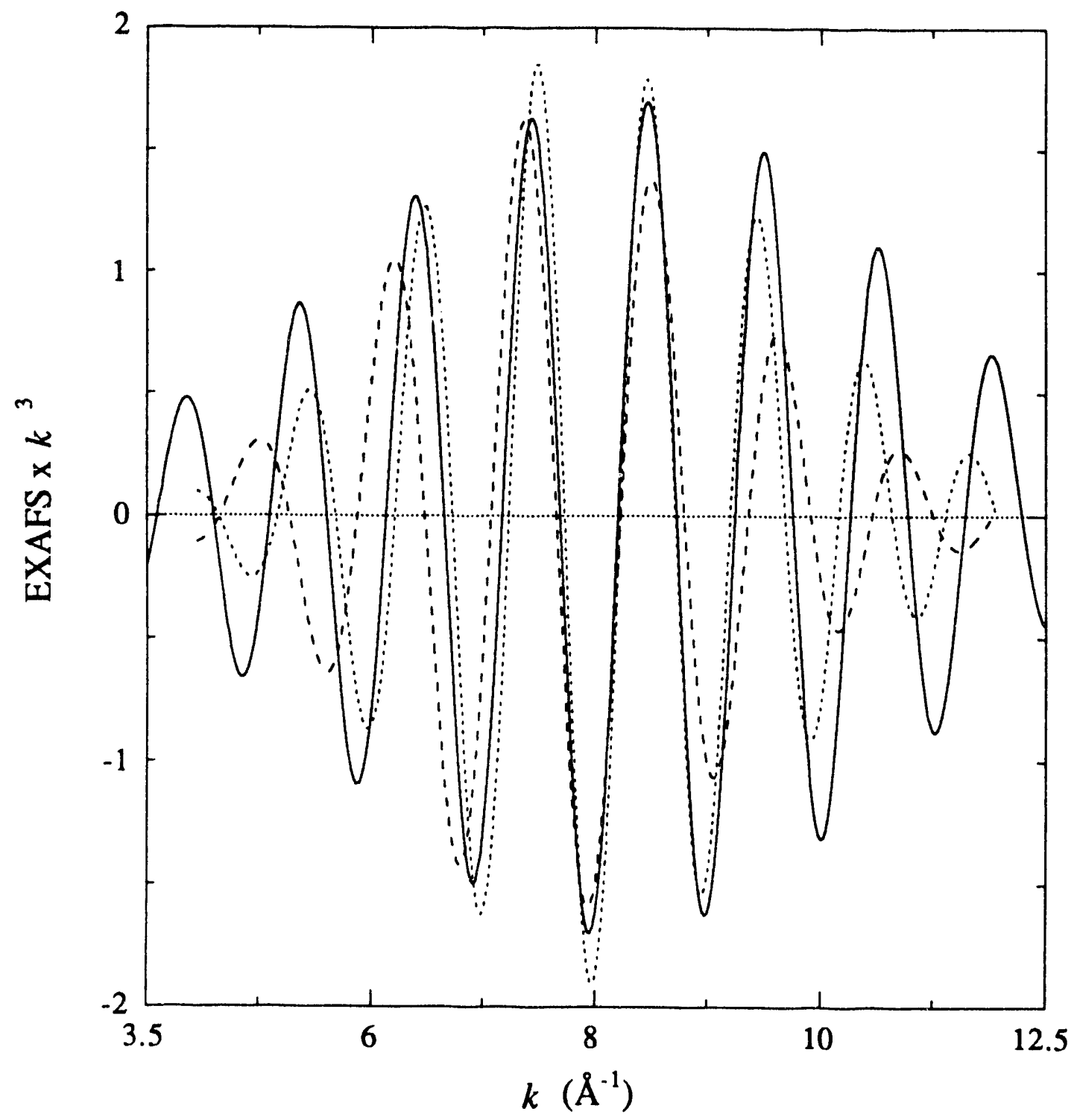

Figure 2.5. Fits to second shell data of EXAFS5 with iron. The fits were done by fixing the coordination number at 1 and varying the distance and Debye-Waller factor (see Table 2.4). Second shell data (solid), $3.42 \AA$ fit (dot), $3.03 \AA$ fit (dash). 
distance. This $3.0 \AA \mathrm{Fe}-\mathrm{C}$ minimum had a lower $\mathrm{F}$ value and was a somewhat better fit to the data than the Fe-only fits for all samples.

For the reduced samples EXAFS3 and EXAFS6, the Fourier transform shows very low intensity in the second shell region compared to the oxidized and semimet hydroxylase Fourier transforms (Figure 2.2d,e). Fits to the reduced hydroxylase data were attempted for a variety of backtransforms. No Fe-only, C-only or $\mathrm{Fe}+\mathrm{C}$ wave could successfully fit the data.

2.3.2.3. Wide Shell Fits. The same trends and distance information were obtained from fits to EXAFS spectra from wide backtransforms of the data for the oxidized and semimet protein samples (Table 2.5). Addition of $\mathrm{Fe}$ to the $\mathrm{N}$ and $\mathrm{O}$ contribution to the fits was necessary to explain the metrical details of the data and improved the fit dramatically (data for EXAFS5, Figure 2.6a,b ). The fit function dropped from a value of 1 for EXAFS5 (Table 2.5, fit 5L) to 0.52 (Table 2.5, fit 5M). The fits which included the short $3.0 \AA \mathrm{Fe}$ contribution were not as good as the fits with the longer Fe distance except for EXAFS4 (Table 2.5, fit $4 \mathrm{M}$ and $4 \mathrm{~N}$ ) which had a slightly lower $\mathrm{F}$ value for the short Fe minimum ( 0.87 for $3.03 \AA$ Fe vs. 0.94 for $3.41 \AA \mathrm{Fe}$ ). A comparison of the final fits to the filtered data with the non-filtered data is given in Figures 2.6d and 2.7.

The ability of the second shell Fe-C parameters to mimic the second shell contribution were tested in fits to the wide backtransforms of the data. Fits consisting of $\mathrm{N}, \mathrm{O}$ and a $3.0 \AA \mathrm{C}$ contribution had lower fit functions than the $\mathrm{N}, \mathrm{O}$ and Fe fits to the data (Table 2.5, $\mathrm{O}$ fits), although fits with a $3.4 \AA \mathrm{C}$ contribution were worse (Table 2.5, $\mathrm{P}$ fits). Addition of $\mathrm{C}$ to the $\mathrm{N} / \mathrm{O} / \mathrm{Fe}$ fits to the data resulted in the same correlation effects between the $\mathrm{Fe}$ and $\mathrm{C}$ coordination numbers and distances noted before in the fits to the second shell data. The addition of $\mathrm{C}$ to the $\mathrm{N}, \mathrm{O}$ and $\mathrm{Fe}$ contributions moderately improved the quality of the fit (Figure 2.6c), illustrating that something in addition to iron is required to adequately explain the data. All of the fits reported in Table 2.5 for the wide filter fit were repeated for the second first shell minimum found, corresponding to $R_{N}<R_{O}$. The same trends were seen with slightly higher $F$ values.

\subsubsection{Model Dependence of EXAFS Results. Given that the Fe-Fe} distance obtained in the fits of the oxidized and semimet protein data was similar to that of the FEHBPZOH model used to extract Fe-Fe backscattering parameters, we investigated the possibility of model dependence of the fit results. These same parameters were therefore used to fit the data of the FEHBPZO model compound, and parameters obtained from this model were used to fit the FEHBPZOH data. In both cases, two Fe-Fe distances were found, the correct one and one at about $0.4 \AA$ away, at 3.43 and $3.06 \AA$ for FEHBPZOH and at 3.15 and $3.52 \AA$ for FEHBPZO (see Chapter 4, Table 4.4). The 


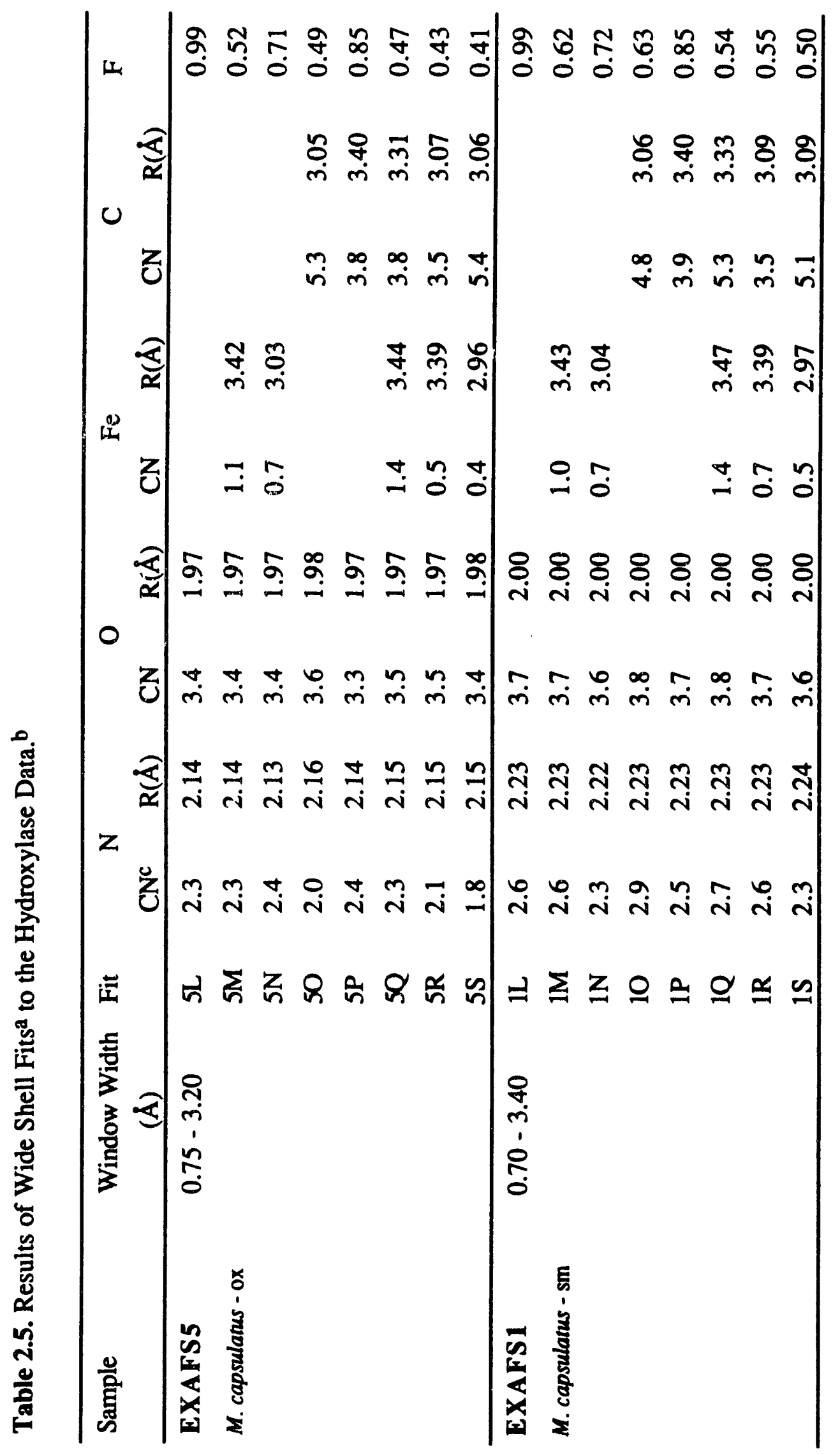




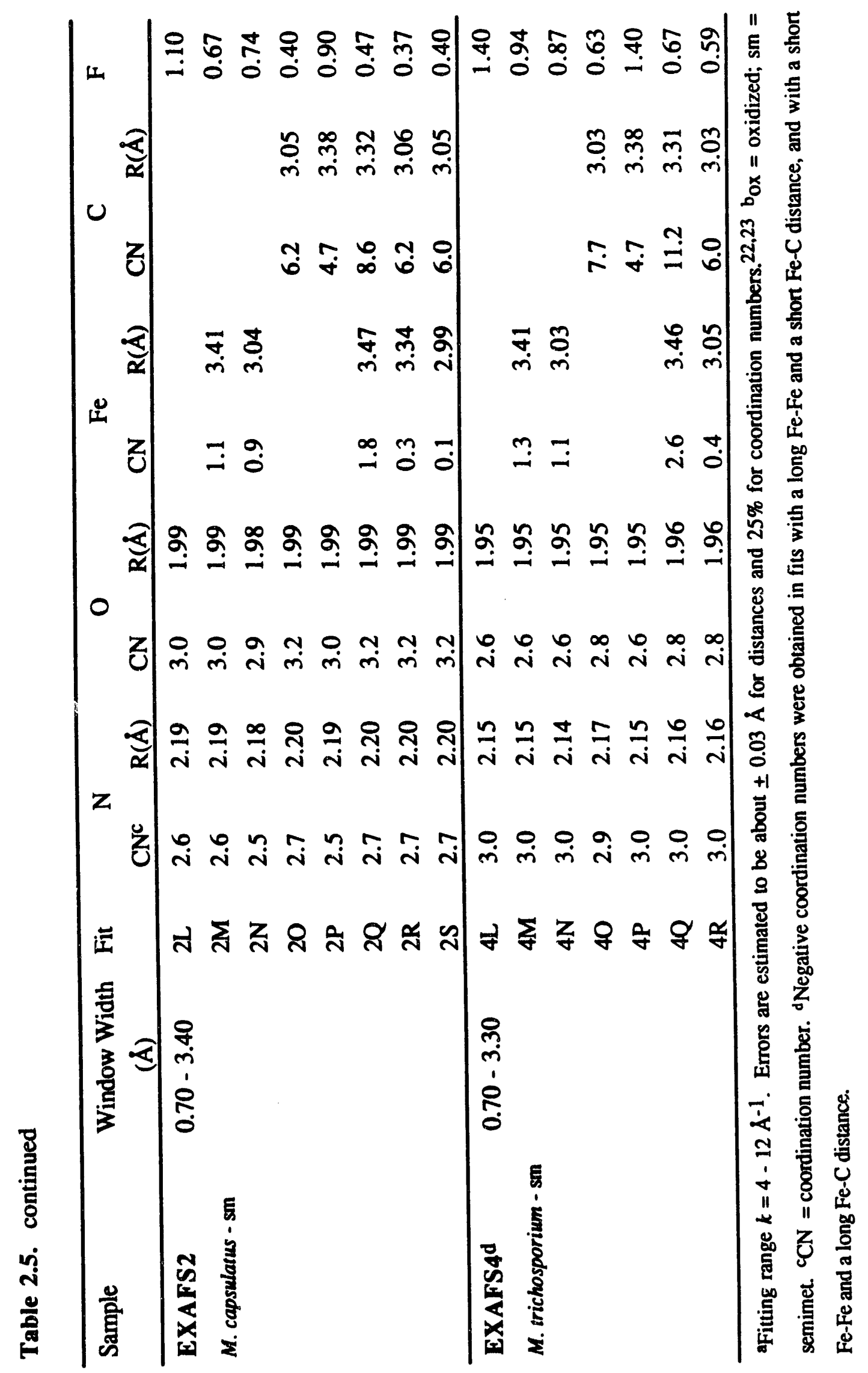




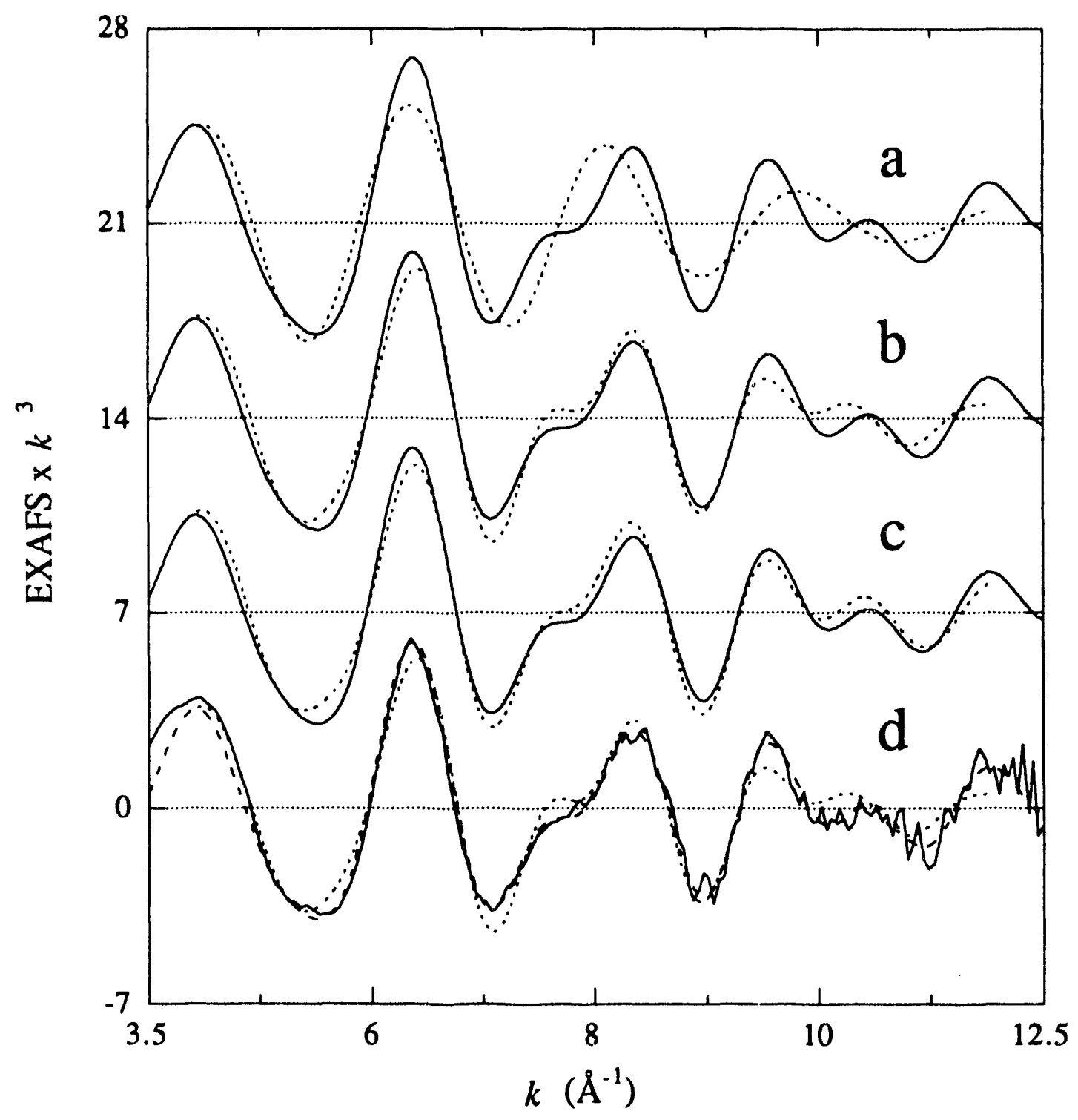

Figure 2.6. Fits to the Fourier filtered data $(0.75-3.2 \AA)$ for EXAFS5. The solid line represents the data and the dotted line is the fit. (a) Fit to the data with $\mathrm{N}$ and $\mathrm{O}$ (Table 2.5, fit 5L), (b) fit to the data with $\mathrm{N}, \mathrm{O}$ and $\mathrm{Fe}$ (Table 2.5, fit 5M), (c) fit to the data with $\mathrm{N}, \mathrm{O}$, Fe and C (Table 2.5, fit 5R), (d) the unfiltered data (solid), the filtered data (dash) and the fit to the filtered data (dot) (Table 2.5, fit 5M). 


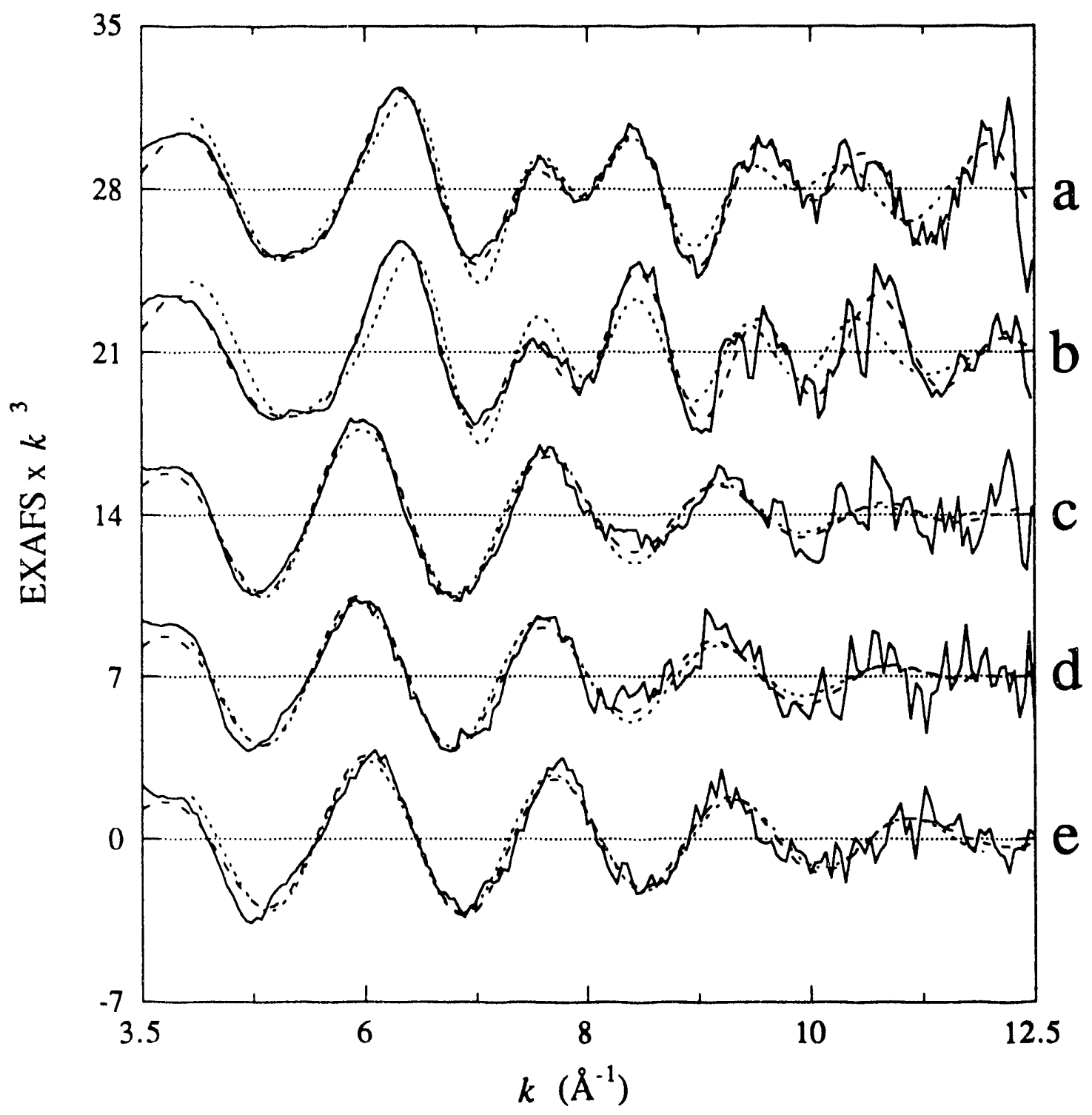

Figure 2.7. A comparison of the unfiltered data (solid), the filtered data (dash), and the fit to the filtered data (dot). (a) EXAFS2 (Table 2.5, fit 2M), (b) EXAFS4 (Table 2.5, fit 4M), (c) EXAFS3 (Table 2.2, fit 3C), (d) EXAFS6 (Table 2.2, fit 6C), (e) RRB2 (Table 2.2, fit RR-C). 
goodness-of-fit parameter, however, was lower for the longer distance obtained for the FEHBPZO model using Fe-Fe parameters from the hydroxo-bridged model (which has a longer Fe-Fe distance) and for the shorter distance obtained for FEHBPZOH using Fe-Fe parameters from the oxo-bridged model (which has a shorter Fe-Fe distance). There is thus an apparent model dependence in which the correct distance is obtained but the results are biased to give preference to the wrong distance if a model much different from the unknown is employed. Since first shell fits showed the absence of an oxo bridge, and the Fe-Fe and Fe-low $Z$ scattering distances are better separated in FEHBPZOH vs FEHBPZO, we believe that the use of parameters from the hydroxo-bridged model, FEHBPZOH, is most appropriate and that the $3.42 \AA$ distance is correct. While the absence of an oxo-bridge is clear, it remains to be established whether our current approach would successfully distinguish highly correlated Fe-Fe and Fe-C backscattering contributions at the same distance for well characterized complexes containing the $\mathrm{Fe}_{2}(\mathrm{OR})_{2}$ bridge unit. 27 The apparent model dependence of the second shell fits to dinuclear non-heme iron data, an effect which has also been addressed by others, 28 warrants careful consideration of the model used in the determination of Fe-Fe distances in dinuclear iron centers. An investigation of this effect is presented in Chapter 4.

2.3.2.5. Fits to the Unfiltered Data. These fits were repeated for the unfiltered data between $k=4$ and $12 \AA^{-1}$ (Table 2.6). Although the quality of the fits was generally worse due to the increased noise level of the unfiltered data, the results of the fits were essentially the same as those reported for the wide shell fits in Table 2.5 for EXAFS1, EXAFS2, EXAFS4, EXAFS5, and for the first shell fits in Table 2.2 for reduced samples EXAFS3 and EXAFS6. Some variation in the coordinaiton numbers of the low $\mathrm{Z}$ atoms were seen, however the total coordination number changed very little. The fits for the raw data, the wide shell data and the filtered data are thus consistent.

2.3.2.6. Fits over a Shorter Data Range. Beyond $k=10 \AA^{-1}$ the EXAFS data became increasingly noisy and beyond $k=13 \AA^{-1}$ the data was unusable. To verify that the results obtained for the $k=4-12 \AA^{-1}$ fits were not influenced by the increasing noise of the data between 10 and $12 \AA^{-1}$, the data from $3.5-10.5 \AA^{-1}$ was Fourier transformed into $R$ space and fit between 3.8 and $10 \AA^{-1}$. In general, the sum of low- $Z$ coordination in the first shell decreased and was closer to 5 than 6 , and the Fe coordination decreased to about 1 for the $3.4 \AA$ distance. Most importantly, fits to the shorter $k$ range for the oxidized and semimet protein samples with $\mathrm{N}, \mathrm{O}$ and $\mathrm{Fe}$ showed a strong preference for the long Fe-Fe distance. The fit with $\mathrm{N}, \mathrm{O}$ and $\mathrm{C}$ was no longer better than the long $\mathrm{Fe}$ Fe fit. In addition, the correlation of the second shell $\mathrm{C}$ and Fe parameters was not as strong over the shorter data range. The Fe-Fe distances stayed closer to the values found 


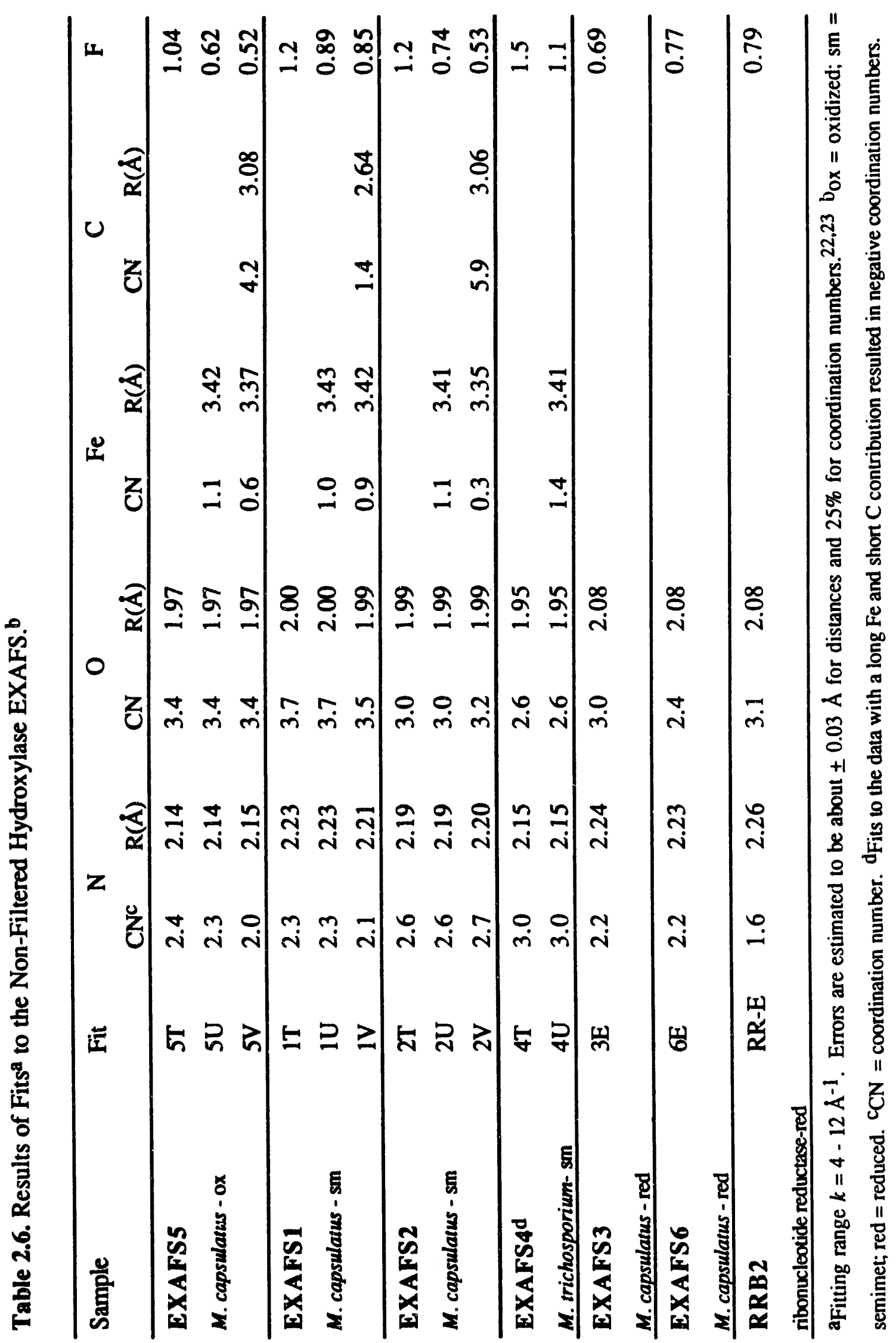


for the $\mathrm{N}, \mathrm{O}, \mathrm{Fe}$ fits to the data when $\mathrm{C}$ was added to the fit.

\subsubsection{Results of Fits to the Ribonucleotide Reductase EXAFS Data}

The EXAFS of the RRB2 sample (Figure 2.2f) and the reduced hydroyxlase samples (Figure $2.2 \mathrm{~d}, \mathrm{e}$ ) are very similar although the quality of the RRB2 data is better in the higher $k$ range, suggesting that the diiron centers in the two proteins have similar structrues There is a slight difference in the depth of the minimum above $k=8 \AA^{-1}$. The Fourier transforms of the data $\left(k=3.5-12.5 \AA^{-1}\right)$ are also very similar (Figure $2.3 \mathrm{~d}-\mathrm{f}$ ). Most interestingly, there is also no indication of a strong second shell contribution to the RRB2 data.

The results of fits to the filtered first shell data are presented in Table 2.2. As noted above, more than one contribution was required to fit the first shell data and in fits with two contributions, more than one minima was found (Table 2.2, fit RR-C and RR-D), depending on the initial values of $R_{N}$ and $R_{O}$. The average first shell coordination was found to be (for $R_{N}>R_{O}$ ) $4.3 \mathrm{~N} / \mathrm{O}$ at $2.13 \AA$. The filtered data, the fit to the filtered data and the non-filtered data are compared in Figure 2.7e.

\subsection{Discussion}

\subsubsection{Nature of the Hydroxylase Active Site}

The first shell data required two contributions to adequately fit the data, suggesting that the first shell contains atoms at different distance distributions. This in turn means the Fe atoms are located in a somewhat distorted environment. The EXAFS analysis clearly indicates the absence of a $\mu$-oxo bridge in the iron center of the diferric and semimet hydroxylase. The results are consistent with mixed $\mathrm{N}$ and $\mathrm{O}$ ligation to the iron atoms in the first coordination sphere. The average first shell distance for the oxidized hydroxylase was found to be $2.04 \AA$, and for the semimet hydroxylase samples, the average first shell distance was $2.06-2.09 \AA$. The EXAFS, Fourier transforms and fit results of the $\mathrm{Fe}$ (II)Fe(III) protein samples from both the $M$. capsulatus (Bath) and $M$. trichosporium OB3b samples (EXAFS1, EXAFS2 and EXAFS4, Figure 2.1d,e and Figure 2.2b,c) are very similar, suggesting that the diiron centers in the two hydroxylases are also structurally similar. For the reduced hydroxylase samples, the average first shell distance was determined to be $2.15 \AA$, and the average coordination number was somewhat lower than the value for the oxidized and semimet samples. 
In fits to the second shell data with either an Fe or a $\mathrm{C}$ contribution, two minima were found at $\sim 3.0$ and $3.4 \AA$. It is difficult to obtain unique fits to second coordination sphere EXAFS in dinuclear metalloproteins because the second shell generally contains carbon, nitrogen and/or oxygen scatterers at radial distances near that of the metal-metal separation. These low- $Z$ contributions are difficult to model adequately because of the typical wide distribution of distances. If well-ordered, the contribution of 4-6 such low-Z scatterers can be approximately of the same magnitude as that of a single metal scatterer. Although the backscattering envelope from Fe should maximize at higher $k$ values than envelopes from low- $Z$ scatterers, interference among several shells of low- $Z$ scatterers can effectively change the expected decreasing contribution to the EXAFS at higher $k$, and make the backscattering envelope of low-Z scatterers appear quite similar to that of the metal. 29 The use of $\mathrm{C}$ backscattering parameters derived from $\mathrm{Fe}$ (acac) 3 to model the low$\mathrm{Z}$ contribution to secoud shell data has often been described in the literature; however, its backscattering envelope is similar to that of a first row transition metal, most likely because of interference of its two closely lying $C$ second shells (Figure 2.8), resulting in the adequate fits to the second shell data with $\mathrm{C}$ and no $\mathrm{Fe}$ contribution. This phenomenon has been observed by others in fits to dinuclear non-heme iron models ${ }^{30}$ and dinuclear copper and mixed copper-iron dimers. 29

For bridged dinuclear model compounds, the second coordination sphere normally contains a large number of low- $Z$ atoms spread around $3.0 \AA$, always at a shorter distance than, and independent of, the Fe-Fe distance. ${ }^{16}$ It is, however, quite unlikely in any $\mathrm{Fe}$ coordination environment that outer $C$ shells would be so ordered that they give an EXAFS contribution as large as those seen for the oxidized and semimet hydroxylase samples. For example, even in Fe(TPP), which has 8 well-ordered $\alpha$-carbon atoms, the second shell peak in the Fourier transform is only about $1 / 3$ the height of the first shell peak. ${ }^{31}$ It would thus be expected that the strength of the $3.0 \AA$ Fe-Fe distance minimum derives from accidental coincidence of the frequency decrease in the phase of the Fe wave with a distance where there is an actual strong contribution to the data from $\mathrm{C} / \mathrm{N} / \mathrm{O}$ backscatterers. The $3.4 \AA$ distance, on the other hand, originates primarily from the Fe backscatterer, most likely mixed with other low-Z backscatterers. As has been pointed out elsewhere, ${ }^{30}$ longer Fe-C distances are expected to contribute less distinctly to the EXAFS because they originate from three-bcind $\mathrm{Fe}-\mathrm{C}$ distances, and thus are expected to have high thermal disorder.

The most reasonable interpretation for the second shell results is that the second shell contains an Fe-backscatterer at a distance of about $3.42 \AA$ for the oxidized and semimet samples, and that there is a shell of low-Z scatterers distributed around $3.05 \AA$. 


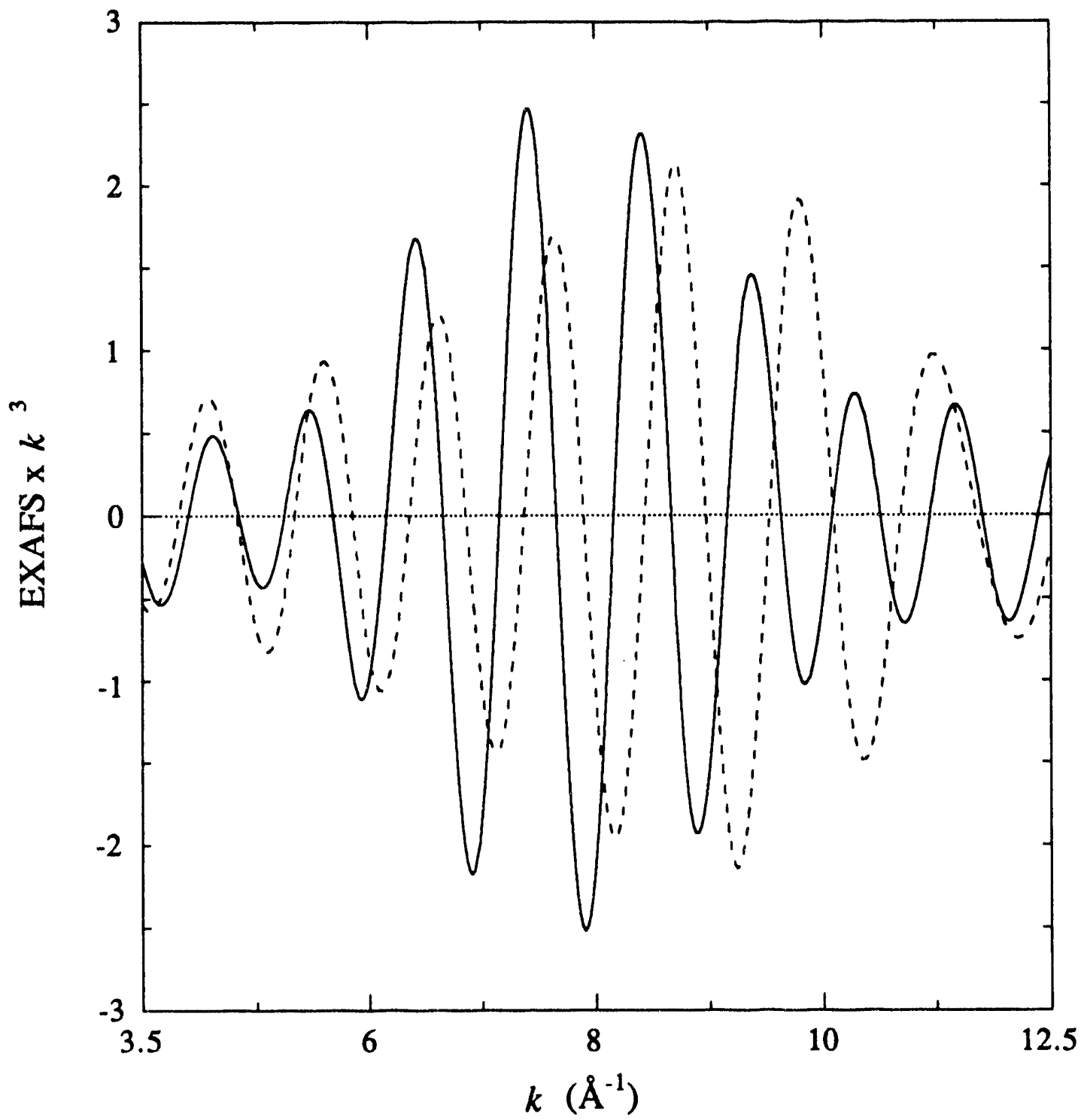

Figure 2.8. A comparison of the Fourier filtered second shell $\mathrm{Fe}-\mathrm{Fe}$ data from FEHBPZOH (solid) and the second shell Fe-C data from $\mathrm{Fe}(\mathrm{acac})_{3}$ (dash). Note that the $\mathrm{Fe}-\mathrm{C}$ wave reaches a maximum at higher $k$ than the $\mathrm{Fe}-\mathrm{Fe}$ wave. 
With Fe-N distances around 2.1-2.2 $\AA$, the Fe-C/N second shell distances lie in the 3.0-3.2 $\AA$ range, in accord with the present observations. The presence of $\mathrm{Fe}$ in the second shell is compatible with EPR data showing the existence of a dinuclear iron center. ${ }^{5 c, 15,18} \mathrm{The} \mathrm{Fe}$ Fe distance should not be affected by intervening-atom multiple scattering effects, because the minimum $\mathrm{Fe}-\mathrm{O}$ bridge distance of $2.0 \AA$ corresponds to a maximum $\mathrm{Fe}-\mathrm{O}-\mathrm{Fe}$ angle of $129^{\circ} .32$ The $3.4 \AA$ distance is compatible with the presence of a monodentate bridging group such as hydroxide, carboxylate or alkoxide. ${ }^{33}$ In addition, the value implies the existence of at least one other bridging group, most likely a syn, syn bidentate carboxylate. ${ }^{33 b}$ The J value of $-32 \mathrm{~cm}^{-1}$ measured for the $\mathrm{Fe}$ (II)Fe(III) form of the hydroxylase indicates weak antiferromagnetic exchange and is consistent with hydroxo, alkoxo, or monodentate carboxylato bridging ligands.

The peak associated with the metal-metal interaction is missing for both reduced hydroxylase samples and the reduced sample of ribonucleotide reductase B2 suggesting that the cause of the reduction in the Fe-Fe backscattering peak is inherent in the structures of the samples, and not in the data collection or analysis. The absence of a resolvable ironiron interaction in diferrous systems is not unusual. In deoxyhemerythrin, the iron-iron peak disappears upon warming the protein from $80 \mathrm{~K}$ to room temperature. ${ }^{34}$ Also, no $\mathrm{Cu}(\mathrm{I})-\mathrm{Cu}(\mathrm{I})$ peak is seen in the Fourier transform of deoxyhemocyanin data. ${ }^{35}$ In addition, the Fe-Fe peak could not be resolved in the semimet form (pink) of beef spleen purple acid phosphatase. ${ }^{36}$ This phenomenon could be caused by the loss of a bridge in the metal center upon reduction causing uncorrelated vibrations of the metal atoms (increased DebyeWaller factor), or by destructive interference in the second shell EXAFS between the $\mathrm{Fe}-\mathrm{Fe}$ wave and second shell $\mathrm{Fe}-\mathrm{N}, \mathrm{Fe}-\mathrm{C}$ and/or $\mathrm{Fe}-\mathrm{O}$ waves.

The change in the $\mathrm{Fe}$ and $\mathrm{C}$ coordination numbers and distances that occur when adding a $\mathrm{C}$ contribution to the $\mathrm{Fe}$ contribution in second shell fits is a result of the correlation between the $\mathrm{Fe}-\mathrm{Fe}$ and $\mathrm{Fe}-\mathrm{C}$ parameters. For model compounds, the correct $\mathrm{Fe}-\mathrm{Fe}$ distance is obtained when fitting with $\mathrm{Fe}$ alone, and a similar correlation effect is seen for fits with the same $\mathrm{Fe}-\mathrm{Fe}$ and $\mathrm{Fe}-\mathrm{C}$ parameters used in the protein fits (see Chapter 4). The strength in the second shell low- $Z$ atom backscattering could arise from second shell atoms arranged in a rigid structure such as the imidazole ring (present in histidine). The presence of rigid ligands has been associated with characteristic features in the outer shell region of Fourier transforms arising from contributions to the data from multiple scattering pathways. The means by which to model the multiple scattering contributions are still being developed. ${ }^{37}$ The relatively weak outer shell features in the hydroxylase samples suggest that such groups are not contributing significantly, therefore these approaches have not yet been attempted. 
In addition to determining the nature of the bridge and the $\mathrm{Fe}-\mathrm{Fe}$ separation in the active site of the MMO hydroxylase, the coordination of the iron atoms in the active site is of interest. The value of the quadropole splitting $\left(\Delta \mathrm{E}_{\mathrm{Q}}\right)$ obtained by Mössbauer studies, was $1.05 \mathrm{~mm} / \mathrm{s}$ which is larger than the quadropole splittings for hydroxo-bridged model compounds and smaller than oxo-bridged iron centers. ${ }^{18}$ The intermediate value can be

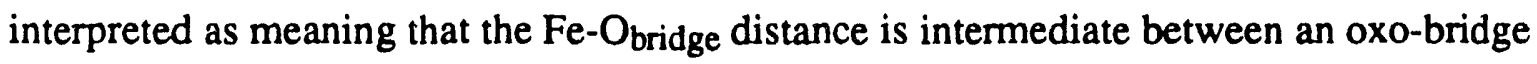
distance and a hydroxo-bridge distance, or that the iron atoms are penacoordinate and/or in a highly distorted octahedral environment. Although fits to the first shell EXAFS of the diferric protein suggest that the coordination number of the iron atoms is approximately six, it should be noted that EXAFS results represent the average of two not necessarily identical iron centers, and therefore may not readily distinguish between five- and six-coordinate iron atoms.

\subsubsection{Comparison to Proteins Containing Diiron Cores}

The spectroscopic similarities of the non-heme dinuclear iron proteins $(\mathrm{Hr}, \mathrm{RRB} 2$, PAP, Uf, and MMO) suggest that the active site structures may also be similar. A comparison of EXAFS studies of RRB230,38 with those on crystallographically characterized $\mathrm{Hr}^{30,34,39}$, have suggested that the two proteins possess similar active sites, consisting of a pair of triply-bridged iron atoms at a separation on the order of $3.2 \AA$ in the diferric form, with a $\mu$-oxo bridge and two syn,syn bidentate carboxylate bridges. Recently, crystallographic characterization of RRB2 revealed the presence of one, not two, bridging carboxylates. ${ }^{40}$ The average first shell coordination of oxyHr consists of $5 \mathrm{~N}$ and $\mathrm{O}$ atoms at $2.15-2.16 \AA$ while the average first shell coordination of diferric RRB2 consists of $5 \mathrm{~N}$ and $\mathrm{O}$ atoms at $2.04-2.06 \AA$ (excluding the oxo-bridge distance). In general, Fe- $\mathrm{N}$ bond lengths are longer than Fe-O bond lengths in iron complexes; the shorter average distance in the first shell coordination of RRB2 compared to oxyHr suggests that there are fewer nitrogens and more oxygens coordinated to the iron atoms in RRB2 than in oxyHr. ${ }^{38}$ The crystal structure has confimed that there are just two histidines ligands coordinated to the iron atoms in RRB2,40 as compared to five histidines in $\mathrm{Hr}{ }^{41}$ The average distance in the first coordination shell of the diferric form of MMO was found to be $2.01-2.04 \AA$, suggesting that $1:$ RRB2, there are more oxygens and fewer nitrogens coordinated to the iron atoms in MMO than in oxyHr. Unlike both diferric centers in RRB2 and oxyHr, there is no $\mu$-oxo bridge in the dinuclear iron center of MMO and the Fe-Fe distance, determined to be $3.42 \AA$, is longer than the Fe-Fe distance in either 
of those proteins, as one would expect for a di- or tri-bridged complex without an oxo bridge.

No evidence for a $\mu$-oxo bridge was found in the diferric form of beef spleen PAP (purple), although the presence of short $\mathrm{Fe}-\mathrm{O}$ (tyrosine) bonds in the protein could have obscured the contribution of the $\mathrm{Fe}-\mathrm{O}$ (bridge) chromophore to the data, 36 and therefore the possibility of an oxo bridge could not be ruled out. An Fe-Fe distance of $3.00 \AA$ was deduced by the FABM curve fitting technique with parameters obtained by using a monobridged ( $\mu$-oxo)diiron(III) model compound. The first shell coordination was similar to that reported here for MMO (Table 2.2, fit $5 \mathrm{C}$ ), with $3.0 \mathrm{~N} / \mathrm{O}$ at $2.13 \AA$ and $3.5 \mathrm{~N} / \mathrm{O}$ at $1.98 \AA$, or an average of $6.5 \mathrm{~N} / \mathrm{O}$ at $2.05 \AA$. EXAFS analysis of the diferric center in phosphate and arsenate bound forms of porcine uterine puple acid phosphatase (uteroferrin, Uf) have established that the diiron center does not contain a $\mu$-oxo bridge. ${ }^{42}$ The average first shell coordination was found to be $6 \mathrm{~N} / \mathrm{O}$ at $2.10-2.14 \AA$, suggesting either that there is greater $\mathrm{N}$ ligation to the iron center in Uf relative to $\mathrm{MMO}$, or that the ligation of the phosphate and arsenate anions distorts the iron center. The Fe-Fe distance was found to be $3.2-3.3 \AA$.

EXAFS analysis of the oxidized hydroxylase from Methylobacterium CRL-26 indicated an Fe-Fe distance of $3.05 \AA$ by using model corrected theoretical amplitude and phase parameters, while no evidence for or against an oxo bridge in the first shell was found owing to the limited range of the data. ${ }^{17}$ This value disagrees with the $\mathrm{Fe}-\mathrm{Fe}$ distance found in the present study for the same protein from a different species. Although it is possible that this difference is real, implying structurally different dinuclear iron centers, the discrepancy may arise from differences in analysis protocol. The first shell coordination of the hydroxylase from Methylobacterium CRL-26 consisted of 4 - 6 N/O at an average distance of $1.92 \AA$. This distance is much shorter than that found for any other diferric protein.

The average increase of $0.04 \AA$ in the first shell coordination distance upon photoreduction to the semimet state of MMO is similar to the increase in the average coordination sphere for oxidized PAP (purple) upon conversion to its one electron reduced (pink) form (6.0 N/O at $2.08 \AA$ ). ${ }^{36}$ The Fe-Fe distance of $3.41-3.43 \AA$ is slightly shorter than the Fe-Fe distance found for semimethemerythrin azide. ${ }^{30}$ Although one would expect the Fe-Fe distance to lengthen upon reduction of the diferric hydroxylase to the semimet form, our fit results do not show this trend. This suggests that the structure of the iron core does not change as much as the hemerythrin core in met- and semimethemerythrin azide $^{30}$ with reduction. It is also possible that improvement of the carbon backscattering parameters could give more precise results. 
Plots of individual contributions to the wide shell fits showed that the differences in the EXAFS at $\sim 7$ and $11 \AA$ for the oxidized and semimet samples are caused by changes in the N/O wave interaction with a more or less constant Fe wave. For the oxidized sample, the Fe wave at $\sim 7$ and $11 \AA$ maximizes almost directly on top of minima in the $\mathrm{N} / \mathrm{O}$ wave, giving rise to the weak shoulder in the data at those two points relative to data for the semimet hydroxylase. For the semimet sample, the N/O wave has shifted to lower frequency and the Fe wave maxima at the two points in question are no longer at a location corresponding to N/O minima, resulting in peak-like features. Due to the limited range of data available, the origin of the differences in the first shell data cannot be determined by EXAFS.

The average first shell coordination distance increased by an average of $0.12 \AA$ from the diferric to the diferrous state. The data and fits for the two diferrous protein samples are essentially identical, although EXAFS3 had higher coordination numbers than EXAFS6. For deoxyhemerythrin, the average first shell coordination was $2.15 \AA$ by EXAFS analysis, similar to the average first shell coordination of the diferrous hydroxylase. The data for reduced RRB2 was very similar to the reduced hydroxylase, with a $2.13 \AA$ average first shell distance. The peak associated with the metal-metal interaction is missing for both reduced hydroxylase protein samples and the reduced ribonucleotide reductase sample, suggesting that the absence of a Fe-Fe backscattering peak is inherent in the structure of the samples, and that therefore the active sites of the reduced hydroxylase of $\mathrm{MMO}$ and B2 subunit of RR may be similar. A $3.57 \AA$ Fe-Fe distance has been reported for deoxyhemerythrin. 28

\subsection{Conclusions}

MMO is similar spectroscopically to the other non-heme dinuclear iron proteins, however the EXAFS results clearly show that the active site structure is different from that in $\mathrm{Hr}$ and RRB2. Specifically, the hydroxylase of MMO does not have a $\mu$-oxo bridge in its iron center. The $3.4 \AA \mathrm{Fe}-\mathrm{Fe}$ separation and the absence of the short Fe-O bridge suggest that the iron atoms may contain an alkoxo, hydroxo or monodentate carboxylato bridge and one or two bidentate carboxylato bridges. The coordination number of the iron atoms is suggested to be 6 , however this determination is not conclusive. In addition, the average first shell distance of $2.04 \AA$ suggests that there are fewer histidine ligands in the iron center of MMO than in $\mathrm{Hr}$.

The determination of the second coordination sphere of the hydroxylase data has proven to be a non-trivial problem. The FEHBPZOH model is more likely to represent 
the protein active site than the FEHBPZO model, however, the apparent modeldependence of second shell fits to the data warrants caution regarding conclusions about Fe-Fe distances in dinuclear centers. Based on the information available, the most reasonable interpretation of the second shell data is that the Fe-Fe distance is $\sim 3.4 \AA$ and that there is a shell of low $\mathrm{Z}$ atoms at $\sim 3.0 \AA$ from the iron center.

\subsection{Acknowledgements}

The data were collected at the Stanford Synchrotron Radiation Laboratory and the National Synchrotron Light Source, Brookhaven National Laboratory, which are supported by the Department of Energy, Office of Basic Energy Sciences, Division of Chemical Sciences and Division of Materials Sciences. SSRL is also supported by the National Institutes of Health, Biomedical Resource Technology Program, Division of Research Resources. Grant support was provided by the National Science Foundation (CHE 8817702 to $\mathrm{KOH}$ ), the Gas Research Institute (5086-260-1209 to $\mathrm{HD}$ ), and the National Institute of General Medical Sciences (GM 32134 to SJL). 


\subsection{References and Notes}

1. Anthony, C. The Biochemistry of the Methylotrophs; Academic Press: London, 1982.

2. (a) Higgins, I. J.; Best, R. C.; Scott, D. Microbiol. Reviews 1981, 45, 556-590.

(b) Whittenbury, R.; Phillips, K. C.; Wilkinson, J. F. J. Gen. Microbiol. 1970, 61, 205218.

3. (a) Prior, S. D.; Dalton, H. J. Gen. Microbiol. 1985, 131, 155-163. (b) Dalton, H.; Prior, S. D.; Leak, D. J. Microb. Growth on Cl Cmpd., Proc. Int. Symp., 1983; Am. Soc. Microbiol.: Washington D. C., 1984; pp. 75-82. (c) Stanley, S. H.; Prior, S. D.; Leak, D. J.; Dalton, H. Biotech. Lett. 1983, 5, 487-492.

4. Tonge, G. M.; Harrison, D. E. F.; Higgins, I. J. Biochem J. 1977, 161, 333344.

5. (a) Colby, J.; Dalton, H. Biochem. J. 1976, 157, 495-497. (b) Stirling, D. I.; Dalton, H. J. Biochem. 1979, 96, 205-212. (d) Patel, R. N. Microb. Growth on C1 Cmpd., Proc. Int. Symp. 1983; Am. Soc. Microbiol.: Washington D. C., 1984; pp. 8390.

6. (a) Colby, J.; Stirling, D. I.; Dalton, H. Biochem. J. 1977, 165, 395-402. (b) Hou, C. T.; Patel, R. N.; Laskin, A. I.; Barnabe, N. FEMS Microbiol. Lett. 1980, 9, 267-270. (c) Leak, D. J.; Dalton, H. J. Gen. Microbiol. 1983, 129, 3487-3497. (d) Fox, B. G.; Borneman, J. G.; Wackett, L. P.; Lipscomb, J. D. Biochemistry 1990, 29, 6419-6427. (e) Stirling, D. I.; Colby, J.; Dalton, H. Biochem. J. 1979, 177, 361-364.

7. Colby, J.; Dalton, H. Biochem. J. 1978, 171, 461-468.

8. (a) Patel, R. N.; Savas, J. C. J. Bacteriol. 1987, 169, 2313-2317. (b) Patel, R. N. Arch. Biochim. Biophys. 1987, 252, 229-236.

9. (a) Colby, J.; Dalton, H. Biochem. J. 1979, 177, 903-908. (c) Lund, J.; Dalton, H. Eur. J. Biochem. 1985, 147, 292-296. (d) Prince, R. C.; Patel, R. N. FEBS 1986, 203, 127-130.

10. (a) Woodland, M. P.; Dalton, H. Anal. Biochem. 1984, 139, 459-462. (b) Woodland, M. P.; Dalton, H. J. Biol. Chem. 1984, 259, 53-59. (c) Fox, B. G.; Lipscomb, J. D. Biochem. Biophys. Res. Comm. 1988, 154, 165-170.

11. (a) Lund, J.; Woodland, M. P.; Dalton, H. Eur. J. Biochem. 1985, 147, 297 305. (b) Green, J.; Dalton, H. Biochem. J. 1986, 236, 155-162. (c) Green, J.; Dalton, H. Biochem. J. 1989, 259, 167-172. 
12. Green, J.; Dalton, H. J. Biol. Chem. 1985, 260, 15795-15801.

13. Woodland, M. P.; Patil, D. S.; Cammack, R.; Dalton, H. Biochim. Biophys. Acta 1986, 873, 237-242.

14. (a) Lippard, S. J. Angew. Chem. Int. Ed. Engl. 1988, 27, 344-361. (b) Sanders-Loehr, J. Iron Carriers and Iron Proteins; VCH Publishers Inc.: New York, 1989; pp. 373-466.

15. (a) Ericson, A.; Hedman, B.; Hodgson, K. O.; Green, I.; Dalton, H.; Bentsen, J. G.; Beer, R. H.; Lippard, S. J. J. Am. Chem. Soc. 1988, 110, 2330-2332. (b) DeWitt, J.; Hedman, B.; Ericson, A.; Hodgson, K. O.; Bentsen, J.; Beer, R.; Lippard, S. J.; Green, J.; Dalton, H. Physica B 1989, 158, 97-98.

16. Hedman, B.; Co, M. S.; Armstrong, W. H.; Hodgson, K. O.; Lippard, S. J. Inorg. Chem. 1986, 25, 3708-3711.

17. Prince, R. C.; George, G. N.; Savas, J. C.; Cramer, S. P.; Patel, R. N. Biochim. Biophys. Acta 1988, 952, 220-229.

18. DeWitt, J. G.; Bentsen, J. G.; Rosenzweig, A. C.; Hedman, B.; Green, J.; Pilkington, S.; Papaefthymiou, G. C.; Dalton, H.; Hodgson, K. O.; Lippard, S. J. J. Am. Chem. Soc. 1991, 113, 9219-9235.

19. This is the same data set as reported in ref. 15a; the data have been reanalyzed over the same range and with the same empirical amplitude and phase parameters used for the other samples in this paper for direct comparison.

20. (a) Stern, E. A.; Heald, S. M. Rev. Sci. Instr. 1979, 50, 1579-1582. (b) Lytle, F. W.; Greegor, R. B.; Sandstrom, D. R.; Marques, E. C.; Wong, J.; Spiro, C. L.; Huffman, G. P.; Huggins, F. E. Nucl. Instr. Meth. 1984, 226, 542-548.

21. Cramer, S. P.; Tench, O.; Yocum, M.; George, G. N. Nucl. Instr. Meth. Phys. Res. 1988, A266, 586-591.

22. (a) Scott, R. A. Meth. Enzymol. 1985, 117, 414-459. (b) Cramer, S. P.; Hodgson, K. O.; Stiefel, E. I.; Newton, W. E. J. Am. Chem. Soc. 1978, 100, 27482761. (c) Cramer, S. P.; Hodgson, K. O. Prog. Inorg. Chem. 1979, 15, 1-39.

23. Scott, R. A.; Hahn, J. E.; Doniach, S.; Freeman, H. C.; Hodgson, K. O. J. Am. Chem. Soc. 1982, 104, 5364-5369.

24. (a) Iball, J.; Morgan, C. H. Acta Cryst. 1976, 23, 239-244. (b) Roof, Jr., R. B. Acta Cryst. 1956, 9, 781-786.

25. Johansson, L. Chem. Scr. 1976, 9, 30-35. The crystal structure of the perchlorate salt has not been determined, but the $\left[\mathrm{Fe} \text { (phenanthroline) }{ }_{3}\right]^{2-}$ complex structure can be 
assumed to be identical with that of the corresponding iodide salt (Johansson, L.; Molund, M.; Oskarsson, A. Inorg. Chim. Acta 1978, 31, 117-123).

26. (a) Armstrong, W. H.; Lippard, S. J. J. Am. Chem. Soc. 1984, 106, 4632-4633.

(b) Armstrong, W. H.; Spool, A.; Papaefthymiou, G. C.; Frankel, R. B.; Lippard, S. J. J. Am. Chem. Soc. 1984, 106, 3653-3667.

27. Snyder, B. S.; Patterson, G. S.; Abrahamson, A. J.; Holm, R. H. J. Am. Chem. Soc. 1989, 11, 5214-5223 and references therein.

28. Zhang, K.; Stern, E. A.; Ellis, F.; Sanders-Loehr, J.; Shiemke, A. K. Biochemistry 1988, 27, 7470-7479.

29. Scott, R. A.; Eidsness, M. K. Comments Inorg. Chem. 1988, 7, 235-267.

30. Scarrow, R. C.; Maroney, M. J.; Palmer, S. M.; Que, L. Jr.; Roe, A. L.; Salowe, S. P.; Stubbe, J. J. Am. Chem. Soc. 1987, 109, 7857-7864.

31. Cramer, S. P. Ph.D. Thesis, Stanford University, 1977.

32. Co, M. S.; Hendrickson, W. A.; Hodgson, K. O.; Doniach, S. J. Am. Chem. Soc. 1983, 105, 1114-1150.

33. (a) Kurtz, D. M., Jr. Chem. Rev. 1990, 90, 585-606. (b) Rardin, R. L., Jr.; Tolman, W. B.; Lippard, S. J. New J. Chem. 1991, 15, 417-430.

34. Elam, W. T.; Stern, E.A.; McCallum, J. D.; Sanders-Loehr, J. J. Am. Chem. Soc. $1983,105,1919-1923$.

35. Co, M. S.; Hodgson, K. O. J. Am. Chem. Soc. 1981, 103, 3200-3201.

36. Kauzlarich, S. M.; Teo, B. K.; Zirino, T.; Burman, S.; Davis, J. C.; Averill, B. A. Inorg. Chem. 1986, 25, 2781-2785.

37. (a) Co, M. S.; Scott, R. A.; Hodgson, K. O. J. Am. Chem. Soc. 1981, 103, 986-988. (b) Hasnain, S. S.; Ed. Synchrotron Radiation and Biophysics; Ellis Horwood Ltd.: Chichester, 1990; Chapters 3 and 4.

38. (a) Scarrow, R. C.; Maroney, M. J.; Palmer, S. M.; Que, L. Jr.; Salowe, S. P.; Stubbe, J. J. Am. Chem. Soc. 1986, 108, 6832-6834. (b) Bunker, G.; Petersson, L.; Sjöberg, B.-M., Sahlin, M.; Chance, M.; Chance, B.; Ehrenberg, A. Biochemistry 1987, 26, 4708-4716.

39. (a) Hendrickson, W. A.; Co, M. S.; Smith, J. L.; Hodgson, K. O.; Klippenstein, G. L. Proc. Natl. Acad. Sci. USA 1982, 79, 6255-6259. (b) Elam, W. T.; Stern, E. A.; McCallum, J. D.; Sanders-Loehr, J. J. Am. Chem. Soc. 1982, 104, 6369-6373. (c) Maroney, M. J.; Scarrow, R. C.; Que, L. Jr.; Roe, A. L.; Lukat, G. S.; Kurtz, D. M. Jr. Inorg. Chem. 1989, 28, 1342-1348. 
40. (a) Nordlund, P.; Uhlin, U.; Westergren, C.; Joelsen, T.; Sjöberg, B.-M.; Eklund, H. FEB 1989, 258, 251-254. (b) Nordlund, P.; Sjöberg, B.-M.; Eklund, H. Nature, 1990, 345, 593-598.

41. (a) Stenkamp, R. E.; Sieker, L. C.; Jensen, L. H.; McCallum, J. D.; SandersLoehr, J. Proc. Natl. Acad. Sci. USA 1985, 82, 713-716. (b) Stenkamp, R. E.; Sieker, L. C.; Jensen, L. H. J. Am. Chem. Soc. 1984, 106, 618-622.

42. True, A. E., Scarrow, R. C.; Holz, R. C.; Que, L., Jr. Inorg. Biochem. 1991, 43,545 , and personal communication. 
Chapter 3

Structural Studies of the Diiron Center in Methane Monooxygenase in the Presence of Substrate and the Regulatory Protein of the MMO Enzyme System 


\subsection{Introduction}

Soluble methane monooxygenase (MMO) is a multi-component enzyme system which catalyzes the $\mathrm{NAD}(\mathrm{P}) \mathrm{H}$ - and $\mathrm{O}_{2}$ - dependent hydroxylation of methane to methanol in methane-metabolizing bacteria. ${ }^{1}$ MMO from Type I methanotroph Methylococcus capsulatus (Bath) ${ }^{2}$ and Type II methanotroph Methylosinus trichosporium (OB3b) ${ }^{3}$ has been resolved into three components. The enzyme system from $M$. capsulatus (Bath) has been the most well-characterized and consists of a dinuclear non-heme iron enzyme ${ }^{4}$ (hy droxvlase, $\mathrm{M}_{\mathrm{r}} 250 \mathrm{kDa}$ ), an $\mathrm{Fe}_{2} \mathrm{~S}_{2}-\mathrm{FAD}$ electron transport protein ${ }^{5}$ (reductase, $\mathrm{M}_{\mathrm{r}} 39$ $\mathrm{kDa}$ ) which mediates the transfer of electrons from $\mathrm{NAD}(\mathrm{P}) \mathrm{H}$ to the hydroxylase, and a small regulatory protein (component $\mathrm{B}, \mathrm{M}_{\mathrm{r}} 16 \mathrm{kDa}$ ) which contains no metals or prosthetic groups. ${ }^{6}$ The soluble enzyme system is not methane-specific and catalyzes the oxidation of a wide variety of alkanes, alkenes and alicyclic, aromatic and heterocyclic compounds. 7 The three components of the $M$. trichosporium (OB3b) MMO system are analagous to those of the $M$. capsulatus (Bath) system, 8 and demonstrate the same lack of substratespecificity. 9

The hydroxylase component from $M$. trichosporium (Ob3b) can oxidize methane to methanol in its chemically mediated fully reduced form in the presence of $\mathrm{O}_{2}$, or in the presence of $\mathrm{H}_{2} \mathrm{O}_{2}$ when fully oxidized ${ }^{10}$ confirming that it is at the hydroxylase component that both substrate binding and oxygen activation occurs. Neither the reductase nor component B are catalytically competent, and all three components of the MMO enzyme system are required for efficient oxidation of substrate.10,11 EPR, Mössbauer, and X-ray absorption spectroscopy have been used to characterize the dinuclear non-heme iron active site in the MMO hydroxylase component from both $M$. capsulatus (Bath) ${ }^{4,12}$ and $M$. trichosporium (OB3b).8,10,12,13 These studies have shown that the hydroxylase from the two methanotrophs are spectroscopically and structurally similar to each other and to the other dinuclear non-heme iron proteins hemerythrin ( $\mathrm{Hr}$ ), ribonucleotide reductase (RR), purple acid phosphatase (PAP) anci uteroferrin (Uf). 14 The oxidized Fe(III)Fe(III) form of the hydroxylase has two high-spin ferric atoms and is EPR silent. The one-electron reduced semimet $\mathrm{Fe}(\mathrm{III}) \mathrm{Fe}$ (II) form has an EPR signal with $\mathrm{gav}_{\mathrm{av}}=1.83$ (for $M$. capsulatus (Bath); $\mathrm{gav}_{\mathrm{av}}=1.85$ for $M$. trichosporium (OB3b)), characteristic of an antiferromagnetically coupled dinuclear non-heme iron center ( $\mathrm{S}=1 / 2$ ground state). The reduced $\mathrm{Fe}(\mathrm{II}) \mathrm{Fe}(\mathrm{II})$ form exhibits a $g=16 \mathrm{EPR}$ signal, attributed to a ferromagnetically coupled 15 integer spin system.

EXAFS studies of the hydroxylase have shown that the first coordination sphere of the iron active site in the oxidized form of the $M$. capsulatus (Bath) hydroxylase consists of 
$\sim 6 \mathrm{~N} / \mathrm{O}$ at an average distance of $2.04 \AA$. The photoreduced semimet form has $\sim 6 \mathrm{~N} / \mathrm{O}$ at $2.08 \AA$ for the $M$. capsulatus (Bath) hydroxylase, and $\sim 6$ N/O at $2.06 \AA$ for the $M$. trichosporium (OB3b) hydroxylase. The reduced hydroxylase from $M$. capsulatus (Bath) has $\sim 5 \mathrm{~N} / \mathrm{O}$ at $2.15 \AA$ in its first coordination sphere. The iron-iron distance for the oxidized and semimet forms of the hydroxylaso was determined to be $\sim 3.4 \AA$, and no ironiron interaction was seen in the EXAFS of the reduced form of the protein, although EPR verified that the dinuclear iron center was intact after exposure to the beam.

These studies have formed the basis for understanding the mechanism of oxygen activation and substrate oxidation. However, studies of the hydroxylase isolated from the other required components of the MMO enzyme system can yield only limited information, as it is known that all three components are required in vivo. Kinetic studies on the roles and interaction of the three components of the MMO system from M. capsulatus (Bath) ${ }^{6,11}$ have suggested the formation of protein complexes during the catalytic cycle which have an effect on the oxygenase activity. Component $B$ appears to serve a strict role in regulating the oxygenase activity of the $M$. capsulatus (Bath) system, preventing reduction of the hydroxylase by the reductase in the absence of substrate, and greatly increasing the electron transfer rate between the two components in the presence of substrate. ${ }^{11}$ In the $M$. trichosporium (OB3b) system, electron transfer between the reductase and the hydroxylase is inhibited, but not prevented by component $B$ in the absence of substrate, and in the presence of substrate, component $B$ greatly increases the yield and the rate of product formation. ${ }^{10}$

Perturbations in the EPR spectra of the hydroxylase in the presence of component B and in the presence of small molecules have been documented, suggesting that complexation with the hydroxylase in some way affects the Fe site. ${ }^{16,17}$ The effects of substrate, component $B$ and the reductase on the redox potentials of the hydroxylase have also been investigated ${ }^{18}$ and show that dramatic changes in the electron affinity of the hydroxylase core occur as a function of the presence of the other components. The change in the redox potentials and the EPR of the complexed forms of the hydroxylase implies a change in the electronic structure of the iron site which could be caused by changes in the ligation of the diiron site, or by conformational changes of the protein near or at the iron center which affect the electronic properties of the diiron core. EXAFS data analysis would be sensitive to any structural changes which occur as a result of the complex formation, and electronic perturbations would have an effect on the edge structure of the hydroxylase complexes.

We have collected Fe K-edge EXAFS data and high-resolution edge data on hydroxylase sample $\dot{j}$ in the diferric and diferrous forms in the presence of component $B$, 
bromopropene, and both component $\mathrm{B}$ and bromopropene to determine the nature of the change in the coordination environment of the iron atoms. These studies show that no dramatic change in the coordination of the iron atoms occurs upon formation of the various complexes. Evidence for a change in the covalency of the diiron center in its semimet form, and inhibition of the reduction of the hydroxylase component to its diferrous form in the presence of component B and bromopropene is directly seen.

\subsection{Experimental}

\subsubsection{EXAFS Sample Preparation}

The soluble hydroxylase of MMO from $M$. capsulatus (Bath) was isolated and purified as previously described. ${ }^{12}$ Component B was produced from a strain of Esherichia coli containing a plasmid with the gene for B from M. capsulatus (Bath). 19 Purified hydroxylase was frozen at $-80^{\circ} \mathrm{C}$ in $50 \mathrm{mM}$ PIPES buffer at $\mathrm{pH}=7.0$ until further use at which point it was thawed and concentrated to approximately $11 \mathrm{mg}$ protein $/ \mathrm{ml}$ using a Centriprep. For EXAFS10, EXAFS14, EXAFS15, EXAFS16, and EXAFS17, component $B$ was added in the stoichiometric molar ratio of 2:1 B:hydroxylase. For EXAFS18, EXAFS19, and EXAFS20, and for EXAFS16 and EXAFS17 after the addition of B, a 1000-fold excess of bromopropene (based on the concentration of protein) was added to insure that the substrate would remain bound to the hydroxylase during the subsequent concentration proceedure. The hydroxylase complexes were dialyzed into a $50 \%$ ethylene glycol solution of $50 \mathrm{mM}$ MOPS buffer $(\mathrm{pH}=7.0)$ for the non-bromopropene containing samples and EXAFS11, and into the above solution with a 10,000-fold excess of bromopropene for EXAFS16, EXAFS17, EXAFS13, EXAFS19 and EXAFS20. After dialysis, the samples were further concentrated on a Centricon centrifugal microconcentrator, after which a 1000 -fold excess of brc nopropene was added to EXAFS16-20. For EXAFS11, no bromopropene was added until after the final concentration on the Centricon, at which point a 1000-fold excess was added.

The concentrated samples were degassed and brought into a wet box. The hydroxylase is isolated in its oxidized form; the reduced form was prepared by adding a 10fold molar excess of sodium dithionite, $100 \mathrm{mM}$ methyl viologen and $10 \mathrm{mM}$ proflavin to samples EXAFS15, EXAFS17 and EXAFS19 and letting the samples incubate for 40 minutes. The samples were loaded into lucite EXAFS cells equipped with caps and with $25 \mu \mathrm{m}$ Kapton windows ( $23 \mathrm{~mm} \times 2 \mathrm{~mm} \times 3 \mathrm{~mm} ; 140 \mu \mathrm{l}$ ), immediately frozen in liquid nitrogen upon removal from the box and stored in a liquid nitrogen refrigerator. 


\subsubsection{EXAFS Data Collection, Reduction and Analysis}

A description of each sample is presented in Table 3.1. EXAFS and high-energy resolution edge scans were collected simultaneously by using a $1 \mathrm{~mm}$ vertical slit opening to maximize energy resolution and taking $0.15 \mathrm{eV}$ steps over the edge region during an EXAFS scan. All of the samples were run on unfocused 8-pole wiggler beamline 7-3 (18 $\mathrm{kG}$ ) at the Stanford Synchrotron Radiation Laboratory (SSRL) operating at $3 \mathrm{GeV}$ and 40 $90 \mathrm{~mA}$. A Si $(220)$ double-crystal monochromator was used, detuned $45 \%$ at the end of the Fe EXAFS scan $\left(7995 \mathrm{eV}, k=15 \AA^{-1}\right)$ to reduce harmonic contamination in the incident beam. The data were measured in fluorescence mode at $10 \mathrm{~K}$ using a continuous-flow $\mathrm{LHe}$ cryostat (Oxford Instruments, model CF1208). The fluorescence signal was monitored with a 13-element $\mathrm{Ge}$ solid-state detector array ${ }^{20}$ (Canberra) windowed on the $\mathrm{Fe} \mathrm{K \alpha}$ signal $(6840 \mathrm{eV})$. Total count rates of between 25,000 and 35,000 per second per channel (measured at $7997 \mathrm{eV}$ detuned 45\%) were maintained throughout the experiments. At these count rates, the detector was below saturation limits. One of the channels showed persistently high count rates; it was therefore not included in further data analysis.

The energy of the individual scans was calibrated using an internal $\mathrm{Fe}$ foil standard, ${ }^{21}$ assigning the first inflection point to $7111.2 \mathrm{eV}$. For every scan, 13 data files (1 from each channel in the detector) were collected; each was inspected individually. Individual channels of data were rejected if there were any discontinuities due to heat load problems for the detector electronics or if any of the channels exhibited poor statistics (erratic dark current values, persistently high count rates). Averages of the remaining data files for each scan were then made and inspected. Individual scans were rejected for signal-to-noise problems caused by low currents, or because of beam instability (short lifet (mes, orbit shifts) or loss of beam, or due to irreproducibility of the scans. The remaining acceptable scans for each sample were then averaged and used for further analysis (summary of averages in Table 3.1).

The data were background subtracted by fitting a polynomial through the EXAFS region which was extrapolated through the pre-edge region and subtracted. A three- or four-segment spline was fit to the post-edge region and subtracted to isolate the EXAFS data and to normalize the edge jump to unity. The spline was chosen so that it removed the low frequency noise without reducing the true EXAFS amplitude; this was checked by monitoring the Fourier transform of the EXAFS data during the spline and normalization process. The normalized data were converted to $k$ space, where $k$ is the photoelectron wavevector defined by $\left[2 m_{e}\left(E-E_{0}\right) / \hbar^{2}\right]^{1 / 2}$. In this expression, $m_{e}$ is the electron mass, $E$ 
Table 3.1. Sample and Data Collection Summarya

\begin{tabular}{|c|c|c|c|}
\hline Sample \# & Description & [Fe] $\mathrm{mu}$ & $\begin{array}{l}\text { scans avg./scans collected } \\
\text { for EXAFS analysis }\end{array}$ \\
\hline EXAFS10 & $\begin{array}{l}\text { Oxidized hydroxylase } \\
\text { w/ component B }\end{array}$ & 2.0 & $269 / 403$ \\
\hline EXAFS11b & $\begin{array}{l}\text { Oxidized hydroxylase } \\
\text { w/ bromopropene }\end{array}$ & 3.2 & $226 / 234$ \\
\hline EXAFS14b & $\begin{array}{l}\text { Oxidized hydroxylase } \\
\text { w/ component B }\end{array}$ & 1.5 & $398 / 442$ \\
\hline EXAFS16 & $\begin{array}{l}\text { Oxidized hydroxylase } \\
\text { w/ component } B \text { and } \\
\text { bromopropene }\end{array}$ & 1.2 & $255 / 286$ \\
\hline EXAFS18 & $\begin{array}{l}\text { Oxidized hydroxylase } \\
\text { w/ bromopropene }\end{array}$ & 1.4 & $230 / 260$ \\
\hline EXAFS20b & $\begin{array}{l}\text { Oxidized hydroxylase } \\
\text { w/ bromopropene }\end{array}$ & $<1.9$ & $124 / 143$ \\
\hline EXAFS7 & $\begin{array}{l}\text { Oxidized hydroxylase } \\
\text { non-complexed }\end{array}$ & 3.4 & $\begin{array}{l}84 / 91 \\
\text { Edge analysis only }\end{array}$ \\
\hline EXAFS15 & $\begin{array}{l}\text { Reduced hydroxylase } \\
\text { w/ component B }\end{array}$ & 1.1 & $448 / 481$ \\
\hline EXAFS 17 & $\begin{array}{l}\text { Reduced hydroxylase } \\
\text { w/ component B and } \\
\text { bromopropene }\end{array}$ & 1.6 & $426 / 481$ \\
\hline EXAFS19 & $\begin{array}{l}\text { Reduced hydroxylase } \\
\text { w/ bromopropene }\end{array}$ & 1.7 & $276 / 286$ \\
\hline EXAFS6 & $\begin{array}{l}\text { Reduced hydroxylase } \\
\text { non-complexed }\end{array}$ & 4 & $\begin{array}{c}91 / 91 \\
\text { Edge analysis only }\end{array}$ \\
\hline
\end{tabular}

aAll samples were run in 1992 at SSRL on unfocused beamline 7-3 by using Si(220) monochromator crystals. A 13-element solid state Ge fluorescence detector was used in every case. bSample was photoreduced to the semimet state by the X-ray beam. Only the scans after photoreduction was complete were averaged for further analysis. ${ }^{c}$ The results of the EXAFS analysis for EXAFS6 have been previously reported, ${ }^{12}$ however edge data were collected during the 1992 run. 
is the photon energy, $\hbar$ is Planck's constant divided by $2 \pi$, and $E_{0}$ is the threshold energy, $7130 \mathrm{eV}$ (where $k$ is defined to be zero).

All of the fits presented in this paper were based on $k^{3}$-weighted data. Non-linear least-squares curve-fitting techniques using empirical amplitude and phase parameters were used to analyze the data, as described in Chapter 1 and elsewhere. ${ }^{22}$ The errors estimated in the EXAFS analysis are $\pm 0.03 \AA$ in the distances and $25 \%$ in the coordination numbers. ${ }^{22}$ The fits proceeded by allowing the initial coordination numbers and distances for an $\mathrm{Fe}-\mathrm{X}$ pair of interest to vary. Empircal amplitude and phase parameters for the $\mathrm{Fe}-\mathrm{X}$ scattering pairs of interest were obtained from the following models: $\mathrm{Fe}-\mathrm{N}$ from $[\mathrm{Fe}(1,10-$ phenanthroline $\left.)_{3}\right]\left(\mathrm{ClO}_{4}\right)_{3} ;{ }^{23} \mathrm{Fe}-\mathrm{O}$ and $\mathrm{Fe}-\mathrm{C}$ from $\left.[\mathrm{Fe} \text { (acetylacetonate) })_{3}\right] ;{ }^{24} \mathrm{Fe}-\mathrm{Fe}$ from $\left[\mathrm{Fe}_{2}(\mathrm{OH})(\mathrm{OAc})_{2}\left(\mathrm{HB}(\mathrm{pz})_{3}\right)_{2}\right]\left(\mathrm{ClO}_{4}\right)_{2}{ }^{25}$ Data for these model compounds were collected as previously described. ${ }^{26}$ For all of the fits described in this paper, the EXAFS data from 3.5 to $12.5 \AA^{-1}$ were Fourier transformed to $\mathrm{R}(\AA)$ space to isolate the first and second shell contributions to the data. The individual contributions were isolated, backtransformed to $k$ space, and fit from 4 to $12.0 \AA^{-1}$. In addition, both shells were backtransformed together and fit between 4.0 and $12.0 \AA^{-1}$, as were the unfiltered data. The windows used to isolate the peaks in the Fourier transforms for the backtransforms are presented in the tables of the fit results. A gaussian window width of $0.1 \AA$ was used to reduce truncation artifacts in the Fourier transforms.

\subsection{Results of XAS Experiments}

The $k^{3}$-weighted EXAFS of the photoreduced semimet complexed samples are presented along with the EXAFS data of the non-complexed semimet hydroxylase in Figure 3.1. The Fourier transforms of the data (from 3.5 - 12.5 $\AA$ ) are presented in Figure 3.2. The data for the non-complexed semimet hydroxylase (EXAFS2) were collected and analyzed as described in Chapter 2 and reference 12. The increased noise level of the EXAFS for the complexed-forms compared to the non-complexed forms reflects the lower iron concentration for the former (1-2 $\mathrm{mM} \mathrm{Fe}$ as compared to $\sim 4 \mathrm{mM} \mathrm{Fe}$ ). The EXAFS of hydroxylase in the presence of component B of the MMO enzyme system (EXAFS10 and EXAFS14 w/ B; EXAFS16 w/ B and bromopropene) are somewhat different from the non-complexed form between $k=7$ and $9 \AA^{-1}$. For the non-complexed hydroxylase (EXAFS2, Figure 3.1a), the maxima at $k>8 \AA^{-1}$ is greater in amplitude than the maxima at $k<8 \AA^{-1}$; in the B-complexed samples (EXAFS10, Figure 3.1b and EXAFS14, Figure 3.1c), the relative amplitudes of these maxima are reversed, and in the $B$ plus bromopropene complex (EXAFS16, Figure 3.1g), the amplitudes are nearly equal. The 


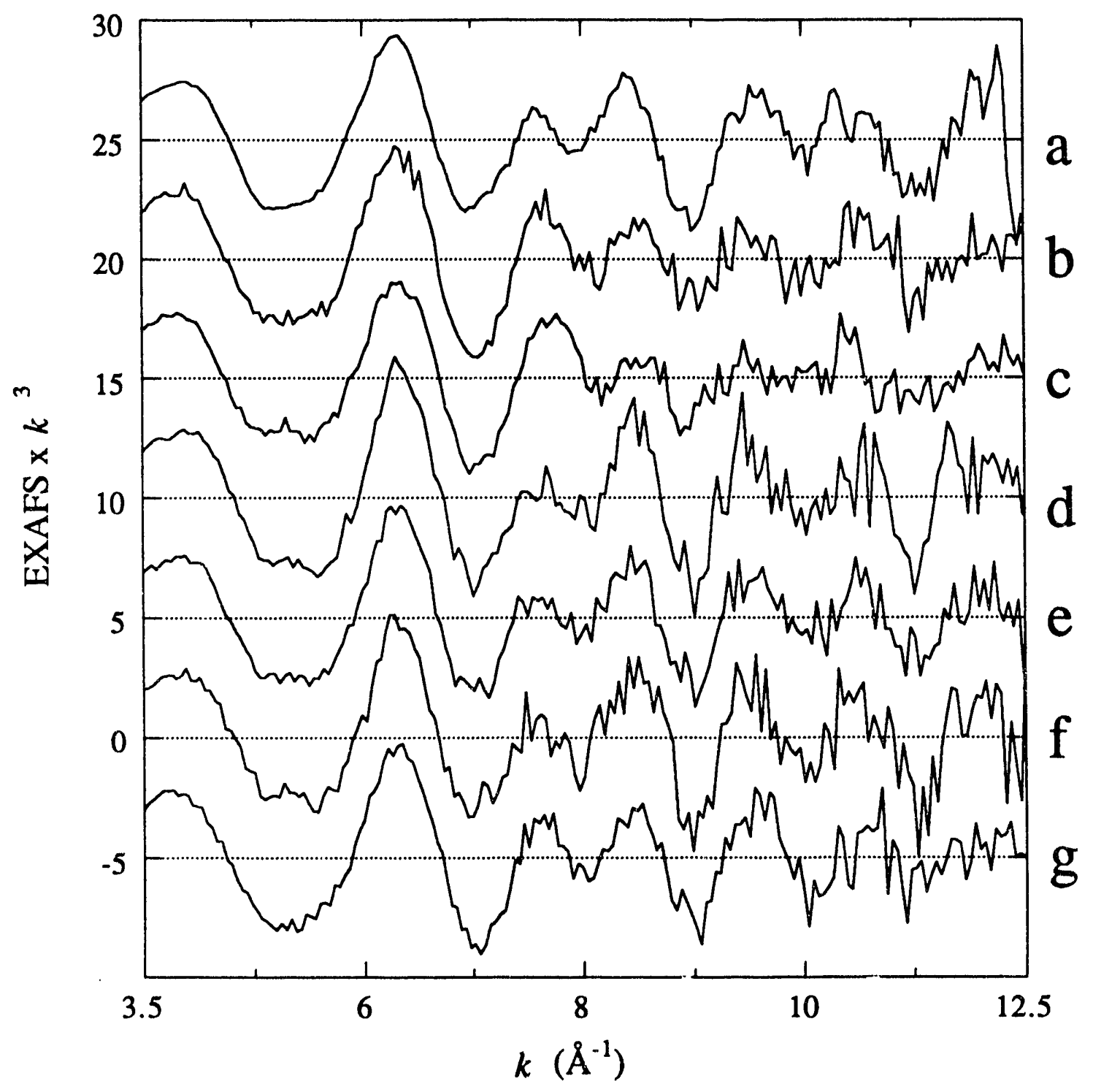

Figure 3.1. EXAFS of the semimet hydroyxlase samples. (a) Semimet hydroyxlase sample EXAFS2; with component B: (b) EXAFS10, (c) EXAFS14; with bromopropene: (d) EXAFS 11, (e) EXAFS18, (f) EXAFS 20; with B and bromopropene: $(\mathrm{g})$ EXAFS16. The data shown here are the data used for Fourier transforms $\left(k=3.5-12.5 \AA^{-1}\right)$. Note the difference in the appearance of the EXAFS between $k=7$ and $10 \AA^{-1}$. 


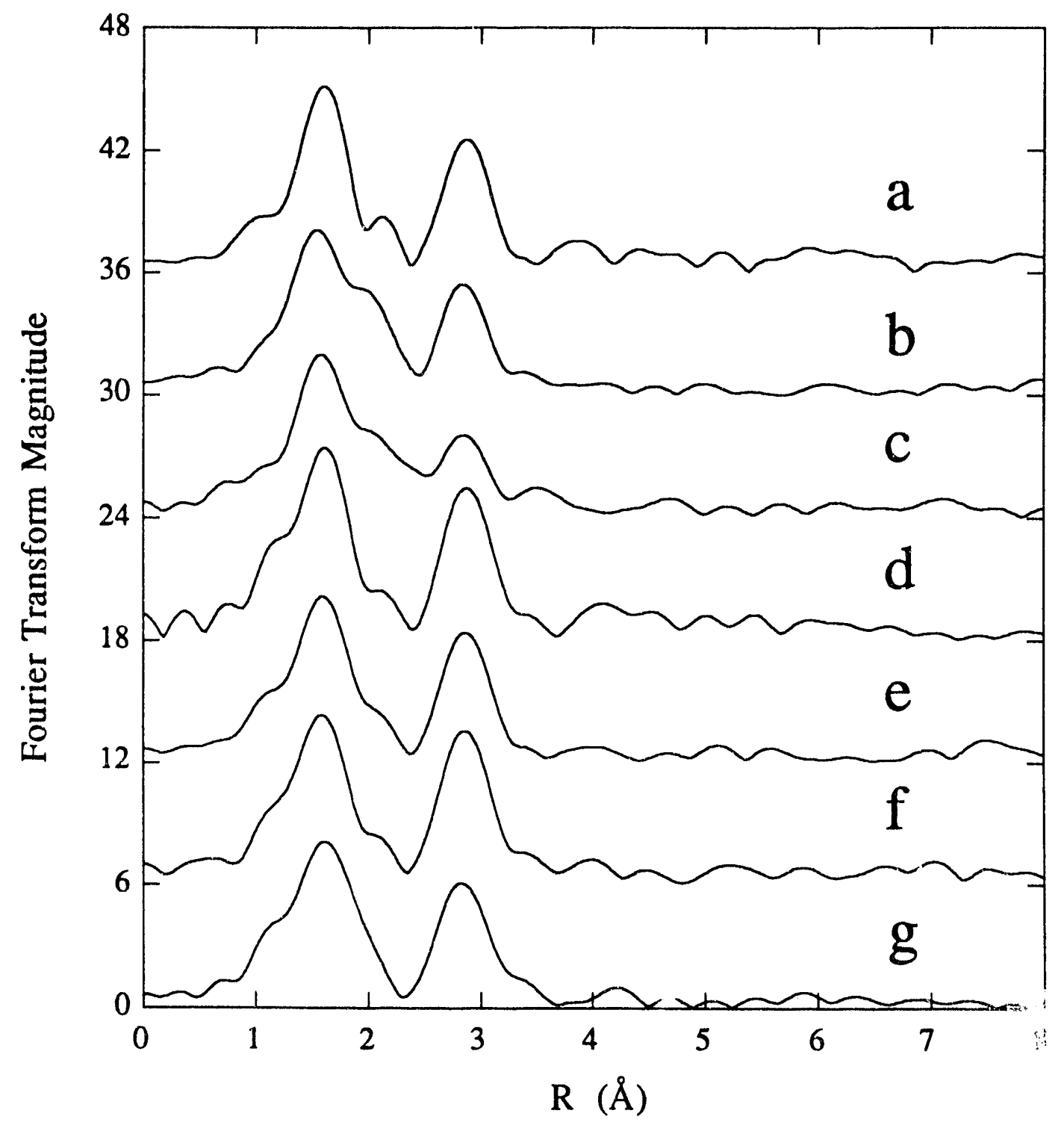

Figure 3.2. Fourier transforms of the semimet hydroxylase EXAFS presented in Figure

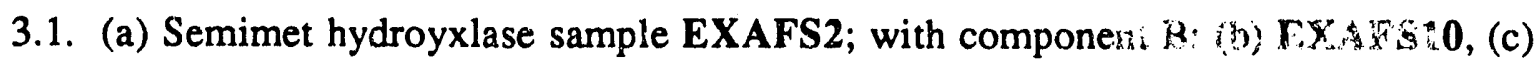
EXAFS14; with bromopropene: (d) EXAFS11, (e) EXAFS18, 13, FAF20; with B and bromopropene: (g) EXAFS16. 
minimum at $k=9 \AA^{-1}$ is deeper for EXAFS10 and EXAFS14 than for EXAFS2 or EXAFS 16. The EXAFS of the hydroxylase in the presence of bromopropene (EXAFS11, Figure 3.1d, EXAFS18, Figure 3.1e EXAFS20, Figure 3.1f) are very similar to the non-complexed form (Figure 3.1a).

Differences are seen in the Fourier transforms of the EXAFS data as well (Figure 3.2). The second shell peaks in the Fourier transform of the B-hydroxylase samples (EXAFS10, Figure 3.2b and EXAFS14, Figure 3.2c) are not as intense as the second shell peaks for the rest of the samples, nor as well resolved from the first shell peaks. The positions of the first and second shell peaks of the complexed forms of the hydroxylase are within $0.05 \AA$ of the positions of the peaks of the non-complexed form, but the relative separation of the first and second sheil peaks is slightly wider for EXAFS10, and slightly narrower for EXAFS16. The intensities of the peaks of the complexed forms (except for EXAFS11 and EXAFS20) are lower than the non-complexed form, but the relative intensities of the second shell peaks to the first shell peaks for all of the samples (except EXAFS14 and EXAFS20, and inciuding EXAFS11) are within 10\% of the value for the non-complexed form (0.72). For EXAFS14, the ratio of the second shell peak to the first shell peak differs from the value for the non-complexed form by $30 \%$, and for EXAFS20, the ratio differs by $20 \%$.

The EXAFS data for the reduced samples are presented in Figure 3.3, and the Fourier transforms of the data given in Figure 3.4. The data for the non-complexed reduced hydroxylase ${ }^{12}$ are also presented for comparison. Here again, there are differences between the complexed vs. the non-complexed forms of the reduced hydroxylase. The EXAFS of the hydroxylase in the presence of component B (EXAFS15 w/ B, Figure 3.3c and EXAFS17 w/ B and bromopropene, Figure 3.3e) are shifted slightly to higher $k$, and the minimum at $k=\sim 8.5 \AA^{-1}$ in the non-complexed samples (EXAFS3, Figure 3.3a and EXAFS6, Figure 3.3b) is not as distinct in the B-hydroxylase samples. The bromopropene-hydroxylase sample (EXAFS19, Figure 3.3d) is more similar to the noncomplexed samples (Figure 3.3a,b); however, the $\sim 8.5 \AA^{-1}$ minimum is shifted to lower $k$ for EXAFS19 relative to EXAFS3 and EXAFS6. These differences in the EXAFS data are clearly seen in the Fourier transforms of the data (Figure 3.4), with the peak for the B-complexed forms of the hydroxylase (Figure 3.4c,e) appearing $0.08-0.09 \AA$ to lower $R$ than the peaks in the non-complexed (Figure 3.4a,b) and bromopropene-complexed (Figure 3.4d) forms. Although the Fourier transforms above $2 \AA$ is different for all of the samples, there is no indication of a second shell $\mathrm{Fe}$-Fe interaction like that seen in the semimet samples (Figure 3.4) for any of the reduced samples. 


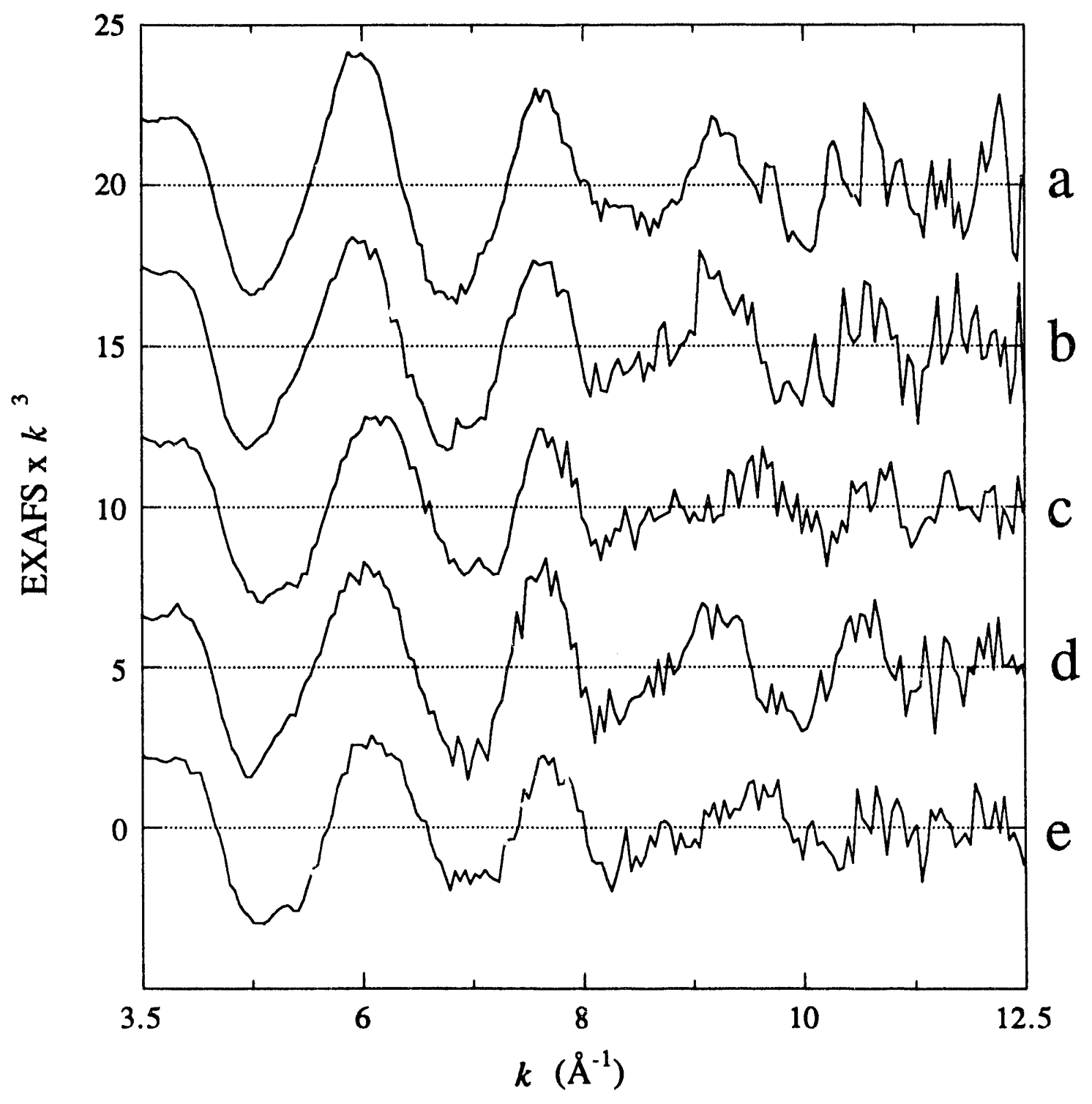

Figure 3.3. EXAFS data of the reduced hydroxylase samples. Reduced hydroxylase samples: (a) EXAFS3, (b) EXAFS6; with component B: (c) EXAFS15; with bromopropene: (d) EXAFS19; with B and bromopropene: (e) EXAFS17. The data shown are the data used for Fourier transforms $\left(k=3.5-12.5 \AA^{-1}\right)$. Note the difference in the appearance of the EXAFS above $k=8 \AA^{-1}$. 


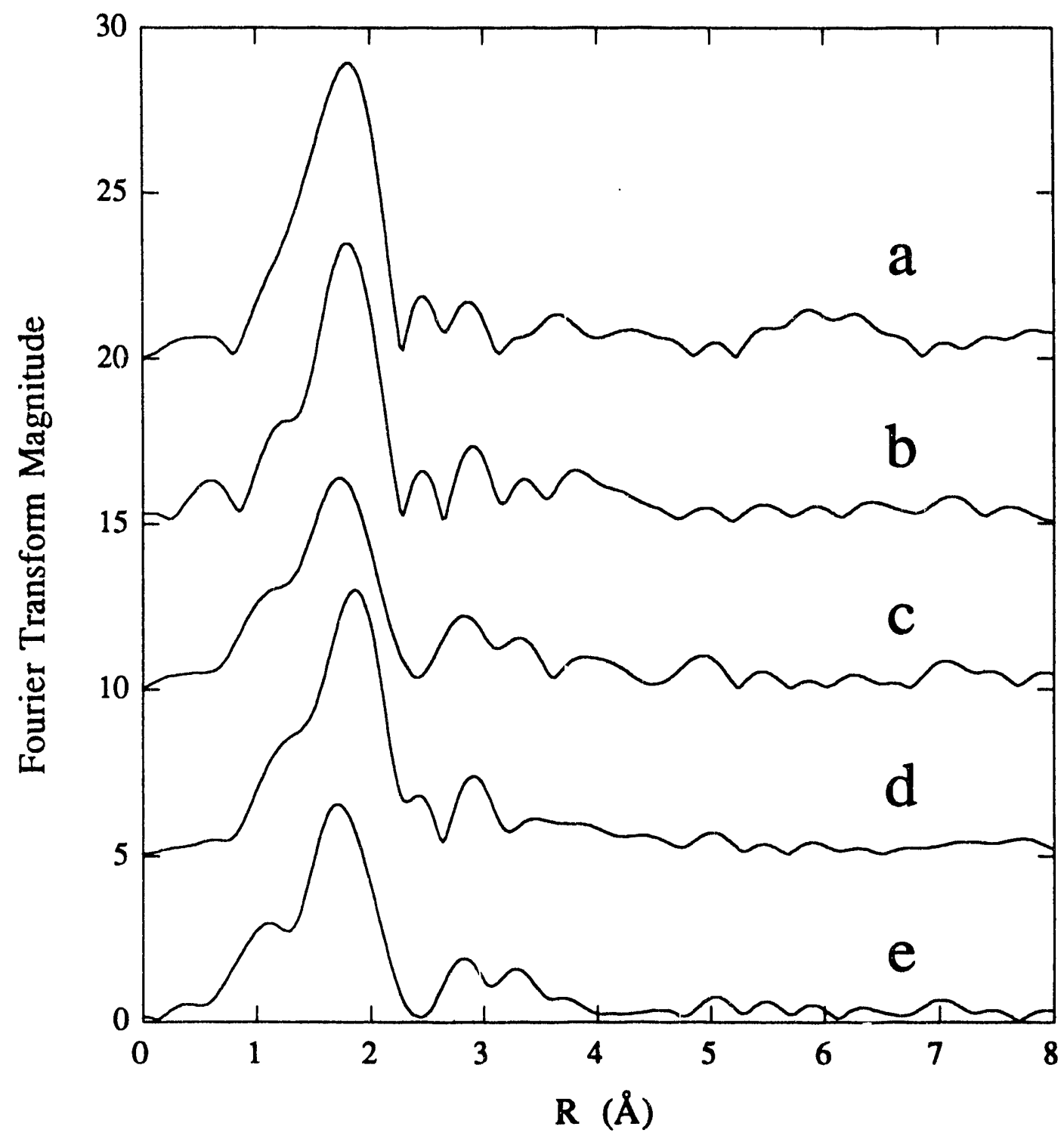

Figure 3.4. Fourier transforms of the reduced hydroxylase EXAFS data presented in Figure 3.3. Reduced hydroxylase samples: (a) EXAFS3, (b) EXAFS6; with component B: (c) EXAFS 15; with bromopropene: (d) EXAFS19; with B and bromopropene: (e) EXAFS17. 


\subsubsection{Photoreduction of the Oxidized Hydroxylase Samples}

As mentioned above, the oxidized samples of the complexed hydroxylase were photoreduced to the 1-electron reduced semimet state (FeIII/FeII) by the X-ray beam, a phenomenon which has been observed before. ${ }^{12,26}$ Anticipating the photoreduction process, SSRL beamline 2-3 (unfocused bending magnet; $\mathrm{Si}(220)$ double crystal monochromator) was used to pre-photoreduce EXAFS14, EXAFS16 and EXAFS18 (temperature $95-100 \mathrm{~K}$, monochromator fully tuned at $7900 \mathrm{eV}$, slits 4 by $15 \mathrm{~mm}$ ), as judged by monitoring the positon of the edge as a function of time $(\sim 2.0 \mathrm{eV}$ shift to lower energy occurs from oxidized to semimet form). Scans were collected every 3-4 hours using a $1 \mathrm{~mm}$ high beam to maximize energy resolution with the monochromator detuned $50 \% \mathrm{ccw}$ at $7400 \mathrm{eV}$. During this process, it was noted that the rate of photoreduction decreased with decreasing ring current; after 21 hours in the beam between $58-30 \mathrm{~mA}$, the edge of EXAFS14 had shifted only $0.8 \mathrm{eV}$. After injection to $\sim 90 \mathrm{~mA}$, an additional $\sim 1$ $\mathrm{eV}$ shift occured in 11 hours. After the pre-photoreduction process, the samples were moved to beamline 7-3 for data collection and in all cases, photoreduction of the samples continued for a few hours. Only the scans collected after the photoreduction process was judged to be complete were used for further data analysis.

\subsubsection{Results of Fits}

3.3.2.1. First Shell Fits. Results of the fits for the Fourier filtered first coordination shell are presented in Table 3.2. For all of the samples, a single $\mathrm{N}$ or $\mathrm{O}$ contribution did not adequately fit the data (fits A and B for all samples), however the data could be fit with two contributions at different bond lengths. This effect has been noted before in fits to structurally characterized model compounds by us and others, ${ }^{27}$ in which single contribution fits resulted in both incorrect coordination numbers and average bond lengths. This has been attributed to interference effects between the $\mathrm{N}$ and $\mathrm{O}$ EXAFS at different but unresolvable distances.

Satisfactory fits were obtained with $2 \mathrm{~N}$ contributions, $2 \mathrm{O}$ contributions, or a mixed N/O contribution (Table 3.2, fits C-F for all samples). The ability of two $\mathrm{N}$ or two $O$ waves to fit the data as well as a mixed N/O contribution reflects the limitation of the EXAFS technique to discriminate between two atoms of similar backscattering strength as is the case with $\mathrm{N}$ and $\mathrm{O}$. EPR studies are consistent with a mixed N/O coordination coordination for the $\mathrm{Fe}$ site; 28 the results with $2 \mathrm{~N}$ or $2 \mathrm{O}$ contributions are therefore more indicative of a first shell consisting of atoms at two different distance distributions, rather 


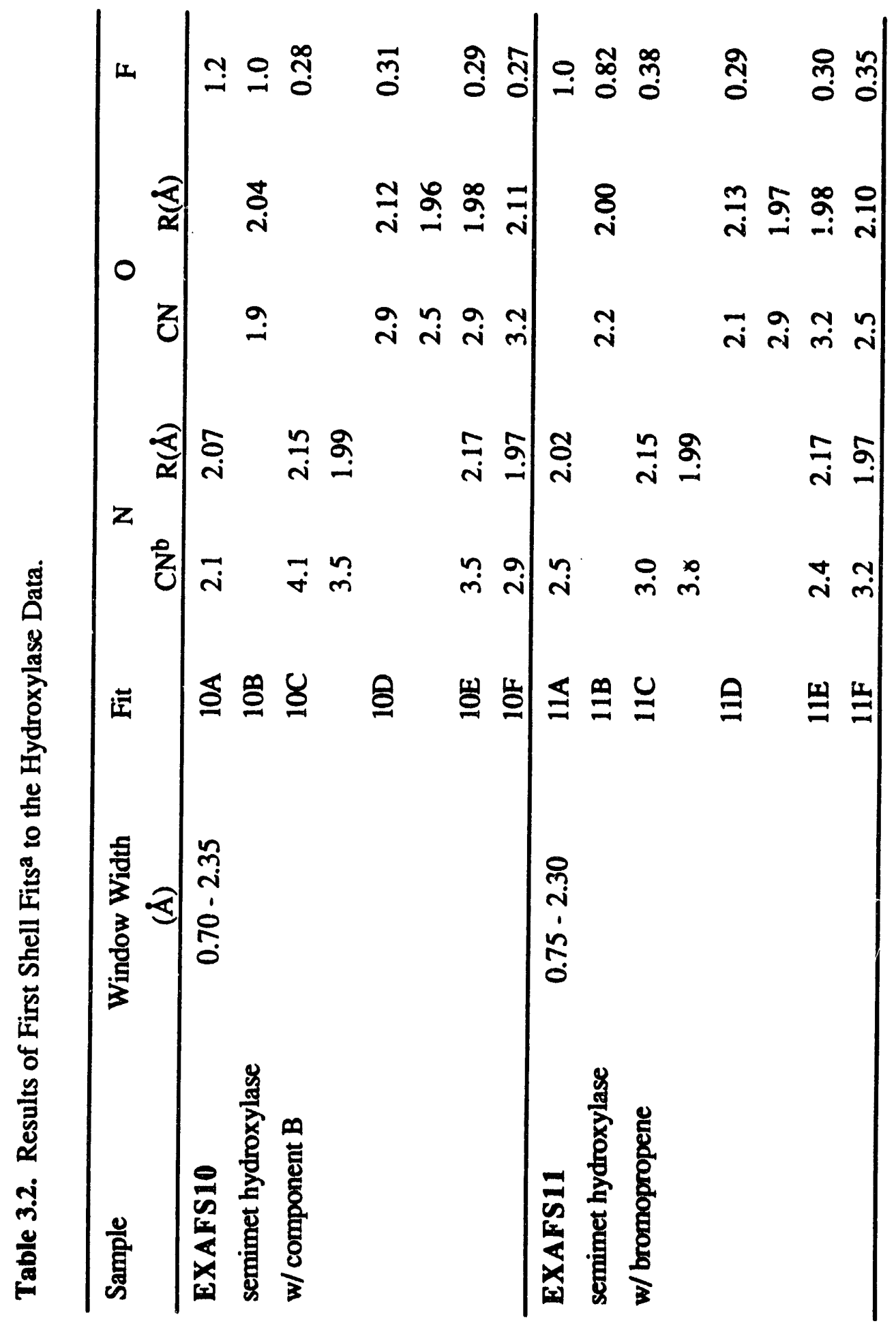




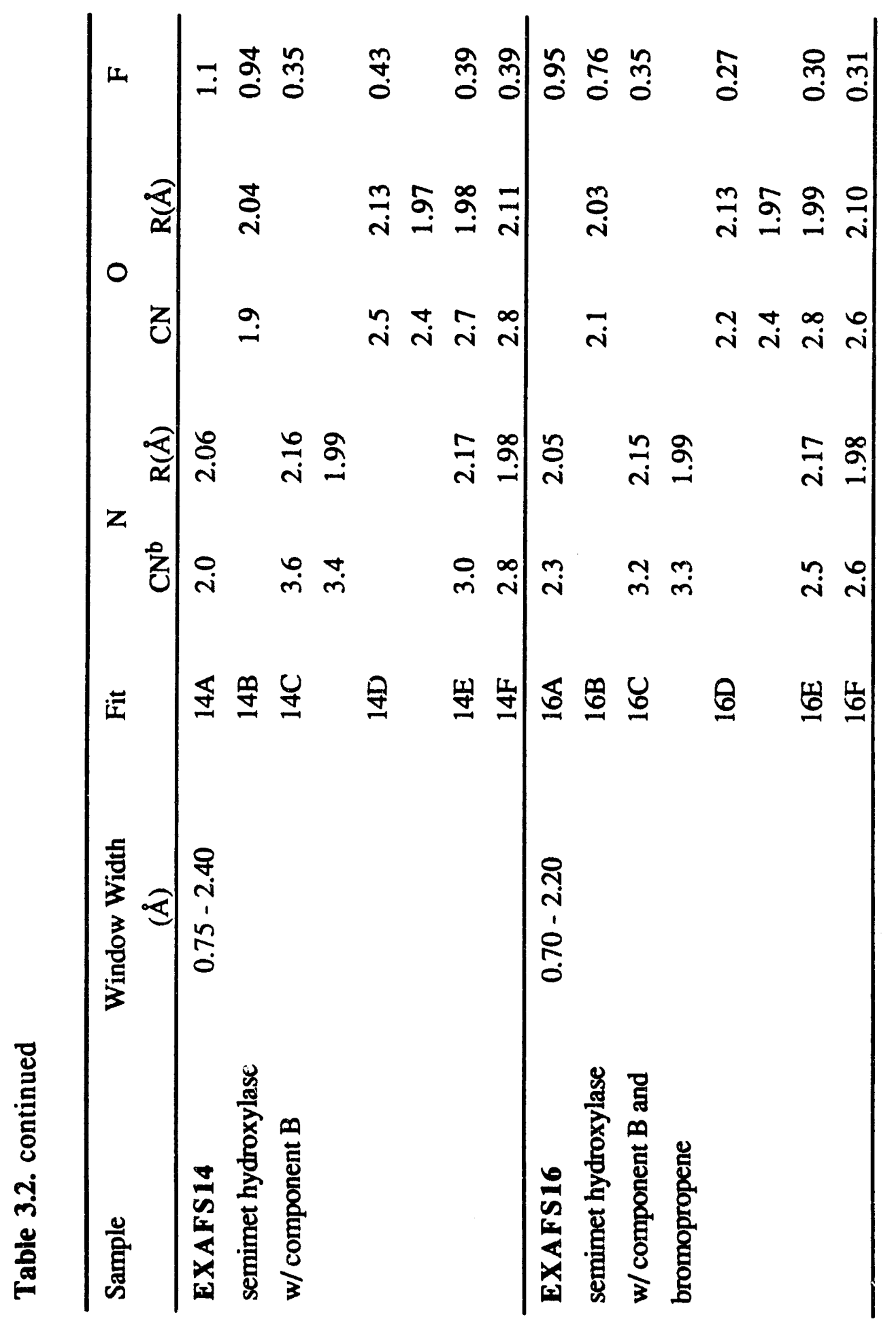




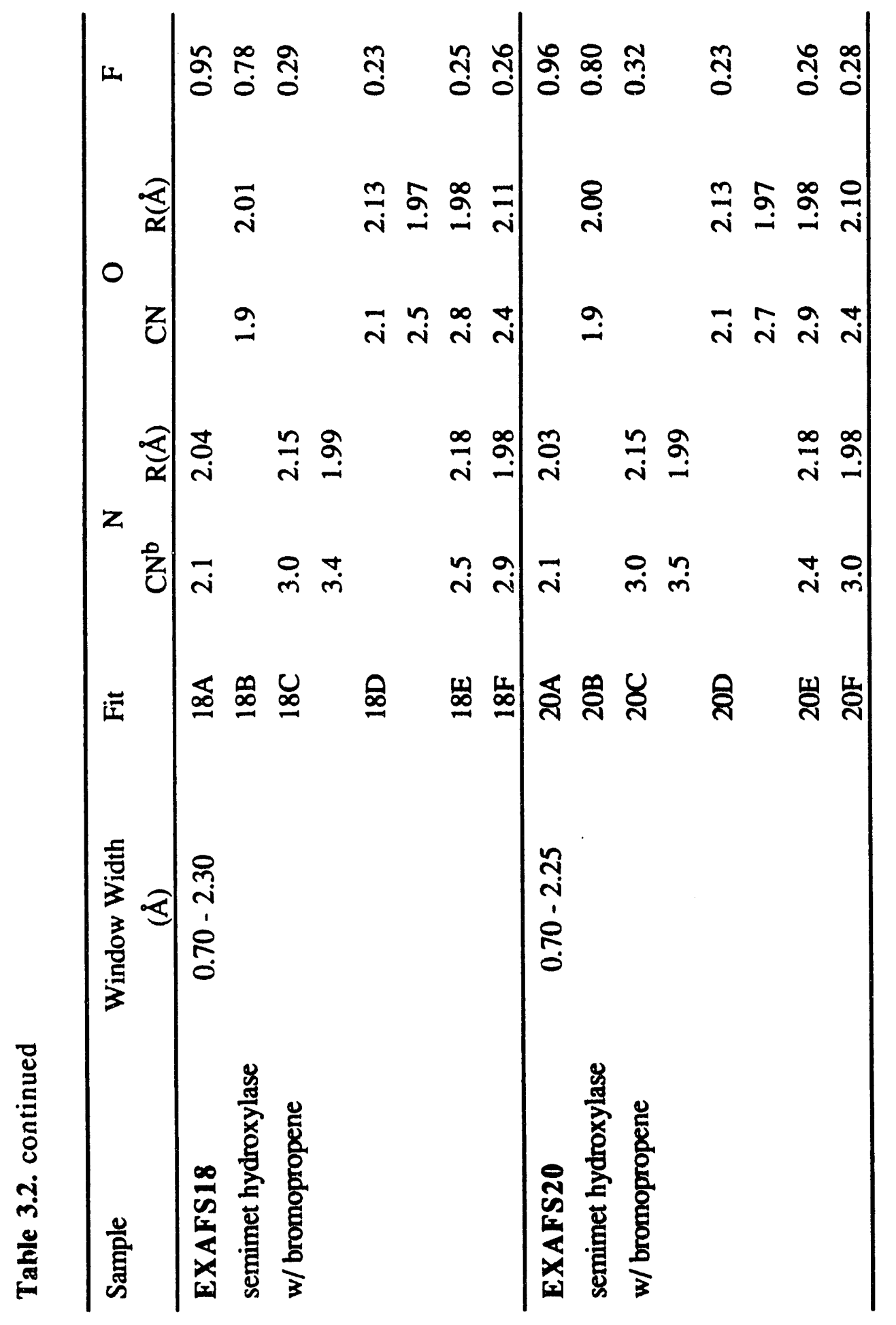




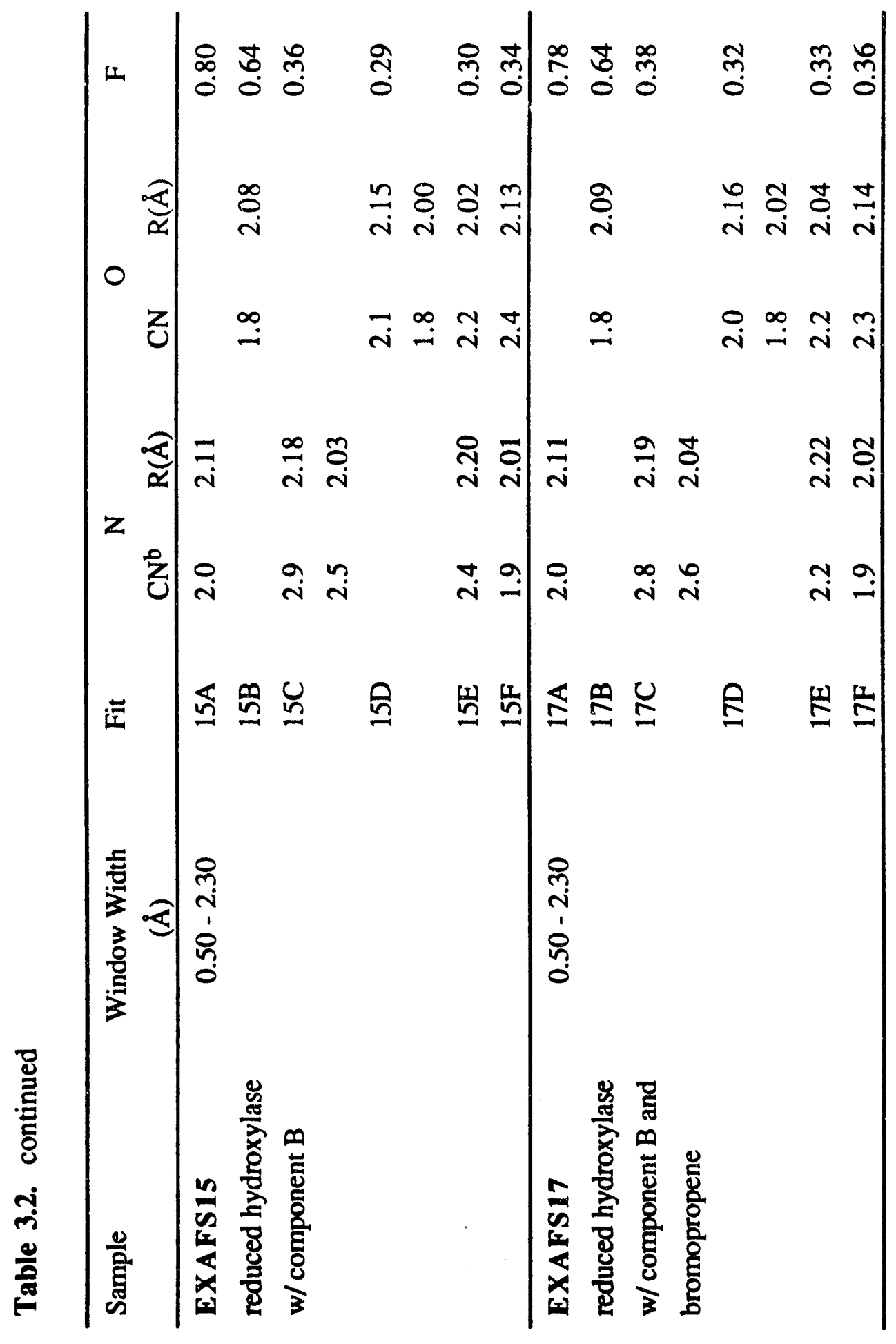




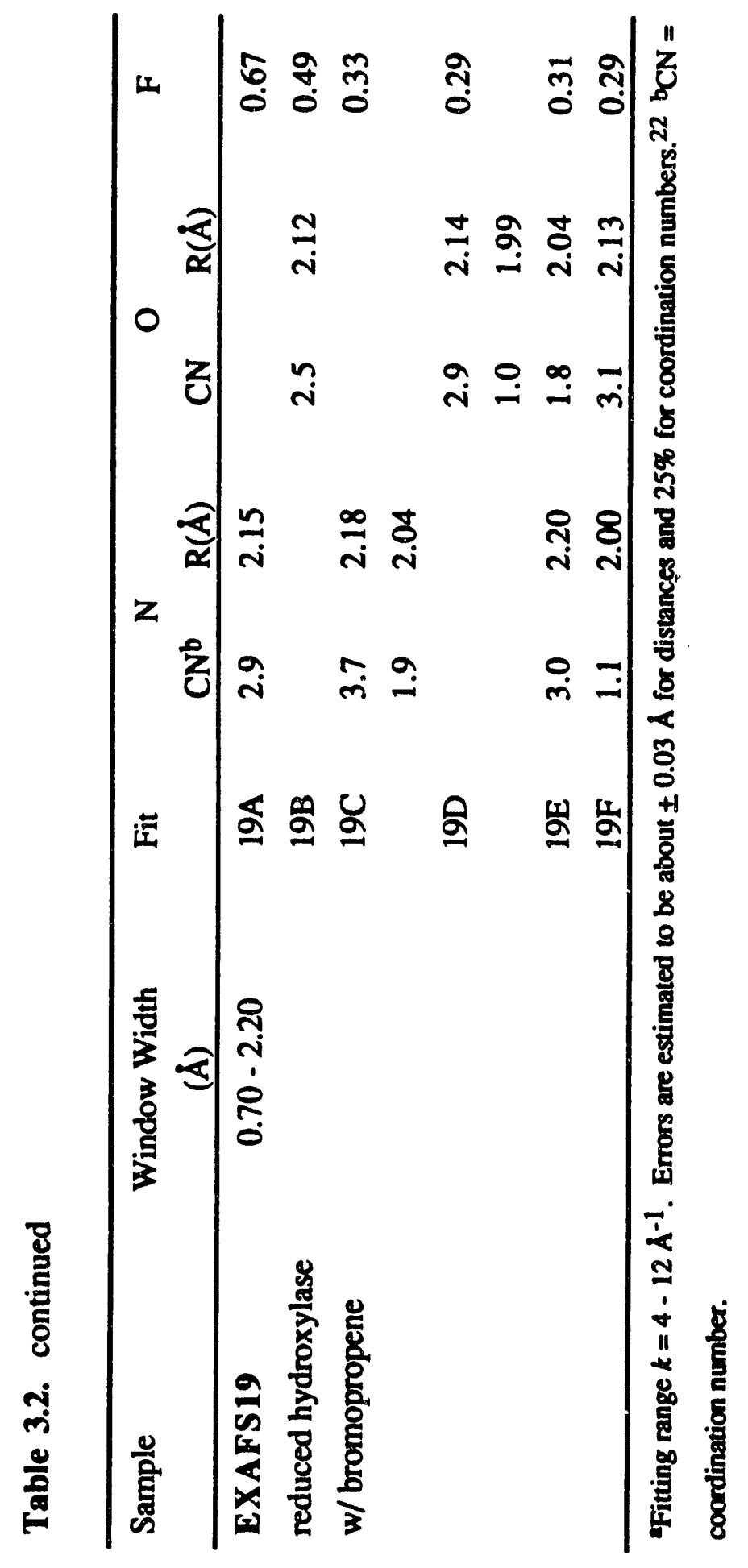


than suggesting an all $\mathrm{N}$ or all $\mathrm{O}$ first shell. Accordingly, the chemically relevant mixed N/O fits will be focused on for the present studies.

For a mixed N/O fit to the data, two possible minima were found in every case depending on the relative starting distances of the $N$ vs. $O$ contributions, one corresponding to $R_{N}>R_{O}$ and one to $R_{N}<R_{O}$ (where $R_{N}$ and $R_{O}$ are the starting $F e-N$ and $\mathrm{Fe}-\mathrm{O}$ bond lengths, respectively). The $\mathrm{N}$ and $\mathrm{O}$ parameters were highly correlated for the limited $k$ range of the data and we therefore place a greater emphasis on the coordination-weighted average bond lengths than on the individual $\mathrm{Fe}-\mathrm{N}$ and $\mathrm{Fe}-\mathrm{O}$ contributions. Since Fe-N bond distances are generally longer than $\mathrm{Fe}-\mathrm{O}$ bond distances in compounds of this type, the fit results with $R_{N}>R_{O}$ were used as the starting point for wide-shell fits to the data. The validity of this approach has previously been confirmed by similar fits to mixed N/O dinuclear iron models ${ }^{12}$ (see Chapters 2 and 4).

The average first shell coordination for all of the complexed forms of the semimet hydroxylase are very similiar to each other. For semimet hydroxylase in the presence of component $\mathrm{B}$, an average coordination of $6.4 \mathrm{~N} / \mathrm{O}$ at $2.08 \AA$ from the iron center (Table 3.2, fit 10E) was found for EXAFS10, and of $5.7 \mathrm{~N} / \mathrm{O}$ at $2.08 \AA$ (Table 3.2, fit 14E) for EXAFS14. The bromopropene-complexed form of the hydroxylase had an average coordination of $5.6 \mathrm{~N} / \mathrm{O}$ at $2.06 \AA$ for EXAFS11 (Table 3.2, fit 11E), and $5.3 \mathrm{~N} / \mathrm{O}$ at $2.07 \AA$ for EXAFS18 and EXAFS20 (Table 3.2, fits $18 \mathrm{E}$ and $20 \mathrm{E}$ respectively). In the presence of both component B and bromopropene (EXAFS16), the average first shell coordination was found to be $5.3 \mathrm{~N} / \mathrm{O}$ at $2.07 \AA$ (Table 3.2, fit $16 \mathrm{E}$ ). No evidence of a short Fe-O contribution which would be consistent with the presence of an oxo-bridged iron center was found. For the reduced forms of the hydroxylase, the average coordination number dropped and the average bond length increased relative to the semimet forms of the hydroxylase. For the reduced hydroxylase with component B (EXAFS15) a first shell coordination of $4.6 \mathrm{~N} / \mathrm{O}$ at $2.11 \AA$ (Table 3.2, fit 15E) was found, consistent with the shift of the first peak in the Fourier transform to shorter $R$ relative to the non-complexed form (average first shell coordination $\sim 5 \mathrm{~N} / \mathrm{O}$ at $2.15 \AA$, reference 12). For reduced hydroxylase with bromopropene, the average coordination of the first shell was $4.8 \mathrm{~N} / \mathrm{O}$ at $2.14 \AA$ (EXAFS 19, Table 3.2, fit 19E), and with both B and bromopropene (EXAFS17), the first shell was found to consist of $4.4 \mathrm{~N} / \mathrm{O}$ at $2.13 \AA$ (Table 3.2 , fit 17E).

Although the average first shell coordinations are similar for the semimet samples and the reduced samples, there is a systematic difference in the individual contributions to the fits discussed above. For the B-complexed samples (EXAFS10 and EXAFS14), the coordination numbers of each contribution is about 3 , but for the bromopropene-complexed 
samples (EXAFS11, EXAFS18 and EXAFS20), the coordination number of the long distance contribution is lower than the coordination number of the short distance contribution (compare the $\mathrm{E}$ fits in Table 3.2). This is similar to the results of the first shell fits for the non-complexed hydroxylase sample (see Chapter 2, Table 2.2, fit 2C). For the reduced samples, the coordination numbers are equal for the two contributions for the Bcomplexed sample (EXAFS15), but the coordination number for the long distance contribution is greater than the short distance contribution for the bromopropene-complexed sample (EXAFS19). The contributions of each wave in the B + bromopropene sample are similar to the bromopropene samples in the semimet state, and to the B samples in the reduced state. The differences in the relative contributions may be responsible for the metrical differences seen in the EXAFS of the various samples at $k \sim 8 \AA^{-1}$ discussed above and illustrated later.

3.3.2.2. Second Shell Fits The results of second shell fits to the data are presented in Table 3.3. Two minima were found for Fe-only fits to the data, one corresponding to $\sim 3.0 \AA$ and one to $\sim 3.4 \AA$, with the better fit corresponding to the $3.4 \AA$ fit. We have previously described the bias of the second shell fits for the $\mathrm{Fe}-\mathrm{Fe}$ distance corresponding to the distance in the model compound from which parameters were obtained. ${ }^{12}$ However, based on the lack of an oxo-bridge in the iron center, it is best to use a non-oxo-bridged model compound for obtaining the empirical parameters. ${ }^{12,29}$ For that reason, the hydroxo-bridged model $\left[\mathrm{Fe}_{2}(\mathrm{OH})(\mathrm{OAc})_{2}\left(\mathrm{HB}(\mathrm{pz})_{3}\right)_{2}\right]\left(\mathrm{ClO}_{4}\right)_{2}(3.4 \AA \mathrm{Fe}-\mathrm{Fe}$ distance $)^{25}$ was used to extract parameters for modeling the second shell $\mathrm{Fe}-\mathrm{Fe}$ interaction. For the semimet hydroxylase in the presence of component B (EXAFS10 and EXAFS14), the best fit Fe-only minimum corresponded to $1.0-0.8 \mathrm{Fe}$ at $3.39 \AA$ (Table 3.3, fits $10 \mathrm{G}$ and $14 \mathrm{G}$ respectively). The Fe-Fe coordination for the hydroxylase with bromopropene was found to be $1.5 \mathrm{Fe}$ at $3.40 \AA$ for EXAFS11 (Table 3.3, fit 11G), 1.2 Fe at $3.41 \AA$ for EXAFS18 (Table 3.3, fit 18G) and $1.4 \mathrm{Fe}$ at $3.41 \AA$ for EXAFS20 (Table 3.3, fit 20G). For the hydroxylase with both B and bromopropene (EXAFS16), the best Fe-only fit corresponded to $1.2 \mathrm{Fe}$ at $3.40 \AA$ (Table 3.3, fit 16G). The $3.0 \AA$ minima (Table 3.3, fits $\mathrm{H}$ for all samples) are believed to be due to coincidence of the $\mathrm{Fe}$ phase with the phase of the contribution from the low $Z$ atoms which are almost certainly present at $\sim 3.0 \AA$ from the iron in bridged, dinuclear iron centers. ${ }^{30}$

The limitations involved with the use of second shell $\mathrm{Fe}-\mathrm{C}$ parameters obtained from $\mathrm{Fe}(\mathrm{acac})_{3}$ have been described elsewhere (Chapter 2 and reference 12). Although the Fe-C parameters are known not to be entirely reliable, we have used them to try to obtain additional information about contributions to the second shell data. 12 For C-only fits to the data, two minima were again found, at $\sim 3.0 \AA$ and $\sim 3.4 \AA$, however the best fit 


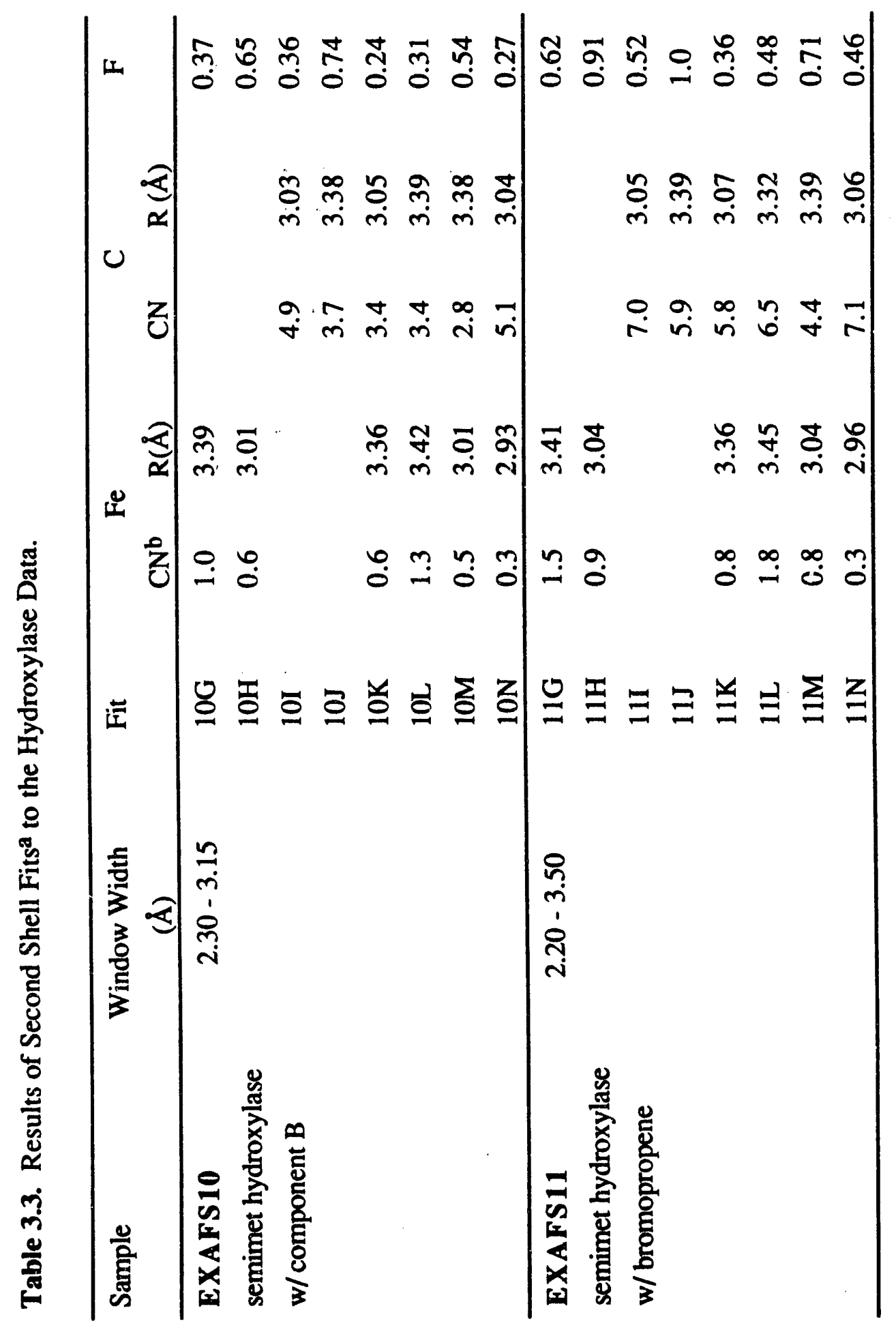




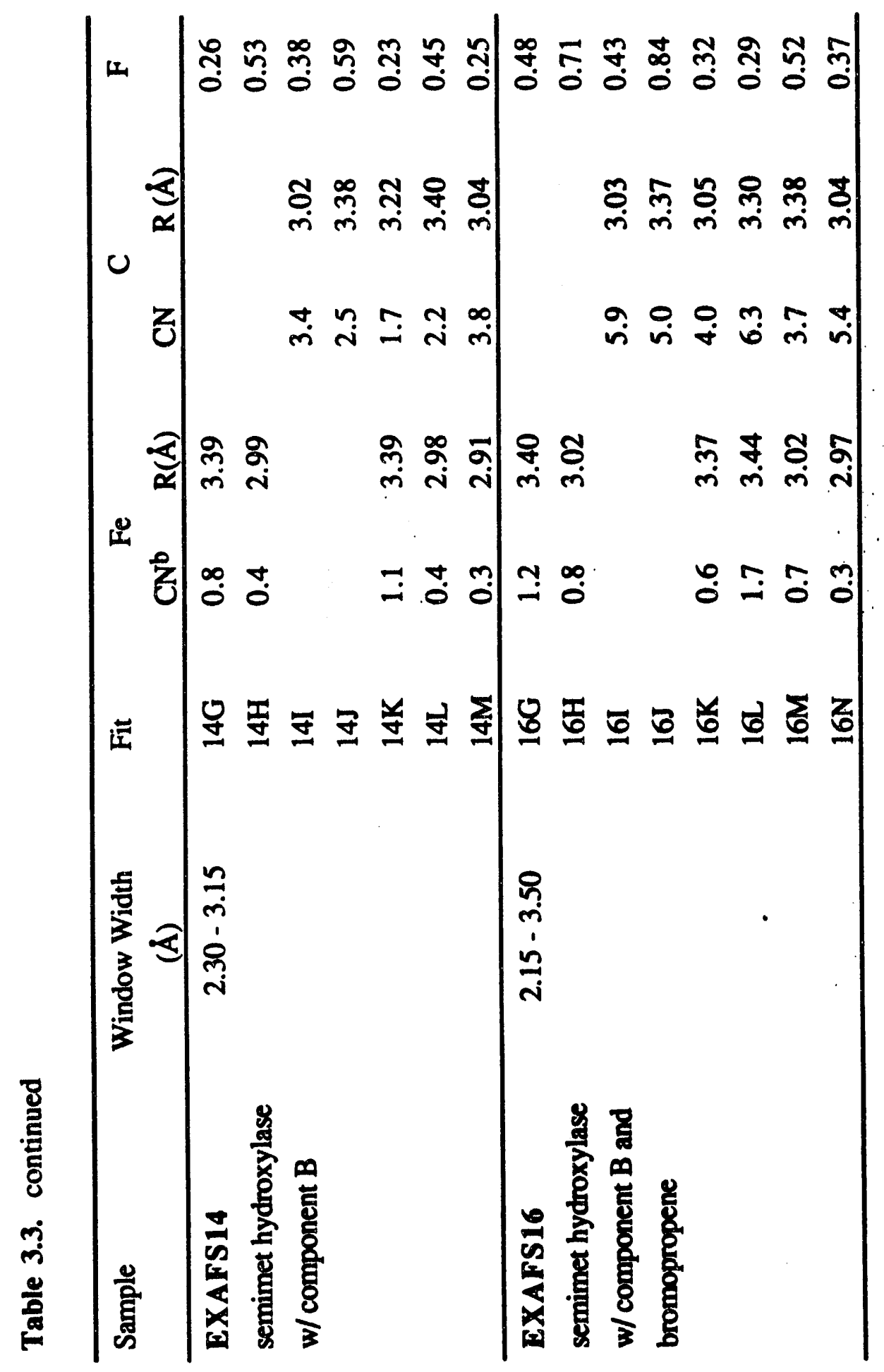




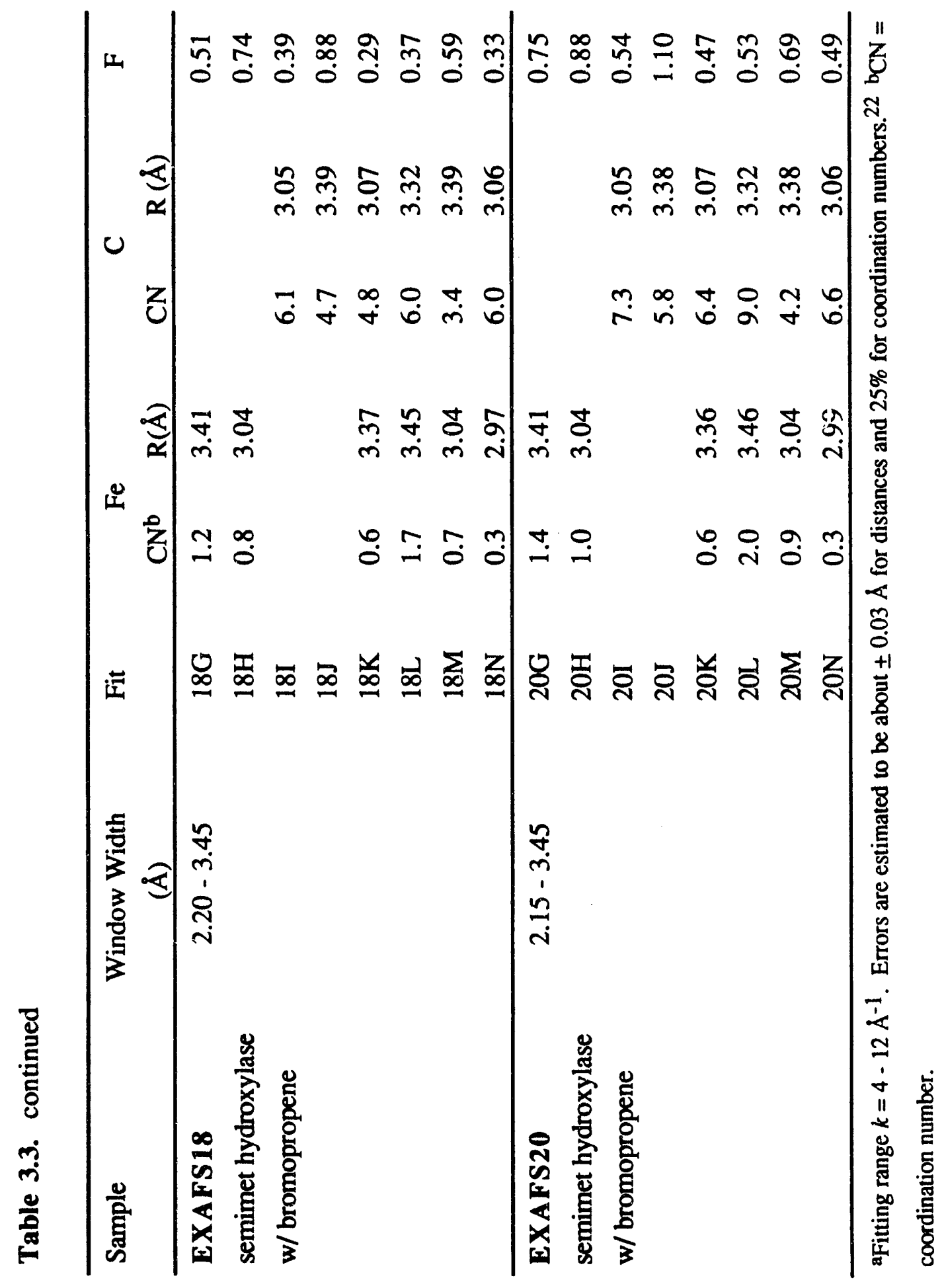


corresponded to the $3.0 \AA$ minimum for the C-only fits (Table 3.3, fits I for all samples). For fits including both $\mathrm{Fe}$ and $\mathrm{C}$, the individual contributions were highly correlated, affecting both coordination numbers and distances. The best fit to the data corresponded to a long $(3.4 \AA) \mathrm{Fe}-\mathrm{Fe}$ distance, and a short $(3.0 \AA) \mathrm{Fe}-\mathrm{C}$ distance for EXAFS10, EXAFS11, EXAFS18, and EXAFS20 (Table 3.3, fits 10K, 11K, 18K, 20K). For these fits, both the $\mathrm{Fe}$ and the $\mathrm{C}$ coordination numbers were lower than the values obtained for the long Fe-only fit and the short C-only fit (fits $\mathrm{G}$ and I, respectively), and the $\mathrm{Fe}-\mathrm{Fe}$ distance decreased while the $\mathrm{Fe}-\mathrm{C}$ distance increased. For EXAFS16, the long $\mathrm{Fe} / \mathrm{short}$ $C$ fit was the second best fit (Table 3.3 fit $16 \mathrm{~K}$ ) and showed the same correlation effect. It should be noted that for EXAFS14, fit 14K was the result for both a long Fe/long $\mathrm{C}$ and a long $\mathrm{Fe} /$ short $\mathrm{C}$ starting point.

These results demonstrate that there are two contributions to the second shell data, from atoms at an average distance of $3.0 \AA$ and $3.4 \AA$ from the iron center. A strong preference for a $3.4 \AA \mathrm{Fe}$-Fe distance and a $3.0 \AA \mathrm{Fe}-\mathrm{C}$ distance was given by the fit results (Table 3.3, fits $\mathrm{G}$ and I respectively). The assignment of the longer distance to $\mathrm{Fe}$ and the shorter distance to $\mathrm{C}$ is consistent with the lack of an oxo-bridged iron center and with the distribution of low $\mathrm{Z}$ atoms in structurally characterized models. ${ }^{25,29}$ To aid in the interpretation of these results, it is useful to compare the Fourier transforms of the fits (4 $12 \AA^{-1}$ ) with the $4-12 \AA^{-1}$ Fourier transform of the second shell contribution. The fits to the filtered second shell data and the Fourier transforms of the fits to the data for EXAFS10 are presented in Figure 3.5. The $3.39 \AA$ Fe fit (Table 3.3, fit 10G; Figure 3.5a) matches the Fourier transform of the second shell wave (at $2.68 \AA$ ) better than the $3.01 \AA$ Fe fit (Table 3.3, fit 10H; Figure 3.5b) both in terms of peak height and position. The Fourier transform of the $3.39 \AA$ Fe fit is shifted $0.11 \AA$ to longer $R$ (at $2.79 \AA$ ), and is $81 \%$ of the height of the data peak, whereas the $3.01 \AA$ Fe fit is $0.27 \AA$ to shorter $R$ (at $2.41 \AA$ ) and only $61 \%$ of the peak height. The Fourier transform of the $3.03 \AA$ C-only fit to the data (Table 3.3, fit 10I; Figure 3.5c) matches the Fourier transform of the data very well, with only a $0.02 \AA$ shift in the peak position of the fit to lower $R$ (at $2.66 \AA$ ), whereas the $3.38 \AA$ C-only fit to the data (Table 3.3, fit 10J; Figure 3.5d) occurs $0.33 \AA$ to higher $R$ (at $3.01 \AA$ ). The height of the peak of the short $C$ fit is $84 \%$ that of the data and the long $C$ fit is $52 \%$ of the data peak height. Clearly, the $3.39 \AA$ Fe fit or the $3.03 \AA \mathrm{C}$ fit better explain the second shell data than the $3.01 \AA \mathrm{Fe}$ fit or the $3.38 \AA \mathrm{C}$ fit, supporting the assignment of the longer distance contribution as iron and the shorter distance contribution as carbon. For the $\mathrm{Fe}+\mathrm{C}$ fits to the data, the fits consisting of long $\mathrm{Fe}$ and short $\mathrm{C}$ (Fit 10K, Figure 3.5e), long $\mathrm{Fe}$ and long $\mathrm{C}$ (Fit 10L, Figure 3.5f) and short $\mathrm{Fe}$ and short $\mathrm{C}$ (Fit 


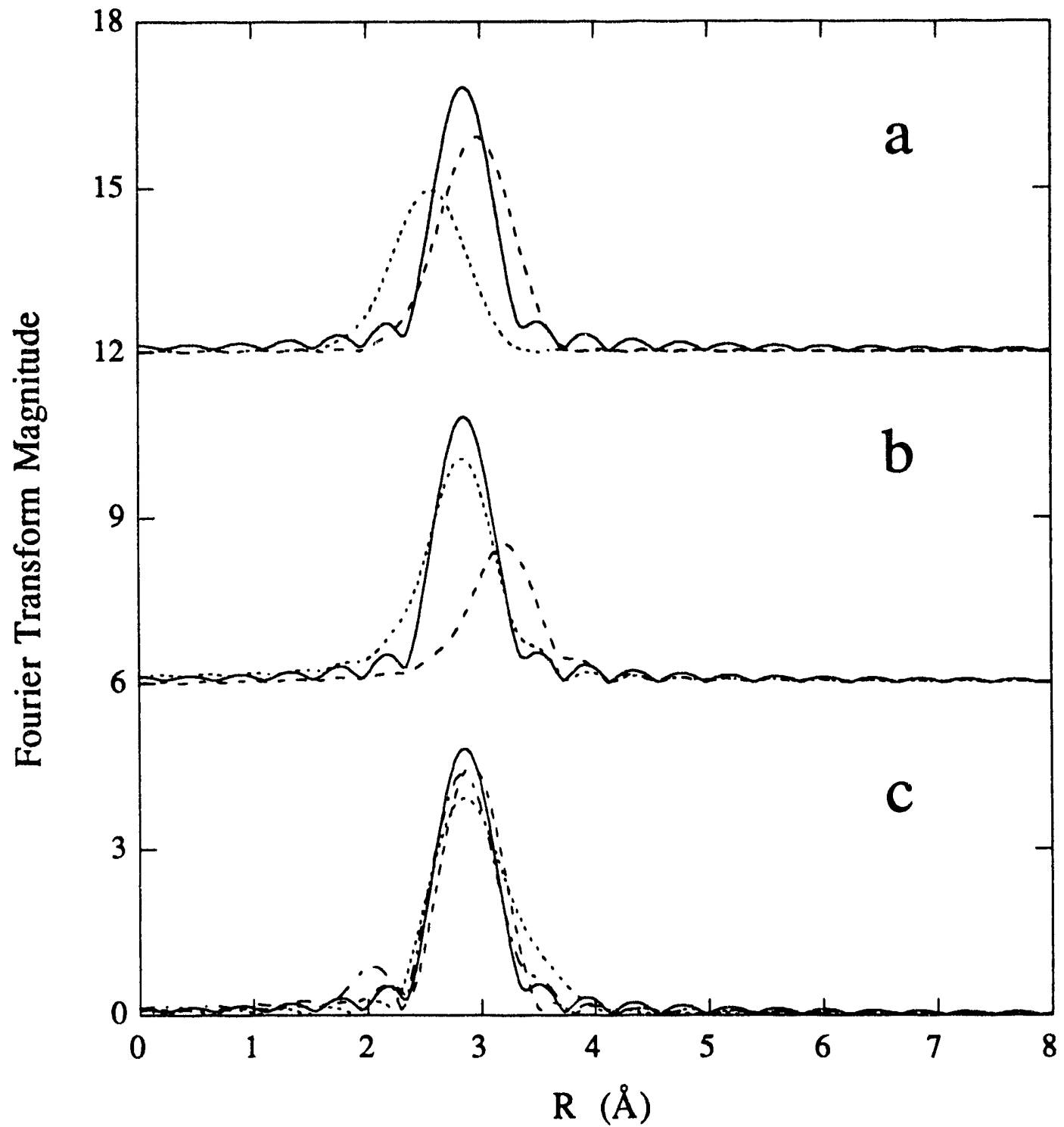

Figure 3.5. Fourier transforms of the second shell fits to the data for EXAFS10 ( $k=$ 4-12 $\AA^{-1}$ ). The solid line is the Fourier transform of the second shell EXAFS in every case. (a) Fe-only fits to the data; $3.39 \AA \mathrm{Fe}$ fit (Table 3.3, fit 10G) (dash) and $3.01 \AA \mathrm{Fe}$ fit (Table 3.3, fit H) (dot). (b) C-only fits to the data; $3.38 \AA \mathrm{C}$ fit (Table 3.3, fit 10J) (dash) and $3.03 \AA \mathrm{C}$ fit (Table 3.3, fit 10I) (dot). (c) $\mathrm{Fe}+\mathrm{C}$ fits to the data; long $\mathrm{Fe}$ and short C (Table 3.3, fit 10K) (dash), long Fe and long C (Table 3.3, fit 10L) (dot), and short Fe and short $\mathrm{C}$ (Table 3.3, fit 10M) (dash dot). 
$10 \mathrm{M}$, Figure $3.5 \mathrm{~g}$ ) are all quite good, however the improvement to the fits simply reflects the correlation of the $\mathrm{Fe}$ and the $\mathrm{C}$ parameters in the latter two fits to the data.

For the reduced data sets, fits to the filtered data over the region between 2.5 and $3.5 \AA$ were attempted with $\mathrm{Fe}$ or $\mathrm{C}$ and could be adequately fit with either less than $0.5 \mathrm{Fe}$ at $\sim 3.4 \AA$ or about $2 \mathrm{C}$ at $\sim 3.0 \AA$. These results are chemically unreasonable for the diiron center based on a comparison of these contributions with the known second shell contributions in structurally characterized diferrous models. In the second shell of the multiply-bridged diferrous model compounds $\left[\mathrm{Fe}_{2} \mathrm{OH}\left(\mathrm{O}_{2} \mathrm{CH}_{3}\right)_{2}\left(\mathrm{Me}_{3} \mathrm{TACN}\right)_{2}\right]\left(\mathrm{ClO}_{4}\right)$ $\mathrm{H}_{2} \mathrm{O}^{31}$ and $\left[\mathrm{Fe}_{2}\left(\mathrm{O}_{2} \mathrm{CH}\right)_{4}(\mathrm{BIPhMe})_{2}\right], 32$ there are $11 \mathrm{C}$ atoms between 2.89 and $3.16 \AA$, and $1 \mathrm{O}$ atom at $3.3 \AA$ (the MezTACN compound also has $\mathrm{Fe}$ at $3.32 \AA$; the Fe distance in the BIPhMe model is $3.57 \AA$ ). The low height of the peak in the second shell region of the Fourier transform, and the low coordination numbers for the fits especially in the 3.0 - 3.2 $\AA$ range, suggest that there is some high frequency contribution to the data that can be mathmatically modeled with the Fe and $\mathrm{C}$ parameters, but does not necessarily reflect the true second shell environment of the ferrous iron center. 33

3.3.2.3. Wide Shell Fits. The same trends seen in the first and second shell fits to the semimet data were $\mathrm{Sen}$ in the fits to the backtransform of both of the peaks in the Fourier transform (Table 3.4). Only the results for $R_{N}>R_{O}$ are reported, although all of the fits with $R_{N}<R_{O}$ were periormed. The fit function for the N/O fit to the data was about 1 for all of the samples (Table 3.4, fits $O$ ), and improved by a factor of 1.8 to 2 with the addition of a either a $3.4 \AA$ Fe contribution (Table 3.4, fits P) or by a factor of 1.3 to 2.6 with a $3.0 \AA \mathrm{C}$ contribution (Table 3.4 , fits $\mathrm{S}$ ). With the addition of both a $3.4 \AA \mathrm{Fe}$ and a $3.0 \AA$ C contribution (Table 3.4, fits T), the fits improved only slightly over the 3.4 $\AA$ Fe-only or the $3.0 \AA \mathrm{C}$-only fits, and the same correlation effects (reduction in the $\mathrm{Fe}$ and $\mathrm{C}$ coordination number and $\mathrm{Fe}$ distance, increase in $\mathrm{C}$ distance) were seen for all samples with the exception of EXAFS14, for which no minimum corresponding to a $3.4 \AA \mathrm{Ae}$ and 3.0 A C initial starting point was found.

The data and fits to the data are presented in Figure 3.6 for EXAFS10. The description here is entirely analagous for the rest of the samples as well. The N/O fit describes both the low frequency contribution to the data and the overall amplitude of the data (Table 3.4, fit 100, Figure 3.6a) but does not explain the higher frequency components of the EXAFS. The effect of adding the high frequency Fe to the $\mathrm{N}$ and $\mathrm{O}$ fit (Table 3.4, fit 10P) is seen in Figure 3.6b, with the beat pattern above $k=7 \AA^{-1}$ in the data now being mimicked by the fit to the data. The inclusion of the short $\mathrm{C}$ contribution to fit 10P (Table 3.4, fit 10T, Figure 3.6c) primarily improves the match in the frequency above $k=10 \AA^{-1}$. 


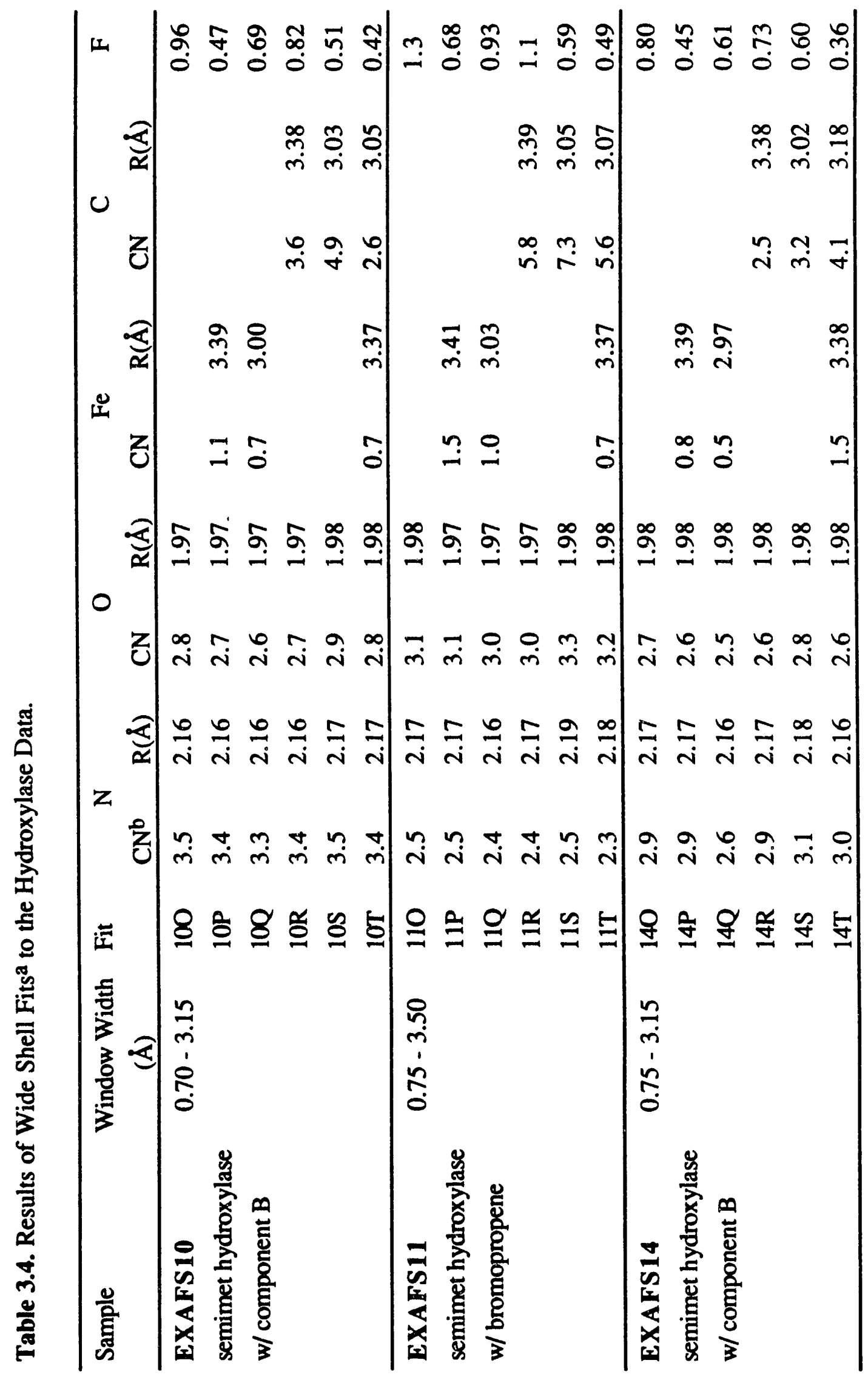




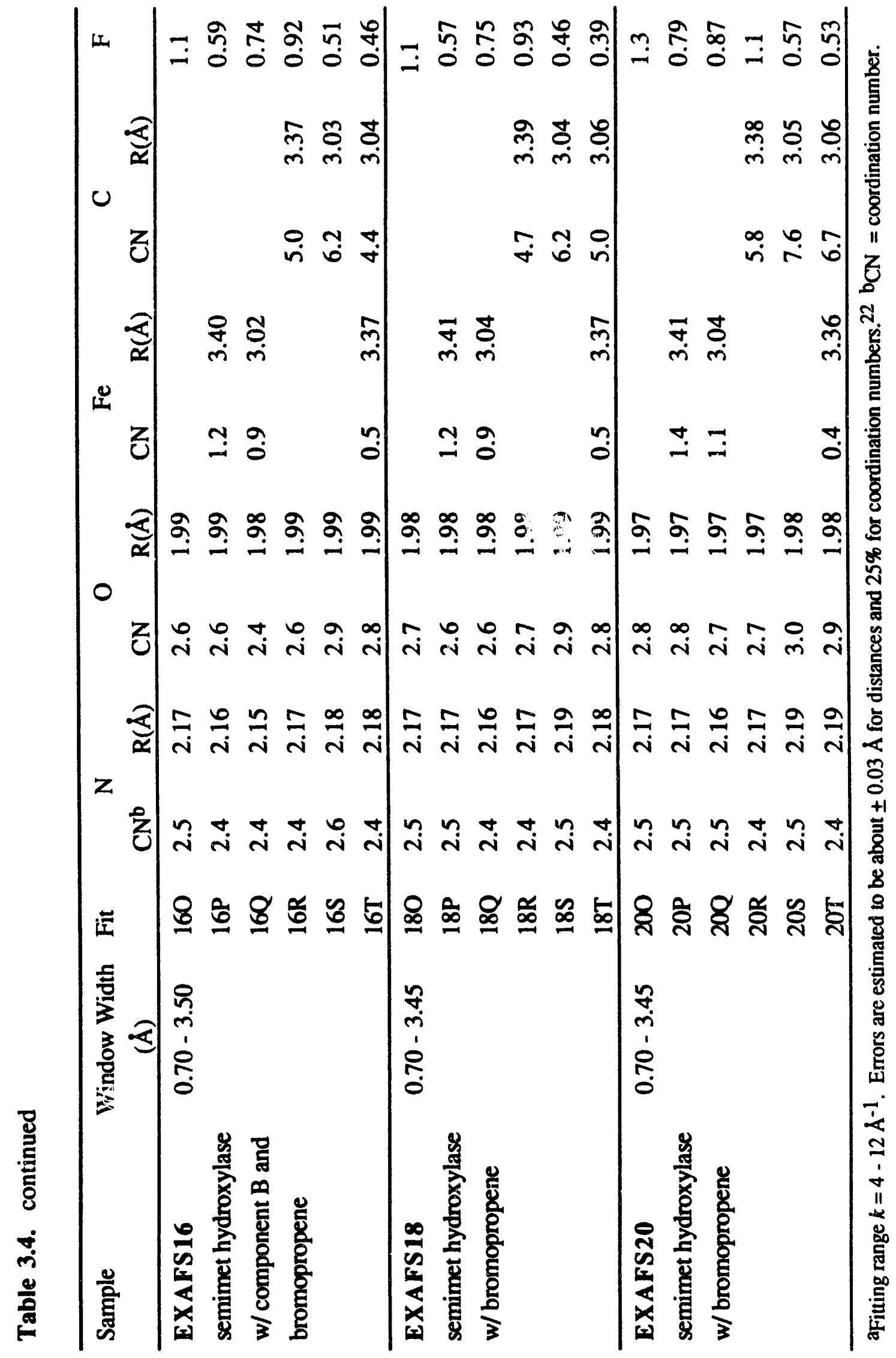




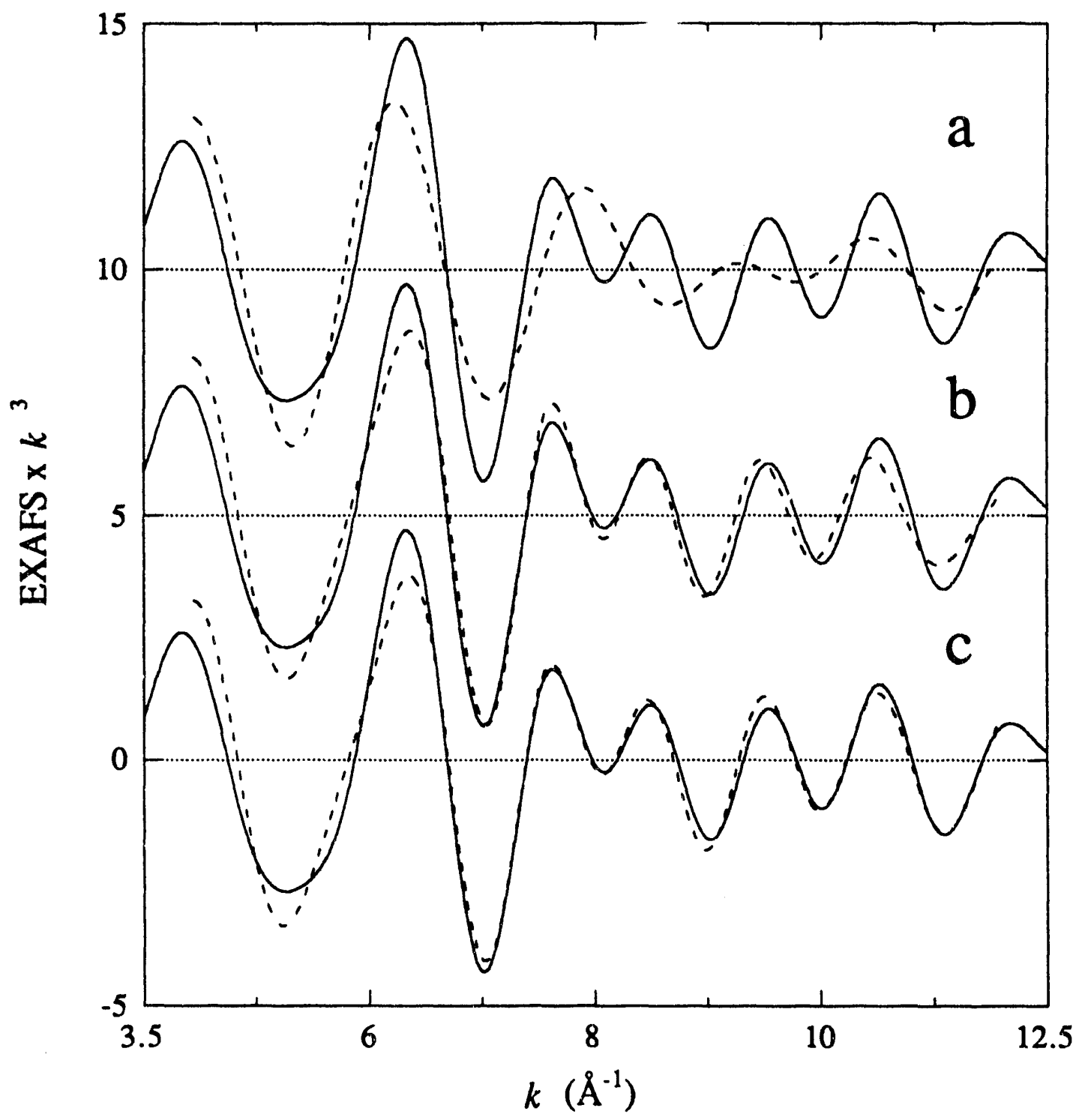

Figure 3.6. Fits to the Fourier filtered first and second shell EXAFS for EXAFS10 ( $k$ $=4-12 \AA^{-1}$ ). The solid line is the data and the dashed line is the fit. (a) Fit to the data with $\mathrm{N}$ and $\mathrm{O}$ (Table 3.4, fit 100), (b) fit to the data with $\mathrm{N}, \mathrm{O}$, and $\mathrm{Fe}$ (Table 3.4, fit 10P), (c) Fit to the data with $\mathrm{N}, \mathrm{O}, \mathrm{Fe}$, and $\mathrm{C}$ (Table 3.4, fit 10T). Note the improvement in the fit with the addition of $\mathrm{Fe}$ to the $\mathrm{N}$ and $\mathrm{O}$ contributions. 
3.3.2.4. The Origin of the Metrical Differences in the EXAFS. There are subtle differences in the heat pattern of the EXAFS data seen above $k=7 \AA^{-1}$ (Figure 3.1) for the hydroxylase complexes vs. the non-complexed hydroxylase as described above. These differences suggest that there may be a structural change that occurs in the complexed forms of the hydroxylase, however the fit results do not support a significant change in the ligation of the iron atoms as a result of the presence of component $B$ or substrate based on a comparison of the average f st shell coordination. Although the differences in the EXAFS cannot be completely accounted for by the fit results, qualitatively it can be shown that the differences arise from differences in the first shell EXAFS. The backtransforms of the first shell data for representative complexed forms of the hydroxylase are compared to the first shell EXAFS of the non-complexed hydroxylase in Figure 3.7, and of the second shell data in Figure 3.8. Significant changes in the first shell EXAFS occur in both amplitude and phase above $k=7 \AA^{-1}$ for all of the samples, although the non-complexed form and the bromopropene-complexed forms are very similar. The second shell data, while nut identical for all of the samples, are certainly more similar to each other than the first shell data. The differences in the beat pattern of the EXAFS can therefore be attributed to the differences in the first shell data above $k=7 \AA^{-1}$ interacting with a relatively constant second shell contribution. The differences in the first shell EXAFS are probably reflected by the differences in the relative coordination numbers of the long and short distance contributions to the first shell fits of the B-complexed vs. bromopropene-complexed samples described above. The effect of the first shell data on the appearance of the EXAFS is illustrated in Figure 3.9, which shows the filtered EXAFS of the B-complexed hydroxylase (EXAFS10), along with the EXAFS from the addition of the first shell data from EXAFS10 to the second shell data of the non-complexed sample and the filtered EXAFS of the non-complexed hydroxylase. Addition of the second shell EXAFS of the non-complexed form to the first shell EXAFS of the B-complexed form (Figure 3.9b) results in EXAFS more like that of the true B-complexed samples (Figure 3.9a) than the non-complexed sample (Figure 3.9c).

3.3.2.5. Fits to the Non-Filtered Data. Fits to the non-filtered data between 4 and $12 \AA^{-1}$ were done for all of the semimet and reduced samples (Table 3.5). All of the trends described above were seen for the fits to the wide filtered data, although the fit functions were higher due to the increased noise level of the data. The data and the fit to the data (Table 3.5, fit $U$ ) for all of the semimet samples are shown in Figure 3.10. The Fourier transforms $\left(4-12 \AA^{-1}\right)$ of the fits in Table 3.5 for EXAFS10 are compared to the $4-12 \AA^{-1}$ Fourier transform of the data in Figure 3.11. The N/O contribution to the data matches the first shell peak in the Fourier transform very well for all of the fits. For 


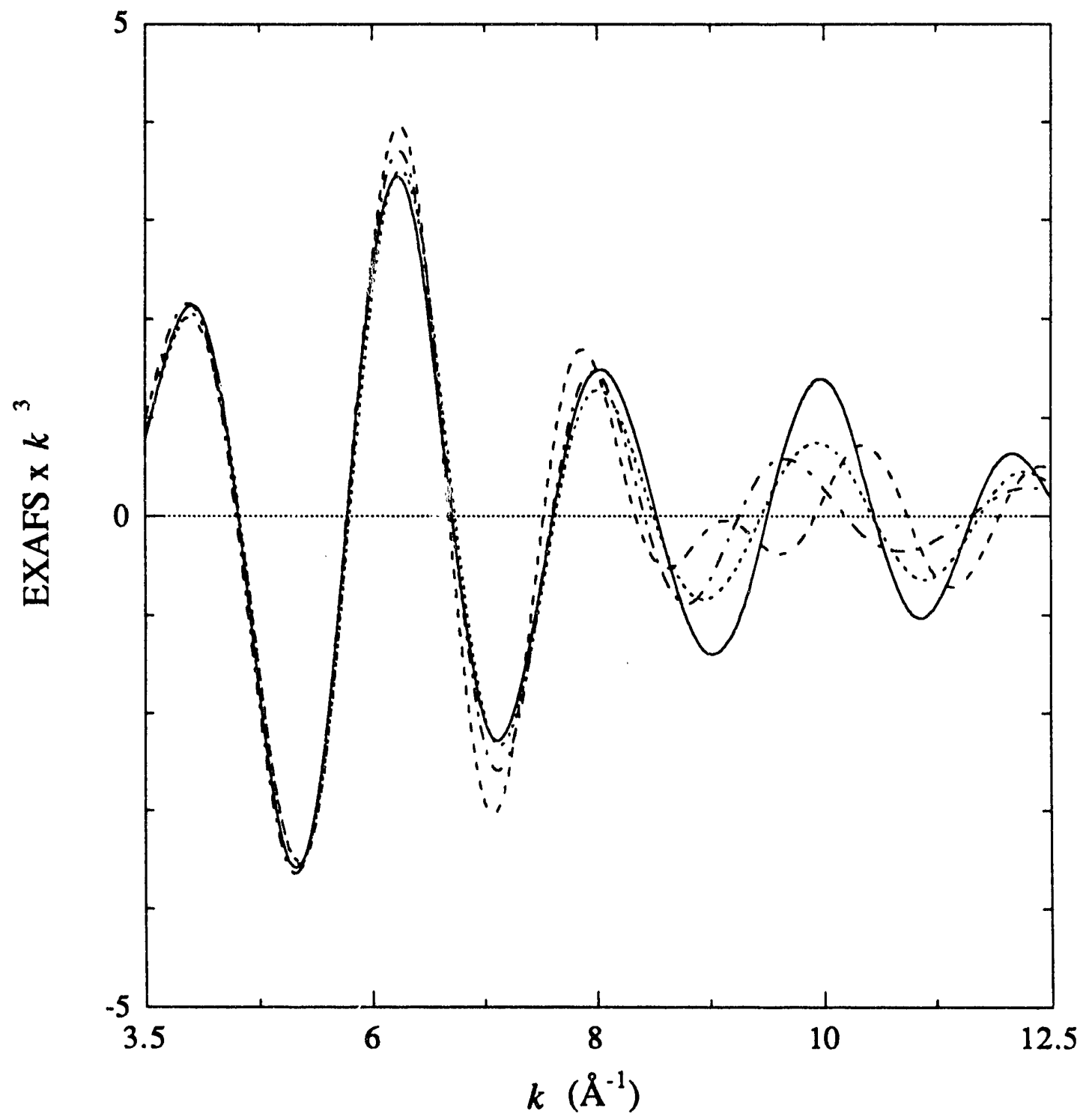

Figure 3.7. Comparison of the Fourier backtransforms of the first shell data (see Figure 3.2) for the semimet hydroxylase samples. Semimet hydroxylase sample, EXAFS2 (solid); with component B, EXAFS10 (dash); with bromopropene, EXAFS18 (dot); and with B and bromopropene, EXAFS16 (dash dot). The Fourier filter window widths used for these backtransforms are given in Table 3.2. Note the difference in the EXAFS above $k=8 \AA^{-1}$, especially for EXAFS10. 


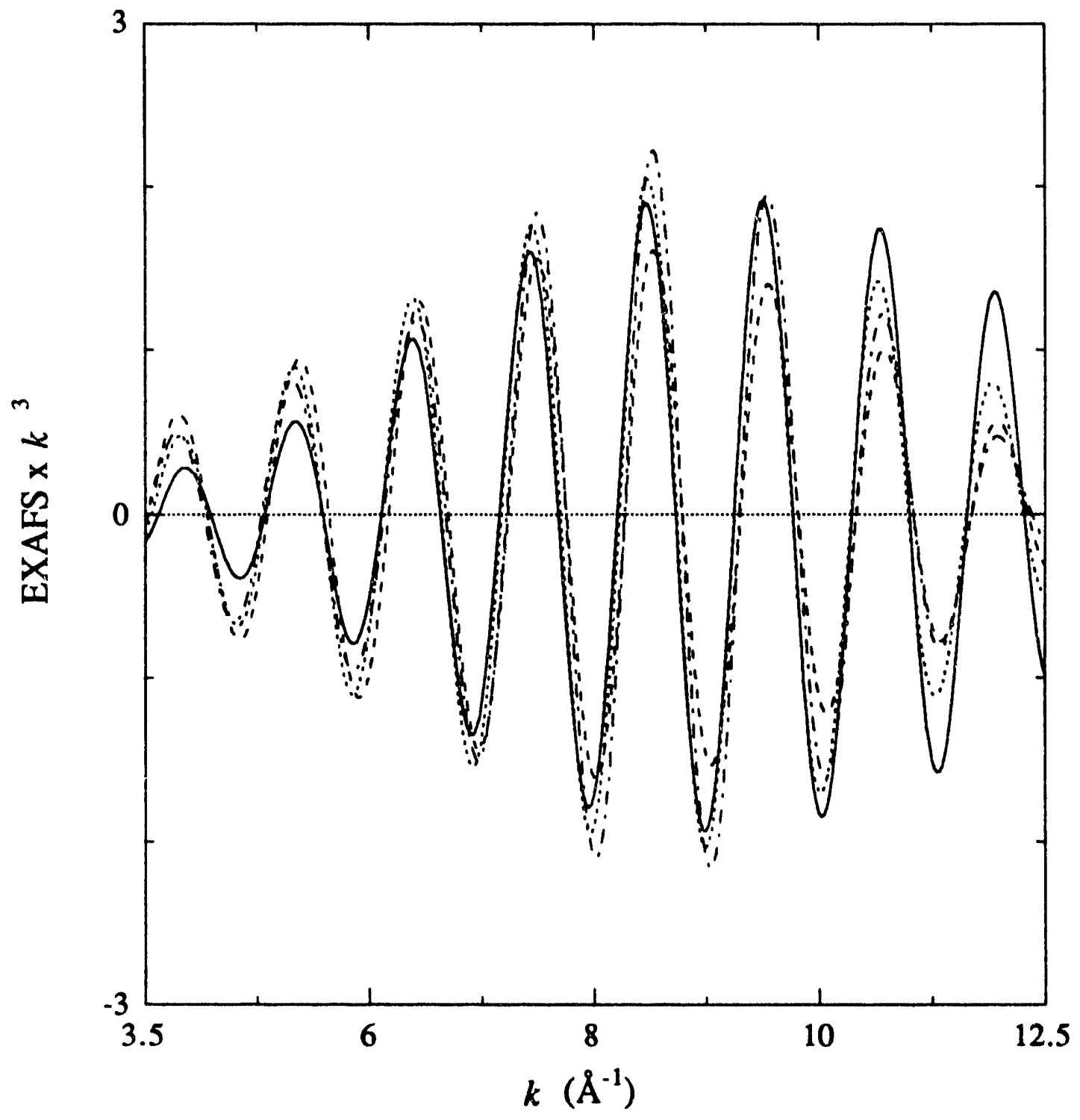

Figure 3.8. Fourier backtransforms of the second shell data (see Figure 3.2) for the semimet hydroxylase samples. Semimet hydroxylase sample, EXAFS2 (solid); with component B, EXAFS10 (dash); with bromopropene, EXAFS18 (dot); and with B and bromopropene, EXAFS16 (dash dot). The Fourier filter window widths used for these backtransforms are given in Table 3.3. 


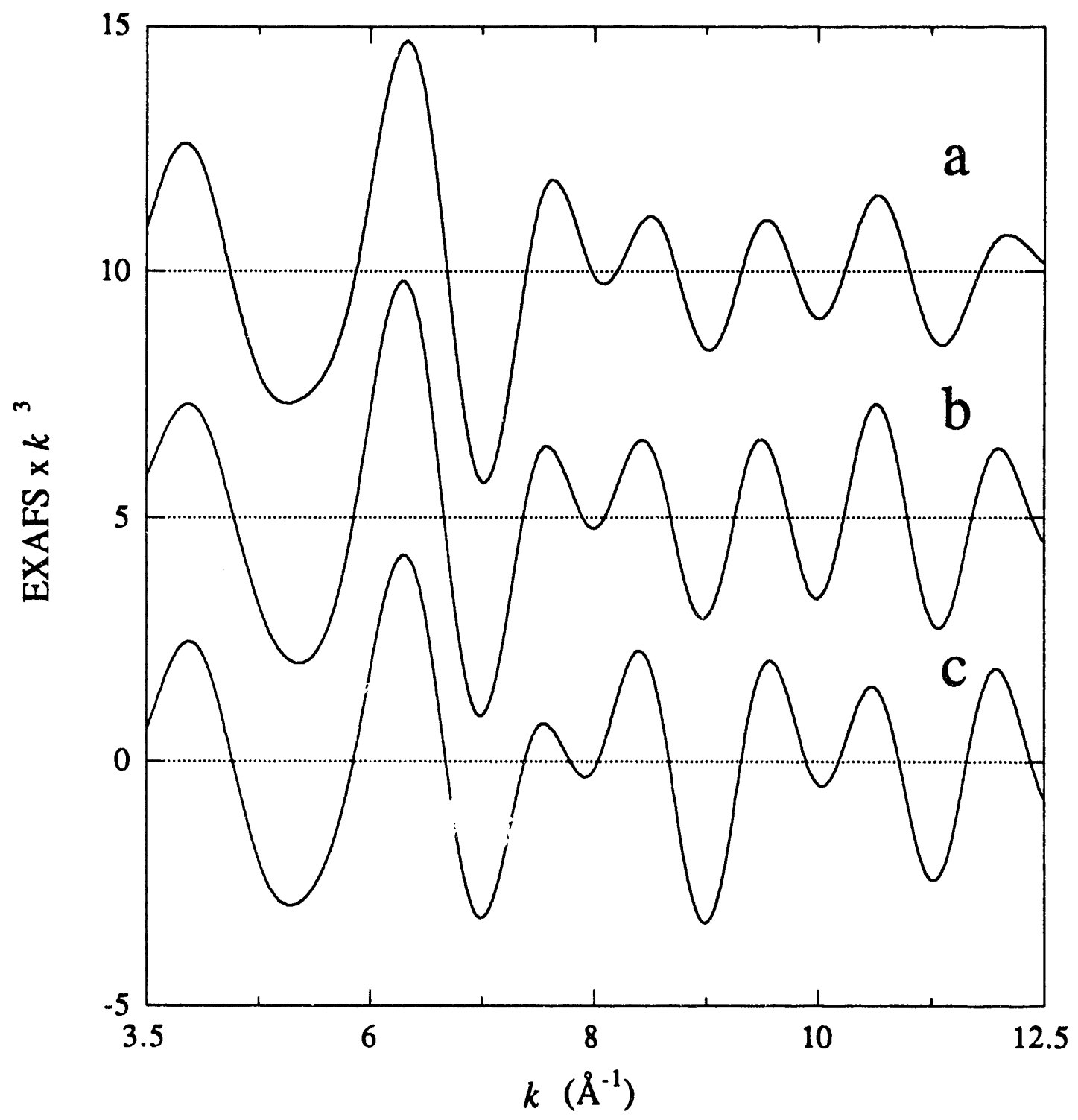

Figure 3.9. A comparison of the interaction between the first and second shell semimet hydroxylase EXAFS data. (a) Fourier filtered EXAFS of EXAFS10 (with component B); (b) the 1:1 addition of the Fourier filtered first shell data of EXAFS10 with the Fourier filtered second shell data of EXAFS2; (c) Fourier filtered EXAFS of EXAFS2 (non-complexed form). 


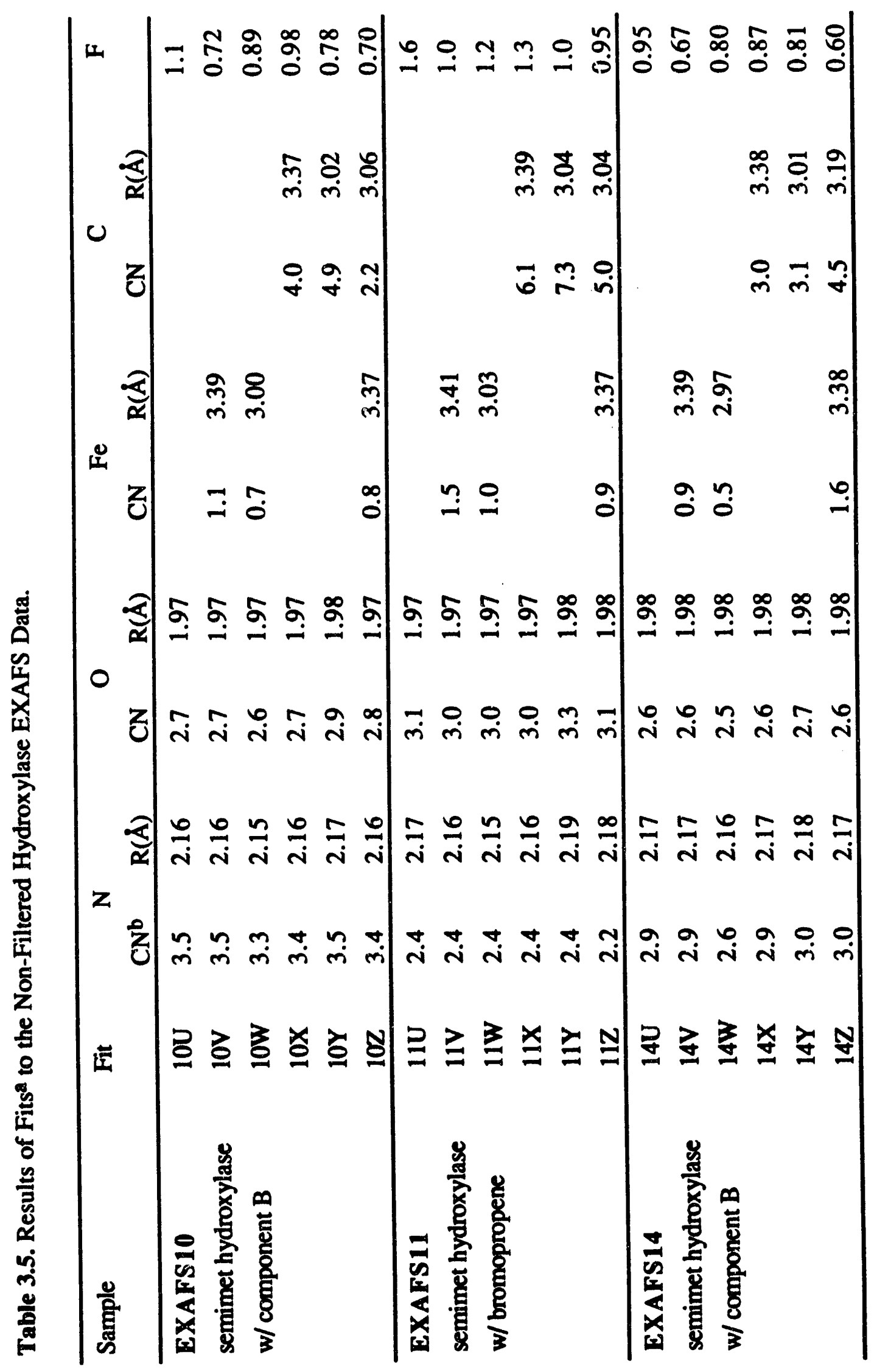




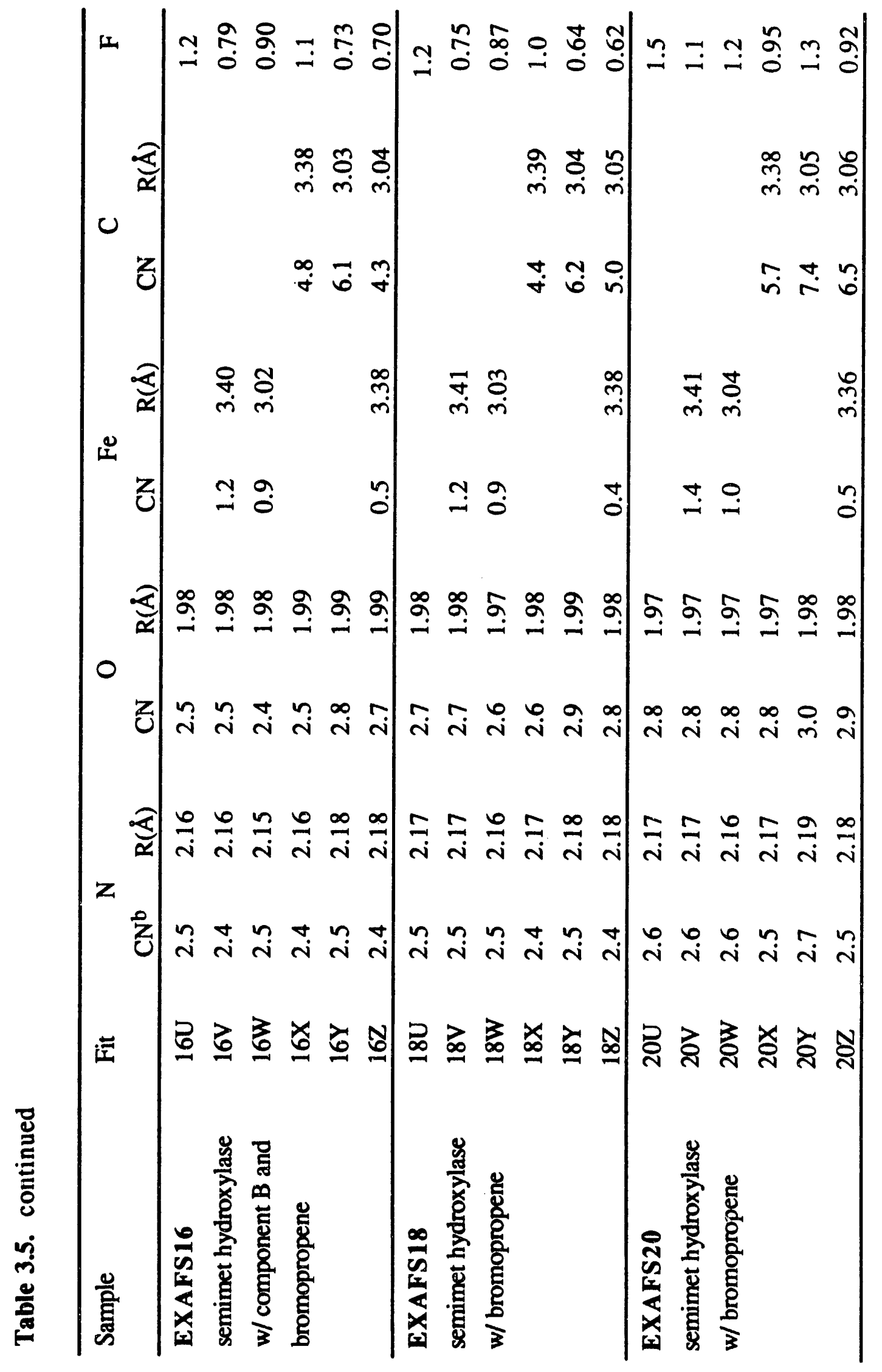




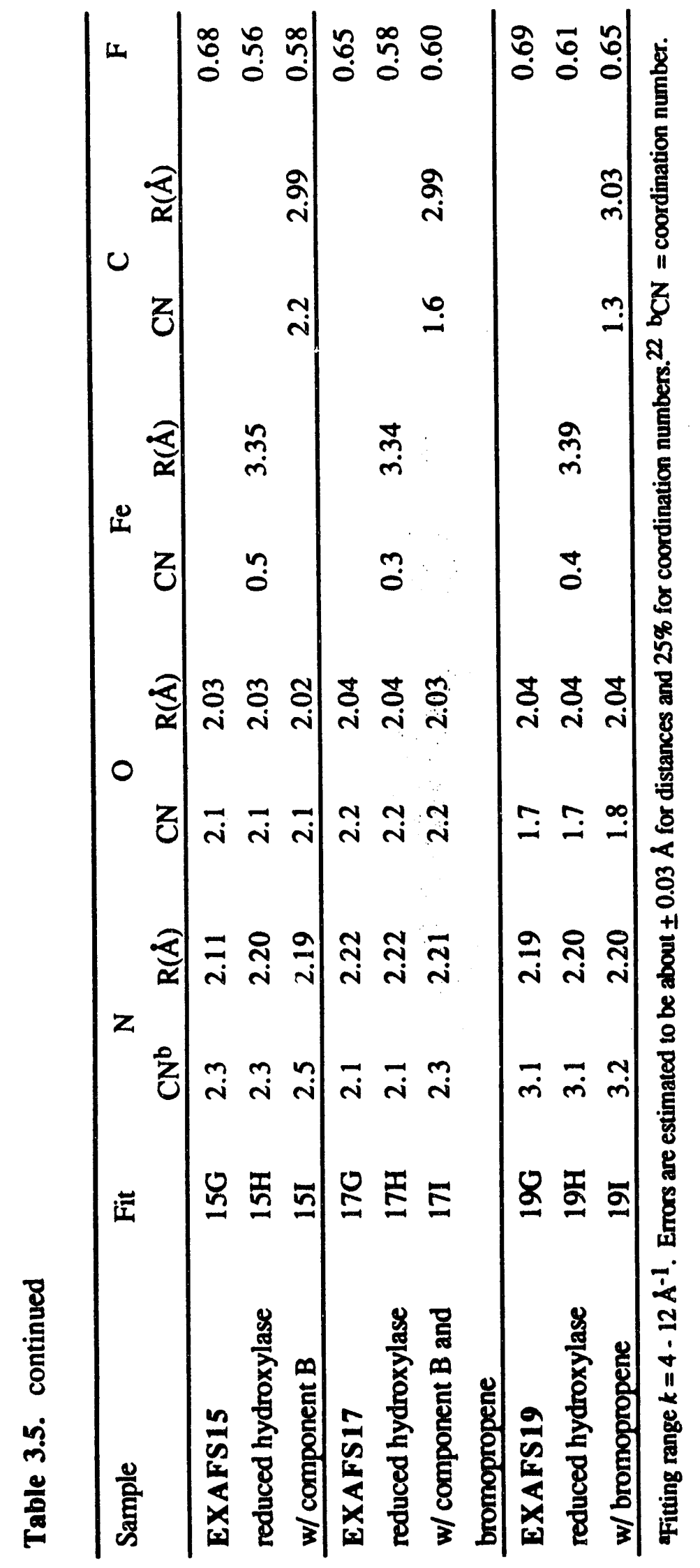




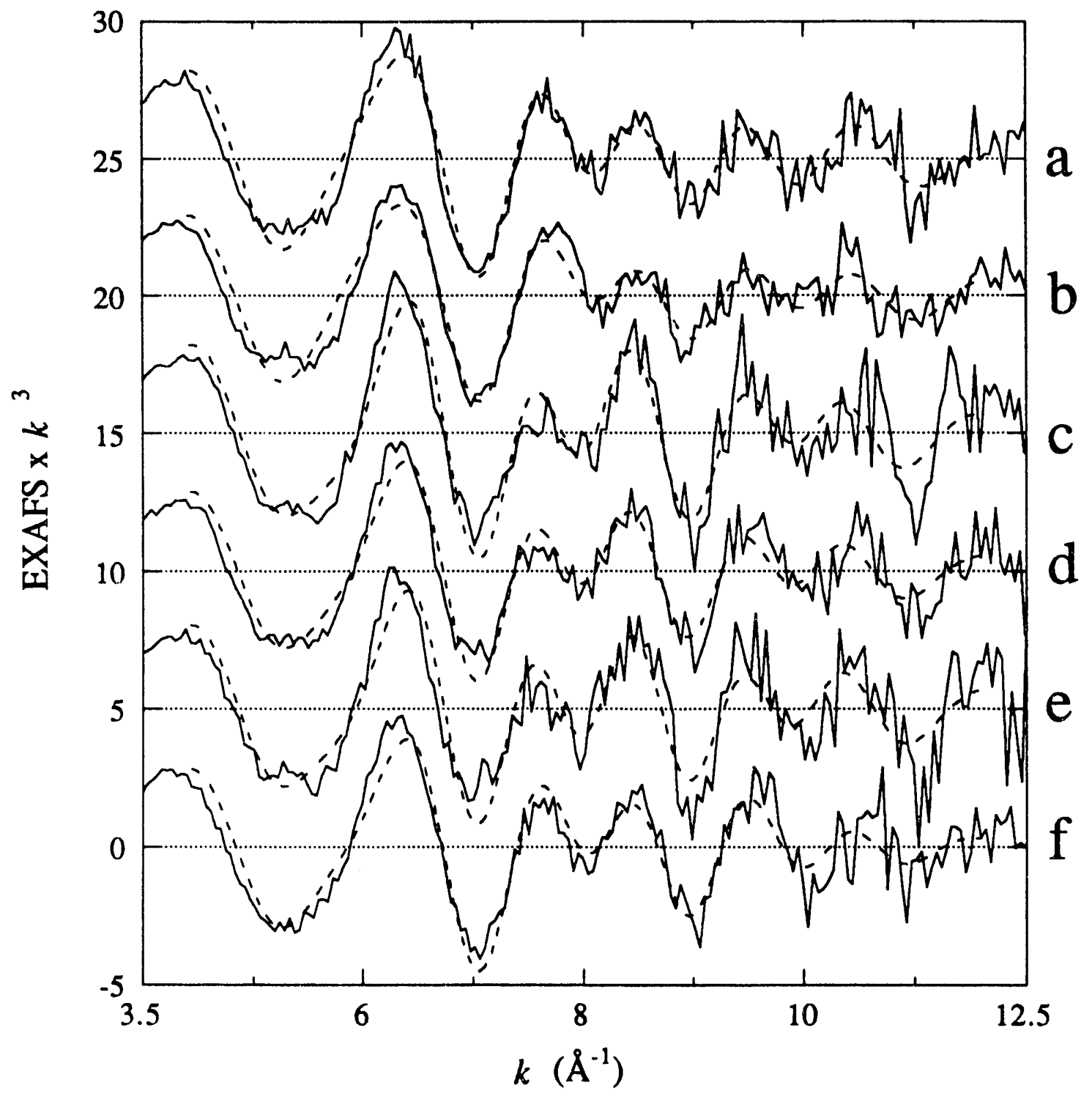

Figure 3.10. Fits to the non-filtered data for the semimet hydroxylase samples (fitting range $k=4-12 \AA^{-1}$ ). The solid line is the data and the dashed line is the fit to the data. The fit shown is the N,O and Fe fit (Table 3.5, fit $U$ for all). (a) EXAFS10, (b) EXAFS14, (c) EXAFS11, (d) EXAFS18, (e) EXAFS20, (f) EXAFS16. 


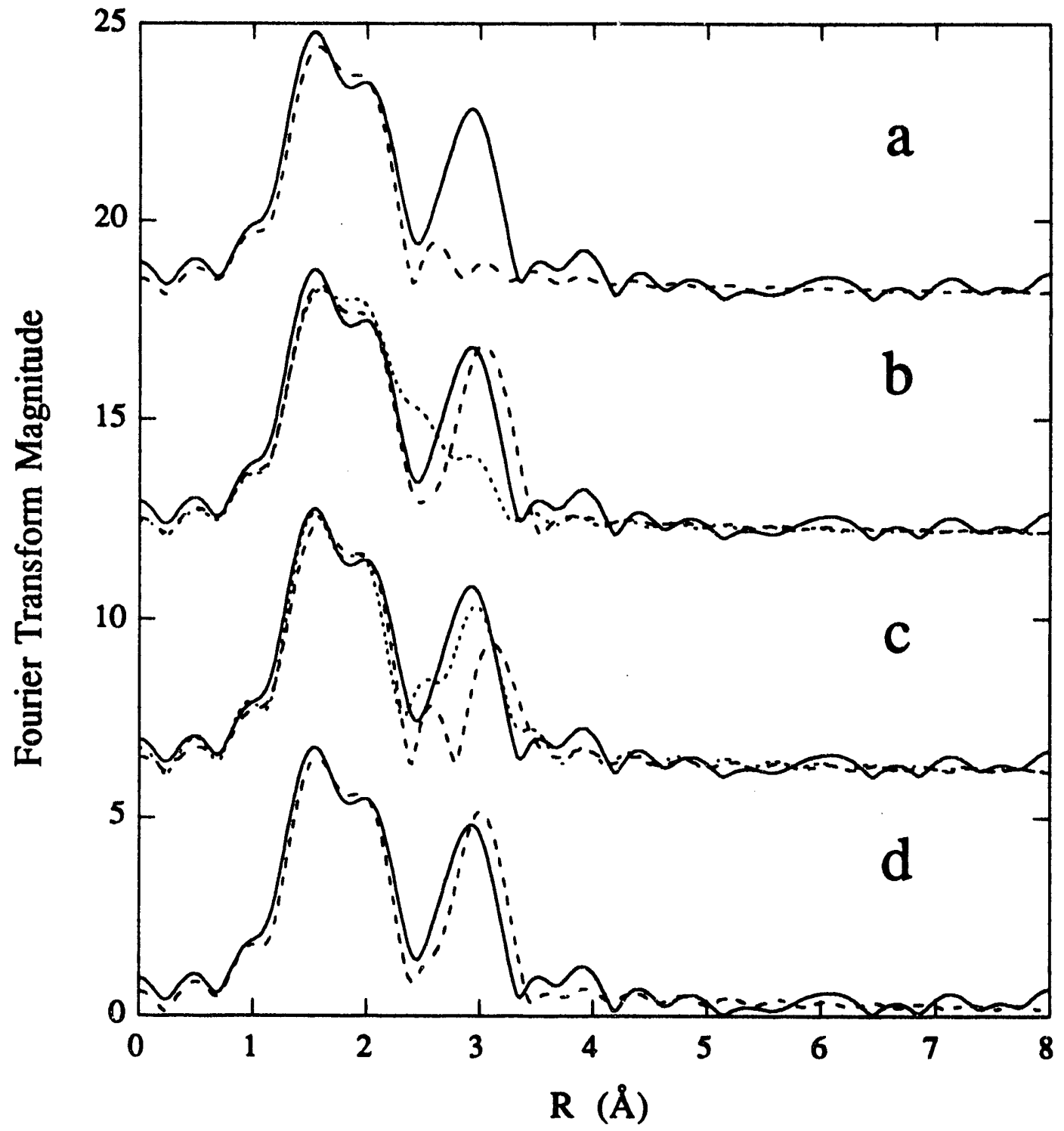

Figure 3.11. Fourier transforms of the fits to the non-filtered data for EXAFS10 (Fourier transform range $k=4-12 \AA^{-1}$ ). The solid line is the data. (a) Fit to the data with $\mathrm{N}$ and $\mathrm{O}$ (dash) (Table 3.5, fit 10U), (b) fit to the data with $\mathrm{N}, \mathrm{O}$ and long $\mathrm{Fe}$ (dash) (Table 3.5, fit 10V) and $\mathrm{N}, \mathrm{O}$ and short $\mathrm{Fe}$ (dot) (Table 3.5, fit 10W), (c) fit to the data with $\mathrm{N}, \mathrm{O}$ and long $C$ (dash) (Table 3.5, fit 10X) and $N, O$ and short $C$ (dot) (Table 3.5, fit 10Y), (d) fit to the data with $\mathrm{N}, \mathrm{O}, \mathrm{Fe}$ and $\mathrm{C}$ (dash) (Table 3.5, fit 10Z). Note that the fit to the data with $\mathrm{N}, \mathrm{O}$ and a long $\mathrm{Fe}$ contribution is better than the $\mathrm{N}, \mathrm{O}$ and short $\mathrm{Fe}$ fit, while the fit including the short $\mathrm{Fe}-\mathrm{C}$ contribution is better than the fit including the long $\mathrm{Fe}-\mathrm{C}$ contribution. 
Fit 10V (Table 3.5, Figure 3.11b), the second shell peak is slightly mismatched in position, with the fit to the data occuring at a slightly higher $R$ value, but the amplitude of the peaks is well-matched, similar to what was described for the second shell fits above. The Fourier transform of the N/O/short Fe fit to the data (Table 3.5, fit 10W) is a poor match for the data (Figure 3.11b). For Fit 10Z, the amplitude of the fit in the second shell region is slightly high, but the addition of the short $\mathrm{C}$ contribution to the N/O/long Fe fit improves the match in the position of the peak of the fit to the data with the second shell peak of the data (Figure 3.11d). Clearly, this shows that the Fe-Fe parameters employed cannot by themselves explain the second shell data completely, and that something in addition to iron needs to be added to the fits.

The same procedure was performed for the reduced data, and the fit results are also presented in Table 3.5. Although the N/O fits to the data were adequate to explain the data, the broad feature suggested on the low $k$ side of the $10 \AA^{-1}$ maximum was not accounted for (Figure 3.12a,c,d). However, with either the addition of a small $3.0 \AA \mathrm{C}$ contribution or a $3.4 \AA \mathrm{Fe}$ contribution, a better fit to the data at higher $k$ was found (illustrated for EXAFS15, Figure 3.12b). As described above in the fits to the second shell data, that while improving the fit to the data mathematically, the actual numerical results of the high frequency contribution should not be interpreted as reflecting a true second shell environment. The Fourier transforms of the data and the fits to the data shown in Figure 3.12 are presented in Figure 3.13. The inclusion of $\mathrm{Fe}$ or $\mathrm{C}$ to the fit models the small peak above $2.5 \AA$.

\subsubsection{X-ray Absorption Near Edge Spectra}

The edge spectra of the semimet hydroxylase samples are presented in Figure 3.14. The spectra consist of a small, low intensity feature at $\sim 7114 \mathrm{eV}$ (Feature A), the main transition at $\sim 7129 \mathrm{eV}$ (Feature B) and a broad maximum at $\sim 7134 \mathrm{eV}$ (Feature C). The intensity of feature B increases in the presence of component B (EXAFS10 and EXAF 16) and a shoulder appears on the rising edge at $\sim 7124 \mathrm{eV}$ (Feature $\mathrm{A}^{\prime}$ ). The energy position of Feature A does not change for the various samples, but its intensity increases for the samples with component B (see Figure 3.14 inset). The shapes of the pre-edge features for the other two complexes of the hydroxylase are not significantly different from the non-complexed form.

Upon reduction to the diferrous state (Figure 3.15), the the main transition moves to lower energy $(\sim 7125 \mathrm{eV})$ and the shape changes dramatically, increasing in intensity and becoming narrow. This change is typical of the reduction of $\mathrm{Fe}$ (III) to $\mathrm{Fe}$ (II) in models 


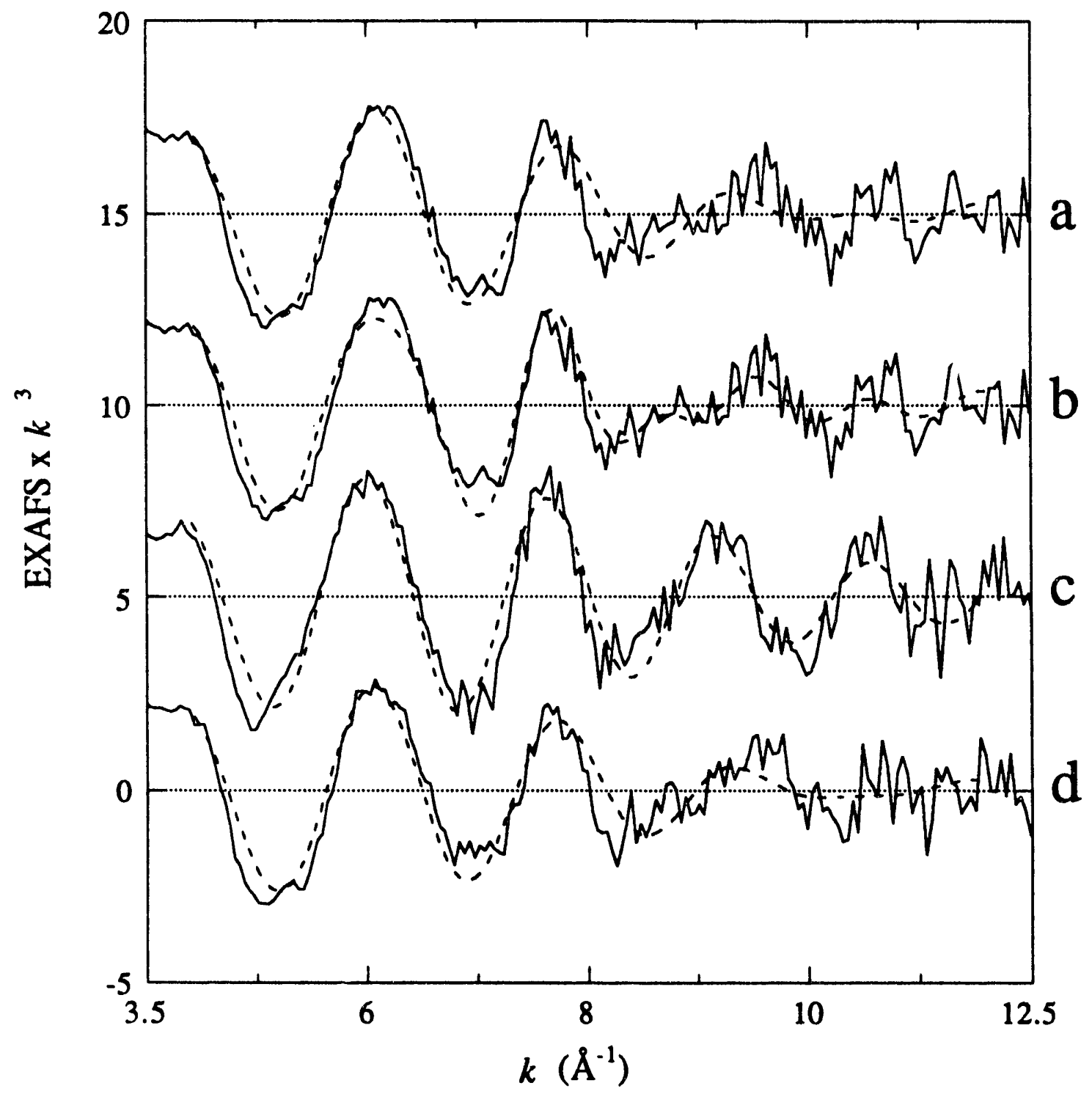

Figure 3.12. Fits to the non-filtered data for the reduced hydroxylase samples (fitting range $k=4-12 \AA^{-1}$ ). The solid line is the data and the dashed line is the fit to the data. (a) Fit to the data for EXAFS15 with $N$ and $O$ (Table 3.5, fit G), (b) fit to the data for EXAFS15 with $\mathrm{N}, \mathrm{O}$ and $\mathrm{Fe}$ (Table 3.5, fit $\mathrm{H}$ ). $\mathrm{N}$ and $\mathrm{O}$ only fits to (c) EXAFS19 (Table 3.5, fit 19G) and (d) EXAFS17 (Table 3.5, fit 17G). Note that the inclusion of Fe to the fit for EXAFS15 accounts for the shoulder in the EXAFS between $k=8$ and $9 \AA^{-1}$. 


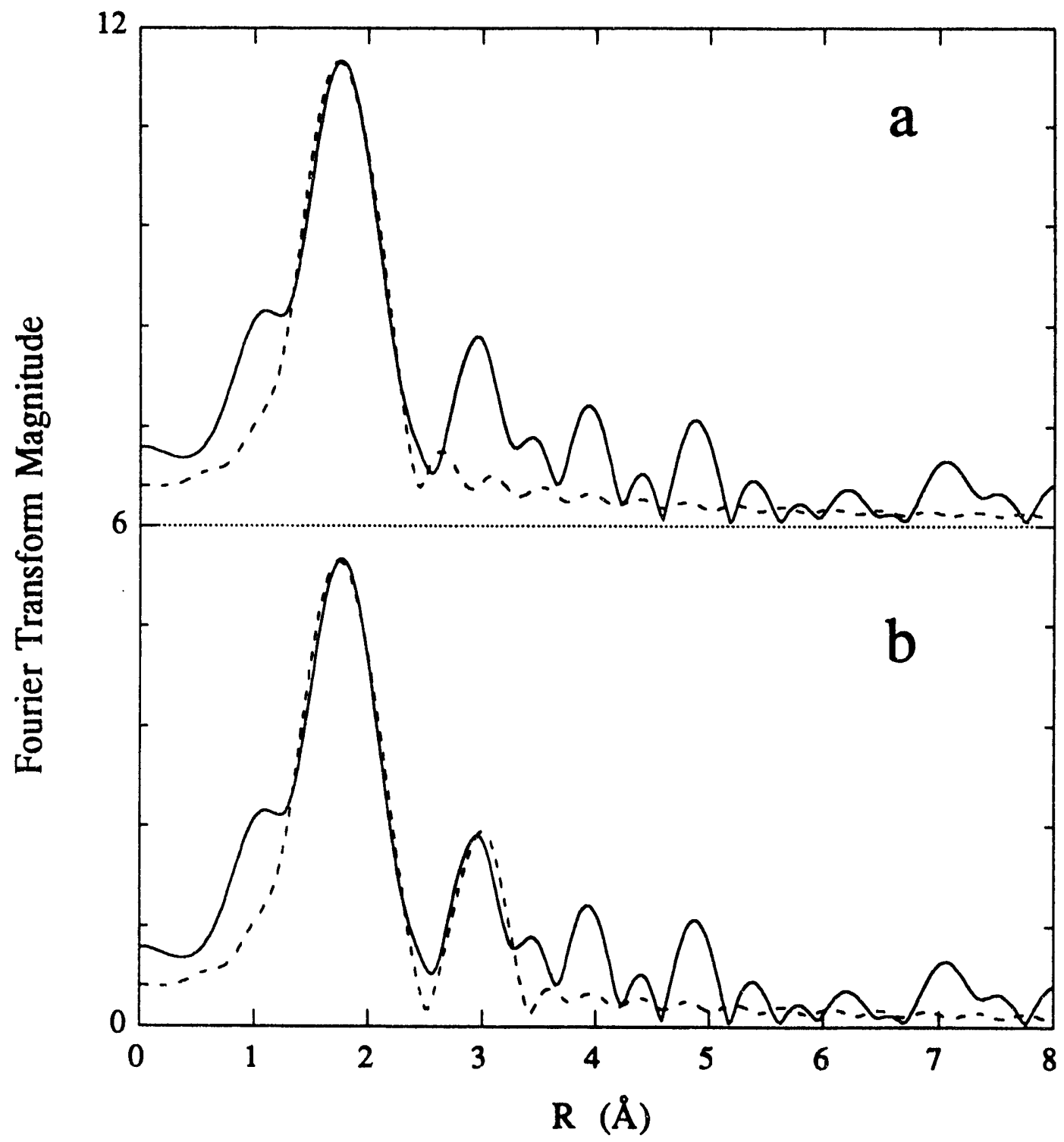

Figure 3.13. Fourier transforms of the fits to the non-filtered data for EXAFS15 (Fourier transform range $k=4-12 \AA^{-1}$ ). The solid line is the Fourier transform of the data and the dashed line is the fit for (a) $\mathrm{N}$ and $\mathrm{O}$ fit (Table 3.5, fit $\mathrm{G}$ ) and (b) $\mathrm{N}, \mathrm{O}$ and $\mathrm{Fe}$ fit (Table 3.5, fit $\mathrm{H}$ ). Note that the inclusion of $\mathrm{Fe}$ to the fit explains the $3.0 \AA$ peak in the Fourier transform. 


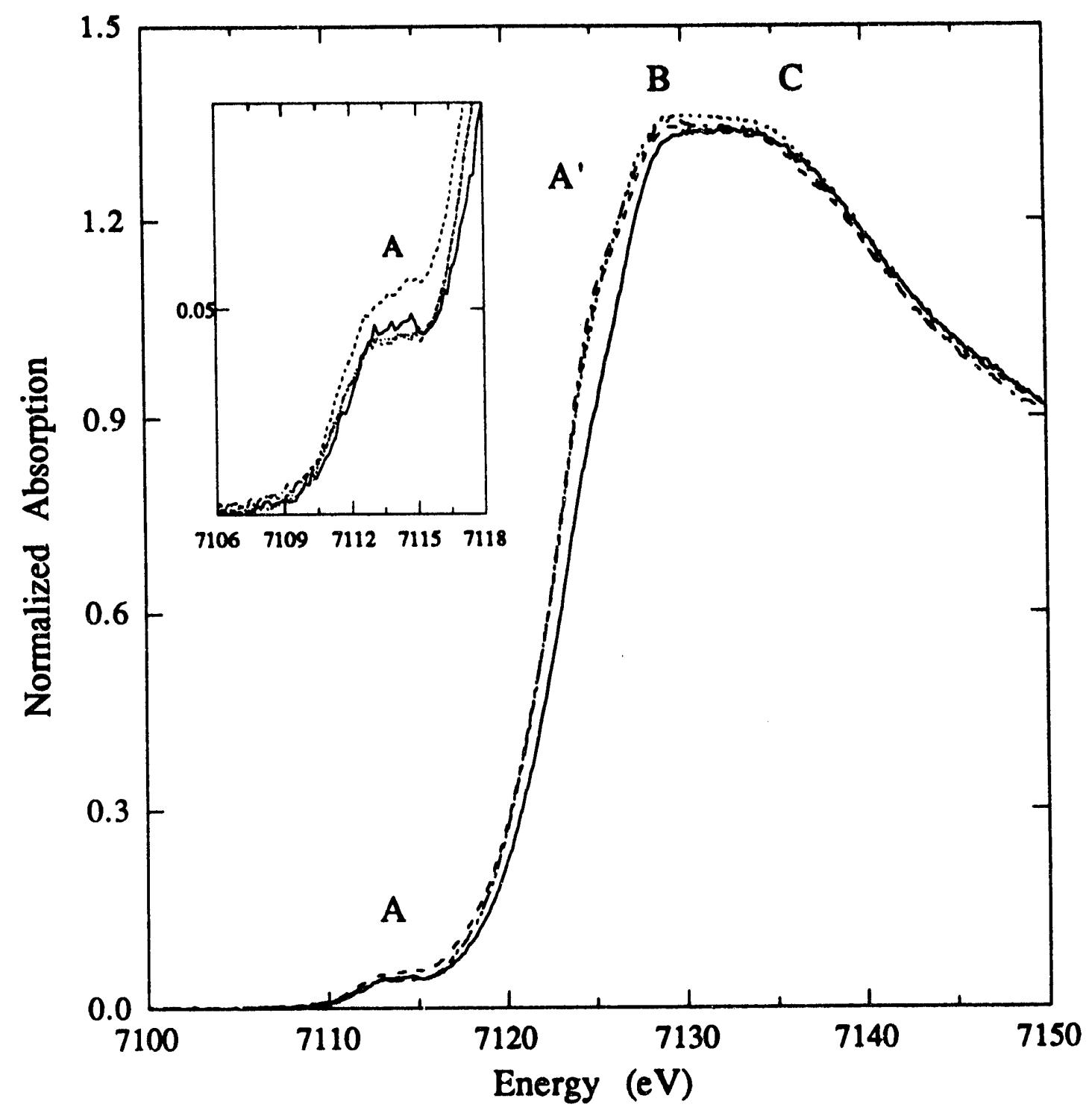

Figure 3.14. Edge spectra of semimet hydroxylase samples. Non-complexed sample, EXAFS7 (solid); sample with component B, EXAFS10 (dash); sample with bromopropene, EXAFS18 (dot); sample with B and bromopropene EXAFS16 (dash dot). Note the appearance of a shoulder on the rising edge in the spectra of the hydroxylase samples in the presence of B and bromopropene. The pre-edge feature of EXAFS10 is more intense than the pre-edge features of the other samples. 


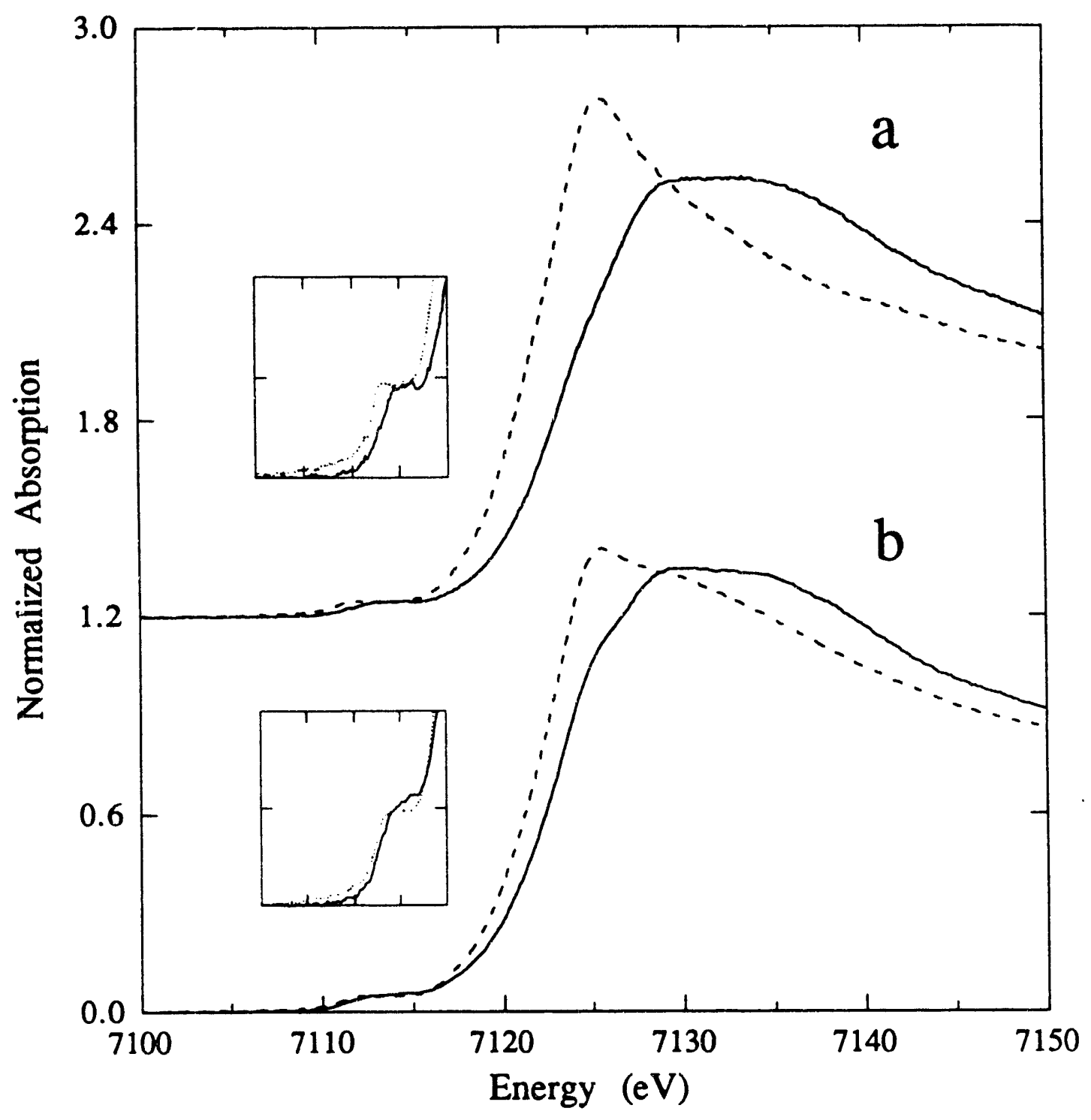

Figure 3.15. Edge spectra of semimet (solid) and reduced (dash) hydroxylase samples. The data shown are for (a) the non-complexed forms of the hydroxylase (EXAFS7 and EXAFS6) and (b) the hydroxylase samples with component B (EXAFS10 and EXAFS15). The change in the appearance of the edge spectra of the hydroxylase samples in the presence of component B is not as great as the change that occurs in the noncomplexed samples. 
with mixed $\mathrm{O}$ and $\mathrm{N}$ ligation (see Chapter 5). The shape of the pre-edge feature (Feature A) changes (Figure 3.15 inset) and the positon of the pre-edge feature moves to lower energy by about $1 \mathrm{eV}$ to $7113 \mathrm{eV}$.

The edges of the reduced hydroxylase complexes are compared in Figure 3.16. The position of Feature A does not change, but the intensity decreases relative to the noncomplexed sample edge. Among the hydroxylase complexes, the intensity of the feature is slightly greater for the hydroxylase sample in the presence of bromopropene than for the other samples. The shape of Feature $A$ is different for the complexes with component $B$ (EXAFS15 and EXAFS17) from the bromopropene-complexed and non-complexed hydroxylase (EXAFS19 and EXAFS6). The pre-edge feature rises to a maximum more steeply for EXAFS6 and EXAFS19 than for EXAFS15 and EXAFS17. The intensity of the main transition decreases and becomes more narrow relative to the edge of the non-complexed samples, and a shoulder to the high energy side of Feature B Fiature C) at $\sim 7131 \mathrm{eV}$ appears for the hydroxylase in the presence of component $\mathrm{B}$.

\subsection{Discussion}

\subsubsection{The Empirical Fitting Proceedure}

The underlying assumption of the empirical fitting approach applied herein is that only single scattering interactions contribute to the EXAFS, and multiple scattering contributions to outer shell data are neglected. The empirical fitting proceedure used for these fits has proven to be very reliable for the first coordination sphere and is especially sensitive to the distance distribution around the $\mathrm{Fe}$ atom, although it may not always accurately reflect the relative numbers of one atom type to another, as has been seen for these samples. Second shell empirical fitting methods are hampered by the difficulty in adequately modeling all of the second-shell low- $Z$ atoms distributed at various distances and in various numbers around metal atoms in dinuclear metalloproteins. The Fe-C parameters obtained from $\mathrm{Fe}$ (acetylacetonate)3, commonly used to model the second shell low- $Z$ atom contribution to the data, describe a wave which mimicks the second shell $\mathrm{Fe}-\mathrm{Fe}$ contribution (Chapter 2, Figure 2.8), resulting in the satisfactory "C-only" fits to the data at the same distances as the Fe-only fits. This also contributes to the high degree of correlation between the $\mathrm{Fe}$ and $\mathrm{C}$ contributions to the fits when both $\mathrm{Fe}$ and $\mathrm{C}$ were used to fit the second shell data. The ability of second shell carbon to mimic a first row transition metal contribution has been noted before in fits to dinuclear copper proteins. ${ }^{34}$ The net result is that it is difficult to isolate the second shell metal-metal interaction from the second 


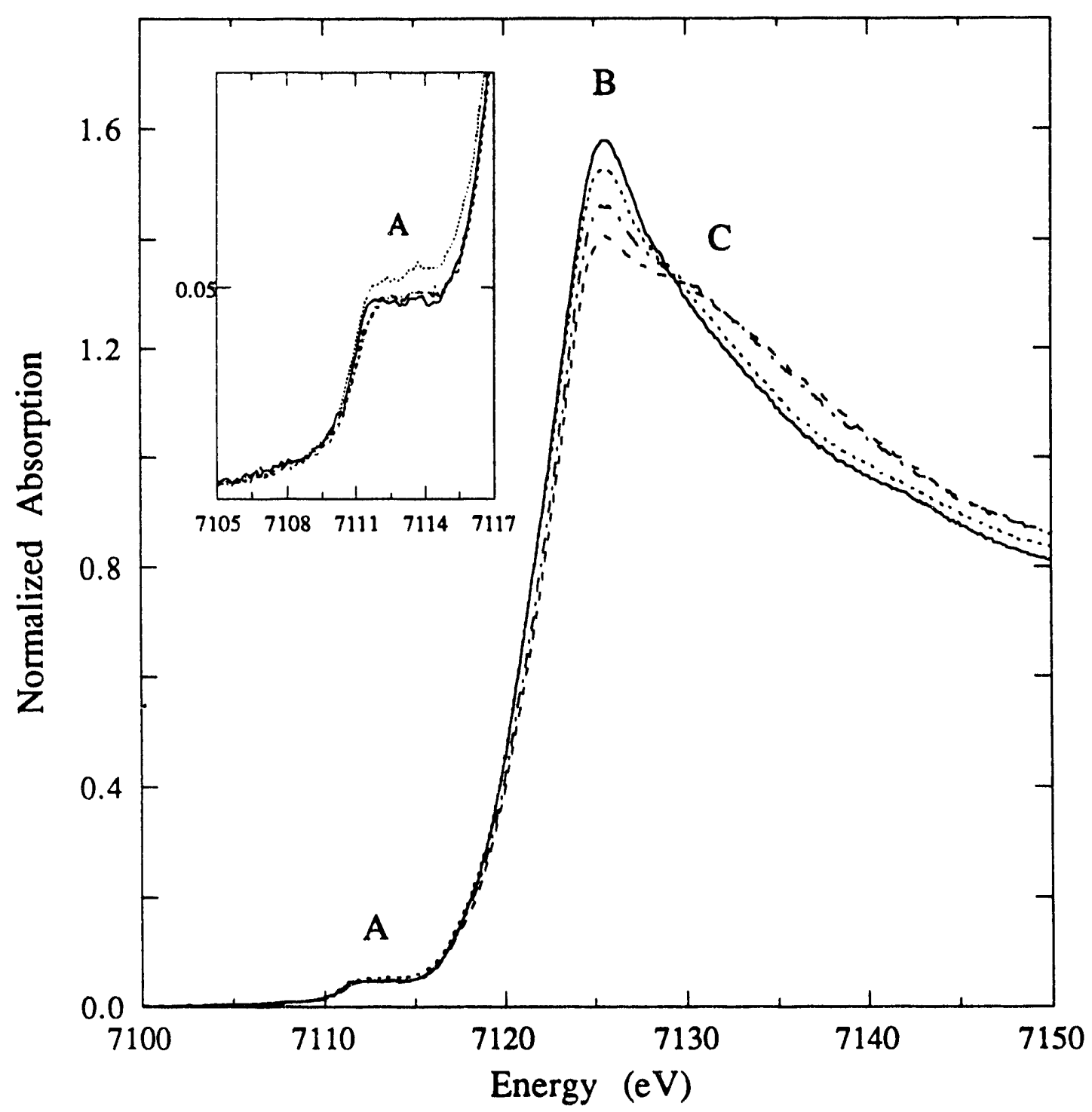

Figure 3.16. Edge spectra of reduced hydroxylase samples. Reduced hydroxyalse sample, EXAFS6 (solid); sample with component B, EXAFS15 (dash); sample with bromopropene, EXAFS19 (dot); sample with component B and brompropene, EXAFS17 (dash dot). Note that the intensity of the main feature decreases in the presence of component $\mathrm{B}$ and bromopropene. 
shell metal-low- $Z$ atom interaction using a single-scattering empirical approach, an effect clearly seen in the fits to the second shell EXAFS data. The inclusion of this shell clearly shows that at least a second contribution is needed to model the second shell properly.

Multiple scattering interactions from the presence of rigid ligand groups such as imidazoles can often contribute significantly to the outer shell data. Semi-empirical methods of dealing with the presence of imidazole groups, in which a set of amplitude and phase parameters which describe the contribution of the imidazole group as a unit, have had limited success. ${ }^{35}$ Recent efforts have focused on including the individual multiplescattering pathways based on theoretical calculations within the imidazole group in fits to the data. ${ }^{36}$ The possibility that multiple scattering from other ligands bound to the iron atom are contributing to the second shell data cannot be ruled out, as it has recently been shown that multiple scattering contributes significantly to the outer shell data in $\mathrm{Fe}(\mathrm{acac}) 3 .{ }^{37}$ This suggests that in addition to imidazole groups, multiple scattering interactions from bidentate coordination of a carboxylate group to a single Fe atom may also contribute to the second shell data. The crystal structure of ribonucleotide reductase B2 38 shows an aspartate group coordinated to an Fe atom in just such a manner and it is certainly possible, based on the similarity between the average first shell data for RR and MMO hydroxylase as determined by EXAFS, 12 that similar coordination of carboxylate groups occur in the hydroxylase active site. The application of a multiple scattering analysis to the second shell data is currently under developement. ${ }^{37}$

\subsubsection{The Effect of Substrate and Component $B$ on the Structure of the Diiron Center}

Perturbations in the EPR spectrum of the semimet hydroxylase in the presence of substrate was the first indication that the hydroxylase component interacted directly with substrate. $4 c, 17$ In the presence of small molecules, the EPR spectrum was generally sharpened and intensified, however the presence of component B caused a dramatic difference in the EPR spectrum of the semimet hydroxylase. ${ }^{16 a}$ The hydroxylase no longer exhibited the $g_{a v}=1.85$ signal $(M$. trichosporium $(\mathrm{OB} 3 \mathrm{~b})$ ), and a different saturation behaviour was observed, resulting in an EPR signal with $\mathrm{gav}_{\mathrm{av}}=1.75$. These results suggest that the interaction of component B with the hydroxylase in its semimet form is more significant than the interaction of substrate. In the fully reduced form, the presence of component B does not affect the EPR as drastically as in the semimet form and only sharpens and intensifies the $g=16$ signal, suggesting that the interaction of $B$ with the reduced hydroxylase is not as significant as its interaction with the semimet hydroxylase. 
These trends are qualitatively seen in the EXAFS of the semimet (Figure 3.1) and reduced samples (Figure 3.3). The EXAFS of the complex formed between semimet hydroxylase and bromopropene (Figure 3.1d-f) is very similar to the non-complexed EXAFS (Figure 3.1a), whereas the EXAFS of the semimet hydroxylase with component B (Figure 3.1b,c) is different from the non-complexed form. The B plus bromopropene sample (Figure 3.1g) resembles a combination of the B-hydroxylase and the bromopropene-hydroxylase samples. The EXAFS of the reduced hydroxylase in the presence of component $B$ (Figure 3.3c) is somewhat different from the non-complexed form (Figure 3.3a,b).

The presence of component $\mathrm{B}$ and substrate also alters the redox potentials of the hydroxylase iron site. ${ }^{18}$ The redox potentials of the $\mathrm{Fe}(\mathrm{III}) \mathrm{Fe}(\mathrm{III}) / \mathrm{Fe}(\mathrm{II}) \mathrm{Fe}$ (III) and $\mathrm{Fe}(\mathrm{II}) \mathrm{Fe}(\mathrm{III}) / \mathrm{Fe}(\mathrm{II}) \mathrm{Fe}$ (II) pairs were slightly lowered in the presence of propylene, suggesting a decrease in the electron affinity of the iron site and a slight inhibition of the reduction process. In the presence of stoichiometric amounts of component $B$ and reductase, no reduction of the hydroxylase occured, suggesting that both component $B$ and reductase are required to inhibit reduction of the hydroxylase. Earlier kinetic studies suggested that component $B$ alone was responsible for the inhibition of the reduction of hydroxylase in the absence of substrate. ${ }^{6,11}$ The XAS sample of the reduced form of the hydroxylase with component B was subjected to the reduction mediators after the protein complex had been formed. The edge spectrum (Figure 3.16) is consistent with the reduced form of a diiron center, therefore reduction of the hydroxylase can proceed in the presence of component $B$ and the absence of substrate. This confirms that both the reductase and component $B$ are required in the absence of substrate to effectively inhibit reduction of the hydroxylase diirion site.

\subsubsection{Changes in the Coordination Sphere of the Iron Center. The} fit results do not show any significant difference in the iron coordination sphere due to the formation of hydroxylase complexes. The average first shell coordination of the complexed forms of the semimet hydroxylase (5.3 - 6.4 N/O at 2.06 - $2.08 \AA$, Table 3.6) do not vary significantly from the average first shell coordination of the non-complexed semimet hydroxylase (5.6 N/O at $2.08 \AA$, Table 3.6). Considering the Fe-only fits to the second shell data, the Fe-Fe distance for all of the semimet complexes is also very similar, varying from $3.39 \AA$ to $3.41 \AA$. Based on a comparison of the coordination weighted average first shell date, the results suggest that no significant change in the coordination of the iron occurs due to the formation of hydroxylase complexes with component $B$ or substrate. However, there is a change in the relative coordination numbers of the long and short distance contributions to the first shell data for the B-hydroyxlase samples. Given the inability of EXAFS to strictly determine the relative numbers of similar strength 


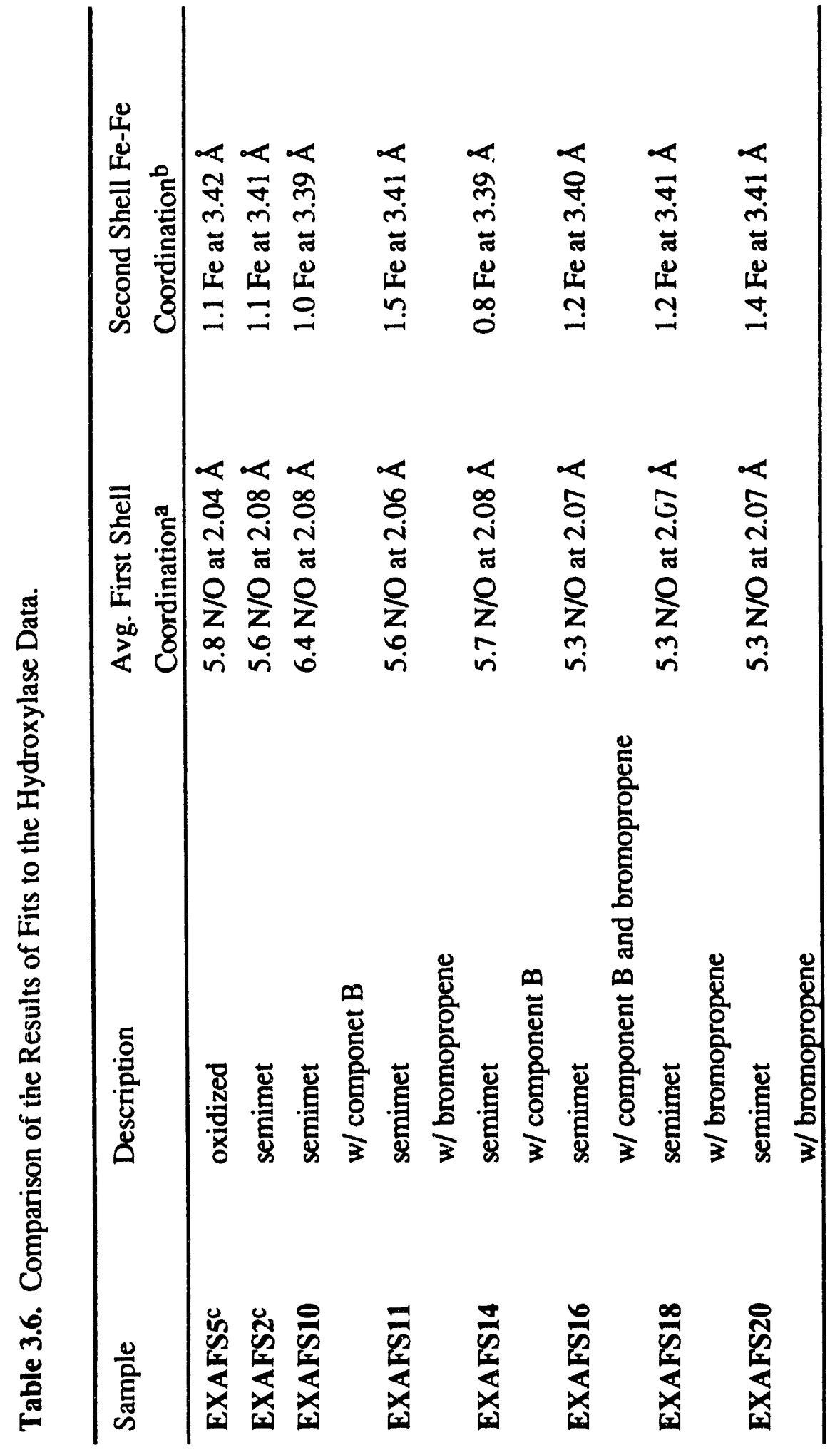




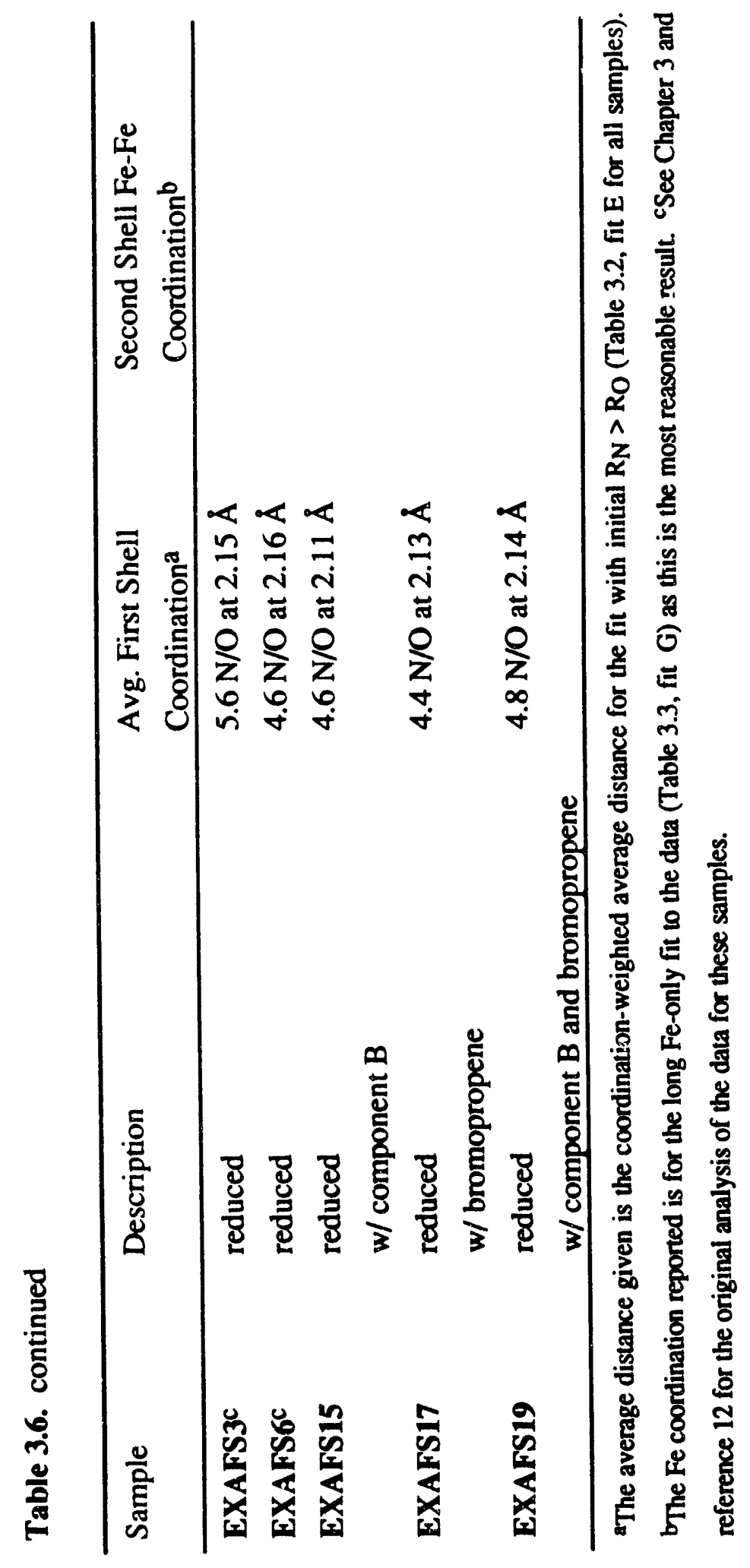


backscattering atoms, we hesitate to interpret the change in the contributions of the individual waves as representing a real change in the relative $\mathrm{N}$ and $\mathrm{O}$ ligation to the iron center, however the results indicate that some minor change does occur in the first coordination sphere. Whether the origin of the difference arises from a change in the ligation of the iron atoms, or a distortion of the coordination environment, or a change in the covalency of the metal site can not be determined based on the EXAFS analysis.

Although it is known that substrate interacts with the hydroxylase component, the location of the binding site has not been determined. A brominated substrate was used for the hydroxylase/substrate complex to provide information about the proximity of the substrate binding site to the iron center. Bromine is a stronger backscatterer than Fe, so an $\mathrm{Fe}-\mathrm{Br}$ interaction should be detected if the substrate were to bind close enough to an iron atom in an ordered configuration (with little rotational or vibrational motion). If 1 bromopropene were to bind to an $\mathrm{Fe}$ atom, the $\mathrm{Fe}-\mathrm{Br}$ distance would be on the order of 2.5-3.75 $\AA$ if the terminal $\mathrm{C}$ to which $\mathrm{Br}$ is attached binds to the iron atom, depending on the angles of the Fe-C to $\mathrm{C}-\mathrm{Br}$ bonds. If the second carbon, at the internal end of the double bond, binds to iron the $\mathrm{Fe}-\mathrm{Br}$ distance would be on the order of 3.0-5.3 $\AA$, again depending on the relative bonding angles. EXAFS should be sensitive to a $\mathrm{Br}$ interaction within $4.0 \AA$. Since $\mathrm{Br}$ is such a strong backscatterer, it might be possible to detect an $\mathrm{Fe}$ $\mathrm{Br}$ interaction at a longer distance and therefore be able to determine the distance to the substrate binding site.

To evaluate this, we have collected EXAFS data on a brominated derivative of $\mathrm{Fe}(\mathrm{acac})_{3}$, [tris-(3-bromo-2,4-pentanedionate) 3 iron (III)] ${ }^{39}$ (or $\mathrm{Fe}(3-\mathrm{Br}$-acac) 3 ) in which the Fe-Br distance is expected to be on the order of $5.2 \AA .40$ The contributions of the three bromine atoms at this distance are clearly seen in the Fourier transform of $\mathrm{Fe}(3-\mathrm{Br}$-acac)3

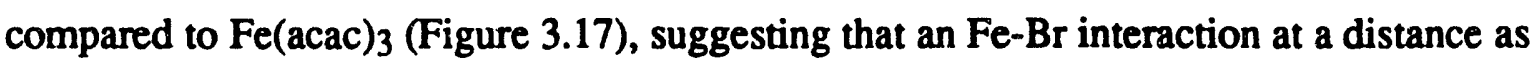
long as $5 \AA$ could be detectable by XAS. Inspection of the Fourier transforms of the semimet and reduced bromopropene complexes (EXAFS11, EXAFS16, EXAFS18, EXAFS20, Figure 3.2; EXAFS17, EXAFS19, Figure 3.4) shows no suggestion of a strong interaction above $3.5 \AA$, suggesting that substrate binds at a distance greater than 5 $\AA$. If the bromine were located between 3 and $3.5 \AA$ from the iron atoms, it would be expected that the fit results to the second shell data would be different for the hydroxylase samples with bromopropene from the non-bromopropene complexed samples, however this was not the case. We have not yet identified an appropriate model for an $\mathrm{Fe}-\mathrm{Br}$ interaction at 3-3.5 $\AA$, so we have been unable to fit the second shell data to test for the presence of bromine. Pending the application of appropriate $\mathrm{Fe}-\mathrm{Br}$ parameters to the fitting proceedure, and given that the data can be well explained by only second shell Fe and C 


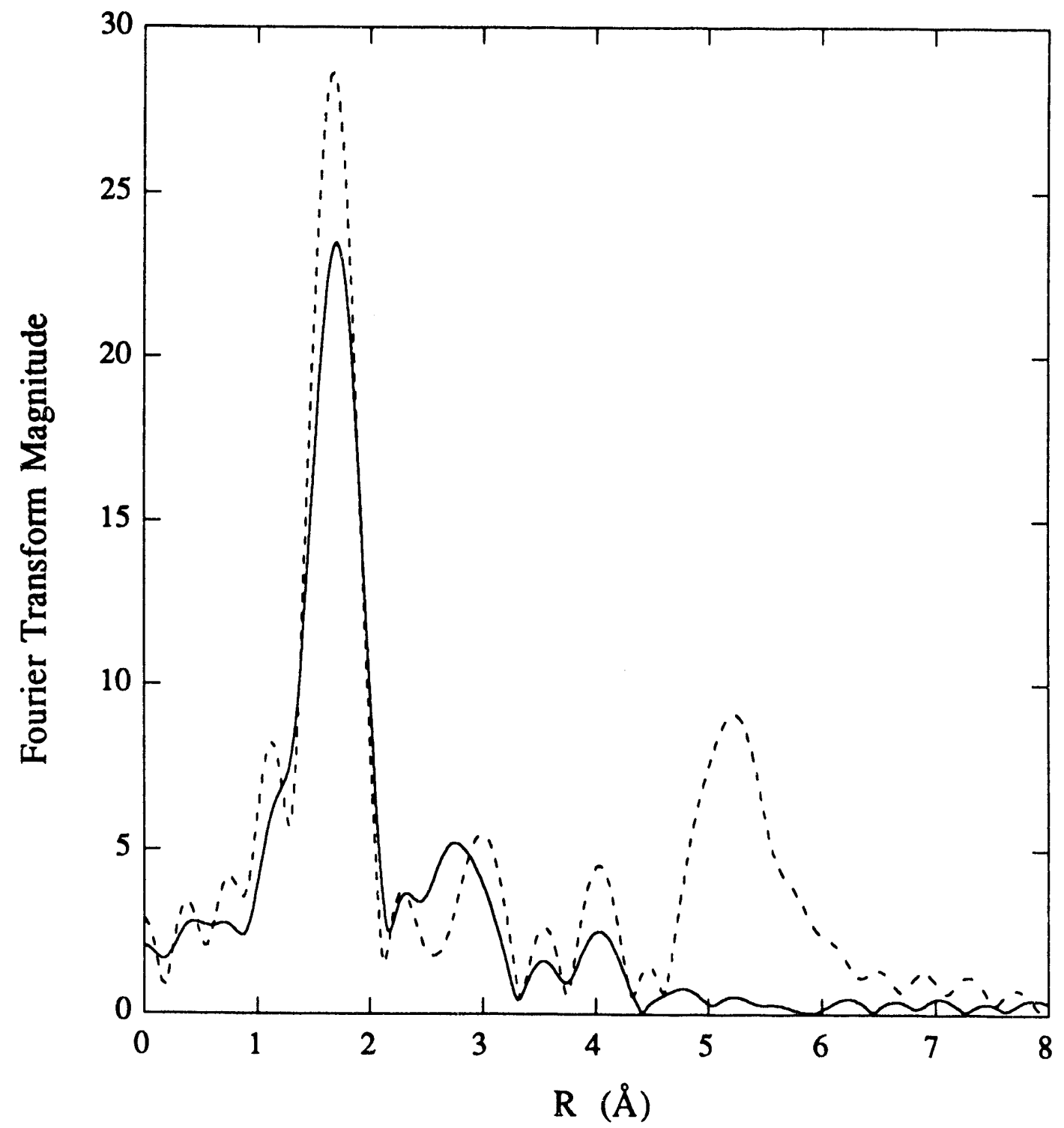

Figure 3.17. Fourier transforms of the EXAFS data for $\mathrm{Fe}(\mathrm{acac})_{3}$ (solid) and $\mathrm{Fe}(3-\mathrm{Br}$ acac) 3 (dash). The peak at $\sim 5 \AA$ in the Fourier transform of $\mathrm{Fe}(3-\mathrm{Br}-\mathrm{acac})_{3}$ is due to the $\mathrm{Fe}-\mathrm{Br}$ interaction. 
contributions, these results suggest that substrate does not bind directly to the iron atoms; instead, it binds at a location such that the $\mathrm{Br}$ atom is more more than $4 \AA$ away from the iron site. This conclusion is consistent with the results of an ENDOR study of the hydroxylase in the presence of methanol, ${ }^{16 \mathrm{~b}}$ in which no evidence was seen for coupling of the methyl protons to the iron site. Coupling with exchangeable imidazole protons 5-6 $\AA$ away from the iron site was seen, so the methanol binding site must be more than $6 \AA$ away from the iron atoms. In addition, the limited deuterium exchange that occured during the course of this study suggested limited access to the diiron site for water; therefore access is probably limited for larger molecules such as bromopropene as well.

3.4.2.2. Interpretation of the Hydroxylase Edge Spectra. The weak pre-edge feature seen well below the $4 p$ transition in transition metal spectra has been assigned to a formally dipole forbidden $1 \mathrm{~s} \rightarrow 3 \mathrm{~d}$ transition made allowed by $4 \mathrm{p}$ mixing into the $3 \mathrm{~d}$ states as a result of symmetry distortions and vibronic coupling. 41.42 The intensity of this feature is inversely proportional to the symmetry of the metal site and increases as the metal site is distorted from octahedral to tetrahedral symmetry. The intensity of the feature can be therefore be used to infer the coordnation number and/or site symmetry of the metal atom. ${ }^{43}$ The presence of the $1 \mathrm{~s} \rightarrow 3 \mathrm{~d}$ feature in the edge spectra of the hydroxylase samples (Figure 3.14 and 3.15, feature $\mathrm{A}$ ) indicates that the $\mathrm{Fe}$ site is distorted from octahedral symmetry. The intensity of this transition is consistent with the proposed 5-6 atom coordination of the iron site in the MMO hydroxylase. The increase in the intensity of the 1s $\rightarrow$ 3d transition for EXAFS10 relative to EXAFS7 suggests that the Fe site is more distorted in the presence of component $B$.

The appearance of the shoulder of the rising edge of the semimet hydroxylase spectra (Feature $A^{\prime}$ ) is similar to changes seen in the edge spectra of $\mathrm{Cu}$ and $\mathrm{Fe}$ systems as a result of the increase in the covalency of the metal site. ${ }^{41.44}$ This feature has been assigned as a ligand-to-metal charge transfer (LMCT) shake-down feature ${ }^{45}$ associated with $1 \mathrm{~s} \rightarrow 4 \mathrm{p}$ transition in the $\mathrm{Cu}$ systems. If Feature $\mathrm{A}^{\prime}$ reflects covalency in the iron site, then the improved resolution of this feature in the hydroxylase complexes suggests that the covalency of the iron site increases as a result of the interaction with component $B$ and/or bromopropene. The decrease in the electron affinity of the iron site in the presence of propylene as measured by the change in the redox potentials is consistent with an increase in the covalency of the $\mathrm{Fe}$ (III) iron center. ${ }^{18}$

For the reduced hydroxylase samples, the $1 \mathrm{~s} \rightarrow 3 \mathrm{~d}$ feature appears to be split by about $2 \mathrm{eV}$ in the complex formed with bromopropene (EXAFS19) and in the noncomplexed form (EXAFS6). Splitting seen in ferrous compounds has been attributed to transitions to the ${ }^{4} \mathrm{~F}$ (lower energy transition) and ${ }^{4} \mathrm{P}$ (higher energy transition) multiplet 
levels of the $\mathrm{d}^{7}$ final state. ${ }^{44}$ The relative intensities of the two features in the reduced hydroxylase spectra are not in accord with predictions $\left({ }^{4} \mathrm{~F}: 4 \mathrm{P} 7: 3\right) .44$ Due to the noise level and the reduction in intensity of the leading edge of the feature for the B-complexed forms, it cannot be established if the pre-edge feature is split for these samples.

During XAS sample preparation, the hydroxylase complexes were made before the samples were reduced. This suggests that the changes in the edge of the reduced samples derive from an interference in the reduction process as a result of the complex formed between the hydroxylase and component $B$ or bromopropene. The intensity difference of the main transition for the semimet and reduced complexes with B (Feature B, Figure $3.15 \mathrm{~b}$ ) is not as great as the intensity difference of the main transition for the noncomplexed semimet and reduced samples (Feature B, Figure 3.15a). If the difference in the edge of the non-complexed forms represents a conversion from semimet to diferrous, then perhaps a lesser degree of change represents an incomplete conversion to the fully reduced form. Therefore, perhaps the intensity of the $1 \mathrm{~s} \rightarrow 4 \mathrm{p}$ transition is directly related to the ability of the hydroxyalse component to undergo chemical reduction.

In the edge spectra of the reduced hydroxylase samples (Figure 3.16), the intensity of the $1 \mathrm{~s} \rightarrow 4 \mathrm{p}$ transition decreases from the non-complexed form (EXAFS6) to the complex with bromopropene (EXAFS19) followed by the complex with both B and bromopropene (EXAFS17) and the complex with only B (EXAFS15). Based on the arguement presented above, this suggests that in the presence of $B$ reduction is inhibited, but the inhibition is lifted if substrate is added to $B$, and further decreased if substrate is added and $B$ is removed. This trend is consistent with the results of kinetic studies 10,11 in which the reduction of the hydroxylase was inhibited or halted by the presence of component $B$ in the absence of substrate, but occured in the presence of substrate. In addition, the reduction of the redox potentials of the hydroxylase in the presence of substrate $\left(E_{1}{ }^{\circ}=30 \mathrm{mV} ; E_{2}{ }^{\circ}=-156 \mathrm{mV}\right)$ as compared to the redox potentials of the hydroxylase alone $\left(E_{1}{ }^{0}=48 \mathrm{mV} ; E_{2}{ }^{0}=-135 \mathrm{mV}\right)^{18}$ suggest a slight decrease in the electron affinity of the iron site in the presence of substrate. These studies suggest that reduction of the hydroxylase in the presence of substrate is very slightly inhibited, and in the presence of component B without substrate, reduction of the hydroxylase is strongly inhibited. The interpretation of the decrease in the intensity of the $1 \mathrm{~s} \rightarrow 4 \mathrm{p}$ transition in the edge spectra of the reduced hydroxylase samples is consistent with these results. It would be interesting to see if longer exposure to the reduction mediators would result in more complete reduction of the complexed forms of the hydroxylase (samples were incubated for approximately 40 minutes). 


\subsection{Conclusions}

The results of the EXAFS analysis for the range of data available do not show any significant change in the average coordination environment of the iron center as a result of complex formation. The interaction of component B and bromopropene therefore occurs at a location other than the iron site. Based on the comparison of the Fourier transforms of the bromopropene-hydroxylase samples and $\mathrm{Fe}(3-\mathrm{Br}-\mathrm{acac})_{3}$, and the results of an ENDOR study of the hydroxylase component, $16 \mathrm{~b}$ the site of the substrate interaction is more than 4 $\AA$ away from the iron site. The presence of component $B$ has an effect on the first shell of the hydroyxlase active site, reflected in the change in the distance distribution of the individual contributions to the first shell data (Table 3.2). The nature of this change cannot be determined for the data range available, but possible explanations include distortion of the iron environment, changes in the ligation of the iron atoms, or a change in the covalency of the iron site due to distant interactions between the hydroxylase and component B.

Direct evidence for the inhibition of the chemical reduction of the hydroxylase in the presence of component B and bromopropene is seen in the edge spectra. The appearance of a shoulder on the rising edge of the spectra of the semimet edges suggests that the covalancy of the diiron center changes due to the presence of component B and substrate. These studies suggest that the changes which occur in the hydroxylase diiron center in the presence of the component $B$ or substrate involve subtle perturbations in the coordination environment of the iron atoms accompanied by changes in the electronic structure of the iron center.

\subsection{Acknowledgements}

The data were collected at the Stanford Synchrotron Radiation Laboratory and the National Synchrotron Light Source, Brookhaven National Laboratory, which are supported by the Department of Energy, Office of Basic Energy Sciences, Division of Chemical Sciences and Division of Materials Sciences. SSRL is also supported by the National Institutes of Health, Biomedical Resource Technology Program, Division of Research Resources (RR-01209) and the Department of Energy, Office of Health and Environmental Research. Grant support was provided by the National Science Foundation (CHE 9121576 to $\mathrm{KOH}$ ). 


\subsection{References and Notes}

1. Anthony, C. The Biochemistry of the Methylotrophs; Academic Press: London, 1982.

2. (a) Colby, J.; Dalton, H. Biochem. J. 1978, 171, 461-468. (b) Colby, J.; Dalton, H. Biochem. J. 1976, 157, 495-497.

3. (a) Stirling, D. I.; Dalton, H. J. Biochem. 1979, 96, 205-212.

4. (a) Woodland, M. P.; Dalton, H. Anal. Biochem. 1984, 139, 459-462. (b) Woodland, M. P.; Patil, D. S.; Cammack, R.; Dalton, H. Biochim. Biophys. Acta 1986, 873, 237-242. (c) Woodland, M. P.; Dalton, H. J. Biol. Chem. 1984, 259, 53-59.

5. Colby, J.; Dalton, H. Biochem. J. 1979, 177, 903-908. (b) Lund, J.; Dalton, H. Eur. J. Biochem. 1985, 147, 291-296.

6. Green, J.; Dalton, H. J. Biol. Chem. 1985, 260, 15795-15801.

7. (a) Colby, J.; Stirling, D. I.; Dalton, H. Biochem. J. 1977, 165, 395-402. (b) Green, J.; Dalton, H. J. Biol. Chem. 1989, 264, 17698-17703.

8. Fox, B. G.; Lipscomb, J. D. Biochem. Biophy. Res. Comm. 1988, 154, 165170.

9. (a) Burrows, K. J.; Cornish, A.; Scott, D.; Higgins, I. J. J. Gen. Microbiol. 1984, 130, 3327-3333. (b) Fox, B. G.; Borneman, J. G.; Wackett, L. P.; Lipscomb, J. D. Biochemistry, 1990, 29, 6419-6427.

10. (a) Fox, B. G.; Froland, W. A.; Dege, J. E.; Lipscomb, J. D. J. Biol. Chem. 1989, 264, 10023-10033. (b) Andersson, K. K.; Froland, W. A.; Lee, S-K.; Lipscomb, J. D. New J. Chem. 1991, 15, 411-415.

11. (a) Lund, J.; Woodland, M. P.; Dalton, H. Eur. J. Biochem. 1985, 147, 297 305. (b) Green, H.; Dalton, H. Biochem. J. 1989, 259, 167-172.

12. DeWitt, J. G.; Bentsen, J. G.; Rosenzweig, A. C.; Hedman, B.; Green, J.; Pilkington, S.; Papaefthymiou, G. C.; Dalton, H.; Hodgson, K. O.; Lippard, S. J. J. Am. Chem. Soc. 1991, 113, 9219-9235.

13. Fox, B. G.; Surerus, K. K.; Münck, E.; Lipscomb, J. D. J. Biol. Chem. 1988, 263, 10553-10556.

14. (a) Lippard, S. J. Angew. Chem., Int. Ed. Engl. 1988, 27, 344-361. (b) Sanders-Loehr, J. Iron Carriers and Iron Proteins; VCH Publishers Inc.: New York, 1989, pp 373-466. (c) Que, L., Jr.; True, A. E. Prog. Inorg. Chem. 1990, 38, 97-200. 
15. Hendrich, M. P.; Münck, E.; Fox, B. G.; Lipscomb, J. D. J. Am. Chem. Soc. 1990, 112, 5861-5865.

16. (a) Fox. B. G.; Liu, Y.; Dege, J. E.; Lipscomb, J. D. J. Biol. Chem. 1991, 266, 540-550. (b) Hendrich, M. P.; Fox, B. G.; Andersson, K. K.; Debrunner, P. G.; Lipscomb, J. D. J. Biol. Chem. 1992, 267, 261-269.

17. (a) Dalton, H. Adv. Appl. Microbiol. 1980, 26, 71-87. (b) Dalton, H. Microbial Growth on $C_{1}$ Compounds; Heyden Press: London, 1980, pp 1-10.

18. Liu, K. E.; Lippard, S. J. J. Biol. Chem., 1991, 266, 12836-12839.

19. Wu, W.; Rosenzweig, A. C.; Lippard, S. J. unpublished results.

20. Cramer, S. P.; Tench, O.; Yocum, M.; George, G. N. Nucl. Instrum. Methods Phys. Res. 1988, A266, 586-591.

21. Scott, R. A.; Hahn, J. E.; Doniach, S. ; Freeman, H. C.; Hodgson, K. O. J. Am. Chem. Soc. 1982, 104, 5364-5369.

22. (a) Cramer, S. P.; Hodgson, K. O.; Stiefel, E. I.; Newton, W. E. J. Am. Chem. Soc. 1978, 100, 2748-2761. (b) Cramer, S. P.; Hodgson, K. O. Prog. Inorg. Chem. 1979, 15, 1-39. (c) Scott, R. A. Methods Enzymol. 1985, 117, 414-459.

23. Johansson, L. Chem. Scr. 1976, 9, 30-35. The crystal structure of the perchlorate salt has not been determined, but the $[\mathrm{Fe} \text { (phenanthroline) } 3]^{2-}$ complex structure can be assumed to be identical with that of the corresponding iodide salt (Johansson, L.; Molund, M.; Oskarsson, A. Inorg. Chim. Acta 1978, 31, 117-123).

24. Iball, J.; Morgan, C. H. Acta Cryst. 1976, 23, 239-244. (b) Roof, R. B. Jr. Acta Cryst. 1956, 9, 781-786.

25. Armstrong, W. H.; Lippard, S. J. J. Am. Chem. Soc. 1984, 106, 4632-4633.

26. Ericson, A.; Hedman, B.; Hodgson, K. O.; Green, J.; Dalton, H.; Bentsen, J. G.; Beer, R. H.; Lippard, S. J. J. Am. Chem. Soc. 1988, 110, 2330-2332.

27. Scarrow, R. C.; Maroney, M. J.; Palmer, S. M.; Que, L. Jr.; Roe. A. L.; Salowe, S. P.; Stubbe, J. J. Am. Chem. Soc. 1987, 109, 7857-7864.

28. The EPR signal at gav $<2.0$ and the value of the coupling constant $\left(J=-32 \mathrm{~cm}^{-1}\right.$, ref. 12) are consistent with an oxo-group bridged diiron center; ENDOR and ESEER studies suggest the presence of $2 \mathrm{~N}$-donating groups coordinated to the iron center (ref. $16 b)$.

29. Zhang, K.; Stern, E. A.; Ellis, F.; Sanders-Loehr, J.; Shiemke, A. K. Biochemistry, 1988, 27, 7470-7479.

30. (a) Hedman, B.; Co, M. S.; Armstrong, W. H.; Hodgson, K. O.; Lippard, S. J. Inorg. Chem., 1986, 25, 3708-3711. 
31. Me3TACN = 1,4,7-trimethyl-1,4,7-triazacyclononane. Hartman, J. R.; Rardin, R. L.; Chaudhuri, P.; Pohl, K.; Wieghardt, K.; Nuber, K.; Weiss, J.; Papaefthymiou, G. C.; Frankel, R. B.; Lippard, S. J. J. Am. Chem. Soc. 1987, 109, 7387-7396

32. BIPHME = bis $(1-$ methylimidazol-2-yl $)$ phenylmethoxymethane. Tolman, W. B.; Bino, A.; Lippard, S. J. J. Am. Chem. Soc. 1989, 111, 8522-8523.

33. EXAFS data has been collected on the above models, however fits to the data were not correct.

34. Scott, R. A.; Eidsness, M. K. Comments Inorg. Chem. 1988, 7, 235-267.

35. Co, M. S.; Scott, R. A.; Hodgson, K. O. J. Am. Chim. Soc. 1981, 103, 986988

36. Hasnain, S. S , Ed. Synchrotron Radiation and Biophysics; Ellis Horwood Ltd.; Chichester, 1990; Chapters 3 and 4.

37. Westre, T. E.; Di Cicco, A.; Filipponi, A.; Natoli, C. R.; Solomon, E. I.; Hedman, B.; Hodgson, K. O. to be submitted.

38. Nordlund, P.; Sjöberg, B.-M.; Eklund, H. Nature 1990, 345, 593-598.

39. Kluiber, R. W. J. Am. Chem. Soc. 1960, 82, 4839-4842.

40. The crystal structure of the Fe complex has not been soi; $e d$; the distance is based on the average $\mathrm{Cr}$ - $\mathrm{Br}$ distance for a crystallographically charactenzed $\mathrm{Cr}$ dimer of the same ligand. Estes, E. D.; Scaringe, R. P.; Hatfield, W. E.; Hoc'gson, D. J. I:org. Thein. 1977, 16, 1605-1610. The sample of $\mathrm{Fe}(3-\mathrm{Br}-\mathrm{acac}) 3$ was diluted with $\mathrm{BN}$ powder, finely ground with a mortar and pestle, and pressid into a $1 \mathrm{~mm} \mathrm{Al}$ spacer windowed with $\mathrm{m}$ iylar tape. The sample was run in transmission mode at SSRL on beamline 7-3 by using a $\mathrm{Si}(220)$ monochromator detuned $50 \%$ at $7997 \mathrm{eV}$. The incident and transmitted beam intensity were monitored by using $\mathrm{N}_{2}$-filled ionization chambers of standard design. The data were collected at $10 \mathrm{~K}$, maintained by a continuous-flow LHe cryostat (Oxford Instruments model CF1208).

41. Shulman, R. G.; Yafet, Y.; Eisenberger, P.; Blumberg, W. E. Proc. Natl. Acad. Sci. USA 1976, 73, 1384-1388.

42. This transition could also be casued by a quadropole transition, in which case the selection rule is \pm 2 and the $1 \mathrm{~s} \rightarrow 3 \mathrm{~d}$ transition is allowed. The authors of reference 41 have estimated that the quadropole transitions would be three orders of magnitude weaker than the intensity of the Fe preedge feature, and concluded that the feature is due to an Fe $1 \mathrm{~s} \rightarrow-3 \mathrm{~d}$ transition made allowed by vibronic coupling of the $4 \mathrm{p}$ and $3 \mathrm{~d}$ states.

43. Roe, A. L.; Schneider, D. J.; Mayer, R. J.; Pyrz, J. W.; Que, L. Jr. J. Am. Chem. Soc. 1984, 106, 1676-1681. 
44. Kau, L.-S.; Spira-Solomon, D. J.; Penner-Hahn, J. E.; Hodgson, K. O.; Solomon, E. I. J. Am. Chem. Soc. 1987, 109, 6433-6442.

45. The LMCT shake-down transiton involves the transfer of an electron from the valence level of the ligand to the metal $3 \mathrm{~d}$ manifold made possible by final state relaxation. This transiton occurs at an energy below that of the metal $1 \mathrm{~s} \rightarrow 4 \mathrm{p}$ transition by an amount equal to the energy difference between the ligand valence and metal 3d orbitals. The position of the LMCT shake-down feature would be expected to move to lower energy as the covalency of the ligands increased because the valence energy level of a more covalent ligand would be higher than that of a less covalent ligand resulting in a larger energy difference between the ligand and metal orbitals. 
Chapter 4

An Investigation of the Model Dependency of EXAFS Data Analysis for Dinuclear Non-Heme Iron Systems 


\subsection{Introduction}

EXAFS has been used for many years to elucidate the local structure around metal atoms in biological systems and has been applied to a wide variety of metalloproteins. ${ }^{1}$ We have been using EXAFS to study the structure of the non-heme dinuclear iron center in the hydroxylase component of methane monooxygenase from Methylococcus capsulatus (Bath) and Methylosinus trichosporium (OB3b). ${ }^{2}$ These early studies on the hydroxylase component and relevant dinuclear iron model compounds ${ }^{3}$ have revealed the sensitivity of the EXAFS technique to the presence or absence of a $\mu$-oxo bridge in the first coordination sphere of a dinuclear iron center, and the accuracy which can be achieved in determining the average first shell coordination of the iron atoms. Additionally, these investigations have revealed various difficulties arising from the applications of experimentally derived amplitude and phase parameters in fits to second shell data. ${ }^{2 a}$ However, the use of amplitude and phase functions derived from EXAFS analysis of appropriate model compounds has been shown to give more accurate results in fits to metal foil EXAFS data than the use of theoretical amplitude and phase functions. ${ }^{4}$ We have therefore used empirically derived amplitude and phase functions for the investigations discussed in this work.

We have previously discussed fits to the second shell data using second shell Fe-C parameters from $\mathrm{Fe}$ (acac) 3 (see Chapters 2 and 3 and reference $2 \mathrm{a}$ ) and have remarked on the ability of the second shell low-Z parameters to mimic a second shell metal-metal interaction, an effect which has been seen by others in fits to binuclear copper and mixed metal systems. ${ }^{5}$ We also have noted the high degree of correlation between the Fe-Fe and Fe-C parameters, which makes the interpretation of the results of second shell fits less definitive. A more interesting effect was noted in fits to the second shell oxidized and semimet hydroxylase data with only an iron contribution, which suggested that the fits to the second shell data may exhibit a model bias. 2 a

In the earlier studies, $\mathrm{Fe}-\mathrm{Fe}$ parameters were obtained from two tribridged models, one which has an oxo bridge and one which has a hydroxo bridge $\left(\left[\mathrm{Fe}_{2} \mathrm{O}(\mathrm{OAc})_{2}\left(\mathrm{HB}(\mathrm{pz})_{3}\right)_{2}\right] \text { and }\left[\mathrm{Fe}_{2} \mathrm{OH}(\mathrm{OAc})_{2}\left(\mathrm{HB}(\mathrm{pz})_{3}\right)_{2}\right]\left(\mathrm{ClO}_{4}\right)\right)^{2}$ Use of the parameters from either one of these models resulted in the same two minima in least-squares fits to the second shell hydroxylase data at distances separated by about $0.4 \AA$.2a It was found that the minimum which corresponded to the best fit to the data depended on which set of parameters were used. Most significantly, it was found that the shorter Fe-Fe distance was a better fit to the hydroxylse data when the oxo-bridged model (Fe-Fe 3.14 $\AA$ ) parameters were used, and the longer Fe-Fe distances was a better fit when the hydroxo-bridged model 
(Fe-Fe $3.44 \AA$ ) parameters were used. Therefore, the best fit to the hydroxylase data corresponded to the fit result with a distance most like the Fe-Fe distance of the model compound used to extract the amplitude and phase parameters employed in the fits, suggesting that the fit results were model-dependent.

To more fully explore the apparent bias of the second shell Fe fits to the hydroxylase data, Fe K-edge EXAFS was collected on 6 dinuclear iron models. The data from these 6 models plus the 3 models previously investigated ${ }^{2}$ were used to investigate model dependence of second shell fit results for dinuclear non-heme iron centers. Di- and tribridged models with a variety of bridging groups and $\mathrm{Fe}-\mathrm{Fe}$ distances were used in this study to determine if the model dependence was a function of the numbers of bridges, the types of bridges, or the $\mathrm{Fe}-\mathrm{Fe}$ distance. Fits to the data which included a low- $\mathrm{Z}$ atom contribution were done for some of the models to test the capabilities of the $\mathrm{Fe}-\mathrm{Fe}$ and $\mathrm{Fe}-\mathrm{C}$ parameters to discriminate between $\mathrm{Fe}$ and $\mathrm{C}$ contributions occuring at the same distance. In addition, the model compounds investigated represented a range of different ligation to the Fe atoms, from $6 \mathrm{O}$ atoms to $3 \mathrm{O}$ and $3 \mathrm{~N}$ atoms. Fits to the first shell data of these models as well as to the first shell data for two iron monomers (with $6 \mathrm{O}$ and $6 \mathrm{~N}$ atoms in the first shell, respectively) were done to investigate the ability of EXAFS to distinguish between different numbers of $\mathrm{N}$ and $\mathrm{O}$ atoms in the first coordination sphere.

\subsection{Experimental}

The model compounds investigated for this study are summarized in Table 4.1.6 The compounds were synthesized according to the references listed in the Table with the following exceptions. All of the tribridged samples were provided by Prof. Stephen Lippard of the Massachusetts Institute of Technology. The SALMP dibridged compounds in all three oxidation states were provided by Prof. Richard Holm of Harvard University.

Data for $\left[\mathrm{Fe}_{2}(\mathrm{OH})(\mathrm{OAc})_{2}\left(\mathrm{HB}(\mathrm{pz})_{3}\right)_{2}\right]\left(\mathrm{ClO}_{4}\right)_{2},{ }^{6 \mathrm{~d}}\left[\mathrm{Fe}_{2} \mathrm{O}(\mathrm{OAc})_{2}\left(\mathrm{HB}(\mathrm{pz})_{3}\right)_{2}\right], 6 \mathrm{e}$ $\left[\mathrm{FeO}\left(\mathrm{O}_{2} \mathrm{CH}\right)_{4}(\mathrm{BIPhMe})_{2}\right]\left(\mathrm{CH}_{3} \mathrm{OH}\right), 6 \mathrm{C}\left[\mathrm{Fe}(\mathrm{acac})_{3}\right]^{6 \mathrm{~h}, \mathrm{i}}$ and the oxidized and semimet forms of the hydroxylase component of MMO from Methylococcus capsulatus (Bath) (EXAFS5 and EXAFS2 respectively) were collected as previously described. ${ }^{2}$ Samples for XAS experiments were diluted with BN powder, finely ground with a mortar and pestle, and pressed into a $1 \mathrm{~mm}$ thick Al sample spacer windowed with Mylar tape (total sample weight, $\sim 55 \mathrm{mg}$ ). The samples were run in transmission mode at the Stanford Synchrotron Radiation Laboratory (SSRL) on unfocused 8-pole wiggler beamlines 4-3 or 7-3 (18 kG) or on unfocused bending magnet beamline X19A at the National Synchrotron Light Source (NSLS) at Brookhaven National Laboratory using either a $\mathrm{Si}(220)$ or a 


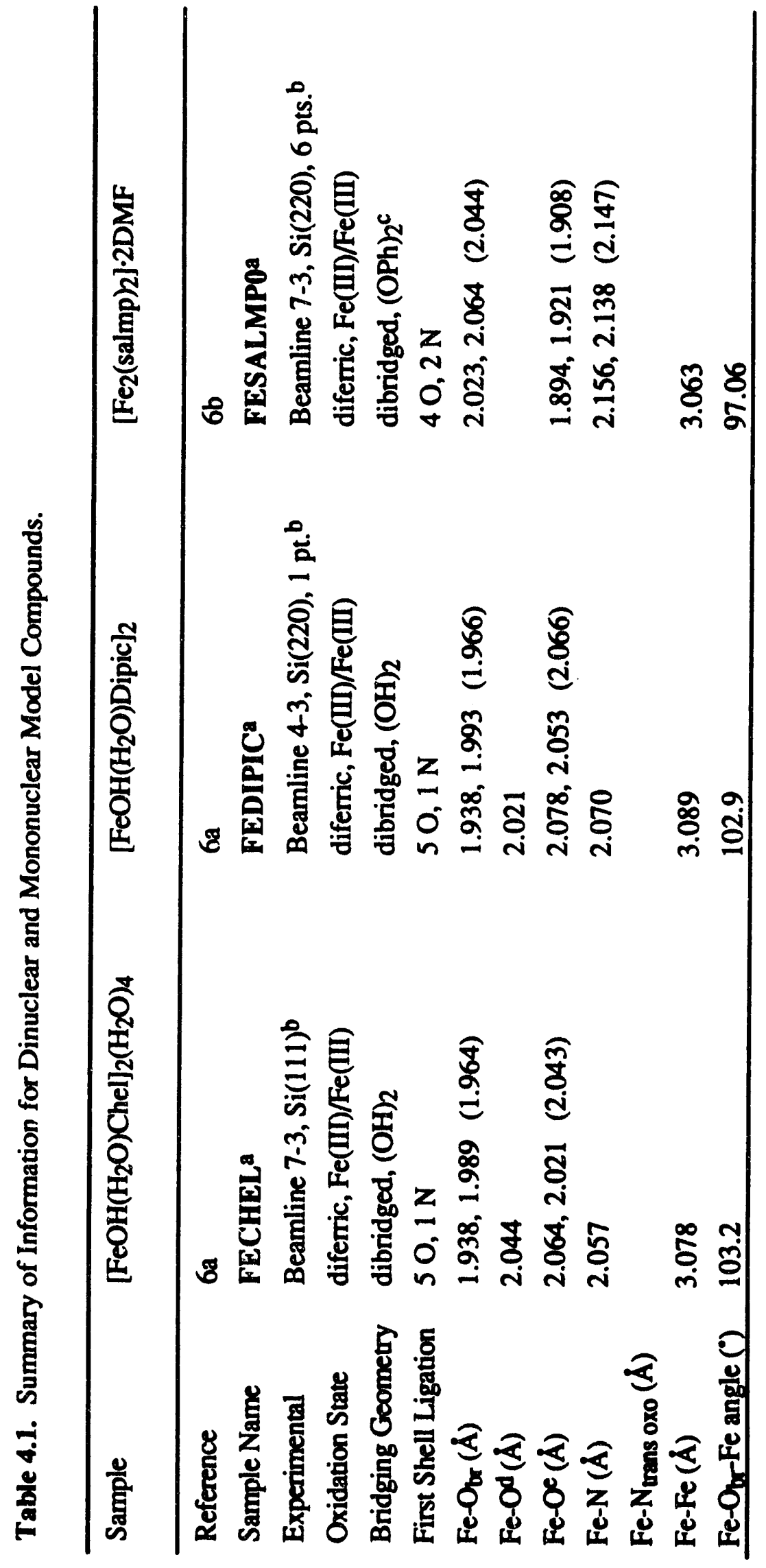




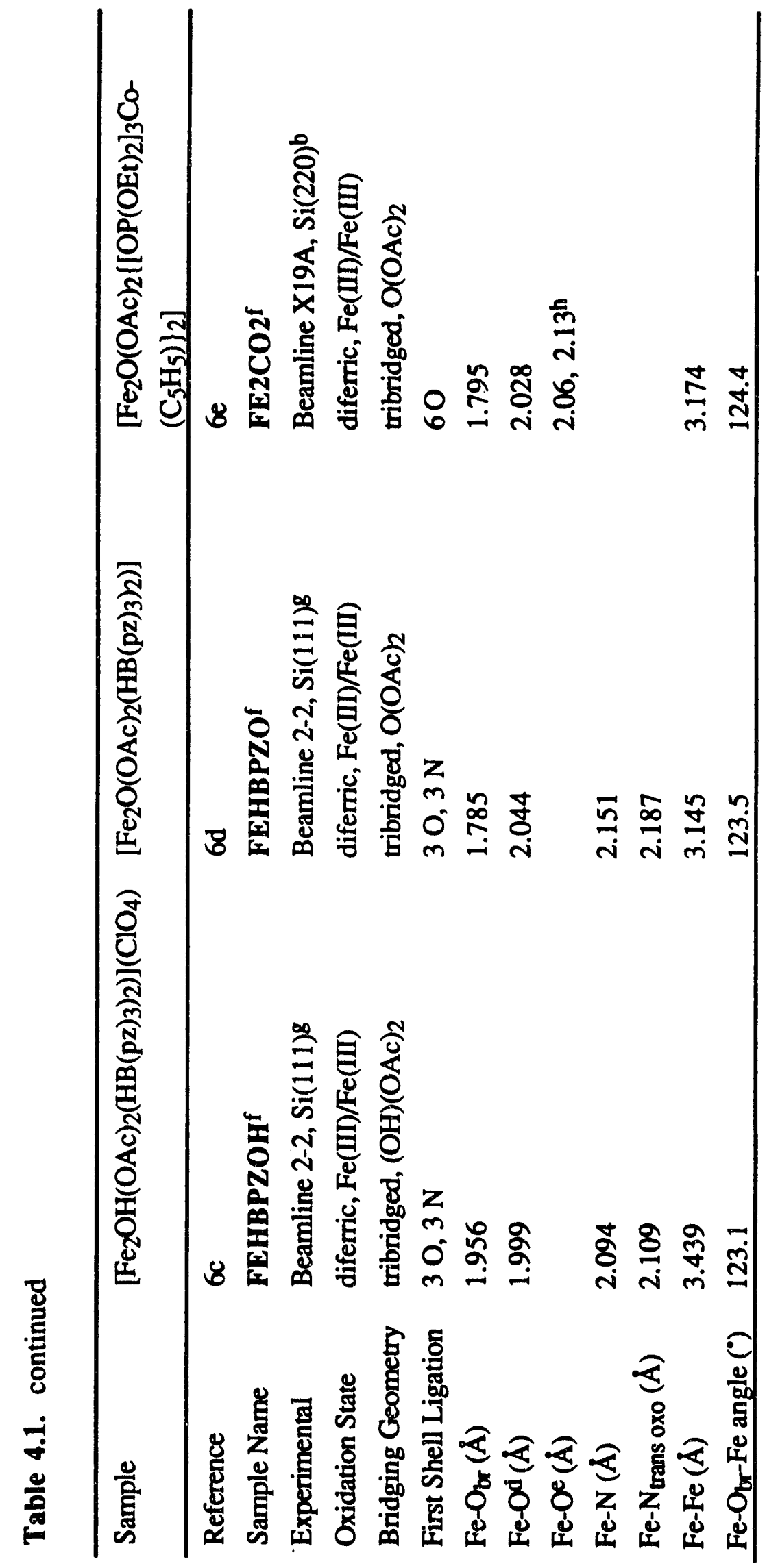




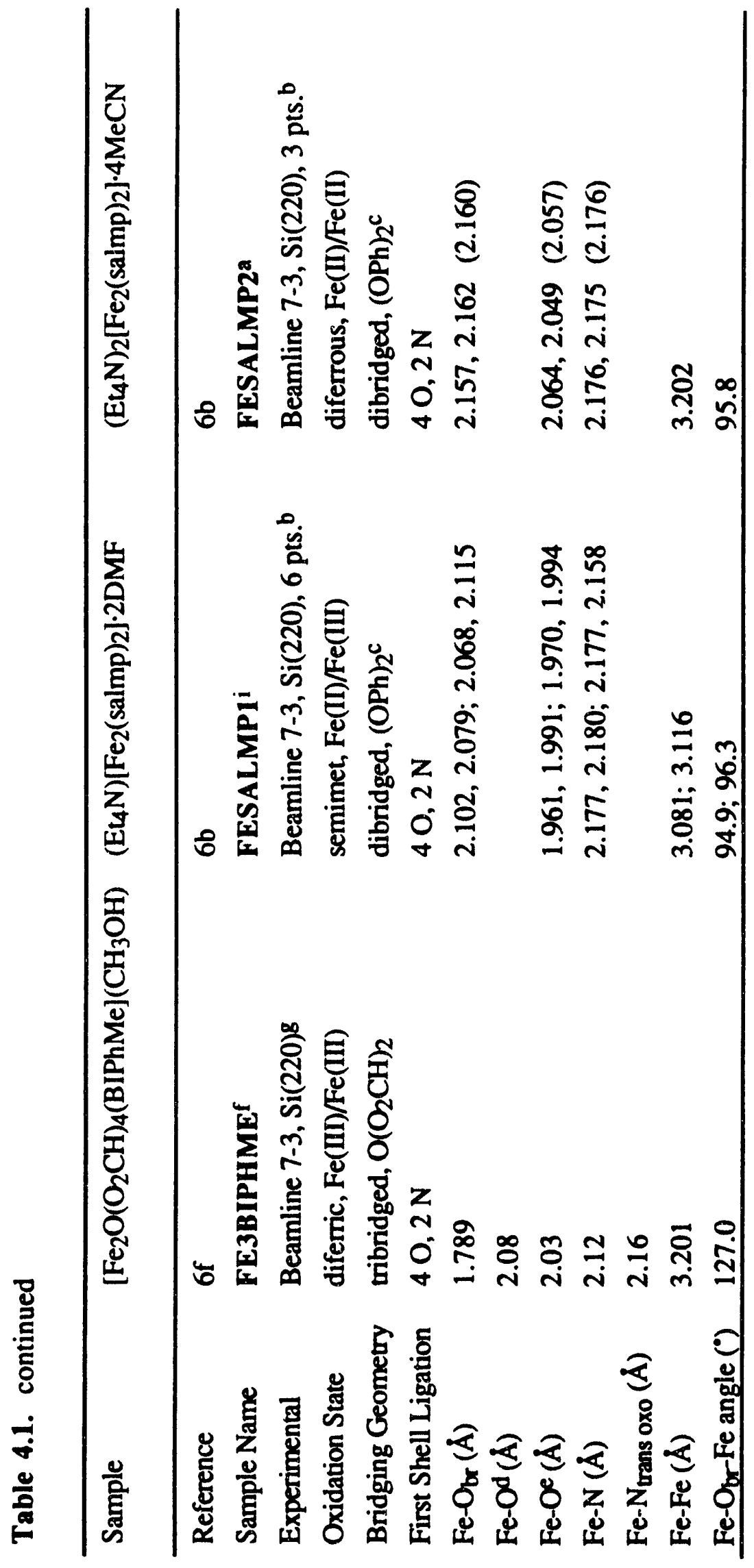




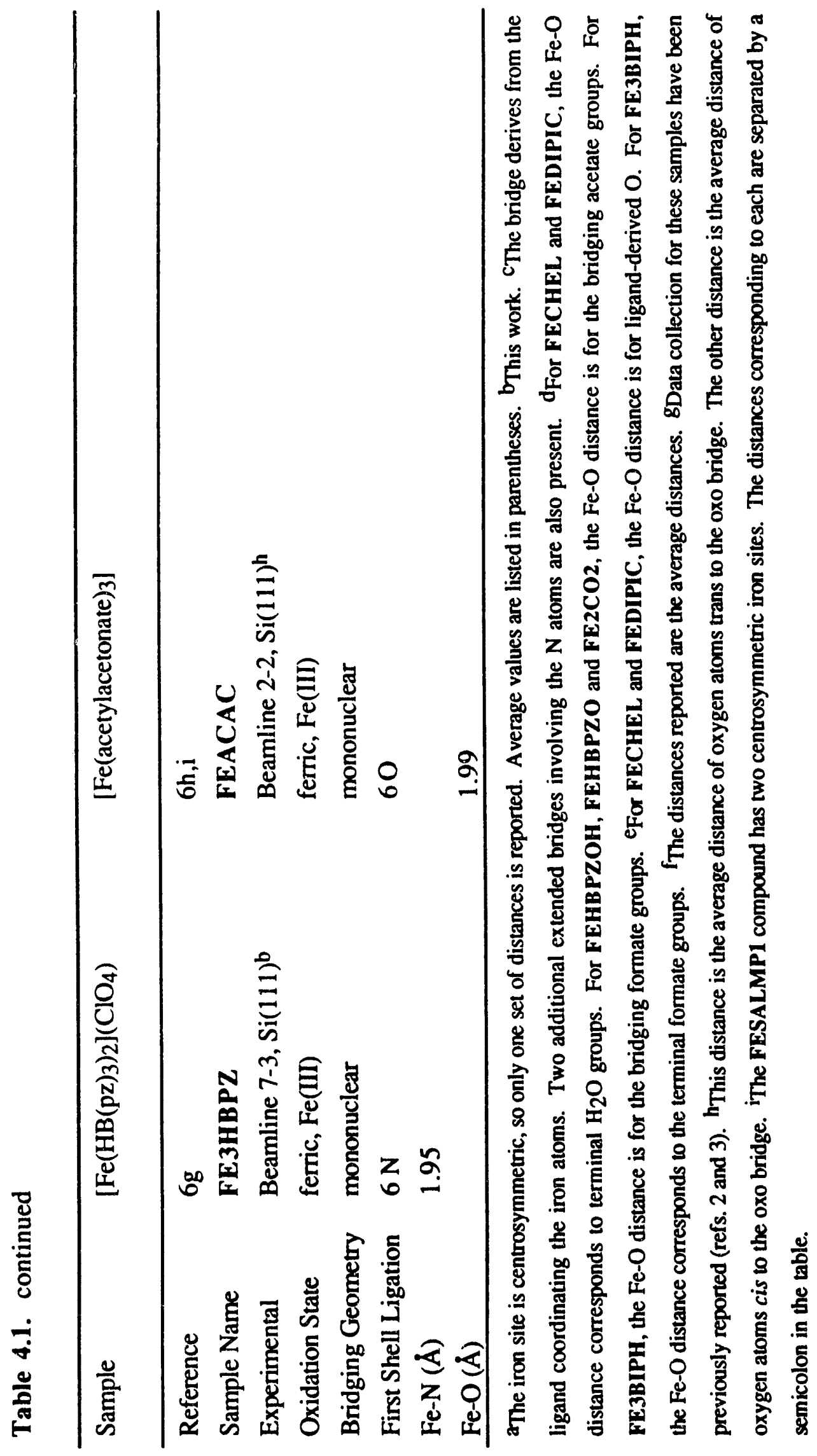


$\mathrm{Si}(111)$ monochromator (see Table 4.1). Ring operating conditions were $3.0 \mathrm{GeV}$ and 40$90 \mathrm{~mA}$ at SSRL, and $2.5 \mathrm{GeV}$ and $90-200 \mathrm{~mA}$ at NSLS. Higher harmonics in the incident beam were rejected by detuning the monochromator $50 \%$ at $7820 \mathrm{eV}$ on beamline X19A, and at $7997 \mathrm{eV}$ on beamlines 4-3 and 7-3. The incident and transmitted beam intensity were monitored by using $\mathrm{N}_{2}$-filled ionization chambers of standard design. The temperature of the samples was $10 \mathrm{~K}$ maintained by a continuous flow LHe cryostat (Oxford Instruments model CF1208).

Energy calibration for each scan was perfomed by using an internal foil calibration method ${ }^{7}$, setting the energy of the first inflection point of an iron foil as $7111.2 \mathrm{eV}$. More than one scan was measured to insure reproducibility of the data. In general, 4 scans were averaged together for each sample and a single-point replacement method was used to remove monochromator glitches in the averaged data where necessary (see Table 4.1). The data were background subtracted by fitting a polynomial to the EXAFS region which was extrapolated through the preedge region and subtracted. A three or four segment spline was fit to the postedge region and subtracted to isolate the EXAFS data and to normalize the edge jump to unity. The spline was chosen so that it removed the low frequency noise without reducing the true EXAFS amplitude; this was checked by monitoring the Fourier transform of the EXAFS data during the normalization process. The normalized data were converted to $k$ space, where $k$ is the photoelectron wavevector defined by $\left[2 \mathrm{~m}_{\mathrm{e}}(\mathrm{E}\right.$ $\left.\left.E_{0}\right) / \hbar^{2}\right]^{1 / 2}$. In this expression, $m_{e}$ is the electron rest mass, $E$ is the photon energy $(e V)$, $E_{0}$ is the threshold energy $(7130 \mathrm{eV}$ for iron, where $k=0)$ and $\hbar$ is Planck's constant divided by $2 \pi$.

The EXAFS data between 3.5 and $12.5 \AA^{-1}$ for all of the models with the exception of FE2CO2 and FESALMP2 were Fourier transformed to R $(\AA)$ space to isolate the first and second shell contributions to the data. For FE2CO2, the data between 3.8 and 12.3 $\AA^{-1}$ were Fourier transformed to $R(\AA)$ space due to the presence of the Co edge above 12.3 $\AA^{-1}$ and duplicated data points in the region between 3.5 and $3.8 \AA^{-1}$ caused by a problem in the data aquisition program. For FESALMP2, the data between 3.5 and $12.3 \AA^{-1}$ were Fourier transformed to $R(\AA)$ space because of a spike in the data above $12.3 \AA^{-1}$ which was too broad to be removed by the standard editing methods without the risk of altering the data.

The individual first and second shell contributions to the data were backtransformed to $k$ space and fit from 4.0 to $12.0 \AA^{-1}$. The windows used to isolate the peaks in the Fourier transforms for the backtransforms are presented in the tables of the fit results. A gaussian window of width $0.1 \AA$ was applied to minimize truncation artifacts introduced by the Fourier transform technique. Non-linear least-squares curve-fitting techniques using 
empirical amplitude and phase parameters were used to analyze the data, as described in Chapter 1 and elsewhere. ${ }^{8}$ All curve fitting was based on $k^{3}$-weighted data and applied to individual filtered shells. Only the structure-dependent parameters, i.e. the distance and the number of atoms in the shell were allowed to vary in the refinements. A "goodness-of-fit" parameter, $F$, was calculated as $F=\left\{\left[k^{6} \text { (data }- \text { fit }\right)^{2}\right] /($ no. of points $\left.)\right\}^{1 / 2}$ for each fit. The fits proceeded by allowing the initial coordination numbers and distances for an $\mathrm{Fe}-\mathrm{X}$ pair of interest to vary. Empircal mplitude and phase parameters for the first coordination shell $\mathrm{Fe}-\mathrm{X}$ scattering pairs of interest were obtained from the following models: $\mathrm{Fe}-\mathrm{N}$ from [Fe(1,10-phenanthroline $\left.)_{3}\right]\left(\mathrm{ClO}_{4}\right)_{3} ;{ }^{9} \mathrm{Fe}-\mathrm{O}$ from [ $\left.\mathrm{Fe}(\text { acetylacetonate })_{3}\right] .{ }^{6}$, i Data for these samples were collected as previously described. ${ }^{2}$

For second shell fits to the data, Fe-Fe parameters were extracted from 6 of the model compounds discussed in this paper, chosen to represent a variety of $\mathrm{Fe}-\mathrm{Fe}$ distances and bridging geometries. The Fe-Fe parameters were extracted by fitting the second shell data using the correct Fe-Fe distance from the crystal structure of the model compound, a coordination number of 1 , and initial amplitude and phase parameters from one of the standards previously used for second shell Fe-Fe fits ( FEHBPZO or FEHBPZOH, see Chapters 2 and 3). The amplitude and phase parameters were allowed to vary in an iterative manner with the distance and coordination number fixed. In the first cycle, all of the amplitude and phase parameters were allowed to vary. The optimized parameters were then used as the starting point for the next iteration by successively allowing just the amplitude or the phase parameters to vary in the fits to the data. Once the sets of amplitude and phase parameters no longer changed during the fits (after 4 cycles), all of the parameters were allowed to vary for the final iteration. The parameters thus obtained were confirmed by repeating the method using initial amplitude and phase parameters from the other Fe-Fe standard, and verifying that the Fe-Fe parameters for the new model compound refined to the same values independently of the initial starting values. These parameters were then applied in fits to the second shell data of all of the dimers and to the second shell data of the oxidized and semimet forms of the hydroxylase of MMO from Methylococcus capsulatus (Bath).

\subsection{Results of Fits to the Model Data}

The model compounds used in this investigation were predominantly ferric, and all were octahedrally coordinated by a mixture of $\mathrm{N}$ and $\mathrm{O}$ atoms, ranging from $6 \mathrm{~N}$ (FE3HBPZ, monomeric ferric compound) to 6 O (FEACAC, monomeric ferric compound and $\mathrm{FE} 2 \mathrm{CO}$, ferric dimer) with various combinations of $\mathrm{N}$ and $\mathrm{O}$ ligation 
represented in the series (Table 4.1). Both di- and tri-bridged compounds were investigated, with the bridging groups consisting of combinations of hydroxo, alkoxo, oxo and carboxylato ligands (Table 4.1). The Fe-Fe distances represented by the compounds discussed below range between 3.06 and $3.44 \AA$, and the $\mathrm{Fe}-\mathrm{O}_{\mathrm{br}}-\mathrm{Fe}$ angles range from $94.9^{\circ}$ to $124.4^{\circ}$. Most of the classes of multiply-bridged diferric non-heme iron models currently available ${ }^{10}$ are represented by the compounds listed in Table 4.1. The exception is the dibridged ( $\mu$-oxo)( $\mu$-carboxylato) models. ${ }^{11}$ The data should provide a sufficient framework to investigate the trends and limitations of the empirical non-linear least-squares fitting approach used in XAS studies of dinuclear non-heme iron centers. The EXAFS data of the model compounds discussed in this paper are presented in Figures 4.1-4.3, and the Fourier transforms of the EXAFS data in Figures 4.4-4.6.

\subsubsection{A Description of the Trends Seen in First Shell Fits.}

The results of the fits to the first shell data are presented in Table 4.2. The results presented in the Table will not be exhaustively discussed; rather, a few models will be discussed to illustrate the general trends noted. Fits to the data were attempted with single $\mathrm{N}$ and single $\mathrm{O}$ contributions, with two $\mathrm{N}$ or two $\mathrm{O}$ contributions, and with a combined $\mathrm{N}$ and $O$ contribution. For the monomeric models FEACAC and FE3HBPZ, which have respectively an octahedral f.rst shell of $\mathrm{O}$ or $\mathrm{N}$ atoms, the fits with more than a single contribution resulted in either unreasonably high or negative coordination numbers. The fits with just a single $\mathrm{N}$ or $\mathrm{O}$ contribution (Table 4.2, fits 4.2-1, 4.2-2, 4.2-3, 4.2-4) were satisfactory in each case, but a better fit was obtained with only $\mathrm{N}$ atoms for FE3HBPZ (Table 4.2, fit 4.2-1). For FEACAC, a better fit was obtained for the O-only fit (Table 4.2 , fit 4.2-4), which is not surprising when one considers that the parameters used for this fit were obtained from FEACAC.

For the dimeric models, which have a mixed $\mathrm{N}$ and $\mathrm{O}$ first shell environment at a range of distances, the fits consisting of just a single $\mathrm{N}$ or $\mathrm{O}$ contribution resulted in low coordination numbers. The best fits to the data corresponded to two separate contributions (either two N, two $\mathrm{O}$, or $\mathrm{N}$ and $\mathrm{O}$ ) at different distances for the non-oxo-bridged models. For example, for FEHBPZOH, the addition of an $\mathrm{O}$ contribution to the $\mathrm{N}$ contribution results in the total coordination number increasing from 4 (Table 4.2 , fit 4.2-23) to -6 (Table 4.2, fits 4.2-27 and 4.2-28) with a drop in the fit function, F, from 0.95 to 0.22 . The improvement seen in the fit to the first shell data of FEHBPZOH with the addition of a second contribution is presented in Figure 4.7. For the oxo-bridged models, a third contribution corresponding to the $\mathrm{Fe}-\mathrm{O}_{0 \times 0}$ distance was required in addition to the other 


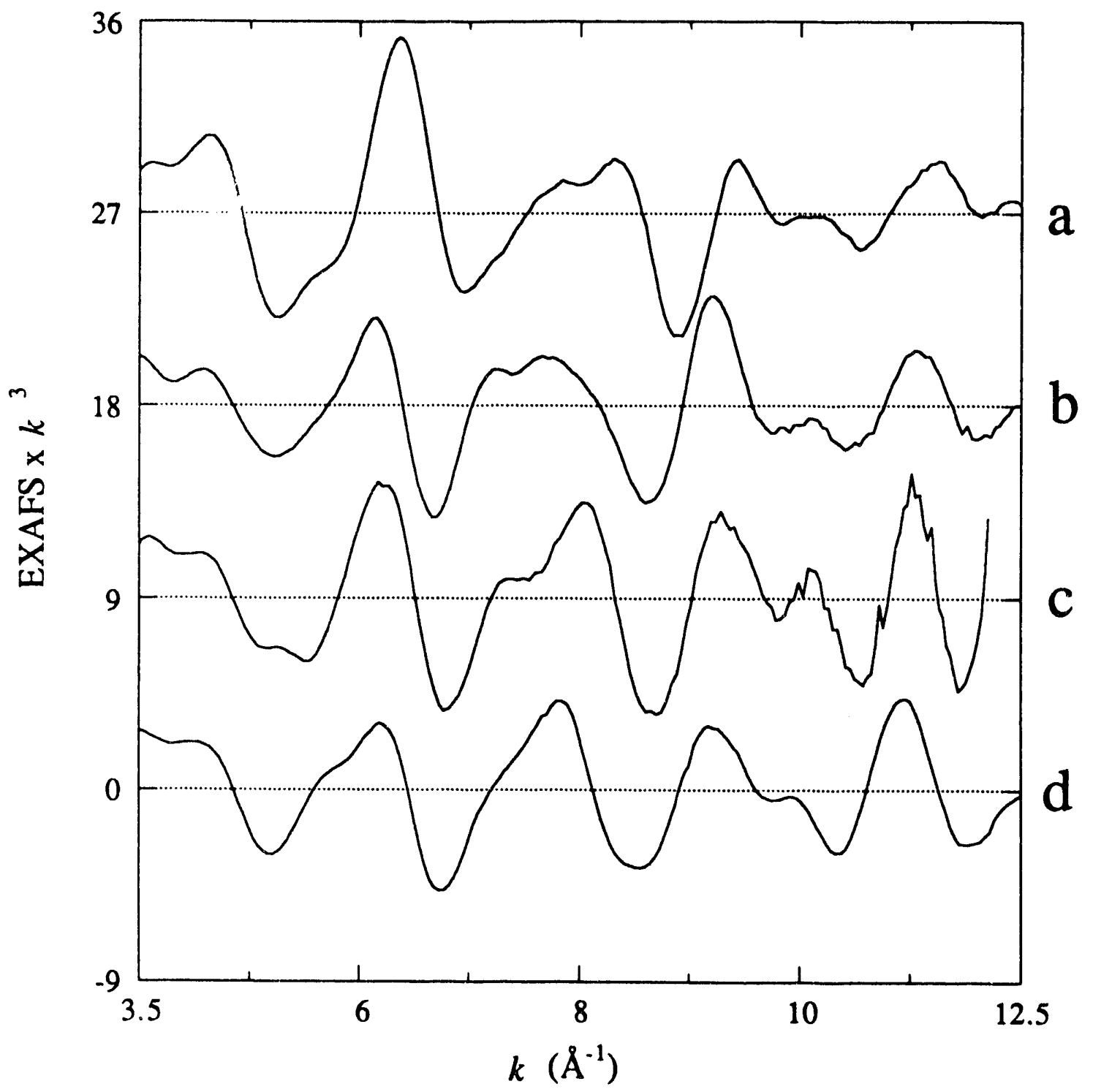

Figure 4.1. EXAFS of tribridged model compounds. (a) FEHBPZOH, (b) FEHBPZO, (c) FE2CO2, (d) FE3BIPHME. 


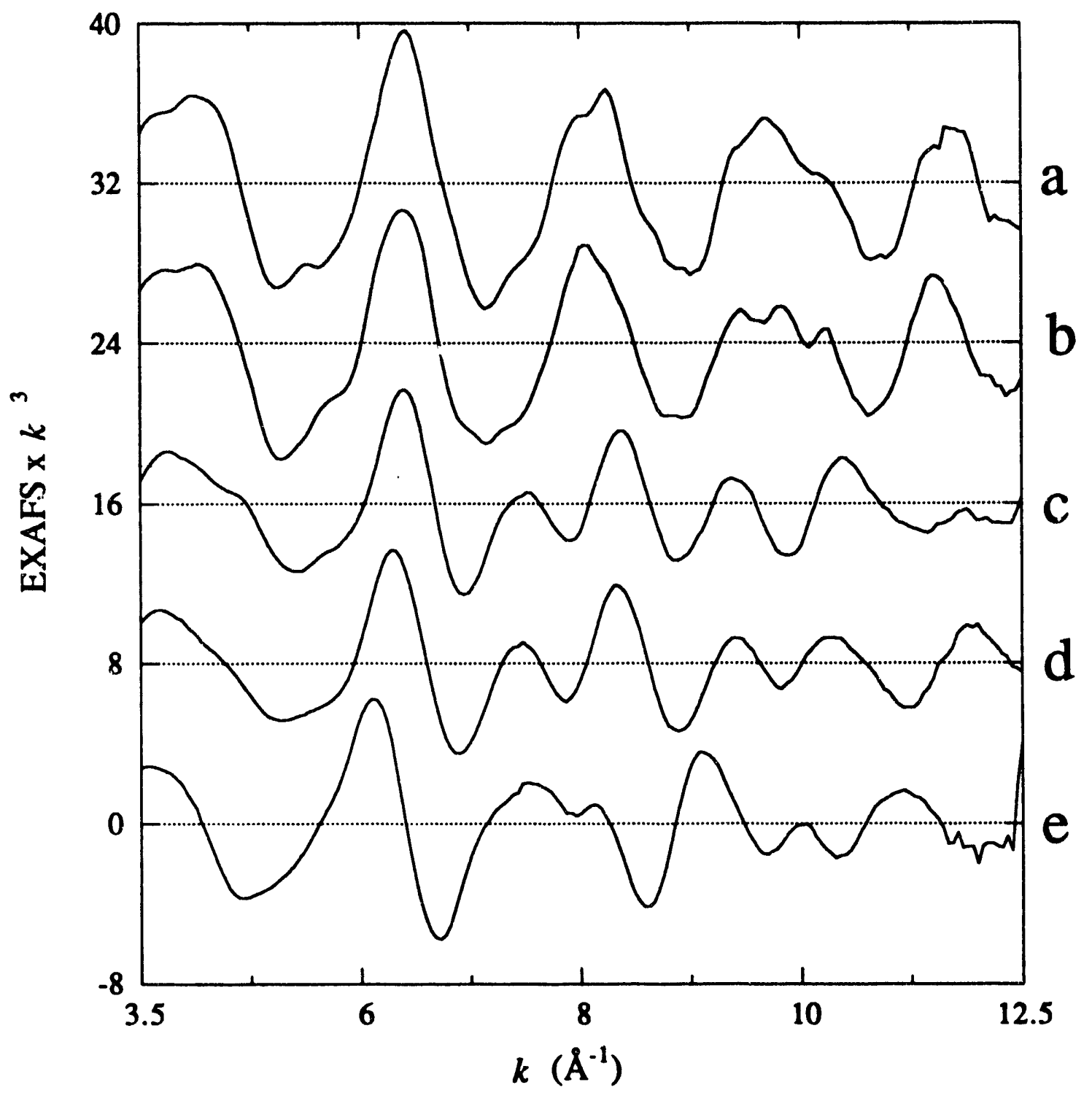

Figure 4.2. EXAFS of dibridged model compounds. (a) FECHEL , (b) FEDIPIC, (c) FESALMP0, (d) FESALMP1, (e) FESALMP2. 


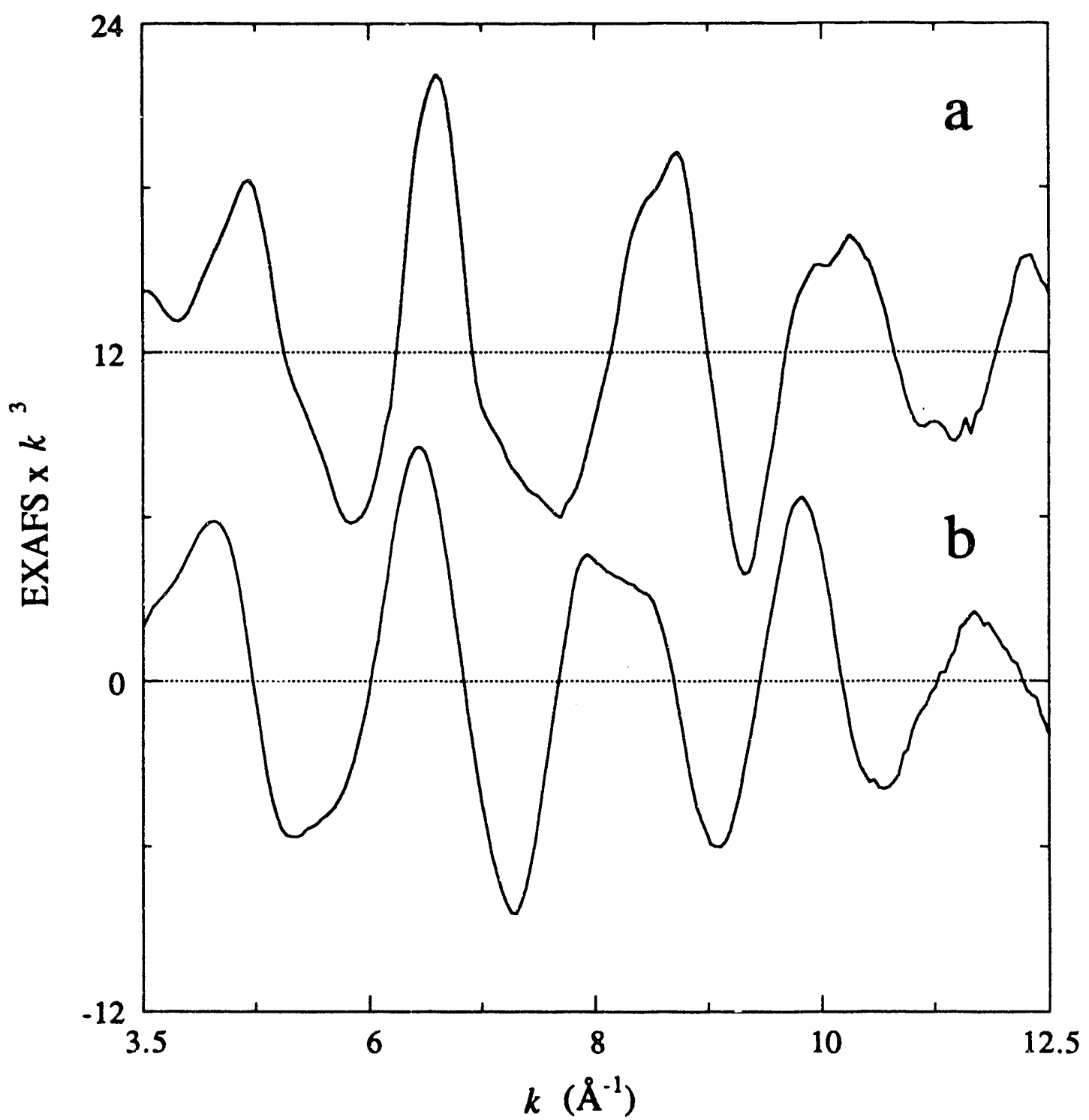

Figure 4.3. EXAFS of monomeric model compounds. (a) FE3HBPZ, (b) FEACAC. 


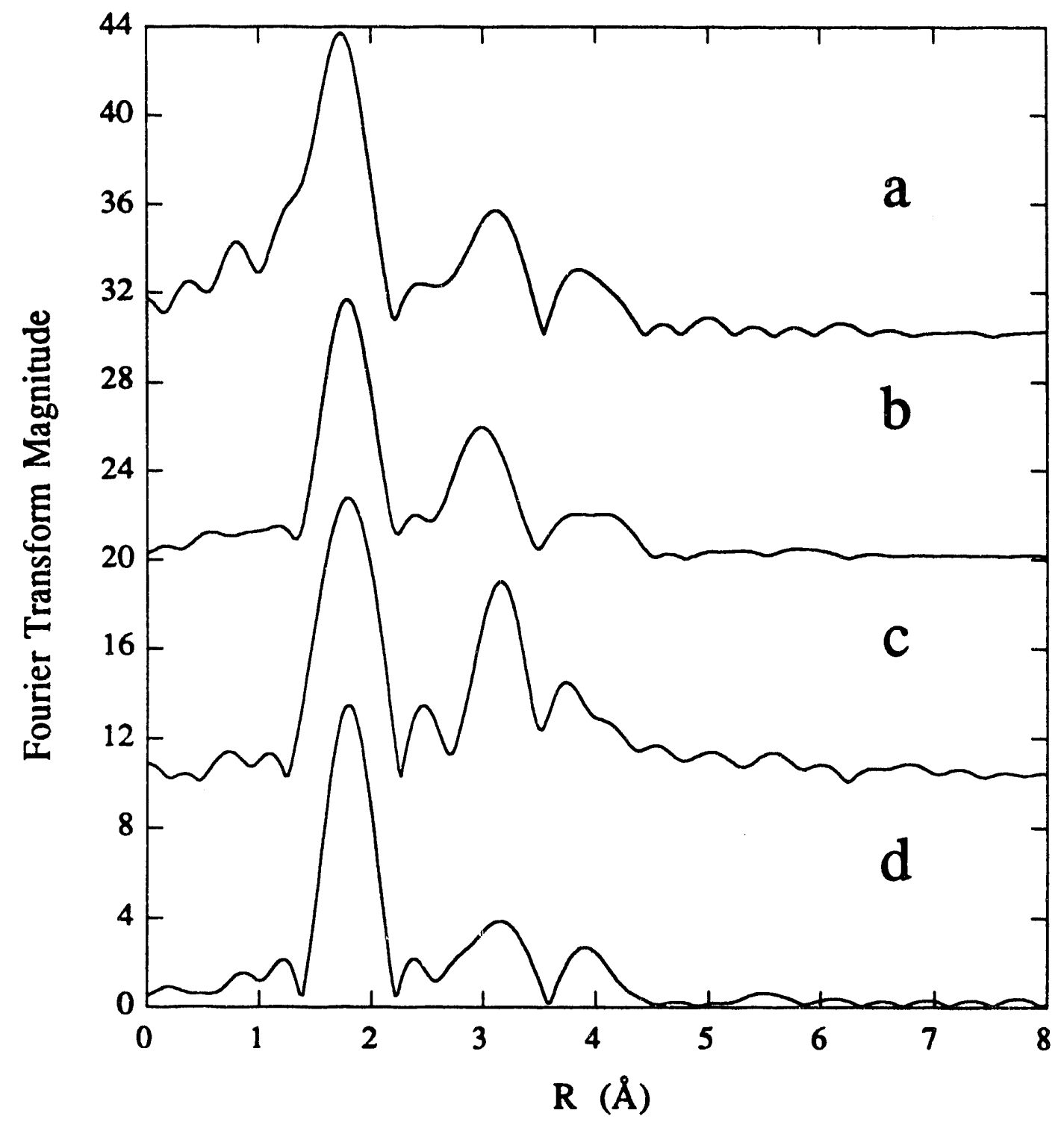

Figure 4.4. Fourier transforms of the EXAFS of tribridged model compounds (Figure 4.1). (a) FEHBPZOH, (b) FEHBPZO, (c) FE2CO2, (d) FE3BIPHME. 


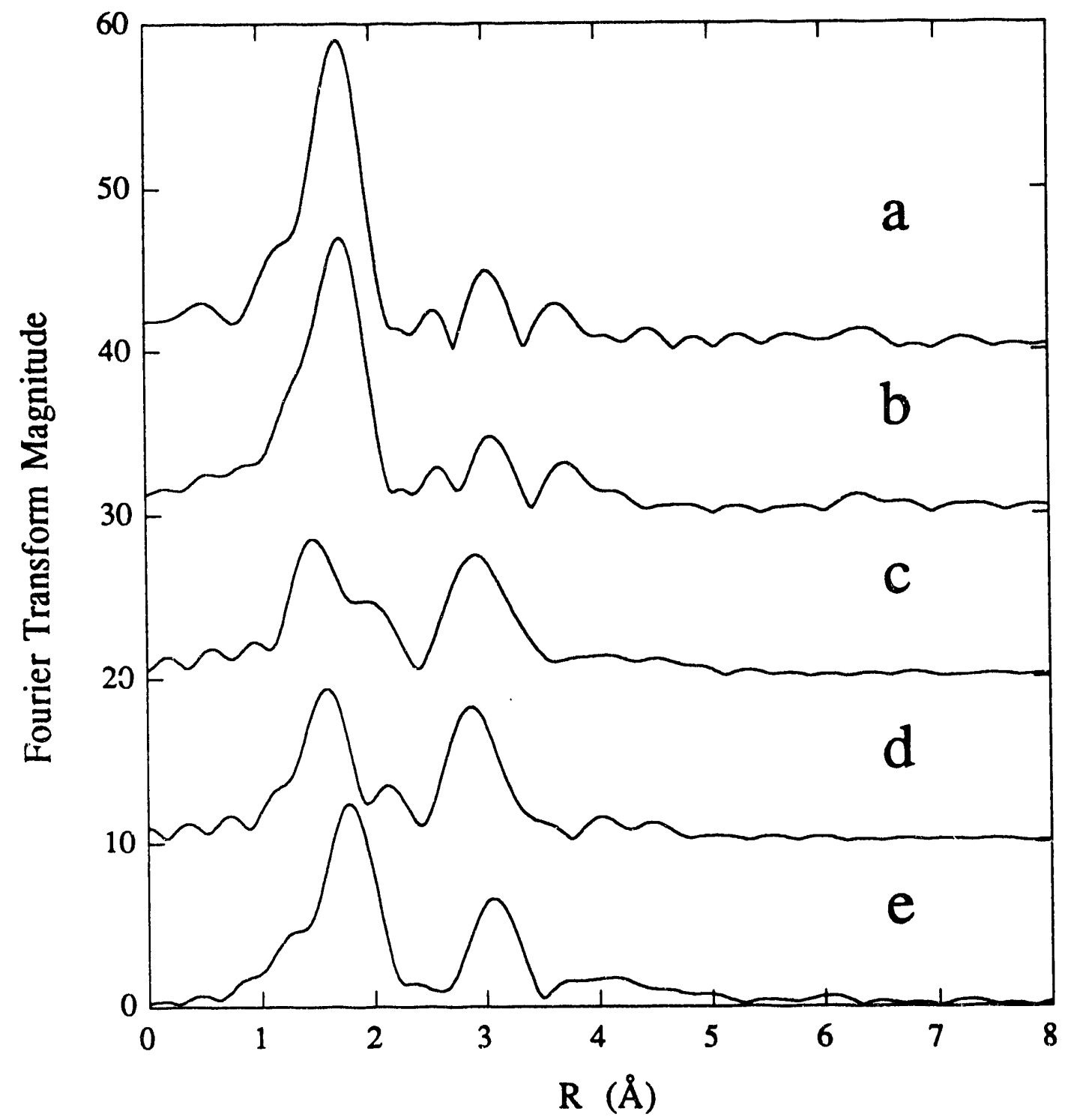

Figure 4.5. Fourier transforms of the EXAFS of dibridged model compounds (Figure 4.2). (a) FECHEL, (b) FEDIPIC, (c) FESALMPO, (d) FESALMP1, (e) FESALMP2. 


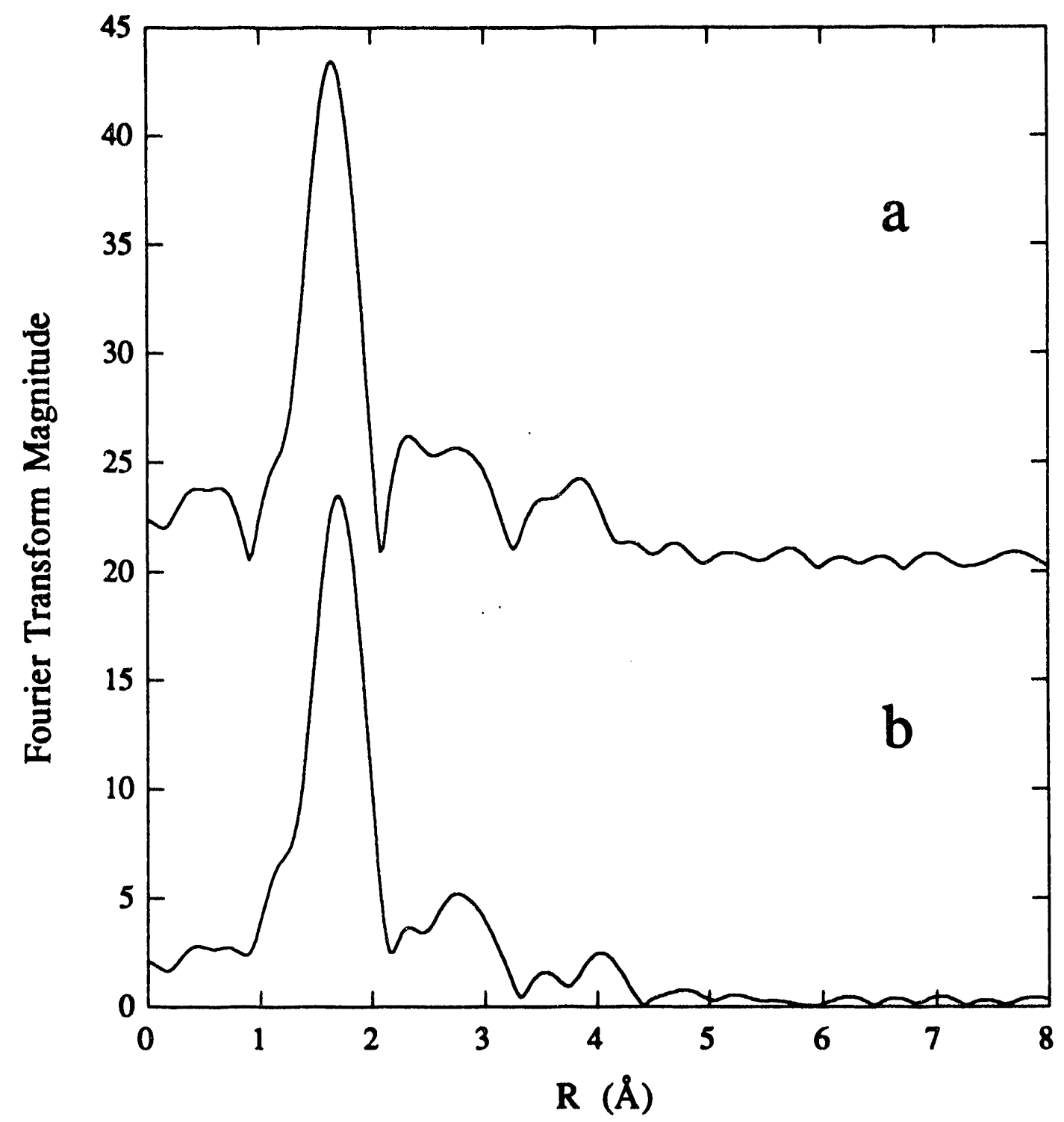

Figure 4.6. Fourier transforms of the EXAFS of monomeric model compounds (Figure 4.3). (a) FE3HBPZ, (b) FEACAC. 


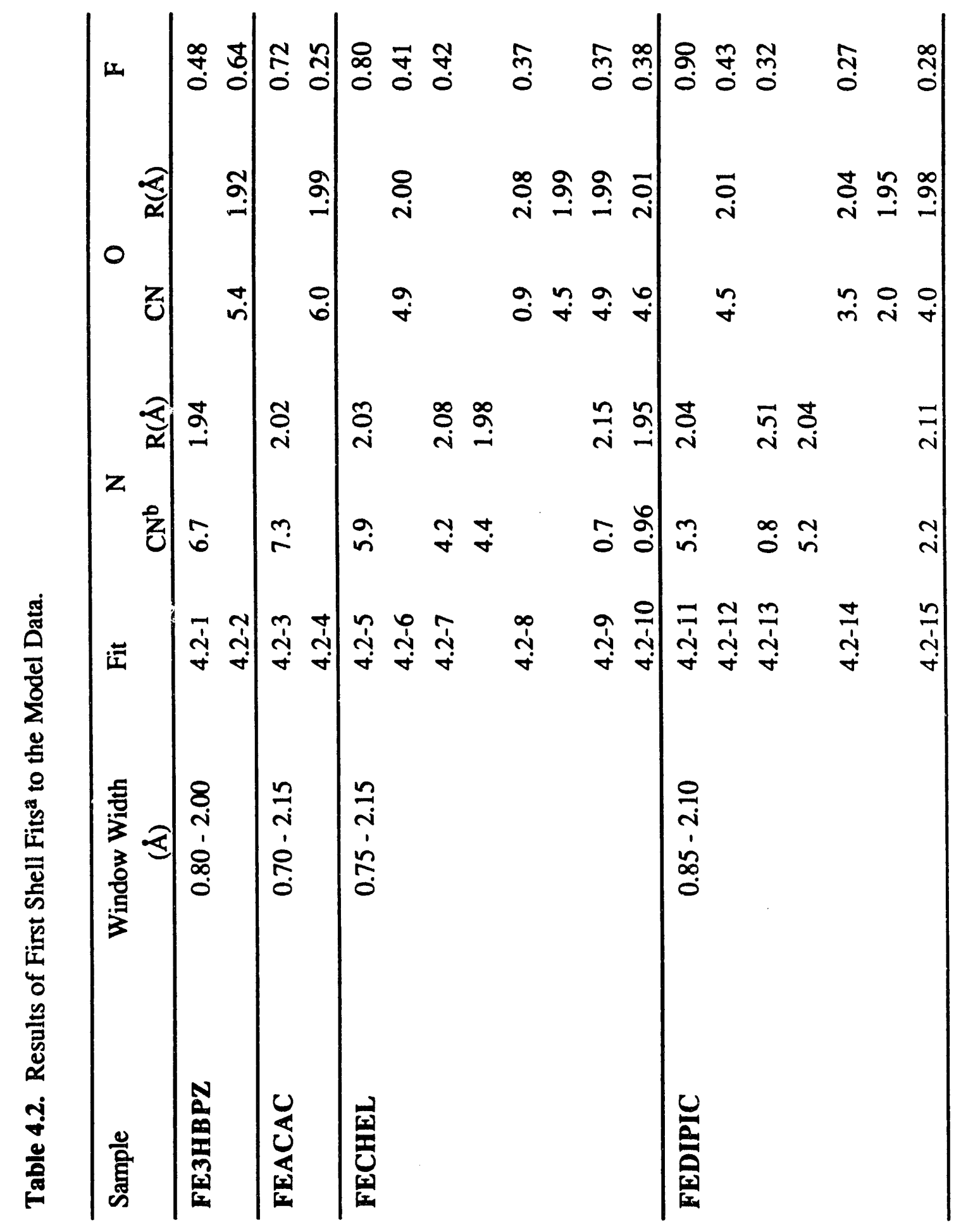




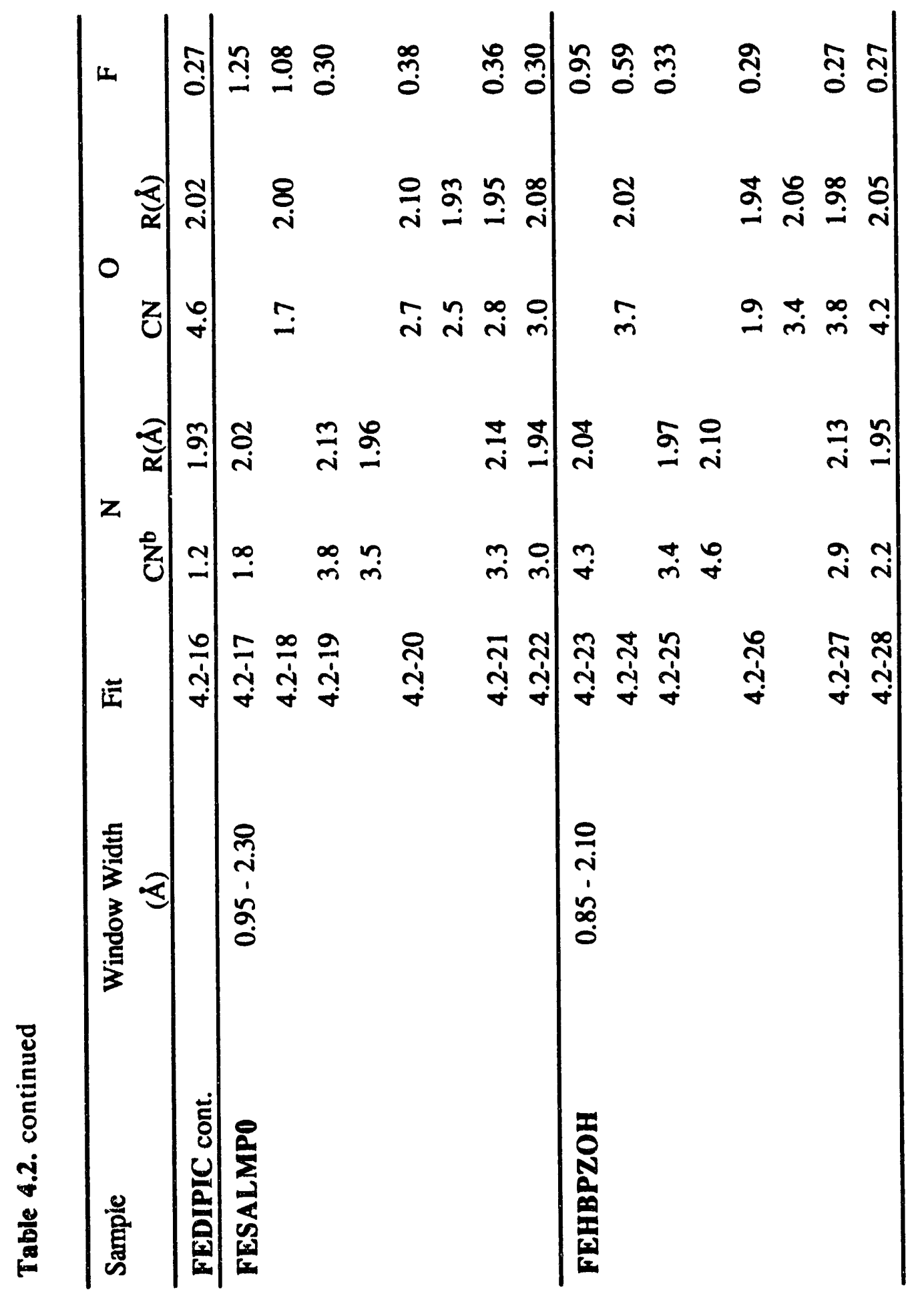




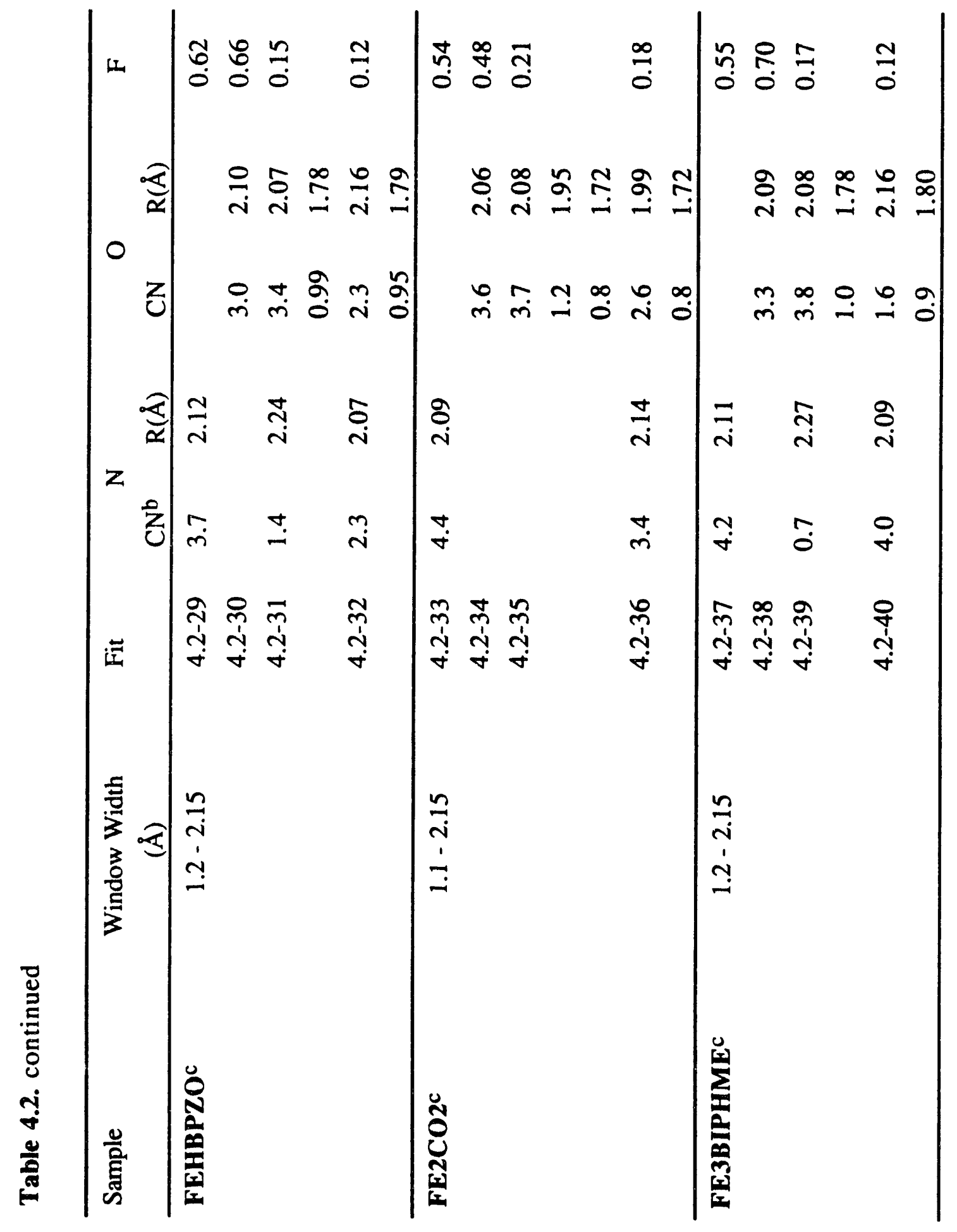




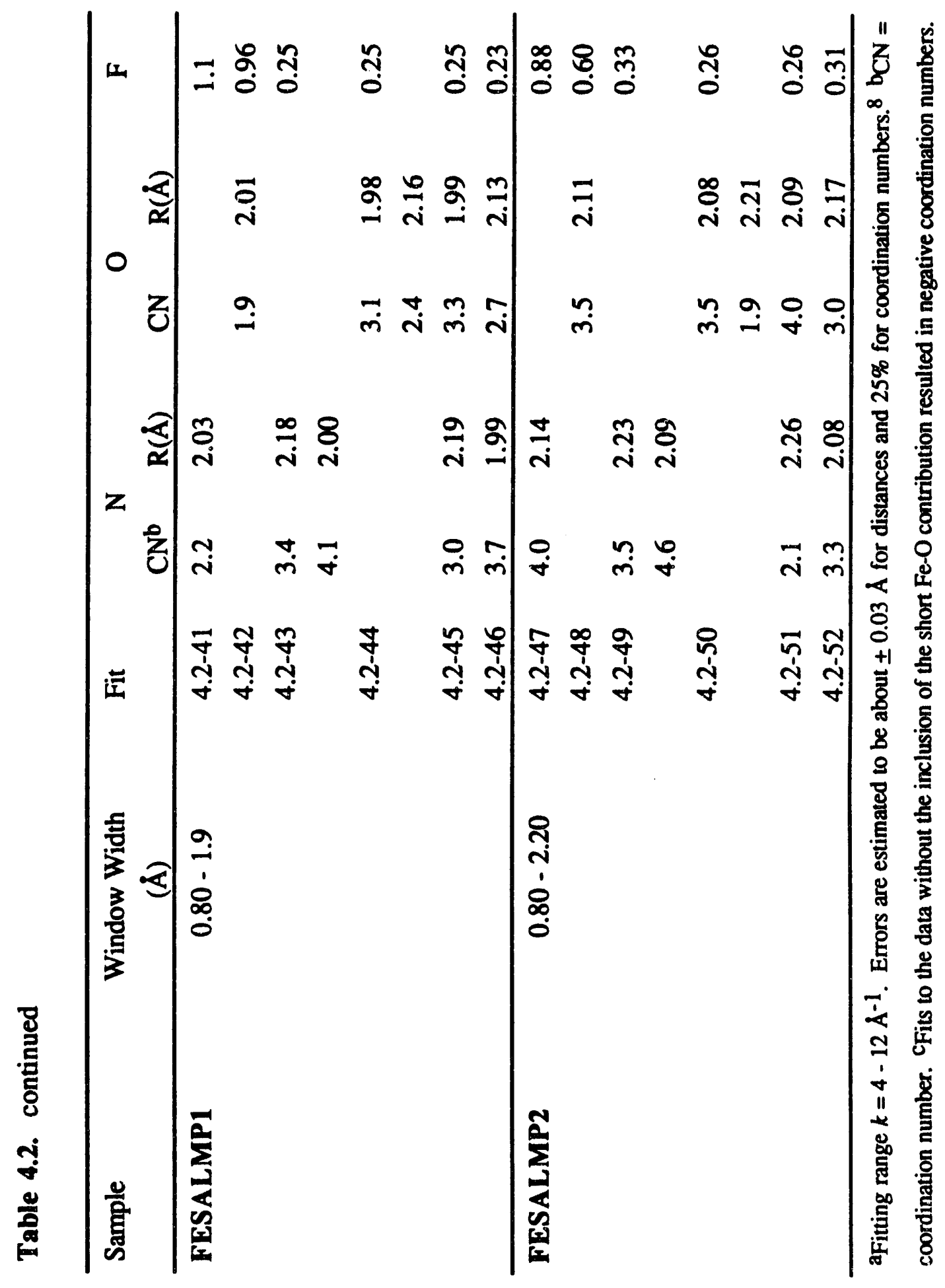




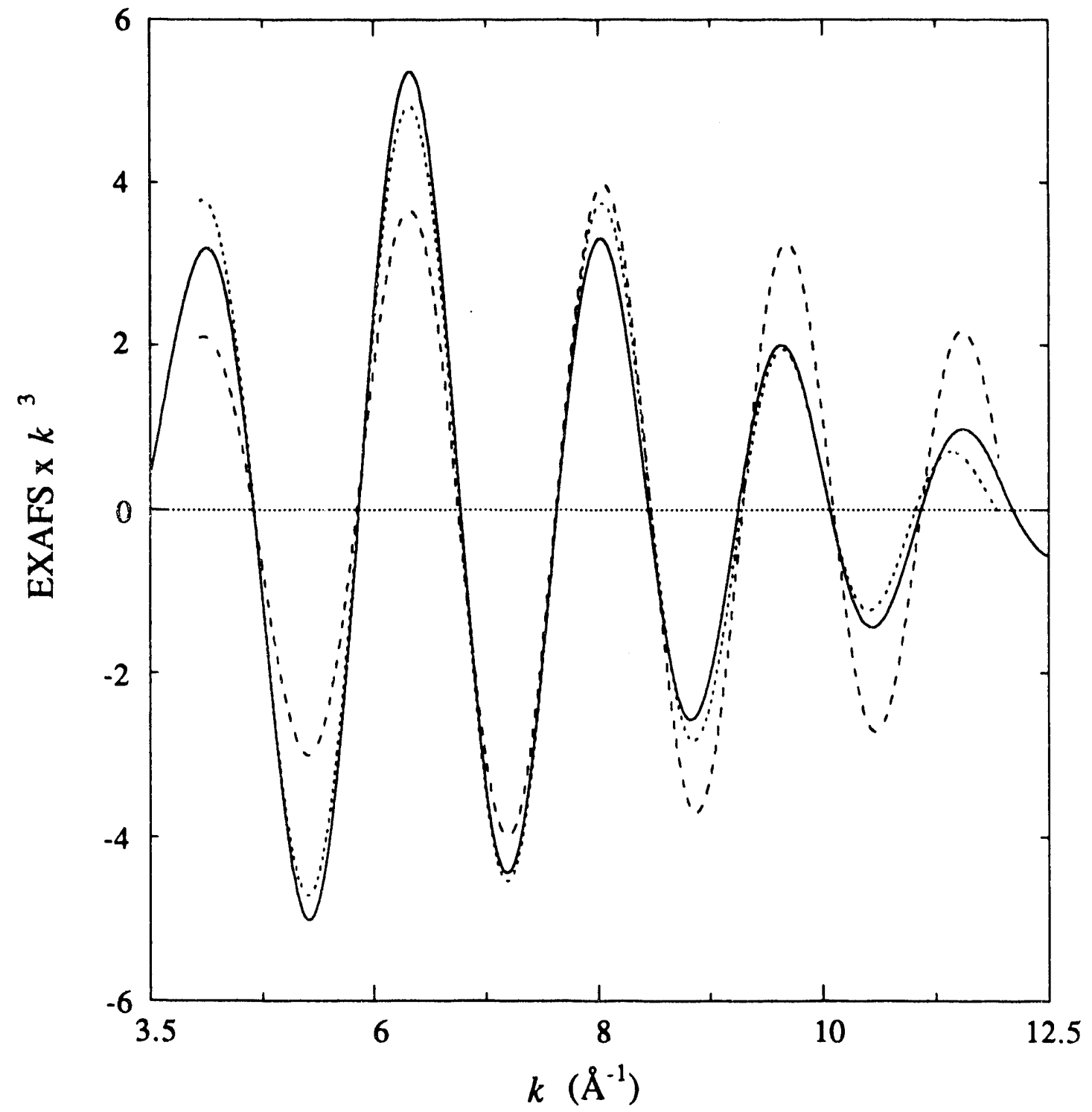

Figure 4.7. Fits to the first shell data of FEHBPZOH. The solid line is the data, the dashed line is the fit to the data with only $\mathrm{N}$ (Table 4.2, fit 4.2-23), and the dotted line is the fit to the data with $\mathrm{N}$ and $\mathrm{O}$ (Table 4.2, fit 4.2-27). Note the improvement in the fit in the high $k$ region with the addition of a second contribution. 
two contributions. Attempts to fit the data with $\mathrm{N}$ and $\mathrm{O}$ only, without the short Fe-O contribution resulted in negative or unreasonably high coordination numbers. However, the addition of a short $\mathrm{Fe}-\mathrm{O}$ contribution resulted in a total coordination number of $\sim 6$ with a fit function of $0.12-0.15$ for FEHBPZO (Table 4.2, fit 4.2-31 and 4.2-32). For the fits to the FEHBPZO data with a single $\mathrm{N}$ or $\mathrm{O}$ contribution, the coordination number was $\sim 3$ and the fit function was $0.62-0.66$ (Table 4.2, fit 4.2-29 and 4.2-30). As noted before in fits to the MMO hydroxylase data (see Chapters 2 and 3), two minima were found for the N/O fits to the data, depending on the relative initial $\mathrm{Fe}-\mathrm{N}$ and $\mathrm{Fe}-\mathrm{O}$ distances used in the fits. This arises from the correlation of the amplitude and phase functions which describe the $\mathrm{Fe}-\mathrm{N}$ and $\mathrm{Fe}-\mathrm{O}$ interactions over the range of data available. The satisfactory results obtained by the two $\mathrm{N}$ and two $\mathrm{O}$ fits to the mixed $\mathrm{N} / \mathrm{O}$ dimers reflects the similar backscattering strength of $\mathrm{N}$ and $\mathrm{O}$ atoms. The distance distribution rather than the atom type appears to be determining factor in the number of contributions required to fit the data.

Dimers with different numbers of $\mathrm{N}$ and $\mathrm{O}$ atoms were fit to determine if the fitting technique could discriminate between different ratios of $\mathrm{N}$ and $\mathrm{O}$ atoms in the first shell. Both FECHEL and FEDIPIC have $5 \mathrm{O}$ and $1 \mathrm{~N}$ in the first coordination sphere, and good fits to the data were obtained with approximately $1 \mathrm{~N}$ at $1.93-1.95 \AA$ and $4.6 \mathrm{O}$ at 2.01-2.02 $\AA$ (Table 4.2, fits 4.2-10 and 4.2-16). The results of the fits agree with the crystallographic distances and coordination numbers (Table 4.1), however the result assigns the wrong atom type to the short distance. The $1.9 \AA$ distance should be an oxygen atom, and the Fe-N distance should be $2.057 \AA$ for FECHEL and $2.070 \AA$ for FEDIPIC. Attempts to obtain a fit with a single short $\mathrm{O}$ contribution and $5 \mathrm{~N}$ contributions at the longer distance were unsuccesful, and in fits to the data with two $\mathrm{O}$ contributions (Table 4.2, fit 4.2-8 and 4.2-14), the lower contribution corresponded to the longer distance instead of to the shorter distance. The O-only fits to the data gave a satisfactory result as well (Table 4.2, fit 4.2-6 and 4.2-12). FESALMPO is coordinated by $4 \mathrm{O}$ atoms at an average distance of $1.98 \AA$ and $2 \mathrm{~N}$ atoms at an average distance of 2.15 $\AA$ (Table 4.1), but the best fits corresponded to either $3.3 \mathrm{~N}$ at $2.14 \AA$ and $2.8 \mathrm{O}$ at $1.95 \AA$ (Table 4.2, fit 4.2-21) or $3.0 \mathrm{~N}$ at $1.94 \AA$ and $3.0 \mathrm{O}$ at $2.08 \AA$ (Table 4.2, fit 4.2-22). The distances are within the expected $0.03 \AA$ error for the crystallographic data based on atom type, but the coordination numbers are inaccurate. These results suggests that the relative numbers of $\mathrm{N}$ and $\mathrm{O}$ atoms cannot be reliably determined from the fits.

The model FE2CO2 has an all O environment with a single oxygen atom at 1.795 $\AA$ and the remaining oxygen atoms at an average distance of $2.06 \AA$. The most reasonable fit to the data suggests that the non-oxo-bridge ligands correspond to a distribution of 1.20 at $1.95 \AA$ and $3.7 \mathrm{O}$ at $2.08 \AA$ (Table 4.2, fit 4.2-35), however there is no Fe-O distance at 
$1.95 \AA$ according to the crystal structure. The coordination-weighted average distance for this fit, $4.9 \mathrm{O}$ at $2.05 \AA$, is in good agreement with the crystallographic data. The coordination-weighted average distances for fits to the model compounds are listed in Table 4.3 along with the average distance calculated from the crystallographic data. There is excellent agreement between the crystallographic average and the EXAFS average for all of the model compounds. This illustrates that accurate information can be obtained by considering the coordination-weighted average distance from the fit results, even if the individual contributions are not accurate.

Fits were also done to the semimet and diferrous model compounds with the SALMP ligand, FESALMP1 and FESALMP2 and the results are also presented in Table 4.2 with the coordination-weighted averages given in Table 4.3. For the corresponding diferric model, FESALMP0, the average first shell distance from the crystal structure was $6 \mathrm{~N} / \mathrm{O}$ at $2.03 \AA$ and the average based on the EXAFS analysis was $6.1 \mathrm{~N} / \mathrm{O}$ at $2.05 \AA$ (Table 4.2, fit 4.2-21). The result of fits to the semimet data was 6.3 N/O at $2.09 \AA$ (Table 4.2, fit 4.2-45), which agrees quite well with the crystallographic average of $6 \mathrm{~N} / \mathrm{O}$ at $2.08 \AA$. For the reduced data, the average coordination was found to be $6.1 \mathrm{~N} / \mathrm{O}$ at $2.15 \AA$ (Table 4.2, fit 4.2-51), also in good agreement with the crystallographic data $(6 \mathrm{~N} / \mathrm{O}$ at $2.13 \AA)$. The average distance in the first coordination sphere increases by $0.05 \AA$ going from the diferric to the semimet oxidation state (2.03 to $2.08 \AA$ ) and by another $0.05 \AA$ upon reduction to the diferrous state (to $2.13 \AA$ ) according to the crystal structures. The fit results reflect this trend, with the average distance increasing by $0.04 \AA$ between the diferric and semimet states, and by an additional $0.04 \AA$ to the diferrous state. The parameters used for the first shell fits, and the method of considering the coordination-weighted average of the fit result, therefore accurately reflect the increase in the Fe-ligand distances which occurs upon reduction of the diiron site.

\subsubsection{The Dependence of Second Shell Fits on Choice of Model Compound}

4.3.2.1. Investigation with Model Data. The model compounds chosen to test the transferability of the $\mathrm{Fe}-\mathrm{Fe}$ parameters and the results of the fits to the second shell model data are listed in Table 4.4. Parameters were extracted from two diferric dibridged models ( $3.063 \AA$ and $3.078 \AA$ Fe-Fe distance), three diferric tribridged models (3.439 $\AA$, $3.143 \AA$, and $3.20 \AA \mathrm{Fe}-\mathrm{Fe}$ distance) and a diferrous dibridged model $(3.20 \AA \mathrm{Fe}-\mathrm{Fe}$ distance) and used in fits to each other as well as to other models. In every case, two minima separated by approximately $0.4 \AA$ were found in fits to the second shell data, 
Table 4.3. A Comparison of the Average First Shell Distances by EXAFS and Crystallography.

$$
\text { Average First Shell Coordination }
$$

\begin{tabular}{lll} 
Sample & from EXAFS analysisa & from crystal structure \\
\hline FE3HBPZ & $6.7 \mathrm{~N}$ at $1.94 \AA$ & $6 \mathrm{~N}$ at $1.95 \AA$ \\
FEACAC & 6.00 at $1.99 \AA$ & $6 \mathrm{O}$ at $1.99 \AA$ \\
FECHEL & $5.6 \mathrm{~N} / \mathrm{O}$ at $2.01 \AA$ & $6 \mathrm{~N} / \mathrm{O}$ at $2.02 \AA$ \\
FEDIPIC & $6.2 \mathrm{~N} / \mathrm{O}$ at $2.03 \AA$ & $6 \mathrm{~N} / \mathrm{O}$ at $2.03 \AA$ \\
FESALMP0 & $6.1 \mathrm{~N} / \mathrm{O}$ at $2.05 \AA$ & $6 \mathrm{~N} / \mathrm{O}$ at $2.03 \AA$ \\
FEHBPZOH & $6.7 \mathrm{~N} / \mathrm{O}$ at $2.04 \AA$ & $6 \mathrm{~N} / \mathrm{O}$ at $2.04 \AA$ \\
FEHBPZO & $4.8 \mathrm{~N} / \mathrm{O}$ at $2.11 \AA ; 10$ at $1.78 \AA$ & $5 \mathrm{~N} / \mathrm{O}$ at $2.11 \AA ; 10$ at $1.79 \AA$ \\
FE2CO2 & 4.90 at $2.04 \AA ; 0.8 \mathrm{O}$ at $1.72 \AA$ & $5 \mathrm{O}$ at $2.06 \AA ; 10$ at $1.79 \AA$ \\
FE3BIPHME & $4.5 \mathrm{~N} / \mathrm{O}$ at $2.11 \AA ; 10$ at $1.78 \AA$ & $5 \mathrm{~N} / \mathrm{O}$ at $2.10 \AA ; 10$ at $1.79 \AA$ \\
FESALMP1 & $6.3 \mathrm{~N} / \mathrm{O}$ at $2.09 \AA$ & $6 \mathrm{~N} / \mathrm{O}$ at $2.08 \AA$ \\
FESALMP2 & $6.1 \mathrm{~N} / \mathrm{O}$ at $2.15 \AA$ & $6 \mathrm{~N} / \mathrm{O}$ at $2.13 \AA$ \\
\hline
\end{tabular}

aThe average distance reported is for the minimum corresponding to $R_{N}>R_{O}$. For FE2CO2, the all oxygen fit is reported. 


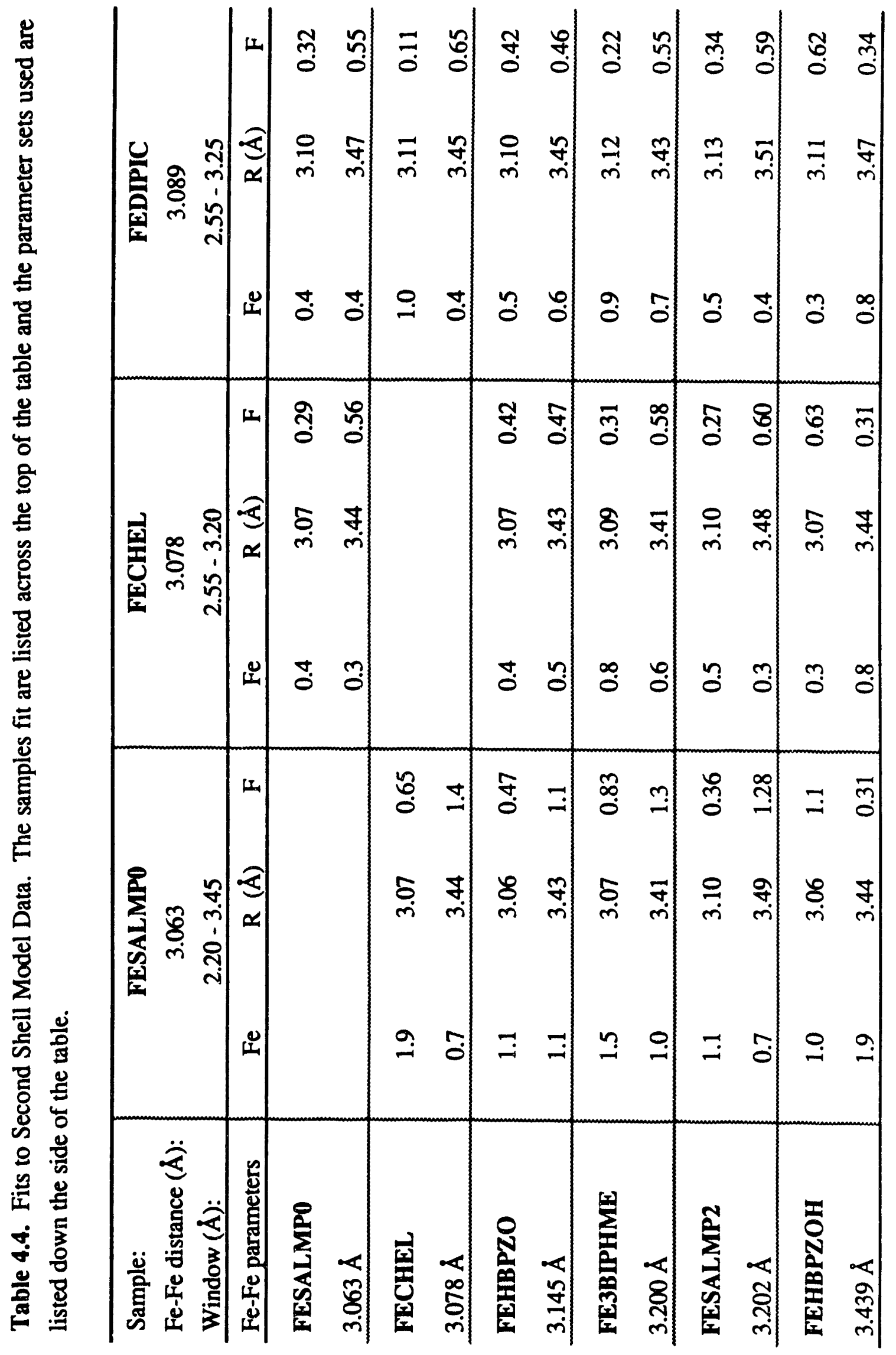




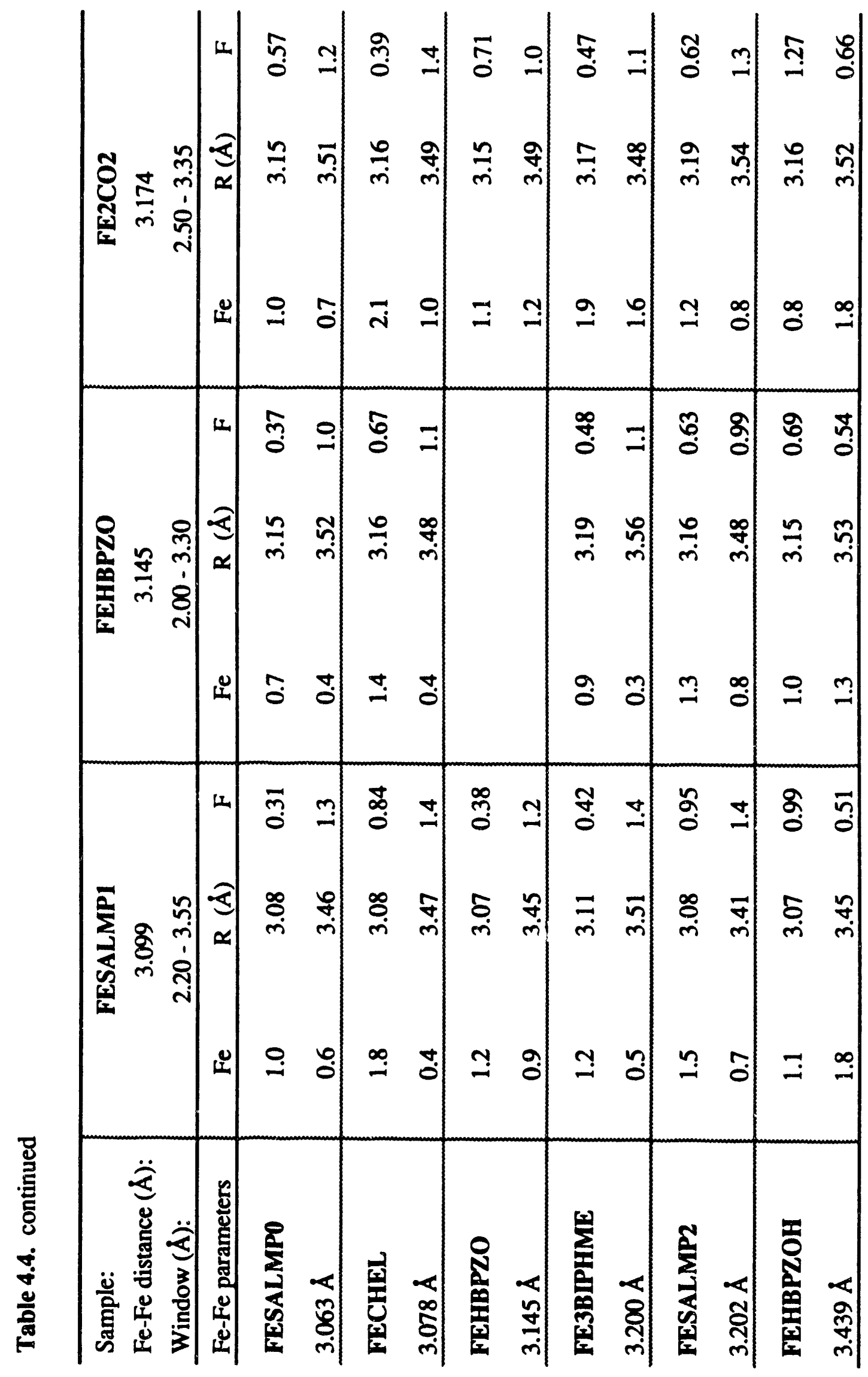




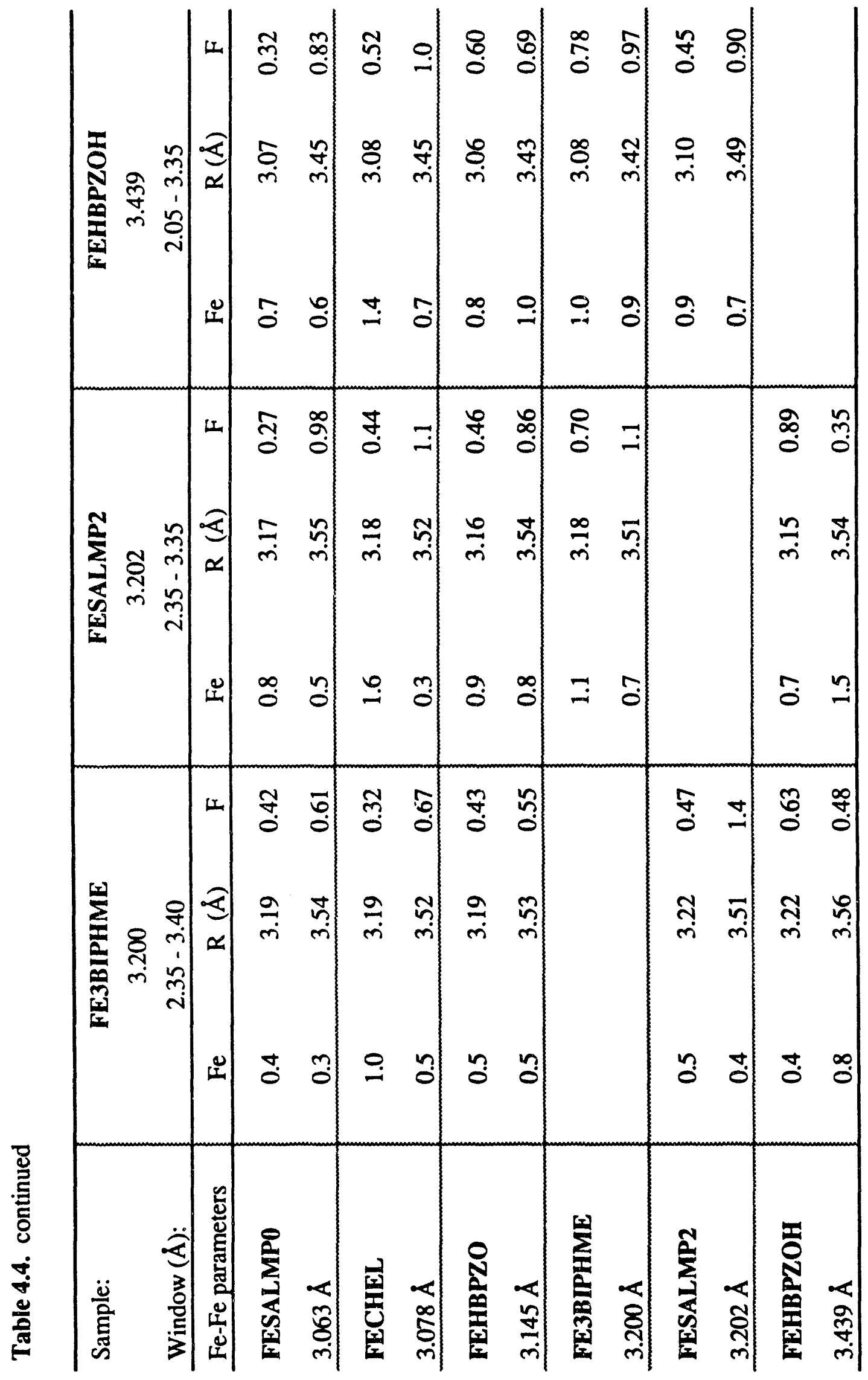


similar to the trend seen in second shell fits to the MMO hydroxylase data (see Chapters 2 and 3). The Fe-Fe distances were consistently longer by $0.03-0.08 \AA$ for the fit results using the diferrous FESALMP2 parameters to fit the diferric model data, except for FE3BIPHME which has the same Fe-Fe distance as FESALMP2 (3.2 $\AA$ ). A distance within $0.05 \AA$ of the correct $\mathrm{Fe}-\mathrm{Fe}$ distance was found for all of the samples, however in some cases the coordination number was off by a factor of two (Table 4.4). Using FECHEL parameters in fits to FESALMP0, which has a much more intense second shell peak relative to the first shell peak than FECHEL (Figure 4.4a and 4.4c), the coordination number was 1.9. In fits to FEDIPIC with the FECHEL parameters (which have similar intensity second shell peaks, Figure 4.4a and 4.4b), a coordination number of 1 was found. Similarly, in fits to FECHEL with FESALMPO parameters, the coordination was $0.4 \mathrm{Fe}$, half of the correct amount. The inaccuracy of the amplitude parameters reflects the relative weakness of the $\mathrm{Fe}-\mathrm{Fe}$ contribution to the data (relative to the strength of the first shell contribution) in some models compared to others (see Figure 4.2). The enhanced amplitude of the second shell peaks in some models over others will be discussed below.

Of the two minima found during the fitting proceedure, the better fit to the data corresponded in every case to the minimum with the $\mathrm{Fe}-\mathrm{Fe}$ distance closer to that of the model from which the parameters were obtained. This result was independent of the number and types of bridges in the iron center as well as the oxidation state of the diiron center. FESALMPO has a dialkoxo-bridged center with an Fe-Fe distance of $3.06 \AA$. Using the FESALMPO parameters in fits to FEHBPZOH data, a tribridged diferric center with a hydroxo-bridge and a $3.44 \AA \mathrm{Fe}-\mathrm{Fe}$ distance, the best fit to the data corresponded to $0.7 \mathrm{Fe}$ at $3.065 \AA$ (Figure 4.8a). In addition, a fit of $0.6 \mathrm{Fe}$ at $3.45 \AA$ was found, but the fit was much worse than the fit at the shorter distance (Figure 4.8a). Likewise, use of the FEHBPZOH Fe-Fe parameters in fits to the FESALMPO data resulted in a better fit at $3.44 \AA$ than at the correct distance of $3.06 \AA$ (Figure $4.8 \mathrm{~b}$ ). Using the parameters from FE3BIPHME, a tribridged center with an oxo-bridge and a $3.20 \AA$ Fe-Fe distance, in fits to FEHBPZOH, fits at $3.08 \AA$ and $3.43 \AA$ were obtained with the better fit corresponding to the shorter distance (Figure 4.8c). The overall worse fit function value obtained in the fits to FEHBPZOH with the FE3BIPHME parameters over the fits with the FESALMP0 parameters reflects the difference in the appearance of the second shell data (discussed below).

The backtransforms of the second shell data of the models from which $\mathrm{Fe}-\mathrm{Fe}$ parameters were obtained are presented in Figure 4.9. The windows used to isolate the second shell data are listed in Table 4.4. The shapes of the amplitude envelopes are quite 


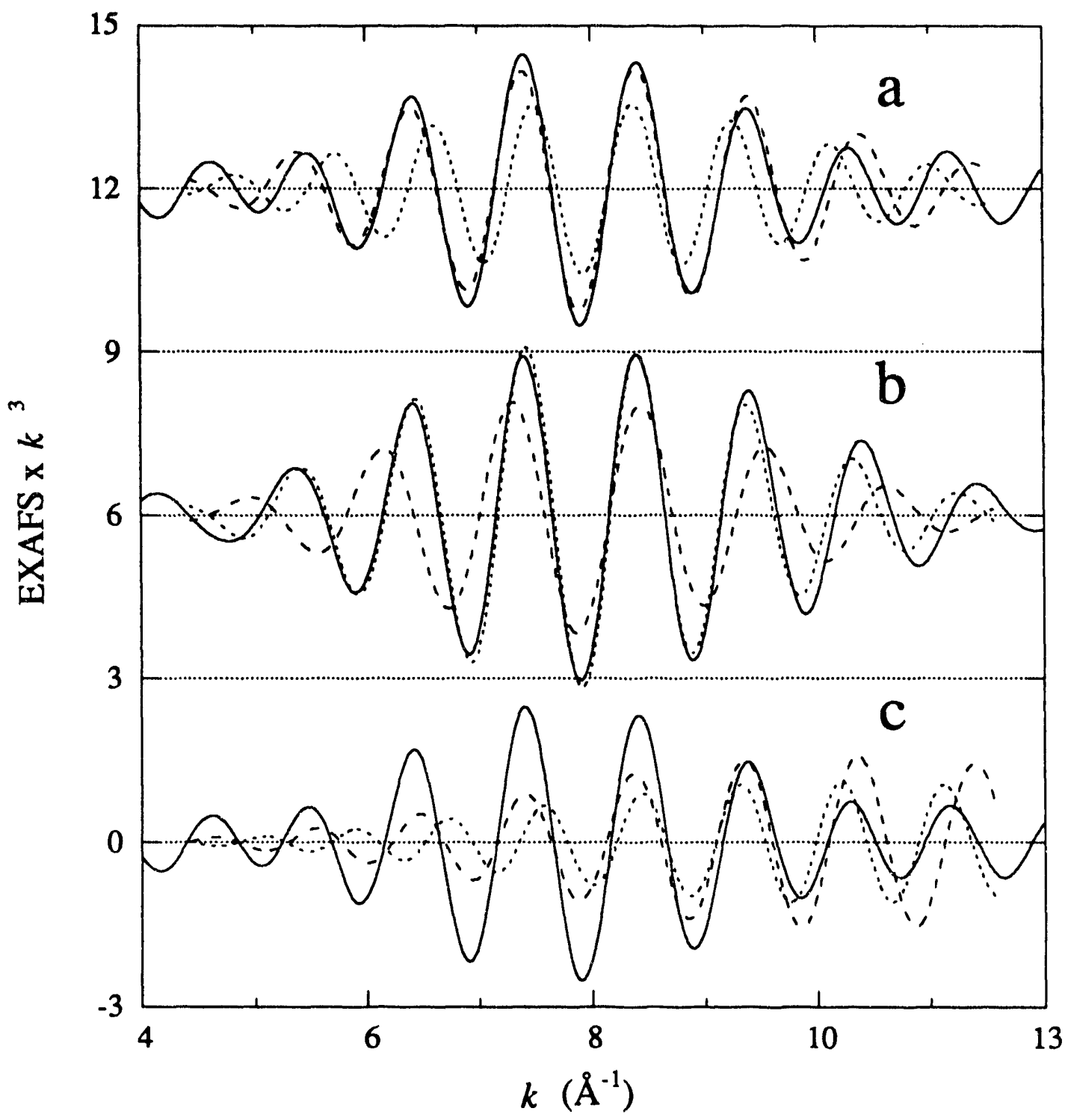

Figure 4.8. Comparison of the model-dependent fits to the second shell data (Table 4.4). The solid line is the data, the dashed line is the fit to the data with a short $\mathrm{Fe}$ contribution and the dotted line is the fit to the data with a long Fe contribution. (a) Fit to FEHBPZOH with FESALMPO Fe parameters. (b) Fit to FESALMPO with FEHBPZOH Fe parameters. (c) Fit to FEHBPZOH with FE3BIPHME Fe parameters. 


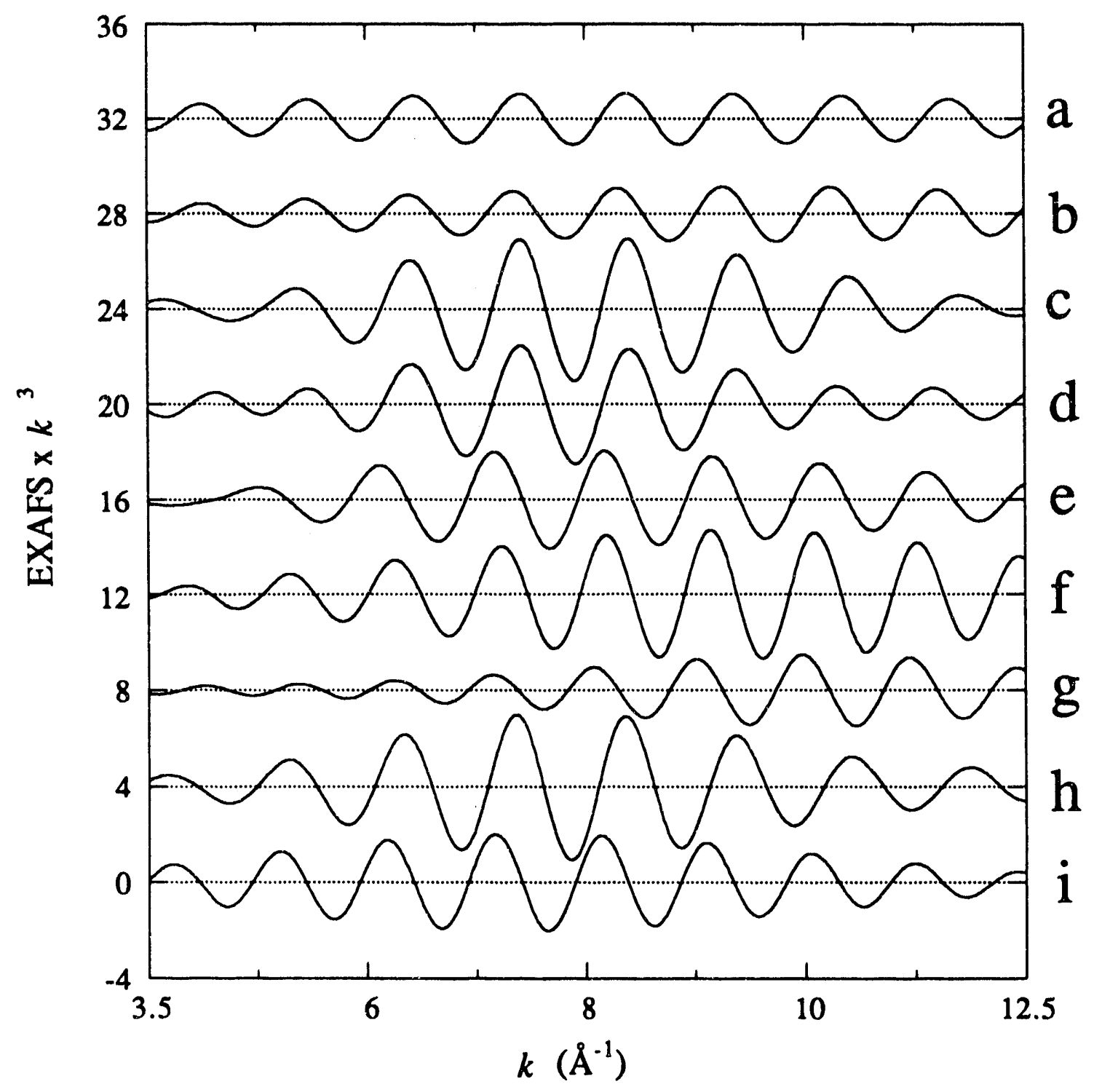

Figure 4.9. Comparison of the filtered second shell data of iron dimers. The windows used to isolate the data from the Fourier transforms are given in Table 4.4. (a) FECHEL, (b) FEDIPICE, (c) FESALMPO, (d) FEHBPZOH, (e) FEHBPZO, (f) FE2CO2, (g) FE3BIHME, (h) FESALMP1, (i) FESALMP2. 
different for each sample, however there does not seem to be any correspondence to the shape of the amplitude envelope with either Fe-Fe distance or bridging geometry. The data for FEHBPZO, FEHBPZOH, and FESALMPO (tribridge oxo, $3.14 \AA \mathrm{Fe}-\mathrm{Fe}$; tribridge hydroxyo, $3.43 \AA \mathrm{Fe}-\mathrm{Fe}$; dibridge alkoxo, $3.06 \AA \mathrm{Fe}-\mathrm{Fe}$, respectively) reach a maximum at around $8 \AA^{-1}$ and dampen out at both ends of the data range (Figure 4.9e, 4.9d and $4.9 \mathrm{c}$, respectively). For FE3BIPHME, another tribridge oxo compound like FEHBPZO, the data are damped in the low $k$ region and reach a maximum above $9 \AA^{-1}$ (Figure $4.9 \mathrm{~g}$ ), while for FECHEL, which has an Fe-Fe distance similar to that of FESALMP0, the data reach a maximum at $8 \AA^{-1}$, but do not dampen out over the data range (Figure 4.9a). In addition, the amplitude is not as great for the FECHEL data as for the FESALMP0 data (Figure 4.9a and 4.9c).

Both FE3BIPHME and FESALMP2 have Fe-Fe distances of $3.2 \AA$, however FE3BIPHME is a diferric tribridged compound with an oxo bridge, while FESALMP2 is a dibridged diferrous compound. The second shell data for FESALMP2 (Figure 4.9i) does not resemble the FE3BIPHME second shell data (Figure 4.9g). Using these parameters in fits to FE2CO2, a $3.185 \AA \mathrm{Fe}-\mathrm{Fe}$ distance was obtained with the FESALMP2 parameters, and a $3.165 \AA$ Fe-Fe distance with the FE3BIPHME parameters (Table 4.4). For both fits, the distance is within $0.01 \AA$ of the correct distance of $3.17 \AA$, however the fit to the data is better with the FE3BIPHME parameters than with the FESALMP2 parameters because the amplitude envelope of the second shell FE3BIPHME data and FE2CO2 data resemble each other (Figure 4.10). The only advantage of making a choice of a model compound based on the shape of the second shell data is a purely cosmetic one. Not surprisingly, better fits to any set of data correspond to the use of parameters from a model with a similarly shaped amplitude envelope, however the same two minima were found using any set of Fe parameters in fits to any data set. Additionally, the shape of the second shell amplitude envelope for FE3BIPHME and FE2CO2, suggests that the second shell data has more than a single contribution of atoms.

\subsubsection{Correlations with Second Shell Low-Z Atoms. Previous} investigations of the hydroxylase data revealed a strong correlation of the second shell low$\mathrm{Z}$ and $\mathrm{Fe}$ parameters which impacted both the distances and coordination numbers. To test whether this fitting approach can distinguish between an $\mathrm{Fe}-\mathrm{Fe}$ interaction and an $\mathrm{Fe}-\mathrm{C}$ interaction at approximately the same distance, as is the case for the structurally characterized $\mathrm{Fe}_{2}(\mathrm{OR})_{2}$ iron center, a second iell low- $\mathrm{Z}$ contribution was added to some of the iron fits in Table 4.4 for FECHEL and FESALMPO. Specifically, a carbon contribution was added to the fits of FECHEL with FESALMP0 parameters, and to fits of FESALMP0 with FECHEL parameters. These models have similar bridging 


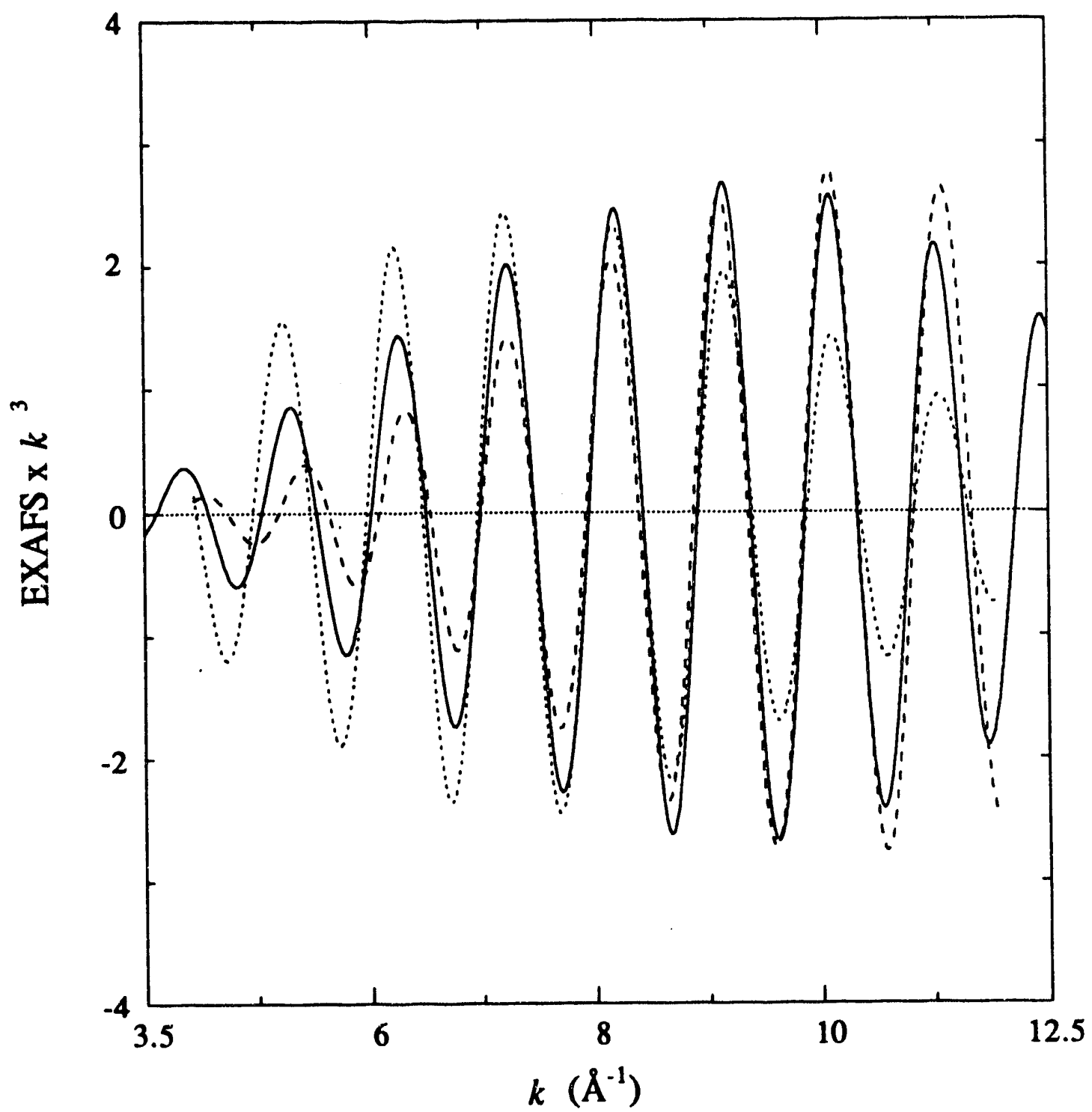

Figure 4.10. Comparison of fits to the second shell data of FE2CO2 (Table 4.4). The solid line is the data, the dashed line is the fit with FE3BIPHME Fe parameters and the dotted line is the fit with FESALMP2 Fe parameters. 
environments and Fe-Fe distances ( 3.08 and $3.06 \AA$, respectively), but the second shell peaks in the Fourier transforms have different intensities (Figure 4.9a and 4.9c). The difference in the amplitudes of the second shell contributions should provide insight into the interference between low- $Z$ and metal contributions which may contribute to the enhancement or reduction of the second shell peak in the Fourier transforms. In addition, a C contribution was added to fits to the second shell data of FEHBPZOH with FECHEL and FESALMPO parameters, and to fits to FECHEL and FESALMPO data with FEHBPZOH parameters to explore the effect the addition of another contribution has on the model dependent results of second shell fits. The results are presented inTable 4.5.

The second shell data for the three models were first fit with only a second shell $\mathrm{C}$ contribution from $\mathrm{Fe}(\mathrm{acac}) 3$. As noted before in fits to the hydroxylase data (Chapters 2 and 3), two minima were found, separated by $0.4 \AA$ at the same distances as the Fe-only fits to the data (Table 4.5, fits 4.5-1, 4.5-2, 4.5-9, 4.5-10, 4.5-17 and 4.5-18, compare with Table 4.4). The better fit to the data corresponded to the shorter Fe-C distance, similar to what was seen for the hydroxylase data. The distance of the short $\mathrm{C}$ fit for FECHEL is slightly long and it should be pointed out that there are no atoms $3.4 \AA$ away from the iron site according to the crystal structure. In general, the distances for the short C minima were slightly longer than the crystallographically determined distances, and the coordination numbers were inaccurate as well for FEHBPZOH and FESALMPO. For example, in the second shell of FEHBPZOH, there is a cluster of $6 \mathrm{~N}$ and $4 \mathrm{C}$ atoms from the pyrazole rings between 3.0 and $3.05 \AA$, however the fit to the data with the $C$ parameters resulted in a coordination number of $5.6 \mathrm{C}$ at $3.06 \AA$ (Table 4.5 , fit 4.5-17), For FESALMPO, the coordination numbers of the low- $Z$ contribution were high. According to the crystal structure, there are $6 \mathrm{C}$ at $3.04 \AA$ and $5 \mathrm{~N} / \mathrm{C}$ at $3.45 \AA$, but the fits determine $8.2 \mathrm{C}$ at $3.07 \AA$ or $7.5 \mathrm{C}$ at $3.43 \AA$, with the longer fit being considerably worse (Table 4.5, fit 4.5-9 and 4.5-10). To properly fit the second shell data, three contributions should be included, consisting of two $\mathrm{C}$ and one Fe wave, however, such a fit would be unreasonable to try due to the correlation of the $\mathrm{C}$ and $\mathrm{Fe}$ parameters and the ability of the $\mathrm{C}$ contribution to mimic the Fe contribution.

In general, the addition of $\mathrm{C}$ to the Fe contribution improved the quality of the fits, resulting in lower values of the fit function $F$ by as much as a factor of 2 . The bias of the best fit for the Fe-Fe distance of the model from which the parameters were derived was maintained in the $\mathrm{Fe}+\mathrm{C}$ fits to the data. The overall best fit using the FEHBPZOH parameters in fits to FECHEL and FESALMPO corresponded to the $3.4 \AA \mathrm{Fe}$ minimum (Table 4.5, fit 4.5-5 and 4.5-13). The Fe contribution remained about the same for both samples (relative to the Fe-only fits, see Table 4.4), but the coordination number of $\mathrm{C}$ 


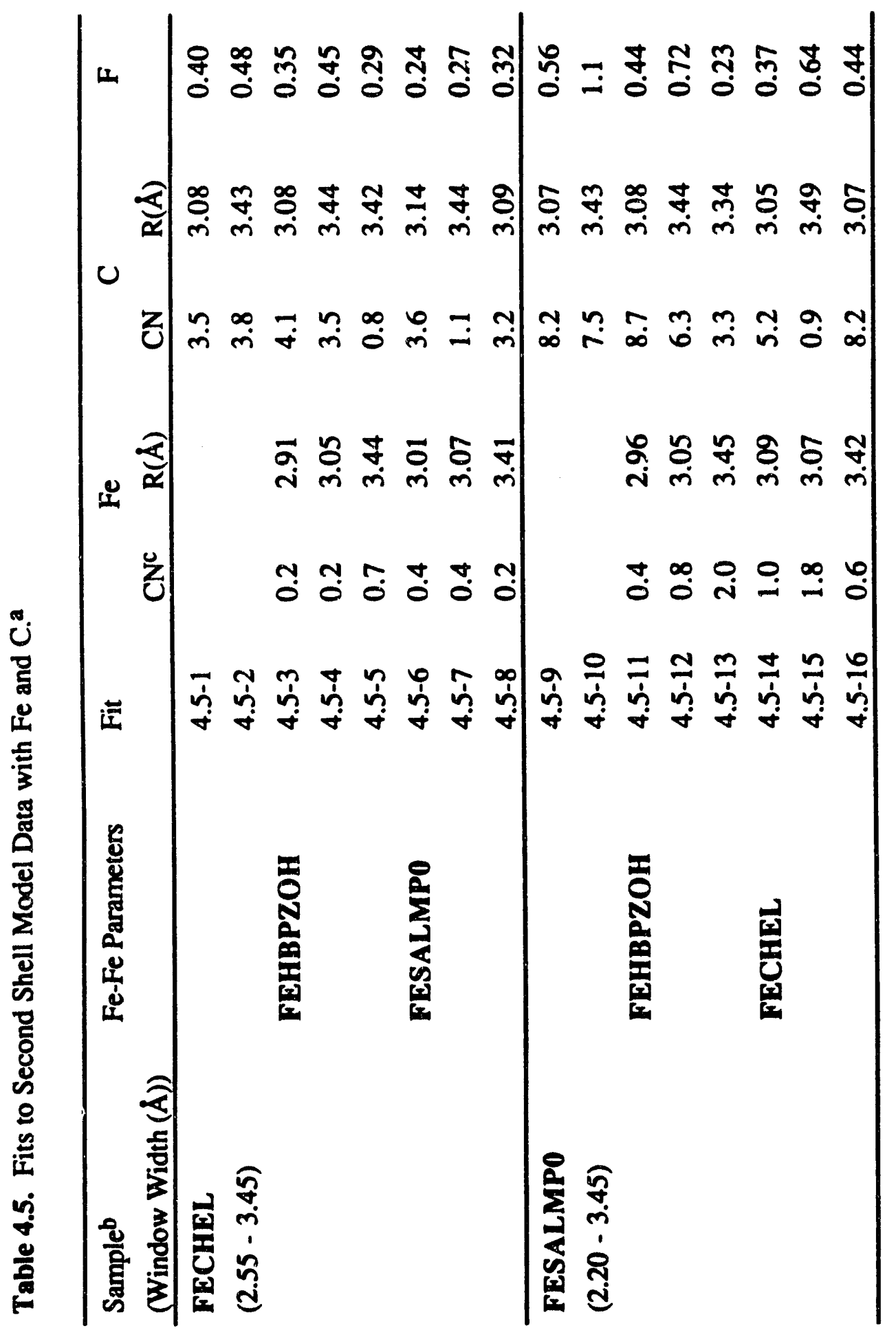




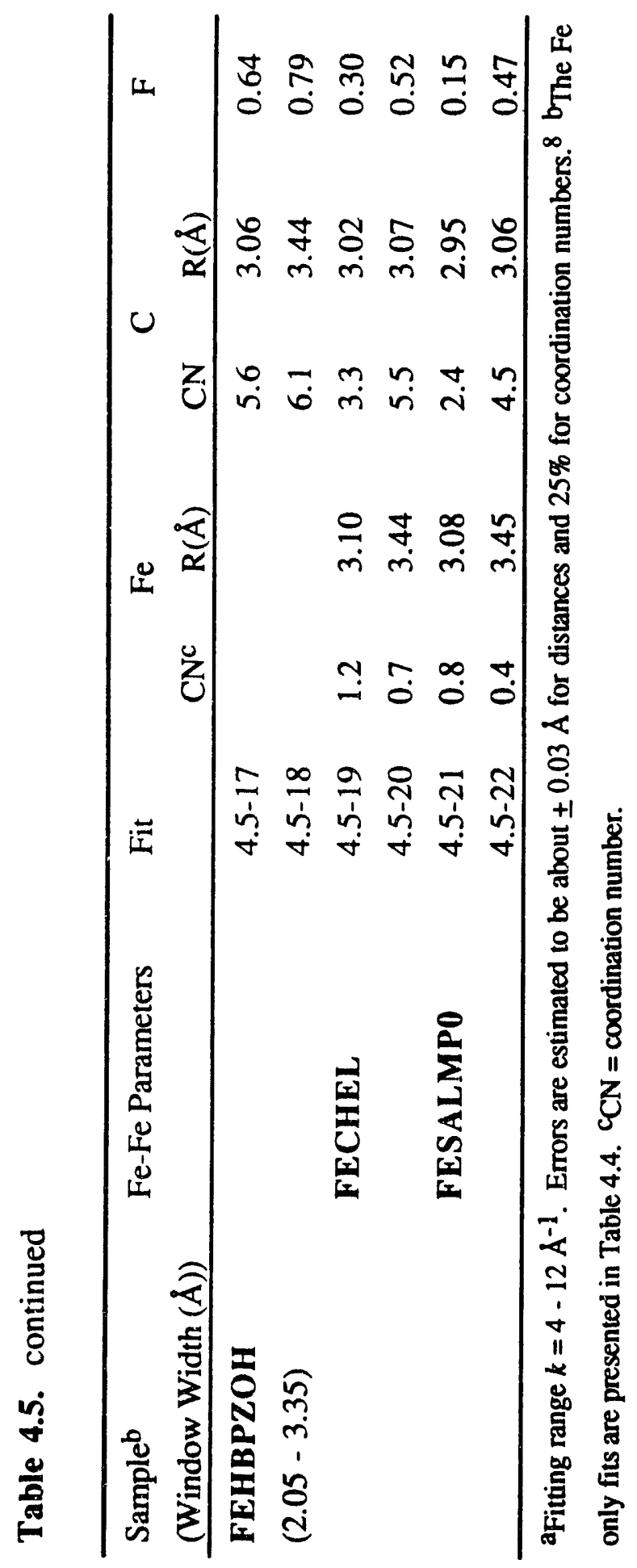


decreased from 3.8 to 0.8 (Table 4.5, fit 4.5-5) for FECHEL. Both the C coordination number and distance decreased for FESALMP0, from 7.5 C at $3.43 \AA$ (fit 4.5-10) to 3.3 $\mathrm{C}$ at $3.34 \AA$ (fit 4.5-13). Fits to the data consisting of a long $\mathrm{Fe}$ and a short $\mathrm{C}$ contribution resulted in negative coordination numbers. The addition of $\mathrm{C}$ to the short $\mathrm{Fe}$ distance resulted in a small decrease in the Fe distance and both the $\mathrm{Fe}$ and $\mathrm{C}$ coordination number (Table 4.5, fit 4.5-3 and 4.5-4 for FECHEL and 4.5-11 and 4.5-12 for FESALMP0). The $\mathrm{C}$ contribution for these fits was dominant over the $\mathrm{Fe}$ contribution.

Using the FECHEL and FESALMPO parameters in Fe + C fits to the FEHBPZOH data, the best fit corresponded to the shorter Fe-Fe distance (Table 4.5, fits 4.5-19 and 4.5-21). The $\mathrm{C}$ coordination number and distance decreased for the short $\mathrm{Fe} / \mathrm{short} \mathrm{C}$ fit to the data (fits 4.5-19 and 4.5-21) while the Fe distance increased by about $0.01-0.02 \AA$. Addition of $C$ to the long Fe distance resulted in a decrease in the coordination number from $0.6 \mathrm{Fe}$ to $0.4 \mathrm{Fe}$ for the fits with FESALMP0 (Table 4.5, fit 4.5-22), while the Fe contribution for the fits with FECHEL stayed the same (Table 4.5, fit 4.5-20). Fits to the FEHBPZOH data with $\mathrm{Fe}$ and a long $\mathrm{C}$ contribution resulted in negative coordination numbers.

The results of the $\mathrm{Fe}+\mathrm{C}$ fits for FECHEL and FESALMP0 using FESALMP0 and FECHEL parameters, respectively, also showed correlation effects. The coordination number of $\mathrm{Fe}$ stayed at 0.4 for the short Fe fits to FECHEL with FESALMPO parameters, but the $\mathrm{Fe}$ distance decreased to $3.01 \AA$ from $3.07 \AA$ with the addition of a short $\mathrm{C}$ contribution (Table 4.5, fit 4.5-6), while the $\mathrm{C}$ distance increased from $3.08 \AA$ to 3.14 $\AA$. In the short $\&$; short $C$ fit to FESALMPO with FECHEL parameters, the Fe coordination number decreased from 1.9 to 1.0 and the distance increased $0.02 \AA$ (Table 4.5, fit 4.5-14), while the $C$ coordination decreased from 8.2 to 5.2. This fit agrees with the distribution of atoms in the second shell of FESALMPO, but the Fe-Fe distance is slightly long. The fits to the data with the $3.4 \AA \mathrm{Fe}$ contribution and a short $\mathrm{C}$ contribution resulted in little or no difference in the $\mathrm{C}$ coordination number and distance, but the $\mathrm{Fe}$ coordination number and distance decreased slightly (Table 4.5, fits 4.5-8 and 4.5-16). The overall best fits to the data were for the short Fe/short $\mathrm{C}$ fit to the data. Fits to the data with a long $\mathrm{Fe}$ and long $\mathrm{C}$ contribution resulted in negative coordination numbers.

4.3.2.3. Investigation with Hydroxylase Data. The parameters from the various models were used in fits to the second shell data of the hydroxylase component of MMO from Methylococcus capsulatus (Bath) in its oxidized and semimet state (EXAFS5 and EXAFS2, respectively). The results using FEHBPZOH and FEHBPZO parameters have been briefly reported (Chapter 2 and ref. $2 a$ ) but are included here in detail. Two minima were found in every case, and the better fit to the data corresponded to 
the minimum with the Fe-Fe distance close to that of the model compound from which the parameters were obtained (Table 4.6). i addition, the coordination number for $\mathrm{Fe}$ was closer to 1 for the short Fe minimum than for the long Fe minimum except for the fits with FE3BIPHME and FEHBPZOH parameters, for which the coordination number was closer to 1 for both Fe minima. In general, the short Fe minimum was strongly preferred over the longer Fe minimum, except for the fits with FEHBPZOH, which are biased for the longer $\mathrm{Fe}$ minimum. I: is interesting to note that while the long $\mathrm{Fe}$ minimum varies from 3.38 to $3.46 \AA(0.08 \AA)$ for EXAFS5 and 3.35 to $3.43 \AA(0.08 \AA)$ for EXAFS2, the short Fe minima varies by only $0.05 \AA$. In addition, in every case, the two minima found were on the order of $3.0 \AA$ and $3.4 \AA$ even with the $3.2 \AA$ parameters from FE3BIPHME, whereas use of the FE3BIPHME fits to the diferric FEHBPZO and semimet FESALMP1 resulted in minima greater than 3.1 and $3.5 \AA$. This result strongly supports the previous conclusion that the second shell contributions to the data occur at 3.0 and $3.4 \AA$. A comparison of the fits to the data for EXAFS5 with the FESALMP0 parameters and FEHBPZOH parameters is presented in Figure 4.11.

\subsection{Discussion}

\subsubsection{Interpretation of First Shell Fits}

The EXAFS technique has long been recognized as being able to accurately determine the first shell coordination of an absorbing atom. The model compounds investigated in this paper were all octahedrally coordinated, but had different numbers of $\mathrm{N}$ and $\mathrm{O}$ atoms coordinating the $\mathrm{Fe}$ atoms as well as different numbers and types of bridges between the Fe atoms. For the monomeric compounds, which contain a shell of nearly equidistant $\mathrm{N}$ or $\mathrm{O}$ atoms, the data could be adequately described by a single contribution at the correct distance (Table 4.2, FE3HBPZ and FEACAC). Fits to the iron dimer data with a single $\mathrm{N}$ or $\mathrm{O}$ contribution resulted in low coordination numbers, although the distance was generally close to the average first shell distance from the crystal structure (excluding the oxo-bridge distance in the average distance). Instead, two contributions at different distances were required to adequately fit the data in the absence of an oxo-bridge, and three contributions if an oxo-bridge was present (the third contribution representing the oxo-bridge itself).

The fit results did not correspond to the crystallographic information based on the numbers of $\mathrm{N}$ or $\mathrm{O}$ atoms in the first shell, but instead seemed to reflect the distribution of atoms in the first shell based on the distance from the iron atom. The distortion of the iron 
Table 4.6. Fits to Second Shell Hydroxylase Data.

\begin{tabular}{l|ccc|ccc}
\hline $\begin{array}{l}\text { Sample: } \\
\text { Window }(\AA):\end{array}$ & \multicolumn{3}{|c|}{ EXAFS5 (diferric) } & \multicolumn{3}{c}{ EXAFS2 (semimet) } \\
\hline $\begin{array}{l}\text { Fe-Fe } \\
\text { parameters: }\end{array}$ & $\mathrm{Fe}$ & $\mathrm{R}(\AA)$ & $\mathrm{F}$ & $\mathrm{Fe}$ & $\mathrm{R}(\AA)$ & $\mathrm{F}$ \\
\hline FESALMP0 & 0.6 & 3.04 & 0.23 & 0.6 & 3.04 & 0.42 \\
$3.063 \AA$ & 0.3 & 3.41 & 0.86 & 0.3 & 3.40 & 0.99 \\
\hline FECHEL & 1.0 & 3.05 & 0.47 & 1.3 & 3.04 & 0.58 \\
$3.078 \AA$ & 0.2 & 3.38 & 0.92 & 0.3 & 3.35 & 1.04 \\
\hline FEHBPZO & 0.8 & 3.03 & 0.22 & 0.8 & 3.03 & 0.26 \\
$3.145 \AA$ & 0.5 & 3.41 & 0.81 & 0.5 & 3.39 & 0.95 \\
\hline FE3BIPHME & 1.0 & 3.05 & 0.52 & 1.2 & 3.04 & 0.43 \\
$3.200 \AA$ & 0.6 & 3.37 & 0.85 & 0.8 & 3.35 & 0.93 \\
\hline FESALMP2 & 0.8 & 3.08 & 0.25 & 0.8 & 3.07 & 0.5 \\
$3.202 \AA$ & 0.2 & 3.46 & 0.90 & 0.2 & 3.43 & 1.0 \\
\hline FEHBPZOH & 0.7 & 3.04 & 0.65 & 0.8 & 3.04 & 0.73 \\
$3.439 \AA$ & 1.1 & 3.42 & 0.40 & 1.1 & 3.41 & 0.61 \\
\hline
\end{tabular}




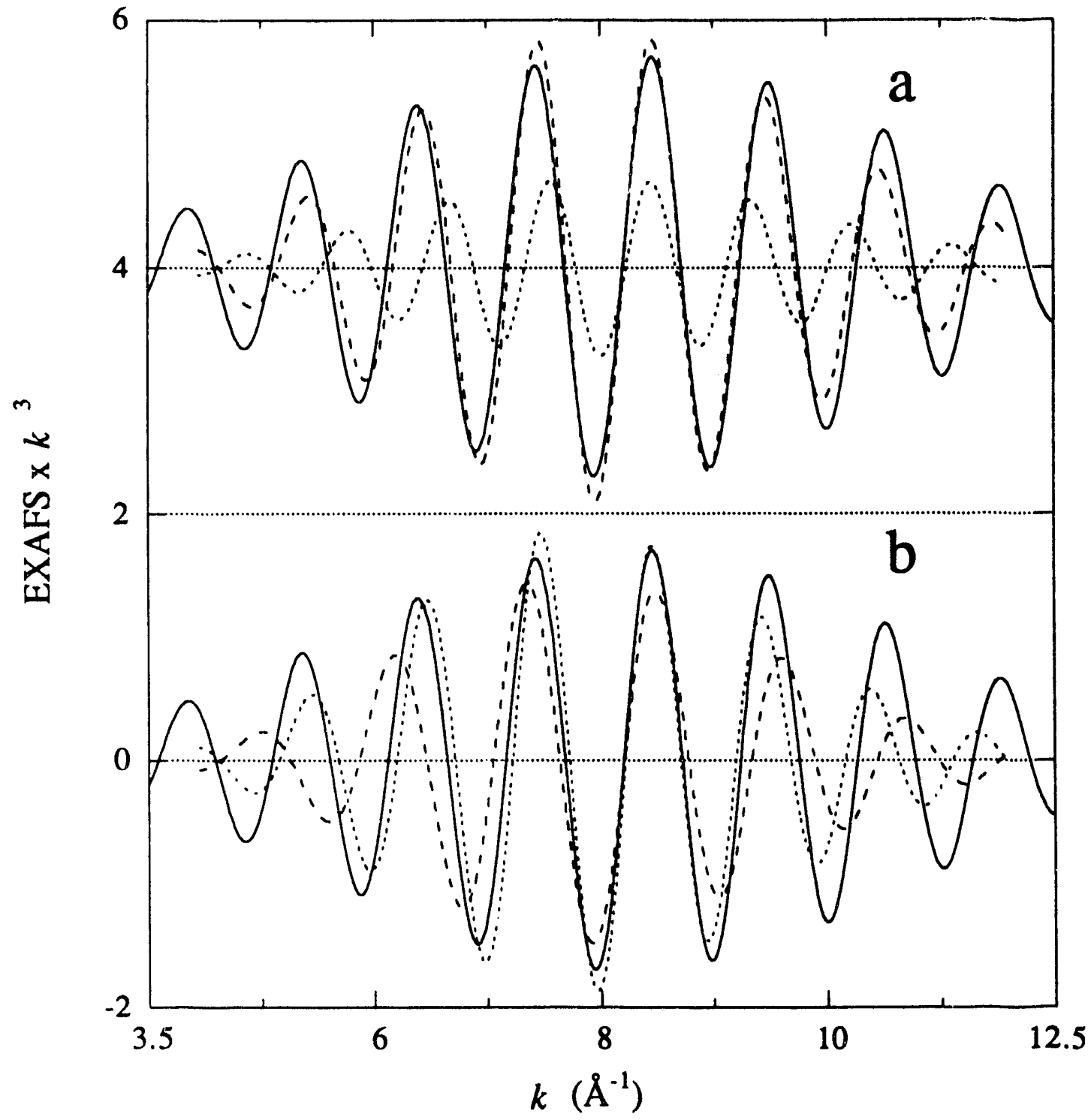

Figure 4.11. Fits to the oxidized hydroxylase data (Table 4.5). The solid line is the data, the dashed line is the fit to the data with a short Fe contribution and the dotted line is the fit to the data with a long Fe contribution. (a) Fit to EXAFS5 data with FESALMP0 Fe parameters. (b) Fits to EXAFS5 data with FEHBPZOH data. 
site from octahedral symmetry imposed by the bridging and ligand geometries, and the mixture of $\mathrm{N}$ and $\mathrm{O}$ ligation results in a range of first shell distances. In the oxo-bridged models, the bond lengths range from $\sim 1.8 \AA$ for the oxo-bridge $\mathrm{Fe}-\mathrm{O}$ distance to over 2.1 $\AA$ for the atom coordinated in a position trans to the oxo bridge (see Table 4.1). The elationship governing the resolution of distances as determined by the EXAFS technique as a function of the range of data available is given by $\Delta k \Delta R=\pi / 2$ where $\Delta k$ is the range of data $\left(\AA^{-1}\right)$ and $\Delta R$ is the corresponding limit on the resolution of the distances determined in the fitting technique $(\AA)$. For data to $k=12.5 \AA^{-1}$, the corresponding resolution of bond lengths is $0.126 \AA$. This explains the sensitivity of the EXAFS chnique to the presence of the $1.8 \AA \mathrm{Fe}-\mathrm{O}$ distance of the oxo-bridge, which is $\sim 0.2 \AA$ shorter than the average bond length of the rest of the atoms. In the model compounds investigated in this study, the range of distances is close to this resolution limit, and although two contributions are required to fit the data, the correct individual contributions cannot be determined due to the limited range of data used for these investigations. In the first shell of FESALMP0, the distribution of atoms can be divided into two sets, corresponding to 3 atoms at an average distance of $1.95 \AA$ and 3 atoms at an average distance of $2.11 \AA$ which differ by $0.16 \AA$ (Table 4.1). The fits to the data (Table 4.2, fits 4.2-21 and 4.2-22) correspond quite well to this interpretation of the crystal structure data. In FECHEL, the range of distances in the first coordination sphere is $0.126 \AA$ with a single $\mathrm{O}$ atom at $1.97 \AA$ and 5 atoms at an average distance of $2.05 \AA$. The fit to the data reflects this distance distribution (Table 4.2, fit 4.2-10) although $\mathrm{N}$ corresponded to the short distance contribution. The first shell fit results thus seem to reflect the distribution of atoms based on their average distance from the iron atom, rather than the contribution of a specific atom type at a given distance.

It was only with the information provided by the crystal structure that the individual contributions based on the fit results could be interpreted for some of the models discussed here. For an unknown structure, such an interpretation would not be possible. The inability of this approach to accurately determine the numbers of $\mathrm{N}$ and $\mathrm{O}$ atoms reflects in part the similar backscattering strength of these atoms. The approach taken in the curvefitting technique employed here assumes that the Debye-Waller factors for the experimentally derived first shell $\mathrm{Fe}-\mathrm{N}$ and $\mathrm{Fe}-\mathrm{O}$ parameters are transferable to the model compounds, however this is not necessarily true. Further investigation of the first shell fits using fixed, correct coordination numbers and allowing the distance and Debye-Waller factor to float would provide additional insight into the ability of the curve-fitting technique to resolve individual contributions to the first shell data. In addition, more than one fit minimum was found, depending on the relative initial distances of the $\mathrm{N}$ and $\mathrm{O}$ 
contributions. The shorter distance was sometimes conserved in the two minima, but the longer distance sometines changed by more tha. $0.1 \AA$ which is greater than the expected distance error for the EXAFS technique $( \pm 0.03 \AA) .8$ This suggests that the individual contributions should not be interpreted as giving accurate information about the distance distribution of atoms in the first coordination sphere for the range of data investigated. Data to a higher $k$ range would provide more accurate and reliable distance information.

The most reliable way to use the results of fits to the first shell data is to consider the coordination-weighted average distance information. For all of the model compounds, the coordination-weighted average of both fit minima falls within the expected error for both the coordination number and distance for the EXAFS technique. ${ }^{8}$ This has been the proceedure used to interpret the results for the hydroxylase data (Chapters 2 and 3). It has generally been assumed that the average distance corresponds to the relative numbers of $\mathrm{N}$ and $\mathrm{O}$ in the first coordination sphere: 12 in addition, the average first shell distance reflects the presence of an oxo-bridge in the center. The presence of an oxo-bridge tends to distort the iron site and results in longer first shell distances, and therefore a longer average distance, than non-oxo-bridged models. This is seen clearly by comparing the average first shell distances for FEHBPZO and FEHBPZOH excluding the shortest bridging distance. FEHBPZO and FEHBPZOH both are coordinated by $3 \mathrm{O}$ and $3 \mathrm{~N}$ atoms and the primary difference in their structure is the presence of the oxo-bridge in FEHBPZO. The average first shell distance in FEHBPZO (excluding the oxo-bridge) is $2.12 \AA$, whereas in FEHBPZOH, the average distance is $2.06 \AA$ (excluding the hydroxo-bridge). If the bridging distance is included, the average for FEHBPZO decreases to $2.06 \AA$ and to $2.04 \AA$ for FEHBPZOH. The average distances in the first coordination sphere for all atoms for a variety of multiply-bridged ferric iron dimers with $\mathrm{N}$ and $\mathrm{O}$ coordination are listed in Table 4.7 and presented graphically in Figure 4.12. In general, the average first shell distance does tend to increase as the number of $\mathrm{N}$ atoms relative to $\mathrm{O}$ atoms increases, and for the same $\mathrm{N}$ and $\mathrm{O}$ ligation, the average first shell distance will be longer if an oxo bridge is present.

\subsubsection{Model-Dependent Results of Second Shell Fits}

4.4.2.1. Model Compounds. It has previously been pointed out that care must be taken when choosing a model compound to mimic the second shell interaction of an unknown compound, and one of the criterion suggested was that the irons in the model have similar bridging oxygens to what is suspected in the unknown. ${ }^{13}$ This does not seem to be the case for the model compounds discussed above. If it were true that the types of 


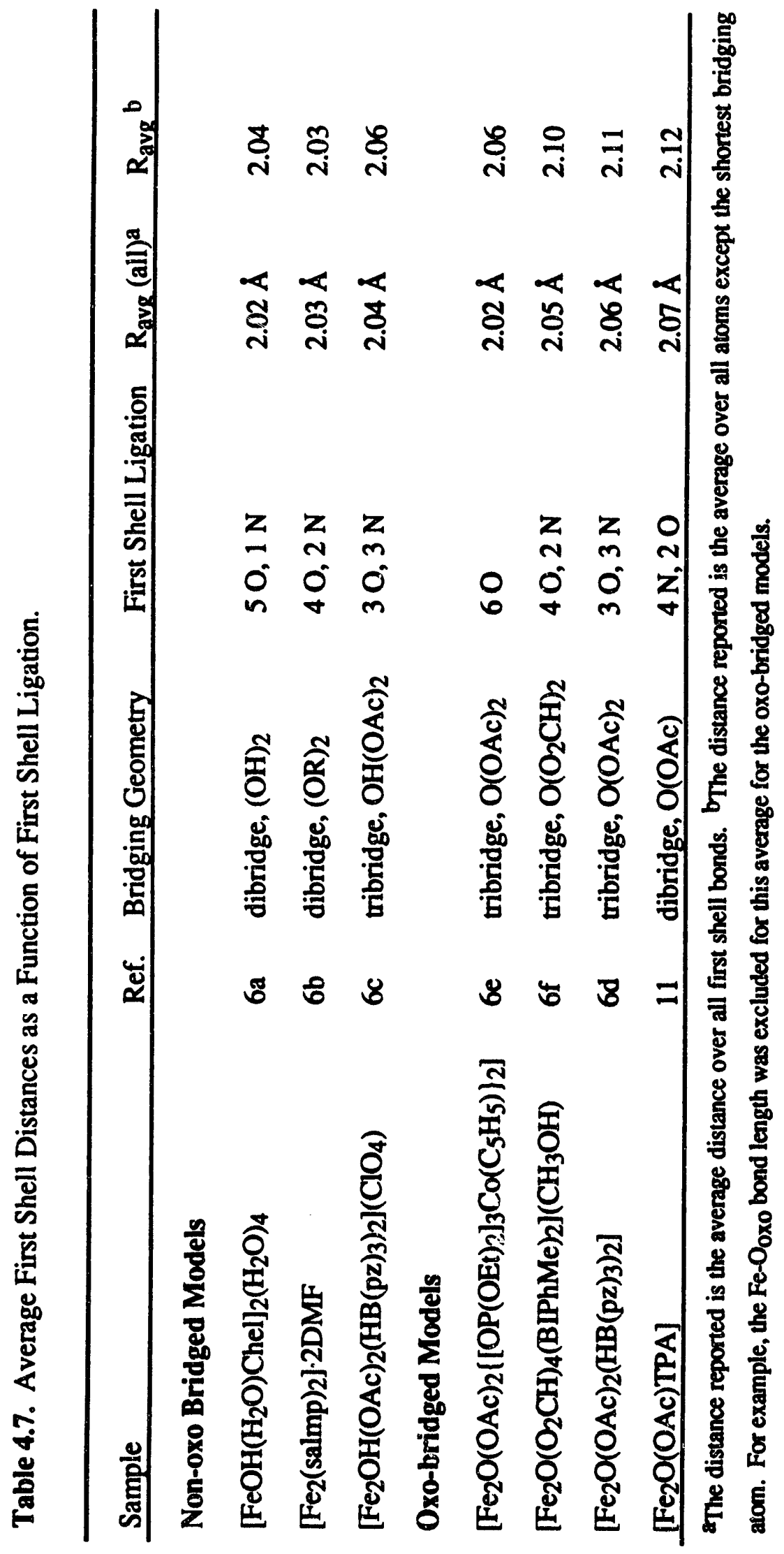




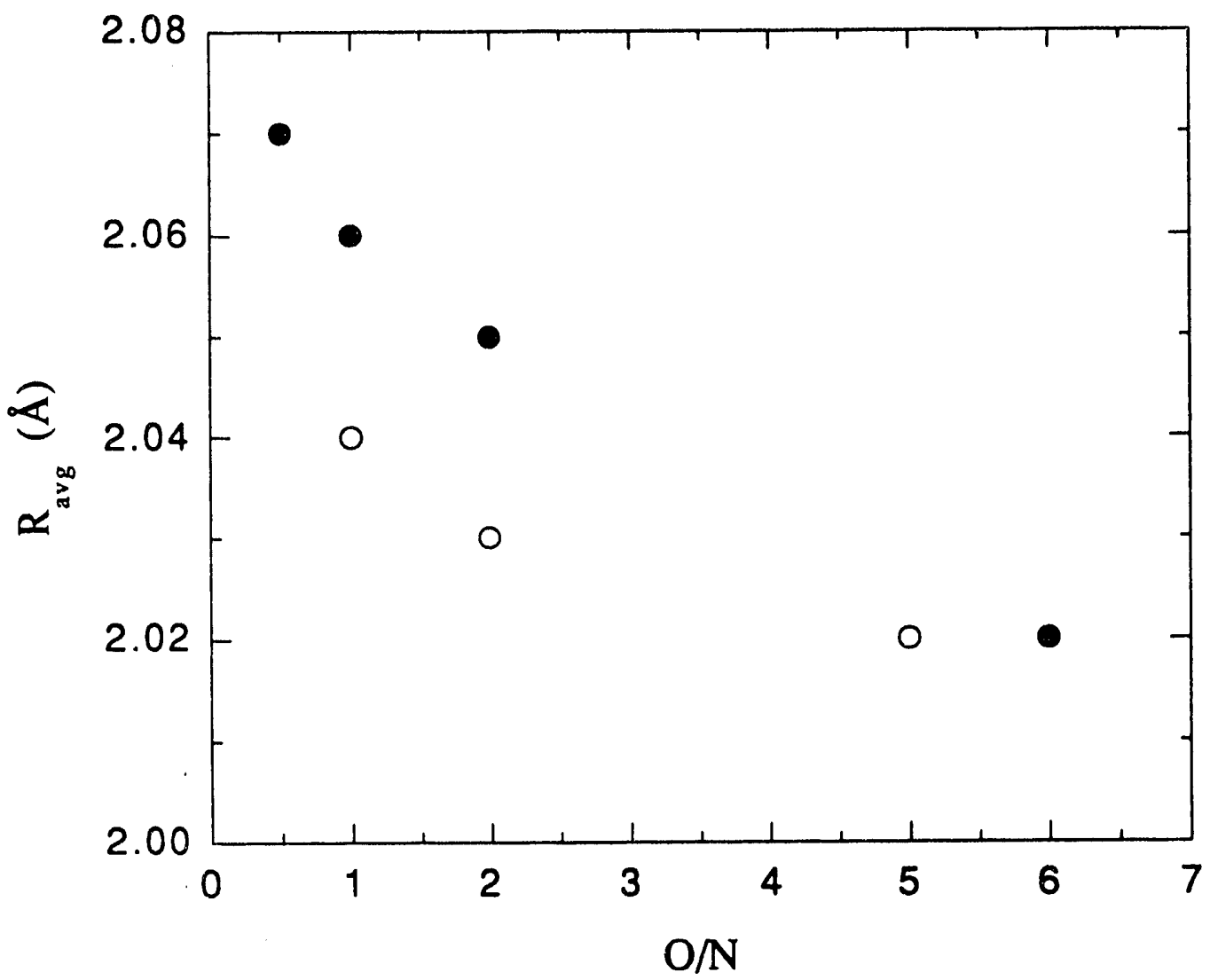

Figure 4.12. Comparison of the average first shell distance with the numbers of $\mathrm{O}$ to $\mathrm{N}$ atoms coordinating the iron atoms (Table 4.7). The solid circles are the oxo-bridged model data and the open circles are the non-oxo-bridged model data. 
bridges determined the choice of an appropriate model, then the tribridged models with an oxo-bridge should be poor choices for the dihydroxo and dialkoxo models, however the fits do not support this hypothesis (Table 4.4). The parameters from both FEHBPZO and FE3BIPHME adequately fit the dialkoxo model FESALMP0 and the dihydroxo models FECHEL and FEDIPIC at the correct distance (the coordination numbers are low for fits to FECHEL and FEDIPIC with the FEHBPZO parameters). Likewise, the FESALMPO and FECHEL parameters adequately fit the tribridged FEHBPZO and FE3BIPHME second shell data.

None of the models tested gave satisfactory fits to the FEHBPZOH second shell data at the correct $3.4 \AA$ Fe-Fe distance. The correct distance was founci in every case, but the better fit corresponded to the shorter distance minimum. All of the models with the exception of FEHBPZOH have Fe-Fe distances of $3.2 \AA$ or less. Using the FEHBPZOH Fe-Fe parameters in fits to the other models, the better fit occured in every case at a distance on the order of $3.4 \AA$ or longer. These results clearly demonstrate that fits to the second shell data are strongly dependent on the compound chosen to model the Fe-Fe interaction. In particular, the bias favors the Fe-Fe distance of the model rather than the number or types of bridges in the diiron center. ${ }^{14}$

One would also expect, if similar bridging geometry determined an appropriate model choice, that the dihydroxo-bridged models FECHEL and FEDIPIC would be excellent models for dialkoxo-bridged FESALMP0, all of which have $\mathrm{Fe}-\mathrm{Fe}$ distances between 3.1 and $3.0 \AA$ and $\mathrm{Fe}-\mathrm{O}_{\mathrm{br}}-\mathrm{Fe}$ angles around $100^{\circ}$. Although the distance information obtained is reasonable in cross-fits between these models, the coordination numbers are off by a factor of two. Inspection of the Fourier transforms shows that the second shell peak in FEDIPIC and FECHEL is much less intense relative to the first shell peak compared to the Fourier transform of FESALMPO. This would lead to different amplitude functions and therefore incorrect coordination numbers for the cross fits. Similar differences in amplitude functions have been noted in fits to iron dimers and has been attributed to multiple scattering contributions to the data arising from the focusing effect of the intervening oxo-bridge, however this effect was determined to be a factor only if the Fe-O-Fe bridging angle were greater than $150^{\circ} .15$ In addition to the focusing effect for models with angles greated than $150^{\circ}$, the various multiple scattering pathways would not be resolveable in the Fourier transform of the data which would also lead to a broadening of the second shell contribution. For FESALMP0, and the other models investigated in this paper, multiple scattering contributions involving the $\mathrm{Fe}_{2} \mathrm{O}$ core should not interfere with the second shell data. 
The various contributions from the multiple scattering pathways should be resolved in the data of FESALMP0, however the second shell peak (Figure 4.5c) is rather broad. The presence of rigid groups such as imidazole also contribute strongly to outer shell data resulting in strong peaks in Fourier transforms. ${ }^{16}$ It is interesting to note that a recent multiple scattering analysis of $\mathrm{Fe}(\mathrm{acac})_{3}{ }^{17}$ has revealed that there is a very strong contribution to the second shell data from a multiple scattering pathway involving the $\mathrm{Fe}-\mathrm{O}$ $\mathrm{C}$ unit (Fe-O-C angle $129.3^{\circ}$ ). This demonstrates that $\mathrm{Fe}(\mathrm{acac})_{3}$ is not a good choice as a model for the single-scattering Fe-C parameters. This kind of geometry may be similar to ligation of metal sites in proteins by carboxylate groups and so may similarly contribute to the data. It would be interesting to investigate the angular dependence of multiple scattering contributions from various chelating groups. Clearly, an understanding of the relationship between the geometries of the ligands coordinating diiron centers and the multiple scattering pathways which contribute significantly to the second sheil data of dinuclear non-heme iron models and protein systems needs to be developed. The determination of the multiple pathways which contribute to the second shell data of model compounds investigated for this paper would provide a great deal of insight into the active site structures of the hydroxylase of $\mathrm{MMO}$, uteroferrin, ribonucleotide reductase and hemerythrin.

The distances of the Fe-Fe contributions in FECHEL ( $3.08 \AA)$ FEHBPZOH (3.44 $\AA$ ) are well resolved from the contributions of second shell low- $Z$ atoms $(2.9 \AA$ for FECHEL and between 3.0 and $3.05 \AA$ for FEHBPZOH). Fits to the second shell data should therefore lead to well defined minima corresponding to the $\mathrm{Fe}$ and the low- $\mathrm{Z}$ atom contribution (modeled as $\mathrm{C}$ ), however this was not the case. As seen before in fits to the hydroxylase data, the second shell $\mathrm{Fe}$ and $\mathrm{C}$ parameters were strongly correlated, with the $\mathrm{Fe}$ and $\mathrm{C}$ distances and coordination numbers changing for the $\mathrm{Fe}+\mathrm{C}$ fits from the values obtained in the Fe-only and $\mathrm{C}$-only fits to the data. The model bias held in the $\mathrm{Fe}+\mathrm{C}$ fits to the data as well, with the best fit corresponding to the iron distance closest to the Fe distance of the model used for Fe parameters. This certainly impacted the results of the fits to FEHBPZOH, as none of the other models are adequate models of this compound. However, a reasonable fit was obtained for FESALMPO using FFCHEL Fe parameters and a short $\mathrm{C}$ contribution. The difference between the Fe and $\mathrm{C}$ shells in FESALMPO is less than $0.1 \AA$. Given the general trends seen in the $\mathrm{Fe}+\mathrm{C}$ fits to the second shell data, it is not entirely clear if this result reflects the quality of the FECHEL parameters in fits of this kind, or if it is simply coincidental.

Moreover, the best fit to the data corresponded to the minimum in which both the $\mathrm{Fe}-\mathrm{C}$ and $\mathrm{Fe}-\mathrm{Fe}$ distance were closest to the $\mathrm{Fe}-\mathrm{Fe}$ distance of the parameter model. In general, the second shell low $-Z$ contribution did not model the total low $-Z$ atom 
contribution to the data at a given distance, nor did the $\mathrm{Fe}+\mathrm{C}$ fits agree with the crystal structure data (with the exception noted above). The use, and the interpretation of results from the use, of $\mathrm{Fe}$ (acac) 3 (and therefore other metal(acac) 3 compounds) as a model of the single scattering Fe-C second shell contribution should be reexamined in light of the multiple scattering analysis discussed above.

4.4.2.2. The Hydroxylse Active Site. In the earlier studies of the hydroxylase active site, the $3.4 \AA$ minimum was assigned as the Fe contribution and the $3.0 \AA$ mimimum was assigned as the low- $Z$ atom contribution due to the presence of clusters of low- $Z$ atoms at $\sim 3.0 \AA$ in model compounds and a recognition that low- $Z$ atoms located at more than $3.0 \AA$ would not contribute strongly to the EXAFS due to the increased disorder of three-bond Fe-C distances (see Chapter 2). The fits to the second shell data with the various model compounds done for this work consistently resulted in a $3.0 \AA$ minimum and a $3.4 \AA$ minimum, supporting the interpretation that there are contributions to the second shell data at those distances, however it is not as easy to dismiss the $3.0 \AA$ minimum as the iron contribution in light of the strong preference for that distance over the longer distance in fits using the $\mathrm{Fe}_{2}(\mathrm{OR})_{2}$ model parameters.

The lack of evidence of an oxo-bridge in the diferric iron center was used as the basis for the choice of the hydroxo-bridged diferric model FEHBPZOH as the model of the Fe-Fe interaction for the hydroxylase data over the oxo-bridged model FEHBPZO. The choice of model compound based on the lack of the presence of an oxo-bridge in the MMO center is certainly a valid one, but it is not clear how to choose between two models with different bridging environments and Fe-Fe distances, but each of which lacks an oxobridge. Such a choice is represented by FEHBPZOH (tribridge, Fe-Fe $3.44 \AA$ ) and FESALMP0 (dibridge, Fe-Fe $3.06 \AA$ ). ${ }^{18}$ Based on the results of fits to the hydroxylase data with FESALMPO parameters, one would conclude that the Fe-Fe distance in the oxidized hydroxylase is $3.04 \AA$, but with FEHBPZOH parameters, the data suggest that the correct Fe-Fe distance is $3.42 \AA$.

If the diiron center in the hydroxylase were a dibridged center similar to that in the FESALMP model compounds, then it would be expected that in the diferrous form of the hydroxylase, the Fe-Fe distance would be on the order of $3.2 \AA$. For such a compact dinuclear iron site, it would be reasonable to expect to see the Fe-Fe interaction in the Fourier transform, similar to that which is seen in the diferrous FESALMP2 data (Figure 4.5e). For the diferrous protein data, however, no Fe-Fe interaction is seen in the Fourier transform (see Figure 2.2 in Chapter 2) suggesting that the Fe-Fe distance in the diferrous form of the hydroxylase is longer than $3.2 \AA$. This in turn suggests that the Fe-Fe distance in the diferric form is longer than $3.0 \AA$, supporting the assignment of the $3.4 \AA$ minimum 
as the Fe contribution. Another possibility, however, is that the Fe distance in the hydroxylase is on the order of $3.0 \AA$ and that upon reduction to the diferrous state, a major rearrangement in the bridging ligands occurs, resulting in an $\mathrm{Fe}-\mathrm{Fe}$ distance in the diferrous state that is longer than what can be detected with EXAFS. Such a rearrangement may involve a shift in the coordinating mode of carboxylate groups ligating the iron center. ${ }^{19}$

Although the only known diferric model with a $3.4 \AA$ Fe-Fe distance is the tribridged FEHBPZOH, the possibility that the hydroxylase is dibridged cannot be eliminated. While the dibridged FESALMP models exhibit different magnetic behaviour from the MMO hydroxylase (being ferromagnetic, ${ }^{6 \mathrm{~b}}$ whereas $\mathrm{MMO}$ is antiferromagnetic ${ }^{2}$ ) which eliminates them as models for the hydroxylase, the other dibridged models (FECHEL, FEDIPIC, FEPIC) are antiferromagnetically coupled. The only dibridged model with a $3.4 \AA \mathrm{Fe}-\mathrm{Fe}$ distance is $\left[\mathrm{Fe}_{2} \mathrm{O}(\mathrm{TPA})_{2} \mathrm{PHT}\right]^{11}$ which has a $(\mu$-oxo $)(\mu$ carboxylato) core (see footnote 14). Since it was possible to synthesize the hydroxobridged derivative of the ( $\mu$-oxo)bis $\left(\mu\right.$-carboxylato) model [ $\left.\mathrm{Fe}_{2} \mathrm{O}(\mathrm{OAc})_{2}\left(\mathrm{HB}(\mathrm{pz})_{3}\right)_{2}\right],{ }^{6} \mathrm{c}$ a model with a ( $\mu$-hydroxo)( $\mu$-carboxylato) core may also be possible to synthesize which would have a longer $\mathrm{Fe}-\mathrm{Fe}$ distance and should exhibit magnetic behaviour similar to that of the hydroxylase active site.

4.4.2.3. Survey of Other Second Shell Analysis Methods. A variety of other techniques have been used to determine the Fe-Fe distances in dinuclear iron centers. The ratio method has been used to determine the $\mathrm{Fe}-\mathrm{Fe}$ distances in various forms of hemerythrin 13,20 in which the amplitude and phase parameters of structurally characterized models are compared the the amplitude and phase parameters of the unknown. The earlier results ${ }^{20}$ determined with this method have been corrected ${ }^{13}$ based on an evaluation of the model compounds employed, and a set of criteria have been described by the authors as the basis for determining the app.opriate model to use whe determination of the correct $\mathrm{Fe}-\mathrm{Fe}$ distance. ${ }^{13}$ In the earlier work, the long Fe-Fe diwa es obtained for oxy-and methemerythrin ( 3.59 and $3.49 \AA$ respectively) were obtaine? the use of a $3.3 \AA \mathrm{Fe}-\mathrm{Fe}$ model. ${ }^{20 \mathrm{a}}$ Using data between 5 and $12 \AA^{-1}$ to eliminate $\mathrm{l}: \mathrm{W}-\mathrm{Z}$ atom interference, the early results were reevaluted using the tribridged oxo-bridged ditmer FEHBPZO which is known based by comparison with the crystal structure to the very similar to the active site of hemerythrin. ${ }^{21}$ A distance of 3.24 and and for oxyhemerythrin, in goud agreement with the crystal structure (the Fis model is $3.14 \AA$, see Table 4.1).

The discrepancy between the earlier and later results was attributed to the dissimilarity of the bridging groups in the $3.3 \AA \mathrm{Fe}$ model to those in hemerythrin, as well as to the inclusion of low $k$ data in the earlier analysis, which would have a strong 
contributions from second shell low-Z scatterers. ${ }^{22}$ Although the the use of the $3.14 \AA$ model could be justified based on the known structure of the protein, there was an indication that the results of this analysis may also be model-dependent. The authors repeated the fits using the $3.30 \AA \mathrm{Fe}-\mathrm{Fe}$ model and th $\mathrm{n}$ new protocol and stated that the use of a $3.30 \AA \mathrm{Fe}-\mathrm{Fe}$ model in fits to the protein data resulted in consistently longer $\mathrm{Fe}-\mathrm{Fe}$ distances by about $0.2 \AA$ than the results obtained by the use of the $3.14 \AA \mathrm{Fe}$-Fe model. 13

A second method employed in the determination of second shell $\mathrm{Fe}-\mathrm{Fe}$ distances is the FABM (fine adjustment based on models) method which uses empirically adjusted theoretical parameters. 12,23 In this approach, fits to a model compound are done with the correct distance information and theoretical parameters, while adjusting both an amplitude reduction factor $(\mathrm{A})$ and shell-specific energy shift $(\Delta \mathrm{E})$ during the fitting proceedure $12,23 \mathrm{a}$ or just an amplitude factor. $23 \mathrm{~b}, 23 \mathrm{c}$ These parameters are then used in non-linear leastsquares fits to the data using the theoretical parameters and allowing the distance and coordination number (or Debye-Waller factor) to vary. Different values of $A$ and $\triangle E$ were obtained in references 12 and 23a, so there is some dependence of these parameters on the model employed. ${ }^{24}$ The dependence of the values of $A$ and $\Delta E$ on the model compound, and the dependence of the results using the different $\mathrm{A}$ and $\Delta \mathrm{E}$ sets has not been explored.

Reliable Fe-Fe distances were obtained in tests on model compounds, ${ }^{12,23 a}$ but in some cases more than one Fe minima was found, similar to results reported here (see Table II in reference 12). It would be informative to explore the range of applicability and any limitations of this approach.

\subsection{Conclusions}

These studies have demonstrated the utility of the EXAFS technique in determining the average first shell coordination of iron atoms in non-heme dinuclear iron centers. The distances obtained with these fits for a variety of model compounds were accurate to within $0.03 \AA$ and the total coordination number to within $10 \%$. The sensitivity of this technique to the presence or absence of a short $\mu$-oxo bridge was once again demonstrated. The exact numbers of $\mathrm{N}$ and $\mathrm{O}$ atoms ligated to the iron center could not be directly determined. The average first shell distance as determined by EXAFS analysis was found to correlate with both the relative numbers of $\mathrm{N}$ and $\mathrm{O}$ atoms and the presence of an oxo-bridge in the iron center (for mixed $\mathrm{N}$ and $\mathrm{O}$ ligation).

The non-linear least-squares curve-fitting method using Fe-Fe parameters derived from model compounds has proven to be extremely model-dependent. The other techniques used for the analysis of second shell data also seem to be susceptible to model 
dependence, although this has not yet been throughly tested. Unlike the conclusion reached from the ratio method of determining Fe-Fe distances, ${ }^{13}$ these studies show that the bias seen reflects the Fe-Fe distance of the model compound from which the parameters were obtained, and is independent of the number or nature of bridges in the diiron center except as those factors determine the Fe-Fe distance. A strong correlation between the second shell $\mathrm{Fe}$ and $\mathrm{C}$ parameters was also observed for the di- and tribridged models tested, impacting both the coordination numbers and the distances of the $\mathrm{Fe}$ and $\mathrm{C}$ contributions. These results illustrate that care must be taken in the analysis and interpretation of the second shell data for dinuclear iron systems. Similar caution should be used in the interpretation of fits for other dimetallic systems as well.

The method employed in fits to the second shell dinuclear iron data assumes that the strongest contribution to the second shell EXAFS will be the single-scattering event between the two iron atoms, however multiple scattering contributions could be contributing as well. Multiple scattering pathways involving the $\mathrm{Fe}_{2} \mathrm{O}$ core should not impact the second shell data of the models investigated here. 15 Rigid coordinating groups such as imidazole also have an effect on second shell data. 16 It has recently been discovered that for monomeric $\mathrm{Fe}$ (acac)3, multiple scattering along the Fe-O-C path contibutes significantly to the second shell amplitude, suggesting that other pathways may be important. The pathways which contribute strongly to the second shell EXAFS need to be determined before the second shell data for dinuclear iron systems can be completely understood. An understanding of the multiple scattering contributions and the developement of a protocol for properly accounting for both the multiple and single scattering interactions will be an important step in the reliable application of the EXAFS technique to second shell data in dinuclear metalloprotein systems. Finally, with the emergence of the theoretical fitting codes FEFF 25 and GNXAS, 26 a systematic investigation of theoretical fitting approaches to the complicated systems discussed in this paper can be done and compared to the results obtained frcm the use of empirically derived amplitude and phase parameters presented here.

\subsection{Acknowledgements}

The data were collected at the Stanford Synchrotron Radiation Laboratory and the National Synchrotron Light Source, Brookhaven National Laboratory, which are supported by the Department of Energy, Office of Basic Energy Sciences, Division of Chemical Sciences and Division of Materials Sciences. SSRL is also supported by the National Institutes of Health, Biomedical Resource Technology Program, Division of Research 
Resources (RR-01209) and the Department of Energy, Office of Health and Environmental Research. Grant support was provided by the National Science Foundation (CHE 9121576 to $\mathrm{KOH}$ ). The author wishes to thank Profs. Stephen Lippard and Richard Holm for providing model compounds. 


\subsection{References and Notes}

1. Cramer, S. P. X-Ray Absorption: Principles, Applications, Techniques of EXAFS, SEXAFS and XANES; John Wiley and Sons, Inc.: New York, 1988; pp 257-320.

2. (a) DeWitt, J. G.; Bentsen, J. G.; Rosenzweig, A. C.; Hedman, B.; Green, J.; Pilkington, S.; Papaefthymiou, G. C.; Dalton, H. J.; Hodgson, K. O.; Lippard, S. J. J. Am. Chem. Soc. 1991, 113, 9219-9235. (b) Ericson, A.; Hedman, B.; Hodgson, K. O.; Green, J.; Dalton, H.; Bentsen, J. G.; Beer, R. H.; Lippard, S. J. J. Am. Chem. Soc. 1988, 110, 2330-2332.

3. Hedman, B.; Co, M. S.; Armstrong, W. H.; Hodgson, K. O.; Lippard, S. J. Inorg. Chem. 1986, 25, 3708-3711.

4. Vaarkamp, M.; Dring, I.; Oldman, R. J.; Stern, E. A.; Koningsberger, D. C. Proc. XAFS VII, Kobe, Japan; August 1992, in press.

5. Scott, R. A.; Eidsness, M. K. Comments Inorg. Chem. 1988, 7, 235-267.

6. (a) Thich, J. A.; Ou, C. C.; Powers, D.; Vasilot, B.; Mastropaolo, D.; Potenza, J. A.; Schugar, H. J. J. Am. Chem. Soc. 1976, 98, 1425-1433. (b) Snyder, B. S.; Patterson, G. S.; Abrahamson, A. J.; Holm, R. H. J. Am. Chem. Soc. 1989, 111, 52145223. (c) Tolman, W. B.; Bino, A.; Lippard, S. J. J. Am. Chem. Soc. 1989, 111, 8522-8523. (d) Armstrong, W. H.; Lippard, S. J. J. Am. Chem. Soc. 1984, 106, 46324633. (e) Armstrong, W. H.; Spool, A.; Papaefthymiou, G. C.; Frankel, R. B.; Lippard, S. J. J. Am. Chem. Soc. 1984, 106, 3653-3667. (f) Feng, X.; Bott, S. G.; Lippard, S. J. J. Am. Chem. Soc. 1989, 111, 8046-8047. (g) Armstrong, W. H.; Lippard, S. J. unpublished results (h) Iball, J.; Morgan, C. H. Acta Cryst. 1976, 23, 239-244. (i) Roof, Jr., R. B. Acta Cryst. 1956, 9, 781-786.

7. Scott, R. A.; Hahn, J. E.; Doniach, S. ; Freeman, H. C.; Hodgson, K. O. J. Am. Chem. Soc. 1982, 104, 5364-5369.

8. (a) Cramer, S. P.; Hodgson, K. O.; Stiefel, E. I.; Newton, W. E. J. Am. Chem. Soc. 1978, 100, 2748-2761. (b) Cramer, S. P.; Hodgson, K. O. Prog. Inorg. Chem. 1979, 15, 1-39. (c) Scott, R. A. Methods Enzymol. 1985, 117, 414-459.

9. Johans on, L. Chem. Scr. 1976, 9, 30-35. The crystal structure of the perchlorate salt has not been determined, but the $[\mathrm{Fe} \text { (phenanthroline)3 }]^{2-}$ complex structure can be assumed to be identical with that of the corresponding iodide salt (Johansson, L.; Molund, M.; Oskarsson, A. Inorg. Chim. Acta 1978, 31, 117-123).

10. Kurtz, D. M. Jr. Chem. Rev. 1990, 90, 585-606. 
11. (a) Norman, R. E.; Yan, S.; Que, L. Jr.; Backes, G.; Ling, J.; Sanders-Loehr, J.; Zhang, J. H.; O'Connor, C. J. J. Am. Chem. Soc. 1990, 112, 1554-1562. (b) Norman, R. E.; Holz, R. C.; M'enage, S.; O'Connor, C. J.; Zhang, J. H.; Que, L. Jr. Inorg. Chem. 1990, 29, 4629-4637.

12. Scarrow, R. C.; Maroney, M. J.; Palmer, S. M.; Que, L. Jr.; Row, A. L.; Salowe, S. P.; Stubbe, J. J. Am. Chem. Soc. 1987, 109, 7857-7864.

13. Zhang, K.; Stern, E. A.; Ellis, F.; Sanders-Loehr, J.; Shiemke, A. Biochemistry 1988, 27, 7470-7479.

14. It should be noted that there is a series of dibridged ( $\mu$-oxo)( $\mu$-carboxylato) model compounds which have Fe-Fe distances from 3.1 to $3.4 \AA$ (ref. 11). These compounds were synthesized and EXAFS data were collected on them twice, however incorrect structural information resulted from fits to the data, so these models were not included in this study. Although the information to date strongly points to a bias based on distance rather than bridging atom type, the inclusion of the model with the $3.4 \AA \mathrm{Fe}-\mathrm{Fe}$ distance would certainly complete the investigation.

15. Co, M. S.; Hendrickson, W. A.; Hodgson, K. O.; Doniach, S. J. Am. Chem. Soc. 1983, 105, 1144-1150.

16. (a) Co, M. S.; Scott, R. A.; Hodgson, K. O. J. Am. Chem. Soc. 1981, 103, 986-988. (b) Hasnain, S. S., Ed. Synchrotron Radiation and Biophysics; Ellis Horwood Ltd.; Chichester, 1990; Chapters 3 and 4.

17. Westre, T. E.; Di Cicco, A.; Filipponi, A.; Natoli, C. R.; Solomon, E. I.; Hedman, B.; Hodgson, K. O. to be submitted

18. FECHEL also meets the criterion of a lack of an oxo-bridge, but the difference in the relative intensites of the second shell peaks in FECHEL and EXAFS5 and EXAFS2 (see Figure 4.5) suggests that FECHEL will not model the amplitude correctly, which is supported by the fits to the hydroxylase data.

19. Rardin, R. L.; Tolman, W. B.; Lippard, S. J. New J. Chem. 1991, 15, 417-430.

20. (a) Elam, W. T.; Stern, E. A.; McCallum, J. D.; Sanders-Loehr, J. J. Am. Chem. Soc. 1982, 104, 6369-6373. (b) Elam, W. T.; Stern, E. A.; McCallum, J. D.; SandersLoehr, J. J. Am.Chem. Soc. 1983, 105, 1919-1923.

21. Stenkamp, R. E.; Sieker, L. C.; Jensen, L. H.; McCallum, J. D.; Sanders-Loehr, J. Proc. Natl. Acad. Sci. USA 1985, 82, 713-716.

22. The use of high $k$ data may not eliminate the second shell low- $Z$ atom contribution as the ability of groups of low- $Z$ atoms to mimic a second shell metal contribution has been noted by is (ref. 2) and others (ref. 5). 
23. (a) True, A. E.; Scarrow, R. C.; Holz, R. C.; Que, L. Jr. Inorg. Biochem. 1991, 43, 545 and personal communication. (b) Kauzlarich, S. M.; Teo, B. K.; Zirino, T.; Burman, S.; Davis, J. C.; Averill, B. A. Inorg. Chem. 1986, 25, 2781-2785. (c) Prince, R. C.; George, G. N.; Savas, J. C.; Cramer, S. P.; Patel, R. N. Biochim. Biophys. Acta 1988, 952, 220-229.

24. The values of $A$ and $\triangle E$ were obtained by fitting the difference of the second shell data for FEHBPZOH and FEHBPZO in ref. 12. The model employed in determining the values of $A$ and $\triangle E$ in ref. 23a was not discussed.

25. Mustre de Leon, J.; Rehr, J. J.; Zabinsky, S. I.; Albers, R. C. Phys. Rev. B $1991,44,4146$.

26. Filipponi, A.; Di Cicco, A.; Tyson, T. A.; Natoli, C. R. Solid State Comm. 1991, $78,265$. 


\section{Chapter 5}

Fe K-Edge X-ray Absorption Edge Spectra of Iron Models Relevant to Dinuclear Non-Heme Iron Enzyme Systems 


\subsection{Introduction}

The appearance of transition metal edge spectra are characteristic of the coordination environment and oxidation state of the metal ion and have been both explained by, and used to demonstrate the utility of, crystal field ${ }^{1}$ and molecular orbital theory. ${ }^{2}$ Transition metal edges have been the subject of investigation for a number of years, however few definitive assignments of the observed transitions have been made. ${ }^{3}$ Systematic investigations of structurally characterized inorganic models by X-ray absorption near edge spectroscopy (XANES) have resulted in correlations of the appearance of the edge spectra with symmetry, oxidation state and ligand environment. These studies have been used to provide insight into the structural environment of the active sites of metalloprotein systems, including the multi-copper oxidase laccase ${ }^{4}$, the mangenese-containing oxygen evolving complex from photosystem II, 5 and the nickel enzymes carbon monoxide dehydrogenase 6 and hydrogenase. ${ }^{7}$ We propose to utilize X-ray absorption spectroscopy to similarly characterize Fe XANES spectra of dinuclear non-heme iron model compounds of relevance to hemerythrin, ribonucleotide reductase, purple acid phosphatase and methane monooxygenase. 8

Fe K-edge X-ray absorption spectra have been collected on a number of dinuclear non-heme iron models. All of the compounds studied have high-spin octahedrally coordinated iron atoms with $\mathrm{N}$ and/or $\mathrm{O}$ ligation. The model compounds investigated cortained tri-, di-, and mono-bridged iron centers with various combinations of oxo, hydroxo, alkoxo and carboxylato bridging groups. Correlations of the appearance of the edge spectra with the numbers and types of bridges, changes in the ligation sphere, and changes in the oxidation state of the diiron center will be discussed. Comparisons will be made with the edge spectra of the hydroxylase component of soluble methane monooxygenase from Methylococcus capsulatus (Bath). ${ }^{9}$

\subsubsection{Interpretation of Transition Metal XANES Spectra}

The dipole-allowed transition for an X-ray absorption $\mathrm{K}$-edge is from an initial is state to a final np state $(\Delta l=1)$ and will correspond to the most intense region in the edge spectrum. Transitions are commonly seen below the $4 p$ threshold for transition metal spectra. The weak pre-edge feature has been attributed to a formally dipole-forbidden $1 \mathrm{~s} \rightarrow-3 \mathrm{~d}$ transition made allowed by $4 \mathrm{p}$ mixing into the $3 \mathrm{~d}$ states as a result of noncentrosymmetric symmetry of the metal site and vibronic coupling. 10,11 This assignment has been confirmed by the absence of this feature in $\mathrm{K}$-edge spectra of $\mathrm{d}^{10}$ transition 
metals, ${ }^{12}$ and by single-crystal polarized studies for other metals. ${ }^{13}$ The intensity of this feature increases as the metal site is distorted from octahedral to tetrahedral symmetry (greater $4 p$ mixing due to symmetry reduction), and can therefore be used as a probe of the coordnation number and/or the site symmety of the absorbing atom. 14

Shoulders have been observed on the rising edge of some transition metal spectra which have been explained as the forbidden $1 \mathrm{~s} \rightarrow-4 \mathrm{~s}$ transition or as the allowed $1 \mathrm{~s} \rightarrow 4 \mathrm{p}$ transition. Single-crystal oriented edge studies have been instrumental in determining the assignment of this feature. Transitions to the spherically symmetric 4 s orbital would not exhibit any polarization dependence unlike transitions to the directional $4 \mathrm{p}$ orbitals. A single-crystal polarized study of a $\mathrm{Cu}(\mathrm{I})$ compound whose edge spectrum exhibited a shoulder on the rising edge showed the feature to exhibit polarization-dependent intensity, 15 and permitted assignment of the transition as occuring to a $4 \mathrm{p}_{z}$ final state orbital. The same conclusion was reached for a similar feature in the edge spectrum of a distorted $\mathrm{O}_{\mathrm{h}} \mathrm{Fe}$ (III) compound using oriented single-crystal measurements. ${ }^{16}$ The sharpness and intensity of this feature has been noted to be quite strong and well-resolved in square planar complexes of $\mathrm{Fe},{ }^{14} \mathrm{Cu}{ }^{15}$ and $\mathrm{Ni}, 6,7$ which reflects the splitting of the $4 \mathrm{p}$ levels as expected for $\mathrm{D}_{4 \mathrm{~h}}$ symmetry metal sites. ${ }^{1}$

The generally accepted explanation of the shoulder is that it is a shake-down feature associated with the $1 \mathrm{~s} \rightarrow-4 \mathrm{p}$ transition resulting from the transfer of an electron from a ligand valence orbital into the metal $3 \mathrm{~d}$ manifold made allowed by final state relaxation. 17,18 This ligand-to-metal-charge transfer (LMCT) shakedown feature would occur to lower energy than the metal $1 \mathrm{~s} \rightarrow 4 \mathrm{p}$ transition by an amount equal to the difference in energy between the ligand $2 p$ and metal $3 d$ levels. The energy of the valence level of a more covalent ligand is higher than that of a less covalent ligand, resulting in a greater energy difference between the ligand orbital and the relaxed metal $3 \mathrm{~d}$ state. The position of the LMCT shake-down feature would therefore be expected to move to lower energy as the covalency of the ligands increased.

\subsection{Experimental}

The model compounds investigated for this study are summarized in Table 5.1.19 The compounds were synthesized according to the references listed in the table with the following exceptions. All of the tribridged samples were provided by Prof. Stephen Lippard of the Massachusetts Institute of Technology. The SALMP dibridged compounds in all three oxidation states were provided by Prof. Richard Holm of Harvard University. 


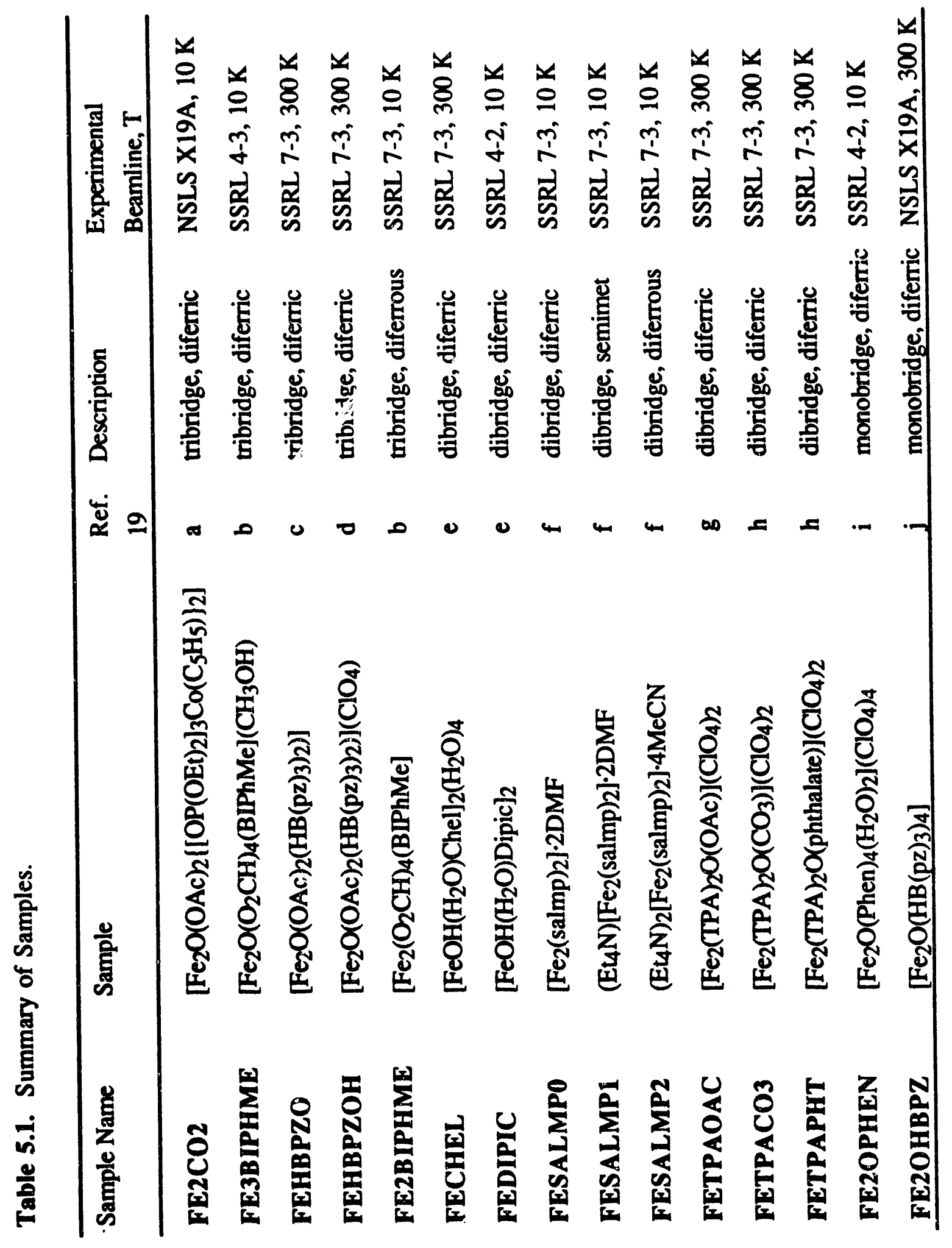


Details of the preparation and data collection for the hydroxylase samples (EXAFS5, EXAFS7, and EXAFS6) have been presented in reference 9 and in Chapters 2 and 3.

Samples for XAS experiments were diluted with BN powder, finely ground with a mortar and pestle, and pressed into a $1 \mathrm{~mm}$ thick $\mathrm{Al}$ sample spacer windowed with Mylar tape (total sample weight, $\sim 55 \mathrm{mg}$ ). The samples were run in transmission mode at the Stanford Synchrotron Radiation Laboratory (SSRL) on unfocused 8-pole wiggler beamlines 4-3 or 7-3 (18 kG), or on unfocused bending magnet beamline X19A at the National Synchrotron Light Source (NSLS) at Brookhaven National Laboratory (see Table 5.1). Ring operating conditions were $3.0 \mathrm{GeV}$ and $40-90 \mathrm{~mA}$ at SSRL, and $2.5 \mathrm{GeV}$ and 90-200 mA at NSLS. The height of the beam was defined to be $1 \mathrm{~mm}$ using slits in front of the monochromator, and a $\mathrm{Si}(220)$ monochromator was used to maximize energy resolution for the edge spectra ( $\sim 2 \mathrm{eV}$ under these conditions for $\mathrm{Fe}$ ). The edge spectra of the samples run at NSLS have worse resolution due to software limitations on the step size in the edge region. The monochromator was detuned $50 \%$ at $7475 \mathrm{eV}$ (the end of the Fe edge scan) to reject higher harmonics in the incident beam. The incident and transmitted beam intensity was monitored by using $\mathrm{N}_{2}$-filled ionization chambers of the standard design. The diferrous and semimet model compounds were run at $10 \mathrm{~K}$ by using a continuous flow LHe cryostat (Oxford Instruments model CF1208). The diferric models were either run at ambient temperature, or at $10 \mathrm{~K}$ if the cryostat was already in use.

Energy calibration for each scan was perfomed by using an internal foil calibration method $^{20}$, setting the energy of the first inflection point of the iron foil as $7111.2 \mathrm{eV}$. Although the signal-to-noise ratio for the model compounds is high enough to obtain satisfactory data with one scan, more than one scan was measured to insure reproducibility of the data. In general, 2 or 3 scans were averaged together for each sample. The data were background-subtracted by fitting a polynomial to the pre-edge region which was extracted through the post-edge region and subtracted. The edge jump was normalized to unity using a polynomial through the post-edge region. Care was taken to insure that the data for each sample were normalized to each other for direct comparison. This process involved adjusting the background subtraction to insure that the slope of the data from sample to sample was the same, and adjusting the normalization to insure that the edge jump from sample to sample was the same.

\subsection{Results and Discussion}

Positions of the features in the edge spectra were determined by measuring the position of the half-width at half-height of the second deriviative of the spectra, and are 
presented in Table 5.2. In many cases, features were suggested by the second derivative that were not clearly resolved in the edge spectrum themselves. The positions of these features are enclosed in parentheses.

\subsubsection{Description of the Edge Spectra}

5.3.1.1. Models Containing a $\mu$-oxo Bridge. In Figure 5.1 are the edge spectra of representative tri-, di-, and mono-bridged models which incorporate a $\mu$-oxo bridge. The spectra represent different ligation of the iron site, from 6 O (FE2CO2) to 5 $\mathrm{N}$ and $1 \mathrm{O}$ (FE2OHBPZ). The most intense pre-edge transition (region $\mathrm{A}$ in the spectra), occurs between 7114.1 - $7114.8 \mathrm{eV}$ (Figure 5.2, top curve). A lower energy transition can be seen in the spectra of the di-and mono-bridged compounds (see FE2OPHEN) and occurs between $7112.2-7112.6 \mathrm{eV}$. The energy splitting between the two features is on the order of $2 \mathrm{eV}$. The resolution of the low-energy shoulder correlates with the increase in the number of $\mathrm{N}$ atoms in the first coordination sphere. It is best resolved in the complexes which have $2 \mathrm{O}$ and $4 \mathrm{~N}$ (FETPAOAC and FE2OPHEN, Figure 5.2; FETPACO3 and FETPAPHT, data not shown). In FEHBPZO (3 O/3 N, Figure 5.2), FE3BIPHME (4 O/2 N, see Figure 5.5) or FE2CO2 (6 O, Figure 5.2), the lower energy transition is not as clearly resolved and leads to the asymmetric apperance of the pre-edge feature. This trend also coincidentally correlates with the decrease in the number of bridges from three to two. It cannot be determined if the ligation or the number of bridges is a more important factor in determining the appearance of the pre-edge feature based on the data available.

A shoulder on the rising edge of the spectra (Feature B) is clearly resolved in the models containing more than $3 \mathrm{~N}$ atoms in the first coordination sphere (Figure 5.1, bottom curve), however the second derivatives suggest the presence of this feature in the other models as well. In the tribridged models, the shoulder occurs between 7125.3 and 7125.9 $\mathrm{eV}$ and in the di- and mono-bridged models, the feature appears between 7123.5 and $7125.6 \mathrm{cV}$. The intensity of this feature is lowest in the $5 \mathrm{~N} / 10$ model FE2OHBPZ and highest in the $4 \mathrm{~N} / 20$ model FE2OPHEN. As seen with the resolution of the shoulder in the pre-edge feature, the appearance of the shoulder correlates both with increasing $\mathbf{N}$ ligation and decreasing number of bridges.

The most intense transition in the edge spectra occurs between 7128.1 and 7130.0 $\mathrm{eV}$ (Feature $\mathrm{C}$ ). This feature is more narrow in the di- and mono-bridged compounds than in the tri-bridged compounds. The position of the feature does not seem to correlate with either the number of bridges or the first shell ligation. The energy difference between the postion of the rising edge shoulder and the primary transition ranges from $3.7 \mathrm{eV}$ for 


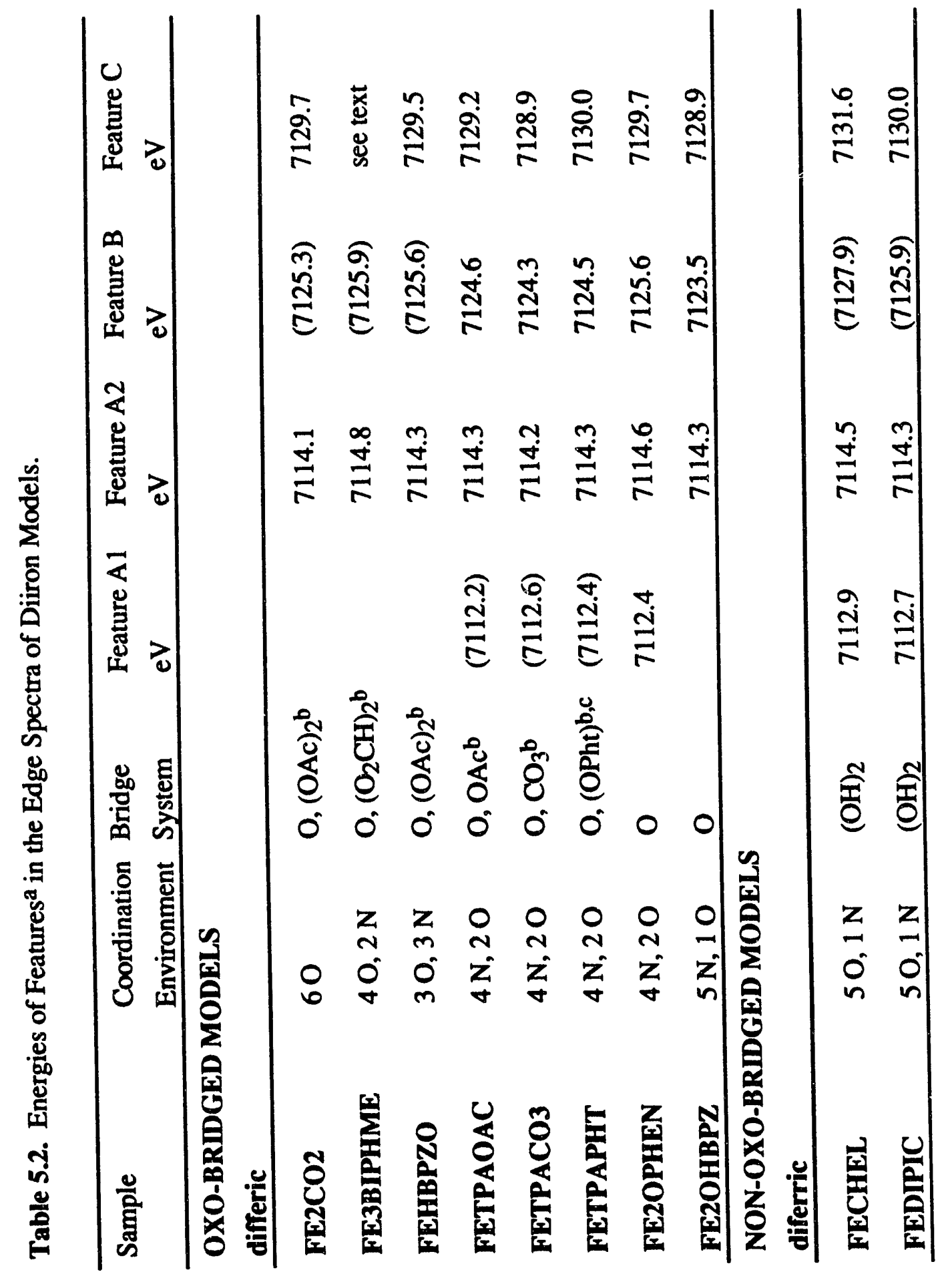




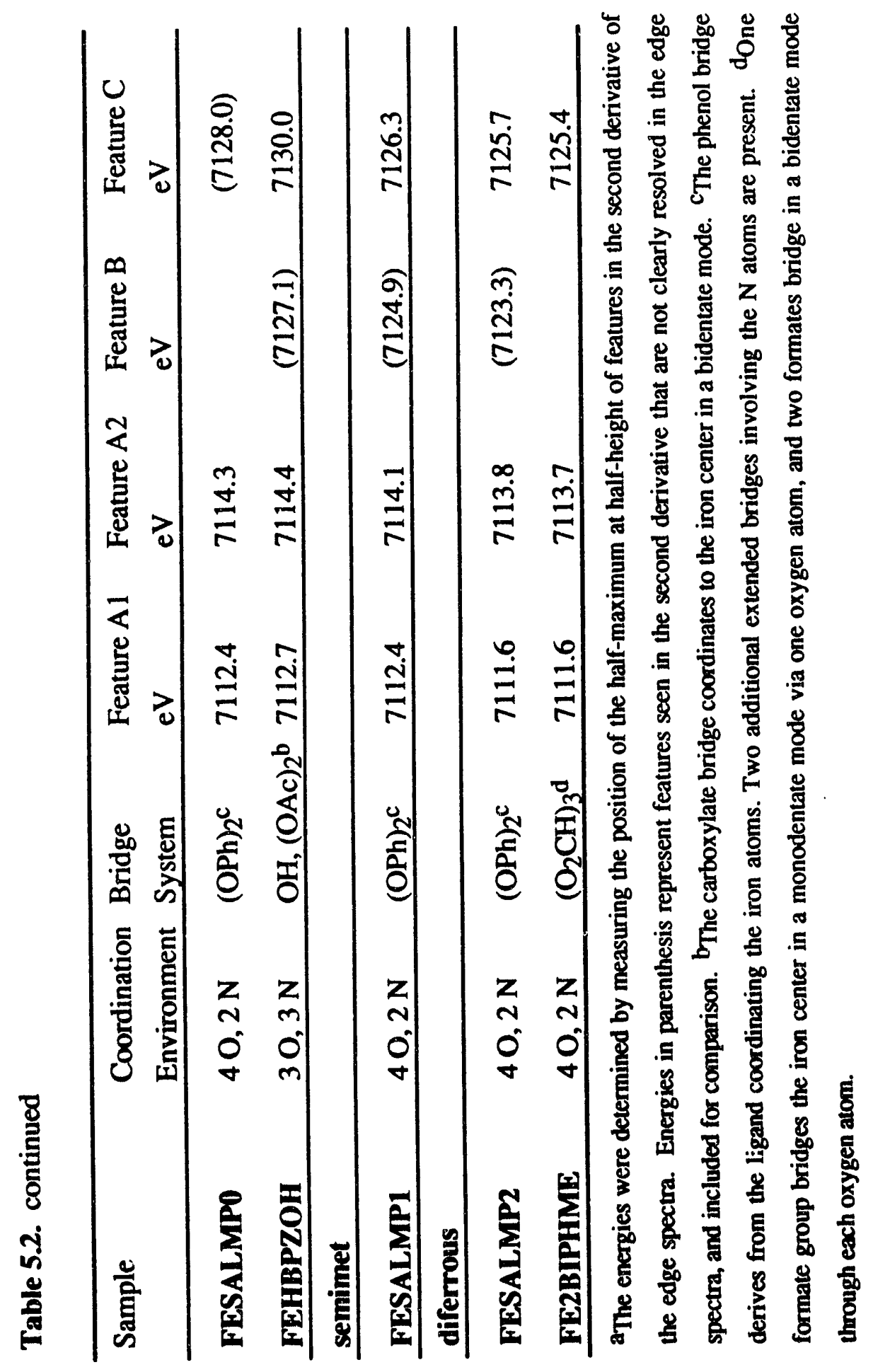




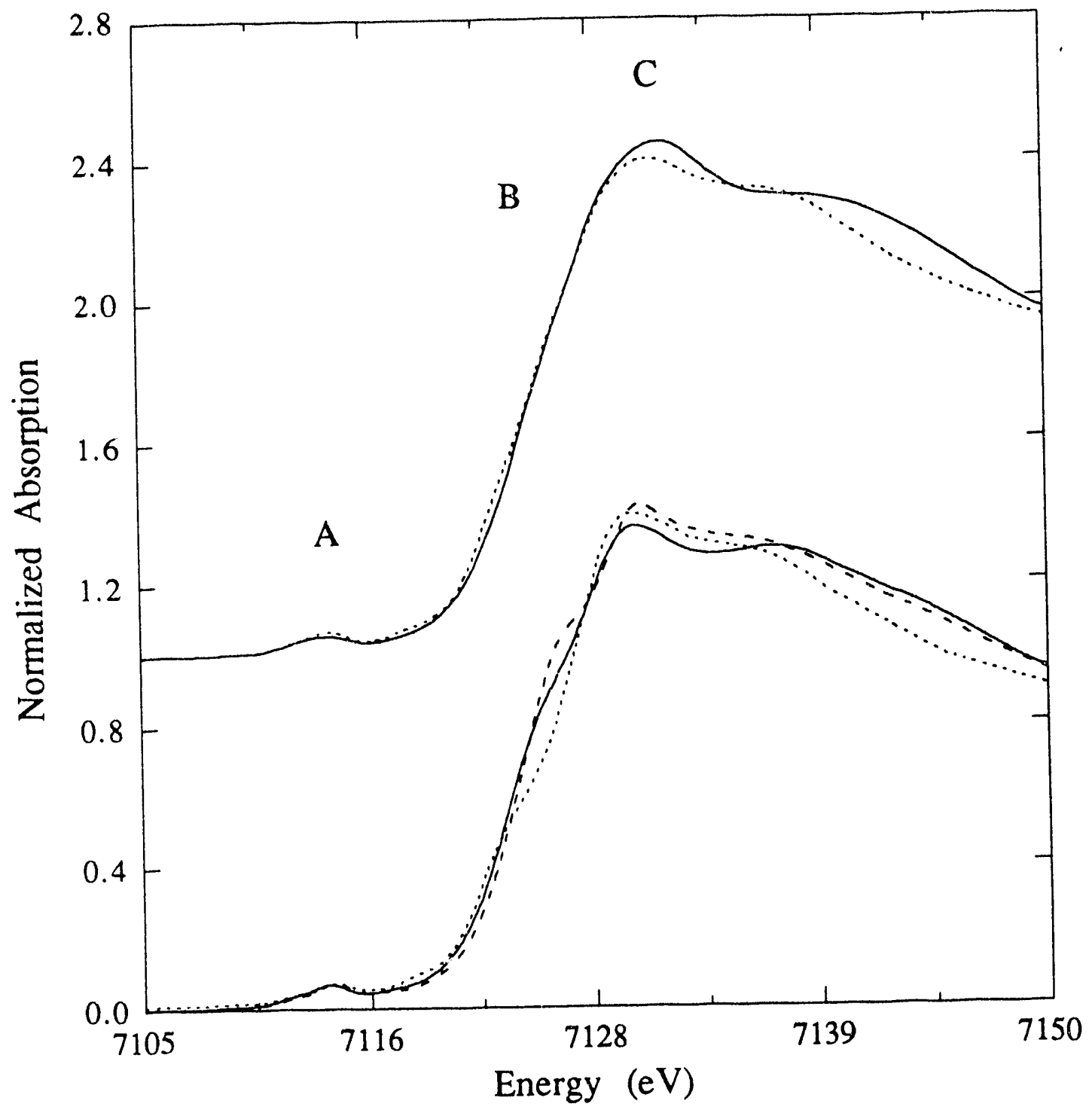

Figure 5.1. The Fe K-edge XANES spectra of oxo-bridged model compounds. Tribridged models FE2CO2 (solid) and FEHBPZO (dot) are presented in the top curve. Dibridged models FETPAOAC (solid), FE2OPHEN (dash) and FE2OHBPZ (dot) are presented in the bottom curve. 


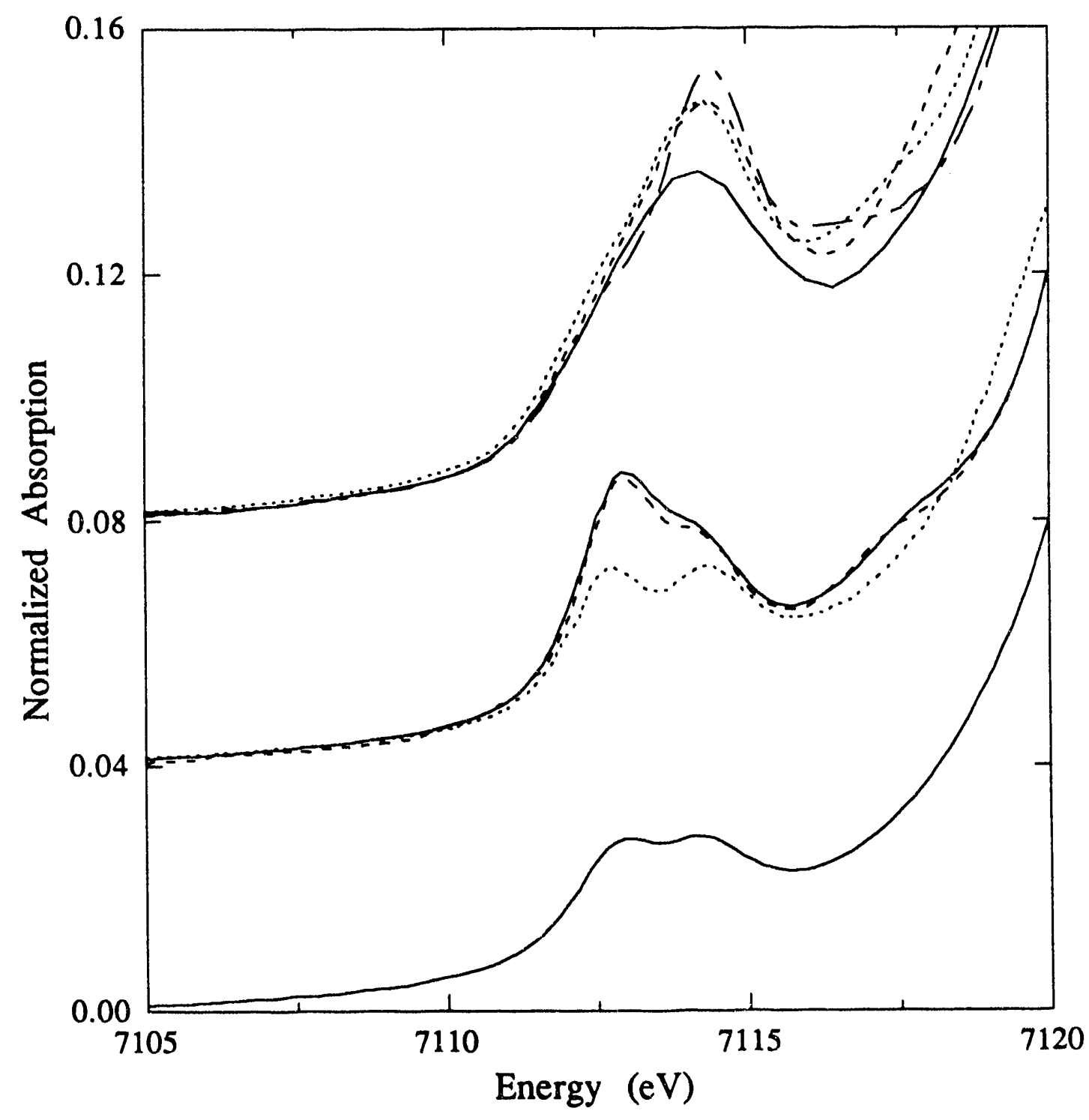

Figure 5.2. The pre-edge features of iron model compounds. The top curve shows the pre-edge transitions of oxo-bridged models FE2CO2 (solid), FEHBPZO (dash), FETPAOAC (dot) and FE2OPHEN (dash-dot) The middle curve shows the pre-edge transitions of non-oxo-bridged models FECHEL (solid), FEDIPIC (dash) and FEHBPZOH (dot). The bottom curve shows the pre-edge feature from monomeric $\mathrm{Fe}$ (acac) 3 (solid). Note the difference in the appearance of the pre-edge feature of the oxobridged models compared to the non-oxo-bridged models. 
FETPACO3 to $5.5 \mathrm{eV}$ for FETPAPHT with the splittings for all of the rest of the compounds falling within this range (Table 5.2). There is also a broad feature above 7135 $\mathrm{eV}$ in all of the compounds whose appearance and position also varies in a non-systematic way from compound to compound.

5.3.1.2. Non-oxo Bridged Models. The edge spectra for representative diand tribridged models which do not contain an oxo bridge are presented in Figure 5.3. These models represent ligation of $5 \mathrm{O}$ and $1 \mathrm{~N}$ (FECHEL and FEDIPIC) and $3 \mathrm{O}$ and 3 $\mathrm{N}$ (FEHBPZOH). The edge spectrum of FESALMPO $(4 \mathrm{O} / 2 \mathrm{~N})$ is presented in Figure 5.4. The shape of the pre-edge feature (region $\mathrm{A}$ ) in these models is is also independent of the number of bridges in the diiron center (Figure 5.2, middle curve) and quite different from the pre-edge feature of the oxo-bridged models. In all of the non-oxo-bridged models, the pre-edge feature is clearly a doublet, with the more intense transition occuring between 7112.4 and $7112.9 \mathrm{eV}$ and the weaker transition occuring between 7114.3 and 7114.6. The difference in relative intensities of these transitions is greater for the $50 / 1 \mathrm{~N}$ compounds than for the $4 \mathrm{O} / 2 \mathrm{~N}$ (see Figure 5.5) or $3 \mathrm{O} / 3 \mathrm{~N}$ compound and the energy splitting between the transitions is in the range of 1.6 to $1.9 \mathrm{eV}$. In addition, a shoulder is seen between the pre-edge transition and the rising edge in the spectra of FECHEL and FEDIPIC at $\sim 7118 \mathrm{eV}$ (Figrre 5.2). A feature is seen in this region of the second derivatives of the edge spectra for all of the models, however it is not clearly seen in the edge spectra thernselves. This vature may be the forbidden $1 \mathrm{~s} \rightarrow-4 \mathrm{~s}$ transition, which would also be made allowed by vibronic coupling.

There is a suggestion of a shoulder on the rising edge of FECHEL $(5 \mathrm{O} / 1 \mathrm{~N})$, which occurs at $7127.9 \mathrm{eV}$ according to the second derivative of the edge spectrum. Unlike the oxo-bridged models, however, the appearance of a rising edge shoulder does not occur with increasing numbers of $\mathrm{N}$ atoms in the first coordination sphere as a shoulder is not seen in FEHBPZOH which has $3 \mathrm{~N}$ and $3 \mathrm{O}$ atoms. In the non-oxo-bridged models, then, the appearance of the shoulder may be due to the reduction in the number of bridges in the diiron center. This is also consistent with the appearance of the rising edge shoulder in the oxo-bridged models, however in the case of the non-oxo-bridged models, the effect is not as dramatic. 21

The main transition in the non-oxo-bridged models (Feature C) occurs at $7130 \mathrm{eV}$ in the spectrum of FEHBPZOH and FEDIPIC, and at 7131.6 in the spectrum of FECHEL. The edge spectrum of FESALMPO is not very well defined (Figure 5.4). The position of Feature $\mathrm{C}$ in the non-oxo-bridged models occurs at slightly higher energies than the position of the corresponding feature in the oxo-bridged models (7128.1 - 7130 


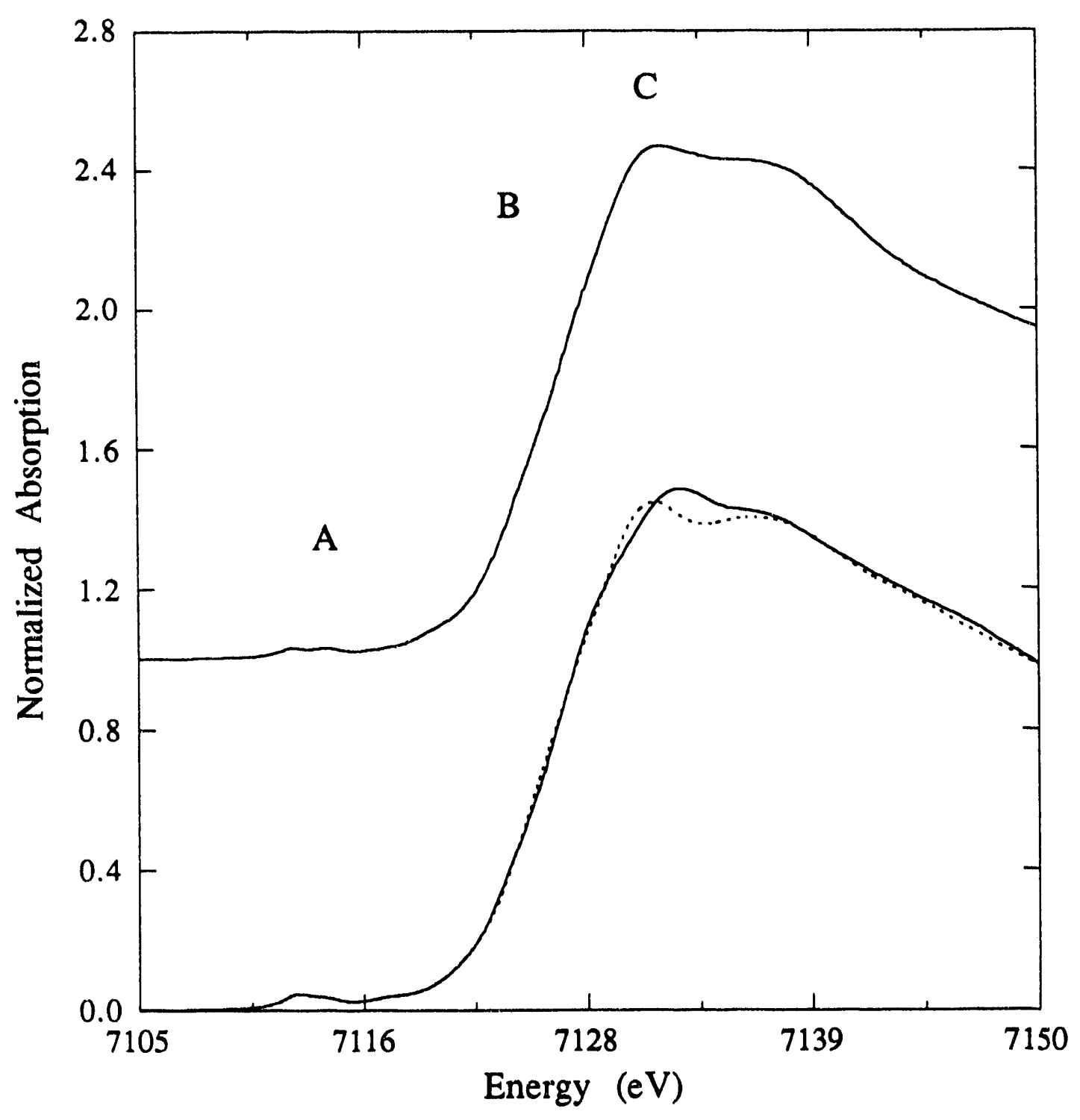

Figure 5.3. The Fe K-edge XANES spectra of non-oxo-bridged model compounds. Tribridged model FEHBPZOH (solid) is presented in the top curve. Dibridged models FECHEL (solid), and FEDIPIC (dot) are presented in the bottom curve. 
eV). As in the edge spectra of the oxo-bridged models, there is a broad feature which occurs above $7135 \mathrm{eV}$ in the spectra of FECHEL, FEDIPIC, and FEHBPZOH.

5.3.1.3. Changes in Spectra with Oxidation State. The spectra of the FESALMP dimer series in all three oxidation states are presented in Figure 5.4 (top curve). Upon reduction from the diferrric Fe(III)Fe(III) state to the semimet $\mathrm{Fe}$ (III)Fe(II) state, the position of the edge (measured at an absorption value of 1 ) moves to lower energy by $2.3 \mathrm{eV}$, the edge broadens, and the intensity of the main transition region (Feature $\mathrm{C}$ ) decreases somewhat. In the diferrous $\mathrm{Fe}$ (II)/Fe(II) state, the position of the edge moves $4.3 \mathrm{eV}$ to lower energy relative to the diferric edge and the shape of the edge changes, increasing in intensity and becoming more narrow. For the BIPHME models (Figure 5.4, bottom curve), the edge moves $3.3 \mathrm{eV}$ to lower energy from the diferric (FE3BIPHME) to the diferrous (FE2BIPHME) state, and the intensity and appearance of the main transitions change in a manner similar to that described for the FESALMP series.

The pre-edge feature in the reduced forms of the models is also a doublet (Figure 5.5 , top curve), and like the non-oxo-bridged difer " mondels, the first transition is more intense than the second transition. The first transition occurs at $7111.6 \mathrm{eV}$ in the diferrous models and the second transition occurs at $7113.7 \mathrm{eV}$ for FE2BIPHME and at $7118.3 \mathrm{eV}$ for FESALMP2, corresponding to a splitting of 2.1 and $2.2 \mathrm{eV}$, respectively. The preedge of the semimet model FESALMP1 is broader and less well-resolved than the preedge of FESALMP0. There is very little change in the intensity of the pre-edge feature in going from the diferric to diferrous forms in the FESALMP models, but in the FEBIPHME models, there is a loss in intensity. This reduction in intensity is counterintuitive to expectation, based on the change in the coordination of the iron atom which occurs in the diferrous form of FE2BIPHME. One of the hexacoordinate iron atoms becomes pentacoordinate in FE2BIPHME, and the decrease in the symmetry should result in an increase in the pre-edge intensity. The loss of the oxo-bridge which occurs upon reduction of FE3BIPHME, which would result in a decrease in the distortion of the iron site, may offset any gain in intensity due to the decrease in the coordination number. The intensities of the diferrous model pre-edge features are similar to each other, therefore it is unlikely that this information can be used to distinguish between symmetric and unsymmetric ferrous diiron sites. 


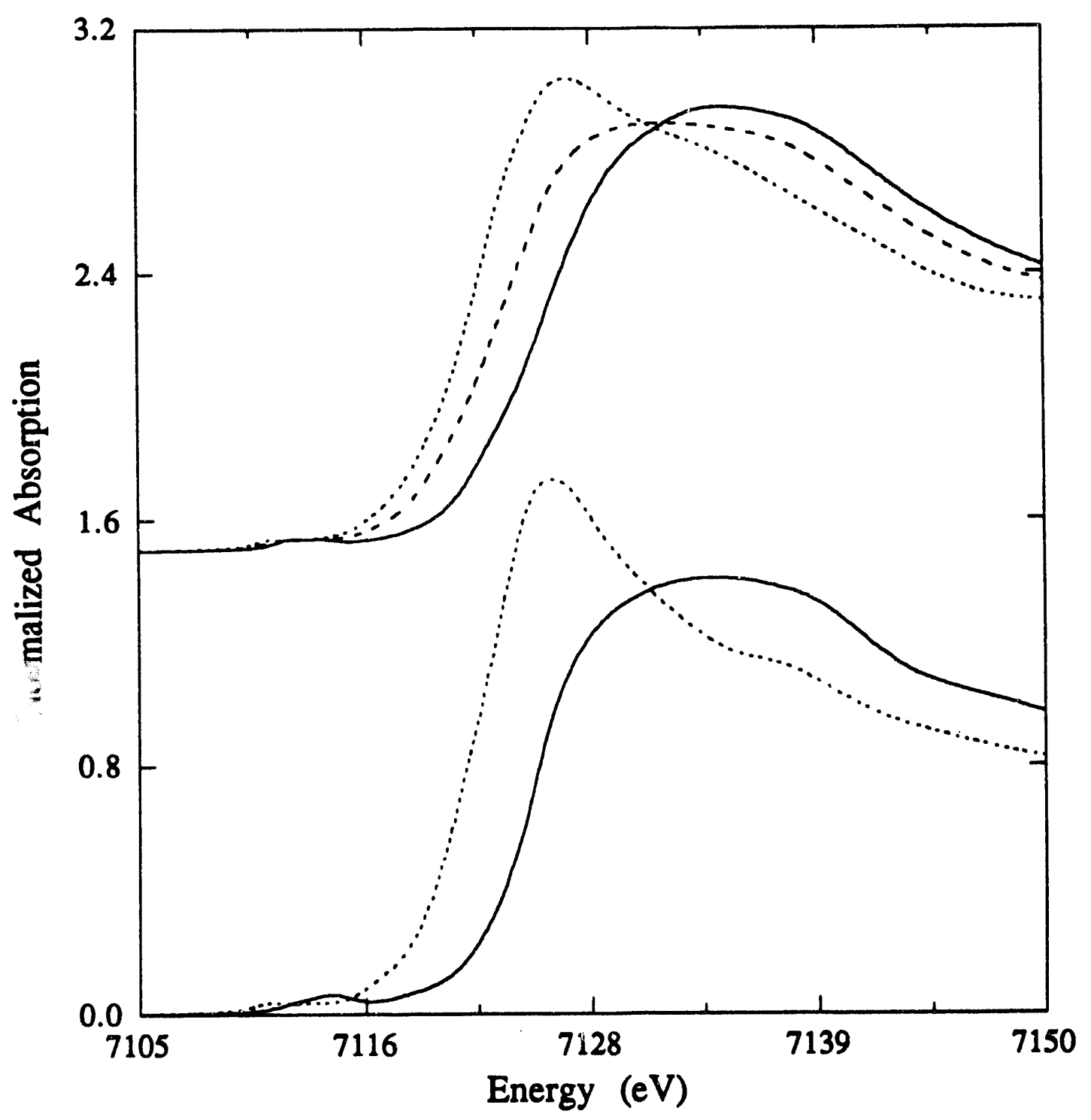

Figure 5.4. Changes in the edge spectra with change in oxidation state. The top curve shows the spectra of diferric Fe(III)Fe(III) FESALMPO (solid), semimet Fe(III)Fe(II) FESALMP1 (dash), and diferrous $\mathrm{Fe}$ (II)Fe(II) FESALMP2 (dot). The bottom curve shows the spectra of diferric FE3BIPHME (solid) and diferrous FE2BIPHME (dot). 


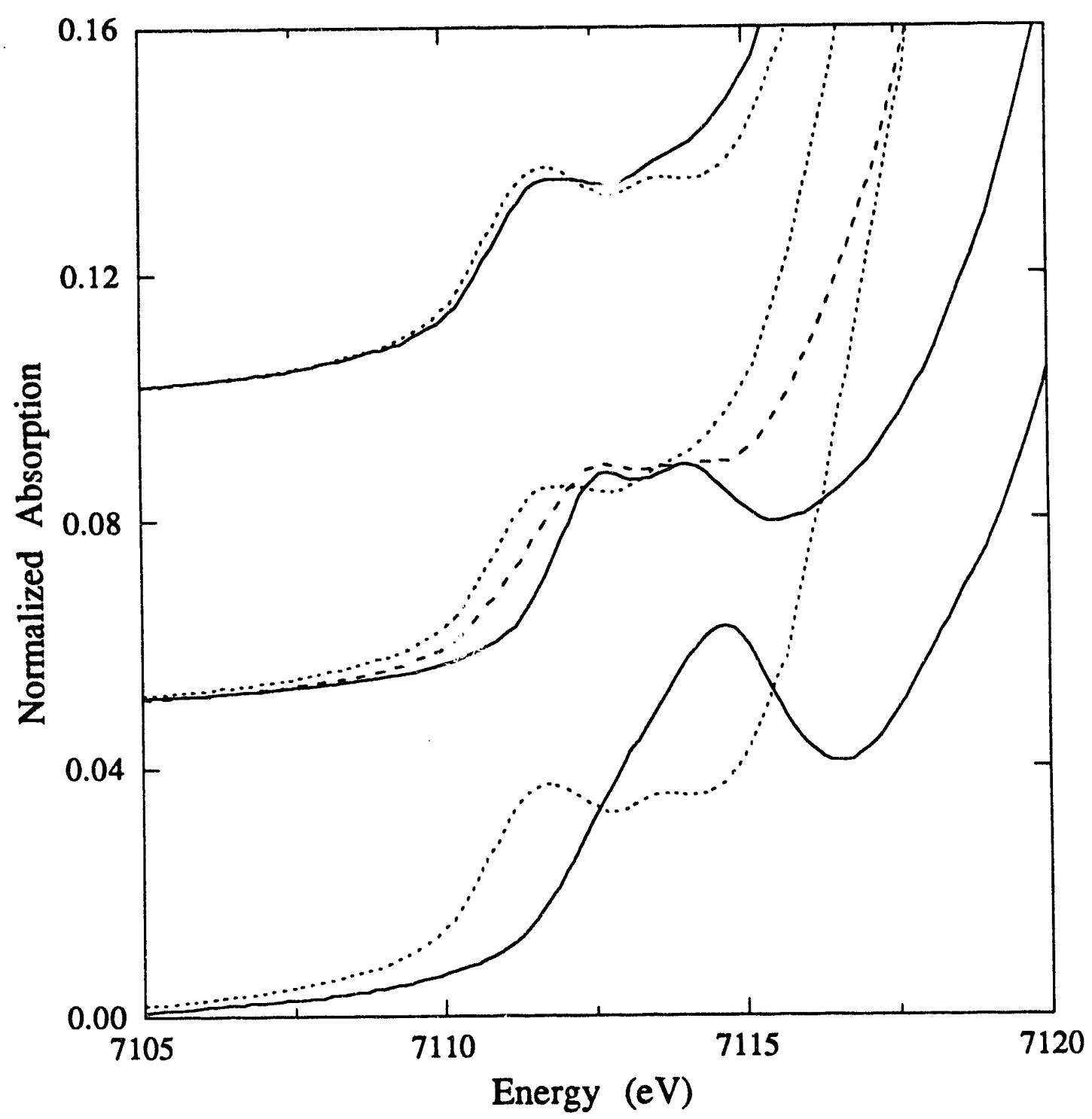

Figure 5.5. Changes in the pre-edge feature with change in oxidation state. The preedge of the diferric models FESALMP2 (solid) and FE2BIPHME (dot) are compared in the top curve. The middle curve shows the pre-edge features of FESALMPO (solid), FESALMP1 (dash) and FESALMP2 (dot). The pre-edge features of FE3BIPHME (solid) and FE2BIPHME (dot) are compared in the bottom curve. 


\subsubsection{Interpretation of Edge Spectra}

All of the compounds investigated had octahedral, or nearly octahedral coordination around the iron atoms and the edge spectra are relatively similar for all of the compounds studied here. The most dramatic change occurs in the pre-edge region, which will be discussed separately. The differences observed in the appearance of the edge structure can be correlated to the nature of ise bridging groups in the diiron center and to changes in the relative numbers of $\mathrm{N}$ and $\mathrm{d}$ atoms ligated to the iron atoms.

5.3.2.1. Changes in the Edge Spectra with Bridging Geometry. The position of the edge (measured at an absorption value of $1 / 3$ ) is correlated to the average first shell bond length for the models stucied (Table 5.3 and Figure 5.6) with the exceptions of FE2CO2 and FESALMPO. In general, the oxo-bridged models have longer average bond lengths than the no:-oxo-bridged models due to the distortion of the site caused by the presence of the short Fe-O bond (see Table 4.1 in Chapter 4). FE2CO2 is an exception to this observation due to the all $\mathrm{O}$ ligation which decreases the distortion effect of the oxo-bridge. The bond length of FE2CO2 is similar to the bond lengths of the non-oxo-bridged models, but the edge position is similar to the oxo-bridged models. The average distance of FESALMPO is consistent with the lack of an oxo-bridge, but the position of the edge of is similar to the positions of the oxo-bridged model edges. Otherwise, however, the position of the edge moves to lower energy as the average first shell bond length increases. The increase in the average bond length is itself loosely correlated to the number of $\mathrm{N}$ and $\mathrm{O}$ atoms ligating the iron center.

The data available for a direct comparison of the effect of an oxo-bridge on the edge spectra of otherwise identical models is limited, however the number of bridges seems to have an effect on the appearance of the edges. In general, the appearance of the features in the edge spectra of the tribridged models are not as well-defined as in the edge spectra of the di- and mono-bridged models, although FESALMPO is an exception to this observation. For example, the edges of oxo-bridged FEHBPZO and hydoxo-bridged FEHBPZOH (both tribridged models) are more similar to each other (Figure 5.7, top curve) than FEHBPZO is to oxo-bridged FETPAOAC or FEHBPZOH is to hydroxobridged FECHEL (Figure 5.7, compare dotted lines and solid lines, respectively). In addition, the edges of FETPAOAC and FECHEL (both dibridged models) are relatively similar to each other (Figure 5.7, middle curve), although the edge of the oxo-bridged model is less intense and broader than the hydroxo-bridged model. Comparing the spectra of FEHBPZO and FEHBPZOH, a similar effect is seen, with the edge of the oxobrid ' $d$ model appearing wider than that of the analagous hydroxo-bridged model (Figure 
Table 5.3. Comparison of Average First Shell Distance with Edge Position.

\begin{tabular}{|c|c|c|c|}
\hline Sainple & $\begin{array}{l}\text { Coordination } \\
\text { Environment }\end{array}$ & $\begin{array}{l}\text { Avg. First Shell } \\
\text { Distance }^{\mathbf{a}}(\AA)\end{array}$ & $\begin{array}{l}\text { Position of } \\
\text { Edge }^{b}(e V)\end{array}$ \\
\hline \multicolumn{4}{|c|}{ OXO-BRIDGED MODELS } \\
\hline FE2CO2 & 60 & 2.02 & 7122.2 \\
\hline FE3BIPHME & $4 \mathrm{O}, 2 \mathrm{~N}$ & 2.05 & 7122.6 \\
\hline FEHBPZO & $3 \mathrm{O}, 3 \mathrm{~N}$ & 2.06 & 7122.0 \\
\hline FETPAOAC & $4 \mathrm{~N}, 2 \mathrm{O}$ & 2.07 & 7121.9 \\
\hline FETPACO3 & $4 \mathrm{~N}, 2 \mathrm{O}$ & 2.07 & 7121.7 \\
\hline FETPAPHT & $4 \mathrm{~N}, 2 \mathrm{O}$ & 2.08 & 7121.5 \\
\hline \multicolumn{4}{|c|}{ NON-OXO-BRIDGED MODELS } \\
\hline FECHEL & $50,1 \mathrm{~N}$ & 2.02 & 7123.4 \\
\hline FEDIPIC & $5 \mathrm{O}, 1 \mathrm{~N}$ & 2.03 & 7123.1 \\
\hline FESALMPO & $40,2 \mathrm{~N}$ & 2.03 & 7122.2 \\
\hline FEHBPZOH & $30,3 \mathrm{~N}$ & 2.04 & 7123.1 \\
\hline \multicolumn{4}{|c|}{ HYDROXYLASE SAMPLE } \\
\hline EXAFS5 & $6 \mathrm{~N} / \mathrm{O}$ & 2.04 & 7122.6 \\
\hline
\end{tabular}

aThe average distance includes all first shell distances from the crystal structures of the models. bThe edge position was measured at an intensity value of $1 / 3$. ${ }^{c}$ The data for the hydroxyalse sample was determined by EXAFS (see Chaper 2). 


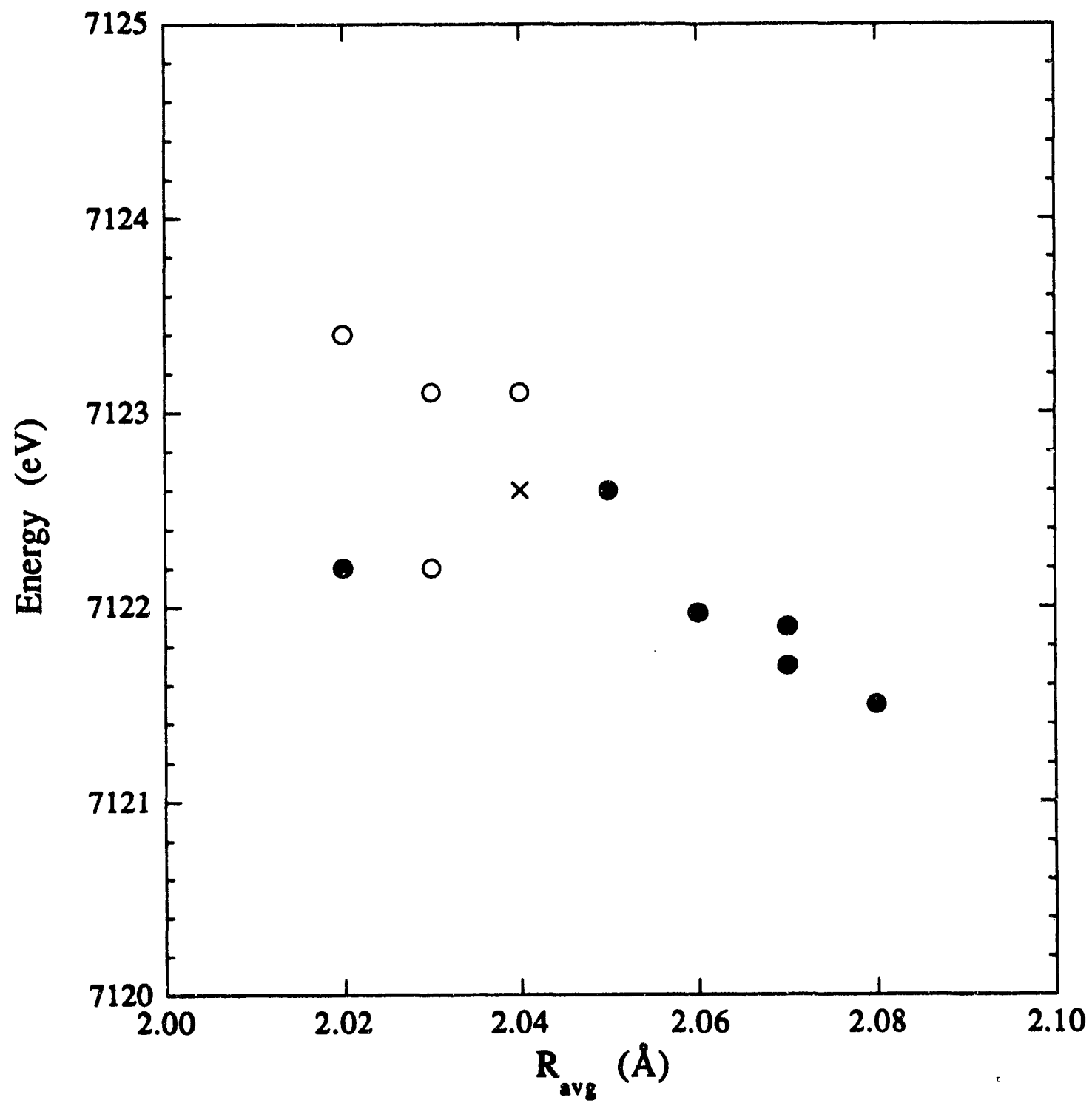

Figure 5.6. A comparison of the average first shell distance with edge position. The solid circles are data for oxo-bridged models. The open circles are data for non-oxobridged models. The $\mathrm{x}$ is the oxidized hydroxyase data. 


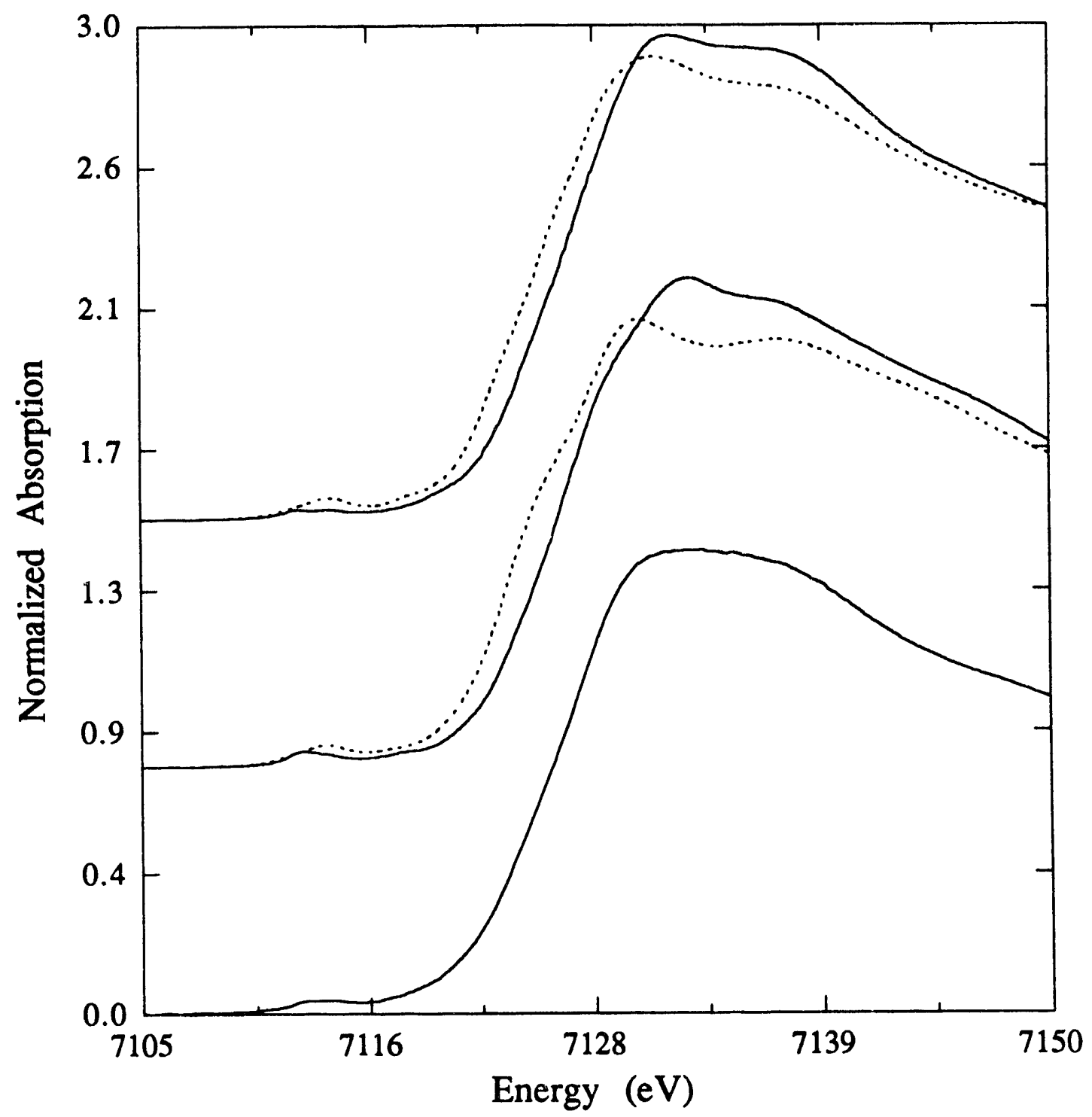

Figure 5.7. Comparisons of model and MMO hydroxylase edges. The solid lines are spectra of non-oxo-bridged models, while the dotted lines are oxo-bridged model spectra. The top curve compares the edge spectra of tribridged models FEHBPZOH (solid) and FEHBPZO (dot). The middle curve compares the edge spectra of dibridged models FECHEL (solid) and FETPAOAC (dot). The edge spectrum of the oxidized hydroxylase sample EXAFS5 is presented in the lower curve, and more resembles the spectra of the tribridged models presented in the top curve. 
5.7, top curve). It should be noted that the ligation of the iron center also changes for FETPAOAC (4 N/2 O) and FECHEL $(5 \mathrm{O} / 1 \mathrm{~N})$ in addition to the types of bridges changing, which introduces another variable into the comparison.

5.3.2.2. Changes in the Edge Spectra with Changes in Ligation. The appearance of the shoulder on the rising edge in the oxo-bridged models (Figure 5.1) corresponded to the increase in the number of $\mathrm{N}$ atoms relative to $\mathrm{O}$ atoms in the first coordination sphere, suggesting that this feature may be related to changes in the covalency of the active site due to differences in the ligation sphere. Similar effects in the edge spectra of $\mathrm{Ni},{ }^{7} \mathrm{Cu},{ }^{4}$ and $\mathrm{Fe}^{9}$ models and proteins have been seen with changes in the ligation of the metal center which have been atributed to an increase in the covalency of the metal site. In the $\mathrm{Ni}$ and $\mathrm{Cu}$ compounds studied, this shoulder has been interpreted as a ligand-tometal charge transfer shake down feature associated with the $1 \mathrm{~s} \rightarrow 4 \mathrm{p}$ transition. A similar interpretation of the feature for the oxo-bridged dinuclear iron centers could apply here as well. No such change in the edge spectra was seen in the non-oxo-bridged compounds as a result of changing ligation sphere, however, the spectrum of dibridged FECHEL has a weak shoulder, whereas the spectrum of tribridged FEHBPZOH does not. This suggests that the decrease in the number of bridges may also impact the presence of the shoulder, perhaps reflecting greater distortion in the diiron center imposed by the bridging geometry. This interpretation is consistent with the greater intensity of the $1 \mathrm{~s} \rightarrow 3 \mathrm{~d}$ pre-edge feature of FECHEL relative to FEHBPZOH (Figure 5.2, middle curve).

5.3.2.3. Pre-edge Feature. The intensity of the pre-edge feature is gained by the non-centrosymmetric character of the Fe site in the iron dimers which allows $4 p$ and $3 d$ mixing to occur. The intensity of the pre-edge feature is related to the amount of $4 \mathrm{p}$ mixing into the $3 \mathrm{~d}$ orbitals and is inversely proportional to the coordination number of the metal site. ${ }^{3,5}$ For the models studied here, the coordination number is 6 in every case so any variation in the intensity of the pre-edge feature reflects the amount of distortion around the iron site. The edge spectrum of oxo-bridged model FEHBPZO and hydroxo-bridged model FEHBPZOH are compared in Figure 5.5a. Both of these compounds are coordinated by $3 \mathrm{~N}$ and $3 \mathrm{O}$ atoms. The intensity of the pre-edge feature in the oxo-bridged model is greater than the intensity in the non-oxo-bridged model, reflecting the increased distortion of the iron site due to the presence of the short oxo-bridge (see Table 4.1 in Chapter 4). In the oxo-bridged model, the average first shell distances range from 1.79 to $2.19 \AA(0.4 \AA$ difference), while in the hydroxo-bridged model, the average first shell distances range from 1.96 to $2.11 \AA$ ( $0.15 \AA$ difference). In addition, the pre-edge features of the non-oxo-bridged models are very similar to the pre-edge feature of $\mathrm{Fe}(\mathrm{acac})_{3}{ }^{22}$ (Figure 5.2, bottom curve), a monomeric octahedral compound coordinated by 60 atoms 
whose bond lengths range from 1.986 to $2.004 \AA$. The presence of the pre-edge feature in the $\mathrm{Fe}(\mathrm{acac})_{3}$ compound reflects the sensitivity of the transition to slight symmetry distortions.

The pre-edge feature is clearly split in all non-oxo-bridged models, and asymmetric in all oxo-bridged models (Figure 5.2). The appearance of this feature is therefore characteristic of the nature of the bridge in the iron center, but not of the number of bridges. There are two possible explanations for the splitting seen in the pre-edge feature. One is that each transition corresponds to the $1 \mathrm{~s} \rightarrow 3 \mathrm{~d}$ transition for each iron atom in the dimer. This doesn't seem very likely as the edge spectra seen will be the average for both iron atoms and the individual iron sites within each dimer are very nearly identical to each other. In addition, the presence of a split pre-edge feature in monomeric $\mathrm{Fe}(\mathrm{acac})_{3}$ argues against this explanation. The alternative explanation is that the transitions seen are to different molecular states in the final d-state configuration.

The transition which occurs in the pre-edge region is from an initial $\left[1 \mathrm{~s}^{2} \ldots 3 \mathrm{~d}^{5}\right]$ state to a final $\left[1 \mathrm{~s}^{1} \ldots 3 \mathrm{~d}^{6}\right]$ state and will occur to states of maximum spin multiplicity. In the free ion limit, only the $5 \mathrm{D}$ state is available, however at somewhat stronger fields (but still in the weak field limit), transitions can occur to the ${ }^{5} \mathrm{~T}_{2 \mathrm{~g}}$ and ${ }^{5} \mathrm{E}_{\mathrm{g}}$ molecular states. The splitting seen in the pre-edge region of the edge spectra is therefore consistent with transitions to the ${ }^{5} \mathrm{~T}_{2 \mathrm{~g}}$ and ${ }^{5} \mathrm{E}_{\mathrm{g}}$ states $\left({ }^{5} \mathrm{~T}_{2 . \mathrm{g}}<{ }^{5} \mathrm{E}_{\mathrm{g}}\right)$. The energy splittings seen in the preedge region of the diferric dimers were betweeen 1.6 and $2.2 \mathrm{eV}\left(\sim 13,000-17,700 \mathrm{~cm}^{-1}\right)$, which correspond to $\mathrm{D}_{\mathrm{q}} / \mathrm{B}$ values in the range from 1.3 to 1.75 . These values are consistent with the high-spin character of these model compounds. The electronic configuration which gives rise to the ${ }^{5} \mathrm{~T}_{2 \mathrm{~g}}$ and ${ }^{5} \mathrm{E}_{\mathrm{g}}$ molecular states are $\left(\mathrm{t}_{2}{ }^{4} \mathrm{e}^{2}\right)$ and $\left(\mathrm{t}_{2}{ }^{3} \mathrm{e}^{3}\right)$, respectively, so the energy splittings observed ir the pre-edge correspond to $10 \mathrm{Dq}$ for the various model compounds.

The overall increase in intensity of the pre-edge feature for the oxo-bridged model spectra over the non-oxo-bridged models can be explained by the increased distortion of the $\mathrm{Fe}$ site due to the presence of the shor $\mathrm{Fe}-\mathrm{O}_{\mathrm{oxo}}$ bridging distance. In the spectra of the non-oxo-bridged models, the transition to the ${ }^{5} \mathrm{~T}_{2 \mathrm{~g}}$ state is the most intense, while the transition to the ${ }^{5} \mathrm{E}_{\mathrm{g}}$ state is the most intense in the spectra of the oxo-bridged models. The intensity differences may reflect a greater amount of $4 \mathrm{p}$ mixing into the ${ }^{5} \mathrm{E}_{\mathrm{g}}$ final state over the ${ }^{5} \mathrm{~T}_{2 \mathrm{~g}}$ state for the oxo-bridged models due to the lowering of the symmetry of the iron atoms caused by the presence of the oxo-bridge.

In the diferrous systems, splitting in the pre-edge region has commonly been attributed to transitions to the free ion ${ }^{4} \mathrm{~F}$ and ${ }^{4} \mathrm{P}$ states of the $\mathrm{d}^{7}$ excited state configuration. 10 The relative intensities of the two transitions has been calculated to be $7: 3$ 
for $F: P\left({ }^{4} F<{ }^{4} P\right)$, so the higher energy transition should be less intense. This is the case for the ferrous models (Figure 5.5, top curve). The magnitude of the splitting seen (2.1 and $2.2 \mathrm{eV}$ ) is consistent with the energy difference expected for the ${ }^{4} \mathrm{~F}$ and ${ }^{4} \mathrm{P}$ levels. Alternatively, the transitions seen could be to the ${ }^{4} \mathrm{~T}_{1 \mathrm{~g}}$ and ${ }^{4} \mathrm{~T}_{2 \mathrm{~g}}$ molecular states which correspond to $D_{q} / B$ values of 1.8 and 1.9. These values of $D_{q} / B$ are consistent with the high-spin character of the model compounds but correspond to $10 \mathrm{D}_{\mathrm{q}}$ values of $\sim 16,500$ $\mathrm{cm}^{-1}$ for FE2BIPHME and $\sim 17,400 \mathrm{~cm}^{-1}$ for FESALMP2, somewhat greater than the corresponding ferric models $\left(10 \mathrm{D}_{\mathrm{q}}\right.$ for FE3BIPHME and FESALMPO was calculated to be $\sim 15,000 \mathrm{~cm}^{-1}$ ). This is not not consistent with the relative positions of $\mathrm{Fe}^{+2}$ and $\mathrm{Fe}^{+3}$ in the spectrochemical series and supports the assignment of the transitions as corresponding to the free ion ${ }^{4} \mathrm{~F}$ and ${ }^{4} \mathrm{P}$ states.

5.3.2.4. Comparisons with Hydroxylase Edge Spectra. The edge spectrum of the oxidized hydroxylase is included in Figure 5.7 for comparison the the dimer edges. The appearance of the hydroxylse spectrum is most similar to tribridged FEHBPZOH and FEHBPZO and to dibridged FESALMP0 (shown in Figure 5.4, top curve). It is not clear why the spectrum of FESALMP0 is less distinct than the other models, but may reflect the extended bridges in the structure (see Table 5.2). The pre-edge features of these four samples are compared in Figure 5.8 (bottom curve). The pre-edge feature of the hydroxylase is asymmetric and the higher energy region is more intense than the lower energy region, similar to oxo-bridged model pre-edge features. The overall intensity of the hydroxylase pre-edge feature is more like the non-oxo-bridged model preedge features. The hydroxylase data are certainly more consistent with the lack of an oxobridge in the iron center, however the exact nature of the bridging geometry is not revealed by this analysis. Inclusion of the diferric hydroxylase on the plot in Figure 5.6 (represented as an $\mathrm{x}$ ) using the average first shell distance of $2.04 \AA$ determined by EXAFS analysis (see Chapter 2) is also consistent with the lack of an oxo bridge in the diiron site. The EXAFS analysis clearly shows that there is no oxo bridge in the iron center (Chapter 2).

The main transition in the differous hydroxylase edge is sharper than in the edge spectrum of either FESALMP2 or FE2BIPHME, and lacks the high energy shoulder seen at around $7135 \mathrm{eV}$ (see Figure 5.4 in Chapter 2). The edge of the semimet hydroxylase appears to be a little broader on the rising edge than the edge of the oxidized hydroxylase. The change in the edge of the hydroxylase with reduction to the semimet and diferrous state is similar to the change seen for the FESALMP and FEBIPHME models (compare Figure 5.4 with Figure 5.4 in Chapter 2). The intensity of the pre-edge features in both the semimet and reduced hydroxylase spectra is greater than the intensity of the 


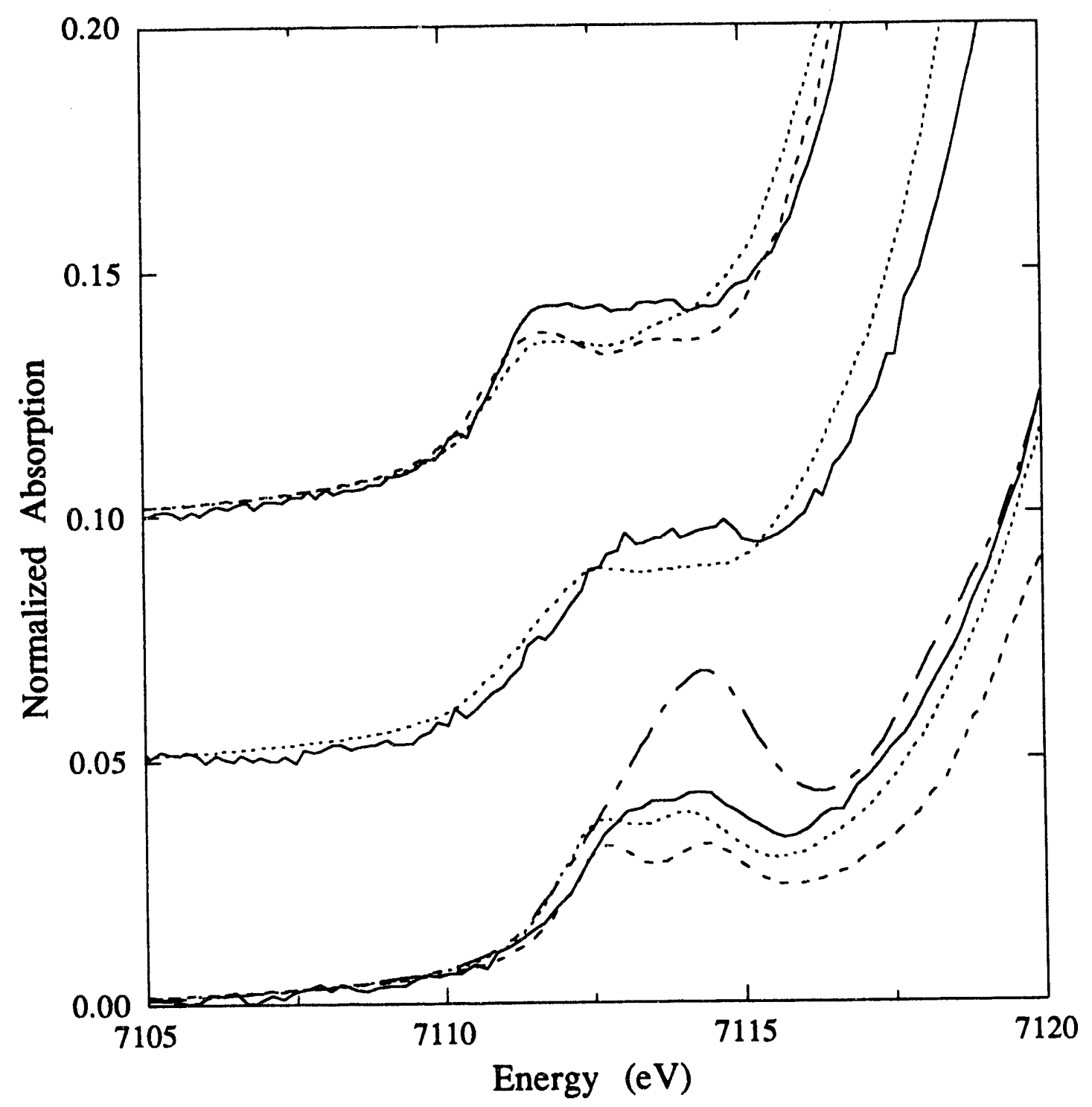

Figure 5.8. A comparison of the pre-edge features of the models and the MMO hydroxylase samples. The solid lines are the protein spectra. The top curve compares the reduced hydroxylase sample EXAFS6 (solid) with FESALMP2 (dot) and FE2BIPHME (dash) and the middle curve compares the semimet hydroxylase sample EXAFS7 (solid) with FESALMP1 (dot). The bottom curve compares the feature of oxo-bridged FEHBPZO (dash-dot) with the oxidized hydroxylase sample EXAFS5 (solid) and the non-oxo-bridged models FEHBPZOH (dash) and FESALMPO (dot). 
corresponding model compounds (Figure 5.8, top curve for reduced samples, middle curve for semimet samples), suggesting that the coordination or symmetry of the hydroxylase diiron center may be different than in the model compounds, however the difference may be due to subtraction of the different background contributions for transmission (model) data and fluorescent (protein) data are different.

\subsection{Conclusions}

The appearance of the Fe K-edge XANES spectra of the model compounds is related to the nature of the bridges in the diiron center and with the ligation of the iron atoms. In general, the features in the spectra are more well-defined for the dibridged models than the tribridged models (compare upper and lower curves in Figures 5.1 and 5.3). The presence of an oxo bridge tends to broaden the edge somewhat over the edges of the non-oxo-bridged models (Figure 5.7). The appearance of a shoulder on the rising edge of the spectra reflects a decrease in the number of bridges in the non-oxo-bridged models. In the oxo-bridged models, the appearance of the shoulder was correlated with the increase in the $\mathrm{N}$ ligation of the iron center. A reasonable interpretation of this feature is that it is a shake down feature associated with the $1 \mathrm{~s} \rightarrow 4 \mathrm{p}$ transition, and reflects an increase in the covalancy of the iron site due to the change in the ligation. The final assignment of this feature as a shake down transition awaits confirmation by XPS/PES experiments. The position of the edge decreases in energy as the average first shell distance in the models decreases, and is related to the presence or absence of a $\mu$-oxo bridge in the center.

The appearance of the pre-edge feature is characteristic only of the presence or absence of a $\mu$-oxo bridge in the diferric center and not of the number of bridges in the diiron site. In oxo-bridgea compounds, the pre-edge feature is asymmetric with the most intense transition occuring at $\sim 7114 \mathrm{eV}$. In the non-oxo-bridged compounds, the pre-edge feature is clearly resolved into a doublet with the lower energy transition $(\sim 7113 \mathrm{eV})$ having more intensity than the $7114 \mathrm{eV}$ transition. The splitting of the transitions is consistent with the assignment of the transition to the ${ }^{5} \mathrm{~T}_{2 \mathrm{~g}}$ and ${ }^{5} \mathrm{E}_{\mathrm{g}}$ molecular states for the excited state $d^{6}$ configuration. The greater overall intensity of the oxo-bridged models is consistent with the increased distortion of the iron site over the non-oxo-bridged models due to the presence of the short $\mathrm{Fe}-\mathrm{O}$ bridge. Additionally, the differences in the relative intensities of the two pre-edge transitions for the oxo- and non-oxo-bridged models probably arises from differences in the site symmetry of the iron atoms which impacts the amount of $4 \mathrm{p}$ mixing into the different molecular states. For the diferrous model, a reasonable interpretation of the split transition is that it occurs to the free ion ${ }^{4} \mathrm{~F}$ and ${ }^{4} \mathrm{P}$ 
molecular states. The intensity of this feature is similar for both symmetrically substituted FESALMP2 and asymmetrically substituted FE2BIPHME, making conclusions about the coordination of the iron atoms in the reduced state difficult based on the pre-edge information.

The diferric hydroxylase edge is most consistent with the lack of an oxo-bridge in the diiron center, supporting the results from the EXAFS analysis (Chapter 2). The shape of the pre-edge feature is reminiscent of an oxo-bridged compound, but the intensity is more like FESALMPO and FEHBPZOH, suggesting the the octahedral symmetry of the iron site in the hydroxylase is more like that in the latter models. The changes in the edge seen with reduction of the diferric state to the diferrous state are similar for the protein and model compounds. The greater intensity of the pre-edge features of the semiinet and reduced hydroxylase samples compared to the semimet and reduced models may reflect lower coordination or symmetry at the Fe center in the protein samples. A systematic study of the edge structure of reduced and semimet model compounds would greatly aid in the interpretation of the edge structure of these forms of the protein samples.

\subsection{Acknowledgements}

The data were collected at the Stanford Synchrotron Radiation Laboratory and the National Synchrotron Light Source, Brookhaven National Laboratory, which are supported by the Department of Energy, Office of Basic Energy Sciences, Division of Chemical Sciences and Division of Materials Sciences. SSRL is also supported by the National Institutes of Health, Biomedical Resource Technology Program, Division of Research Resources (RR-01209) and by the Department of Energy, Office of Health and Environmental Research. Grant support was provided by the National Science Foundation (CHE 91-21576 to $\mathrm{KOH}$ ). The author wishes to thank Profs. Stephen J. Lippard and Richard Holm for the gifts of model compounds, and Susan E. Shadle for helpful discussions. 


\subsection{References and Notes}

1. Cotton, F. A.; Ballhausen, C. J. J. Chem. Phys. 1956, 25, 617-623.

2. Seka, W.; Hanson, H. P. J. Chem. Phys. 1969, 50, 344-350.

3. Srivastava, U. C.; Nigam, H. L. Chem. Coord. Rev. 1972, 9, 275-310 and references therein.

4. Kau, L.-S.; Spira-Solomon, D. J.; Penner-Hahn, J. E.; Hodgson, K. O.; Solomon, E. I. J. Am. Chem. Soc. 1987, 109, 6433-6442.

5. Penner-Hahn, J. E.; Fronko, R. M.; Pecoraro, V. L.; Yocum, C. L.; Betts, S. D.; Bowlby, N. R. J. Am. Chem. Soc. 1990, 112, 2549-2557.

6. Tan. G. O.; Ensign, S. A.; Ciurli, S.; Scott, M. J.; Hedman, B.; Holm, R. H.; Ludden, P. W.; Korszun, Z. R.; Stephens, P. J.; Hodgson, K. O. Proc. Natl. Acad. Sci. USA 1992, 89, 4427-4431.

7. Colpas, G. H.; Maroney, M .J.; Bagyinka, C.; Kumar, M.; Willis, W. S.; Suib, S. L.; Baidya, N.; Mascharak, P. K. Inorg. Chem. 1991, 30, 920-928.

8. (a) Lippard, S. J. Angew. Chem. Int. Ed. Engl. 1988, 27, 344-361. (b) SandersLoehr, J. Iron Carriers and Iron Proteins; VCH Publishers Inc.: New York, 1989; pp. 373-466. (c) Que, L., Jr.; True, A. E.. Prog. Inorg. Chem. 1990, 38, 97-200.

9. DeWitt, J. G.; Bentsen, J. G.; Rosenzweig, A. C.; Hedman, B.; Green, J.; Pilkington, S.; Papaefthymiou, G. C; Dalton, H.; Hodgson, K. O.; Lippard, S. J. J. Am. Chem. Soc. 1991, 113, 9219-9235.

10. Shulman, R. G.; Yafet, Y.; Eisenberger, P.; Blumberg, W. E. Proc. Natl. Acad. Sci. USA 1976, 73, 1384-1388.

11. This feature could also be due to a quadropole transition, in which case the selection rule is $\Delta l=2$ and the $1 \mathrm{~s} \rightarrow 3 \mathrm{~d}$ transition is allowed. The authors of reference 10 have estimated that the quadropole transitions would be three orders of magnitude weaker than the intensity of the Fe preedge feature, and concluded that the feature is due to an Fe $1 \mathrm{~s} \rightarrow 3 \mathrm{~d}$ transition made allowed by vibronic coupling of the $4 \mathrm{p}$ and $3 \mathrm{~d}$ states.

12. Cotton, F. A.; Hanson, H. P. J. Chem. Phys. 1958, 28, 83-87.

13. Hahn, J. E.; Scott, R. A.; Hodgson, K. O.; Deniach, S.; Desjardins, S. E.; Solomon, E. I. Chem. Phys. Lett. 1982, 88, 595-598.

14. Roe, A. L.; Schneider, D. J.; Mayer, R. J.; Pyrz, J. W.; Que, L. Jr. J. Am. Chem. Soc. 1984, 106, 1676-1681. 
15. Smith, T. A.; Penner-Hahn, J. E.; Hodgson, K. O.; Berding, M. A.; Doniach, S. Springer Proc. Phys. 1984, 2, 58-60.

16. Waychunas, G. A.; Brown, G. E. J- Phys. Chem. Minerals 1990, 17, 420-430.

17. Blair, R. A.; Goddard, W. A. Phys. Rev. B 1980, 22, 2767-2776.

18. Shadle, S. E.; Penner-Hahn, J. E.; Schugar, H. J.; Hedman, B.; Hodgson, K. O.; Solomon, E. I. J. Am. Chem. Soc. accepted for publication, 1992.

19. (a) Feng, X.; Bott, S. G.; Lippard, S. J. J. Am. Chem. Soc. 1989, 111, 80468047. (b) Armstrong, W. H.; Spool, A.; Papaefthymiou, G. C.; Frankel, R. B.; Lippard, S. J. J. Am. Chem. Soc. 1984, 106, 3653-3667. (c) Tolman, W. B.; Bino, A.; Lippard, S. J. J. Am. Chem. Soc. 1989, 111, 8522-8523. (d) Armstrong, W. H.; Lippard, S. J. J. Am. Chem. Soc. 1984, 106, 4632-4633. (e) Thich, J. A.; Ou, C. C.; Powers, D.; Vasilou, B.; Mastropaolo, D.; Potenza, J. A.; Schugar, H. J. J. Am. Chem. Soc. 1976, 98, 1425-1433. (f) Snyder, B. S.; Patterson, G. S.; Abrahamson, A. J.; Holm, R. H. J. Am. Chem. Soc. 1989, 111, 5214-5223. (f) Bertrand, J. A.; Breece, J. L.; Eller, P. G. Inorg. Chem. 1974, 13, 125-131. (g) Norman, R. E.; Yan, S.; Que, L. Jr.; Backes, G.; Ling, J.; Sanders-Loehr, J.; Zhang, J. H.; O'Connor, C. J. J. Am. Chem. Soc. 1990, 112, 1554-1562. (h) Norman, R. E.; Holz, R. C.; M'enage, S.; O'Connor, C. J.; Zhang, J. H.; Que, L. Jr. Inorg. Chem. 1990, 29, 4629-4637. (i) Gaines, A.; Hammett, L. P.; Walden, G. H. J. Am. Chem. Soc. 1936, 58, 1668-1674. (j) Armstrong, W. A.; Lippard, S. J. unpublished results

20. Scott, R. A.; Hahn, J. E.; Doniach, S.; Freeman, H. C.; Hodgson, K. O. J. Am. Chem. Soc. 1982, 104, 5364-5369.

21. The second derivative of the edge spectra of all of the models does indicate the presence of a feature in the 7122.7 to $7124.8 \mathrm{eV}$ range which is not seen in the edge spectra themselves.

22. (a) Iball, J.; Morgan, C. H. Acta Cryst. 1976, 23, 239-244. (b) Roof, Jr., R. B. Acta Cryst. 1956, 9, 781-786. 
Chapter 6

Sulfur and Chlorine K-Edge X-ray Absorption Spectroscopic Studies of Photographic Materials 


\subsection{Introduction}

Sulfur is present in several important components of the photographic system including spectral sensitizing dyes and chemical sensitizing centers. These components exist at the surface of the photoactive silver halide crystals and are involved in electron and energy transfer processes. It is therefore likely that the surface structure and electronic environment of these sensitizing dyes and centers will have an effect on photographic performance. X-ray absorption spectroscopy (XAS) studies can directly probe the local geometric and electronic structure of these important surface-active species on the photoactive substrate.

Previous sulfur XAS studies have shown that the S K-edge has sharp absorption features in the edge and near-edge region arising from bound-state transitions from the sulfur 1s orbital to low-lying unoccupied or partially occupied atomic or molecular orbit. These features have been shown to be very dependent on the local geometry and oxidai:its state of the absorbing atom. $1,2,3$ With a spectrometer energy resolution of $0.5 \mathrm{eV}$ at these energies (2-3 keV), shifts of as much as $13 \mathrm{eV}$ have been seen with a change in the oxidation state of sulfur from -2 to +6 .

Studies of the sulfur K-edge of numerous organic and inorganic compounds have revealed characteristic absorption spectra dependent on the nature of sulfur in the compound, making XAS a useful tool in determining the forms and relative amounts of sulfur present in petroleum asphaltenes and coal. ${ }^{4}$ Polarized K-edge measurements together with theoretical calculations using a multiple-scattered wave $X-\alpha$ formalism have resulted in assignments of some of these S K-edge features. 5 A number of soft X-ray studies have been done on gaseous ${ }^{6}$ and surface-adsorbed $6 a, 7$ organic and inorganic sulfurcontaining molecules, such as thiols, thioethers, and sulfuryl halides. These studies provide further insight into the origin and nature of the $\mathrm{S} \mathrm{K}$-edge features, including information about the symmetry of the final state orbitals to which the transition occurs, and the orientation dependence of the edge features of adsorbed species. $6 \mathrm{a}, 6 \mathrm{~b}, 8$

Spectral sensitizing dyes are used in many applications such as non-linear optics, solar energy conversion, electrophotography and silver halide photography. Merocyanine and cyanine dyes extend the response of photographic materials beyond the intrinsic blue/ultraviolet absorption of the silver halide photoconductor into the visible and infrared regions. The spectral sensitivity imparted to the silver halide substrate depends on the visible absorption properties of the dye molecules. Spectral shifts of the visible absorption features to longer wave-lengths result from the aggregation of the dye molecules on the surface, and depend on both the concentration and the orientation of the dye molecules. 
The degree and extent of aggregation, and consequently of the spectral shift, is in part determined by silver halide morphology, the presence of other adsorbates, and structural features of the dye molecules themselves. ${ }^{9}$ Structural properties of dye molecules which are important for aggregation formation include extended delocalization of $\pi$ electrons, planarity of the molecule, and reduction of positional isomers.

The sensitization process involves the transfer of electrons or energy as an exciton from the photoexcited state of the dye molecule aggregate into the conduction band of the silver halide substrate. For well-ordered, strongly interacting molecular systems, excitons are transferred via coupled oscillations of the molecules in a time period shorter than the period of the molecular vibrations. The transfer of the exciton from the dye aggregate to the substrate conduction band requires that the dye molecules exist in an adsorbed state on the surface of the silver halide grains. The nature of the interaction between the dye aggregates and the silver halide substrate has been the subject of many studies. For dyes containing benzthiazole groups (belonging to the cyanine dye class), it has been postulated that the adsorbed dye molecules are oriented with their sulfur atoms directed toward the silver halide surface. ${ }^{10,11}$, XPS experiments of dyed $\mathrm{AgCl}$ crystals ${ }^{12}$ and electrophoretic mobilities of dyed silver halide grains ${ }^{13}$ have suggested that direct interaction between the sulfur atoms and the silver ions on the crystal surface is a driving force in the adsorption process and a determining factor in the orientation of dye molecules on silver halide crystal faces.

Another important process in photography is latent image formation. Latent image formation involves the reduction of $\mathrm{Ag}^{+}$to $\mathrm{Ag}^{0}$ which appear as dark areas on negatives and are invisible until the film is exposed to developer. The aggregates of $\mathrm{Ag}$ metal are referred to as latent image centers. The efficiency of formation of latent image centers is related to the size of the $\mathrm{Ag}$ metal aggregates and the competition between combination of the photoelectron with $\mathrm{Ag}^{+}$and electron-hole recombination. The efficiency of latent image formation, which means that less exposure time is required to form an image, can be improved by the formation of chemical sensitization centers on the silver halide surface.

Chemical sensitization centers are formed by the treatment of silver halide crystals with aqueous solutions of labile sulfur-containing compounds such as thiosulfate and thioureas. The sensitization centers are believed to be silver sulfide specks on the silver halide surface which act as electron traps for photoelectrons. ${ }^{14}$ The electron-trapping capabilities of these centers result in a reduction in the electron-hole recombination rate which improves the efficiency of latent image formation, and they may also promote or stabilize latent image center formation. The highest degree of chemical sensitization is 
achieved with a combination of sulfur compounds and gold-containing salts, however the nature of these chemical sensitization centers is not well understood.

We have used S and Cl K-edge XAS to characterize the electronic and geometric structure of a wide variety of compounds important to the photographic system, including cyanine and merocyanine dyes and dye intermediates, and thiol- and thione-containing molecules. The nature of the interaction between the metal ions and the forms of sulfur of importance in the photographic system have been studied by S K-edge XAS measurements on silver and gold complexes of sulfides, thiazoles, and thiol- and thione-containing ligands. Oriented single-crystal polarized studies of representative dye nuclei and a metalsulfur compound have been used to determine the angular dependence of the features seen the in powder spectra. These studies permit assignments of the features and provide a basis for interpreting polarized measurements of dye molecules and chemical sensitization centers on silver bromide sheet crystals.

\subsection{Experimental}

The samples discussed in this paper are presented in Tables 6.1-6.4. The dye samples (Tables 6.1 and 6.2) were measured to be $90 \%$ pure by HPLC. All of the samples with the exception of 9,10-dichloroanthracene were supplied by Eastman Kodak Co. Single crystals of 2-thiohydantoin ${ }^{15}$ (IM4) were obtained from ethanol as orange rhombic plates with sides 1-2 mm long and $0.3-0.5 \mathrm{~mm}$ thick. Yellow needles of 3,3'diethylthiacyanine bromide ${ }^{16}$ (DC5) were obtained from propanol (1-2 $\mathrm{mm} \mathrm{x} \sim 0.25 \mathrm{~mm} \mathrm{x}$ $\sim 0.25 \mathrm{~mm}$ ). Single crystals of 9,10-dichloroanthracene ${ }^{17}$ (DCA) were obtained from saturated petroleum ether solutions of the compound placed in a dessicator containing scraps of paraffin. The crystals were yellow plates of dimensions $1-2 \mathrm{~mm} \times 0.3 \mathrm{~mm} \times<$ $0.3 \mathrm{~mm}$. White single crystals of bis(ethylenethiourea) gold (I) chloride hydrate ${ }^{18}$ were obtained from water. The crystals were parallelograms, $2 \mathrm{~mm} \times 1 \mathrm{~mm} \times 0.5 \mathrm{~mm}$. The integrity of the crystals after exposure to synchrotron radiation was verified by checking that degradation of the diffraction quality of the crystal had not occurred, and by confirming the unit cell dimensions using a diffractometer after the conclusion of the experiments. $\mathrm{AgBr}$ sheet crystals ( $50 \mu$ thick) were grown on quartz plates using a growth gradient technique. The dyed surface samples were prepared by submerging the sheet crystal in 10$5 \mathrm{M}$ aqueous solutions of the dyes $\left(20\right.$ minutes at $\left.40^{\circ} \mathrm{C}\right)$. The chemically sensitized samples were prepared by submerging the sheet crystals in $10^{-5} \mathrm{M}$ aqueous solutions of sodium thiosulfate or aurous dithiosulfate $\left(20\right.$ minutes at $\left.50^{\circ} \mathrm{C}\right)$. 


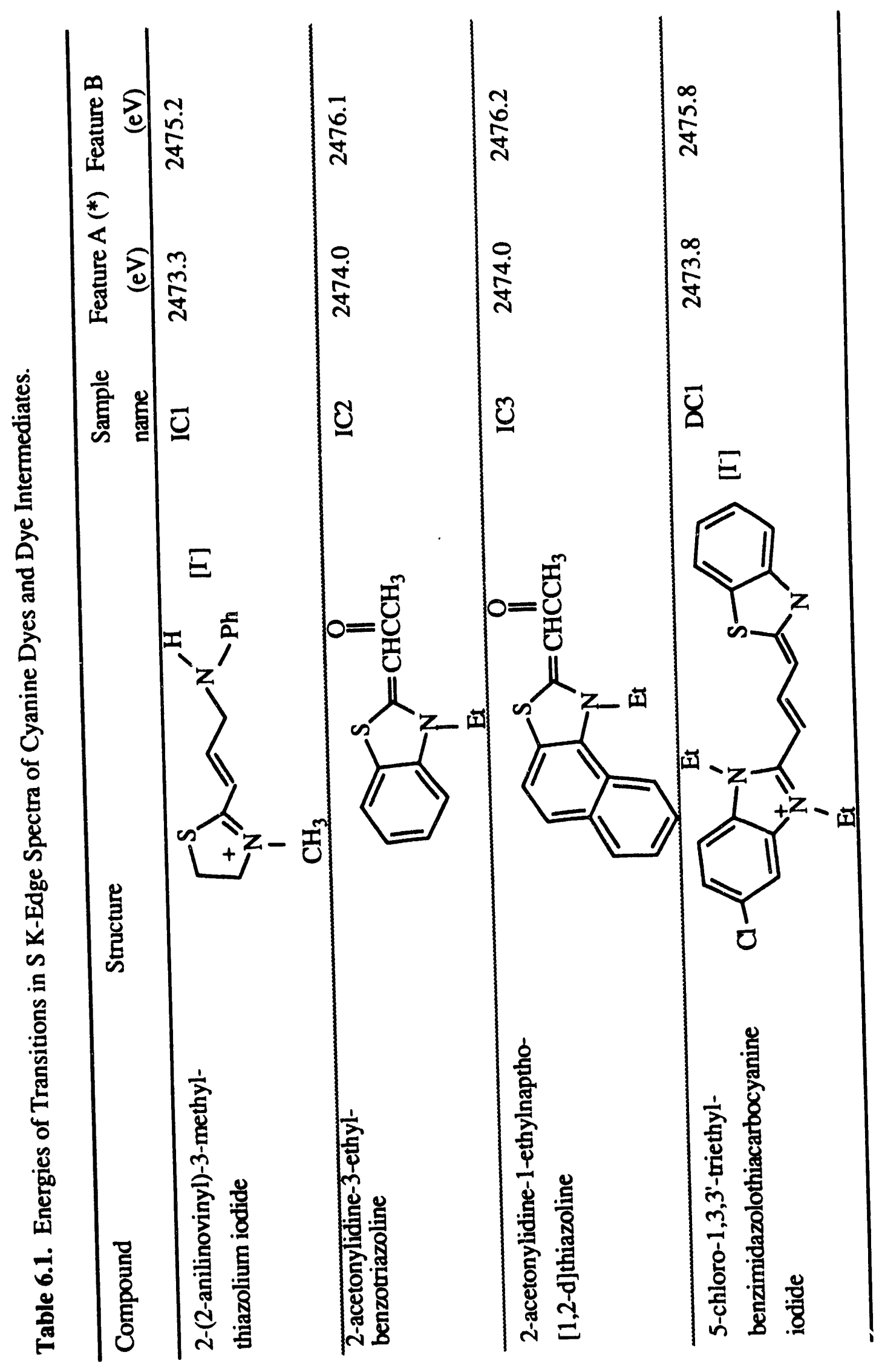




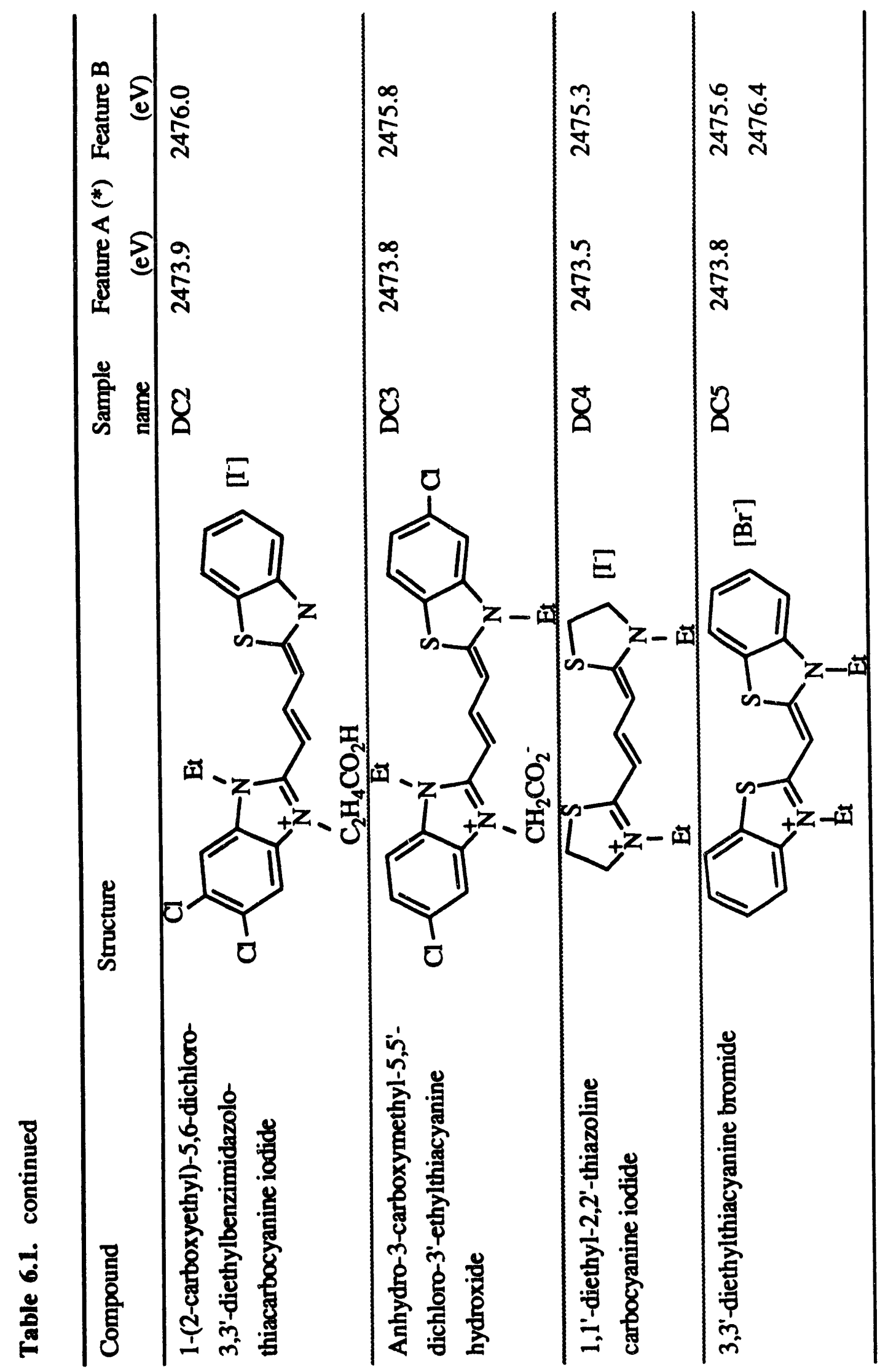




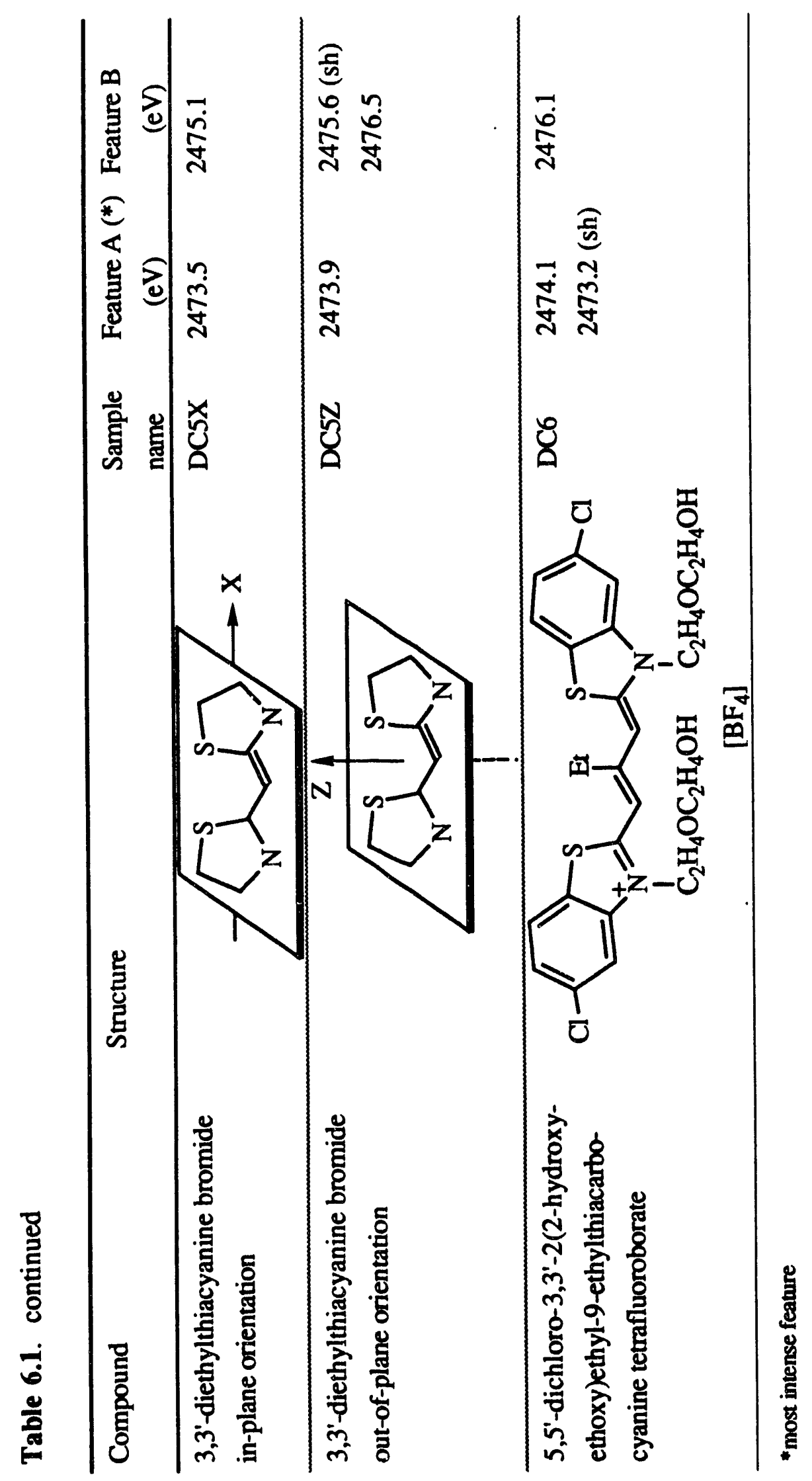




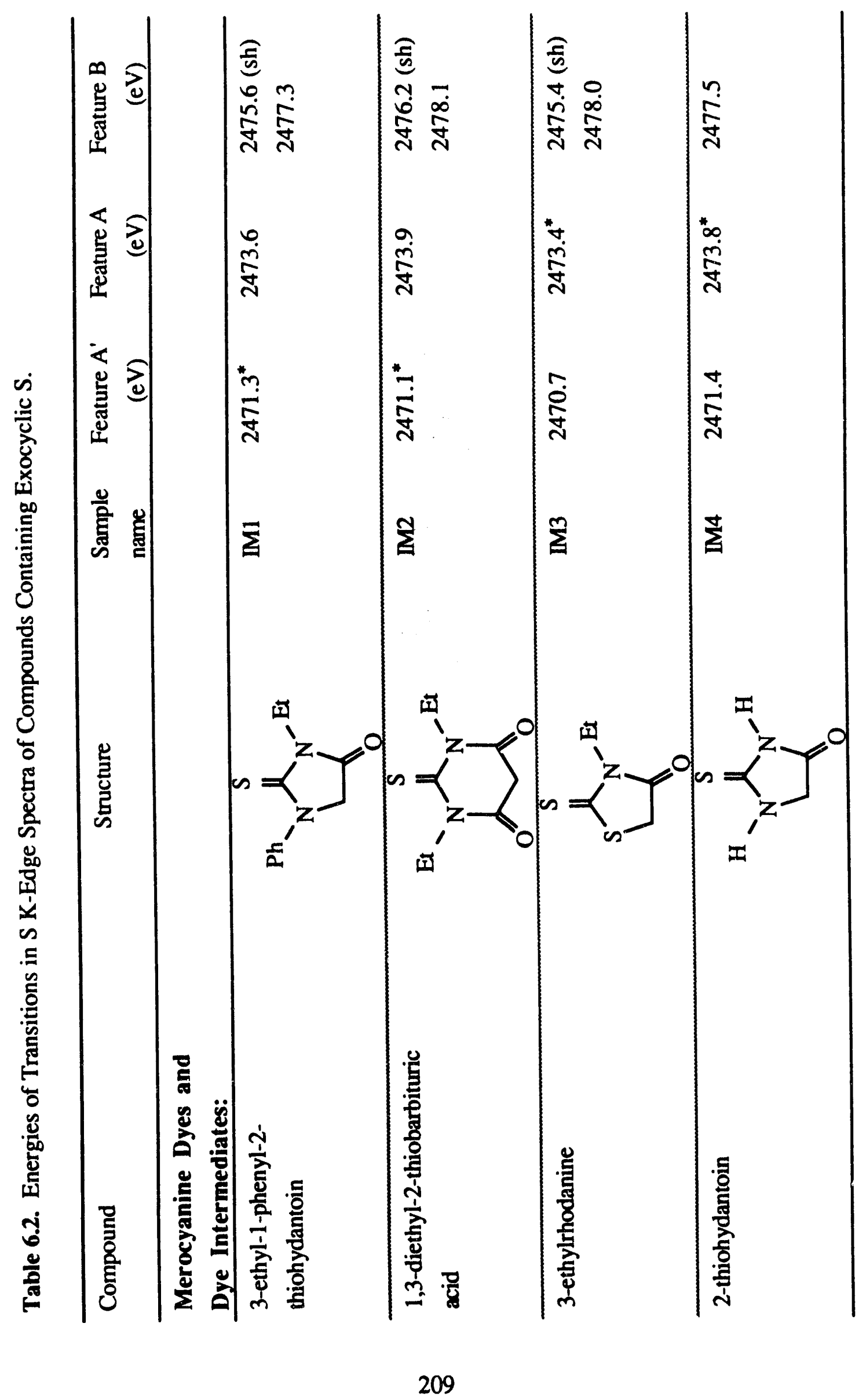




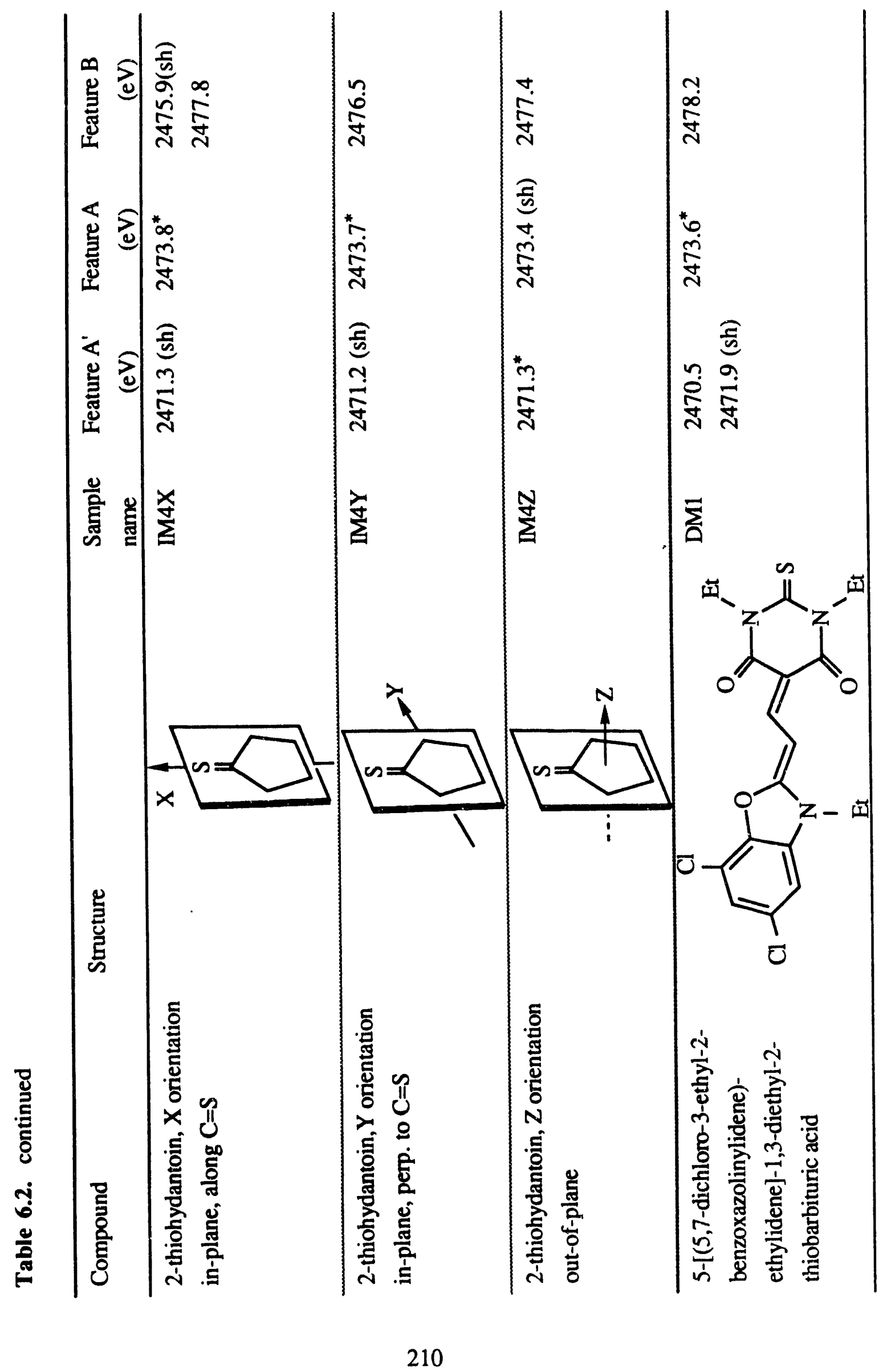




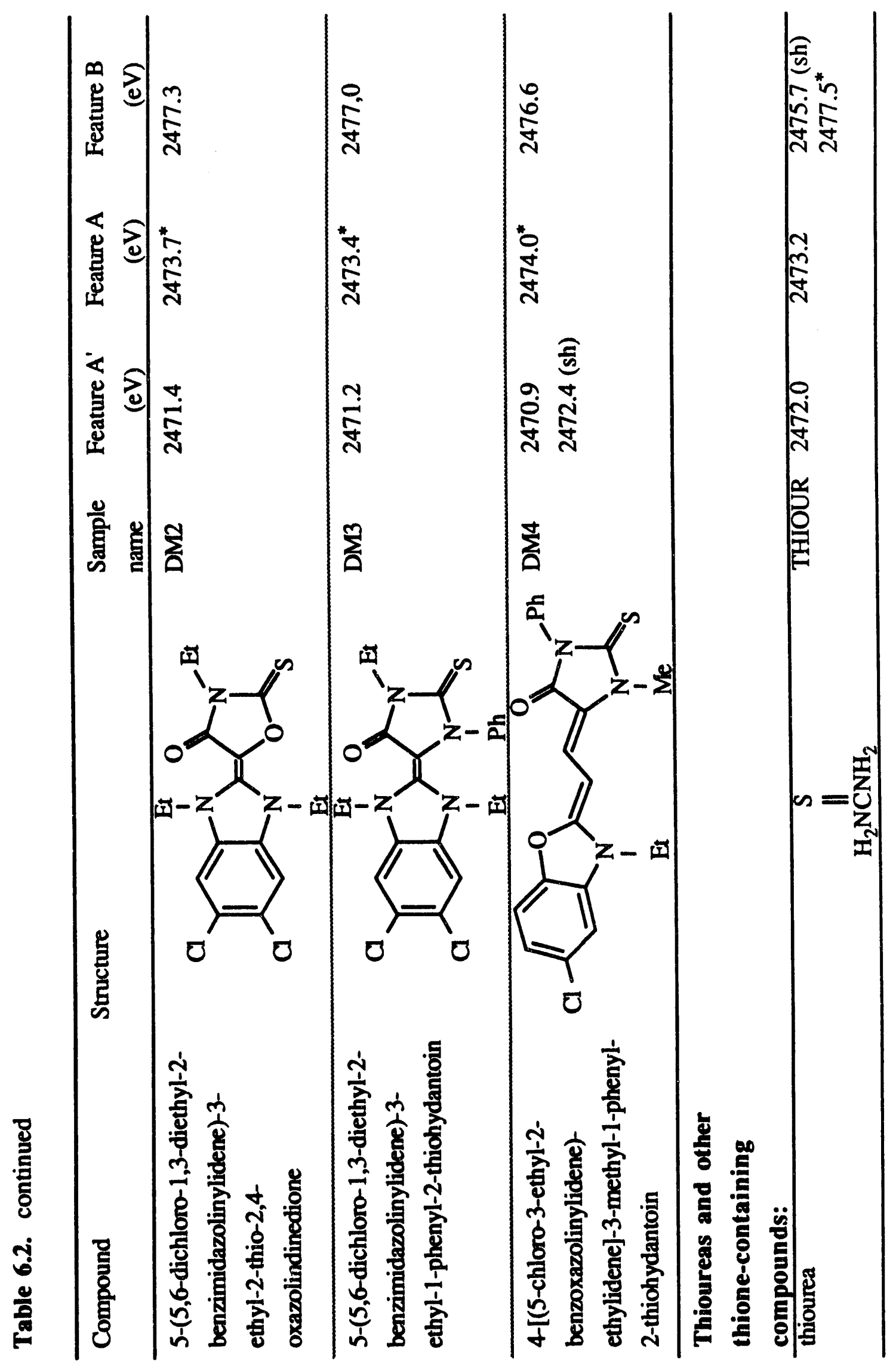




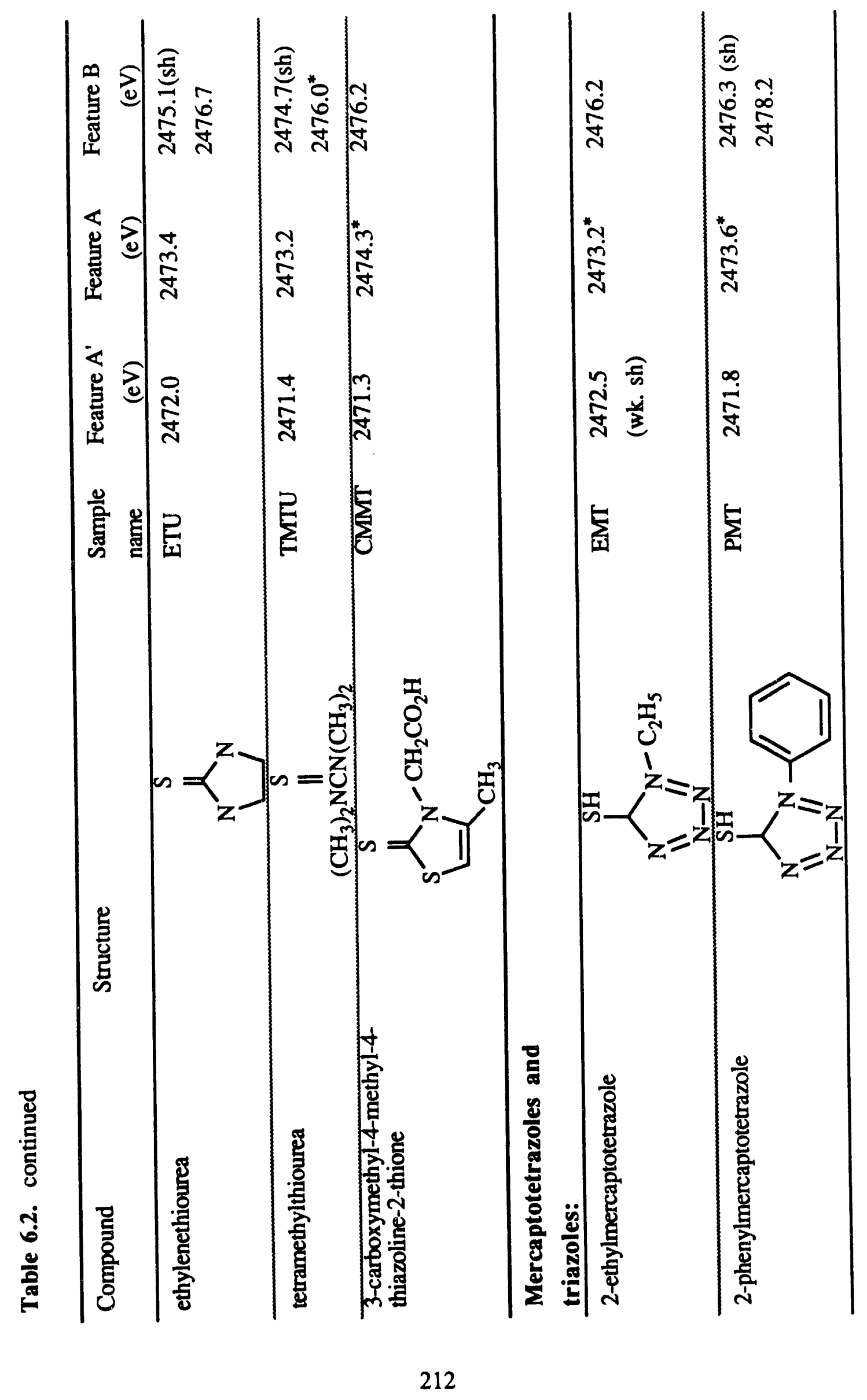




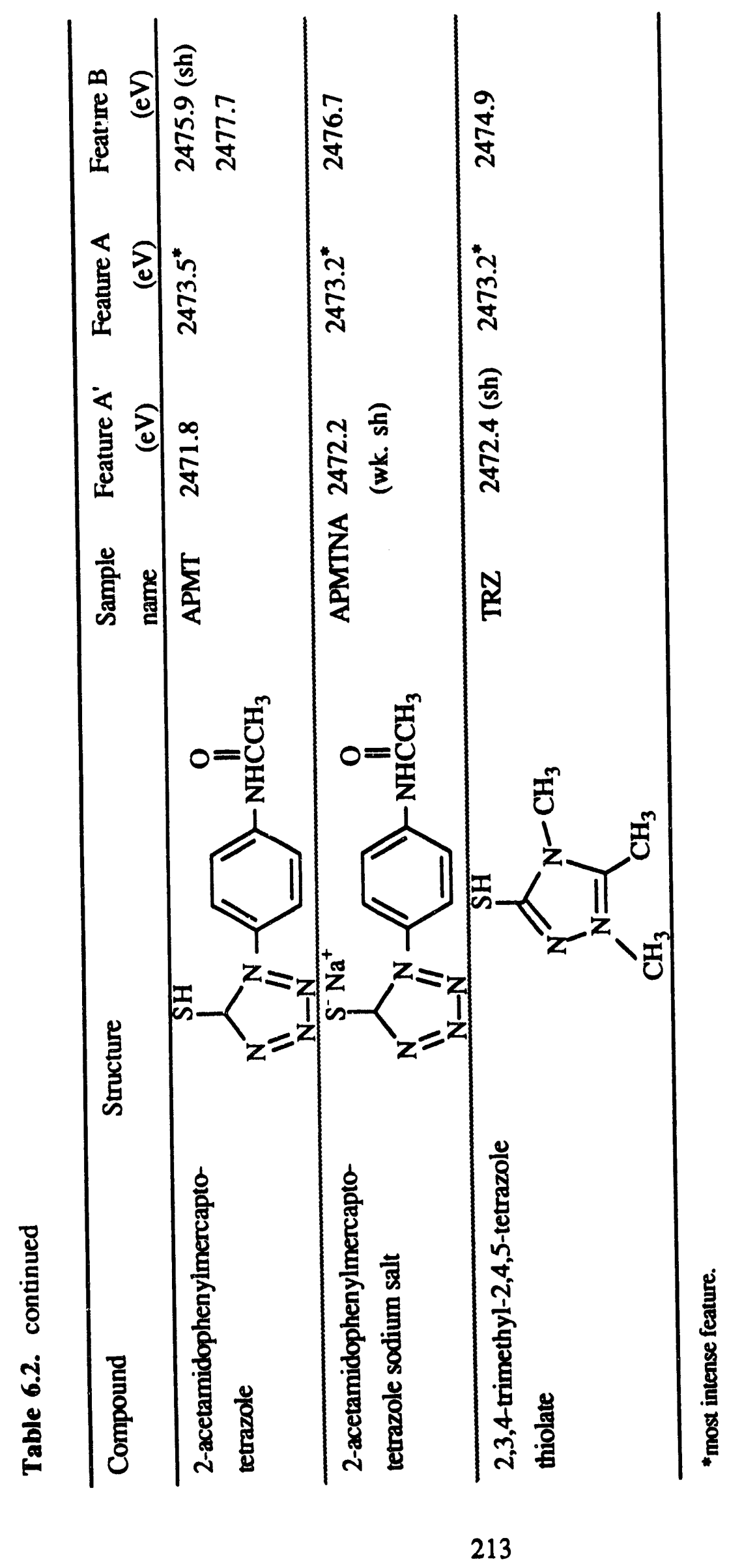




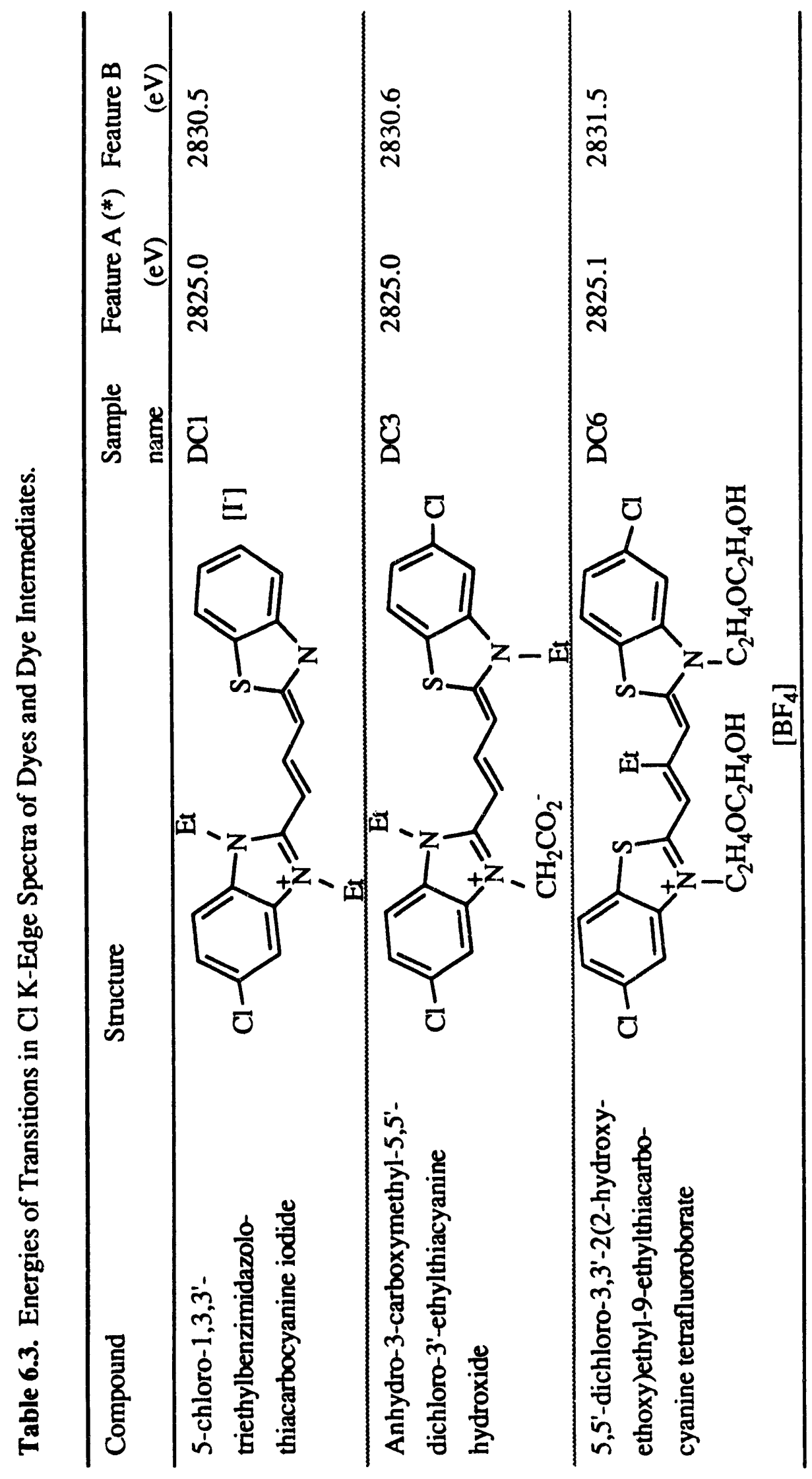




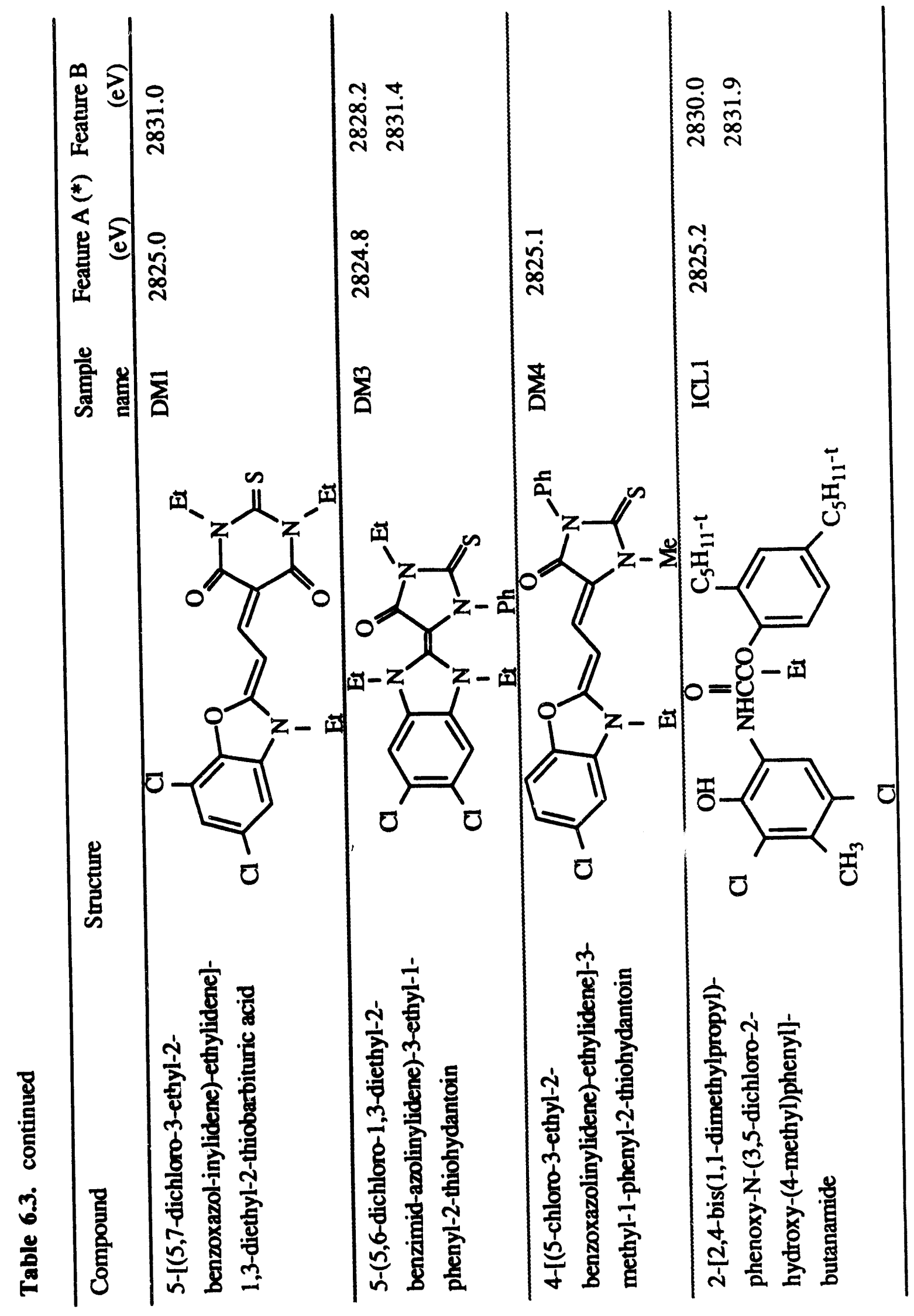




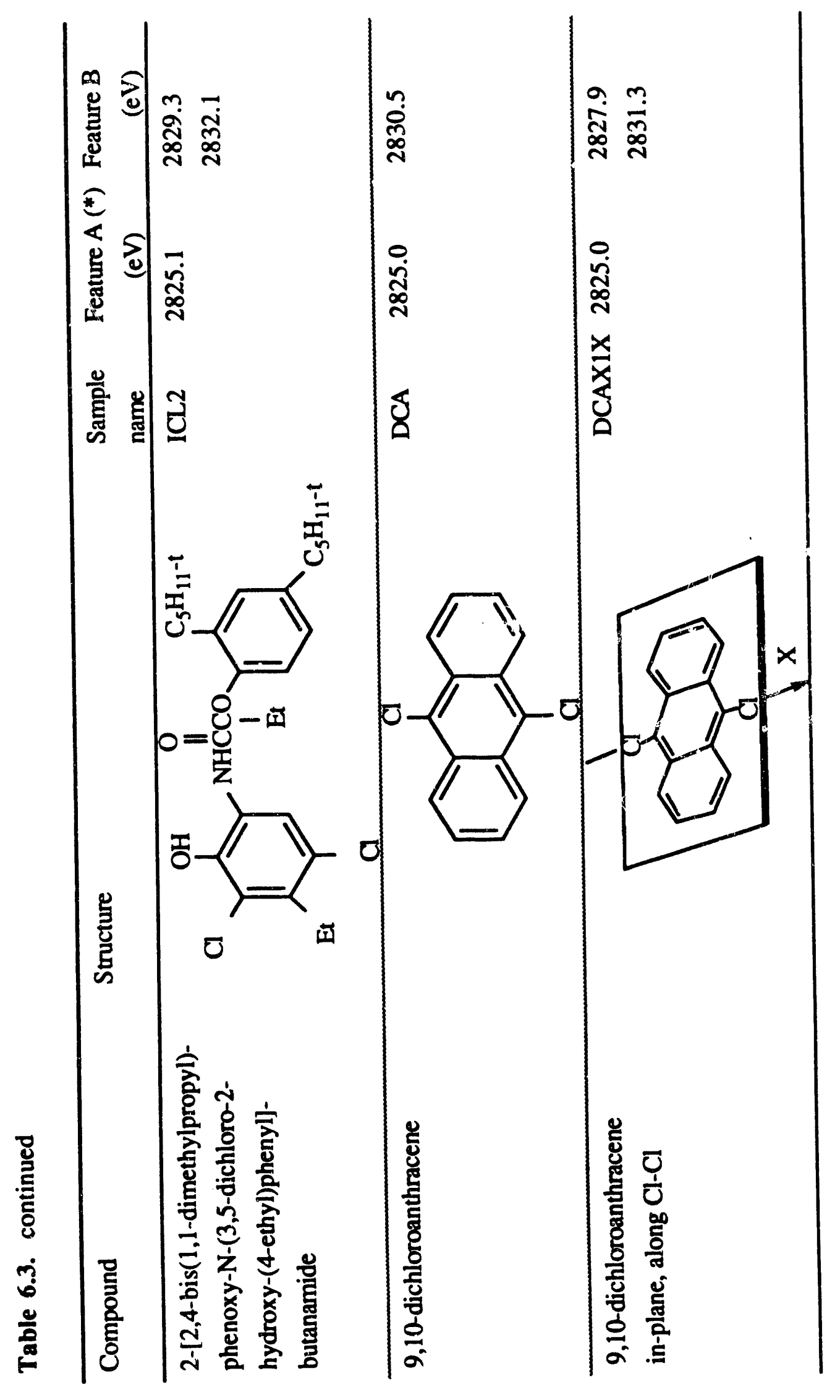




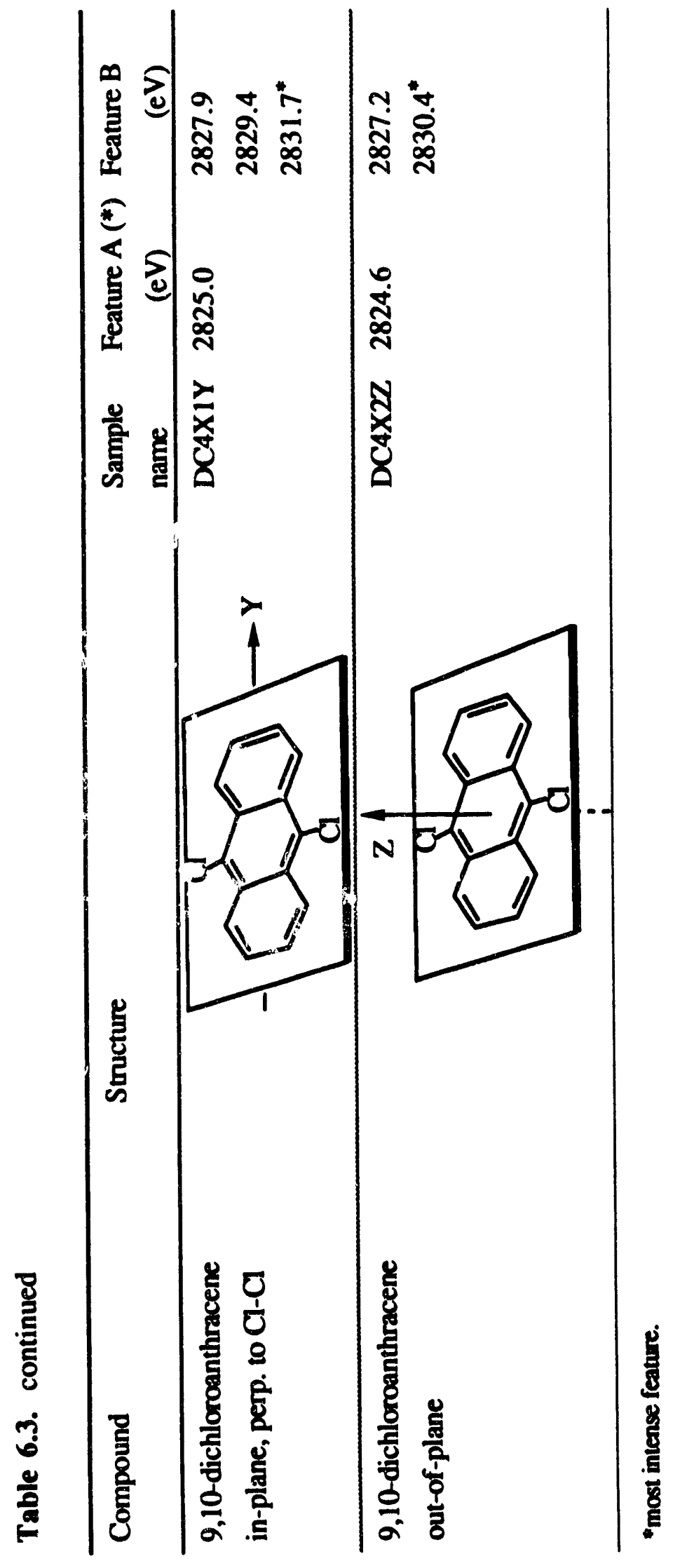


Table 6.4. Energies of Transitions in Silver- and Gold-Sulfur Complexes.

\begin{tabular}{|c|c|c|c|}
\hline Compound & $\begin{array}{l}\text { Sample } \\
\text { name }\end{array}$ & $\begin{array}{c}\text { Feature A } \\
(\mathrm{eV})\end{array}$ & $\begin{array}{c}\text { Feature B } \\
(\mathrm{eV})\end{array}$ \\
\hline $\begin{array}{l}\text { silver (I) benzthiazole } \\
\text { tetrafluoroborate }\end{array}$ & AGBENZ & $2473.3^{*}$ & 2474.5 \\
\hline $\begin{array}{l}\text { silver (I) 3-carboxymethyl-4-methyl-4- } \\
\text { thiazoline-2-thione hydrate } \\
\text { tetrafluoroborate }\end{array}$ & AGCMMT & $\begin{array}{l}2473.7 \\
2471.8(\mathrm{sh})\end{array}$ & 2476.1 \\
\hline $\begin{array}{l}\text { silver (I) phenylmercaptotetrazole } \\
\text { tetrafluoroborate }\end{array}$ & AGPMT & $2473.3^{*}$ & \\
\hline $\begin{array}{l}\text { silver (I) bis(trimethyltriazolium- } \\
\text { thiolate) tetrafluoroborate, monomer }\end{array}$ & AGTRZ & $\begin{array}{l}2473.6^{*} \\
2473.0(\mathrm{sh})\end{array}$ & 2474.8 \\
\hline $\begin{array}{l}\text { silver (I) bis(trimethyltriazolium- } \\
\text { thiolate) tetrafluoroborate, polymer }\end{array}$ & AGTRZ2 & $2473.3^{*}$ & \\
\hline $\begin{array}{l}\text { silver (I) tetris(trimethyluriazolium- } \\
\text { thiolate) tetrafluoroborate, monomer }\end{array}$ & AGTRZ4 & $2473.2^{*}$ & $\begin{array}{l}\text { (sh at } 2472.7 \\
\text { pk at } 2473.6) \\
\end{array}$ \\
\hline $\begin{array}{l}\text { gold (I) bis(ethylenethiourea) chloride } \\
\text { hydrate }\end{array}$ & AUETU2 & $2473.3^{*}$ & 2475.3 \\
\hline $\begin{array}{l}\text { gold (I) bis(ethylenethiourea) chloride } \\
\text { hydrate } \\
\text { in-plane, along C-S }\end{array}$ & AUETU2X & 2473.5 & $2475.3^{*}$ \\
\hline $\begin{array}{l}\text { gold (I) bis(ethylenethiourea) chloride } \\
\text { hydrate } \\
\text { in-plane, along Au-S }\end{array}$ & AUETU2Y & $2473.4^{*}$ & 2475.4 \\
\hline $\begin{array}{l}\text { gold (I) bis(ethylenethiourea) chloride } \\
\text { hydrate } \\
\text { out-of-plane }\end{array}$ & AUETU2Z & $2473.2^{*}$ & $\begin{array}{l}2474.5 \\
2475.6\end{array}$ \\
\hline gold (I) thiourea tetrafluoroborate & AUTHIOUR & $2473.3^{*}$ & 2475.0 \\
\hline $\begin{array}{l}\text { gold (I) tetramethylthiourea } \\
\text { tetrafluoroborate }\end{array}$ & AUTMTU & $\begin{array}{l}2473.3^{*} \\
2472.6(\mathrm{sh})\end{array}$ & 2474.9 \\
\hline $\begin{array}{l}\text { gold (I) bis(trimethyluriazolium thiolate) } \\
\text { tetrafluoroborate }\end{array}$ & AUTRZ2 & $2473.5^{*}$ & 2475.1 \\
\hline $\begin{array}{l}\text { silver (I) gold (I) bis(trimethyl- } \\
\text { triazolium thiolate) tetrafluoroborate }\end{array}$ & AGAUTRZ2 & $2473.6^{*}$ & 2475.4 \\
\hline
\end{tabular}


Table 6.4. continued

\begin{tabular}{lccc}
\hline Compound & $\begin{array}{l}\text { Sample } \\
\text { name }\end{array}$ & $\begin{array}{c}\text { Feature A } \\
(\mathrm{eV})\end{array}$ & $\begin{array}{r}\text { Feature B } \\
(\mathrm{eV})\end{array}$ \\
\hline Metal Sulfides & & & \\
\hline silver (I) sulfide & AG2S & 2471.8 & $2474.1^{*}$ \\
\hline gold (I) sulfide & AU2S & $2472.6^{*}$ & \\
\hline gold (III) sulfide & AU2S3 & $2472.3^{*}$ & \\
\hline (Ph4P)4[Au $\left.12 \mathrm{~S}_{8}\right]$ & & & \\
\hline
\end{tabular}

- most intense feature 
Experiments were conducted under dedicated conditions on the unfocused 8-pole wiggler beamline 4-1 and the focused 54-pole wiggler beamline 6-2 (in low magnetic field mode) at Stanford Synchrotron Radiation Laboratory (3.0 GeV, $40-80 \mathrm{~mA}$ ), and on unfocused bending magnet beamlines X19A and X10C at the National Synchrotron Light Source $(2.5 \mathrm{GeV}, 90-200 \mathrm{~mA}$ ) by using Si (111) double crystal monochromators. Higher harmonics were rejected by detuning the monochromator $80 \%$ at $2740 \mathrm{eV}$ for the sulfur edge and $60 \%$ at $3250 \mathrm{eV}$ for the chlorine edge on beamline 4-1. On beamline 6-2, higher harmonics were rejected by placing 3-4 layers of Al foil between the sample holder and the detector and detuning the monochromator at $2740 \mathrm{eV}$ for $\mathrm{S}$ and $3150 \mathrm{eV}$ for $\mathrm{Cl}$ until the fluorescent signal approached dark current level, resulting in $\sim 20 \%$ detuning. Incident radiation was detuned $20 \%-30 \%$ at $2740 \mathrm{eV}$ on beamlines X19A and X10C. Beam size for the powder samples was defined to be $2 \mathrm{~mm} \mathrm{x} \sim 15 \mathrm{~mm}$ except on beamline 6-2 which was defined by the focusing mirror to be $\sim 1.5 \mathrm{~mm} \times 4.0 \mathrm{~mm}$. For the single crystal samples, the incident beam size was defined to be slightly larger than the size of the crystal in the appropriate orientation. For the polarized surface measurements (performed on beamline 6-2 at SSRL), the width of the beam was determined by the size of the focusing optics. Slits were set to allow the maximum amount of sample to be illuminated by a narrow band of beam $\left(7 \times 1 \mathrm{~mm}\right.$ for $\chi=0^{\circ}, 5 \times 2 \mathrm{~mm}$ for $\left.\chi=90^{\circ}\right)$.

Data were collected at room temperature in fluorescence mode using a $\mathrm{N}_{2}$-filled gas ionization detector of the Stern/Heald/Lytle design. 2,19 Powder samples were finely ground in a mortar and dusted onto Mylar tape to avoid self-absorption effects. At the low energies at which these experiments were conducted $(\sim 2460 \mathrm{eV}$ to $\sim 3200 \mathrm{eV})$, reduction in incident radiation intensity by air absorption is of major concern. To alleviate this problem, the experiment was conducted under a helium beam path for the incident radiation and sample fluorescence. Polypropylene windows of thickness $6.3 \mu \mathrm{m}$ were used where necessary. Scans of $\mathrm{Na}_{2} \mathrm{~S}_{2} \mathrm{O}_{3} \cdot 2 \mathrm{H}_{2} \mathrm{O}$ collected between sample measurements were used to calibrate the energy of the sample spectra by assigning the position of the first peak in the thiosulfate scan at $2472.02 \mathrm{eV}$. For each sample, 2-5 calibrated scans were averaged and the inherent background in the data was removed by fitting a polynomial to the pre-edge region which was extrapolated through the entire spectrum and subtracted. The data were normalized to an edge jump of unity for direct comparisons of intensities of features. The positions of the edge features were determined by locating the position of the half-width at half-maximum of the second derivative in the region of the feature of interest, and are listed in Tables 6.1-6.4. 


\subsubsection{Single-Crystal Polarized Studies}

For dipole-allowed transitions, the features seen in the K-edge spectra are governed by the following relation:

$$
\sigma=K \Psi_{\text {fle }} \cdot r\left|\Psi_{\mathrm{i}}\right\rangle \mid 2
$$

which can be approximated by:

$$
\sigma=\cos ^{2} \theta\left|\left\langle\Psi_{\mathrm{f}}|\mathbf{r}| \Psi_{\mathrm{i}}\right\rangle\right| 2
$$

where $\sigma$ is the photoabsorption cross section, $\Psi_{\mathrm{f}}$ is the final state wave function, $\Psi_{\mathrm{i}}$ is the initial state wave function ( $\mathrm{S}$ or $\mathrm{Cl}$ 1s orbital), $\mathrm{e}$ is the polarization vector of the incident radiation (perpendicular to the direction of the incident radiation and in the plane of the synchrotron ring), $\mathbf{r}$ is the transition dipole operator $(\mathbf{x}, \mathbf{y}$, or $\mathbf{z})$ and $\theta$ is the angle between $e$ and $r$. The dipole-allowed transition from an initial $1 \mathrm{~s} \mathrm{state} \mathrm{is} \mathrm{to} \mathrm{a} \mathrm{final} p$ state $(\Delta l= \pm 1)$, and the maximum in the photoabsorption cross section is obtained when $\theta=0^{\circ}$, when the polarization vector and the dipole operator are parallel. By aligning a molecular orientation in the sample (for example, a C-S bond) with the direction of the incident radiation polarization vector, one can selectively excite transitions into the orbitals along that molecular orientation. The symmetry of the transitions ( $p \sigma$ or $p \pi$ ) seen in the anisotropic powder spectrum can be therefore be determined by observing the polarization properties of the various transitions as a function of molecular alignment.

Single crystal samples were mounted on a glass fiber using polymethylmethacrylate in methylene chloride as the adhesive for the S-containing crystals, and a standard 5 minute epoxy (which contains S) for the Cl-containing crystals, and attached on a standard goniometer head. On the beamline, a lid to the sample chamber containing a goniometer mount was used allowing full rotation in phi with chi fixed at $180^{\circ}$. The samples were preoriented on a Syntex P2 1 4-circle diffractometer in such a way that the molecular orientation of interest could be isolated solely by rotations in phi. This geometric restriction meant that more than one crystal was required to isolate all of the orientations of interest. For 2-thiohydantoin and 9,10-dichloroanthracene, two crystals were used to isolate three orientations. For bis(ethylenethiourea) Au (I) and 3,3'-diethylthiacyanine bromide, one crystal per orientation was required, for a total of three crystals for AUETU2, and two crystals for DC5. The largest source of error in these polarized measurements is due to 
misalignment of the samples in the beam, however we estimate that the maximum misalignment of the goniometer mount on the beamline is $2^{\circ}$, resulting in less than a $5 \%$ loss of polarization. After the data at the proper phi setting were measured, the crystal was misaligned $2-5^{\circ}$ to insure that the features noted were not due to Bragg diffraction peaks from the crystal lattice planes.

\subsubsection{Polarized Surface Measurements}

Surface $X$-ray measurements at glancing angle configurations take advantage of the fact that $X$-rays undergo total external reflection below the critical angle, $\Theta_{c}$, and therefore penetrate only 20 - $30 \AA$ into the surface. ${ }^{20}$ At angles less than the critical angle, the signal of an adsorbed species on the surface of a substrate is enhanced relative to that of the substrate due to the decreasing penetration depth of the incident radiation. The critical angle is dependent on the type of substrate the $X$-rays reflect from and is governed by the following equation for $\Theta_{c}$ in radians: 21

$$
\Theta_{c}=\lambda\left(5.4 \times 10^{10} \frac{Z \rho}{A}\right)^{1 / 2}
$$

where $\lambda$ is the wavelength of radiation $(\mathrm{cm})$, and $Z$ is the atomic number, $\rho$ the densi, and A the atomic weight of the substrate. For a $\mathrm{AgBr}$ substrate, the critical angle (in degrees) ranges from 1.13 at the $S \mathrm{~K}$-edge $(2470 \mathrm{eV})$, to 1.02 at the end of a typical sulfur scan $(2740 \mathrm{eV})$, and from 0.987 at the $\mathrm{Cl} \mathrm{K}$-edge $(2820 \mathrm{eV})$ to 0.883 at the end of a chlorine scan $(3150 \mathrm{eV})$. The measurements reported for this work were done at angles of $1^{\circ}$, which was sufficient at all energies to obtain an acceptable signal-to-noise level. There has been a study of low- $Z$ adsorbates on high- $Z$ substrates using fluorescence detection in which the authors found that a glancing angle configuration was not required for the characterization of the surface species. 22 This will be tested on this system as well. The surface samples were mounted on a sample plate attached to a rotation stage, thereby allowing full phi rotations to be made (in the azimuthal plane). The sample plate and rotation stage were placed on a $90^{\circ}$ chi circle mounted perpendicular to the beam to allow both in-plane and out-of-plane orientations to be collected at grazing incidence angles (Figure 6.1). 
a

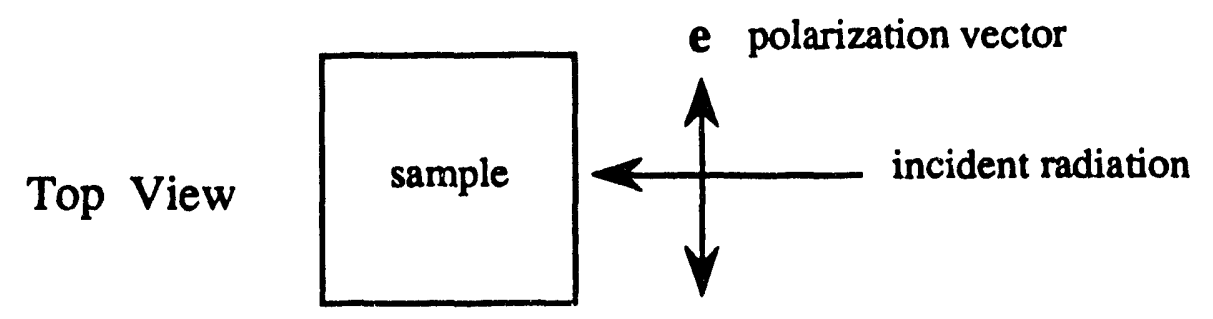

Side View

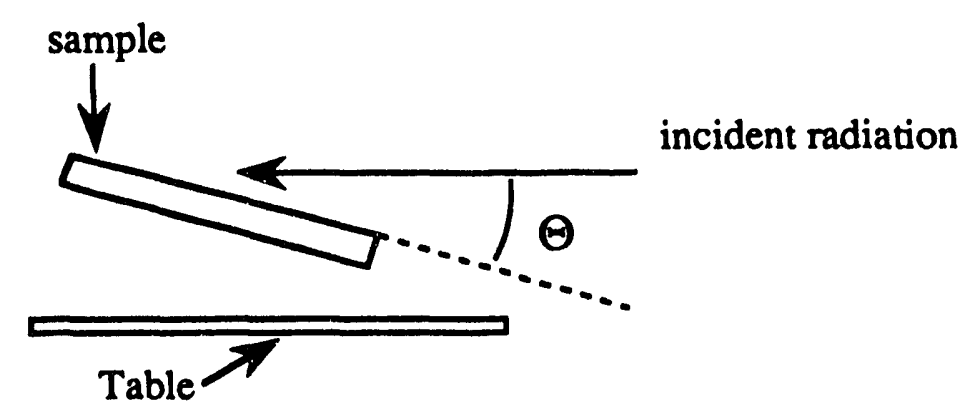

b

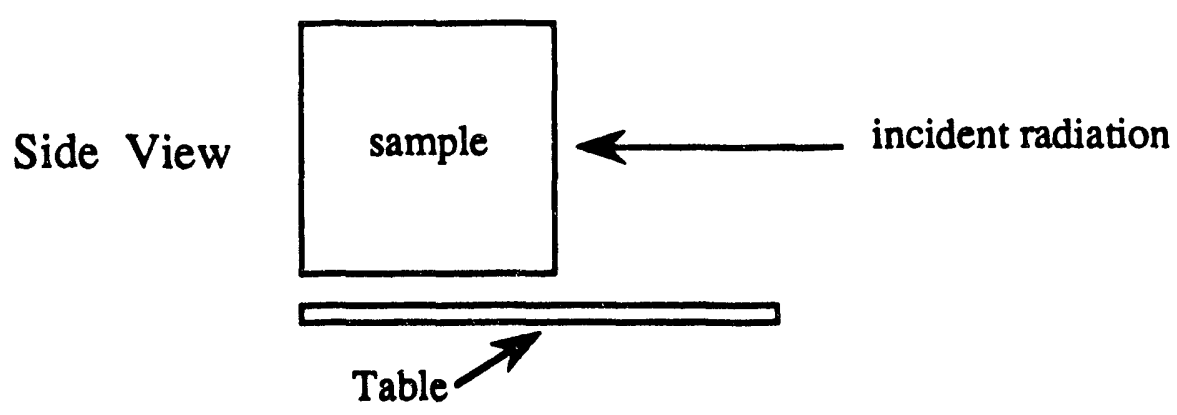

Figure 6.1. Schematic of the geometry utilized for the surface measurements. $\chi$ refers to the orientation of the surface relative to the table and $\theta$ refers to the angle of the surface relative to the incident radiation. (a) $\chi=0^{\circ}$ orientation. (b) $\chi=90^{\circ}$ orientation. 


\subsection{Results}

\subsubsection{S K-Edge Spectra of Dye Molecules}

6.3.1.1. Cyanine Dye Molecules. Cyanine spectral sensitizing dyes contain cyclic sulfur in 5-membered rings such as thiazole, benzthiazole and napththiazole with various ring substituents and bridges between the dye nuclei (Table 6.1). The sulfur spectra of cyanine molecules consists of two features (Figure 6.2), a high-intensity feature at $2473.3-2474.1 \mathrm{eV}$ (Feature A) and a lower-intensity transition at $2475.2-2476.4 \mathrm{eV}$ (Feature B). The relative intensities of these features and the shape of the multiple scattering regime (above $\sim 2478 \mathrm{eV}$ ) reflects changes in the local environment of the $S$ atom. The energy of Features A and B are relatively invariant, however the features appear at a slightly higher energy for cyanine-type molecules which contain aromatic groups, such as the benzthiazole (IC2, DC1, DC2, DC3, DC5, DC6) and napththiazole (IC3) derivatives (Feature A at 2473.8 - 2474.0 eV; Feature B at $2475.8-2476.4 \mathrm{eV}$ ) relative to the nonaromatic thiazole compounds (IC1, DC4: Feature A at 2473.3 - $2473.5 \mathrm{eV}$; Feature B at $2475.2-2475.3 \mathrm{eV}$ ). This suggests that the presence of the electron-withdrawing aromatic groups results in an effective positive charge on the sulfur atom relative to the non-aromatic group-containing dyes and dye intermediates.

Single-crystal polarized spectra of the benzthiazole-containing dye molecule 3,3'diethylthiacyanine bromide are presented in Figure 6.3. Spectra were obtained for two polarizations, one corresponding to polarization in the plane defined by the benzthiazole group (DC5X), and the other corresponding to polarization along the average normal to that plane (DC5Z). The molecule is not completely planar, with a dihedral angle of $\sim 13^{\circ}$ between the thiazoline rings, therefore the polarizations of this molecule could not be completely isolated. In the powder spectrum, the most intense feature occurs at $2473.8 \mathrm{eV}$, and Feature B appears at 2475.6 with a shoulder at $2476.4 \mathrm{eV}$. In the in-plane polarized spectrum, the white-line feature occurs at $2473.5 \mathrm{eV}$, and in the out-of-plane polarized spectrum at $2473.9 \mathrm{eV}$. The shoulder at around $2475.6 \mathrm{eV}$ is also present in both polarized spectra, however it occurs as a single feature in the in-plane polarized spectrum at 2475.1 $\mathrm{eV}$, and as a doublet in the out-of-plane spectrum at 2475.6 and $2476.5 \mathrm{eV}$. The white-line feature is somewhat more intense in the in-plane polarized spectrum than in the out-of-plane spectrum, suggesting that the contribution to the S K-edge absorption features of 3,3'diethylthiacyanine bromide, and by extension to the other molecules with $\mathrm{S}$ heterocyclic groups, involves transitions to final states with po type symmetry. The presence of the $2473.9 \mathrm{eV}$ feature in the out-of-plane spectrum might be due to the inability to completely 


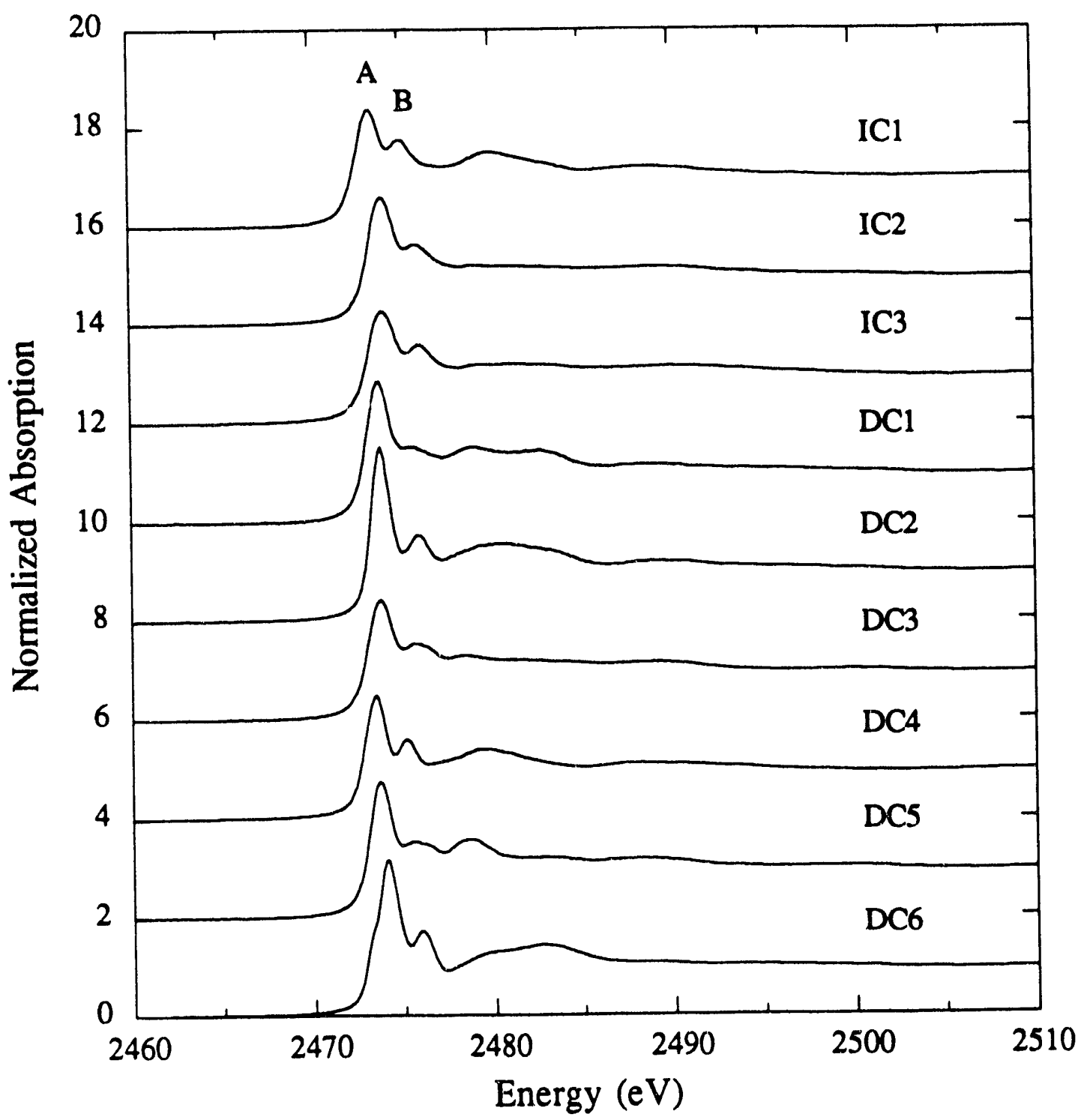

Figure 6.2. The S K-edge spectra of cyanine dyes and dye intermediates (Table 6.1). 


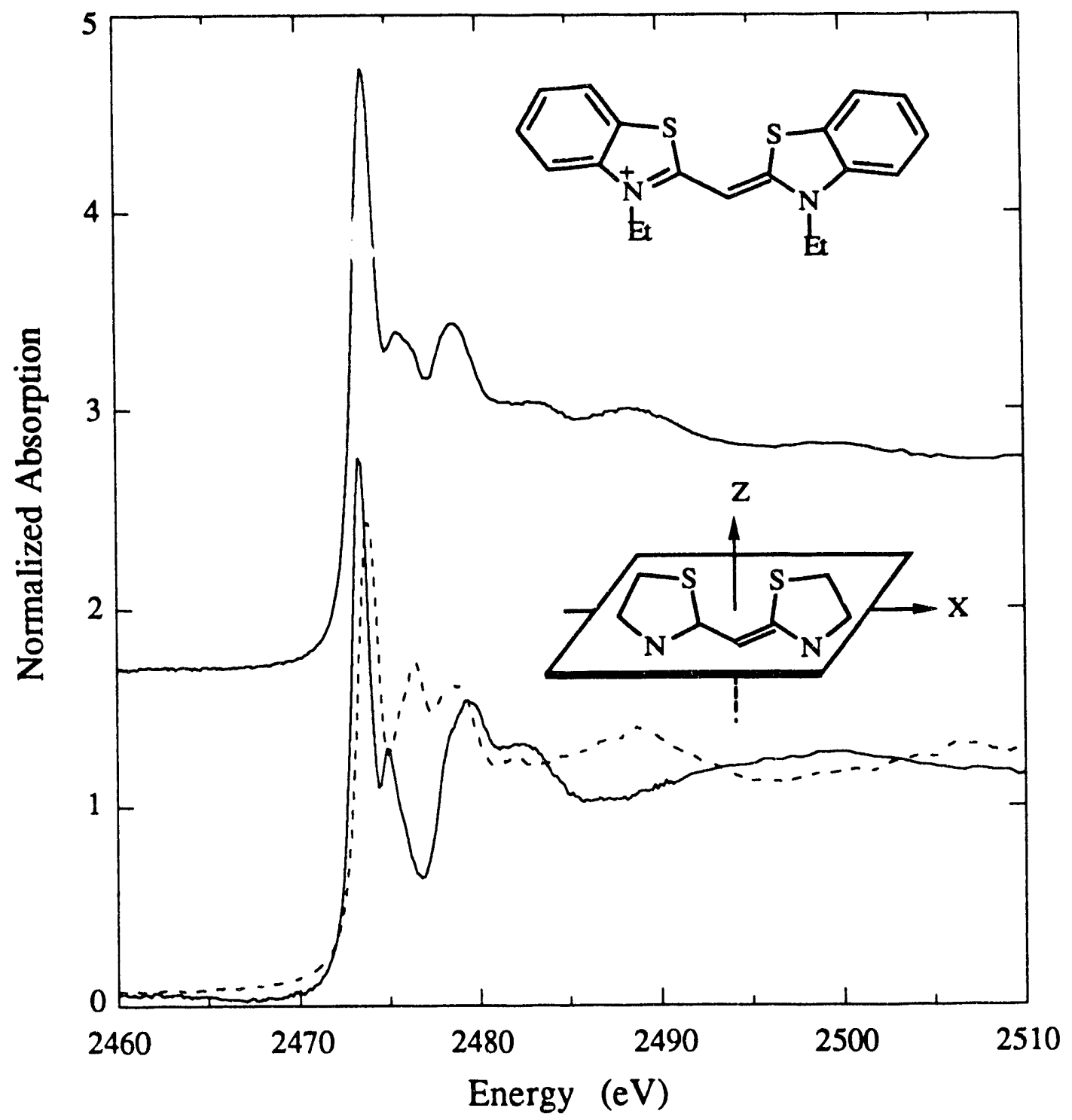

Figure 6.3. The powder spectrum (top) of DC5, a cyanine dye molecule, compared with the single-crystal spectra (bottom) corresponding to polarization along the average $\mathrm{X}$ (solid) and $\mathrm{Z}$ (dash) molecular axes. 
isolate the out-of-plane orientation from the in-plane orientation due to the packing of the molecules in the crystal structure of this compound, or it could reflect a transition to an orbital oriented along the normal to the benzthiazole plane (discussed below).

These studies on the cyanine dye molecules are consistent with the results of the soft $\mathrm{X}$-ray studies and calculations done for a variety of $\mathrm{S}$ heterocycles, thiols and thioethers. 6,7 In these studies, the dominant feature in the $\mathrm{S} \mathrm{K}$-edge spectra occurs between 2472.3 and $2473.4 \mathrm{eV}$ (relative to the $\mathrm{t}_{1 \mathrm{u}}$ resonance of $\mathrm{SF}_{6}$ at $2486 \mathrm{eV}$ ), similar to the location of the strongest feature in the cyanine dyes and dye intermediaries. This feature has been assigned to a transition to a S-C final state with $\sigma^{*}$ symmetry, $2,6,7$ which supports the qualitative determination of the symmetry of this feature based on our polarized singlecrystal study. One explanation for the $2473.9 \mathrm{eV}$ feature in the $Z$, or out-of-plane orientation of 3,3'-diethylthiacyanine bromide is that there is a final state orbital with $\pi$ character that accounts for some of the intensity of the primary feature in the powder spectrum. In a study of the aromatic molecule thiophene, ${ }^{6 \mathrm{a}} \mathrm{MS}-\mathrm{X} \alpha$ calculations show that the lowest unoccupied valence orbital has $\pi^{*}$ symmetry and occurs at approximately the same energy as the S-C $\sigma^{*}$ orbital. The aromatic character of thiophene allows appreciable delocalization of the $\pi^{*}$ level onto the sulfur atom, accounting for approximately $25 \%$ of the intensity of the $2473 \mathrm{eV}$ feature, with the balance being accounted for by the transition to the S-C $\sigma^{*}$ orbital. It is therefore likely that there is a contribution to the white-line feature from a transition to a final state with p $\pi$ symmetry due to the extended conjugated system of 3,3'-diethylthiacyanine bromide, and that the presence of the $2473.9 \mathrm{eV}$ feature in the outof-plane polarized spectrum is not due solely to the crystallographic limitations on isol..ing the out-of-plane orientation from the in-plane orientation.

6.3.1.2. Merocyanine Dye Molecules. Merocyanine spectral sensitizing dyes contain sulfur as a terminal thione group on 5- or 6-membered heterocyclic rings such as thiohydantoin, benzoxazole, benzimidazole, or thiobarbituric acid (Table 6.2). Like cyanine dyes, the merocyanine dye spectra have a feature between 2473.4 and $2474.0 \mathrm{eV}$ (Feature A), which has been attributed to a transition to a final state with po symmetry (Figure 6.4). However, this feature is less intense relative to the edge jump in the merocyanine dye spectra than in the cyanine dye spectra (see Figure 6.6a). The shoulder on the high energy side of Feature $A$ in the cyanine dye spectra is absent in the merocyanine dye spectra, however a broad feature which in some cases has a low energy shoulder, is seen in all of the merocyanine dye spectra (2475.4 - $2478.2 \mathrm{eV}$, Feature B). The most striking difference between the merocyanine and cyanine dye spectra occurs to the low energy side of Feature A. The merocyanine dye spectra are characterized by a sharp feature 


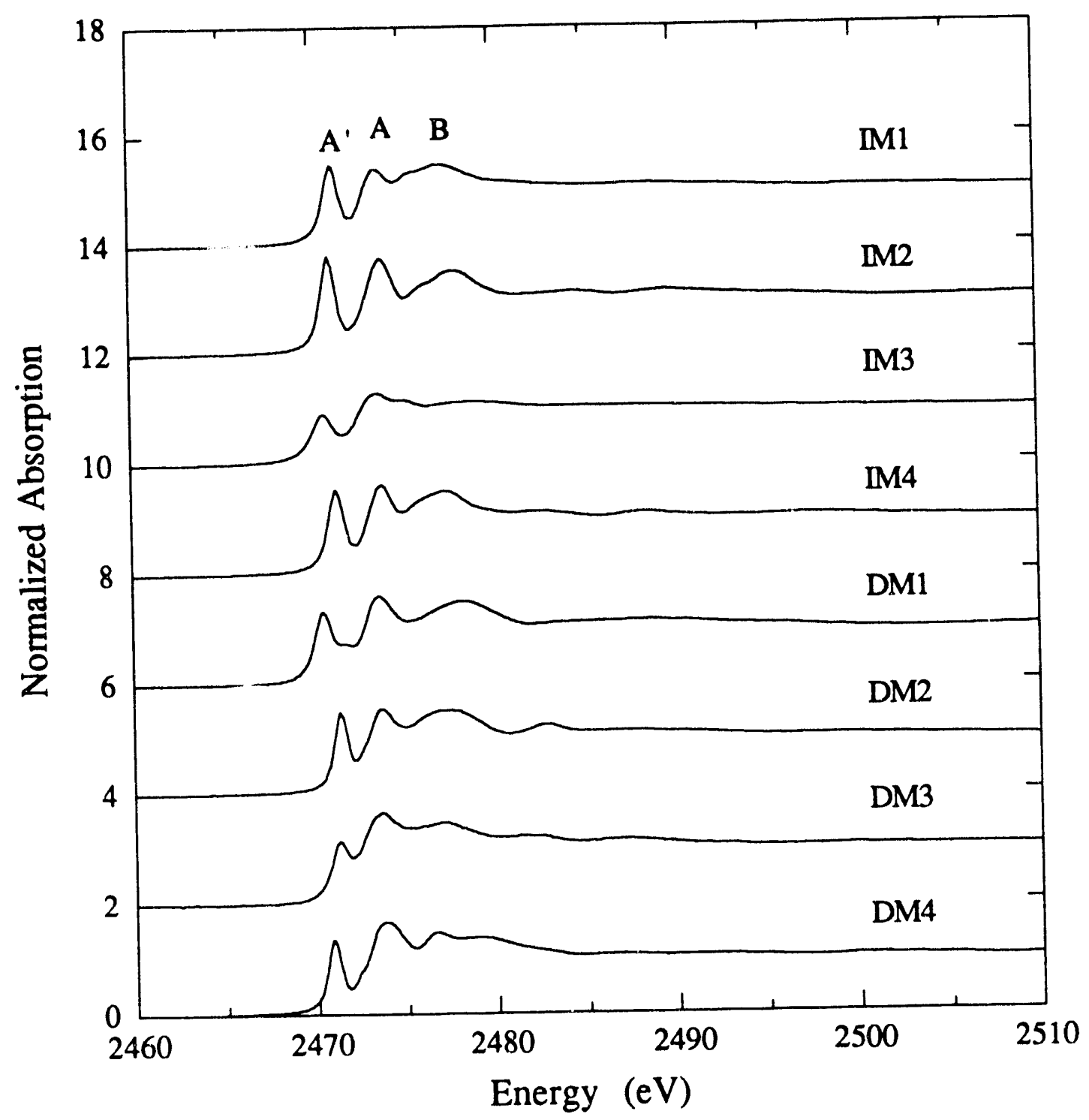

Figure 6.4. The S K-edge spectra of merocyanine dyes and dye intermediates (Table 6.2 ). 
(Feature $\mathrm{A}^{\prime}$ ) at $2470.5-2472.5 \mathrm{eV}$, which is unique to the presence of the terminal thione group.

The single-crystal polarized spectra for 2-thiohydantoin, a merocyanine dye intermediate, are presented in Figure 6.5. Three orientations were isolated, corresponding to polarization in the plane of the ring and along the $\mathrm{C}=\mathrm{S}$ bond ( $\mathrm{X}$ polarization), in the plane and perpendicular to the $\mathrm{C}=\mathrm{S}$ bond ( $\mathrm{Y}$ polarization), and perpendicular to the plane and the $\mathrm{C}=\mathrm{S}$ bond ( $\mathrm{Z}$ polarization). The $\mathrm{Z}$ orientation is $100 \%$ polarized normal to the plane of the 2-thiohydantoin molecule, while the $\mathrm{X}$ and $\mathrm{Y}$ orientations are each $70 \%$ polarized. The spectra reveal that the features in the powder spectrum have different polarization properties. The sharp pre-edge feature at $2471.2 \mathrm{eV}$, characteristic of the thione sulfur, is polarized almost entirely along the normal to the plane of the 2thiohydantoin molecule, while the white-line feature at $2473.7 \mathrm{eV}$ is polarized in the plane of the molecule, predominantly along the $\mathrm{C}=\mathrm{S}$ bond. This is consistent with the results of the single-crystal polarized study of the cyanine dye molecule, in which the $\sim 2474 \mathrm{eV}$ white-line feature corresponds to a transition to a final state with po symmetry. The sharp pre-edge feature, on the other hand, can be accounted for by a transition to a state with $\mathrm{p} \pi$ symmetry due to the presence of unfilled $\mathrm{p} \pi^{*}$ orbitals for molecules of this type.

The compounds 3-ethylrhodanine and 3-carboxymethyl-1,4-methyl-4-thiazoline-2thione contain sulfur in both a heterocyclic and exocyclic form (IM3 and CMMT, respectively, Table 6.2). The spectra are a superposition of the features characteristic of both the thione and thiazole $S$, with a pre-edge feature characteristic of the thione $S$ and a white-line intensity similar to that of the cyclic sulfur cyanine dyes (Figure 6.6).

In addition to the merocyanine dye and dye intermediates discussed above, spectra were collected on a variety of thioureas, mercaptotetrazoles and mercaptotriazoles (Figure 6.7). In the thioureas, the sulfur is present as a thione, similar to the merocyanine dyes, while the mercaptotetrazoles and triazoles contain sulfur as a thiol (Table 6.2). Although Feature $A^{\prime}$ is present in all of the spectra, its position and resolution from Feature $A$ is sample dependent. In the thione-containing compounds, the feature occurs between 2470.5 and $2472.0 \mathrm{eV}$. Upon protonation of the thione sulfur to a thiol group, the position of the foatlure shifts to higher energy and occurs at $2471.8 \mathrm{eV}$ for the phenyl-substituted mercaptotetrazoles (PMT and APMT) and at $2472.5 \mathrm{eV}$ for ethylmercaptotetrazole and trimethyltriazolium thiolate (EMT and TRZ). Upon deprotonation of the thiolate in the sodium salt of 2-acetamidophenylmercaptotetrazole (APMTNA), the pre-edge feature appears as a poorly resolved shoulder on the rising edge at $2472.4 \mathrm{eV}$ (Figure 6.8).

There have been a few $\mathrm{S}$ K-edge studies of compounds containing a terminal sulfur group. $6 \mathrm{~b}-\mathrm{c}, 7$ Both $\mathrm{CS}_{2}$ and SCO have features below $2473 \mathrm{eV}$ (at 2470.8 for $\mathrm{CS}_{2}$ and 


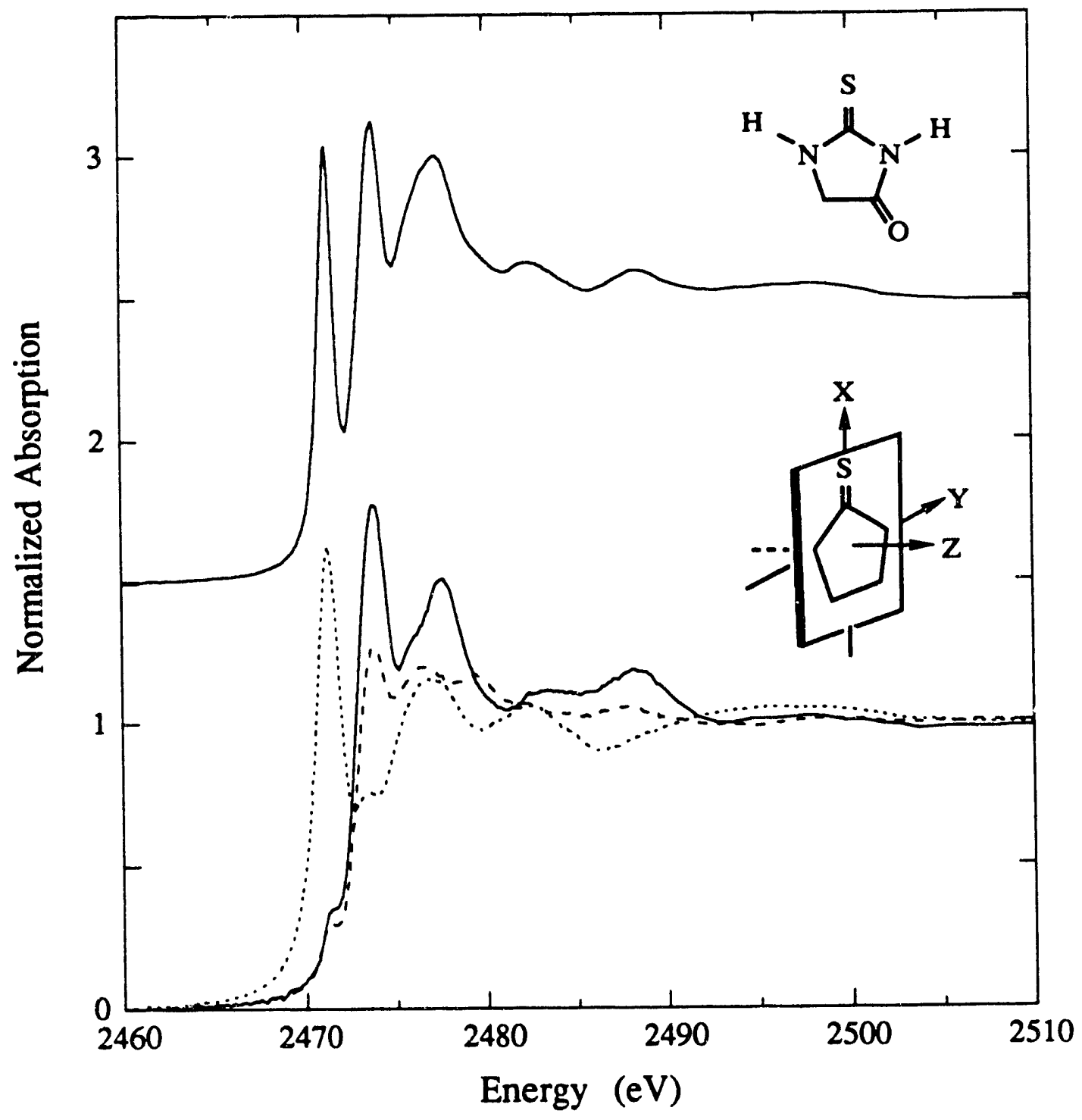

Figure 6.5. The powder spectrum (top) of IM4, a merocyanine dye intermediate, compared with the single-crystal spectra (bottom) corresponding to polarization along the average $\mathrm{X}$ (solid), $\mathrm{Y}$ (dash), and $\mathrm{Z}$ (dot) molecular axes. 


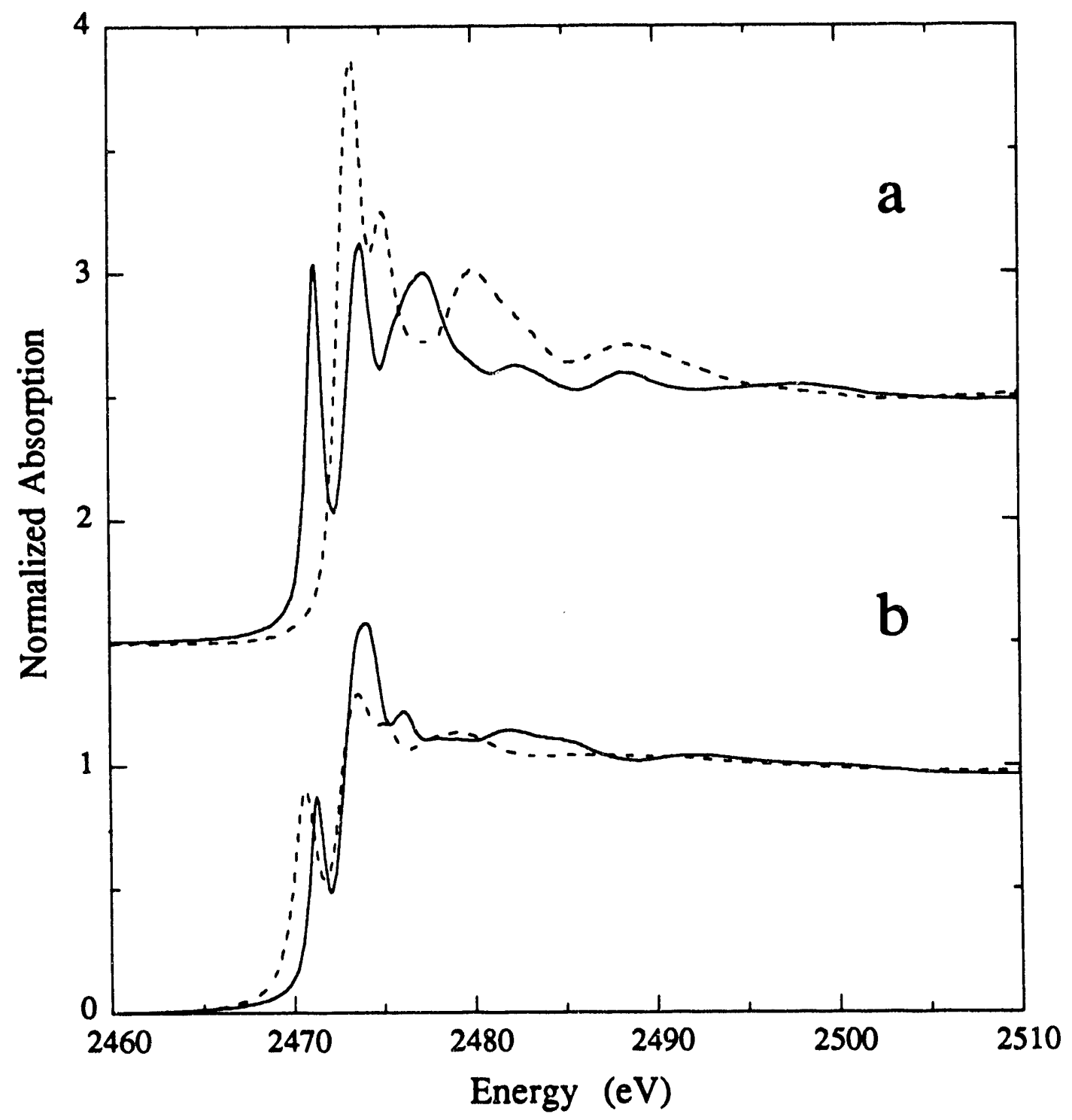

Figure 6.6. The spectra (a) of IM4 (solid), a merocyanine dye intermediate, and ICl (dash), a cyanine dye intermediate compared to the spectra (b) of CMMT (solid) and IM3 (dash) which contain $\mathrm{S}$ both in a cyclic and exocyclic form. 


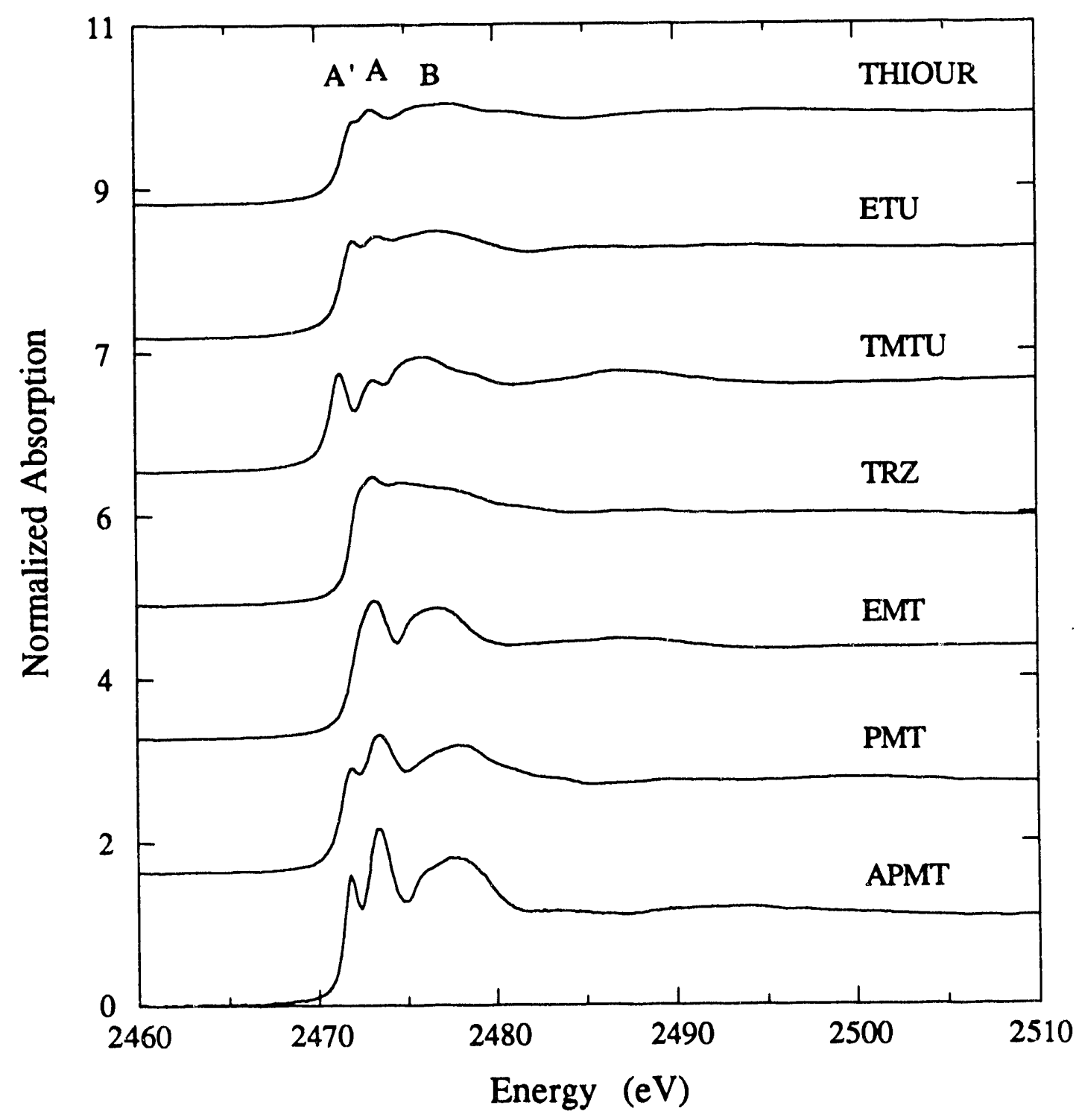

Figure 6.7. The S K-edge spectra of thioureas, mercaptotetrazoles and mercaptotriazoles (Table 6.2). 


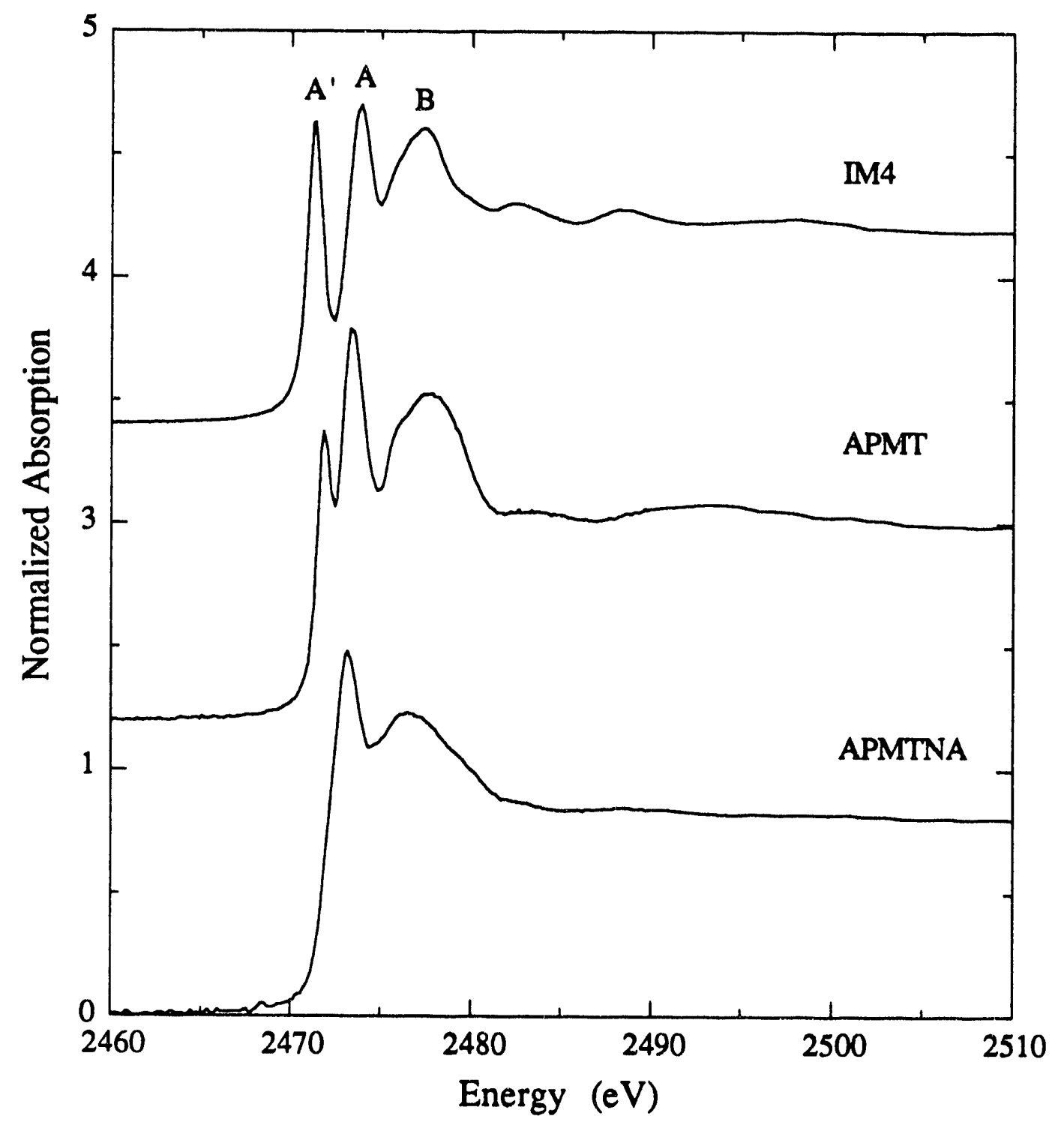

Figure 6.8. The position of the thione-feature is compared for $\mathbf{S}$ present as a thione (top, IM4), a thiol (middle, APMT), and a thiolate (bottom, APMTNA). See Table 6.2 for the structures. 
2472.0 for SCO), which have been assigned to transitions with final states of $\pi$ symmetry, consistent with the qualitative results of the polarized single-crystal measurements on 2thiohydantoin reported herein. In contrast to the thiols studied here, there is no low energy pre-edge feature seen in the S K-edge spectra of the alkyl thiols studied by Dezarnaud et. al. $6 \mathrm{~b}$ The primary difference between the alkyl thiols and the thiols studied in this work is the presence of the conjugated system in the mercaptotetrazoles and -triazoles. The low energy feature may thus reflect the involvement of the terminal $S$ group in some sort of a $\pi$ system, whether it be a double bond to $\mathrm{C}$ as in the merocyanine dye molecules and the thioureas, or the presence of a conjugated $\pi$ system such as in the mercaptotetrazoles and -triazoles. The factors involved in determining the relative intensities and the energies of the terminal sulfur low energy feature studied in this work have not yet been established. Qualitatively, the position of this feature moves to higher energy from thione to thiol to thiolate (Figure 6.8), suggesting that the position of the low energy feature is related at least in part to the accumulation of negative charge on the terminal sulfur.

\subsubsection{Cl K-Edge Spectra of Dye Molecules}

Chlorine is present as a substituent on both merocyanine and cyanine dye molecules (Table 6.3) and exiatances the aggregation of the dye molecules resulting in the desired spectral shift to longer wavelengths. The $\mathrm{Cl}$ spectra are similar in all cases (Figure 6.9), with a white line transition between 2824.8 and $2825.1 \mathrm{eV}$, and a broad feature occurring between 2829.1 and $2830.6 \mathrm{eV}$.

To understand the polarization properties of $\mathrm{Cl}$ as a substituent on dye molecules, single-crystal polarized studies on 9,10-dichloroanthracene (DCA) were performed. The powder spectrum of DCA has the same spectral features as the $\mathrm{Cl} \mathrm{K}$-edge spectra of the dye and dye intermediate samples, making it an appropriate choice for the single crystal studies. The polarized spectra are presented along with the powder spectrum in Figure 6.10. The three spectra correspond to having the polarization vector in the plane of the molecule and along the $\mathrm{Cl}-\mathrm{Cl}$ vector ( $\mathrm{X}$ orientation), in the plane of the molecule and perpendicular to the $\mathrm{Cl}-\mathrm{Cl}$ vector ( $\mathrm{Y}$ orientation), and perpendicular to the plane of the molecule ( $\mathrm{Z}$ orientation). The features in the powder spectra are present in all three of the polarized spectra, however the $\mathrm{X}$ orientation shows the highest intensity of the three spectra. In the $\mathrm{Z}$ polarized spectrum, the white-line feature is shifted to slightly lower energy, to $2824.6 \mathrm{eV}$ and is a much narrower transition than for the in-plane oriented spectra, indicating that there may be multiple transitions occurring in the in-plane orientation. These results suggest that the white-line feature of compounds containing $\mathrm{Cl}$ in this kind of an environment is primarily 


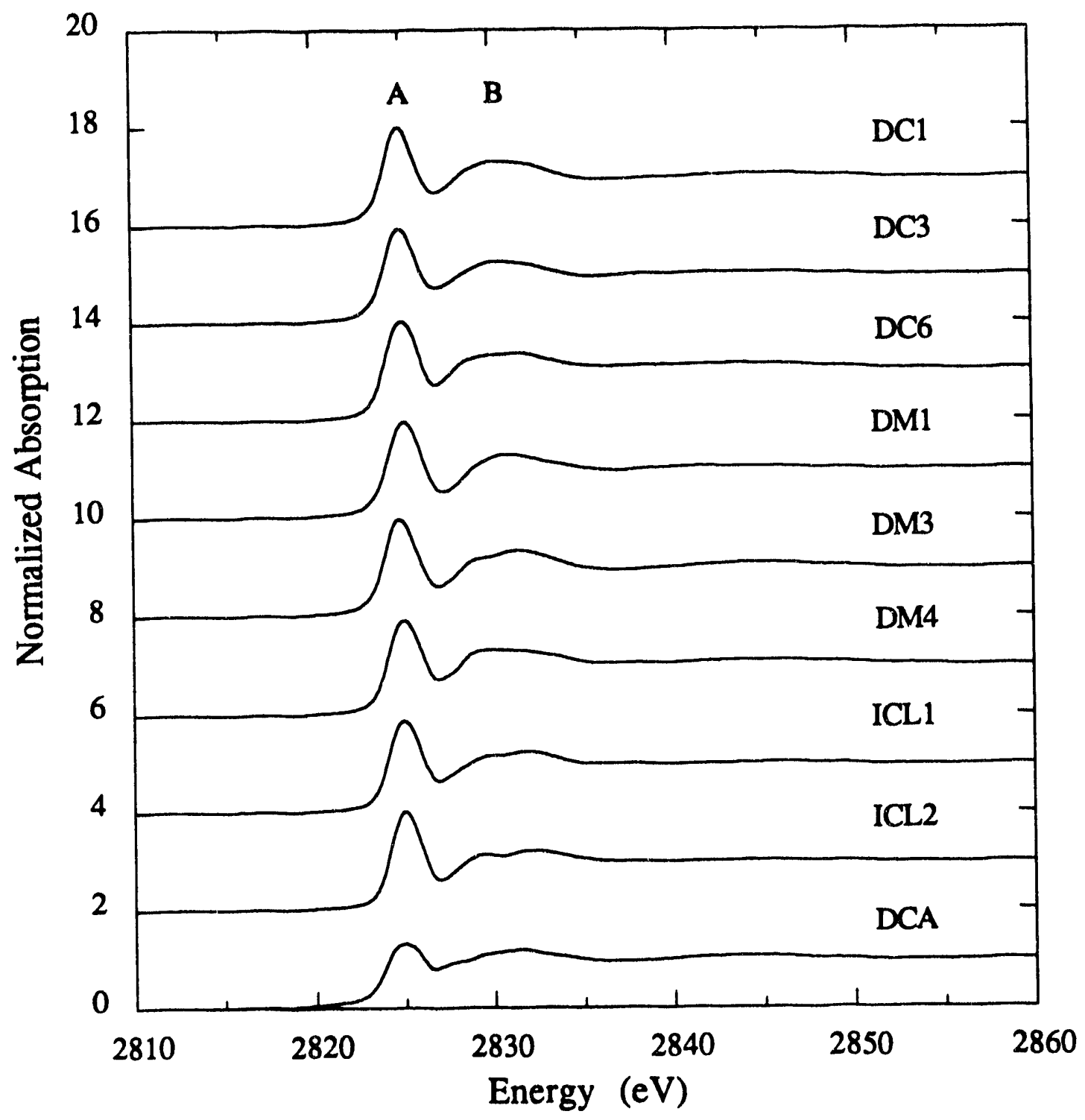

Figure 6.9. The $\mathrm{Cl} \mathrm{K}$-edge spectra of dyes and dye-intermediates (Table 6.3). 


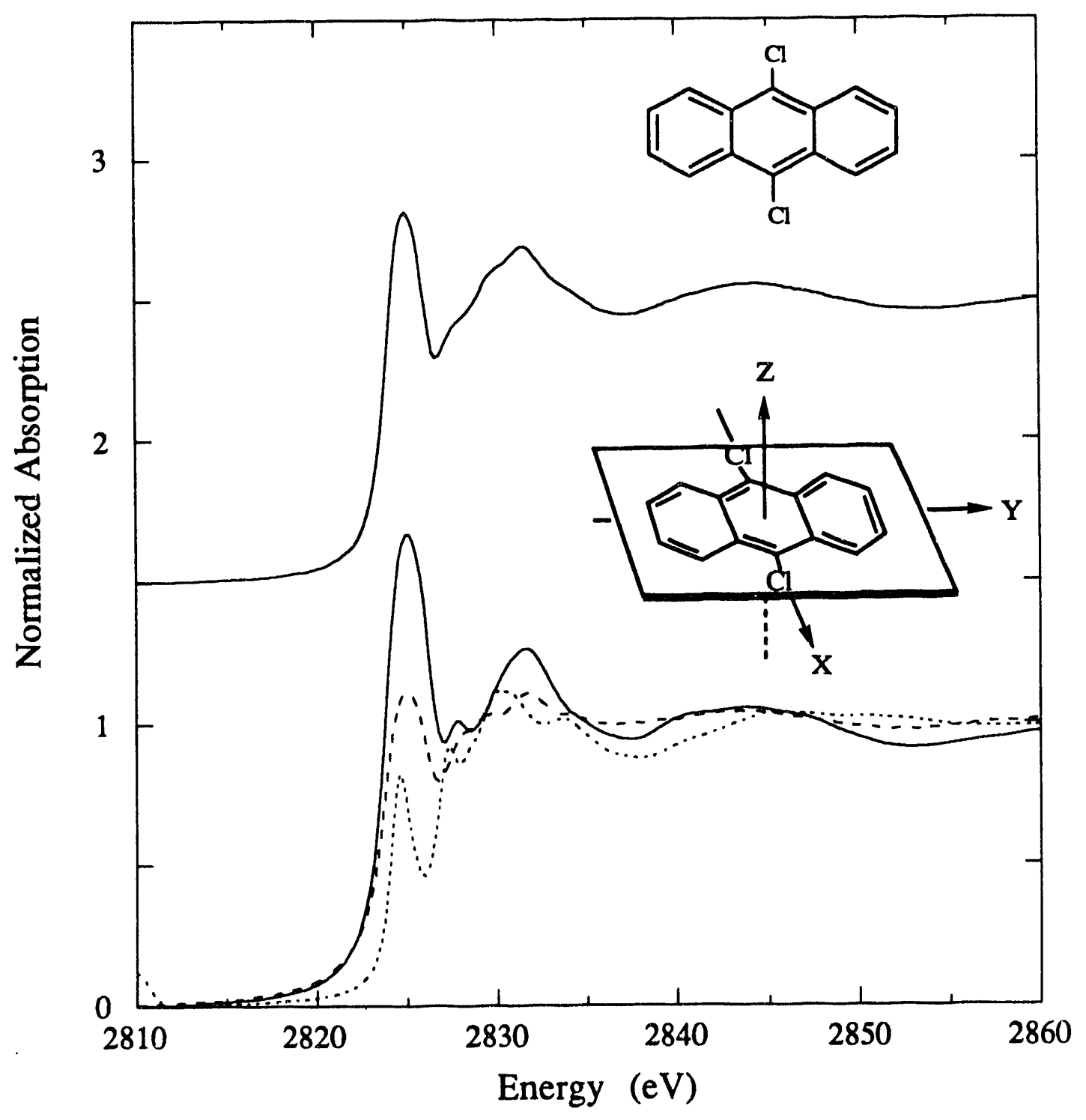

Figure 6.10. The $\mathrm{Cl}$ K-edge powder spectrum (top) of DCA, compared with the singlecrystal spectra (bottom) corresponding to polarization along the average $X$ (solid), $Y$ (dash), and $\mathrm{Z}$ (dot) molecular axes. 
due to a transition to a final state orbital with $\sigma$ symmetry. The presence of the feature in the out-of-plane polarized spectrum could he due to a transition to a $\pi$ symmetry orbital due to the aromatic nature of 9,10-dichloroanthracene, similar to the case of thiophene. $6 \mathrm{a}$

There have been no other studies of $\mathrm{Cl}$ present as it is in the dye systems studied here, however $\mathrm{Cl} \mathrm{K}$-edge spectra have been reported for $\mathrm{SCl}_{2}, \mathrm{~S}_{2} \mathrm{Cl}_{2}, \mathrm{SOCl}_{2}$, and $\mathrm{SO}_{2} \mathrm{Cl}_{2}$. ${ }^{\mathrm{d}}$-e For these compounds, the $\mathrm{Cl} \mathrm{K}$-edge spectra is similar to the $\mathrm{Cl} \mathrm{K}$-edge spectra of the dye molecules studied here, with the most intense feature occurring between 2821.4 and $2822.5 \mathrm{eV}$. This feature has been assigned as a transition to a final state with $\mathrm{S}-\mathrm{Cl} \sigma^{*}$ symmetry, supporting the qualitative conclusion that the white line feature in the $\mathrm{Cl}$ $\mathrm{K}$-edge of the dye molecules can be attributed to a transition to a final state with $\sigma$ symmetry.

\subsubsection{Au and Ag Complexes with Sulfur-Containing Ligands}

$\mathrm{S}$ K-edge XAS measurements were done on a wide variety of complexes formed between $\mathrm{Ag}$ or $\mathrm{Au}$ and $\mathrm{S}$ present as a thione group (3-carboxymethyl-4-methyl-4thiazoline-2-thione, thiourea, ethylenethiourea and trimethylthiourea), a thiol group (phenylmercaptotetrazole, trimethyl-1,2,4-triazolium thiolate) or S present in a ring (benzthiazole), as well as on a variety of metal sulfides (Table 6.4). These compounds were chosen to investigate the effect that $\mathrm{Ag}$ or Au metal complexation would have on the electronic structure of photographic dyes, and to investigate the nature of the chemical sensitization centers. A comparison of representative ligand and metal-ligand complexes is presented in Figure 6.11. In all of the metal-sulfur compounds, the $2473-2474 \mathrm{eV}$ whiteline feature changes as a result of metal complexation and a shoulder to the high-energy side of the white-line feature appears at $\sim 2475 \mathrm{eV}$. For the complex between Ag and a benzthiazole group (AGBENZ), the white-line feature broadens and decreases in intensity (Figure 6.11a). In complexes between $\mathrm{Ag}$ and terminal sulfur-containing ligands, such as AGCMMT (Figure 6.11b), AGPMT (Figure 6.11c), AGTRZ (Figure 6.11d), AGTRZ2 and AGTRZ4 (Figure 6.11e), the white-line feature increases in intensity and the pre-edge feature between 2470.5 and $2472.0 \mathrm{eV}$ is absent. The pre-edge feature has been attributed to a transition to a p $\pi^{*}$ orbital by our single-crystal polarized studies; it's absence in the metal complexes suggests that the $\mathrm{p} \pi^{*}$ orbital of $\mathrm{S}$ is directly involved in the covalent metalsulfur interaction.

The presence of gold has a much stronger effect on the S K-edge spectra than silver (Figure 6.12). In general, the intensity of the white-line feature of the Au complexes studied here (AUTMTU, AUETU2, AUTHIO, AUTRZ2, Figure 6.12a-d, respectively) is 


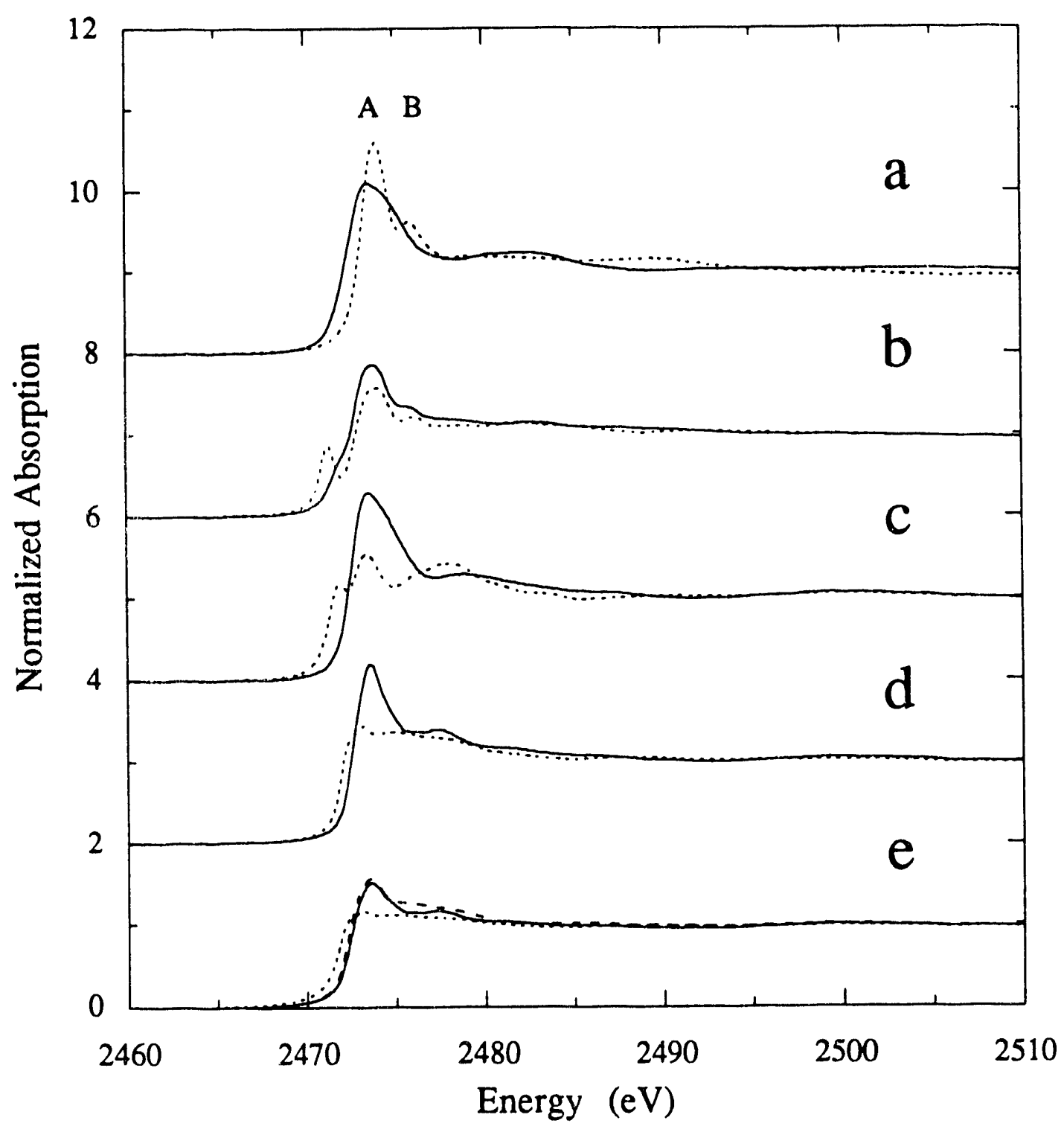

Figure 6.11. The spectra of Ag-S complexes. The solid lines are the spectra of the metal complexes and the dotted lines are the spectra of the ligands. (a) AGBENZ and BENZ, (b) AGCMMT and CMMT, (c) AGPMT and PMT, (D) AGTRZ and TRZ, (e) AGTRZ2, AGTRZ4 (dash) and TRZ. 


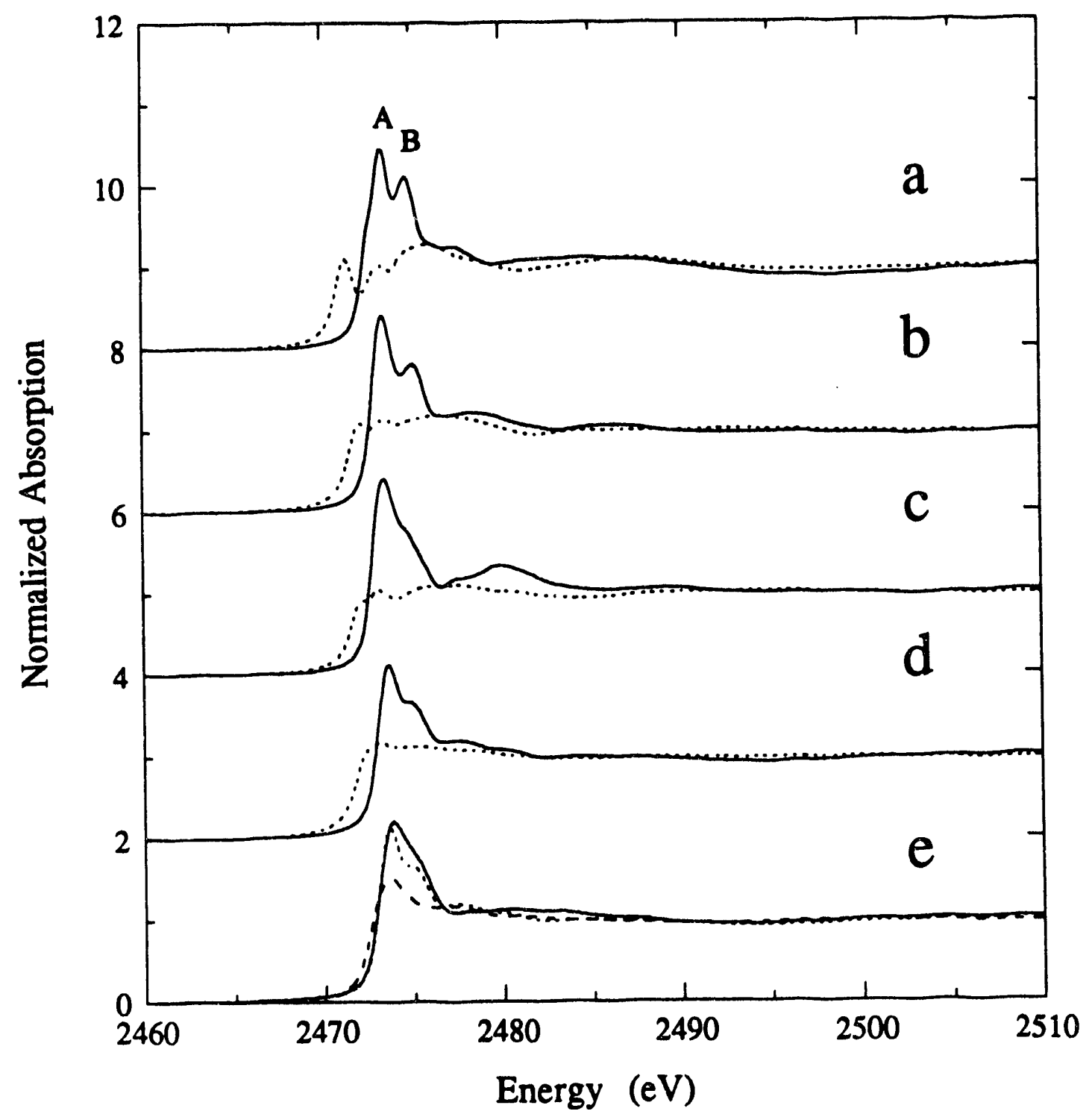

Figure 6.12. The spectra of Au-S complexes. The solid lines are the spectra of the metal complexes and the dotted lines are the spectra of the ligands. (a) AUTMTU and TMTU, (b) AUETU2 and ETU, (c) AUTHIO and THIOUR, (d) AUTRZ2 and TRZ. In (e), the spectra of a mixed metal complex (AUAGTRZ, solid) is compared to the spectra of a Au (AUTRZ2, dot) and Ag (AGTRZ2, dash) complex with the same ligand. 
greater than that of the Ag complexes. The white-line transition in the Au complexes is also more narrow than in the $\mathrm{Ag}$ complexes and a shoulder can be seen on the high energy side of the main transition which is sometimes well-resolved. In the $\mathrm{Ag}$ complexes, the main transition is broadened to the high energy side, however no shoulder is resolved from this feature. In a comparison of Ag vs. Au complexation with the same ligand (AUTRZ2 and AGTRZ2, Figure 6.12e), the Au-containing complex has a much more intense white-line feature than the analogous Ag complex, and in the mixed metal AGAUTRZ compound, the spectrum is more similar to that of AUTRZ2 than AGTRZ2 (Figure 6.12e).

Data were collected on a series on $\mathrm{Ag}, \mathrm{Au}$ and mixed metal sulfides (AG2S, $A U 12 S 8, A U 2 S, A U 2 S 3$ ) as models of the chemical sensitization centers. AU2S and AU2S3 are indistinguishable from each other, but the larger cluster, AU12S8, has a narrower white-line with a second transition to higher energy (Figure 6.13). The position of the white-line occurs at a higher energy than the white-line position of the smaller clusters as well. Silver sulfide is different from all of the gold sulfides (Figure 6.13), with a split, less intense and broader white-line. Although the S K-edge spectra do not provide enough information to determine the size of the metal sulfide clusters formed on the photographic substrate, it may be possible to decipher between $\mathrm{Au}$ and $\mathrm{Ag}$ sulfide clusters based on the shape and intensity of the white-line feature.

Single-crystal polarized S K-edge measurements were done on bis(ethylenethiourea) Au(I) hydrate hydrochloride (AUETU2), in which the Au metal forms a complex with the thione $S$. The powder spectra of AUETU2 and ethylenthiourea are compared in Figure 6.12b. For the powder spectrum of AUETU2, the low energy feature attributable to the thione $S$ seen in the ligand spectrum is absent, and the spectrum contains an intense feature at $2473.3 \mathrm{eV}$ and a lower-intensity feature at $2475.3 \mathrm{eV}$. For the polarized measurements, $\mathrm{X}$ was defined to be in the plane of the ligand rings along the average $\mathrm{C}-\mathrm{S}_{\text {thione }}$ bond, $\mathrm{Z}$ was defined to be the average of the ligand plane normals, and $Y$, defined to be the crossproduct of $\mathrm{X}$ and $\mathrm{Z}$, is directed roughly along the $\mathrm{S}-\mathrm{Au}-\mathrm{S}$ bond. As a result of this definition, the $Y$ orientation will contain some $X$ character.

The polarized spectra reveal that the features in the powder spectra of AUETU2 are polarized primarily in the plane of the ligand rings (Figure 6.14). In the Y-oriented spectrum, the sharp white-line feature occurs at the same energy as in the powder spectrum, but in the X-oriented spectrum, the feature is broad and occurs at $2473.9 \mathrm{eV}$. This suggests that the white-line feature of the powder spectra of AUETU2 can be attributed to transitions to two final states, one with Au-S $\sigma^{*}$ character (2473.3 eV), and one with C-S $\sigma^{*}$ character $(2473.9 \mathrm{eV})$. The shoulder at $2475.3 \mathrm{eV}$ is the dominant feature in the X-polarized spectrum and it can therefore be concluded that this feature is due to a 


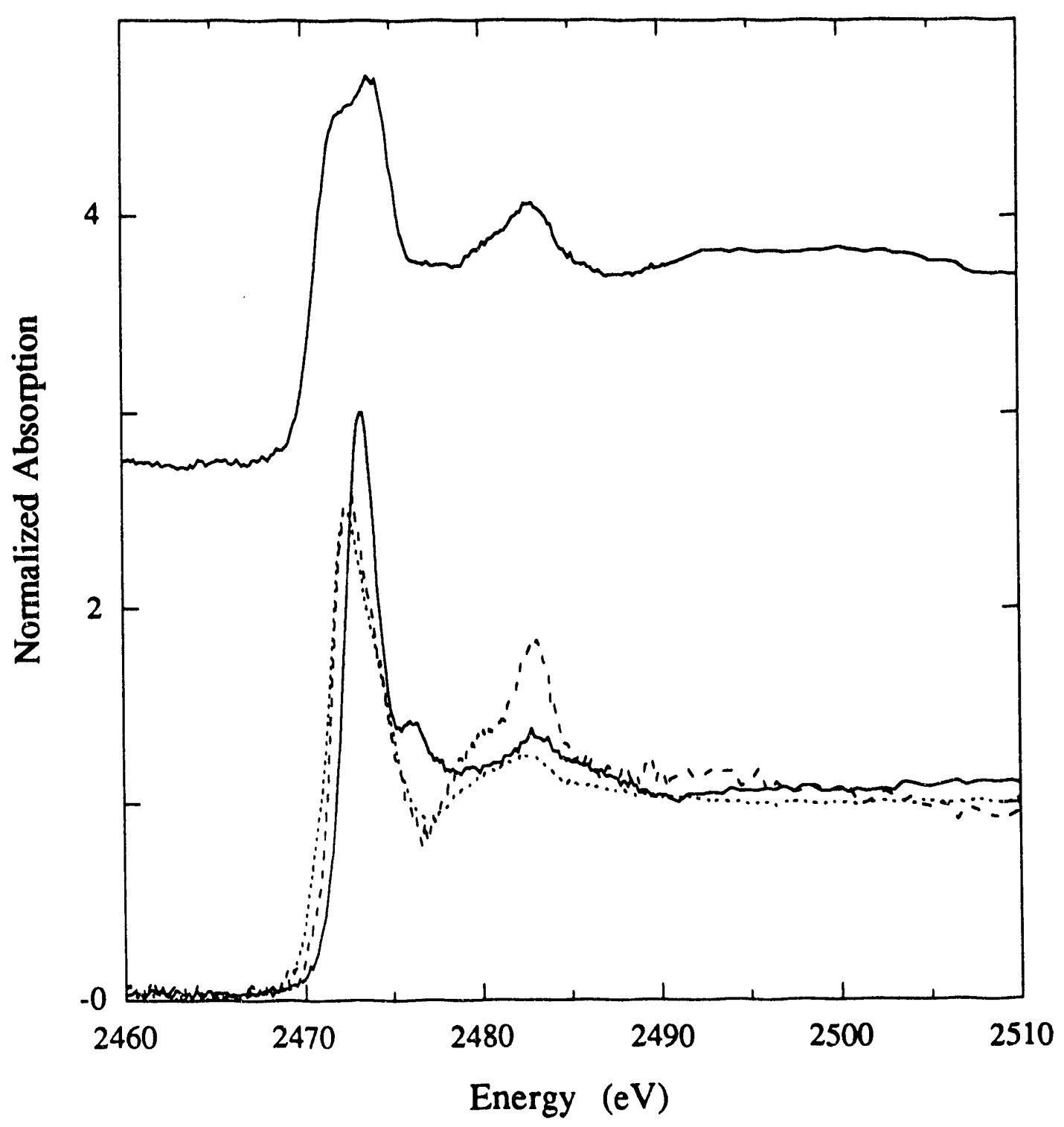

Figure 6.13. The $\mathrm{S} \mathrm{K}$-edge spectra for $\mathrm{Ag}$ and $\mathrm{Au}$ sulfides. (a) the powder spectrum of AG2S, (b) the powder spectra of AU12S8 (solid), AU2S (dash) and AU2S3 (dot). 


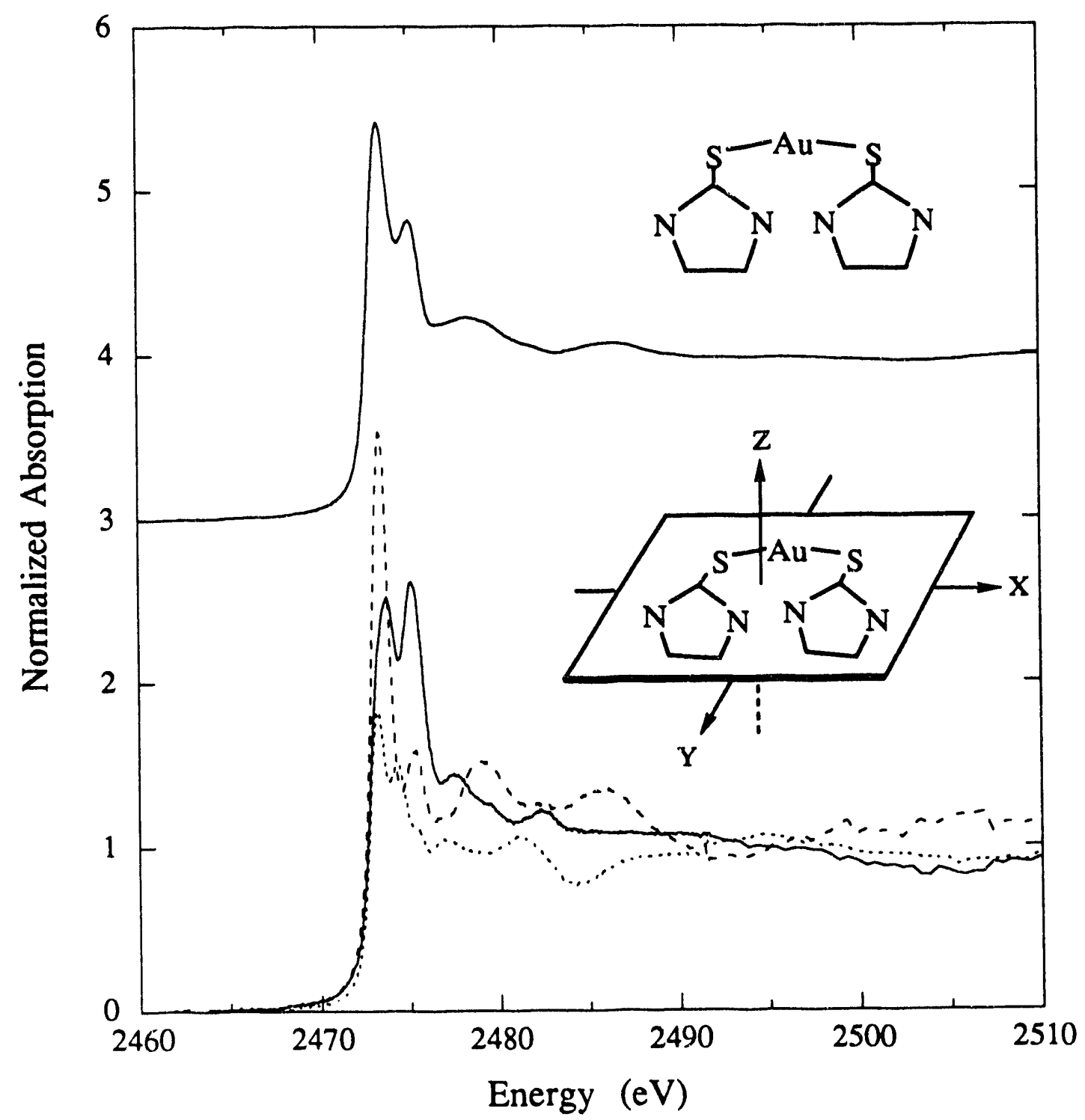

Figure 6.14. The powder spectrum (top) of AUETU2 compared with the single-crystal spectra (bottom) corresponding to polarization along the average $\mathrm{X}$ (solid), $\mathrm{Y}$ (dash), and $\mathrm{Z}$ (dot) molecular axes. 
transition to a C-S $\sigma$-type orbital. In the Z-polarized (out-of-plane) spectrum, there is a feature at $2473.2 \mathrm{eV}$, whereas in the out-of-plane orientation of 2-thiohydantoin, no transition occurs at this energy (see Figure 6.5). The presence of the $2473.2 \mathrm{eV}$ feature in the out-of-plane spectrum of bis(ethylenethiourea) $\mathrm{Au}(\mathrm{I})$ must therefore be due to the metalsulfur interaction, and either reflects a transition to a final Au-S state with p $\pi^{*}$ symmetry which occurs at energies similar to those of the metal- and C-S o-type final states, or represents a shift in the energy of the C-S $p \pi^{*}$ final state as a result of the interaction of the metal and sulfur atoms.

Extending these results to the other metal-sulfur complexes, it can be concluded that the intense feature at $2473 \mathrm{eV}$ is due to transitions to two final states of $\sigma$ symmetry, one with metal-S $\sigma$ character, and the other with C-S $\sigma$ character, while the shoulder at $2475 \mathrm{eV}$ is due to a transition to a C-S o-type orbital. A comparison of the spectra of a thiol-ligand (APMT), a Ag-thiol complex (AGPMT), and the sodium salt of a thiol group (APMTNA) is shown in Figure 6.15. In contrast to the dramatic change seen in the $\mathrm{S} \mathrm{K}$-edge spectra as a result of a covalent interaction between $\mathrm{Ag}$ or $\mathrm{Au}$ and $\mathrm{S}$, an ionic interaction between $\mathrm{S}$ and a metal results in the loss of the pre-edge peak, but no change in the shape or intensity of the white-line feature. This suggests that the ionic interaction between $\mathrm{Na}$ and $\mathrm{S}$ has little or no effect on the $\sigma$-symmetry final state orbitals, whereas the covalent interaction between $\mathrm{Ag}$ and $\mathrm{S}$ has a significant effect. As noted before, the loss in the pre-edge feature from thione to thiol to thiolate seems to be correlated to the accumulation of negative charge on the $S$ atom, perhaps as a result of destabilization of the $p \pi^{*}$ orbitals.

\subsubsection{Surface Measurements}

Data were collected on two dyed and three chemically sensitized AgBr sheet crystal samples at glancing angle incidence. The dyed samples were treated with a cyanine dye (DC6) or a merocyanine dye (DM4), both of which contain $\mathrm{Cl}$, thereby allowing $\mathrm{Cl} \mathrm{K}$-edge polarized measurements to be undertaken as well. Polarized measurements were collected on the merocyanine-dyed sample, corresponding to in-plane $\left(\chi=0^{\circ}\right)$ and out-of-plane $(\chi=$ $90^{\circ}$ ) orientations. For the cyanine-dyed sample, the signal-to-noise level was very poor in the out-of-plane orientation, so only in-plane measurements were made. The chemically sensitized samples consisted of a thiosulfate-treated $\mathrm{AgBr}$ sheet crystal, and two $\mathrm{Au} / \mathrm{S}$ treated sheet crystal samples with differing Au:S ratios (Au:S 1:2 and 1:4). Data for these samples were collected in the in-plane orientation.

The in-plane polarized DC6 sample is compared to its powder spectrum in Figure 6.16. Although the noise level of the surface sample is very high, the intensity of the main 


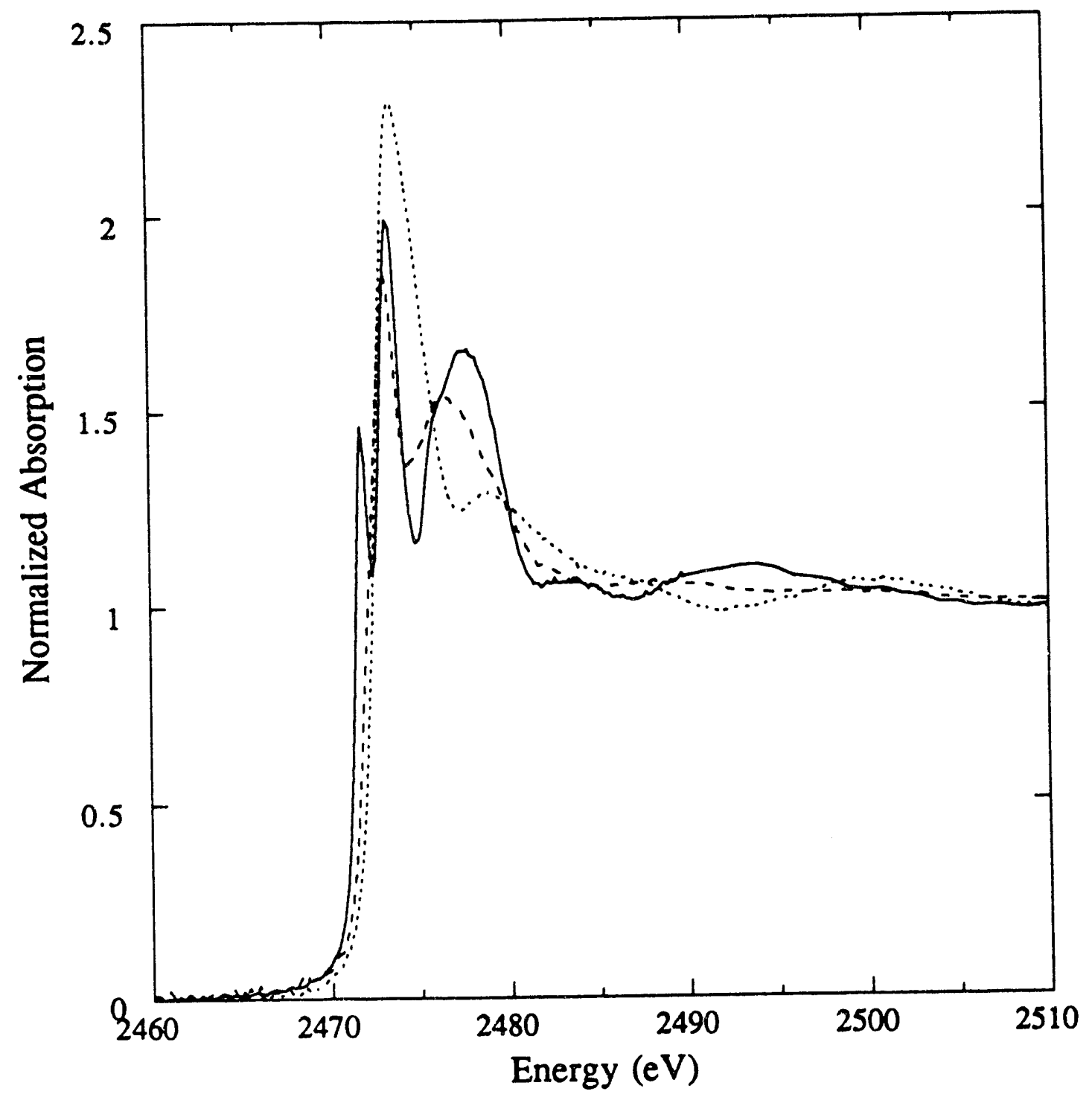

Figure 6.15. The $\mathrm{S}$ K-edge spectra of an exocyclic S-containing molecule (APMT, solid) compared with the spectra of an ionic (APMTNA, dash) and covalent complex (AGPMT, not) with a similar molecule. 


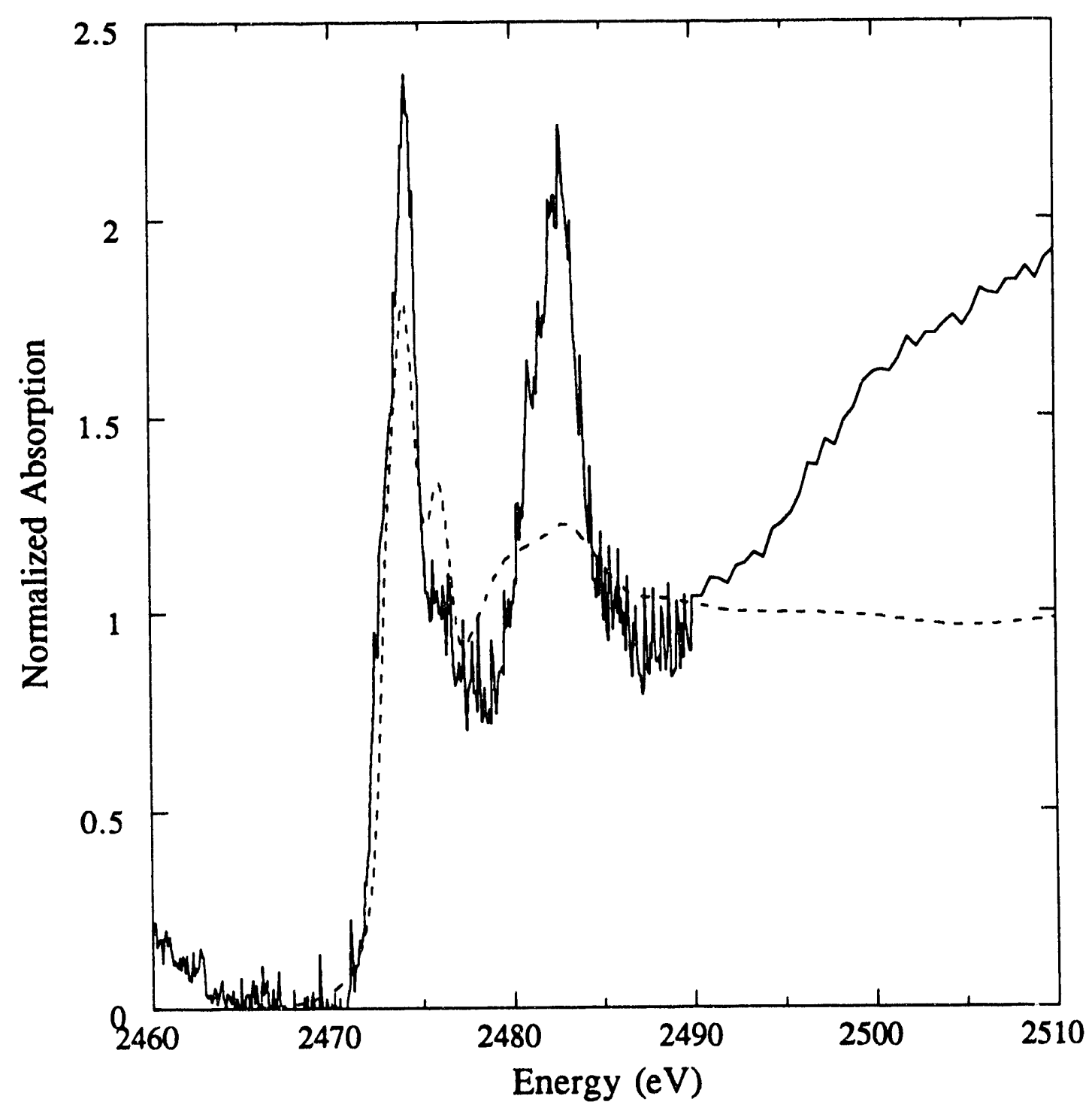

Figure 6.16. $\mathrm{S}$ K-edge surface spectrum of a $\mathrm{AgBr}$ sheet crystal treated with cyanine dye DC6 measured at $\chi=0^{\circ}$ (solid), compared to the powder spectrum of DC6 (dash). 
transition increases relative to the intensity of the same transition in the powder spectrum. The change in the surface DC6 spectrum is different from the change seen due to the interaction between $\mathrm{Ag}$ and benzthiazole sulfur (see Figure 6.12a), in which the intensity of the ligand feature decreases and the feature broadens. This suggests that the interaction between the cyanine dye $\mathrm{S}$ and the $\mathrm{AgBr}$ sheet crystal does not involve the same kind of interaction which occurs in silver benzthiazole. The same is observed for the merocyanine dyed surface sample. In both the in- and out-of-plane orientations (Figure 6.17), the thione pre-edge feature is present, and the white-line feature does not increase in intensity, therefore no bond between the thione $\mathrm{S}$ and the $\mathrm{Ag}$ in the $\mathrm{AgBr}$ sheet crystal is formed (see AGCMMT, Figure 6.13b; AUTMTU, AUETU2, and AUTHIOUR, Figure 6.13a-c). The features in the DM4 sample show an orientational dependence. The thione feature is more pronounced in the in-plane polarization than in the out-of-plane orientation. For polarization studies, intensity enhancement occurs when the polarization vector of the incident radiation is codirectional with the orbital of interest. The pre-edge feature is due to a transition to a S p $\pi^{*}$ orbital oriented out of the plane of the molecule. For the intensity of this feature to increase, the molecule is oriented on the surface so that this orbital is perpendicular to the direction of the beam and parallel to the polarization vector (Figure 6.1). This can only happen if the dye molecules are oriented edge-on, with the plane of the ring perpendicular to the surface and in the plane defined by the direction of the beam. If the orbital of interest is $100 \%$ parallel to the polarization vector in the in-plane orientation, one would expect the intensity of this feature to vanish in the out-of-plane orientation. The pre-edge feature does not vanish in the out-of-plane orientation, but it is about half as intense as in the in-plane spectrum. This suggests that the dye molecules may be tilted with respect to the plane of the surface with the result that the overlap of the $p \pi^{*}$ orbital with the polarization vector is not $0^{\circ}$ in the out-of-plane configuration, resulting in some amount of excitation into this orbital.

The $\mathrm{Cl} \mathrm{K-edge} \mathrm{data} \mathrm{for} \mathrm{the} \mathrm{DC6} \mathrm{dyed} \mathrm{sample} \mathrm{is} \mathrm{presented} \mathrm{in} \mathrm{Figure} \mathrm{6.18.} \mathrm{For} \mathrm{this}$ sample, the white-line is very intense in the in-plane orientation and much less intense in the out-of-plane configuration. This suggests that the dye molecules are oriented so as to maximize the overlap of the $\mathrm{C}-\mathrm{Cl}$ bond with the polarization vector for the in-plane orientation suggesting that the molecule is oriented with a ring axis perpendicular to the direction of the beam and parallel to the sheet crystal surface. It cannot be determined from these data if the dye molecules are oriented edge-on (with the plane of the ring perpendicular to the surface) or flat-on (with the plane of the ring parallel to the surface) as these orientations are identical with respect to the $\mathrm{C}-\mathrm{Cl}$ bond. The polarized $\mathrm{Cl} \mathrm{K}$-edge spectra of the DM4 samples do not exhibit much polarization dependence (Figure 6.19), 


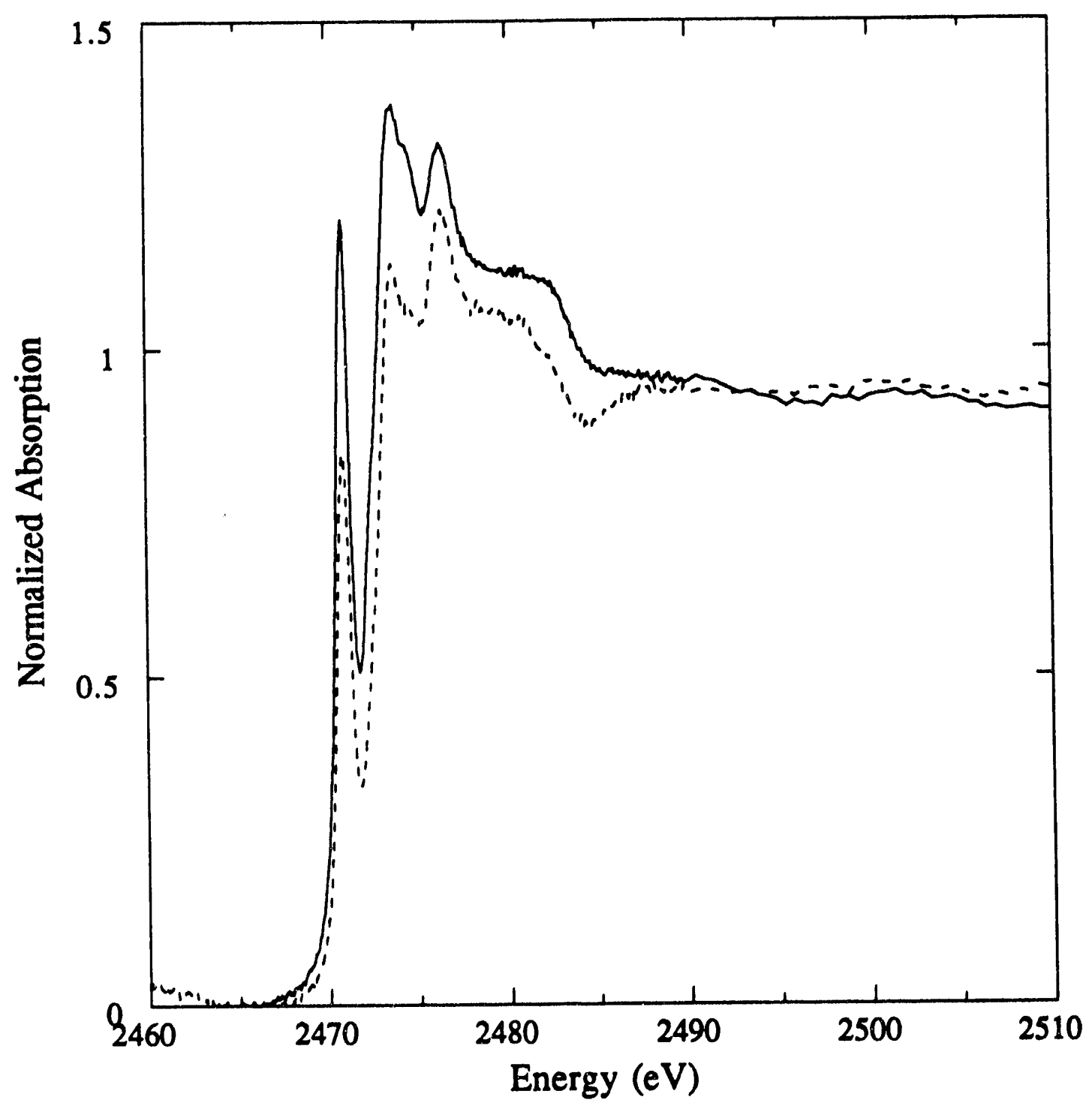

Figure 6.17. S K-edge surface spectra of a $\mathrm{AgBr}$ sheet crystal treated with merocyanine dye DM4, measured at $\chi=0^{\circ}$ (solid) and $\chi=90^{\circ}$ (dash). 


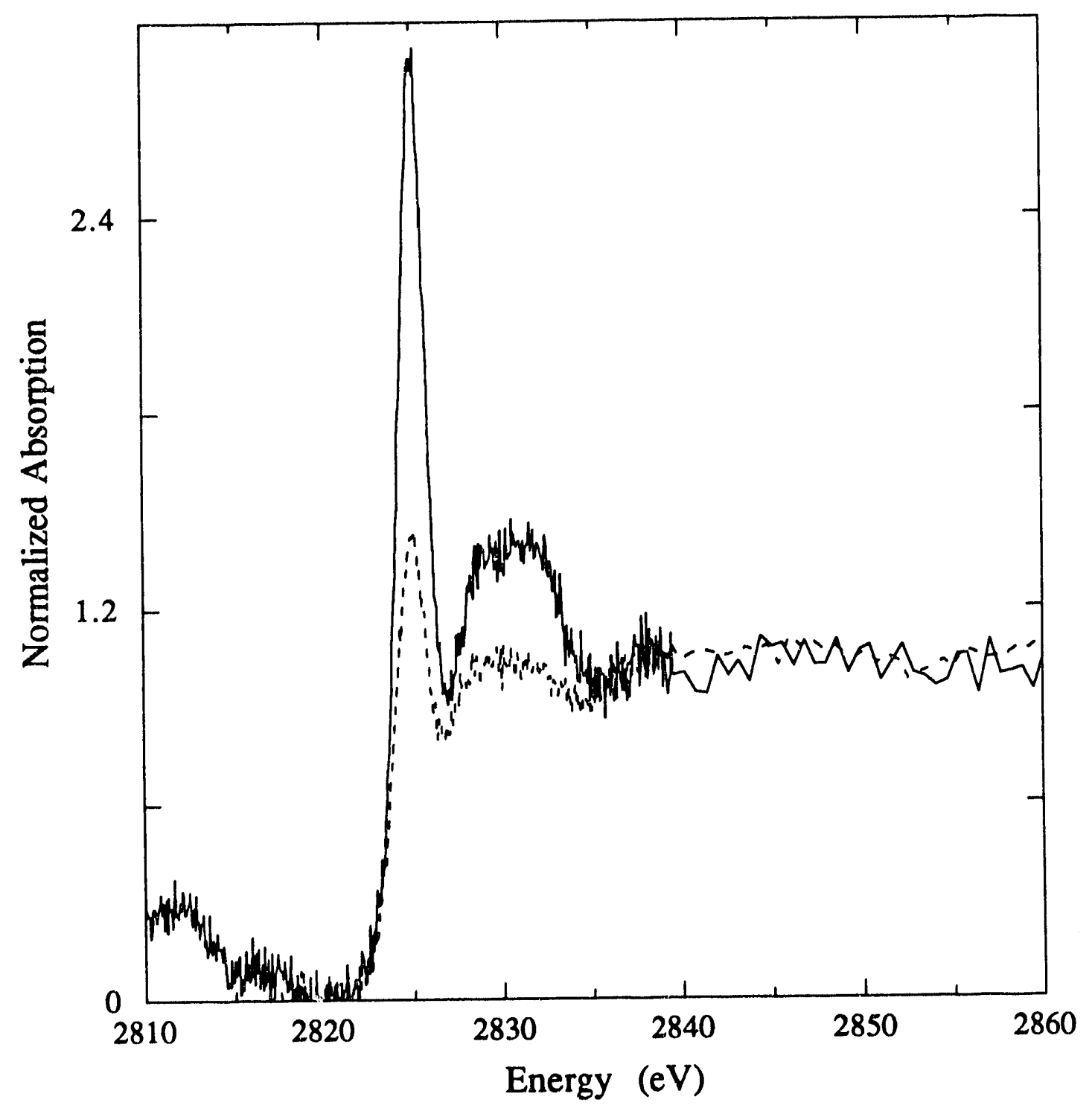

Fig are 6.18. $\mathrm{Cl} \mathrm{K}$-edge surface spectra of a $\mathrm{AgBr}$ sheet crystal treated with cyanine dye DC6, measured at $\chi=0^{\circ}$ (solid) and $\chi=90^{\circ}$ (dash). 


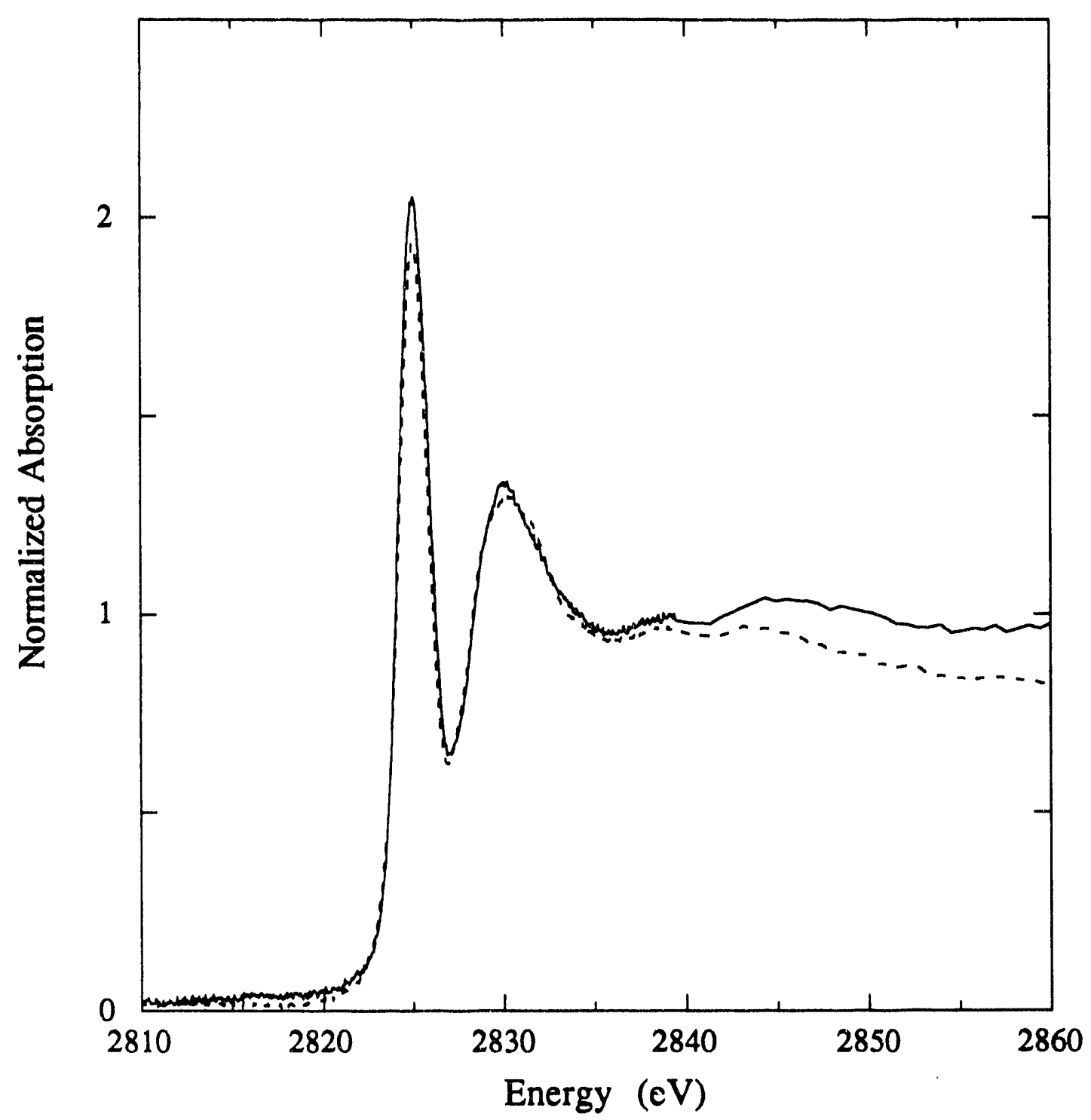

Figure 6.19. $\mathrm{Cl} \mathrm{K}$-edge surface spectra of a $\mathrm{AgBr}$ sheet crystal treated with merocyanine dye DM4, measured at $\chi=0^{\circ}$ (solid) and $\chi=90^{\circ}$ (dash). 
suggesting that there may be some disorder in the alignment of the dye molecules on the $\mathrm{AgBr}$ substrate.

The thiosulfate-treated $\mathrm{AgBr}$ sheet crystal sample has an edge very similar to that of $\mathrm{Ag}_{2} \mathrm{~S}$ powder, and also similar to $\mathrm{H}_{2} \mathrm{~S}$-treated $\mathrm{Ag}$ on quartz, suggesting that silver sulfide is the species formed during the sensitization process (Figure 6.20a, see Figure 6.13). The presence of $\mathrm{Au}$ in the 4:1 (Figure 20b) and 2:1 (Figure 20c) S: Au samples modifies the appearance of the edge features, with an increase in the intensity of the lower energy feature of the split main transition as the amount of gold increases. The edge spectra of the Autreated samples are also different in appearance from the edge spectra of pure $\mathrm{Au}_{2} \mathrm{~S}_{3}$ or $\mathrm{Au}_{2} \mathrm{~S}$ (Figure 6.13b), suggesting that the species formed during the sensitization treatment of the $\mathrm{AgBr}$ sheet crystals is distinct from $\mathrm{Ag}_{2} \mathrm{~S}$ and $\mathrm{Au}_{2} \mathrm{~S}_{3}$ or $\mathrm{Au}_{2} \mathrm{~S}$.

\subsection{Discussion}

These studies have shown that $S$ and $\mathrm{Cl} \mathrm{K}$-edge $\mathrm{X}$-ray absorption spectroscopy can be used to characterize the electronic and geometric structure of these low- $Z$ atoms in components of the photographic system. The $\mathrm{Cl} \mathrm{K}$-edge spectra are essentially identical and do not depend on the type of molecule or on the position of $\mathrm{Cl}$ on the molecule. There are, however, striking differences in the spectra of molecules containing sulfur in an exocyclic (thione(ol)) position compared to a cyclic sulfur (thiazole). The white-line feature occurs at about the same position for all of the compounds studied, but is more intense for the thiazole compounds than for those containing thione(ol). This feature is polarized along the C-S bond and corresponds primarily to a transition to C-S p $\sigma^{*}$ orbital. X $\alpha$ calculations on thiophene, however, suggest that a transition to a $\mathrm{p} \pi^{*}$ also contributes. ${ }^{6 \mathrm{a}}$ This was also seen from our single-crystal polarized studies in which a transition in the region of the white-line feature was seen in the out-of-plane orientation. For the exocyclic $S$ compounds, only a transition to the C-S p $\sigma^{*}$ orbital contributes to the white-line feature. This difference could account for the increase of the white-line feature intensity of the thiazoles relative to the thione(ol)s.

A pre-edge feature below the position of the white-line is seen for compounds in which $\mathrm{S}$ is present as a thione or a thiol; this feature is absent in the cyclic sulfur compounds. The presence of this feature is due to a transition to a final state $p$ orbital arising from the involvement of the exocyclic sulfur in a p $\pi$ system. This has been confirmed by our single-crystal polarized measurements, in which this feature was shown to be polarized perpendicular to the $\mathrm{C}=\mathrm{S}$ bond and the plane of the ring, and by $\mathrm{X} \alpha$ calculations by others. ${ }^{6 b-c, 7}$ For molecules containing exocyclic sulfur, the position of the 


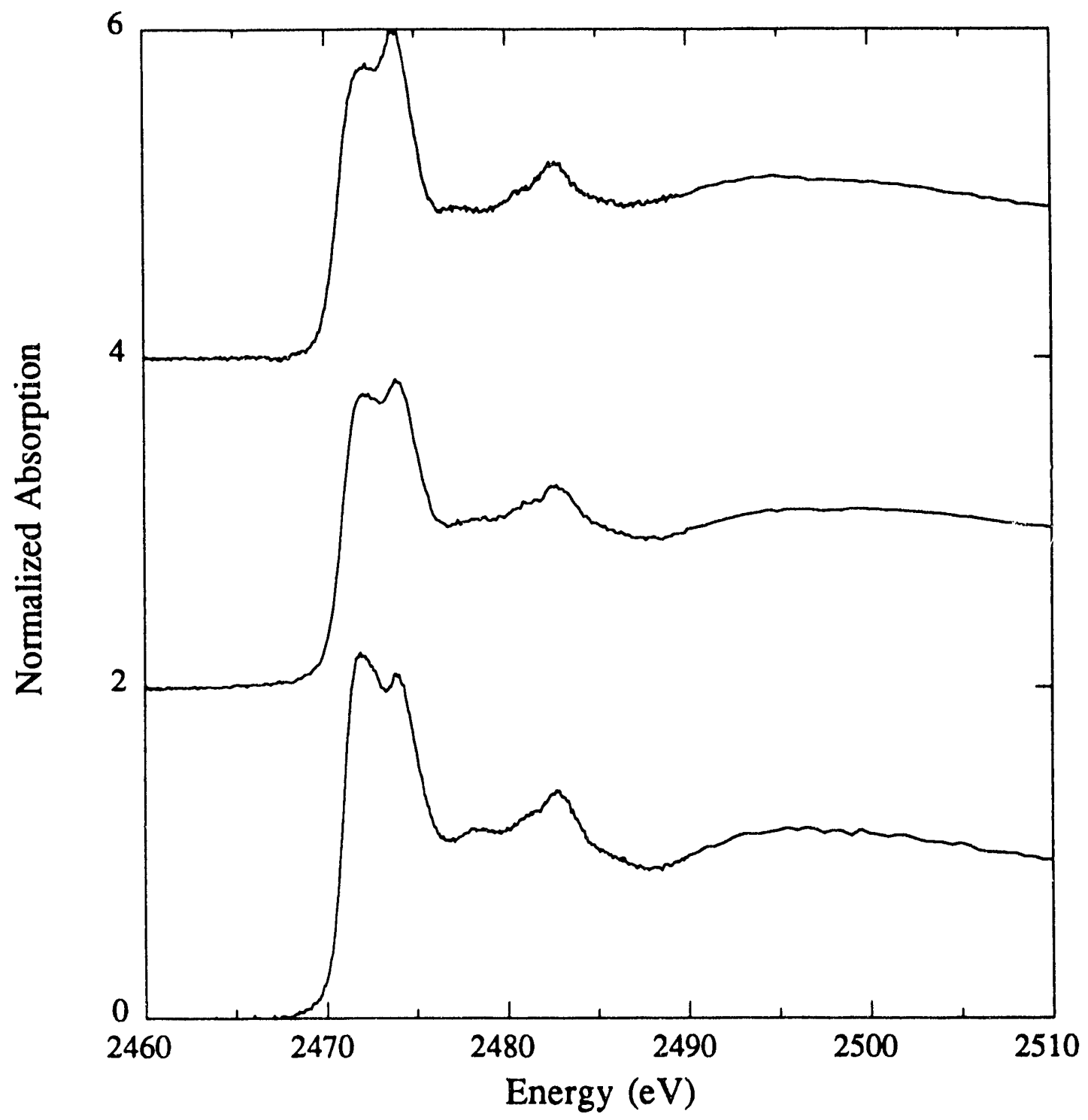

Figure 6.20. Surface spectra of (a) thiosulfate treated $\mathrm{AgBr}$ sheet crystal measured at $\chi$ $=0^{\circ}$, and gold dithiosulfate treated AgBr sheet crystals in a S:Au ratio of (b) 4:1 and (c) $2: 1$, measured at $\chi=0^{\circ}$. 
pre-edge feature moves to higher energy from thione to thiol to thiolate (Figure 6.8) and the position of the white-line feature moves to lower energy in accordance with the accumulation of negative charge on the sulfur atom. The movement of the thione feature to higher energy suggests that the change in the $S$ environment from thione to thiol to thiolate must effect the final state C-S p $\pi^{*}$ orbital as well. The involvement of this orbital either in a covalent bond with $\mathrm{H}$ (thiol) or an ionic bond with $\mathrm{Na}^{+}$(thiolate) results in the partial or total filling of the otherwise empty $\mathrm{p} \pi^{*}$ orbital, resulting in a destabilization of this orbital relative to the core level, hence the transition to this orbital appears at a higher energy.

There is also some variation seen in the intensity and position or the pre-edge feature within the thiourea molecules, in which the sulfur is present only as a thione, and within the tetrazoles, in which the $S$ is present as a thiol. The differences in the intensity of the pre-edge feature from sample to sample probably reflect the amount of $S \mathrm{p} \pi_{\mathrm{z}}$ mixing in the final state orbital, whereas the differences in the position of the feature might reflect the accumulation of electron density on the $S$ atom as a result of structural differences within the two classes of compounds. For example, EMT has an ethyl group in the 2 position, PMT has a phenyl group at the same location, and APMT has an acetamido-substituted phenyl group in the same position. In Figure 6.21, the S K-edge spectra of these compounds is compared. EMT has almost no pre-edge feature, whereas APMT has a wellresolved pre-edge feature, and PMT lies somewhere in between these two. It has previously been demonstrated that in going from a thione to a thiol to a thiolate (Figure 6.8) the pre-edge peak moves to higher energy and the white-line transition moves to lower energy as the electron density on the $S$ atom increases. One possible explanation is that destabilization of the $\mathrm{p} \pi^{*}$ orbital relative to the core level occurs due to the partial filling of that orbital by the interaction with $\mathrm{H}$ or $\mathrm{Na}$, with the result that the transition occurs at higher energy. If the accumulation of electron density results in a shift to higher energy of the thione(ol) pre-edge feature, one would expect that EMT, which has an electrondonating group in the 2 position, would have the least resolved pre-edge feature, whereas APMT, which has a better electron-withdrawing group than PMT, would have the best resolved pre-edge feature. At the same time, the white-line transition of EMT should occur at a lower energy than that of APMT. These trends are seen in the data (Table 6.2).

The nature and extent of the interaction between $\mathrm{S}$ in photographic materials and $\mathrm{Ag}$ and $\mathrm{Au}$ metals, in conjunction with single-crystal polarized measurements, have been characterized. Covalent or ionic interactions with a metal $\left(\mathrm{Ag}, \mathrm{Au}\right.$ or $\mathrm{Na}^{+}$) results in a dramatic change in the S K-edge (Figures 6.12,6.13 and 6.15). The white-line feature for all exocyclic S-containing ligands increases in intensity due to a $\mathrm{C}-\mathrm{S}$ p $\sigma^{*}$ interaction between $S$ and the metal, and the pre-edge feature vanishes due to an interaction between 


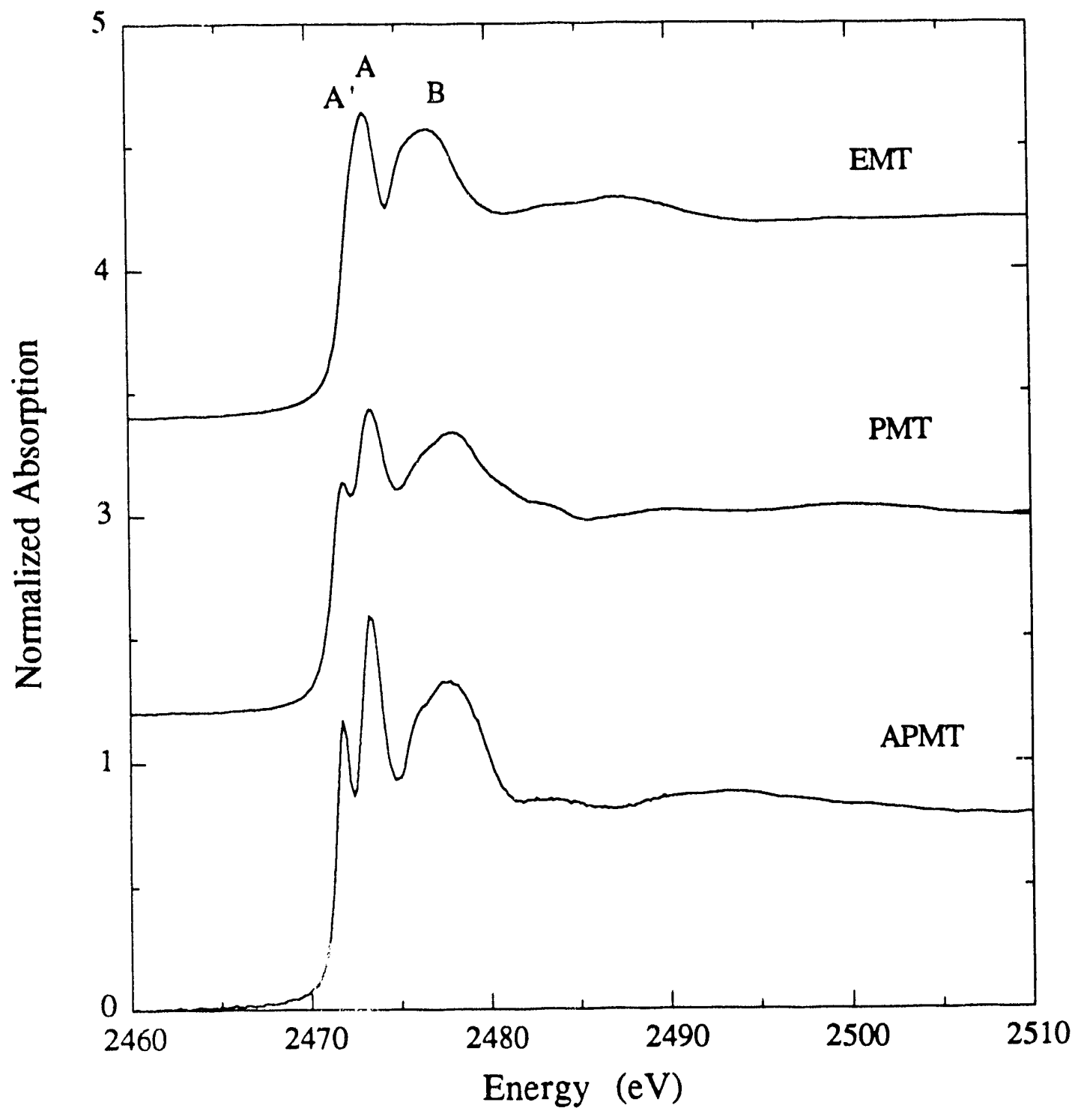

Figure 6.21. The powder spectra of EMT (top), PMT (middle) and APMT (bottom). 
the $\mathrm{S} p \pi^{*}$ orbital and the metal. The white-line feature has been shown by single-crystal polarized studies to have contribution from transitions to two $\sigma$ orbitals, one with metalsulfur character and one with carbon-sulfur character. This could explain the increase in the intensity of this feature upon complexation with a metal. There is also a feature in this same energy region for the out-of-plane oriented spectrum (Figure 6.14), which could be due to a transition to a $\mathrm{Au}-\mathrm{S}$ p $\pi$-type orbital occurring at the same energy as the $\sigma$ transitions, or it could be that the involvement of the $S \mathrm{p} \pi^{*}$ orbital with the metal results in a shift of the C-S p $\pi^{*}$ orbita' of the non-complexed thione(ol) group (Figure 6.5) to this energy. Both of these possibilities would also contribute to the intensity of the white-line feature. For the silver benzthiazole compound, in which the silver metal interacts with a cyclic $S$ compound, the white-line feature broadens and decreases in intensity. It is clear from these studies that the covalent interaction between $\mathrm{Ag}$ and $\mathrm{Au}$ with $\mathrm{S}$ in photographic materials results in a significant degree of change in the electronic structure of the $S$ atoms and that studies of this type can be used to characterize the extent of the interaction between these metals and sulfur-containing components of the photographic system.

The dyed $\mathrm{AgBr}$ sheet crystal samples did not show the dramatic change in the S Kedge features associated with a bonding interaction between the metal and the sulfur (Figure 6.16 and Figure 6.17). This suggests either that the nature of the interaction in the systems studied is of a physical, rather than a chemical nature, or that any direct bonding interaction which does occur between the dye molecules and the $\mathrm{AgBr}$ substrate does not involve the $\mathrm{S}$ atoms. Although we believe that there was only a monolayer of dye on the surface, we cannot eliminate the possibility that we were sampling a multilayer region of the substrate and not the substrate/adsorbate interface. Unlike the study done by Stöhr et. al, 22 we found that at angles greater than the glancing angle, there was a tremendous increase in the background which swamped the signal of interest. For these types of systems, a glancing angle configuration is required.

In the in-plane polarized spectrum of the merocyanine-dyed $\mathrm{AgBr}$ sheet crystal (thione $S$ ), the pre-edge feature is enhanced relative to the powder and the out-of-plane spectra. The enhancement of this feature in the in-plane polarized spectrum means that the dye molecules are oriented such that this orbital is parallel to the polarization vector; this in turn means that the plane of the ring of the molecule is in the plane defined by the direction of the beam and that the molecules stack together with the rings parallel. The presence of this feature in the out-of-plane polarized spectrum suggests that the alignment of the p $\pi^{*}$ orbital is not perfectly parallel to the surface; if the molecule were tilted with respect to the surface, there would be some amount of overlap with the polarization vector in the out-ofplane orientation resulting in some possibility of a transition occurring. The best insight 
into the orientation of the DC6 molecules (thiazole S) is obtained from the polarization properties of the $\mathrm{Cl} \mathrm{K}$-edge, as we were unable to obtain a scan of the $\mathrm{S} \mathrm{K}$-edge in the $\chi=$ $90^{\circ}$ orientation. The white-line feature for the $\mathrm{Cl}$ edge of DC6 was most intense in the inplane spectrum, and decreased in the out-of-plane spectrum (Figure 6.18), suggesting that the $\mathrm{C}-\mathrm{Cl}$ bond is parallel to the polarization vector in the in-plane orientation. However, we cannot determine if the molecule is oriented edge-on or flat-on based on the results of the $\mathrm{Cl}$ $\mathrm{K}$-edge studies. The treatment of the $\mathrm{AgBr}$ sheet crystals with gold dithiosulfate results in a sulfide species distinct from pure $\mathrm{Ag}_{2} \mathrm{~S}, \mathrm{Au}_{2} \mathrm{~S}$ or $\mathrm{Au}_{3} \mathrm{~S}_{2}$, although the exact nature of the metal sulfide cluster formed cannot be determined from the data available.

\subsection{Conclusions}

X-ray absorption spectroscopy is particularly sensitive to the different environment of $S$ in the merocyanine and cyanine dyes and dye intermediates, and single-crystal polarized studies have allowed determination of the origin of the transitions seen in the spectra to be made. XAS can distinguish between covalent and ionic interactions between a metal and exocyclic sulfur. A distinctive change occurs in the $\mathrm{S} \mathrm{K}$-edge spectra of $\mathrm{Ag}$ and Au metal complexes, allowing predictions of the nature of the dye adsorbate/ $\mathrm{AgBr}$ substrate interaction to be made, and clearly showing that there is no covalent interaction between the $\mathrm{Ag}$ in the $\mathrm{AgBr}$ sheet crystals and the $\mathrm{S}$ in the cyanine and merocyanine dye molecules. In addition, the sensitivity of XAS to the polarization properties of the $\mathrm{S} \mathrm{K}$-edge features provides insight into the orientation of the dye molecules on the $\mathrm{AgBr}$ substrate. $\mathrm{X}$-ray absorption spectroscopy has proven to be a valuable technique for characterizing and understanding the nature of $\mathrm{S}$ and $\mathrm{Cl}$ present in elements of the photographic system, as well as the nature of the interaction between $\mathrm{S}$ and $\mathrm{Ag}$ and $\mathrm{Au}$ metals.

\subsection{Acknowledgements}

This work was supported by research funds from Eastman Kodak Company. These data were collected at the Stanford Synchrotron Radiation Laboratory (SSRL) and at the National Synchrotron Light Source (NSLS), Brookhaven National Laboratory, which are supported by the Department of Energy, Division of Materials Sciences and Division of Chemical Sciences. SSRL is also supported by the National Institutes of Health, Biomedical Resource Technology Program, Division of Research Resources and by the Department of Energy, Office of Health and Environmental Research. 


\subsection{References and Notes}

1. Hedman, B.; Frank, P.; Penner-Hahn, J. E.; Roe, A. L.; Hodgson, K. O.; Carlson, R. M. K.; Brown, G.; Cerino, J.; Hettel, R.; Troxel, T.; Winick, H.; Yang, J. Nucl. Instr. and Meth. 1986, A246, 797.

2. Lytle, F. W.; Greegor, R. B.; Sandstrom, D. R.; Marques, E. C.; Wong, J.; Spiro, C. L.; Huffman, G. P.; Huggins, F. E. Nucl. Instr. and Meth. 1984, 226, 542.

3. Sugiura, C. J. Chem. Phys. 1983, 79, 4811.

4. (a) Huffman, G. P.; Huggins, F. E.; Mitra, S.; Shah, N.; Pugmire, R. J.; Davis, B.; Lytle, F. W.; Greegor, R. B. Energy \& Fuels 3, 1989, 200-205. (b) Spiro, C. L.; Wong, J.; Lytle, F. W.; Greegor, R. B.; Maylotte, D. H.; Lamson, S. H. Science 226, 1984, 48-50. (c) George, G. N.; Gorbaty, M. L. J. Am. Chem. Soc. 111, 1989, $3182-$ 3186.

5. Tyson, T. A.; Roe, A. L.; Hodgson, K. O.; Hedman, B. Phys. Rev. B 1989, 39, 6305.

6. (a) Hitchcock, A. P.; Horsley, J. A.; Stöhr, J. J. Chem. Phys. 85, 1986, 48354848. (b) Dezarnaud, C.; Tronc, M.; Hitchcock, A. P. Chem. Phys. 142, 1990, 455462. (c) Perera, R. C. C.; LaVilla, R. E. J. Chem. Phys. 81, 1984, 3375-3382. (d) Hitchcock, A. P.; Bodeur, S.; Tronc, M. Chem. Phys. 115, 1987, 93-101. (e) Hitchcock, A. P.; Tronc, M. Chem. Phys. 121, 1988, 265-277. (f) Sze, K. H.; Brion, C. E.; Tronc, M.; Bodeur, S.; Hitchcock, A. P. Chem. Phys. 121, 1988, 279-297.

7. (a) Yakata, Y.; Yoloyama, T.; Yagi, S.; Happo, N.; Sato, H.; Seki, K.; Ohta, T.; Kitajima, Y.; Kuroda, H. Surf. Sci. 259, 1991, 266-274. (b) Seymour, D. L.; Bao, S.; McConville, C. F.; Crapper, M. D.; Woodruff, D. P.; Jones, R. G. Surf. Sci. 189/190, 1987, 529-534. (c) Stöhr, J.; Kollin, E. B.; Fischer, D. A.; Hastings, J. B.; Zaera, F.; Sette, F. Phys. Rev. Lett. 1985, 55, 1468-1471.

8. Stöhr, J.; Outka, D. A. Phys. Rev. B 1987, 36, 7891-7905.

9. Herz, A. H. Adv. Coll. Inter. Sci. 1977, 8, 237.

10. Bird, G. R.; Norland, K. S.; Rosenoff, A. E.; Michaud, H. B. Phot Sci. Engr. 1968, 12, 196.

11. Ficken, G. E. J. Phot. Sci. 1973, 21, 11.

12. Saijo, H.; Kitamura, T.; Ohtani, H. Surf. Sci. 1986, 177, 431-443.

13. Weiss, G.; Ericson, R.; Herz, A. J. Coll. Inter. Sci. 1967, 23, 277.

14. Spencer, H. E.; Atwell, R. E.; Levy, M. J. Phot. Sci. 1983, 31, 158. 
15. Walker, L. A.; Folting, K.; Merritt, L. L. Jr. Acta Cryst. 1969, B25, 88-93.

16. Nakatsu, K.; Yoshioka, H.; Aoki, T. Chem. Lett. 1972, 339-340.

17. Trotter, J. Acta Cryst. 1986, C42, 862-864.

18. Jones, P. G.; Guy, J. J.; Sheldrick, G. M. Acta Cryst. 1976, B32, 3321-3322.

19. Stern, E. A.; Heald, S. M. Rev. Sci. Instr. 1979, 50, 1579.

20. Heald, S. M.; Keller, E.; Stern, E. A. Phys. Lett. 1984, 103a, 155-158.

21. Agarwal, B. K. in X-ray Spectroscopy, Springer Series in Optical Sciences vol.

15; D. L. MacAdam, Ed.; Springer-Verlag, Berlin, Heidelberg; 1979; pp 130 - 137

22. Stöhr, J.; Kollin, E. B.; Fischer, D. A.; Hastings, J. B.; Zaera, F.; Sette, F. Phys.

Rev. Lett. 1985, 55, 1468-1471. 

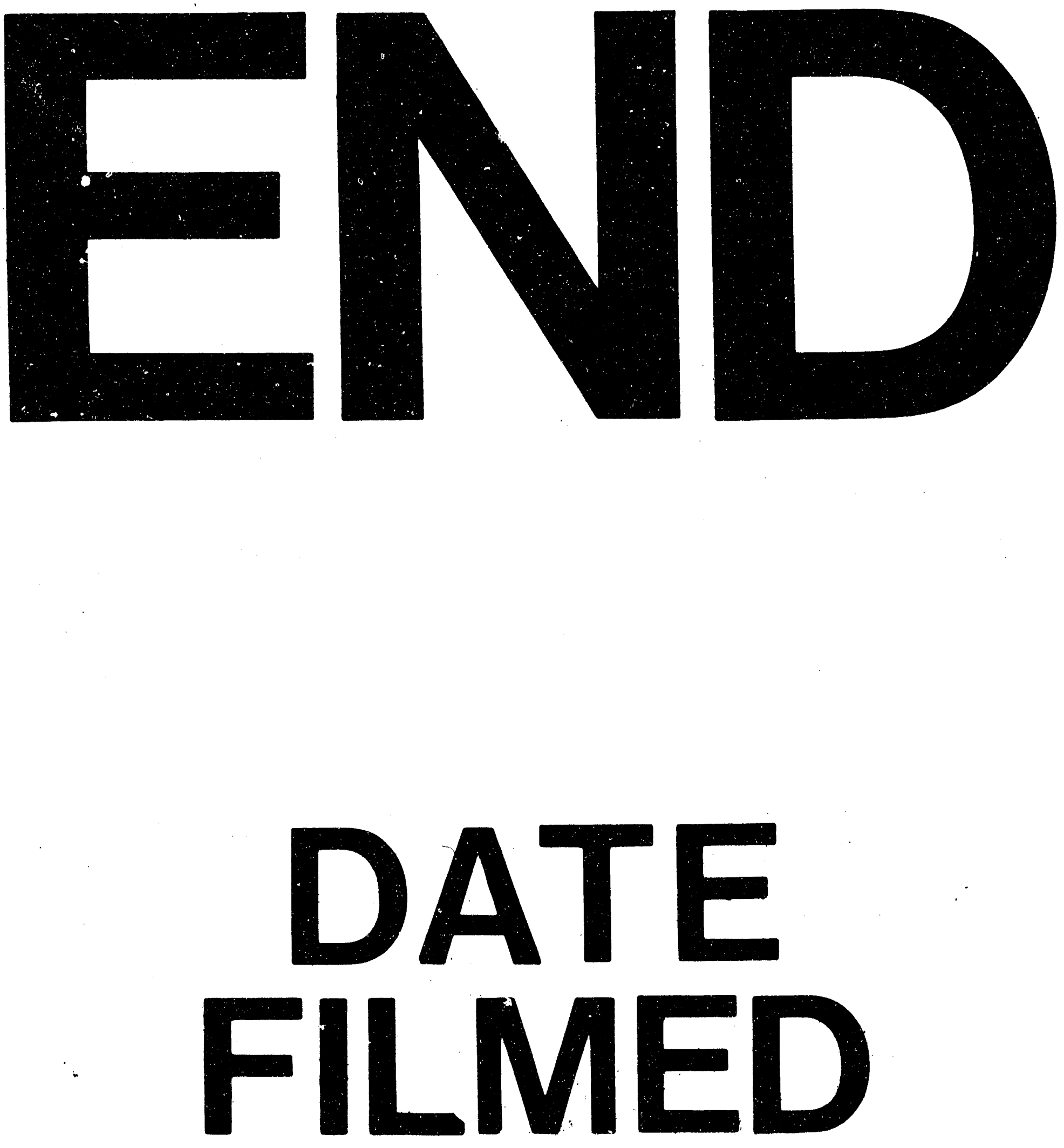

1

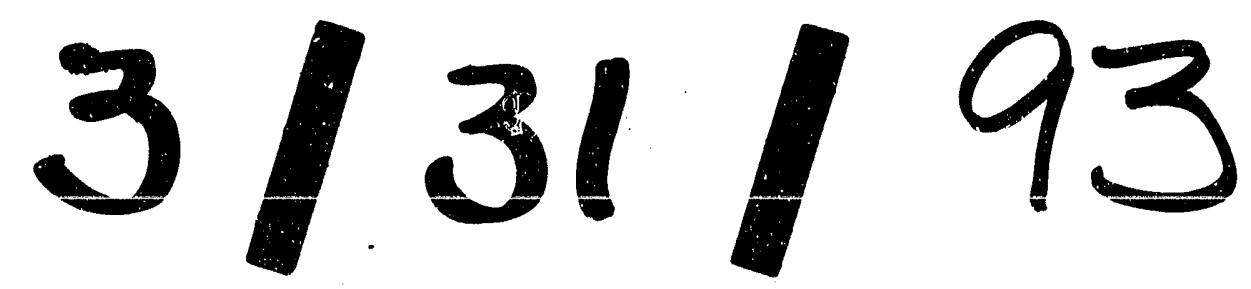


